Genderkonstruktionen und

$$
\text { »kulturelle Differenz« in }
$$

den Lebensentwürfen

binationaler Paare 
Margarete Menz

Biographische Wechselwirkungen 
Margarete Menz (Dr. phil.) ist wissenschaftliche Mitarbeiterin am Institut für Allgemeine Pädagogik an der Helmut-Schmidt-Universität in Hamburg. Ihre Forschungsschwerpunkte sind interkulturelle Geschlechterforschung, Migrationspädagogik und qualitative Forschung. 
MARGARETE Menz

\section{Biographische Wechselwirkungen}

Genderkonstruktionen und »kulturelle Differenz«

in den Lebensentwürfen binationaler Paare 
Gefördert durch die Deutsche Forschungsgemeinschaft durch ein Promotionsstipendium im Rahmen des Graduiertenkollegs »Migration im modernen Europa« der Universität Osnabrück.

Bibliografische Information der Deutschen Bibliothek

Die Deutsche Bibliothek verzeichnet diese Publikation in der Deutschen Nationalbibliografie; detaillierte bibliografische Daten sind im Internet über http://dnb.ddb.de abrufbar.

(C) 2008 transcript Verlag, Bielefeld

\section{(c) $\$($ This work is licensed under a Creative Commons BY NC ND Attribution-NonCommercial-NoDerivatives 3.0 License.}

Umschlaggestaltung: Kordula Röckenhaus, Bielefeld Lektorat \& Satz: Margarete Menz

Druck: Majuskel Medienproduktion GmbH, Wetzlar ISBN 978-3-89942-767-7

Gedruckt auf alterungsbeständigem Papier mit chlorfrei gebleichtem Zellstoff.

Besuchen Sie uns im Internet: http://www.transcript-verlag.de

Bitte fordern Sie unser Gesamtverzeichnis und andere Broschüren an unter: info@transcript-verlag.de 
INHALT

Einleitung

\section{Anblicke}

Einführung: Dokumentation der Datenerhebung und Vorstellung des Samples

Strategien zur Samplerekrutierung $\quad 19$

Verlauf der Interviewerhebung $\quad 22$

1. Phase: Erzählaufforderung und Ratifizierung 23

2. Phase: Haupterzählung 24

3. Phase: interne und externe Nachfragen 25

$\begin{array}{ll}\text { Das Kernsample: drei Paare } & 27\end{array}$

Das Forschungsfeld: binationale Paare in Deutschland 31

Erste Annäherung: zur Lebenssituation binationaler Paare in Deutschland $\quad 31$

Begriffsklärungen: inter-, multi-, bi-nationale Paare 33

Binationale Paare in Deutschland: ein statistischer Überblick 35

$\begin{array}{ll}\text { Partnerwahltheorien } & 38\end{array}$

Intermarriage als Folge demographischer Faktoren $\quad 39$

Intermarriage als Beitrag zur Statuserhöhung bzw. -stabilisierung 41

Intermarriage als Folge spezifischer Persönlichkeitsmerkmale 43

Heterogame Irritationen: binationale Paare in der deutschen Gesellschaft 46

Ver-rechtliche Subjekte $\quad 46$

Kulturalisierungen und Neuentdeckungen: Studien zu Lebenswirklichkeiten binationaler Paare $\quad 50$

Zweite Annäherung: Folgen von Migrationsprozessen 65

Zumutungen im Migrationsprozess $\quad 66$

Das Female Breadwinner Modell: Konkretisierung der Zumutungen 71

(Binationale) Beziehungen als soziale Systeme und Orte der gemeinsamen Produktion von Wirklichkeit 
Die Forschungsperspektive: Biographie, gender und natio-ethno-kulturelle Zugehörigkeit

Erste Annäherung: Biographie als Ort der Konstruktion

Erhöhte Anforderungen und Kontingenz: vom institutionalisierten

Lebenslauf zur „Biographisierung von Erleben und Handeln“ $\quad 81$

$\begin{array}{ll}\text { Biographie als ,Ort' von Subjekt und Struktur } & 87\end{array}$

Biographie als Produkt von Erzählungen 94

Zweite Annäherung: gender und natio-ethno-kulturelle

Zugehörigkeit

Gender als biographische Konstruktion

Gender ethnographisch: doing gender

Gender biographisch: doing biography

Natio-ethno-kulturelle Zugehörigkeit als

biographische Konstruktion

Wechselwirkungen: gender und natio-ethno-kulturelle

Zugehörigkeit als verflochtene Kategorien

Biographien binationaler Paare: erste Erkenntnisse und weiterführende Forschungsfragen

\section{Einblicke}

Das methodologische und methodische Design der Studie

Gegenstandsbezogene Theoriebildung: die Forschungslogik 125

$\begin{array}{lr}\text { Der abduktive Forschungsprozess } & 128\end{array}$

Die Auswertung der Interviews $\quad 130$

Erste Schritte: Transkription, Erstellung des Verlaufprotokolls, biographisches Portrait 130

Kernstellenanalyse $\quad 132$

Biographische Interviews binationaler Paare: Besonderheiten der Interpretation und der Darstellung $\quad 134$

Biographische Interviews mit Migranten $\quad 135$

Besonderheiten in der Darstellung der Interpretationsergebnisse 138

Was für ein Text entsteht? Ein methodologisches Verständnis der Interpretationen

Einführung in das Analysemodell: Formen biographischen

Lernens in sozialen Kontexten

Dimensionen biographischen Lernens $\quad 143$

Lernen und Bildung: zwei Seiten eines Zusammenhangs $\quad 143$

Lernen in Biographien 
Das heuristische Analysemodell: Formen biographischen Lernens in sozialen Kontexten

Dimension der Bearbeitung

Der Beziehungshorizont der Paare 161

Thema und Bearbeitungsfelder $\quad 165$

Schematische Darstellung des Interpretationsmodells $\quad 168$

\section{Der Fremde und die Andere:}

Susanne Frei und Rachid Frei-Heidarzadeh

Biographische Portraits Susanne Frei und Rachid Frei-Heidarzadeh 169

Susanne Frei 169

Rachid Frei-Heidarzadeh 172

Der Horizont der Paare: Beziehung als

handlungserweiterndes Moment

Konstruktion des Paarhorizontes bei Susanne: Fremdheit als Ressource

Konstruktion des Paarhorizontes bei Rachid: die Andere als Katalysator

Der Fremde und die Andere: Komplementarität 182

Thema der Beziehung: Nachhaltigkeit $\quad 183$

Bearbeitungsfelder 184

Bearbeitungsfelder bei Susanne: Innere und Äußere

(Un-)Abhängigkeit

184

Bearbeitungsfelder bei Rachid: (Re-)Positionierungen $\quad 189$

Form biographischen Lernens: natio-ethno-kulturelle

Zugehörigkeit als Handlungsfeld für Konstruktionen von

Geschlechterbeziehungen - Individualisierung und Integration

\section{Sehnsucht und Vertrautheit:}

Nicole und Carlos Ortega Garcia

Biographische Portraits Nicole und Carlos Ortega Garcia 199

Nicole Ortega Garcia $\quad 199$

Carlos Ortega Garcia 201

Der Horizont der Paare: Beziehung als integratives Moment 203

Konstruktion des Paarhorizonts bei Nicole: das Vertraute im Fremden

Konstruktion des Paarhorizonts bei Carlos:

Gelegenheitsstruktur Beziehung

Gesehnt und gewartet: Integration 210

Thema der Beziehung: Realisierung 210 
Bearbeitungsfelder bei Carlos: Desillusionierung und

$$
\text { Re-Migration }
$$

Form biographischen Lernens: natio-ethno-kulturelle Zugehörigkeit

als Handlungsfeld für Konstruktionen von

Geschlechterbeziehungen - Individualisierung als Retraditionalisierung und die Entwicklung eines Gegenhorizontes

\section{Programmatik und Lebenswirklichkeit:}

Maria Wesel und Pablo Marmani Rodriguez

Biographische Portraits Maria Wesel und Pablo Marmani Rodriguez 227

Maria Wesel

Pablo Marmani Rodriguez

Der Horizont der Paare: Beziehung als bestätigendes Moment

Konstruktion des Paarhorizonts bei Maria: interkulturelle Begegnungen

Konstruktionen des Paarhortizontes bei Pablo: Fortsetzungen

Interkulturelle Begegnungen: Öffnungen und Grenzen

Thema der Beziehung: Transmigration

Bearbeitungsfelder

Bearbeitungsfelder bei Maria: Konkretisierungen

Form biographischen Lernens: gender als Ausdruck natio-ethno-

kultureller Zugehörigkeit - Transmigration als Integrationsmodus 253

\section{Ausblicke}

Neue soziale Wirklichkeiten? Biographien binationaler Paare

Zum Verhältnis von ,Kultur' und ,Geschlecht' als Ressourcen der Biographiegestaltung 


\section{EINLEITUNG}

Binationale Paare stehen in Deutschland unter vielfältiger und durchaus paradoxer Beobachtung durch die Gesellschaft. Die einen betrachten sie argwöhnisch, sehen kulturelle und nationale Grenzen überschritten, Auflösungserscheinungen der deutschen Gesellschaft und haben Angst vor Überfremdung. Andere sehen in binationalen Paaren die Realisierung des konkreten Dialogs der Kulturen und die Vorreiter einer zukünftigen modernen, multikulturell handlungsfähigen Gesellschaft. Parallel dazu verhindern ausländerrechtliche Regelungen die unproblematische Realisierung von Lebensplänen dieser Paare. Innerhalb solcher Spannungsfelder müssen binationale Paare ihre Form der Biographiegestaltung entwickeln. Gleichzeitig aber haben sie, anders als mononationale Paare, die Möglichkeit, auf mehrere Bezugshorizonte zurückzugreifen, können stärker eigene biographische Modelle entwickeln und sich damit über nationale und kulturelle Grenzen hinaus in kreativer und mehrdeutiger Weise verorten. Als Forschungsfeld bieten binationale Paare insofern vielfache Zugangsmöglichkeiten. Umso erstaunlicher ist es, dass sie in Deutschland nach wie vor vergleichsweise selten Gegenstand wissenschaftlicher Forschung sind. Erst in den letzten Jahren ist, im Zuge des Paradigmenwechsels von der Differenz- zur Konstruktionsperspektive in der Migrationsforschung und der interkulturellen Geschlechterforschung, einige Forschung zum Beispiel zu Reisebeziehungen (Schlehe 2000a) oder über binationale Paare in der Schweiz (Waldis 1998) durchgeführt worden, die sich abgrenzt von der Form von Forschungsliteratur, wie sie in den 80er und 90er Jahren betrieben wurde. Zu dieser Zeit untersuchten häufig selbst binational verheiratete Wissenschaftlerinnen ebensolche Paare. Eine solche Konstellation gibt zum Teil eher Auskunft über die persönliche Lebenssituation der Forscherin, als dass sie Einblick in die spezifischen Lebenssituationen binationaler Paare geben (vgl. dazu auch Thode-Ahora 1999: 89). 
Diese Studie schließt eine Lücke in der Forschungslandschaft, indem sie Handlungs- und Orientierungsmuster in Biographien binationaler Paare analysiert. Mit insgesamt acht binationalen Paaren wurden jeweils einzelne biographisch-narrative Interviews geführt. Eine häufig bei binationalen Paaren vorkommende Konstellation wird zum Anlass genommen, nach Realisierungsmöglichkeiten und Modifikationsnotwendigkeiten der Biographiegestaltung zu fragen: Aufgrund der fehlenden offiziellen Anerkennung von Berufsabschlüssen, aufgrund mangelnder Sprachkenntnisse oder diskriminierender Einstellungspraxen möglicher Arbeitgeber(innen) müssen Migrant(inn)en, wenn sie nach Deutschland kommen, mit einer Phase der Arbeitslosigkeit rechnen. In dieser Zeit ist der deutsche Partner bzw. die deutsche Partnerin alleine für den Verdienst der Familie zuständig. Die Konstellation einer Beziehung zwischen einer deutschen Frau und einem nicht-deutschen Mann führt unter diesen Voraussetzungen häufig zum female breadwinner Modell. Mit diesem Modell wird der Umstand bezeichnet, dass die Frau in finanzieller Hinsicht Haupternährerin des Paares bzw. der Familie ist. Es beschreibt eine spezifische Konstellation, die im Gegensatz zu der in Deutschland und auch weltweit üblichen Arbeitsteilung steht. Im Unterschied dazu wird die traditionelle geschlechtsspezifische Arbeitsteilung auch als male breadwinner Modell bezeichnet. ${ }^{1}$

Von Interesse ist hierbei der Umgang mit solchen Herausforderungen: Was passiert in der Auseinandersetzung mit solchen strukturellen Zwängen, versuchen die Paare möglichst schnell zu einer ,normalen“ Arbeitsteilung zurückzukehren, oder nutzen die Paare die (oft ungewollte) Konstellation, um neue, vielleicht sogar emanzipative Lebensmodelle zu entwickeln? Werden ,kulturelle Differenzen“ dramatisiert oder deessentialisiert, wird die Arbeitslosigkeit des migrierenden Partners oder der migrierenden Partnerin als schwieriger erlebt, weil die berufliche Partizipation nicht möglich ist und sich so die Abhängigkeit vom deutschen Partner oder der deutschen Partnerin vergrößert? Oder erleichtert der Migrationshintergrund die Auseinandersetzung, weil externe Faktoren als Ursachen für die aktuelle Situation herangezogen werden können? Ziel der Arbeit ist daher die Konstruktion, (Re-)Konstruktion und Modifikation von gender und von natio-ethno-kultureller Zugehörigkeit. Beide Kategorien werden als biographisch gebundene Kategorien verstanden, die in der Biographiekonstruktion selbst an Bedeutung gewinnen, modifiziert oder verstärkt werden können. Strukturelle Ordnungskategorien, wie die beiden sie darstellen, werden in einer jeweils sub-

1 Frauen werden komplementär dazu häufig als cakewinner bezeichnet, als diejenigen, die das Geld für die kleinen Extras und den nicht unbedingt notwendigen Luxus, den Kuchen also, verdienen. 
jektspezifischen Art und Weise verarbeitet. Sie sind für die soziale Positionierung eines Subjekts relevant und daher auch für die Biographiekonstruktion bedeutsam. Die Biographiekonstruktion produziert keine Abbilder von gesellschaftlichen Verhältnissen, sondern eigenwillige Formen, die wiederum Rückwirkungen auf gesellschaftliche Verhältnisse haben. Um solche Entwicklungs- und Veränderungsprozesse von Positionierungen nachzeichnen und benennen zu können, wird in der vorliegenden Arbeit mit der Perspektive der Formen biographischen Lernens gearbeitet. Lernen als biographisch verfasstes Lernen zu verstehen, ermöglicht den empirischen Nachvollzug von Lernprozessen über die Lebenszeit hinweg. Die Form biographischen Lernens bezeichnet das jeweilige, sich im Verlauf der Bearbeitungsprozesse entwickelnde Verhältnis zwischen Geschlechterbeziehungen und der Bedeutung, die ,kulturelle Differenz' zugesprochen bekommt. Durch die Analyse solcher Formen kann etwas ausgesagt werden über den Umgang von Subjekten mit gesellschaftlichen Herausforderungen. ${ }^{2}$

Die Studie ist in drei große Blöcke unterteilt: In den Anblicken werden die theoretischen und empirischen Zugänge zu dem Forschungsfeld, binationale Paare in Deutschland, vorgestellt. Nach der Dokumentation der Entwicklung der Forschungsidee und der Erhebung der Interviews stehen im Abschnitt über das Forschungsfeld Untersuchungen zu binationalen Paaren im Mittelpunkt. Die Entscheidung für die Bezeichnung binationale Paare (in Unterscheidung zu interkulturellen oder multikulturellen Paaren) wird ebenso begründet wie vorliegende Forschung zu binationalen Paaren dokumentiert und in Hinblick auf mein eigenes Forschungsinteresse hin analysiert. Während insbesondere in den USA Forschung zur ,Intermarriage“ vorrangig quantitativ ausgerichtet ist und mit Bezug darauf Partnerwahltheorien entwickelt wurde, wird in Deutschland vorrangig qualitativ geforscht. Die Forschung der 1980er Jahren geht von einem vorgängigen Kulturproblem aus, welches sich in der Paarbeziehung widerspiegele: Binationale Beziehungen werden als Begegnung der Kulturen im Kleinen gesehen, die Partner(innen) in gewisser Weise nur als verlängerter Arm und Ausdruck ihrer jeweiligen Herkunftskultur. Aus der neueren Forschung, die solche Kulturkonstruktionen nicht unhinterfragt voraussetzt, sondern vielmehr in ihren Wir-

2 Mit dem Begriff der Geschlechterbeziehungen lehne ich mich an den Knapp an, die Begriffsdifferenzierungen entlang der Mikro-, Meso- und Makroebene vornimmt. Analysen von Geschlechterbeziehungen beziehen sich demnach ,auf Charakteristika der Relation zwischen Frauen und Männern. [...] Analysiert werden in diesem Horizont interpersonale Bezüge und deren Spezifika als Beziehung“ (Knapp 2001: 22). Geschlechterordnung meint die Dimension der ,normativen Konfigurationen, ritualisierten Interaktionen und kulturellen Konventionen“ (vgl. ebd.). 
kungs- und Konstruktionsweisen erst untersucht, ließen sich für meine eigene Arbeit wertvolle Anregungen und Überlegungen entwickeln. So wird zum Beispiel deutlich, dass die Entwicklung von Paarbeziehungen mit komplexen Prozessen einhergeht, die durch das imaginierte ,ganz Andere' (das romantisierte Stereotyp des fremden Anderen) wie durch das imaginierte Gemeinsame (die scheinbaren kulturelle Differenzen stellen tatsächlich Gemeinsamkeiten dar) strukturiert sind. Ebenfalls wird deutlich, wie ,kulturelle Differenz' in ihrer Bedeutung, je nach Paar und je nach Lebenslage, in den Vordergrund geschoben oder relativiert werden kann. An diesen allgemeinen Überblick über spezifische Lebenslagen und Anforderungen an die Paare und die derzeitige Forschungslage schließt sich die Konkretisierung der Zumutungen im Migrationsprozess für , meine' Paare an. Inwiefern das female breadwinner Modell in der deutschen Gesellschaft gegen die klassische geschlechtsspezifische Arbeitsteilung verstößt und was das für die Paare bedeuten kann, ist hier Thema. Das Kapitel endet mit einer theoretischen Perspektive auf Paarbeziehungen als Ort der gemeinsamen Wirklichkeitsproduktion. Darauffolgend wird die biographietheoretische Forschungsperspektive vorgestellt: Die Gestaltung der eigenen Biographie und die Übernahme der Verantwortung für die eigene Biographie, für das Scheitern und für Erfolge, lassen sich als Merkmale moderner Gesellschaften bestimmten. Binationale Paare stehen in besonderer Weise vor der Anforderung, eigene Lebensmodelle zu entwickeln, sie können sich weniger leicht auf ihnen bekannte Handlungs- und Orientierungsmuster zurückziehen. Für sie gilt also in besonderer Weise, dass sie den Modernisierungsanforderungen, der Verantwortung für die eigene Biographiegestaltung, ausgesetzt sind und in den Spannungsfeldern von Herausforderung und Möglichkeit der Biographiegestaltung ihren Weg finden müssen. Dies ermöglicht es in besonderer Weise, subjektive Gestaltungsprozesse zu rekonstruieren. Diesen ersten Block schließt ein vorläufiges Fazit $\mathrm{ab}$, innerhalb dessen die ersten Erkenntnisse zusammengefasst und vorläufige Forschungsfragen formuliert werden.

Der zweite große Block ist mit Einblicke überschrieben. Hier hat die eigentliche empirische Studie ihren Platz. Die Einführung in die methodologische Anlage der Studie enthält zum einen die methodologische Verortung in der qualitativen Sozialforschung. In dieser qualitativen Studie, die den methodologischen Prinzipien der Entwicklung einer Grounded Theory folgt, werden Theorien nicht in feststehende Hypothesen heruntergebrochen, sondern als Aufmerksamkeitsrichtungen, als sensibilisierende Konzepte, verwandt: Sie lenken, aber bestimmen nicht den Blick auf die Interviews. Ein solcher fortdauernder Kommunikationsprozess macht auf die Notwendigkeit zur Reflexivität aufmerksam, 
zum beständigen kritischen Beobachten der eigenen Interpretationen. ${ }^{3}$ Zum anderen werden die Besonderheiten in der Auswertung der Interviews dargestellt und kritisch diskutiert. So waren die Interviews mit den Migranten häufig eher problemzentrierte Interviews als biographisch-narrative Interviews, auch wurde ein Interview auf Spanisch geführt und dann ins Deutsche übersetzt. Diese Erhebungsmodalitäten führten insofern zu Modifikationen in der Auswertung, als dass die Interviews stärker thematisch ausgewertet wurden. Anschließend wird das Analysemodell entwickelt. Dieses folgt der abduktiven Forschungslogik der Grounded Theory und ist daher ebenso Ergebnis der bisherigen theoretischen Überlegungen, wie es Phänomene und Konstruktionslogiken aus den ersten Interpretationen der Interviews aufnimmt. Es fokussiert zwei Dimensionen. Im gemeinsam konstruierten Paarhorizont entwickelt sich eine spezifische Bedeutung der Beziehung für den bzw. die Einzelne(n). Die Beziehung lässt sich als Möglichkeit hinsichtlich einer biographischen Anschlussfähigkeit verstehen: Gerade die Binationalität der Beziehung eröffnet Möglichkeiten, die in einer mononationalen Beziehung nicht gesehen wurden. Aus diesem Paarhorizont ergibt sich die zweite Dimension: das jeweilige Thema der Beziehung und notwendig werdende Bearbeitungsfelder. Aus dem Verständnis und der Bedeutung der Beziehung heraus lässt sich jeweils ein Thema der Beziehung benennen, welches als ,Leitmotiv“ über den Bearbeitungsfeldern steht. Dieses kann bei einem Paar die Realisierung eines gewünschten Geschlechtsmodells sein, bei einem anderen dreht es sich um das Konzept der Transmigration. Analog dazu entstehen jeweils unterschiedliche Bearbeitungsnotwendigkeiten. So ist das female breadwinner Modell nicht zwangsläufig als ,Bruch“ in der Lebensplanung zu verstehen, es kann auch gewollter oder zumindest antizipierter Teil der Biographiegestaltung sein. Die Konzeptualisierung der Formen biographischen Lernens, die Form des sich über den Verlauf der Biographien hinweg entwickelnden Verhältnisses zwischen gender und natio-ethno-kultureller Zugehörigkeit, schließt das Interpretationsmodell ab. Den Mittelpunkt dieses zweiten Abschnitts stellen die Interpretationen der Paare dar. Drei der interviewten Paare wurden intensiv analysiert und werden hier vorgestellt. Die verschiedenen Formen der Bearbeitung zeigen wie binationale Beziehungen als Möglichkeit gesehen werden, eigene und individuelle

3 Als Korrektiv und zur Erweiterung der eigenen Zugangsweisen und Analyseperspektiven zu den Interviews ist die Mitarbeit in Forschungswerkstätten hilfreich. Ich konnte so insbesondere durch das von mir mitbegründete Doktorandinnennetzwerk qualitative Sozialforschung (DINQS) und durch die Forschungswerkstatt der Arbeitsgruppe Biographie- und Lebensweltforschung (ABL) an der Uni Göttingen profitieren. 
Lebensmodelle realisieren zu können, wie aber auch strukturelle Zwänge Korrekturen und Modifikationen erforderlich machen. Drei verschiedene Formen biographischen Lernens können rekonstruiert werden: Beim ersten Paar, Susanne Frei und Rachid Frei Heidarzadeh, ${ }^{4}$ stellt die natio-ethno-kulturelle Zugehörigkeit des und der anderen das Handlungsfeld für eine Konstruktion von Geschlechterbeziehungen dar, die vorrangig auf Individualisierung ausgerichtet ist. Individualisierung ist hier im Sinne von Distanzierung von traditionellen Geschlechterbeziehungen gemeint. In dem Versuch, ein eigenes Modell zu entwickeln, wird die natio-ethno-kulturelle Zugehörigkeit des/der jeweils anderen als Ermöglichungsstruktur genutzt. Ähnlich verhält es sich beim zweiten Paar, Nicole und Carlos Ortega Garcia. Die Gestaltung bzw. der Wunsch nach Individualisierung ist jedoch auf eine Rückkehr zu bzw. Verstärkung von traditionellen Geschlechterbeziehungen gerichtet. Das dritte Paar hingegen, Maria Wesel und Pablo Marmani Rodriguez, bringt von vornherein in seine Beziehung eine transnationale Perspektive unter interkulturellen Vorzeichen ein. Diese Perspektive ermöglicht es, natioethno-kulturelle Zugehörigkeit als Ausdruck kultureller Sozialisation zu externalisieren (interkulturelle Vorzeichen) und gleichzeitig ein Leben in beiden kulturellen Räumen anzustreben (transnationale Perspektive).

Der letzte große Abschnitt enthält den Ausblick der Arbeit. Zunächst werden die zu Beginn der Arbeit gestellten Fragen wieder aufgegriffen und mit Blick auf die empirischen Ergebnisse diskutiert: Sind binationale Paare wirklich ,Prototypen der Moderne“? Inwiefern lassen sich hier neue soziale Wirklichkeiten beobachten, und was lässt sich über die Bedeutung natio-ethno-kultureller Zugehörigkeit für die Aushandlungsund Bearbeitungsprozesse der Paare sagen? Die Ergebnisse der Interpretationen machen deutlich, dass die Beziehung den Paaren Möglichkeiten der Biographiegestaltung bietet, die sie in einer mononationalen Beziehung nicht sahen. Infolgedessen ist auch die Bedeutung, die das female breadwinner Modell in der Beziehung zugesprochen bekommt, wesentlich von dem konstruierten Paarhorizont und den impliziten und expliziten Erwartungen an die Beziehung abhängig. Natio-ethno-kulturelle $\mathrm{Zu}$ gehörigkeit wird flexibel und kreativ ,instrumentalisiert“ (Waldis 2003) und kann so als Ermöglichungsstruktur verstanden werden. Es wird aber auch deutlich, dass die erweiterten Bezugsmöglichkeiten zum Rückzug führen können, wenn konkrete Schwierigkeiten, wie sie die Folgen des female breadwinner Modell darstellen können, als biographisch nicht anschlussfähig erscheinen. Die von mir interviewten binationalen Paare können insofern die Anforderung der biographischen Selbstgestaltung

4 Die Namen der Personen sind anonymisiert. 
für ihre Entwürfe nutzen, die Hoffnung, binationale Paare seien sozusagen Vorreiter einer demokratischen Gesellschaft, ist damit aber nicht zwangsläufig erfüllt.

Dissertationen, qualitative Arbeiten erst recht, sind in gewisser Weise Gemeinschaftsprodukte. Ebenso wie die Interpretation von Daten konstitutiv auf Forschungswerkstätten und den Austausch mit Kolleg(inn)en angewiesen ist, bleibt die Forscherin im gesamten Forschungsprozess und während der Arbeit des Nachdenkens, Verwerfens, vorsichtigen Reformulierens und freudigen Entdeckens von Kategorien und Zusammenhängen aus den empirischen Analysen auf andere angewiesen. Einige seien hier stellvertretend genannt.

Grundlage der Arbeit sind Interviews mit binationalen Paaren. Ohne die Bereitschaft der Männer und Frauen, ihre Lebensgeschichte mit mir zu teilen und mir Einblick in ihr Leben, ihre Wünsche und ihre Erwartungen zu geben, hätte es diese Arbeit nicht gegeben. Dafür danke ich ihnen. Mit dem DINQS, dem Doktorandinnen Netzwerk Qualitativer Sozialforschung, ist eine Zusammenarbeit entstanden, die die gesamte Phase der Promotion mit allen dazu gehörigen beruflichen und privaten Entwicklungen intensiv begleitet hat und in den entscheidenden Momente der Arbeit motivierend und korrigierend gewirkt hat. Grundlegenden Anteil an der Arbeit haben also: Sandra Glammeier, Manuela KaiserBelz, Anja Nordmann, Vera Reinartz, Daniela Rothe, Ruth Slomski, Christine Thon, Inga Truschkat. Die Dissertation wurde von der Deutschen Forschungsgemeinschaft (DFG) durch ein Promotionsstipendium im Graduiertenkolleg des IMIS, dem Institut für Migrationsforschung und Interkulturelle Studien in Osnabrück, gefördert. Die Förderung hat es mir ermöglicht mich ohne finanzielle und zeitliche Sorgen auf die Studie zu konzentrieren. Dem Graduiertenkolleg und meinen Mitgraduierten danke ich für den produktiven Kontext, in dessen Rahmen ich meine Ideen entwickeln und meine Argumente schärfen konnte. Mein Forschungsaufenthalt bei Prof. Dr. Madeline Kalbach an der University of Calgary ermöglichte mir den Zugang zur englischsprachigen Literatur zum Forschungsfeld Intermarriage. Durch die Gastfreundschaft und die spannenden Begegnungen hat der Aufenthalt weit über den akademischen Ertrag hinaus für bleibenden Eindruck gesorgt. Prof'in Eva Breitenbach und HD Paul Mecheril, meinen beiden Betreuer(inne)n, danke ich für das Vertrauen und die Unterstützung bei der Entwicklung meines eigenen Weges und für die Offenheit, mit der sie meine Vorstellungen begleitet haben: keineswegs eine Selbstverständlichkeit. Interkultur, dem Theorie- und Forschungskreis an der Universität Bielefeld, danke ich für lebhafte Diskussionen, kritische Fragen und Aufmunterungen zur richti- 
gen Zeit. Zwei Hamburger(innen) habe ich besonders zu danken: Prof. Lutz-Rainer Reuter für die Selbstverständlichkeit, mit der er mir Zeit für die Fertigstellung der Arbeit eingeräumt hat und Alke-Marit Fingerhut für die Übernahme der unerfreulichen Arbeit der Rechtschreibkorrektur und für ihre stilsicheren Anmerkungen. Ohne all diese Menschen wäre die Durchführung der Studie in der vorliegenden Form nicht möglich gewesen, Péter Szász aber hat mich nun schon durch die zweite Qualifikationsarbeit begleitet, Texte gelesen, Gedanken geordnet und Ver(w)irrungen aufgefangen, ohne sich allzu sehr durch die stattfindenden Metamorphosen in den verschiedenen Prozessphasen abschrecken zu lassen. Ohne ihn hätte ich wohl manches Mal die Verbindung zu den realen Seiten des Lebens verloren. Und Paul Szász Menz steuert zu diesen realen Seiten nicht nur völlig neue Welteinsichten bei, er hat glücklicherweise auch schon sehr früh begriffen, dass nur ausgeschlafene Eltern entspannte Eltern sind. 
Anblicke 



\section{EINFÜHRUNG: DOKUMENTATION DER DATENERHEBUNG UND VORSTELLUNG}

\section{DES SAMPLES}

In den Anblicken wird durch die Einführung in das Forschungsfeld und die Forschungsperspektiven der theoretische und konzeptuelle Rahmen entwickelt, innerhalb dessen die Interviewanalysen durchgeführt wurden.

\section{Strategien zur Samplerekrutierung}

Das Forschungsvorhaben baute auf dem Vorhaben auf, auf jeden Fall beide Partner(innen) zu interviewen. Ursprüngliche Absicht war es zudem, beide Konstellationen zu untersuchen: sowohl deutsche Frauen, die mit Migranten verheiratet sind, als auch deutsche Männer, die mit Migrantinnen verheiratet sind. Auch Migrantinnen haben Schwierigkeiten bei der Eingliederung in den Arbeitsmarkt, und auch hier lassen sich, so die Vermutung, interessante Formen der Bearbeitung wieder finden. Letztendlich jedoch haben sich ausschließlich Paare gemeldet, bei denen die Konstellation des female breadwinner Modells, der weiblichen Haupterwerbstätigkeit, bestand. Bei der Suche nach den Interviewpartner(inne)n habe ich drei Hauptquellen genutzt. Zum einen konnte ich in den vierteljährlich erscheinenden IAF-Informationen eine Kurzbeschreibung meines Projektes zu veröffentlichen. Neben dieser schriftlichen Veröffentlichung nutzte ich einige Internetseiten für die Verbreitung von Informationen und Anfragen. Der Verband binationaler Paare und Familie hatte in seinem Internetauftritt für einige Zeit ein Forum eingerichtet. Daneben gibt es eine Homepage für binationale Paare in Deutschland (www.binational-in.de), welche ein großes und gut besuchtes Forum für 
Austausch und Ratsuchende bietet. Ebenfalls nutzte ich zum Beispiel einen Treffpunkt für deutsch-afrikanische Partnerschaften (afrikanisch.multikulti-in.de/). Als dritter erfolgreicher Weg erwies sich Mundzu-Mund-Propaganda. Binationale Paare verwiesen mich an Freunde weiter, die eventuell Interesse hätten und die für mich ebenfalls interessant sein könnten, und auch der Freundes- und Kolleg(inn)enkreis half bei der Suche.

Schwierigkeiten gab es weniger hinsichtlich der Verbreitung meines Interesses und der Kontaktaufnahme $\mathrm{zu}$ potentiellen Interessenten. Schwieriger war es vielmehr, tatsächlich Paare zu finden, die sich zu einem (biographischen) Interview bereit erklärten. So haben sich auf meine Beiträge hin vergleichsweise viele Interessent(inn)en gemeldet, daraus ergaben sich jedoch nicht unbedingt Interviews. $\mathrm{Zu}$ einem großen Teil lag das an divergierenden Interessenslagen. Überwiegend meldeten sich die (deutschen) Frauen bei mir. Viele waren eher auf der Suche nach einer Beratung für ihre aktuellen Probleme, als dass sie ein Interview durchführen wollten. Andere wiederum waren an einem generellen Erfahrungsaustausch interessiert. Waren die Frauen mit einem Interview einverstanden, scheiterte die Durchführung oftmals noch an der Einwilligung des Mannes. ${ }^{1}$ Über deren Gründe lässt sich aus erster Hand nichts sagen. Mir wurden die Absagen von den Frauen mitgeteilt, von denen häufig mangelndes Interesse seitens des Mannes oder aber auch Scham über die derzeitige Situation genannt wurde. Ein weiteres Hindernis stellten die biographischen Interviews dar, die in Länge und Art der Durchführung deutlich von dem abweichen, was normalerweise unter Interviews verstanden wird. Die Hemmschwelle, in aller Ausführlichkeit Auskunft über sein Leben zu geben, war vergleichsweise groß, zumal die Partner(innen) einzeln befragt wurden. Eine letzte Schwierigkeit stellten zudem meine Sprachkenntnissen dar. Da ich nur Englisch und Deutsch gut genug spreche, um Interviews durchzuführen, ließen sich Interviews mit Migranten anderssprachiger Länder nicht durchführen. Eine Ausnahme bildet hier das Spanische. Da ich gute Kontakte zu spanisch sprechenden Kolleginnen hatte, die ebenfalls über eine Interviewausbildung verfügen, ließ sich das Angebot machen, Interviews auf Spanisch durchzuführen. Eines der Interviews aus dem Kernsample ist so zustande gekommen: Es wurde auf Spanisch geführt und später von einer spanischen Muttersprachlerin übersetzt. ${ }^{2}$ Letztendlich gab es etwa

1 Ein Interview führte ich daher auch nur mit der Frau, ein anderes Mal willigte eine geschiedene Frau erst ein, zog dann aber später ihre Einwilligung zurück.

$2 \mathrm{Zu}$ den Modifikation, die ein solches Vorgehen bei der Auswertung mit sich brachte, s. das methodische Vorgehen. 
dreimal so viele Anfragen, wie Interviews zu Stande gekommen sind. Diese Erfahrung führte zu der Entscheidung, keinerlei Einschränkungen hinsichtlich der Herkunftsregionen der Männer, der Aufenthaltsdauer in Deutschland oder anderer Merkmale vorzunehmen. Eine solche Auswahl vorab zu treffen, erschien zum einen aufgrund der geringen Zahl der Interviews nicht sinnvoll. Zum anderen aber gilt es bei solchen Entscheidungen auch aus theoretischer Sicht, vorsichtig zu sein. Relevante Auswahl- und Unterscheidungskriterien entwickeln sich im Prozess der Interpretation, und eine Festlegung auf so etwas wie Abhängigkeit zwischen Kulturen und Verhaltensweisen sollte nicht erfolgen: Für mein Forschungsinteresse schien das konkrete Herkunftsland des Mannes nicht eines der entscheidenden Merkmale zu sein.

Für die Vorbereitung der Interviews wurde ein Kurzfragebogen entwickelt, der neben Fragen zu sozialstatistischen Daten auch die Bitte enthielt, den bisherigen Lebenslauf kurz zu beschreiben. Dieser wurde neben einem ausführlichen Begleitschreiben, in welchem mein Promotionsthema und der Interviewverlauf erläutert wurden, jedem interessierten Paar zugeschickt und sollte vor dem Interviewtermin zurückgeschickt werden. Ein solches Vorgehen bietet den Vorteil des ersten Überblickes in biographische Rahmendaten vor den Interviews und ermöglicht die Konzentration auf Narrationen im Interview. Auch können so interne und externe Nachfragen besser auf die konkrete Biographie abgestimmt werden. Die Ausführlichkeit, mit der der Fragebogen ausgefüllt wurde, war sehr unterschiedlich. Zum Teil wurden ausführliche Lebensläufe mit der Hervorhebung zentraler Lebensereignisse und -erfahrungen formuliert, deren Umfang häufig mehrere Seiten umfasste. Zum Teil erhielt ich aber auch chronologische Kurzlebensläufe. Als nächster Vorbereitungsschritt und wenn das Einverständnis zu den Interviews vorlag, wurden längere Telefongespräche geführt, um das Vorhaben genau zu erläutern und eventuelle Fragen möglichst schon vorab klären zu können. Ebenfalls geklärt wurde die Anonymität der Auswertung. ${ }^{3}$ Insgesamt konnten so Interviews mit neun Paaren geführt werden. $\mathrm{Zu} \mathrm{Be}$ ginn wurde zudem ein einzelnes Interview einer mit einem Tunesier verheirateten Frau geführt. Die Interviews fanden in ganz Deutschland statt und wurden hauptsächlich 2003 geführt.

3 Hinsichtlich der Anonymität wurde den Paaren frei gestellt, ob das Herkunftsland des Mannes ebenfalls anonymisiert werden sollte oder nicht. Keines der Paare hatte etwas gegen die Veröffentlichung des richtigen Ländernamens. 


\section{Verlauf der Interviewerhebung}

Die Interviews wurden alle in den Wohnungen der Paare geführt bzw. bei einem nicht zusammenlebenden Paar in der jeweiligen Wohnung der Person. Zudem wurde ein Paar jeweils an einem Tag interviewt, so dass wir in der Regel den ganzen Tag zusammen verbrachten. Diese zeitliche Einteilung hatte den Vorteil, dass so Vertrauen aufgebaut und Skepsis hinsichtlich des biographischen Interviews abgebaut werden konnte. Diese erneute Vorbereitung auf das biographische Interview war auch deswegen wichtig, da der Kontakt und die Vorgespräche, wie schon beschrieben, häufig nur über die Frauen liefen (auch wenn die z.B. E-Mails immer an beide adressiert wurden), und die Männer so auf jeden Fall persönlich auf das Gespräch vorbereitet werden konnten. Zum anderen konnte ich durch die vergleichsweise langen Aufenthalte Einblicke in das Alltagsleben der Paare gewinnen, häufig haben wir danach noch zusammengesessen und geredet. Die Reihenfolge, in der die Interviews durchgeführt wurden, war den Paaren freigestellt.

Die Interviews selbst waren als biographisch-narrative Interviews geplant. Das biographisch-narrative Interview, als eine besondere Form des narrativen Interviews, ist die am wenigsten strukturierte Form eines Interviews. Es dient dazu, die bzw. den Interviewte(n) zur ausführlichen Erzählung einer Lebensphase bzw. der eigenen Lebensgeschichte zu motivieren (vgl. Fischer-Rosenthal/Rosenthal 1997: 414; zuerst Schütze 1977), und eignet sich so in besonderer Weise zur „Analyse sozialer Phänomene in ihrer Prozesshaftigkeit“" (Jacob 1997: 446).

„Das narrative Interview ist ein sozialwissenschaftliches Erhebungsverfahren, welches den Informanten zu einer umfassenden und detaillierten Stegreiferzählung persönlicher Ereignisverwicklungen und entsprechender Erlebnisse im vorgegebenen Themenbereich veranlasst“ (Schütze 1987 in Marotzki 1995: $62)$.

Das so erhobene Datenmaterial erlaubt es, ,individuumsnahe Erfahrungsräume zu explorieren in dem Sinne der Analyse der Ordnung sozialer Wirklichkeit, wie sie von den Subjekten in unhintergehbar ablaufenden Interpretationsprozessen hergestellt wird" (Marotzki 2003: 23, Herv. d. Verf.). Biographisch-narrative Interviews gliedern sich in drei Phasen: die Erzählaufforderung und die Ratifizierung der Erzählaufforderung durch die oder den Interviewte(n), die Haupterzählung und die Phase der Nachfragen, die zuerst erzählgenerierend und textimmanent sein (interne Nachfragen) zum Abschluss aber auch Fragen im Hinblick auf Bereiche enthalten sollten, die für das Thema interessant sind aber 
bislang nicht angesprochen wurden (externe Nachfragen). Ebenfalls kann in dieser Phase eine Gesamtevaluation des Lebens durch die/den Biographieträger(in) stattfinden. Die Interviews dauerten zwischen eineinhalb und drei Stunden. Alle wurden auf Band aufgenommen. Zusätzlich wurden nach dem Besuch bei dem Paar meine ersten Eindrücke und Erfahrungen protokolliert.

\section{Phase: Erzählaufforderung und Ratifizierung}

Die Erzählaufforderung für das Interview war bewusst sehr allgemein und offen gehalten. Wichtig war in diesem Kontext, ebenso wie bei der Vorbereitung der Interviews, dass der Impuls, der für die Erzählung gegeben wird, sich nicht auf die Konstellation des female breadwinner Modells richtet, sondern allgemein auf die Lebensgeschichte der Person. Die Erzählaufforderung wurde daher wie folgt gegeben:

„Ich möchte Sie bitten, mir Ihre Lebensgeschichte zu erzählen, all die Ereignisse und Geschichten, die Ihnen einfallen. Am besten fangen Sie mit Ihrer Kindheit an, mit dem, woran Sie sich als Erstes erinnern.“

An die Erzählaufforderung soll sich die narrative Ratifizierung der Erzählaufforderung anschließen. Diese kann in unterschiedlicher Art und Weise vorgenommen werden, wie die beiden Interviewausschnitte zeigen:

Christa: (hustet) --- „Also wir waren zu Hause vier Kinder, --- ich war das erste, ---- unnnd, äähm ---- meine Mutter waaar ---- seehr streng --- der General, -- so heißt sie ((beide lachen)), -- der General, -- uund äähm --- mein Vater war so der klassische Pantoffelheld, --- äähm, -- hatte allerdings -- deen Vorteil, dass man -- bei ihm immer, -- da ging's immer locker zu -- und es war immer lustig, ja, während bei meiner Mutter alles immer ganz schlimm war ((gespielt betroffen intoniert)). --- Jaa, in meiner Kindheit erinner' ich mich als Allererstes daran, dass sie mir so ständig einen Riesenstress wegen der Schule gemacht haben, -- ja, -- $\underline{\text { der }}$ brutale Leistungsdruck, -- also es gab zwei Sachen: 'ne Eins in der Schule und am Sonntag in die Kirche gehen! --- Ja? Alsoo ---- erst später, so mit elf, -- ääh, --- isses dann richtig furch'bar geworden, -- weil dann ham wir also zu saudi-arabischen Familienverhältnissen gewechselt. -- Also meinee -- Eltern sind beide so, -- so --- oberkatholisch“ (Christa Müller 1/13-22). 
Carlos: „Ganz von vorne, ok. Ich bin in - - ähm erstmal entschuldigen für mein Deutsch, so schlecht aber

Interviewerin: „Ach so, und wenn du_wenn dir irgendwas in Englisch leichter fällt, dann kannst du auch gerne - wechseln.“

Carlos: „Ja. Ok, alles klar. Ok, ich bin in - 1973 geboren, in A-Stadt, aber - dann hab ich - meine Eltern sie haben, also mein Vater hat versucht eine bessere Lebensqualität uns zu geben, und dann wir sind nach B-Stadt umgezogen, B-Stadt ist ein nordwestlich Stadt in A-Stadt, äh von A-Stadt, ja - äh und ähm - ja, das ist am Meer, Strand, Palmen, alles. Sehr schön. - - äh und ja, dann bin ähm, dann hab ich studiert, ähm, mein Grundsch_äh hab meine Grundschule beendet - und - Junior High School, das gibt es nicht in Deutschland“" (schmunzelnd, Carlos Ortega Garcia 1/12-21).

Im ersten Interview steigt die Interviewte nicht, wie vielleicht zu erwarten wäre, mit Informationen zu sozialen Daten ein, dem Ort der Kindheit, ihr Alter und das ihrer Geschwister, sondern schildert vielmehr sofort und sehr anschaulich Erinnerungen aus ihrer Kindheit und das Verhältnis zu ihren Eltern. Ihre Erzählung folgt dabei nicht unbedingt einer Chronologie, sondern dient als Belegerzählung für das Klima, das zu Hause herrschte. Ganz anders das zweite Interview. Hier fängt die Haupterzählung mit einer kurzen Interaktion an. Carlos entschuldigt sich für sein schlechtes Deutsch, und die Interviewerin erweitert ihre Erzählaufforderung. Offensichtlich hat sie vorher vergessen, ihn von der Möglichkeit in Kenntnis zu setzten, das Interview auch auf Englisch zu führen. Diese neue Information sichert er ab und steigt dann in die Haupterzählung mit seinen sozialen Daten (Geburtsjahr und -ort) ein. Der Unterschied im Erzählstil ist zu diesem Zeitpunkt deutlich: Während Heidi die Struktur ihrer Erzählung an Erinnerungen und Episoden festmacht, orientiert sich Carlos an eher äußeren Daten.

\section{Phase: Haupterzählung}

Die zweite Phase besteht aus der Haupterzählung der oder des Interviewten. Die interviewte Person bestimmt dabei selber die temporalen und inhaltlichen Kriterien der Lebensgeschichte, sie bestimmt die jeweiligen Relevanzstrukturen. Die Länge einer solchen Haupterzählung ist sehr unterschiedlich, im meinem Sample hat sie zwischen 15 Minuten und eineinhalb Stunden gedauert. In dieser Phase soll die Erzählung nicht durch Interventionen gesteuert oder in einer anderen Weise eingegriffen werden. Vielmehr soll nur durch unterstützende Eingriffe der Erzählfluss aufrechterhalten werden („,Und wie ging's dann weiter, an was können sie sich noch erinnern?"), wenn die/der Interviewte ins Stocken gerät. Mit dem Beginn des Erzählflusses, gekennzeichnet durch die Rati- 
fizierung der Erzählaufforderung, wird eine Dynamik wirksam, „die quasi automatisch gewissen Strukturprinzipien Rechnung trägt“ (Diekmann 1998: 449). Erzählungen sind durch Schemata gekennzeichnet, die Kallmeyer/Schütze 1977 als „Zugzwänge des Erzählens“ beschrieben haben.

„Die Zugzwänge sorgen dafür, dass der Interviewpartner um eine für seinen Zuhörer verstehbare und plausible Erzählung bemüht ist. Zugleich setzen sie eine Eigendynamik des Erzählvorgangs in Gang, in dessen Folge sich der Erzähler mehr und mehr in die früheren Ereignis- und Erfahrungsabläufe verstrickt und eine autobiographische Darstellung hervorbringt“ (Jacob 1997: 449). ${ }^{4}$

Schütze unterscheidet zwischen dem Gestaltschließungszwang (eine einmal angefangene Geschichte muss zu Ende erzählt werden und kann nicht mittendrin abgebrochen werden), dem Detaillierungszwang (eine Geschichte muss, um plausibel zu sein, genügend Einzelheiten und Verständnisinformationen enthalten), und dem Relevanz- und Festlegungszwang (er sorgt in einer Erzählung für eine überschaubare Darstellung und Konzentration auf das Wesentliche; vgl. z.B. Schütze 1984). Die Haupterzählung endet häufig mit einer Koda, einem bilanzierenden Abschluss:

Helena: - - ,und irgendwie instinktiv hab ich ja das Gleiche - gefühlt. Irgendwann dann (auflachend). Und ihn nimmt er an und äh - er sieht natürlich auch, dass es harmonisch läuft mit mit meinem Sohn und das ist scheinbar auch so nen Punkt. Also es ist äh - - ich hab das Gefühl angekommen zu sein“ (Helena Aristides, 40/15-17).

Helena beendet ihre Haupterzählung im Hier und Jetzt. Mit dem Gefühl, angekommen zu sein, charakterisiert sie nicht nur ihren derzeitigen $\mathrm{Zu}$ stand in ihrem Leben, sondern auch den Stand des Interviews.

\section{Phase: interne und externe Nachfragen}

Die dritte Phase des Interviews ist in interne und externe Nachfragen unterteilt. Interne Nachfragen beziehen sich auf den Hauptteil. FischerRosenthal/Rosenthal unterscheiden drei Grundtypen narrativen Nachfragens: 1. Das Ansteuern einer Lebensphase („Können Sie mir über die

4 Hier wird eine Analogie zwischen Erfahrung und Erzählung hergestellt, die nicht unproblematisch ist. Vgl. dazu ausführlich das Kapitel zur Forschungsperspektive. 
Zeit (Kindheit etc.) noch etwas mehr erzählen?"); 2. Das Ansteuern einer benannten Situation („Sie erwähnten vorhin die Situation X. Können Sie darüber noch etwas mehr erzählen?“); 3. Das Ansteuern einer Belegerzählung zu einem Argument (,Können Sie sich noch an eine Situation erinnern, in der Sie nicht mehr an Ihren Erfolg glaubten?“). Da diese internen Nachfragen weitere Erzählungen generieren sollen, empfiehlt es sich, Warum- und Wieso-Fragen zu vermeiden und vielmehr die Möglichkeit zu eröffnen einen Handlungsablauf zu erzählen, der immer an konkrete Situationen gebunden ist (vgl. ebd. 1997: 418).

Der externe Nachfrageteil ist jedoch auf das spezifische Thema der Untersuchung zugeschnitten und enthält zum einen Fragen zu (biographischen) Themen, die nicht im Interview vorgekommen sind. Zum anderen können in dieser Phase auch allgemeine Fragen angesprochen werden, die für die Analyse interessant sein könnten. Solche externen Nachfragen können flexibel genutzt werden, d.h. je nach Verlauf des Interviews und angesprochenen Themen können Fragen weggelassen werden, einige Fragen richteten sich jeweils nur an eine(n) Partner(in). Zum Teil haben auch diese Fragen noch weitere Erzählungen generiert, zum Teil sind sie auch in eine Diskussion mit mir gemündet, in der auch an mich Fragen gestellt wurden oder ich zur Stellungnahme aufgefordert wurde zu rassistischen Erfahrungen oder ,typisch deutschen“ Mentalitäten und Einstellungen. ${ }^{5}$

Der geplante Ablauf der Interviews ließ sich jedoch nur bedingt realisieren. Die Interviews mit den Frauen verliefen in der Regel wie geplant. Die Interviews mit den Männern lassen sich allerdings nicht durchgehend als tatsächlich biographisch-narrative Interviews verstehen. Insbesondere mit denjenigen, deren Sprachkenntnisse in Englisch oder in Deutsch für ein biographisches Interview nicht ausreichten, wurden eher problemzentrierte Interviews geführt. Das Prinzip der Nicht-Intervention in der Haupterzählung ließ sich nicht aufrechterhalten, da nur wenige und kurze Erzählungen produziert wurden und die Haupterzählungen nicht besonders lang waren. Die Textsorten, die so produziert wurden, sind also sehr unterschiedlich.

I(nterviewerin): „And you're here for only five months.“

F(ernando): „Ja, 5 months. - I really wanted to make this interview in Deutsch, but.“

5 Die Interviews wurden so zu einer gegenseitigen Lern- und auch Zuweisungssituation, in der ich das Verhalten mancher Deutscher erklären sollte, umgekehrt mir aber auch die Erfahrungen der Nicht-Zugehörigkeit von Migranten deutlich gemacht wurden. 
I: „Jeah, probably next year or in two years.“

F: „No, next year. The next year. For June in next year I think I will be able to" -

I: „Hm.“

F: „And Deutsch ist difficult. Deutsch is difficult. - - I learn English a little bit easier. I don't speak very good English but it was easier.“

I: „Ja, English is easier.“

F: „And Deutsch, with a lot of complex gr_grammar.“

I: „Ja.“

F: „Die Grammatik is very complex.“

I: „I know“ (Fernando Rodriguez 7/11-22).

Solche Einschübe waren nicht ungewöhnlich, häufig wurden von den Interviewten kurze Diskussionen in Erzählungen oder Argumentationen eingeflochten. Die Präsenz solcher und ähnlicher Textsorten, die deutlich von einem biographisch-narrativen Interview abwichen, hatten auch Modifikationen in der Auswertungsstrategie zur Folge: Die formale Textanalyse, wie sie von Schütze entwickelt wurde (vgl. ebd. 1981), ließ sich in der von ihm vorgeschlagenen Art und Weise nicht durchführen.

\section{Das Kernsample: drei Paare}

Ingesamt wurden acht Paare und eine einzelne Frau interviewt. Aus diesem Hauptsample heraus, aus acht Paaren bestehend, sollten drei Paare als Kernsample für die intensive Fallanalyse ausgewählt werden. Dem methodologischen Verständnis der Grounded Theory folgend richtete sich die Auswahl der ersten beiden Paare nach dem Prinzip der maximalen Kontrastierung. Entscheidungskriterien waren zum einen sozialstrukturelle Daten wie die Dauer des gemeinsamen Lebens in Deutschland, das Alter, (gemeinsame) Kinder und die Frage, ob sie sich zukünftig ein Leben in einem anderen Land als in Deutschland vorstellen könnten. Zum anderen wurden durch erste interpretatorische Zugänge in Forschungswerkstätten, die über die ,äußeren` Daten hinaus in die biographischen Interviews selbst Einblick nahmen, die beiden ersten Paare ausgewählt. Das erste Paar, Susanne Frei und Rachid Frei-Hardarzadeh, lebt schon seit 22 Jahren in Deutschland und hat zwei schon etwas ältere Kinder. Das female breadwinner Modell kam für sie nicht unerwartet, und es bestand über einen relativ langen Zeitraum. Das zweite Paar hingegen, Carlos und Nicole Ortega Garcia, ist erst seit ein paar Jahren in Deutschland und wurde von Carlos Arbeitslosigkeit (unangenehm) überrascht. Sie haben keine Kinder. Nach der Interpretation dieser beiden Fälle wurde als drittes Paar eines ausgewählt, welches in den entwickel- 
ten Kernkategorien wichtige Unterschiede bzw. Ergänzungen vorwies und daher weitere wichtige Interpretationsergebnisse versprach. Maria Wesel und Pablo Marmani Rodriguez sind erst seit zwei Jahren in Deutschland und haben zwei kleine Kinder. Für sie steht der Umzug nach Mexiko von vorneherein fest, zum Zeitpunkt des Interviews stehen sie kurz davor. Das female breadwinner Modell in Deutschland ist insofern von vorneherein durch die transnationale Perspektive zeitlich begrenzt. Alle Paare haben sich im Herkunftsland des Mannes bzw. (wie in dem Fall der Familie Frei-Heidarzadeh) in einem dritten Land kennen gelernt. Der Migrationsanlass für die Männer lässt sich daher in allen drei Fällen als Heiratsmigration bezeichnen. ${ }^{6}$ Diese drei Paare, die im empirischen Teil intensiv interpretiert und analysiert worden sind, werden an dieser Stelle in der Art eingeführt, dass die Gemeinsamkeiten und die Unterschiede zwischen den drei Paaren in Bezug auf relevante Kategorien tabellarisch erfasst werden. Zur späteren detaillierteren Vorstellung der Paare dienen biographische Portraits, die vor den jeweiligen Interpretationen stehen.

6 Ich unterscheide zwischen Migrationsanlass und -grund. Die Gründe für eine Migration sind vielfältig und lassen sich oft nicht so klar benennen, zum Teil bestand schon vor der Begegnung mit der zukünftigen Partnerin eine Migrationsdisposition, zum Teil wurde erst danach über eine mögliche Migration nachgedacht. Den konkreten Anlass zur Migration aber stellt in allen Fällen die Beziehung bzw. die Heirat dar. 
Tabelle 1: Das Kernsample

\begin{tabular}{|c|c|c|c|}
\hline & $\begin{array}{l}\text { Susanne Frei } \\
\text { und Rachid } \\
\text { Frei- } \\
\text { Heidarzadeh }\end{array}$ & $\begin{array}{l}\text { Nicole und Car- } \\
\text { los Ortega Gar- } \\
\text { cia }\end{array}$ & $\begin{array}{l}\text { Maria Wesel } \\
\text { und Pablo } \\
\text { Marmani } \\
\text { Rodriguez }\end{array}$ \\
\hline $\begin{array}{l}\text { Herkunfts- } \\
\text { land des } \\
\text { Mannes }\end{array}$ & Iran & Mexiko & Mexiko \\
\hline $\begin{array}{l}\text { Alter zum } \\
\text { Zeitpkt. des } \\
\text { Interviews }\end{array}$ & $\begin{array}{l}\text { M: } 45 \mathrm{~J} . \\
\text { F: } 47 \mathrm{~J} .\end{array}$ & $\begin{array}{l}\text { M: } 30 \mathrm{~J} . \\
\text { F: } 27 \mathrm{~J} .\end{array}$ & $\begin{array}{l}\text { M: } 29 \\
\text { F: } 33\end{array}$ \\
\hline $\begin{array}{l}\text { Schul- und } \\
\text { Berufsaus- } \\
\text { bildung }\end{array}$ & $\begin{array}{l}\text { M: Abitur in } \\
\text { Iran, Lehre als } \\
\text { KFZ-Mechaniker } \\
\text { u. Erzieher in D } \\
\text { F: Realschule, } \\
\text { Ausbildung zur } \\
\text { Erzieherin in D }\end{array}$ & \begin{tabular}{|l|} 
M: Abitur, Stu- \\
dium der Kom- \\
munikations- \\
wissenschaften in \\
Mexiko \\
F: Realschule, \\
Ausbildung zur \\
Bauzeichnerin in \\
D
\end{tabular} & $\begin{array}{l}\text { M: Abitur, Archi- } \\
\text { tekturstudium in } \\
\text { Mexiko } \\
\text { F: Abitur, Jour- } \\
\text { nalistikstudium in } \\
\text { D }\end{array}$ \\
\hline $\begin{array}{l}\text { Seit wann } \\
\text { gemeinsam } \\
\text { in Deutsch- } \\
\text { land }\end{array}$ & seit 22 Jahren & seit vier Jahren & seit zwei Jahren \\
\hline $\begin{array}{l}\text { Migrations- } \\
\text { anlass }\end{array}$ & Heiratsmigration & Heiratsmigration & Heiratsmigration \\
\hline Kinder & $\begin{array}{l}\text { zwei (15 u. } 12 \\
\text { Jahre) }\end{array}$ & keine & $\begin{array}{l}\text { zwei (Zwillinge, } \\
\text { zwei Jahre) }\end{array}$ \\
\hline $\begin{array}{l}\text { Dauer des } \\
\text { female bre- } \\
\text { adwinner } \\
\text { Modells } \\
\end{array}$ & $\begin{array}{l}\text { mit Unterbre- } \\
\text { chungen } 15 \text { Jahre }\end{array}$ & seit vier Jahren & seit zwei Jahren \\
\hline $\begin{array}{l}\text { Umzug in } \\
\text { ein anderes } \\
\text { Land }\end{array}$ & nein & $\begin{array}{l}\text { gerne in vier bis } \\
\text { fünf Jahren nach } \\
\text { Mexiko }\end{array}$ & $\begin{array}{l}\text { kurz nach dem } \\
\text { Interview ziehen } \\
\text { sie nach Mexiko }\end{array}$ \\
\hline
\end{tabular}

7 Alle zeitlichen Daten haben als Bezugspunkt den Zeitpunkt der Interviews. 



\section{DAS FORSCHUNGSFELD: Binationale PaARE in DEUTSCHLAND}

\section{Erste Annäherung: zur Lebenssituation binationaler Paare in Deutschland}

Nicole: „Also es war wirklich, ich weiß man kann sich das schlecht vorstellen, so Liebe auf den ersten Blick, es war so. Also ich bin mit ihm ausgegangen und - - ja, ich weiß nicht, er war sofort - es war so vieles, also - wie er mich behandelt hat. - - sehr zuvorkommend, also wirklich, es is auch heut noch so. . sind so Sachen, die ich glaub ich an den deutschen Männern - vermiss. Also das is dann nich so, mir wird der Stuhl zurecht gerückt, aus der Jacke geholfen, - ich darf nicht auf der Straßenseite gehen, weil des is gefährlich, sozusagen. Des is jetzt nicht ein Bevormunden oder so, sondern einfach, dass er Rücksicht auf mich nimmt, dass er auf mich aufpasst. Das find ich - echt Klasse. - und, das kam schon am ersten Abend, als wir ausgegangen sind, is mir das schon aufgefallen. [...] Und - ja das war am Anfang, später auch in Deutschland so_so 'n blödes Thema - das ich bezahlt hab. Daran hat er sich auch erst gewöhnen müssen. Weil es einfach, er lebt, also in Mexiko ist es einfach, da zahlt keine Frau, also - nich dass - das hat damit nix zu tun, dass sie das Geld nicht hätte, sondern - - es schaut doch besser aus, meinen se, wenn der Mann sie einlädt, das is doch einfach, wenn ich dich frag, ob wir zum Essen gehen, dann lad ich dich doch mit der Frage ein, meint er immer, und nicht .[...] dass du dann selbst bezahlst, sonst würd ich dich nicht fragen“" (Nicole Ortega Garcia 6/12-32).

Carlos: „Die ersten drei Monate war alles wunderbar, also Deutschland wunderschön. - war auch Sommer. - ja, wunderbar, wir war'n in Neusch_Neuschwanstein, wunderschöner Ort, [...] in Füssen, - ähm - - ja, also war alles toll. Deutschland ganz anders, andere Sprache, alles wunderbar. Und das Essen, alles schmeckt toll und - ja, dann dann kommt das Problem - wie lange kann ich in Deutschland bleiben? Äh, natürlich ich habe nur ein Touristenvi- 
sum. - und mehr als drei Monate kann ich nicht bleiben“" (Carlos Ortega Garcia, 15/16-23).

Nicole und Carlos erzählen in diesen Interviewpassagen von dem Beginn der Beziehung, von der Besonderheit, die diese Beziehung von anderen unterscheidet, und von den ersten gemeinsamen Monaten in Deutschland. Die Beziehung zu Carlos ist, das wird aus der Interviewpassage von Nicole deutlich, für sie von Anfang an anders als eine Beziehung zu einem Deutschen. Nicole verliebt sich , auf den ersten Blick ${ }^{*}$ und ist fasziniert von der altmodischen Höflichkeit und Rücksichtsnahme, die Carlos ihr gegenüber zeigt. Solche Verhaltensweisen kennt sie von deutschen Männern nicht, und sie hat sie, wie sie selbst sagt, bislang vermisst. Kontrastierend zu diesen positiven Erfahrungen, die sie in Mexiko mit Carlos gemacht hat, erinnert sie sich an die erste Zeit in Deutschland, in der Carlos erst hatte lernen müssen, mit der anderen Art der Geschlechterbeziehungen umzugehen. Die in Deutschland üblichen Konventionen hinsichtlich der Bezahlung von Restaurantbesuchen sind für Carlos eben nicht selbstverständlich, Nicole und er müssen sich daher mit ihren unterschiedlichen Erwartungen an die Situation auseinandersetzen. Auch Carlos erinnert sich in dieser Textstelle an Konsequenzen, die sich für ihn aus der Migration nach Deutschland ergeben haben. Für ihn stellen sich jedoch an dieser Stelle vor allem die rechtlichen Restriktionen eine Schwierigkeit dar, die massiv in die Beziehung einwirken. Mit seinem Touristenvisum darf er nur drei Monate in Deutschland bleiben, danach muss er entweder das Land wieder verlassen, oder aber Nicole und er heiraten, und er kann so eine Aufenthaltsgenehmigung erhalten.

In den hier zitierten Interviewpassagen lassen sich zwei Dimensionen binationaler Beziehungen unterscheiden: Zum einen formuliert Nicole einige für sie relevante Gründe und Motive, eine solche Beziehung einzugehen. Sie unterscheidet dabei deutlich zwischen deutschen und mexikanischen Männern und sieht gerade in der Herkunft Carlos` die entscheidenden Gründe, eine Beziehung eben nicht zu einem deutschen, sondern zu einem mexikanischen Mann einzugehen. Zum anderen werden sowohl von Nicole als auch von Carlos verschiedene Aspekte benannt, die ihre Beziehung zu einer besonderen mit spezifischen Lebensumständen werden lässt. Nicole formuliert diese an dieser Stelle eher auf der Ebene der innerpartnerschaftlichen Auseinandersetzung, Carlos auf der Ebene der Einwirkung durch rechtliche Rahmenbedingungen. Beider Erfahrungen aber lassen Unterschiede zu mononationalen Beziehungen deutlich werden, die von dem Paar bearbeitet werden müssen. 
Diese und weitere Phänomenbereiche werden in der folgenden ersten Annäherung an das Forschungsfeld diskutiert. Dazu wird in einem ersten Schritt die in dieser Arbeit verwendete Bezeichnung ,binationale Paare' in Auseinandersetzung mit anderen Begriffsvorschlägen begründet. Zusätzlich wird mit Hilfe der (allerdings nur ungenügend zur Verfügung stehenden) Statistiken ein Überblick über binationale Partnerschaften in Deutschland geliefert. In dem sich anschließenden Überblick zur Forschungsliteratur zu binationalen Paaren fällt auf, dass insbesondere im angloamerikanischen Bereich intensiv Theorien und Konzepte zur Partnerwahl diskutiert werden. Spezifische Lebensumstände und/ oder Problembereiche binationaler Partnerschaften werden hingegen eher in deutschen Studien analysiert.

\section{Begriffsklärungen: inter-, multi-, bi-nationale Paare}

„Intermarriage, then, will be defined as marriage of persons deriving from those different in-groups and out-groups other than the family which are culturally conceived as relevant to the choice of a spouse“ (Merton 1972 [1942]: 13, Herv. d. Verf.). ${ }^{1}$ In der deutschen Forschung zum Phänomen der Intermarriage (so die Begriffsverwendung in der englischen Sprache) finden sich vergleichsweise viele unterschiedliche Bezeichnungen, die jeweils verschiedene Perspektiven auf die untersuchte Gruppe einnehmen. Begriffe wie interethnische Ehen, bi- oder interkulturelle Partnerschaften beziehen sich daher in der Regel zwar auf das gleiche Phänomen der Intermarriage, fokussieren jedoch durchaus unterschiedliche Dimensionen der Differenz. Die Fokussierung ist einerseits sicherlich abhängig von der disziplinären Zugehörigkeit der Autorin/des Autors oder der theoretischen Relevanzsetzung der Untersuchung, stellt jedoch auf der anderen Seite insofern eine künstliche Definition dar, als dass sich Zugehörigkeiten ja nur ,idealtypisch' in kulturelle, ethnische oder nationale Zugehörigkeiten trennscharf unterscheiden lassen. Die Entscheidung für eine Bezeichnung lässt sich insofern eher als eine Art Annäherung begreifen, um im Kontext des eigenen Interesses einen Begriff zu wählen der, mit dem Wissen um die Unzulänglichkeit der Bezeichnungen, als Orientierungsrahmen für die spezifische

1 Einige Autor(inn)en weisen auf eine grundlegende Differenz zwischen Männern und Frauen hin, die jede Form der Beziehung zu einer mixed marriage werden ließe (vgl. z.B. Davis 1941 in Thode-Ahora 1999: 25). Insofern sollte, so die Schlussfolgerung, eine solche Bezeichnung nur für eine die Heiratsregeln einer Gesellschaft verletzende Ehe benutzt werden. 
Aufmerksamkeitsrichtung fungiert (vgl. zur Diskussion um die Bezeichnung auch Molnár 2004: 7ff).

In der Literatur werden die Begriffe folgendermaßen unterschieden ${ }^{2}$ :

- Kulturelle Zugehörigkeit bezieht sich auf Gemeinschaften, deren Verbundenheit häufig auch in einer gemeinsamen religiösen Zugehörigkeit gesehen wird. ,Kultur' wird hierbei verstanden als Wertegemeinschaft mit gemeinsamen Normen und Handlungsorientierungen (vgl. dazu z.B. Waldis 1998).

- Ethnische Zugehörigkeit bezieht sich auf Gemeinschaften, deren Verbundenheit durch eine „Ideologie einer gemeinsamen Abstammung und Kultur" hergestellt wird, die sich von anderen Gemeinschaften abgrenzt und abgegrenzt wird. „Als Markierungen dieser Abgrenzungen dienen bestimmte kulturelle Merkmale“ (ThodeAhora 1999: 21, vgl. dazu z.B. Pusitz/Reif 1996). Schon beim Definitionsversuch wird die Schwierigkeit der Trennung zwischen Ethnie und Kultur deutlich: Ethnische Zugehörigkeit erklärt sich durch kulturelle Merkmale. Die beiden Begriffe werden daher auch häufiger synonym benutzt, beziehungsweise werden sie häufig, wie in der hier vorgestellten Definition, in einer Art argumentativen reflexiven Schleife genutzt, die, je nach Zusammenhang, die eine Differenzlinie mit der anderen begründet.

- Nationale Zugehörigkeit wiederum bezieht sich auf die Zugehörigkeit zu zwei verschiedenen Staaten und charakterisiert damit die juristisch-nationalstaatliche Perspektive auf Intermarriage dar. Eine solche Bestimmung bringt jedoch, abhängig von der untersuchten Gruppe, ebenfalls Probleme mit sich: Staatsangehörigkeiten wechseln und zwei oder sogar mehr Staatsangehörigkeiten sind keine Seltenheit (mehr) (vgl. dazu z.B. Kriechhammer-Yagmur/Pfeiffer-Pandey/Saage-Fain et al. 2001). ${ }^{3}$

Für mein Sample spreche ich von binationalen Paaren, da die Binationalität das für meinen Analysefokus (mit-)entscheidende Kriterium ist. Ist hingegen das allgemeine Phänomen gemeint, verwende ich den übergeordneten Begriff der Intermarriage. Mit der Entscheidung für die Bezeichnung, binational' folge ich vor allem der Selbstbeschreibung des

2 Der Begriff der ,Mischehe“ (im Englischen mixed marriage) ist aufgrund seiner Verwendung während des Nationalsozialismus im Deutschen nicht mehr in Gebrauch.

3 Die Zahl der Doppelstaatler wird auf mehr als zwei Millionen geschätzt. Es handelt sich vorrangig um Kinder aus binationalen Ehen, deren eines Elternteil die deutsche Staatsbürgerschaft besitzt (vgl. Schily 1999). 
wohl größten deutschen Lobbyverbandes dieser Gruppe, der IAF, dem Verband binationaler Paare und Familien. Zum anderen ist das Distinktionsmerkmal ,binational', also die Zugehörigkeit zu zwei verschiedenen Staaten, auch für die von mir analysierte Gruppe von Paaren in besonderer Weise relevant. Die Zugehörigkeit des Partners zu einem ,anderen“ Staat als Deutschland bzw. als zu dem Staatenbund EU strukturiert die ,Problemlage“ der Paare in besonderer Weise. Diese Paare sind in besonderem Maße ver-rechtlichte Subjekte. Dies zum einen aufgrund der Nicht-Zugehörigkeit des männlichen Partners zu Deutschland. Die Entscheidung zur Heirat muss vor dem Hintergrund getroffen werden, dass in der Regel, ohne Arbeits- oder Studienvisum, ein nur dreimonatiger Aufenthalt in Deutschland möglich ist. Zum anderen entsteht das female breadwinner Modell aus der Tatsache heraus, dass Bildungsabschlüsse anderer Staaten nicht ohne weiteres anerkannt werden. Die von mir interviewten Paare haben ihren Schul- bzw. Berufsabschluss allesamt in ihrem Herkunftsland erworben und sind daher mit dieser fehlenden Anerkennung konfrontiert.

\section{Binationale Paare in Deutschland: ein statistischer Überblick}

\section{Abbildung 1: Eheschließungen nach der Staatsangehörigkeit der}

Ehepartner von 1955 - 2000

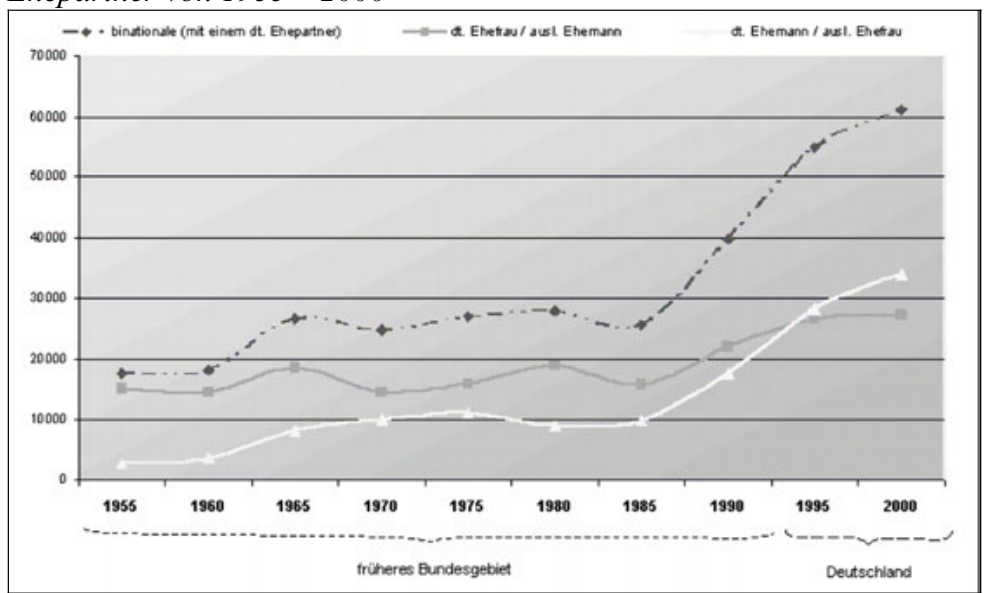

(Quelle: IAF 2006)

Statistische Angaben über binationale Paare sind vor allem aufgrund der fehlenden Datenerfassung unvollständig. Die IAF, der Verband binationaler Paare und Familien, veröffentlicht jährlich die aktuellen Statistiken 
über die Anzahl der Eheschließungen binationaler Paare. Erfasst werden jedoch zum einen nur diejenigen, die tatsächlich zwei unterschiedliche Staatsangehörigkeiten haben, so dass eingebürgerte Personen mit Migrationshintergrund oder auch Doppelstaatler(innen) nicht aufgenommen werden können. Zum anderen werden auch nur diejenigen erfasst, die in Deutschland heiraten. Da aber gerade binationale Eheschließungen aufgrund der organisatorischen Hürden häufig im Ausland geschlossen werden, ist davon auszugehen, dass die tatsächliche Anzahl von binationalen Eheschließungen noch höher ist. Hinzu zu zählen sind desweiteren auch eingetragene Partnerschaften.

Der Anteil binationaler Eheschließungen unter Beteiligung einer/s deutschen Partnerin/s an allen Eheschließungen ist seit der ersten Erfassung 1955 stark angestiegen und betrug 2003 15,72\%. ${ }^{4}$ Waren es früher vor allem deutsche Frauen, die binational geheiratet haben, sind es seit 1995 mehr Männer als Frauen.In den Herkunftsländern der Partner mit nicht-deutscher Staatsangehörigkeit spiegeln sich Migrationsentwicklungen wieder. So verwundert es nicht, dass 2003 der Anteil der Eheschließungen zwischen deutschen Frauen und türkischen Männern mit 22,3\% mit Abstand am größten ist, gefolgt von 8,3\% der Eheschließungen mit jugoslawischen Männern und 6,8\% mit italienischen Männern. Deutsche Männer wiederum haben 2003 vorrangig polnische Frauen geheiratet $(15,3 \%)$, gefolgt von russischen und thailändischen Frauen mit je 7,2\% (vgl. IAF 2006, eig. Berechnungen) Die steigende Zahl von deutschen Männern, die binational heiraten, ist sicherlich auch eine Folge der zunehmenden Migration von Frauen. Waren die ersten Arbeitsmigranten aus der Türkei, Italien und dem ehemaligen Jugoslawien vorrangig Männer, verschiebt sich jetzt das Geschlechterverhältnis in Migrationsprozessen. Die IAF vermutet allerdings, dass die Eheschließungen mit polnischen und russischen Frauen auch eine Art Heiratsmigration darstellt: ,Hinter den deutsch-polnischen Heiraten verbergen sich häufig Aussiedler, die sich eine Polin aus dem Heimatland holen', sagt Wießmeier" (TAZ vom 05.06.1998, S. 21).

Neben den Eheschließungen in Deutschland geben auch die Zahlen zum Familiennachzug Hinweise über die quantitative Bedeutung binationaler Partnerschaften. Für Ehegatten, die im Ausland leben (da zum Beispiel die Eheschließung im Ausland erfolgt ist), muss in der Regel ein Visum für den Familiennachzug beantragt werden.

Die ,,ausländerrechtlichen Regelungen [geben] den örtlichen Ausländerbehörden [allerdings] in Einzelfällen die Möglichkeit, im Inland eine Aufenthalts-

4 Soweit nicht anders angegeben, beziehen sich die Zahlen auf die Veröffentlichungen der IAF auf deren Homepage www.verband-binationaler.de 
genehmigung zu erteilen, auch wenn der Betroffene mit einem Touristenvisum oder zu einem Kurzaufenthalt eingereist ist. Diese Fälle der Familienzusammenführung gehen nicht in die Statistik des Auswärtigen Amtes ein. Insofern „unterschätzt“ die Visastatistik den Ehegatten- und Familiennachzug. Angaben zur Größenordnung dieser Ausnahmefallgruppen lassen sich jedoch nicht machen“ (Ausländerbeauftragte 2003: 24).

\section{Abbildung 2: Erteilte Visa zum Zweck des Ehegatten- und}

\section{Familiennachzugs nach Deutschland von 1996 bis 2003}

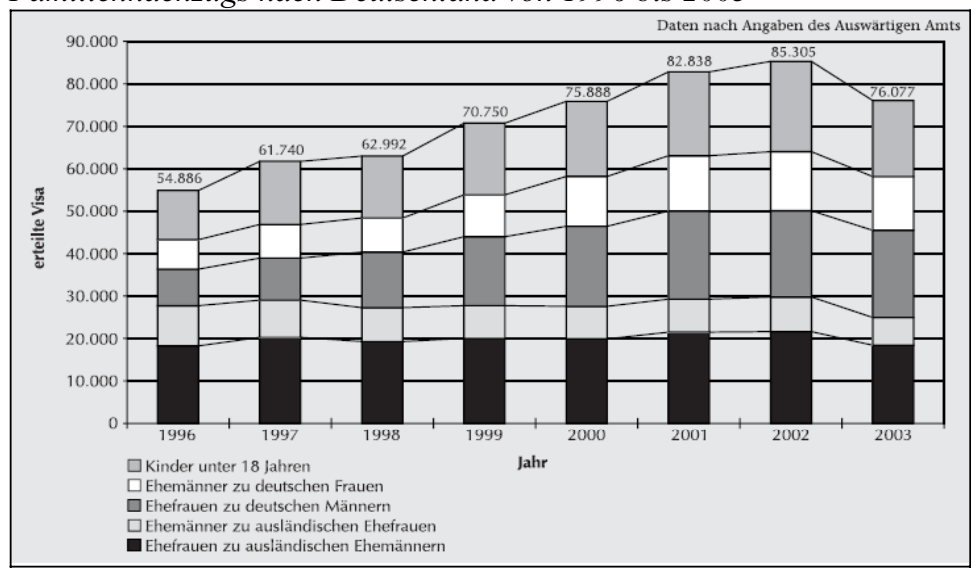

(Quelle: Beauftragte der Bundesregierung für Migration 2004: 14)

Auch diese Statistik zeigt, dass die Anzahl der nachziehenden Frauen zu deutschen Männern deutlich größer ist als die der nachziehenden Männer zu deutschen Frauen. Gleichzeitig steigt die Zahl der erteilten Visa, ein Hinweis unter anderem darauf, dass Eheschließungen im Ausland an Bedeutung gewinnen. Die absolute Zahl der binationalen Partnerschaften und Ehen in Deutschland lässt sich aus diesen Statistiken nicht ermitteln. Überhaupt gibt es bislang nur wenige Forscher(innen) in Deutschland, die sich quantitativ mit der Intermarriage beschäftigen (vgl. dazu vor allem Klein 2001a, b). Dies unterscheidet die hiesige Forschungslandschaft deutlich von der US-amerikanischen, in der quantitative Forschung über Heiratsverhalten von Gruppen und zur Erklärung von Segregation und Assimilation eine lange Tradition hat. ${ }^{5}$ Die im Fol-

5 Dies liegt auch daran, dass Intermarriage als ein deutlich sichtbares Zeichen von Assimilation verstanden wird. Die Heirat mit einer Person insbesondere aus der Mehrheitsgruppe stelle einen wichtigen Abschnitt (vgl. dazu Merton 1972 [1942]). Ein solch ,naturwüchsiger‘ Prozess ließ sich allerdings empirisch nicht bestätigen, dennoch ist die Annahme von Intermarriage als Assimilationsindikator geblieben (vgl. dazu z.B. Richard 1991). 
genden dargestellten Konzepte zu Partnerwahltheorien sind daher auch zu einem großen Teil in den USA entwickelt worden.

\section{Partnerwahltheorien}

Die große Mehrzahl insbesondere der anglo-amerikanischen Literatur zur Intermarriage beschäftigt sich mit Partnerwahltheorien und den Gründen für das Übertreten des Homogamiegebotes, der Heterogamie. ${ }^{6}$ Intermarriage wird als Folge von Migrationsprozessen verstanden, die die Einheitlichkeit einer Mehrheitsgesellschaft durch den Zuzug einzelner Personen bzw. ganzer sozialer Gruppen aufbricht. Analysiert werden also Bedingungen der Partnerwahl unter der Perspektive eines auf Dauer angelegten Zusammenlebens verschiedener sozialer Gruppen. Ein solches Verständnis hat Konsequenzen für die methodologische Ausrichtung empirischer Studien: Während insbesondere in den USA und Kanada Intermarriage als strukturelles Moment der Gesellschaft verstanden wurde und die Frage nach ,who marries whom' im Mittelpunkt steht, die Studien demzufolge zwangsläufig in der Mehrzahl quantitativ sind, interessieren in Deutschland eher die sozialen Lebensbedingungen, die ,Besonderheiten“ in der Lebensführung, in der Beziehung und in der Interaktion mit der sozialen Umwelt. Die (wenigen) Studien dazu sind infolgedessen fast ausschließlich qualitativ angelegt und rekurrieren, wenn notwendig, auf existierende Partnerwahltheorien.

Hilke Thode-Ahora, die 1999 ein Handbuch zur Forschungslage zu binationalen Partnerschaften herausgegeben hat, beschäftigt sich ausführlich mit existierenden Theorien zur Partnerwahl und fasst auf über 130 Seiten die Ergebnisse zusammen (ebd.: 94-243). Bei der Rekapitulation ihre Vorgehens wird deutlich, dass sämtliche Theorien zwar einerseits den Anspruch erheben, allgemeingültige Faktoren für die Partnerwahl identifiziert zu haben, sich die unterschiedlichen Erklärungsmodelle gleichwohl zum Teil ausschließen. Zudem sind einige der Theorien aufgrund ihrer Entstehungsgeschichte explizit auf spezifische Gruppen oder historische Phasen bezogen und können in der Form nicht verall-

6 Homogamie wird prinzipiell als Organisationsprinzip von sozialen Gruppen angenommen. Gemeint ist dabei die Heirat innerhalb der eigenen sozialen Gruppe, um deren Weiterbestehen zu sichern. Heterogamie als Heirat außerhalb der sozialen Gruppe soll demgegenüber oftmals entweder durch normative Strukturen oder auch faktische Sanktionen verhindert werden. Was dabei als Heterogamie gilt, ist abhängig von historischen Verläufen ebenso wie von sozialen Prozessen. So ist, zumindest pro forma, für die Ehe eines/r Katholiken/in mit einer/m Protestanten/in nach wie vor ein Ehedispens notwendig. 
gemeinert werden. Insofern erscheint es an dieser Stelle wenig sinnvoll, solche Überblicke, wie sie zudem an anderer Stelle vorliegen, zu wiederholen (vgl. dazu z.B. auch Buba/Ueltzen/Vaskovics 1984; Scheibler 1992; Kienecker 1993). Hilfreicher ist es, die unterschiedlichen Perspektiven auf das Phänomen Intermarriage, welche durch die verschiedenen Theorien transportiert werden, darzustellen und analytisch einzuordnen. Dazu wurden die Erklärungsansätze in drei Dimensionen aufgeteilt: in das Verständnis von Intermarriage als Folge demographischer Faktoren, in Intermarriage als Beitrag zur Statuserhöhung oder -stabilisierung und schließlich in Intermarriage als Folge spezifischer Persönlichkeitsmerkmale. Jede dieser drei Dimensionen wird anhand von exemplarischen Vertreter(inne)n dieser Theorieansätze diskutiert.

\section{Intermarriage als Folge demographischer Faktoren}

Diese erste Perspektive versteht Intermarriage als Folge demographischer bzw. struktureller Faktoren. Als demographische Faktoren gelten hierbei zum Beispiel die Größe der Minderheiten- und Mehrheitsgruppen oder auch die Geschlechterstruktur der Mitglieder dieser Gruppen. Als strukturelle Faktoren werden mögliche ,Kontaktbereiche' wie Nachbarschaft oder Arbeitsplatz in Betracht gezogen. Ausgangspunkt dieser Perspektive sind insofern „fields of availability“ (Kerckhoff 1974 in Thode-Ahora 1999: 103f). Die Idee eines vorstrukturierenden Heiratsmarktes wird ebenfalls von Blau/Becker/Fitzpatrick 1984: unter Bezug auf Simmels Konzept der „crosscutting social circles“ (ebd.: 558) formuliert. Sie gehen von der Beobachtung aus, dass Personen verschiedenen sozialen Gruppen angehören, wobei in der Regel nur selten dieselben Personen zu jeder einzelnen dieser Gruppen gehören. Daher bietet sich hier die Gelegenheit der Überschneidung sozialer Kreise, die wiederum Intermarriage ermöglichen und unterstützen. Wichtige Kriterien für die Entwicklung der Heterogamieraten seien die relativen Gruppengrößen, die Geschlechter- und Altersstruktur und die räumliche Verteilung. Sozialstrukturelle Faktoren als bestimmende Faktoren der Intermarriage sehen ebenfalls Buba/Ueltzen/Vaskovics 1984, die als Erste einen statistischen Überblick über ,gemischt-nationale Ehen“ (ebd.: 421) in Deutschland erstellten. Auch sie verstehen die demographische bzw. strukturelle Verteilung der potentiellen Partner(innen) und die Möglichkeiten bzw. Felder der Begegnung, wie zum Beispiel Bildungseinrichtungen, als grundlegende Voraussetzung für binationale Beziehungen. Eher ungünstig sind in diesem Modell Felder räumlicher Segregation, da diese Begegnungen erschweren. Zusätzlich vermuten sie auch die Existenz von Kulturkreisen, deren Nähe oder Ferne zur deutschen 
Referenzkultur eine Eheschließung mit Deutschen erleichtert bzw. erschwert (Buba/Ueltzen/Vaskovics 1984: 428f). ${ }^{7}$ Ein Vertreter dieses Ansatzes in Deutschland ist Thomas Klein (z.B. Klein 2000, 2001b), der quantitative Forschung zu binationalen Paaren in Deutschland durchführt. Klein sieht die Struktur des Heiratsmarktes als bestimmend für die homogame oder heterogame Partnerwahl an. Der Heiratsmarkt bestimmt sich aus der Gesamtsumme potentieller Ehepartner(innen), diese Gesamtsumme entspricht jedoch nicht der Summe aller heiratsfähigen Mitglieder einer Gesellschaft. Vielmehr sind Begegnungsmöglichkeiten entscheidend durch soziale und geographische Strukturen ,vororganisiert durch die fields of availability. Heterogamie, die Wahl eines/r Ehepartners/in außerhalb der eigenen sozialen Gruppe, stellt sich für die Vertreter dieses Erklärungsansatzes als eine Art Ausweichmöglichkeit dar. Intermarriage tritt dann auf, wenn aus bestimmten Gründen die Realisierung einer homogamen Partnerschaft nicht möglich sei. ${ }^{8}$ Solche Gründe liegen für Klein einerseits in der Größe der Einwanderergruppen: So lässt sich bei zwei Einwanderergruppen, bei Spaniern und Türken, ein typischer U-förmiger Verlauf der Heterogamierate zeigen: Aufgrund der geringen Größe der Einwanderungsgruppen müssen die Heterogamieraten zu Beginn der Migrationen eher größer sein. Mit wachsender Größe der ethnischen Gruppen sinkt die Rate wieder, um dann im Zuge von Integration der Einwanderergruppen wieder anzusteigen (vgl. Klein 2001a: 337). ${ }^{9}$ Andererseits bezieht Klein individuelle Präferenzen und handlungstheoretische Motive mit ein: Heterogamie entsteht dann, wenn die Maxime des Wunsches nach größtmöglicher Attraktivität des Partners oder der Partnerin nicht erfüllbar ist. Ein solches Heiratsmuster findet seinen Ausdruck auch in der Tatsache, dass die Heterogamieraten mit steigendem Lebensalter zunehmen: „Grund dafür ist, dass sich der Partnermarkt mit zunehmendem Lebensalter verändert, wenn die Präferenzen auf einen altersnahen Bereich potentieller Partner konzentriert sind“ (Klein 2000: 233). Partner(innen) der eigenen Altersgruppe sind oft ver-

7 Damit erklären sie die vergleichsweise geringe Anzahl deutsch-türkischer Eheschließungen Anfang der 80er Jahre. Zwar sei die Zahl solcher Ehen „,dennoch von Bedeutung“, dies hänge aber damit zusammen, ,dass in den letzten Jahren sehr viele Türken in die Bundesrepublik gekommen sind“ (Buba/Ueltzen/Vaskovics 1984: 429). Die Gruppengröße wirke sich also stärker aus als kulturelle Differenzen.

8 Homogamie ist für ihn demzufolge auch einer der entscheidenden Faktoren der Strukturpersistenz: „Allgemein lässt sich sagen, dass die Reproduktion sozialer Strukturen von den Mustern der Partnerwahl abhängt“ (Klein 2000: 229).

9 Dies ist ein Verlauf, wie er in den USA auch von anderen Einwanderungsgruppen bekannt ist. 
geben, zudem ,verschlechtere“ sich (in den Augen der Beteiligten) der Partnermarkt, da die noch zur Verfügung stehenden nicht diejenigen mit der größtmöglichen Attraktivität sind.

\section{Intermarriage als Beitrag zur Statuserhöhung bzw. -stabilisierung}

Eine zweite, eher subjektorientierte Perspektive, versteht Intermarriage als Folge bewusste oder unbewusster Entscheidungen der Ehepartner(innen). Zwei Theoriestränge verfolgen diese Perspektive. Beide verstehen Heirat und Ehe als eine Art Austausch-Prinzip, indem jede(r) Partner(in) bestimmte sozial erwünschte Merkmale in die Beziehung mit einbringt und dafür von dem/der Anderen ebensolche Merkmale verlangt. Dieses Phänomen der Auswahl läßt sich, analog zu oben, mit den „fields of desirability“ verstehen (in Thode-Ahora 1999: 104). Die Theorie des kompensatorischen Austauschs lässt sich dabei mit dem alltagswirklichen Wissen um das Partnerwahlprinzip ,Gegensätze ziehen sich an' beschreiben, die Summationstheorie hingegen mit dem Prinzip des ,Gleich und Gleich gesellt sich gern'. Zwar schließen sie sich in ihrer Grundannahme aus, beide zielen aber auf die Vorstellung von Ehe als einem Tausch von Qualitäten und Qualifizierungen ab.

Das Konzept des kompensatorischen Austauschs ist insbesondere durch Robert Merton (Merton 1972 [1942]) bekannt geworden. Er legt seinen Überlegungen die strukturelle Aufteilung des Heiratsmarktes zugrunde und fragt darauf aufbauend nach Entscheidungskriterien der Paare. Für ihn ist dieser Austausch von Statusmerkmalen kein rationaler und auch nicht zwangsläufig ein bewusster Akt. Vielmehr könnten die beteiligten Parteien die Beziehung durchaus als ,reine Liebesbeziehung' verstehen, der Statusaustausch bilde sich dennoch in den Charakteristika der jeweiligen Ehe ab (vgl. Merton 1972 [1942]: 29). Anhand der Analyse von „,black white marriage“ überprüft er seine Thesen, nach denen Intermarriage zwischen sozialen Stratifikationsmerkmalen einer Gesellschaft wie ethnische Zugehörigkeit, Geschlecht, Einkommen und Klasse stattfindet. ${ }^{10}$ Das typische Beispiel eines solch kompensatorischen Austausches bildet die Ehe zwischen einem schwarzen, wirtschaftlich erfolgreichen Mann der eine weiße, aus eher ärmlichen Verhältnissen

10 Hierbei gilt es zu unterscheiden zwischen Gesellschaften wie Indien, in denen Hypergamie, die Heirat einer Frau in eine höhere Klasse oder Kaste, institutionalisiert ist, und Gesellschaften wie der US-amerikanischen, in denen ein solches System nicht ,offiziell' implementiert ist. In letzterer Gesellschaft lassen Analysen jedoch trotzdem auf ein implizites System des Austauschs schließen (vgl. Merton 1972 [1942]: 16). 
kommende Frau heiratet. Hier ,tauscht‘ der Mann seinen wirtschaftlichen Erfolg gegen die Zugehörigkeit zu einer als höherwertig verstandenen ethnischen Zugehörigkeit. Umgekehrt gibt die Frau diese Zugehörigkeit für mehr wirtschaftliche Sicherheit her:

„We should expect pairing [...] lower class white women and upper class Negro man to occur most frequently for it involves a reciprocal compensatory situation in which the Negro male ,exchanges" his higher economic position for the white female's higher caste status“ (Merton 1972 [1942]: 28).

Die Summationstheorie wiederum, von Boalt (in Thode-Ahora 1999: 123) in den Diskurs eingebracht, geht von dem Prinzip einer zu erreichenden Gleichheit bzw. Gleichwertigkeit relevanter Merkmale der Partner(innen) aus. Die Gemeinsamkeit kann zum einen in Merkmalen oder Einstellungen liegen. Zum anderen aber können auch einzelne, als ,Nachteile“ empfundene Merkmale einer Person ausgeglichen werden durch andere, positive Charakteristika der geheirateten Person, so dass am Ende ein ausgewogenes Verhältnis zwischen beiden Partner(inne)n besteht. Das Austauschprinzip bleibt also bestehen, folgt aber einer anderen Logik. Anders als bei Merton können nach Boalt mehrere Merkmale kombiniert werden, einzelne Nachteile, wie beispielsweise ein geringer Bildungsstand, lassen sich durch andere Merkmale kompensieren. Zudem bezieht Boalt nicht nur äußerlich ,zuordenbare“ Merkmale wie Schicht- und Religionszugehörigkeit oder Sprache mit ein, sondern auch Persönlichkeitsmerkmale wie die Einstellungen gegenüber anderen sozialen Gruppen oder die Solidarität mit der eigenen Gruppe. An dieser Stelle werden die Grenzen zu Theorien fließend, die Intermarriage als Folge spezifischer Persönlichkeitsmerkmale verstehen. So gibt es zum Beispiel Studien, die die Homogenität der Partner(innen) in Bezug auf die Ablehnung der eigenen (natio-ethno-kulturellen) Herkunftsgruppe sehen (vgl. Hecht-El Minshawi 1988), oder Studien, nach denen die Gemeinsamkeit der Ehepartner(innen) darin liegt, dass sie beide in zweiter Ehe miteinander verheiratet sind (dazu z.B. Aldrige 1978). Die Studien, die mit der Summationstheorie arbeiten, beziehen sich jedoch explizit auf die Gleichwertigkeit als das spezifische Ergebnis des Partnerwahlprozesses und betonen nicht so sehr die persönliche Besonderheit der Partner(innen). 


\section{Intermarriage als Folge spezifischer Persönlichkeitsmerkmale}

Diese dritte Perspektive basiert auf den oben genannten Theoriesträngen und pointiert gewissermaßen die Vorstellung einer Gemeinsamkeit zwischen den Partner(inne)n. Sie lenkt den Blick auf Intermarriage als Folge spezifischer Persönlichkeitsmerkmale, die beide Partner(innen) jenseits von Homogamie-Grenzen füreinander ,passend" machen. Zwei Aspekte werden untersucht. Zum einen werden spezifische, quantifizierbare Kriterien wie Einkommen, Familienstatus, Alter etc. miteinander verglichen. Zum anderen aber werden auch Studien durchgeführt, die biographische Erfahrungen wie ,abweichende' Sozialisationserfahrungen, eine große Distanz zur eigenen Kultur oder etwa spezifische Sehnsüchte nach einem anderen Land als entscheidendes Kriterium benennen. Wie schon oben formuliert, sind die Grenzen zwischen den beiden letzten Perspektiven fließend: Kompensatorischer Austausch oder Summationstheorie können sich auch auf individuelle Persönlichkeitsmerkmale richten. Umgekehrt werden oft auch ,objektiv` messbare Kriterien wie Einkommen, Bildungsstand oder das Alter zum Zeitpunkt der EheschlieBung als Persönlichkeitsmerkmal verstanden.

Eines der Grundlagenwerke und gleichzeitig eine der Untersuchungen, die nicht auf psycho-soziale Besonderheiten abstellt, ist die frühe Analyse von Kennedy (Kennedy 1944). In Auseinandersetzung mit dem idealisierenden Konzept der USA als ,melting pot' der Rassen, Religionen und Schichten entwickelte sie die These des ,triple melting pot". Zwar steige die Intermarriage Rate und bestätige damit auf den ersten Blick den melting pot, die Rate steige allerdings restringiert entlang dreier religiöser Grenzen: der protestantischen, der katholischen und der jüdischen Konfessionszugehörigkeit. Religionszugehörigkeit erwies sich daher bis in die 40er Jahre als nur schwer zu überwindende Grenze. In der jetzigen Zeit, das zeigen übereinstimmend US-amerikanische und kanadische Studien, ist es die Kategorie ,race‘, anhand deren Grenze Intermarriage-Prozesse verlaufen (vgl. dazu und auch zu den ,gender gaps ${ }^{6}$, dem Phänomen der zunehmenden Ehen zwischen Schwarzen und Weißen, Close 2003). ${ }^{11}$

Die zweite Perspektive analysiert die Bedeutung biographischer Erfahrungen und vermuteter oder tatsächlicher ,Rollenvorteile' für binationale Beziehungen (vgl. dazu den Überblick bei Thode-Ahora 1999:

11 Dies ist zwar sicherlich auch eine Folge des Heiratsverbotes zwischen Weißen und Schwarzen, welches in einzelnen Staaten der USA bis 1967 bestand, dennoch zeigt es die Beständigkeit der ethnischen Trennung in den USA. 
235ff.). Anders als bei vorangegangenen Argumentationen, in denen Intermarriage eher als Ausweichmöglichkeit denn als ,erste Wahl' verstanden wird, erscheint hier natio-ethno-kulturelle Zugehörigkeit bzw. als natio-ethno-kulturell markierte Persönlichkeitsmerkmale des/der Partners/in als spezifischer Entscheidungsgrund für die Partnerschaft. Bei der Analyse solcher Beziehungskonzepte kommen verstärkt qualitative Studien zum Zuge. Dazu gehört beispielsweise die Studie von Hecht-El Minshawi über Ehen zwischen deutschen Frauen und muslimischen Männern (Hecht-El Minshawi 1988). Sie führt biographische Interviews mit den Frauen, um etwas über deren Motivationen und biographischen Dispositionen zu erfahren. Als Ergebnis erkennt sie bei den Frauen eine biographisch bedingte Außenseiterrolle in der Gesellschaft. Distanziert von ,herkömmlichen' kulturellen und sozialen Handlungsorientierungen bestärken sie ihre Außenseiterrolle, indem sie sich mit einem anderen „Outsider“ (Hecht-El Minshawi 1988: 237) zusammen tun. „Sie befinden sich im Anderssein und Anderswo“ (ebd.: 238). Diese Außenseiterrolle kann unterschiedlich ausgeformt sein, sie kann zum Beispiel aus häufigen Umzügen in der Kindheit oder auch aus einer politischen Motivation heraus entstanden sein. Relevant ist jedoch ihrer Ansicht nach, dass die Frauen sich schon vor ihrer Beziehung aus der deutschen Kultur gelöst hätten, der Akkulturationsprozess hat also schon vorher eingesetzt.

Betrachtet man die unterschiedenen Konzepte der Partnerwahltheorien fällt auf, dass in diesen Intermarriage als Sonderfall angesehen wird, der erklärungsbedürftig ist. Analysen, die mit dem Heiratsmarkt als bestimmendem Faktor der Partnerwahl operieren, untersuchen die Wahrscheinlichkeiten bzw. Möglichkeiten der Begegnung zwischen potentiellen Ehepartner(inne)n verschiedener natio-ethno-kultureller Herkunft. Sind solche fields of availability vorhanden, ist die Wahrscheinlichkeit der Intermarriage eine Frage der Alternativmöglichkeiten. Intermarriage wird in dieser Perspektive als ,zweite Wahl‘ konzipiert, als Ausweichmöglichkeit, wenn andere, homogame Partnerschaften aus verschiedenen Gründen nicht möglich sind. Auch das Konzept des kompensatorischen Austauschs oder die Summationstheorie versuchen zu erklären, warum Personen unter bestimmten Umständen eine heterogame Ehe in Betracht ziehen, um ihren sozialen Status entweder zu erhöhen oder zu stabilisieren. Die Ehe erscheint hier als Mittel zum Zweck, der über eine homogame Ehe nicht erreicht werden kann.

Ein Phänomen nehmen beide Ansätze nicht in den Blick: Heiratsmigration, wie es für mein Sample den Regelfall darstellt. Paare, die sich im Herkunftsland des einen kennen lernen, dann aber beschließen im 
Herkunftsland des anderen zu heiraten. Sie bewegen sich damit außerhalb des Heiratsmarktes des jeweiligen Aufnahmelandes, konstituiert sich der Heiratsmarkt doch aus Personen, die durch Migration oder Geburt dauerhaft in diesem Land leben. Ansätze, die sich mit biographischen Erfahrungen und gegenseitigen Imaginationen auseinandersetzen, sind daher für meinen Zugang gewinnbringender. Diese machen auf Prozesse der Partnerwahl aufmerksam, die von mehr strukturiert werden als von räumlichen Gelegenheitsstrukturen und sozialstatistischen Daten. Anlehnungen an Tauschtheorien finden sich auch hier, allerdings nicht als zweite Wahl verstanden, sondern als explizite Entscheidung für eine bestimmte Person. Nicoles kulturalisierende Deutungen, ihr Bedürfnis nach einem ,passenden“ Mann und ihre Faszination hinsichtlich des altmodischen Verhaltens von Carlos lassen sich durchaus durch die oben genannten vermuteten Positionsvorteile und -verschiebungen erklären, die sie in einer binationalen Beziehung sieht. Hier handelt es sich nicht um einen sozialen Status, einen Zugang zu einem anderen Milieu, eine wirtschaftliche Absicherung o.ä., welche sie durch die Heirat mit einem Mexikaner erreichen will. Vielmehr geht es um die biographische Anschlussfähigkeit spezifischer Lebenskonzepte. Imaginationen über , andere‘ Kulturen und über vermutete oder bekannte Geschlechterverhältnisse gewinnen so in biographischen Erzählungen in der Retrospektive als handlungsleitende Motive Relevanz. ${ }^{12}$

Für die genauere Betrachtung des Kontextes, innerhalb dessen die Biographiegestaltung binationer Paare in Deutschland stattfindet, werden im Weiteren zum einen die rechtlichen Rahmenbedingungen vorgestellt, mit denen sich binationale Paare in Deutschland auseinandersetzen müssen. Anders als für mononationale Paare spielen Gesetze und verwaltungsrechtliche Vorschriften eine entscheidende Rolle. Auch das female breadwinner Modell ist eine Folge diskriminierender Vorschriften. Zum

12 Die Perspektive von Hecht-El Minshawi ist noch aus einem anderen Grund höchst problematisch: Mindestens implizit ist bei ihr die Annahme einer falsch verlaufenen Sozialisation der deutschen Frauen vorhanden. Würden sich die Frauen wegen biographischer Faktoren nicht als Außenseiterinnen fühlen, wäre es für sie nicht notwendig, einen Mann mit muslimischer Religionszugehörigkeit zu heiraten. Eine solch defizit-orientierte Sichtweise lässt diese Frauen als Opfer ihrer Sozialisation zurück und die Männer als gesellschaftliche Außenseiter, die nur über andere Außenseiter Zugang zur deutschen Gesellschaft finden können. Darauf lässt auch ihre Formulierung schließen, dass sich die Frauen mit anderen „Outsidern“ zusammentun. Da sie nur die Frauen interviewt hat, kann sie für die Männer keine biographische Distanzierung analysieren, sondern schließt von ihrer Religionszugehörigkeit auf ihren gesellschaftlichen Status. 
anderen lassen sich anhand von Ergebnissen qualitativer Studien zur Intermarriage relevante Aushandlungsfelder und spezifische Lebensumstände dieser Paare beschreiben. Dazu wird vorrangig auf deutsche Studien zurückgegriffen. Zusätzlich sind jedoch auch Studien aus der Schweiz, Österreich und den USA relevant. Die älteren deutschen Studien sind allerdings häufig aufgrund ihrer methodologischen Anlage als nicht unproblematisch einzuschätzen. Ihre Ergebnisse sind insofern vor allem als sensibilisierende Hinweise auf mögliche relevante Phänomene $\mathrm{zu}$ betrachten, weniger als Vorlagen für die eigene empirische Untersuchung oder als feststehende Hypothesen.

\section{Heterogame Irritationen: binationale Paare in der deutschen Gesellschaft}

Heterogame Irritationen lassen sich auch in den eingangs zitierten Interviewpassagen finden: Carlos fühlt sich zwar in Deutschland wohl, muss sich aber von vornherein mit seinem unsicheren Aufenthaltsstatus auseinandersetzen. Und auch in Bezug auf die Unterschiede in Geschlechterbeziehungen formuliert Nicole Irritationen, die sie kulturell deutet: Carlos sei es als Mexikaner nicht gewohnt, dass eine Frau für ihn bezahlt. Die Lebenssituation der beiden, Carlos hat zu dem Zeitpunkt kein eigenes Einkommen, lässt aber eine andere Lösung nicht zu. Spezifische Lebensumstände bestehen für dieses binationale Paar also auf (mindestens) zwei Ebenen: zum einen auf der gesellschaftlichen und rechtlichen Ebene, die Anforderungen und Rahmenbedingungen schaffen, die ungleich denen mononationaler Paaren sind. Zum anderen auf der Ebene der individuellen Aushandlungspraxis, die für Nicole (auch) durch kulturalisierende Wahrnehmungen strukturiert sind.

\section{Ver-rechtliche Subjekte}

Im Unterschied zu binationalen Paaren können mononationale heterosexuelle deutsche Paare in Deutschland vergleichsweise unbehelligt vom Wissen um Gesetze und rechtliche Regelungen leben. Nur im Ausnahmefall bzw. zu besonderen Gelegenheiten (wie Heirat, Scheidung, etc.) werden Lebensentwürfe durch Gesetze und staatliche Vorgaben strukturiert. Dies ist bei binationalen Paare anders: Sie werden von Beginn ihrer Beziehung an mit rechtlichen Einschränkungen und Besonderheiten konfrontiert. Die Wahl der Beziehungsform, verheiratet oder nicht, zusammenlebend oder in getrennten Wohnungen, kann nicht von dem Paar selbst getroffen werden. Wie Betty de Hart in ihrer Studie über binatio- 
nale Paare in den Niederlanden zeigt, sind häufig insbesondere die niederländischen Partner(innen) überrascht vom Verlust der für selbstverständlich gehaltenen Selbstbestimmtheit:

„As a consequence of their choice of spouse, rights that they have always thought self-evident, like the right to determine the form of family life (cohabition or marriage) and the right to live this family life in the Netherlands, became contested rights“ (de Hart 2003: 279).

Die Schwierigkeiten der Paare beginnen schon vor der Eheschließung. Es ist nicht möglich, eine Aufenthaltsbewilligung, die Vorstufe der Aufenthaltsgenehmigung, für eine Liebesbeziehung ohne ,offiziellen' Status der Beziehung zu erlangen (vgl. hierzu und im Folgenden Kugler 2000). ${ }^{13}$ Aufenthaltsbewilligungen zum Zwecke der Erwerbsarbeit werden seit dem Anwerbestopp in den 70er Jahren nicht mehr erteilt. Es gibt zwar einen umfangreichen Katalog von Ausnahmen, der aber bezieht sich fast ausschließlich auf Hochqualifizierte wie Wissenschaftler, die für eine begrenzte Zeit in Deutschland forschen wollen, Künstler oder Sportler (Kugler 2000: 44ff). Den Paaren, die in Deutschland leben wollen, bleibt nur die Beantragung eines Touristenvisums, welches für drei Monate gültig ist. ${ }^{14}$ Neben der Überprüfung der Kostendeckung durch den oder die Gastgeber(in) wird dabei insbesondere die Rückkehrbereitschaft des/der Touristen/in überprüft. ${ }^{15}$ Einen Rechtsanspruch gibt es nicht, Touristenvisa werden durch die Konsulate nach Ermessen ausgestellt oder verweigert (vgl. dazu Kriechhammer-Yagmur/PfeifferPandey/Saage-Fain et al. 2001: 54f). Zudem wird das Visum in der Regel nicht verlängert, so dass die Ausreise nach drei Monaten verpflich-

13 „Die Aufenthaltsgenehmigung wird als Aufenthaltsbewilligung erteilt, wenn der Aufenthalt nur zu einem bestimmten Zweck dient und zeitlich befristet ist. Eine unbefristete Verlängerung ist nicht möglich. Der Aufenthaltstitel der Aufenthaltsbewilligung soll eine Verfestigung $\mathrm{zu}$ einem rechtlich gesicherten Aufenthalt verhindern“ (Kugler 2000: 25). Hier wird noch auf das alte Ausländergesetz Bezug genommen, da sich die Paare der vorliegenden Studie mit diesem auseinandersetzen mussten. Das neue Zuwanderungsgesetz bringt allerdings hinsichtlich der Stellung binationaler Paare kaum Erleichterung (vgl. dazu die Stellungnahme der IAF auf ihrer Homepage).

14 Ausgenommen von der Visumspflicht sind lediglich Mitglieder der EUStaaten und des Europäischen Wirtschaftsraumes, der USA, Honduras, Monaco, San Marino und der so genannten Positivstaatler, unter die Staatsangehörige von Ländern wie Japan, Israel, Südkorea und Polen fallen (Kugler 2000: 37).

15 Die besuchte Person muss dafür eine Erklärung ausstellen, die die Verpflichtung zur Übernahme sämtlicher durch den Besuch entstehender Kosten wie Unterhalts- oder Krankenkosten beinhaltet. 
tend ist. Eine nicht-eheliche Lebensgemeinschaft in Deutschland zu führen, wie es für deutsch-deutsche Paare selbstverständlich ist, ist insofern so gut wie unmöglich. Ausnahmen bilden Beziehungen während des Studiums. Hier kann zumindest bis Ende des Studiums die Beziehung unbehelligt von rechtlichen Einschränkungen geführt werden, da der Aufenthaltsstatus während der Ausbildung (in der Regel) gesichert ist. Alle anderen Paare stehen sehr schnell vor der Wahl die Beziehung entweder als Fernbeziehung zu führen, sie zu beenden oder sie, im wahrsten Sinne des Wortes, zu ,legalisieren'. Insbesondere Paare, die sich im Urlaub kennengelernt haben, sind so gezwungen schon nach wenigen Wochen oder gar Tagen weitreichende Entscheidungen zu treffen. Vor dem Hintergrund dieser Restriktionen hat die Debatte um die so genannte Scheinehe, die Eheschließung zum Zweck der Aufenthaltsermöglichung die IAF daher auch zum Begriff der ,Zwangsehe ' bewogen. Mit diesem Begriff soll der Zwang zur Eheschließung deutlich gemacht werden, der die meisten binationalen Paare zur schnellen Heirat drängt. Dass eine rasche Eheschließung mit sehr kurzer vorheriger Beziehung von Standesbeamten jedoch als Indiz für eine Scheinehe angesehen werden soll, lässt die Absurdität der Situation deutlich werden. Oft bleibt den Paaren gar nichts anderes übrig, als rasch zu heiraten, genau diese Schnelligkeit führt jedoch auf Seiten der Standesämter zur kritischen Überprüfung der Beziehung.

Die Vorbereitung zur Eheschließung in Deutschland ist für deutsche Paare in aller Regel relativ unkompliziert und eine freudige Angelegenheit, die vom Standesbeamten in angemessener Art und Weise vollzogen werden soll. Auch dies ist für binationale Paare, die in Deutschland heiraten wollen, anders. Sie müssen in oft langwierigen und komplizierten Prozeduren notwendige Papiere beschaffen, deren Übersetzung und die Genehmigungen der Übersetzung organisieren. Die Vorbereitung wird zum einen durch die zuständigen Standesbeamten erschwert, die häufig unklare oder unvollständige Angaben machen, so dass sich häufig erst im Laufe der Beantragung die vollständigen Anforderungen herausstellen. Zum anderen aber erschwert auch die Situation in den Herkunftsländern die Beantragung. Migrant(inn)en aus Ländern, in denen Geburtenregistrierung nicht selbstverständlich ist oder in denen durch politische Entwicklungen Unterlagen verschwinden oder vernichtet werden, haben oft große Schwierigkeiten, die benötigten Dokumente zu beschaffen.

Ein Antrag auf Familienzusammenführung muss immer dann gestellt werden, wenn ein Partner oder eine Partnerin nach der Heirat aus dem Ausland wieder einreisen will. Dies ist zum Beispiel dann der Fall, wenn im Ausland (möglicherweise im Herkunftsland des/der einen Part- 
ners/in) geheiratet wurde. Es kann aber auch dann passieren, wenn beispielsweise im Zuge eines Asylanerkennungsverfahrens der Asylantrag abgelehnt wurde und der/die Partner(in) „vollziehbar ausreispflichtig“ ist. Dann ist eine Ausreise ,selbst nach einer erfolgten Eheschließung im Bundesgebiet notwendig, um die anschließende Aufenthaltserlaubnis zu erhalten" (Kriechhammer-Yagmur/Pfeiffer-Pandey/Saage-Fain et al. 2001: 73). Je nach Fall und Ermessensentscheidung der Behörden muss in diesem Fall auch ein Antrag auf Familienzusammenführung gestellt werden. Diese Anträge machen einen immer größeren Anteil an binationalen Eheschließungen aus: So stieg, wie schon gezeigt, die Zahl der erteilten „Visa zum Zweck des Ehegattennachzugs nach Deutschland“ in den Jahren 1996 bis 2003 von 15.651 auf 33.222 (Beauftragte der Bundesregierung für Migration 2004: 14). Die Bearbeitung der Genehmigung zur Familienzusammenführung kann unter Umständen bis zu zwei Jahre dauern: „Die deutschen Auslandsvertretungen überprüfen dabei alle nichtdeutschen Dokumente auf ihre Glaubwürdigkeit, setzen Vertrauensanwälte hierfür ein und befragen intensiv das Paar über die Gründe ihrer Partner(-innen)wahl“" (Kriechhammer-Yagmur/Pfeiffer-Pandey/Saage-Fain et al. 2001: 111). Die Zeitdauer und auch die Intensität der Überprüfung sind dabei allerdings aufgrund der verschiedenen Zuständigkeiten und der Ermessensentscheidungen der zuständigen Beamt(-inn)en sehr unterschiedlich. Eine von mir interviewte Frau hat eine Familienzusammenführung aus Kuba innerhalb weniger Wochen erfolgreich hinter sich gebracht. ${ }^{16}$ Heiraten im Ausland, insbesondere in Dänemark, ist daher nach den Erfahrungen der IAF eine beliebte Alternative. In der Regel ist kein explizites Anerkennungsverfahren der Eheschließung notwendig. Schwierigkeiten entstehen eher auf der Ebene der Aus- und Einreise in die Bundesrepublik: Personen mit Duldung oder Asylbewerber(innen) gefährden durch die Ausreise die Möglichkeit der sofortigen Wiedereinreise. Solche Paare müssen vom Ausland aus einen Antrag auf Familienzusammenführung stellen (Kriechhammer-Yagmur/ PfeifferPandey/Saage-Fain et al. 2001: 111). Schwierig wird es ebenfalls bei der Heirat mit einem Migranten, der sich ohne Aufenthaltsstatus in Deutschland aufhält. Hier droht die Abschiebung bei der Beantragung der Eheschließung.

16 Um die Existenz einer ,Scheinehe' ausschließen zu können, werden in diesem Verfahren intime Fragen gestellt, deren Beantwortung zur Erlaubnis oder Verweigerung der Einreise führen können: „If a young couple says that they do not want any children, although they have no health reason for not having a family, permission to marry is always refused" (Breger 1998: 140). 
Wenn die Paare verheiratet sind und in Deutschland leben, werden sie mit den verschiedenen Formen der Aufenthaltsgenehmigungen und den damit verbundenen Arbeitserlaubnissen oder -verboten konfrontiert. Einerseits gelten für ausländische Ehepartner einer Deutschen dabei im Unterschied zu anderen Ausländergruppen begünstigende Regelungen. Wurde einmal die Familienzusammenführung erfolgreich beantragt, erhalten ausländische Ehepartner eine auf drei Jahre befristete Aufenthaltserlaubnis. ${ }^{17}$ Im Unterschied zu anderen Migrant(inn)en brauchen sie dann keine Arbeitsgenehmigung. ${ }^{18}$ Andererseits aber lassen sich eine Reihe von institutionellen und gesellschaftlichen Schranken identifizieren, die der Arbeitsaufnahme des Migranten entgegenstehen. Wesentliches Instrument der Exklusion ist dabei die fehlende Anerkennung ausländischer Berufsausbildungen und akademischer Grade. Ausländer, die kein entsprechendes Zertifikat vorweisen können, werden in Deutschland als Arbeitssuchende „ohne Berufsausbildung“ eingestuft: „Mögliche Arbeitgeber müssen in diesem Fall persönlich von den beruflichen Fähigkeiten überzeugt werden“ (Kriechhammer-Yagmur/Pfeiffer-Pandey/Saage-Fain et al. 2001: 200). Sind Berufsabschlüsse nachweisbar, wird überprüft, ob sie auf das deutsche System übertragbar sind und damit anerkannt werden können. Häufig müssen aber Teile einer Ausbildung nachgemacht werden, dies gilt auch für Studienabschlüsse. Diese rechtlichen Vorgaben führen dazu, dass das female breadwinner Modell regelmäßiger, in zeitlich unterschiedlicher Länge, Teil der Lebenssituation der meisten binationalen Paare ist.

\section{Kulturalisierungen und Neuentdeckungen: Studien zu Lebenswirklichkeiten binationaler Paare}

Hinsichtlich der rechtlichen Einbindung erleben binationale Paare starke Einschränkungen, die erheblich in die persönliche Lebensplanung einwirken und zum Teil auch in Frage stellen. Neben diesen staatlichen und rechtlichen Irritationen stellt auch der Alltag im Zusammenleben Irritationen bereit. Wenn sich binationale Familien selbst als ,bunt' bezeichnen (vgl. Krüger 1997), beziehen sie sich dabei vor allem auf die Lebensbedingungen und Lebensumstände, die solche Beziehungen und

17 Voraussetzung dafür ist, dass die Ehe in der Bundesrepublik geführt wird und eine eheliche Lebensgemeinschaft besteht (vgl. Kugler 2000: 70). Nach drei Jahren konnte, unter ähnlichen Voraussetzungen, eine unbefristete Aufenthaltserlaubnis beantragt werden, nach fünf Jahren eine Aufenthaltsberechtigung, die höchste Stufe der Aufenthaltsverfestigung.

18 Für EU-Angehörige gelten andere, begünstigende Regeln. Hier gibt es eine Reihe von Abkommen, die die gegenseitige Anerkennung von Zeugnissen und Abschlüssen regeln. 
Familien von homogamen Familien unterscheiden. Das Zusammentreffen unterschiedlicher Biographien, unterschiedlicher Sozialisationsbedingungen und unterschiedlicher Lebenspläne findet sich in jeder Familie, jede Familie muss ihre eigene Wirklichkeit herstellen und divergente Erwartungen integrieren können. Die Besonderheit binationaler Paare und Familien wird demgegenüber jedoch zum einen in der ,größeren Unterschiedlichkeit aufgrund der unterschiedlichen kulturellen Bezugsgruppen gesehen. Zum anderen wird diesen Paaren, aufgrund der homogenen Struktur der Gesellschaft, die heterogenen Beziehungen skeptisch bis ablehnend gegenüber steht, ihre Besonderheit als ,anderes' Paar deutlich bzw. deutlich gemacht. Die (wenigen) Studien zu binationalen Paaren in Deutschland analysieren solche Besonderheiten. Vor der Referierung der Ergebnisse wird jedoch in einem Exkurs ein Ordnungsversuch der existierenden Studien vorgenommen: Forschung zu binationalen Paaren ist eng mit politischen und gesellschaftlichen Entwicklungen verflochten, zum Teil sogar als explizite politische Auftragsforschung entstanden. Durch die folgende Unterteilung der Forschung in drei Phasen lassen sich daher Schwerpunkte in der wissenschaftstheoretischen Ausrichtung festmachen, die den gesellschaftspolitischen Diskurs begleiten (vgl. zur Diskursentwicklung in der Migrationsforschung Bukow/Heimel 2003).

\section{Exkurs: im Auftrag des Gesundheitsamtes - Forschung über Intermarriage in Deutschland}

Die existierende Forschung seit dem II. Weltkrieg lässt sich in drei Phasen unterteilen. In die erste Phase der 50er bis 70er Jahre, in der der Schutz der Frauen vor den Gastarbeitern im Vordergrund stand. In die zweite Phase bis Mitte der 90er Jahre, in der die kulturellen Differenzen zwischen den Paaren im Fokus der Analysen standen, und in die derzeitige Phase, in der schwerpunktmässig Potentiale und Ressourcen binationaler Paare und Familien untersucht werden.

\section{Gastarbeiter und der Schutz der Frau (1955 - 1978)}

Buba, Ueltzen und Vaskovics weisen in ihrer statistischen Untersuchung zum Verlauf der Intermarriage Rate einen deutlichen Anstieg ab 1955 nach. Dieser Anstieg ist im wesentlichen Folge der zunehmenden (zu 2/3 männlichen) Migration nach Deutschland im Zuge der Anwerbung so genannter Gastarbeiter. 1955 wurden 16.000 Ehen zwischen deutschen Frauen und ausländischen Männern geschlössem und nur knapp 4000 Ehen zwischen deutschen Männern und ausländischen Frauen (vgl. ebd 1984: 425). Intermarriage war bis in die 80er Jahre vorrangig ein weibliches Phänomen. Statistisch gesehen wurden diese Ehen also für das Heiratsverhalten der Deutschen nach dem II. Weltkrieg relevanter, und auch in der Gesellschaft fanden sie bald (negative) Aufmerksamkeit. Ebenso wie die deutsche Gesellschaft in den 50er Jahren nicht auf dauerhaft sesshafte Migranten eingerichtet war, hatte niemand ernsthaft mit 
dem Phänomen der binationalen Ehen zwischen den männlichen Gastarbeitern bzw. den Besatzungsmächten und den ,inländischen' Frauen gerechnet. Die wissenschaftliche Forschung nimmt die Angst vor der ,Überfremdung' der Gesellschaft in ihren Arbeiten auf. Es existieren nur wenige Studien aus der Zeit, diese beschäftigen sich ausgiebig mit der Frage der sozialen Deprivation und mit der Angst um den ,guten Ruf ' der deutschen Frauen. Diese Art der Forschung knüpft im Grunde nahtlos an die Haltung in der Gesellschaft gegenüber Mischehen und insbesondere gegenüber Ehen deutscher Frauen mit ausländischen Männern an. Während Ehen deutscher Männer mit ausländischen Frauen zumindest toleriert, wenn nicht sogar mit einem gewissen Verständnis angesehen wurden, wurde und wird das ,Fremdgehen' der Frauen als Verrat an der deutschen Kultur und der deutschen Nation verstanden. 1978 lehnten laut einer Umfrage 79\% der deutschen Bevölkerung eine solche Ehe ab (Wolf-Almanasreh 1979: 6). ${ }^{19}$ Oder, um es sehr plastisch mit den Worten eines Leserbriefes zu sagen: „Jawohl, Flittchen sind sie und nichts anderes. Eine anständige Frau hängt sich nicht an einen so fremden, hergelaufenen, schwarzen Kerl. Diese Frauen sind die Totengräber des Deutschen Volkes" (zit. n. Wolf-Almanasreh 1979: 4). In zwei wissenschaftlichen Dokumenten jener Zeit spiegelt sich diese Haltung wider. Zum einen wurde im Auftrag eines Gesundheitsamtes eine sozialstatistische Untersuchung von „Ehen zwischen deutschen und ausländischen Arbeitnehmern" (Schramm/Steuer 1965) durchgeführt. Die Untersuchung war als Folgeuntersuchung konzipiert, da 1964 in einer Studie festgestellt wurde, dass ,ausländische Arbeitnehmer gehäuft in ,asoziale' deutsche Familien einheiraten, und dass die Kinder aus diesen Ehen zu einem grossen Prozentsatz wiederum ,asoziale“ Züge aufweisen“ (Kienecker 1993: 63). Die Untersuchung konzentrierte sich also auf die Frage der sozialen Devianz solcher Ehen. Letztendlich ließ sich diese These jedoch nicht aufrechterhalten. Die Forscher fanden keinerlei Hinweise auf verstärkte Asozialität oder soziale Deprivation. In der Anlage subtiler ist der Ratgeber von Becker, der 1974 überarbeitet und weiterentwickelt in seiner zweiten Auflage erschien. Unter dem Hinweis der Informationsvermittlung werden junge Frauen selbst, aber auch die Mütter der potentiell bedrohten jungen Mädchen, angesprochen. Der Ratgeber beschäftigt sich vorrangig mit den Folgen für deutsche Frauen, die in ein fremdes, insbesondere „orientalisches“ Land auswandern wollen. ${ }^{20}$ Interessant ist diese populärwissenschaftliche Veröffentlichung dabei insbesondere hinsichtlich zweier Merkmale: Zum einen bestimmen diskriminierende, verallgemeinernde Darstellungen und gleichzeitig eine große Furcht vor dem Islam und fremden Ländern Stil und Aufbau des Ratgebers: „Wir fassen zusammen: Die größten Schwierigkeiten entstehen dadurch, dass in den Ländern des Südens und in den Ländern des Orients ganz andere Sitten herrschen und dass die Bindungen der Religion viel stärker sind als bei uns" (Becker 1974 : 23). Zum anderen wird ein Bild implementiert, welches das deutsche, freie und gleichberechtigte Gesellschaftssystem dem rückständigen, patriarchalen, starren muslimischen Gesellschaftssystem gegenüber stellt:

19 Die Ablehnung einer Intermarriage ist zu dem Zeitpunkt unter der Bevölkerung in Deutschland ohne deutschen Pass bei weitem nicht so stark. 1985 antworteten knapp 50\% zustimmend auf die Frage, ob sie eine oder einen Deutsche(n) heiraten würden (Bundesministerium für Arbeit 1995: 242, vgl. auch Nauck 2001).

20 ,Orientalisch ' wird hier nicht weiter differenziert. Es bleibt der Phantasie der Leserin überlassen, sich konkrete Länder vorzustellen. 
„Keinem Zweifel kann es unterliegen, dass in allen südlichen Ländern der Frau eine geringere persönliche Freiheit zugestanden wird als in Deutschland. Fest steht auch, dass in den Ländern des Islams der Einfluss der Verwandtschaft und der religiösen Überzeugung viel stärker ist als bei uns" (Becker 1974: 16) ${ }^{21}$ Die muslimischen Männer, die unglücklicherweise auch noch als junge und zudem häufig auch noch außerordentlich ,attraktiv[e]“ (ebd.: 13) Männer nach Deutschland kommen, versuchten durch Bitten, Schmeicheln und Täuschen die deutschen Frauen auf ihre Seite und in ihr Heimatland zu ziehen. Becker ist dabei aber durchaus verständnisvoll. Schließlich seien die Randgebiete Europas und der Orient kulturell anders geprägt und zudem noch wirtschaftlich rückständig. So sei es auch kein Wunder, dass den Menschen dort das Wissen um die Würde der Frau fehle. Anlasten könne man es daher auf keinen Fall den Männern, vielmehr seien es faktische Tatsachen, mit denen zu rechnen sei und die die Entscheidung für oder gegen eine Ausreise entscheidend beeinflussen sollten. ${ }^{22}$

\section{Integrationsbemühungen und das Leben in zwei Kulturen (1979 bis Mitte der 90er)}

Intermarriage in Deutschland bleibt zu diesem Zeitpunkt ein vorwiegend weibliches Phänomen. Binationale Ehen werden vor allem mit Migranten aus den Anwerbeländern geschlossen. In dieser Zeit konzentriert sich die ,Ausländerpädagogik' auf die Aufgabe der Integration (gemeint ist Assimilation) von Migranten und Ausländern in die deutsche Gesellschaft. Nach der ersten Phase der ,Pathologisierung' durch offizielle Stellen und Sozialämter finden jetzt zunehmend die Erfahrungen von betroffenen Frauen Eingang in die wissenschaftliche Literatur. Die durchgeführten Untersuchungen sind daher häufig Diplom- und andere Abschlussarbeiten von Frauen, die selber in einer binationalen Partnerschaft leben. Die Arbeiten sind fast ausschließlich qualitativ angelegt und scheinen zum Teil eher die persönliche Lebenssituation der Forscherin wiederzugeben, als dass sie Auskunft geben über Lebensumstände und Situationen binationaler Paare: „Am Beispiel der Arbeit Streckers (1982) wur-

21 Dies erscheint grade vor dem Hintergrund der derzeit wieder auflebenden Debatte um die Demokratietauglichkeit muslimischer Migrant(inn)en interessant. Deutschland als Musterland für die Gleichberechtigung der Geschlechter zu sehen, spricht allerdings eher für eine ideologische Verblendung.

22 Hinsichtlich der rechtlichen ,Behandlung' der Ehen stellten sich Besonderheiten insbesondere bei Ehen deutscher Frauen mit Migranten dar. Das deutsche Familienrecht, entstanden aus dem BGB von 1900, stellt auf den Mann als Familienoberhaupt ab. Bis 1953 verloren deutsche Frauen automatisch ihre Staatsangehörigkeit bei einer Heirat mit einem Nicht-Deutschen, auch wurde ihnen danach die Ausreise in das Heimatland des Mannes regelmäßig nahe gelegt. Kinder aus solchen Verbindungen bekamen nur die Staatsangehörigkeit des Mannes. Ebenso wie der Frau die Ausreise nahe gelegt wurde, war die Aufenthaltserlaubnis des Mannes stets nur eine provisorische. Ständig von der Ausweisung bedroht, ohne eine langfristige Perspektive, sahen viele Paare nur in der Ausreise die Möglichkeit einer stabilen Lebensperspektive. Die ausländischen Frauen deutscher Männer hingegen konnten bis 1970 durch eine einfache Erklärung vor dem Standesbeamten die deutsche Staatsangehörigkeit erwerben (vgl. dazu MüllerDincu 1981: 39f). 
de gezeigt, dass persönliche Betroffenheit und zusätzlich schlechte methodische Arbeitsweise den Untersuchenden nur bemerken lassen, was er voraussetzt" (Thode-Ahora 1999: 89, Herv. d. Verf., s. a. Kienecker 1993: 67 für einen Überblick über die Untersuchungslage in dem Zeitraum). Strukturiert sind diese Studien alle durch einen Integrationsbegriff, der kulturelle Differenz als Ansatzpunkt seiner Bemühungen versteht und, aufbauend auf dieser Differenz, den Versuch unternimmt, identitäre Unterschiede anzuerkennen und zu akzeptieren: „Es entsteht der Mythos vom Leben und Zerbrechen zwischen zwei Kulturen“" (Bukow/Heimel 2003: 17).

Als zwei Studien, die den Ansprüchen wissenschaftlicher Forschung eher genügen und zugleich als charakteristisch für diese Phase in der Forschung anzusehen sind, können Hecht-El Minshawi, veröffentlicht 1988, und Kienecker, veröffentlicht 1993, genannt werden. Die ältere Untersuchung, Hecht-El Minshawi, steht stellvertretend für Studien, die die Gründe für die Partnerwahl in der gemeinsamen Exklusion der Partner(innen) aus der Gesellschaft sehen. Beide Partner(innen) seien in ihren jeweiligen Kulturen Außenseiter und ,Fremde', dies verbinde sie und schaffe die Basis für die Partnerschaft. Intermarriage ist also kein selbstverständlicher Bestandteil einer Einwanderungsgesellschaft, sondern wird als Ausnahme gesehen, deren Gründe in der mangelnden Eingliederung der Frauen in der Gesellschaft liegen. Die Partner(innen) selbst sind Repräsentant(inn)en ihrer Kultur: „In der hautnahen Auseinandersetzung des Paares steckt zugespitzt der schärfste Druck zweier kulturell verschiedener individueller gesellschaftlicher Normen und Wertesysteme" (Hecht-El Minshawi 1988: 9). Kienecker hingegen untersucht nicht primär die Gründe für die Partnerwahl, sondern interessiert sich für die Ausgestaltung der Beziehung und mögliche Konfliktfelder in der Beziehung. ${ }^{23}$ Problemfelder wie die Sprache, die Erziehung der Kinder, Koch- und Essensgewohnheiten oder Religionsausübung werden identifiziert und als potentielle Krisenherde in der Beziehung erkannt. Symptomatisch ist diese Untersuchung vor allem hinsichtlich des theoretischen Hintergrundes. Auch Kienecker geht von der Begegnung zweier Menschen aus, die durch ihre Kultur definiert und damit sozusagen als Repräsentanten der Kultur, als verlängerter Arm, ihres jeweiligen Herkunftslandes gelten können. In dieser rollentheoretischen Ausrichtung ist für die individuelle Aneignung und Abweichung wenig Platz, vielmehr dienen die Eigenschaften der beteiligten Partner(innen) als Illustration der Verhältnisse der jeweiligen ethnischen Gruppen. Die Begegnung zweier Menschen unterschiedlicher Sozialisation wird dabei zugespitzt auf die Begegnung zweier Kulturen und damit auf ein essentialistisches Verständnis von Kultur und kultureller Identität. Neben dieser Art von Forschung findet sich ebenfalls eine zunehmende Anzahl von Studien, die sich mit bikulturellen und bilingualen Kindern beschäftigt. Insbesondere die Frage der Bilingualität wird unter dem Gesichtspunkt der identitären Verunsicherung bzw. des Gewinnes durch Bilingualität diskutiert. So geht Adler (1977) davon aus, dass Bilinguale weit mehr Anpassungsprobleme (problems of personal adjustment) haben als Monolinguale. Er folgert, dass Bilinguale sozusagen „Diener zweier Herren sind“ (in Aleemi 1991:130). Dagegen steht Forschung, die positive Korrelationen zwischen Zweisprachigkeit und kognitiver Entwicklung bei Kindern nachweisen (vgl. Lüdi 1996: 236).

23 Methodisch problematisch ist dabei, dass sie nur die (deutschen) Frauen interviewt und so kein Gesamtbild der Beziehung zeichnen kann. Ein Manko, das im Übrigen die meisten Studien teilen. 


\section{Binationale Paare als Prototypen für die multikulturelle Gesellschaft und die De-Konstruktion des ,Fremden' (seit Mitte der 90er)}

Nach wie vor wird in der Forschung zu binationalen Paaren vorwiegend mit qualitativen Methoden gearbeitet. Zusätzlich dazu werden jedoch seit 1996 Daten zur Familienzusammenführung erhoben, so dass die statistische Datenlage differenziertere Analysen zulässt. ${ }^{24}$ Die Erhebungen zeigen den Anstieg der Anträge auf Ehegattenzusammenführung, ein Indiz für die zunehmende Zahl von Heiratsmigration. Zusätzlich steigt auch die Zahl deutscher Männer, die über nationale Grenzen hinweg heiraten.

In der methodologischen Anlage der Forschungen setzt sich ein Paradigmenwechsel durch, der in der (qualitativen) Migrationsforschung ebenfalls zu einer Neuorientierung geführt hat: „Der Konstruktivismus avanciert hier als interpretativ geprägter Ansatz zur Antithese gegenüber dem überkommenden normativ geprägten Defizitansatz, nach dem Differenz nur als Defizit, ja als Devianz, und Integration nur als prolongierte Assimilation denkbar ist" $(\mathrm{Bu}-$ kow/Heimel 2003: 26). Kennzeichnend für eine solche wissenschaftstheoretische Richtung ist sicherlich die Studie von Barbara Waldis (Waldis 1998). Sie untersucht unter kommunikationstheoretischer Perspektive die Herstellung und Aufrechterhaltung von Fremdheit und Differenz in binationalen Beziehungen. Der und die ,Fremde' werden also nicht mehr vorausgesetzt, vielmehr kann in der (Re-)Konstruktion nachvollzogen werden, wie Grenzziehungen ausgehandelt, verschoben und neu definiert werden. Natio-ethno-kulturelle Zugehörigkeiten werden bei dieser Art von Untersuchung nicht als vorgängige identitäre Strukturen verstanden, sondern in ihrer Wirkungsweise und ihren Funktionen analysiert. Auch der gesellschaftspolitische Wandlungsversuch hin zu einem Einwanderungsland Deutschland durch die rot-grüne Regierung ab 1998, machte auf die Notwendigkeit aufmerksam, Migrant(inn)en nicht auf eine scheinbare kulturelle Differenz zurückzuverweisen, sondern vielfältige Lebens- und Zugehörigkeitskonzepte als konstitutiven Bestandteil Deutschlands zu verstehen. Dabei werden binationale Paare, sozusagen in Umkehrung des Defizitansatzes, häufig genug als Prototyp einer postmodernen Gesellschaft idealisiert, als freie Individuen, deren romantische Liebe alle Hindernisse überwindet. So sieht Beck-Gernsheim in binationalen Paaren „moderne Romeos und Julias“, die nicht den Gesetzen „,von Herkunft und Heimat" folgen, sondern „dem Gesetz ihres Herzens“ (Beck-Gernsheim 2001: 120). Auch ohne solche Romantisierungen setzten die Interessensverbände der binationalen Paare besondere Hoffnungen in interethnische Beziehungen und deren Abkömmlinge für die Herausforderungen einer multikulturellen Gesellschaft. Insbesondere bilingual und bikulturell aufgewachsene Kinder werden als besonders handlungsfähig im Hinblick auf die Anforderungen einer multikulturellen Gesellschaft verstanden (vgl. z.B. Khounani 2000). Gründe dafür seien in der größeren Ambiguitätstoleranz der Kinder zu sehen, da sie von Beginn der primären Sozialisation an mit mehreren und zum Teil sich widersprechenden Bezugs- und Ordnungssystemen konfrontiert werden. Ob diese Hoffnungen wirklich tragen und ob nicht eine Ideologie die nächste ablöst, wird sich noch zeigen. Wesentlich allerdings scheint die neue Perspektive zu sein, die Migrationsforschung im Allgemeinen und Forschung zur Intermarriage im Be-

24 Nach wie vor basieren die vorhandenen Statistiken allerdings auf den Nationalitäten der beteiligten Personen. Da diese Staatsangehörigkeitswechsel und doppelte Zugehörigkeiten nicht erfassen, bleibt die Dunkelziffer groß. 
sonderen einnimmt. Nicht mehr die Defizite und die nachzuholenden Modernisierungsprozesse werden unterstellt (und damit in der Regel auch rekonstruiert). Der Blick wird vielmehr auf Potentiale und kreative Handlungsweisen der beteiligten Personen gerichtet.

Wie der Exkurs zeigt, hat sich die wissenschaftstheoretische Grundlage der Studien zu binationalen Paaren deutlich verändert. Auch die inhaltlichen Foki verschieben sich. Ältere Studien beschäftigen sich weitestgehend mit der Ausgestaltung der Ehe und der Untersuchung und/oder Definition von spezifischen Problembereichen in der Partnerschaft (z.B. Müller-Dincu 1981; Scheibler 1992; Kienecker 1993). Neuere Studien dagegen analysieren, oft mit Hilfe rekonstruktiver Verfahren, in welcher Weise Kultur als Deutungsmuster in der Beziehung verwendet wird, wie in Aushandlungen (natio-ethno-kulturelle) Differenzen bestätigt oder verschoben werden, oder inwiefern sich durch binationale Partnerschaften neue Geschlechterkonstruktionen beobachten lassen (z.B. Larcher 2000; Schlehe 2000b, a; Waldis 2001; Molnár 2004). Die Referierung der Studien folgt daher dieser Zweiteilung: Zuerst werden die von den älteren Studien als Konfliktfelder identifizierten Bereiche dargestellt, bevor in einem zweiten Schritt die Ergebnisse der neueren Studien hinsichtlich einiger Phänomenfelder skizziert werden.

Den oben vorgestellten Studien ist sicherlich zugute zu halten, dass sie binationalen Paaren als Forschungsgegenstand Aufmerksamkeit zukommen lassen und dass insbesondere Kienecker die spezifische Situation der „Deutschen Frauen mit ausländischem Partner“ (so der Untertitel ihrer Studie) analysiert. ${ }^{25}$ Das Interesse der deutschen Wissenschaftler(innen) an ,abweichenden Lebensbedingungen “ und den daraus resultierenden Besonderheiten in der Lebensführung hat dabei jedoch eine theoretische und thematische Engführung und eine Fokussierung auf kulturelle Differenzen zur Folge. Die Partnerschaft ist insgesamt durch das „Leben in zwei Kulturen“ (Kienecker 1993: 95) strukturiert, welches größere und konfliktträchtigere Aushandlungen insbesondere in Bereichen wie dem Umgang mit der Herkunftsfamilie, Erziehung der Kinder und Geschlechterbeziehungen nahe legt und erwarten lässt. Die Autor(inn)en identifizieren solche Konfliktfelder und ordnen sie jeweils dem Innen- oder Außenbereich der Beziehung zu. Während mit dem Innenbereich der Bezug der Partner(innen) aufeinander gemeint ist, ist mit

25 Anfang der 90er Jahre existiert eine solch spärliche Anzahl an Untersuchungen zu dieser Gruppe, dass sie von einer „Tabuisierung“ des Themas spricht (Kienecker 1993: 8). 
dem Außenbereich die Auseinandersetzung des Paares mit seiner näheren sozialen Umgebung (Familie, Freunde, Behörden etc.) gemeint.

Im Innenbereich der Beziehung entstehen Konflikte insbesondere durch verschiedenartige Erziehungsvorstellungen, durch unterschiedliche Modelle der Geschlechterbeziehungen und durch die Konsequenzen des „,biographischen Bruchs“ (Scheibler 1992: 107), der durch die Migration ausgelöst wurde. Scheibler, die binationale Paare aus der EU interviewt hat, stellt dabei u. a. fest, dass durch die Migration der/die autochthone Ehepartner(in) eine zentrale Rolle für den sozialen Kontakt zugeschrieben bekommt, die überfordernd und belastend wirken kann (vgl. ebd.: 109). Kienecker, die sich wie Hecht-El Minshawi für die Partnerwahlmotivation der Frauen interessiert, kommt zu dem Ergebnis, dass sich die Motivation aus einer Verbindung von kompensatorischem Tauschmodell (soziale und rechtliche Absicherung der Frau vs. ,andere' Kultur des Mannes) und dem Wunsch nach einem anderen Leben und anderen Erfahrungen, als sie deutsche Männer zu bieten haben, speist (vgl. Kienecker 1993: 105). Beide, Scheibler und Kienecker, interpretieren die Aushandlungen der Partner(innen) vor dem Hintergrund eines Arrangement-Modells, welches von Tseng (in Scheibler 1992: 44, Tseng/Mc Dermott/Maretzki 1984, auch in McFadden/Moore 2001) entwickelt wurde. Drei mögliche Arrangements lassen sich in einer binationalen Ehe finden: das einseitige Arrangement, in welchem sich ein(e) Partner(in) an die andere Kultur anpasst und diese die dominierende Rolle zugesprochen bekommt, der Kompromiss, durch welchen in verschiedenen Bereichen der Partnerschaft jeweils einer der beiden Kulturen Geltung verschafft wird, und schließlich das kreative Arrangement, in welchem aus beiden Kulturen eine eigenständige dritte konstruiert wird. Die Mehrzahl der untersuchten Paare, so das Ergebnis beider Studien, praktiziere jedoch ein einseitiges Arrangement und orientiert sich an der Kultur des Landes, in dem sie leben. Ein Kompromiss sei zudem häufiger im außerpartnerschaftlichen Bereich als im Innenbereich der Beziehung zu finden.

Hinsichtlich der Auseinandersetzungen im außerpartnerschaftlichen Bereich (Verwandte, Freunde, Nachbarn, Behörden) werden die starken Ressentiments und Ablehnungen deutlich, denen binationale Paare ausgesetzt sind. Insbesondere auf Seiten der Eltern der (deutschen) Frau begegnen die Paare deutlicher Abwehr. Hier scheint, liest man die Interviewzitate in Kieneckers Studie, das Tauschkonzept der Partnerwahltheorien ins Alltagsbewusstsein gesunken zu sein. Die Wahl eines ,Ausländers' wird als sozialer Abstieg gesehen, der in den meisten Fällen 
durch den finanziellen oder sozialen Status des Partners eben nicht aufgewogen wird: „Aber am Anfang waren sie eher skeptisch, aus gesellschaftlichen Gründen. [...] Weil ich mein Klassenziel auch nicht erreicht habe. Weder bin ich Doktorin geworden, noch habe ich einen geheiratet" (zit. n. Kienecker 1993: 81). Insbesondere beim ,offensichtlich“ migrationsgezeichneten Partner wird die Ablehnung der Familie stärker, ist hier doch auch noch für die nächste Generation mitzudenken, in der die ,Mischlingskinder' alltägliches Anschauungsmaterial für die ungebührliche Entscheidung der Tochter liefern. Neben diesen familiären Reaktionen, in ähnlicher Weise reagieren auch Freunde und weitere Verwandtschaft, stellt sich insbesondere der Bereich der rechtlichen und institutionellen Auseinandersetzung als relevantes Konfliktfeld dar. Wie schon skizziert, sind binationale Paare verstärkt staatlichen und rechtlichen Eingriffen ausgesetzt. Dies gilt im Übrigen, wie Scheibler feststellt, auch für die Paare aus der EU. Auch hier sei die Diskriminierung durch Behörden sehr groß (vgl. ebd. 1992: 115ff). In diesem Kontext finden alltägliche Diskriminierungserfahrungen und rassistische Begegnungen statt, welche die Paare in ihrer Lebensführung behindern und ihnen zudem immer wieder den Status der , anderen` Beziehung zuschreiben.

Die zitierten Studien erlauben einen Blick in relevante Konfliktfelder einer binationalen Partnerschaft. Die Felder scheinen sich nicht so sehr von denen mononationaler Paare $\mathrm{zu}$ unterscheiden. Auch diese müssen sich mit der Reaktion ihrer Eltern auf ihre Partnerwahl auseinandersetzen, und die Ablehnung der (zukünftigen) Schwiegertochter durch die (zukünftige) Schwiegermutter ist Gegenstand unzähliger Witze. Vielmehr liegt die Unterscheidung in der Aufladung der Konstellation mit kultureller Differenz, und zwar sowohl durch die Paare selbst als auch durch ihre soziale Umgebung. Über solche Konstruktionen lässt sich durch diese Studien allerdings nur wenig erfahren. Wie es dazu kommt, dass sich die Paare in Kieneckers und Scheiblers Studien als Vertreter(innen) ihrer Kultur verstehen, die ihre Konfliktfelder als Kulturkonfliktfelder deuten, bleibt im Dunkeln. Sind solche Handlungsorientierungen und Deutungsmuster konstant oder verändern sie sich oder entstehen sie gar erst im Lauf der Beziehung? Zwar lässt sich einiges über Partnerwahlmotive erfahren (die ,andere' Kultur als Attraktion und Gegenentwurf zu Deutschland), jedoch bleiben die Konkretisierung dieses Gegenentwurfes und seine Folgen für den weiteren Biographieverlauf unbeachtet. Unklar bleibt mit einer solchen Perspektive beispielsweise, welche Folgen Nicoles hohe Erwartungen an Carlos' ,männliches“ Verhalten haben und welche Funktion die von ihr formulierten gender-Konzepte und Konzepte von natio-ethno-kultureller $\mathrm{Zu}$ gehörigkeit in dem gemeinsamen Leben in Deutschland (und der Kon- 
frontation mit dem female breadwinner Modell) haben werden bzw. in welcher Weise Nicole auch zu Modifikationen gezwungen sein wird. Das Verständnis von Kultur und Ethnizität als grundlegende, Handlungen strukturierende und Differenzen stabilisierende Kategorien verhindert den Blick auf interaktive Prozesse, in denen kulturalisierende Begründungen erst im Zusammenspiel mit anderen Kategorien wie gender und/oder Milieu an Bedeutung gewinnen bzw. durch hybride oder grenzüberschreitende Handlungsorientierungen modifiziert und abgelöst werden. Solche Grenzüberschreitungen kann die bis jetzt vorgestellten Literatur zu binationalen Paaren nicht oder nur kaum analysieren, greift doch schon die Konzeption der Analyse auf feststehende Kulturkonzepte zurück, anstatt sie aus dem Verständnis (und dem sich ändernden Verständnis) der Paare zu rekonstruieren. ${ }^{26}$ Neuere Studien über binationale Paare, wie zum Beispiel Breger/Hill 1998; Waldis 1998; Larcher 2000; Schlehe 2000b; Molnár 2004, verfolgen jedoch einen solchen rekonstruktiven Ansatz, der kulturelle Differenzen nicht als gegeben voraussetzt, sondern sie in ihrer Funktion und Bedeutung für spezifische Aushandlungsprozesse analysiert. Die Ergebnisse der Studien haben auch für meine Untersuchung wichtige Hinweise geliefert und lassen sich in vier Phänomenbereiche aufteilen.

\section{,Liebe‘ als Chiffre}

Liebe, wie Beck-Gernsheim empathisch betont, überspringt Klassen, Rassen und nationale Grenzen (ebd. 2001, vgl. in ähnlicher Weise auch Root 2001). In einer solch romantisierenden Vorstellung bekommt ,Liebe' die entscheidende Funktion als Movens der Entscheidung für die Beziehung. Die Chiffre der ,Liebe“ kann allerdings komplexere Prozesse und Orientierungen verbergen, die bei der Entscheidung für oder gegen eine Beziehung Relevanz zugesprochen bekommen. Larcher, der binationale Paare in Österreich interviewt hat, verweist bei der Analyse der „Inszenierungen des Kennenlernens“ (ebd. 2000: 49ff) auf unterschiedliche, kulturell gebundene Begegnungsrituale, die Begegnungen zwischen potentiellen Liebespaaren vorstrukturieren. Solche Rituale lassen sich bei binationalen Paaren nicht ungebrochen weiterführen. Ob die Irritation aber als positiv oder als negativ erlebt wird, entscheidet auch über den Fortbestand der Beziehung. Nicht nur Anfangsrituale sind Bestandteil des Liebesmotivs, auch Imaginationen über die , anderen` Män-

26 Vgl. dazu auch die Examensarbeit von Burian 1999, die Ehen mit Schwarzund Weißafrikanern einerseits und Österreichern andererseits untersucht, um Unterschiede hinsichtlich der Ehezufriedenheit und der Geschlechtsrollenausprägung zu analysieren. 
ner bzw. Frauen strukturieren den Entscheidungsprozess, wie Schlehe am Beispiel von Reiseromanzen zwischen westlichen Frauen und indonesischen Männern analysiert:

„Bei den Frauen tritt deutlich das romantisierte Stereotyp des fremden Anderen zutage. Sie tragen die spezifisch westlichen Imaginationen vom ,Orient ${ }^{6}$ mit sich, und die Männer suchen aus strategischen Gründen, diesen Orientbildern und den damit verbundenen Projektionen nachzukommen“ (Schlehe 2000a: 134).

Solche Imaginationen können zum einen, wie bei Schlehe, als kulturalisierte Stereotype, als Attraktivität des Fremden an Bedeutung gewinnen. Ein solcher Konstruktionsprozess lässt sich ja auch bei Nicole beobachten. Gerade weil Carlos Mexikaner ist, hebt er sich positiv von den deutschen Männern ab. Zum anderen können aber auch imaginierte Gemeinsamkeiten Basis für die Beziehung sein. So zeigt Molnár in ihrer Untersuchung binationaler Paare in Deutschland, dass insbesondere der/die nicht-deutsche Partner(in) den/die Partner(in) der eigenen Kultur zuordnet:

„Die nicht-deutschen Partner hingegen beziehen sich in ihrer Erklärung der Attraktivität des Partners nicht auf die deutsche Kultur. Sie ordnen den Partner vielmehr der eigenen Kultur zu oder nennen dessen Interesse an fremden (nicht-deutschen) Kulturen als Grund für seine Attraktivität“ (Molnár 2004: 108). ${ }^{27}$

Molnár verweist darauf, dass die Verwendung von kulturellen Zuschreibungen selten eindeutig ist. Vielmehr verschwimmt Kultur, je nach Themenfeld oder Interaktionssituation dient es mal als Referenzgrösse, mal nicht (ebd.: 111). Ähnlich sind hingegen die Deutungsmuster innerhalb einer Partnerschaft. Die Paare scheinen also in ihrer gemeinsamen Wirklichkeitskonstruktion auch kulturelle Deutungsmuster auszuhandeln und anzugleichen. ${ }^{28}$

27 Ähnliche Ergebnisse finden sich auch bei Breger und Hill, wobei hier eher einzelne Merkmale (wie gemeinsame religiöse Überzeugungen, ein gemeinsamer (hoher) Bildungsstatus oder eine emanzipative Einstellung) der Paare betont werden (Breger/Hill 1998: 19ff.). Dies erinnert nicht zufällig an die Partnerwahltheorien, wie sie ja auch von Hecht-El Minshawi vorgestellt werden.

28 Diese Beobachtungen kann sie auch deshalb machen, weil sie beide Partner interviewt hat. Der Notwendigkeit, die Perspektive beider Partner aufzunehmen, tragen die meisten der neueren Studien Rechnung. Auch Waldis, Larcher und Schlehe haben beide Partner interviewt. 


\section{Gender und Kultur als verflochtene Zusammenhänge}

Für meine eigene Untersuchung höchst relevant ist die Beobachtung der Studien, dass gender und Kultur in einem engen, interdependenten $\mathrm{Zu}-$ sammenhang verstanden werden müssen.

„Ilse Lenz formuliert die These, dass Subjekte in interkulturellen Begegnungen „konfigurative Handlungsstrategien“ verfolgen, d.h. dass sie einzelne Elemente der Geschlechtsrollen, ihres ethnischen Hintergrundes usw. auswählen, kombinieren oder aber herunterspielen und vermeiden können (Lenz 1996: 219), wobei in diesem konfigurativen Handeln neue kulturelle Mischformen und Synthesen gebildet werden: das Hybride. Dieses kann also weder einheitlich sein, noch ist es zufällig zusammengesetzt, sondern es oszilliert - innerhalb spezifischer politischer Bedingungen - entsprechend den Interessen der Subjekte und der jeweiligen diskursiven Konstruktion ihrer ethnischen und Geschlechtsidentität“ (Schlehe 2000b: 10).

Solche engen Zusammenhänge kann auch Molnár analysieren. Gender wird kulturspezifisch analysiert, d.h. dass Unterschiede in geschlechtsspezifischem Verhalten oder in den Erwartungen auf kulturelle Unterschiede zurückgeführt werden (vgl. Molnár: 111). Zu einem ähnlichen Schluss kommt auch Waldis: „Die kulturelle Differenz überdeckt andere Differenzen“" (Waldis 1998: 298). Auf die große Bedeutung, die gerade das Zusammenspiel von natio-ethno-kultureller Zugehörigkeit und gender hat, verweisen auch Diehm und Radtke:

„Theoretikerinnen, die feministische Theoriebildung und Ethnizitätsforschung verknüpfen, können die wechselseitig sich verstärkende Essentialisierung freilegen, wenn die beiden Kategorien Geschlecht und Ethnizität im Spiel sind. Nicht einfach Verdopplung, sondern ein komplexes Ineinandergreifen lässt nach dem Geschlecht die holistische Ethnizitätskonstruktion zu einer quasi naturhaften Größe werden und umgekehrt: Die Geschlechtskonstruktion wird im Zusammenspiel mit der fixierten Kulturdifferenz-Annahme einem Härtungsund Festigungsprozeß unterworfen, auf den sich Goffmanns Begriff der „,institutionellen Reflexivität“" passend anwenden lässt. Die Verankerung von Geschlecht in einem als defizitär beschriebenen kulturellen Kontext bringt die Essentialismen zum Zirkulieren: Die Geschlechterdifferenz nährt sich von der Kultur-Differenz, diese erhält durch jene eine zusätzliche Verankerung“ (Diehm/Radtke 1999: 95, Herv. d. Verf.). ${ }^{29}$

29 Larcher sieht in dieser Verflechtung aber auch den Vorteil der Reflexionsmöglichkeit. Wenn das „Quasi-Natürliche“ als kulturell entlarvt werden kann, ist Raum für Emanzipation und Veränderungen (ebd. 2000: 134). 


\section{Funktionshorizont von Kultur: Ermöglichung und Begrenzung}

Anders als in den früheren Studien suggeriert, ist der Funktions- und Bedeutungshorizont von Kultur und natio-ethno-kultureller Zugehörigkeit sehr viel komplexer und widersprüchlicher. Grundsätzlich lässt sich zeigen, wie Waldis deutlich macht, dass es sich bei natio-ethnokultureller Zugehörigkeit um eine Kategorie handelt, welche bei binationalen Partnerschaften quasi , auf der Hand liegt‘ und von den Partner(inne)n als nahe liegend verstanden und eingesetzt wird. In dieser großen Bedeutung, die die Kategorie für die Paare hat, können zwei differenzierende Dimensionen analysiert werden. Zum einen schränkt die Kulturalisierung der Beziehung Reflexions- und Diskussionsmöglichkeiten ein. Waldis zeigt, wie Konflikte von den Paaren als Kulturkonflikte interpretiert werden, insbesondere wenn es eher ,um ein Machtverhältnis des Paares [geht] als um kulturelle Unterschiede“" (ebd. 1998: 190). Insofern wird häufig, insbesondere wenn die Beziehung beendet ist bzw. wenn Probleme in der Beziehung auftauchen, der Vorwurf an den/die andere(n) Partner(in) gerichtet, dass Kultur als „metakommunikative Ressource“ (Molnár 2004: 108) eingesetzt wird. Kultur und Kulturdifferenz kann also in allen kommunikativen Zusammenhängen als grundlegendes Deutungsmuster genutzt werden, Verständigungen und insbesondere Veränderungen sind so allerdings kaum möglich. Diese Form der Kulturalisierung sieht auch Larcher als Problem einer binationalen Beziehung an (vgl. ebd. 2000: 92). Auf der anderen Seite bietet die Kategorie ,Kultur‘ auch die Möglichkeit zu Reflexionsanstößen und für Veränderungen. Etwas als in seiner Herkunft kulturell zu verstehen, heißt, es als sozial und nicht-natürlich einzuordnen: „Es muss zunächst als kulturell erkannt werden, damit es auch als veränderbar erkannt wird“ (Larcher 2000: 12). Breger/Hill können zudem zeigen, dass die Kulturalisierung der Partner(innen) im Laufe der Beziehung abnehmen kann: „Otherness is therefore no longer percieved in the partner, but is externalized and seen only in the outside culture" (ebd. 1998: 11). Gerade der Bezug auf die Kategorie ,Kultur' muss damit also nicht in einer Sackgasse enden, sondern kann produktiv gewendet werden, indem sich von der Herkunftskultur distanziert wird und eigene neue Räume und Wege eröffnet werden. Einen solchen Prozess der Entkulturalisierung sieht auch Waldis als notwendige Bedingung für eine erfolgreiche Beziehung:

„Die Analyse interkultureller Kommunikation bei binationalen Paaren [...] hat gezeigt, dass ein Gegeneinander der Herkunftskulturen und - im Fall von Paarbeziehungen - der Geschlechter als Perspektive nicht fruchtbar ist. Es 
müssen für eine binationale Lebenswelt eines Paares gewisse gemeinsame Kodes vorhanden sein“ (ebd. 1999: 190).

\section{Idealisierung und Verfremdung}

Ein letztes Phänomen, welches sich sozusagen ,quer ${ }^{6} \mathrm{zu}$ den bisher genannten Feldern bewegt, ist die Beobachtung des Spannungsverhältnisses zwischen der Idealisierung und der Verfremdung des/der Partners/Partnerin. Die Auflösung dieses Spannungsfeldes zur einen oder zur anderen Seite scheint zum Teil abhängig zu sein vom Verlauf der Beziehung:

„Sich auf Fremdes einzulassen, in andere Lebensformen einzutauchen, ist bis zu einem gewissen Grad reizvoll. Wenn sich dann aber entweder das Fremde als ,zu fremd“ erweist oder aber, ganz im Gegenteil, sich auch dort die allzu vertrauten Muster in den Geschlechterbeziehungen ausmachen lassen, dann erscheint auch der Freund nicht mehr als der ganz Besondere, als die große Ausnahme. [...] Der ,ganz Andere“ wird zum ,ganz Typischen““ (Schlehe 2000a: 135).

Auch Molnár analysiert solche Verläufe. Andere, fremde Kulturen sind solange spannend und können als Gegenfolie zu Deutschland gelesen werden, solange sie ,aus einer touristischen Sichtweise wahrgenommen“ werden.

„Sobald aber das Zusammenleben mit einer fremden Kultur verpflichtend und unausweichlich wird, da es zur alltäglichen Routine der Partnerschaft wird, schwindet die Attraktivität und die problematischen Seiten treten hervor“ (ebd. 2004: 108).

Die Referierung der Literatur zur Intermarriage zeigt, dass durch den Paradigmenwechsel in der wissenschaftstheoretischen Ausrichtung differenzierte Analysen von Konstruktionsprozessen möglich werden. Die Ergebnisse der Studien wie auch die Auseinandersetzung mit den rechtlichen Rahmenbedingungen wurden daher auch für die Entwicklung des Interpretationsmodells der vorliegenden Untersuchung herangezogen. Sie haben die Aufmerksamkeit insbesondere auf zwei Ebenen geschärft: Zum einen wurde die Notwendigkeit deutlich, sowohl den Innenbereich einer Partnerschaft als auch den Außenbereich in die Analyse mit einzubeziehen. Irritationen können nicht nur in der binationalen Beziehung entstehen, natio-ethno-kulturelle Zugehörigkeit kann nicht nur in der Partnerschaft als Relevanzkategorie herangezogen werden. Insbesondere in der Interaktion mit dem sozialen Nahraum, den Herkunftsfamilien 
oder den Freund(inn)en, kann die ,Ungebührlichkeit‘ der Entscheidung virulent werden. Hier ist auch die Funktion des/der Partners/Partnerin als gatekeeper zu beachten, der/die einerseits zwischen seinem/ihrem Herkunftsland und der/dem Ehepartner(in) vermittelt, andererseits durch diese Vermittlung aber auch die Rolle als Interpret übernimmt und entscheidet, welches Wissen zugänglich ist. Neben diesem doppelten Bezug machen vor allem die neueren Studien auf Dimensionen aufmerksam, die bei (Re-)Konstruktionen von gender und natio-ethno-kultureller $\mathrm{Zu}$ gehörigkeit relevant sein können. So scheint zum Beispiel die Beobachtung der Dechiffrierung von ,Liebe“ durchaus fruchtbar zu sein, um gegenseitige Imaginationen und Sehnsüchte analysieren zu können. Dies setzt in gewisser Weise an der Frage der Gemeinsamkeiten der beiden Partner(innen) an. Auch hier ist die Partnerwahl nicht zufällig. Dennoch unterscheidet sich ein solcher Zugang deutlich von der Analyse HechtEl Minshawis, die beide Partner(innen) als Außenseiter konzipiert. Denn gefragt wird nach gegenseitigen Konstruktionen, die gerade die individuelle biographische Gestaltung bei gleichzeitiger Partizipation in der Aufnahmegesellschaft, in meinem Fall Deutschland, oder auch, wie in den Analysen von Schlehe, während einer Reiseromanze ermöglichen. Interessant für die vorliegende Untersuchung ist also die biographische Anschlussfähigkeit der Beziehung. Dafür ist es zudem notwendig, beide Partner(innen) zu interviewen. Gegenseitige Imaginationen und Konstruktionen lassen sich nicht aus einem Interview heraus erklären, vielmehr müssen Wirklichkeitskonstruktionen in der und durch die Beziehung analysiert werden. Des Weiteren ist die Analyse des Verlaufs der Bearbeitungsprozesse notwendig, um Bedeutungszuschreibungen und -verschiebungen der Kategorien beobachten zu können. Sonst läuft man leicht Gefahr, Konstruktionen als statisch und essentiell zu verdoppeln.

Die weitere Konkretisierung des Aufmerksamkeitsfokus erfolgt im nächsten Abschnitt mit der Beschreibung der biographischen Zumutungen im Migrationsprozess, die für mein Sample Bedeutung haben. Dazu gehört in besonderer Weise das female breadwinner Modell, welches sich als Konkretisierung oder Zuspitzung der Zumutung verstehen lässt. 


\section{Zweite Annäherung: Folgen von Migrationsprozessen}

Pablo: „Und ich bearbeitete meine Arbeiten nochmals, ich layoutete alles neu, ich machte auch eine CD-Rom, um sie vorzustellen zu können, [...] ich hatte auch nicht viel Erfolg, weil sie schrieben immer zurück: Ja, meine Arbeit wäre sehr gut, sehr schön, aber es gäbe zur Zeit keine Möglichkeit, und sie wünschten mir immer viel Glück für die Zukunft. $\mathrm{Na}$ ja, es ging nicht. Ja, sehr schwer... [...] ich dachte, was kann noch gemacht werden? Vielleicht kann ich hier in Deutschland studieren, mit einem Aufbaustudium weitermachen - - ich setzte mich mit der, mit der Universität in Verbindung - und sie fragten: Aus welchem Land kommst du? Ja, aus Mexiko, aus Lateinamerika. Hm, hm, nein, was du studiert hast, ist hier nicht gültig, es wird hier nicht akzeptiert. [...] Nein, nein, sie akzeptieren es nicht. Und ich sagte, ich habe eine Diplomarbeit, ich war an der Universität, das ist ein hohes Niveau, es ist eine, eine Universität. Kann es nicht als technisches Niveau akzeptiert werden? Nein, nein, die Diplomarbeit interessiert uns nicht und was weiß ich. Na ja, an der Universität hatte ich keine Chance, aber ich bat doch um ein Papier für die Bestätigung, [...] für die Anerkennung hier von A-Stadt oder Deutschland. Ja, sie gaben es mir aber für die Fachhochschule, das ist dieses praktisches Niveau und ich nahm es an. Das heißt, ich habe ein Papier, ja, wo steht: Sie sind Architekt, Architekt, aber sie müssen dazu Mex. schreiben, das heißt mexikanischer Architekt. [...] Und, ähh, ich fing auch damit an, für mich als Architekt Werbung zu machen. Zurzeit mache ich Arbeiten ohne, ohne, wie heißt es? Ohne, dass ich dafür bezahlt werde“ (Pablo Marmani Rodriguez 12/9 - 13/13).

Pablo kommt mit einem abgeschlossenen Architekturstudium und ersten Berufserfahrungen nach Deutschland. Hier aber macht er die Erfahrung, dass seine berufliche Qualifikation auf dem freien Arbeitsmarkt, trotz seiner aufwendigen Bewerbungen, nicht gefragt ist. Alternative Überlegungen, wie ein Zusatzstudium, lassen sich ebenfalls nicht realisieren: Sein universitärer Abschluss wird nicht als gleichwertig anerkannt, die einzige Möglichkeit bestünde in einem erneuten Studium. Auch die Anerkennung seines Universitätsabschlusses als Fachhochschulabschluss kann er nicht durchsetzen. Zwar kann er seinen Abschluss offiziell führen, muss diesen aber durch einen Zusatz als mexikanischen Abschluss kennzeichnen. Zurzeit arbeitet er unentgeltlich, um Berufserfahrung zu sammeln und um auf diese Weise weitere berufliche Kontakte knüpfen zu können.

Solche Migrationsgeschichten wie Pablos sind weder für das von mir in den Blick genommene Sample noch für Migrationsprozesse allgemein ungewöhnlich. Migration verändert soziale, berufliche und kulturelle Positionierungen von Subjekten. Ressourcen, die bislang nutzbringend verwandt werden konnten, soziales Kapital wie Kontakte oder kulturel- 
les Kapital wie Abschlüsse oder Berufserfahrungen, verlieren an Wertigkeit bzw. werden, wie in Pablos Fall, einem Dequalifizierungsprozess unterworfen. ${ }^{30}$ Entwicklungsprozesse und Veränderungen wie die hier beschriebenen lassen sich als Zumutungen verstehen, die die Migranten und ihr soziales Umfeld vor die Herausforderung stellen, neue Handlungsmuster zu entwickeln und bisherige Selbstverständlichkeiten in Frage zu stellen. Zumutungen meint dabei nicht, dass sie zwangsläufig ungewollt oder unerwartet geschehen, wohl aber sind es strukturelle Folgen von Migrationsprozessen, die Eingang in die Bearbeitungen finden. Welche Bedeutung diese Zumutungen für die Biographiegestaltung des Einzelnen bekommen und in welcher Weise sie bearbeitet werden, lässt sich damit jedoch noch nicht klären.

In dem folgenden Kapitel wird der Fokus auf einige der Zumutungen gerichtet, mit denen sich das Sample auseinandersetzt. Dazu gehört, neben der beruflichen Eingliederung, insbesondere das Phänomen des female breadwinner Modells. Es lässt sich als ,Konkretisierung der Zumutungen' konzeptualisieren, da es ein gemeinsames Charakteristikum der Paare ist und im Gegensatz zur geschlechtlichen Arbeitsteilung in Deutschland steht. Auch in den Herkunftsländern der Männer meines Samples ist die Trennung zwischen Reproduktions- und Produktionsbereich geschlechtspezifisch.

\section{Zumutungen im Migrationsprozess}

Ein Migrationsprozess bedeutet für die beteiligten Subjekte Positionsverschiebungen auf unterschiedlichen Ebenen. Migranten kommen aus ihren bisherigen Positionen und Verflechtungen in neue Verortungszusammenhänge. In der Regel sind diese Re-Positionierungen mit Dequalifizierungsprozessen verbunden, insbesondere bei Arbeits- oder Heiratsmigrant(inn)en, wie sich ja auch bei Pablo zeigt. ${ }^{31}$ Die beschriebenen

30 Der Prozess der Verschiebung und Entwertung von Ressourcen lässt sich auch mit Bourdieus Konzept der Kapitalsorten erklären (vgl. zum Überblick Schwingel 1998): Alle vier Sorten - das kulturelle, ökonomische, soziale und symbolische Kapital - sind konstitutiv durch das sie umgebende soziale Feld strukturiert. Zwar sind alle Sorten im begrenzten Umfang auf andere Felder und in andere Gesellschaften übertragbar, doch sind dabei Entwertungsprozesse zu erwarten, wie sich beim Versuch zeigt, kulturelles Kapital in Form von Bildungsabschlüssen zu übertragen, oder beim Verlust der sozialen Netzwerke durch Migration. Auch Weiß weist auf die Delegitimierung und Abwertung hin, die die Kapitalsorten ethnischer Minderheiten erfahren (vgl. Weiß 2001).

31 Dies ist sicherlich bei hochqualifizierten Migranten anders. So findet ein solcher Migrationsprozess für Personen, die zum Beispiel für mehrere Jah- 
rechtlichen Schwierigkeiten machen zum Teil tiefgreifende Um- und Neuorientierungsleistungen der Subjekte erforderlich.

Die Zumutungen, denen sie durch den Migrationsprozess begegnen, werden auf verschiedenen Ebenen sichtbar. Besonders deutlich zeigt sich die Entwertung der bisherigen Ressourcen auf der Ebene der Bildungs- und Arbeitsmarktintegration. Deutschlands Arbeitsmarktstruktur ist, das ,gehört zu den gut gesicherten Erkenntnissen der sozialwissenschaftlichen Arbeitsmarktforschung“ (Konietzka/Kreyenfeld 2001: 267), stark formalisiert, und zwar auf zweierlei Ebenen: Nicht nur sind Zugänge zu Berufen und Ausbildungen eng an Ausbildungen und zertifizierte Kenntnisse gebunden, auch sind Übergänge zwischen verschiedenen Berufs- und Tätigkeitsbereichen ohne entsprechende Weiter- und Umschulungen sehr schwierig. ${ }^{32}$ Insbesondere in den klassischen Ausbildungsberufen sind fachfremde Einsatzmöglichkeiten nur sehr begrenzt möglich. Migranten, die auf dem deutschen Arbeitsmarkt Fuß fassen wollen, stehen also vor vielfachen Schwierigkeiten: Die Anerkennung der Ausbildungen ist in der Regel nur sehr schwer bis gar nicht möglich, oft werden nur Teile einer Ausbildung anerkannt, wenn z.B. das iranische Abitur als Fachabitur für das Studium von technischen Berufen an einer Fachhochschule anerkannt wird. Zudem sind, auch wenn die Zertifikate anerkannt werden, die Abschlüsse häufig nicht mit dem deutschen Arbeitsmarkt kompatibel. Konietzka/Kreyenfeld weisen in ihrer Analyse der Verwertbarkeit von Berufsabschlüssen von Aussiedler(inne)n nach, dass diesen trotz des gleichen Bildungsniveaus der Einstieg in den Arbeitsmarkt nur unter bestimmten Umständen möglich ist:

„Migranten können aus ihren Hochschulabschlüssen generell Kapital schlagen, berufliche Abschlüsse zahlen sich jedoch nur bei einer Beschäftigung in den Berufen aus, in denen sie ausgebildet wurden. Aussiedler mit beruflichem Ausbildungsabschluss, die in Deutschland nicht im erlernten Beruf tätig wer-

re im Auftrag ihrer Firma in ein anderes Land gehen, dort einen ,Relocator' zur Seite gestellt bekommen und Wohnung, Arbeitsplatz und Kindergartenplatz zur Verfügung gestellt bekommen, sicherlich unter ungleich günstigeren Voraussetzungen statt. Auch fehlende oder mangelnde Deutschkenntnisse müssen hierbei kein Hindernis sein, wenn die Firmenund Kundenkommunikation auf Englisch stattfindet.

32 Dies gilt in abgeschwächter Form auch für Hochschulabschlüsse. Insbesondere bei den ,weichen" Wissenschaften wie Sozial- und Geisteswissenschaften ist allerdings der formale Abschluss relevanter als die Fachrichtung. Letzterer Aspekt wird insbesondere in der aktuellen Debatte um die ,soft skills‘ hochgehalten, die Absolventen dieser Fachrichtungen vielfältiger einsetzbar machen und einem berufsspezifischen Ausschluss entgegenwirken. 
den (können), werden dagegen im Großen und Ganzen so behandelt, als verfügten sie über keine berufliche Ausbildung. Ihre Arbeitsmarktchancen unterscheiden sich nicht von denen ungelernter Arbeitskräfte“ (ebd. 2001: 279, Herv. d. Verf.).

Demzufolge arbeiteten 1998 zwar 65\% der Westdeutschen, aber nur $41 \%$ der Aussiedler(innen) mit Berufsabschluss in dem erlernten Beruf (ebd.: 279). Diese Analyse, die in ihrer strukturellen Aussage auch für Arbeits- oder Heiratsmigranten Gültigkeit besitzt, weist auf die erheblichen Schwierigkeiten hin, denen Migranten bei der Eingliederung in den Arbeitsmarkt begegnen. Der Dequalifizierungsprozess ist für Aussiedler und andere Migranten insgesamt ein weit realeres Risiko als für Deutsche. Aus der oben erwähnten starken Formalisierung des deutschen Arbeitsmarktes ergibt sich ein weiteres Problem. So finden sich in anderen Ländern durchaus Lebenswege mit Aufstiegsmöglichkeiten ohne formalisierte Ausbildungen. Die Berufsbiographie eines von mir interviewten tunesischen Migranten verdeutlicht einen solchen Verlauf. Er hat nach der siebten Klasse die Schule abgebrochen und danach in verschiedenen Bereichen gejobbt. Nach dem Ableisten seines Militärdienstes hat er wiederum gejobbt und ist nach einiger Zeit in eine größere Stadt mit hohem Touristenanteil gezogen. Dort konnte er sich, dank guter Englischkenntnisse, vom Kellner bis zum Junior Manager eines Hotels hocharbeiten. Hier allerdings bricht die berufliche Erfolgsgeschichte ab. Nach der Heiratsmigration nach Deutschland findet er keine Stelle bzw. muss als ungelernter Arbeiter ohne Deutschkenntnisse und ohne zertifizierte Arbeitserfahrungen wieder von vorne anfangen. Schwierigkeiten bestehen also insbesondere für im deutschen Sinne geringqualifizierte Migranten ohne Schulabschluss oder Ausbildung. Daneben können auch soziale Ressourcen nicht ohne weiteres aktiviert und wie gewohnt genutzt werden. Zwar sind Migrationen nicht Prozesse einzelner Individuen, sondern meist eingebettet in soziale und familiäre Netzwerke, jedoch ist hier ebenfalls mit Re-Positionierungs- und in der Regel Abwertungsprozessen der Kapitalien zu rechnen. ${ }^{33}$ So besitzt Pablo zwar

33 Hier allerdings gilt es zwischen dem Nutzen des sozialen Kapitals bei Migrationsentscheidungen, insbesondere Kettenmigration, und dem Nutzen nach der Ankunft in der Aufnahmegesellschaft zu differenzieren. Bei ersterem wird von einigen Forscher(inne)n auf die große Bedeutung sozialen Kapitals hingewiesen, als ,ability to command scarce means by virtue of membership in social structures" (Faist 1995 in Haug 2000: 22, s. a. Haug 1997). Die Eingebundenheit in soziale Netzwerke und die Fähigkeit, daraus Nutzen für seine individuelle Position zu ziehen, ist insbesondere für illegale Migrant(inn)en nicht zu unterschätzen. Hinsichtlich möglicher Abwertungsprozesse sozialen Kapitals bei Heiratsmigranten findet sich 
Kontakte auch zu Mexikanern in Deutschland, diese können ihm aber nicht bei der Arbeitssuche helfen und die Abwertung seiner beruflichen Qualifikationen auffangen. Die Arbeitslosigkeit von Personen ohne deutschen Pass ist etwa doppelt so hoch wie die bei Personen mit deutschem Pass. Sie

„ist - nach einem Maximum im Jahr 1997 - zwischen 1997 und 2000 deutlich zurückgegangen, steigt seit dem Jahr 2001 jedoch wieder stetig an. Der Anstieg von 2000 auf 2004 beläuft sich auf $22 \%$. Derzeit sind 538.139 Ausländer in Deutschland ohne Job, darunter $37 \%$ Frauen. Die Arbeitslosenquote beträgt $20,1 \%$ - im Vergleich dazu liegt die Arbeitslosenquote in Deutschland insgesamt bei 11,7\%“(AiD - Integration in Deutschland 2004).

Solche Beobachtungen der Anforderungen, die in Migrationsprozessen entstehen, haben in der neueren Migrationsforschung zu einer Verschiebung der Analyseperspektiven geführt: Nicht mehr die einseitige Assimilation oder Integration in das ,Aufnahmeland“ steht im Mittelpunkt des Interesses, sondern die produktive Bearbeitung der Zumutungen und die dadurch entstehenden ,neuen sozialen Wirklichkeiten'. Ähnlichkeiten mit Modernisierungsprozessen drängen sich auf, die Bommes zu folgender Feststellung gebracht haben: Er kritisiert, dass

„übersehen wird, dass Migranten in den kulturellen Formen ihres Lebens in der Gesellschaft, in die sie eingewandert sind, ebensolche strukturell eingebauten Individualisierungszumutungen erleben, wie sie auch ansonsten als bestimmend für das Leben in modernen Industriegesellschaften gezeichnet werden [...]. Das muss aus theoretischer Sicht allein deshalb überraschen, weil doch insbesondere bei den Migrantengruppen Umbruchprozesse der kulturellen Bedeutung von Zumutungen, wie sie mit Modernisierungsschüben verbunden sind, geradezu verdichtet zu beobachten sind" (Bommes 1992 zit. in Herwartz-Emden 1997: 895f).

Die zu beobachtenden , verdichteten“ Modernisierungsschübe stellen auf die erhöhten Risiken von Migrationsprozessen und Migrationsbiographien ab, da diese in ,gesteigertem Maße allgemeine Signaturen der reflexiven Modernen wie Ungewissheit, Unsicherheit, Nicht-Wissen und Risiko“ (Seitter 1999: 34) verkörpern:

jedoch eine andere Situation. Zwar sind auch Heiratsmigrationen nicht nur Einzelentscheidungen von Individuen, sondern können in Zusammenhang mit Familienentscheidungen stehen. Dennoch wird das soziale Kapital des Ehepartners sehr viel relevanter, nicht zuletzt da die zentrale Bezugsgruppe in der Regel das soziale Umfeld der/des deutschen Partners/in ist. Das eigene soziale Kapital erfährt so einen Entwertungsprozess. 
„Migration kann [...] als das gleichzeitige Verwiesensein auf die Herkunftsund Aufnahmegesellschaft beschrieben werden mit großen Unsicherheitspotentialen und ungewissen Zukunftsperspektiven - gerade auch gemessen an den relativ bekannten Ausgangsbedingungen in der Herkunftsgesellschaft. Zugleich impliziert der gesellschaftliche Kontextwechsel eine Steigerung von Begründungsnotwendigkeiten und -pflichten sowohl dem Heimat- als auch dem Aufnahmeland gegenüber“ (ebd.). ${ }^{34}$

Betrachtet man binationale Paare, gewinnen die erhöhten Modernisierungsanforderungen für beide Partner(innen) an Bedeutung. Auch für die deutschen Ehepartnerinnen bedeuten die Herausforderungen der Migration und die spezifische Situation in Deutschland eine Risikoerhöhung und eine gesteigerten Unsicherheit hinsichtlich der Gestaltung der weiteren Paarbiographie. Nicht nur die Situation der Migration stellt dabei jedoch die Herausforderung dar. Binationale Paare stehen, wie aus den Forschungsergebnissen deutlich wurde, unter besonderer Beobachtung durch das gesellschaftliche und das persönliche Umfeld, insbesondere wenn deutsche Frauen Migranten heiraten. „Während Männern die ,Evasion` und die ,Suche nach Exotik' erlaubt zu sein scheint, müssen sich Frauen sehr oft mit der Etikettierung der ,Sitzengelassenen“, der ,Untreuen der Gruppe', ja sogar der ,Verräterin der Rasse' herumschlagen" (Waldis 1998: 46). Freund(inn)e(n) und insbesondere die Verwandten vermitteln häufig das Unbehagen, einen Partner aus dem falschen Land, oder noch schlimmer, mit der falschen Hautfarbe gewählt $\mathrm{zu}$ haben. Neben solchen rassistischen und diskriminierenden Begegnungen im sozialen Nahfeld stellen ebenfalls strukturelle Erfahrungen wie die oben beschriebene Ver-rechtlichung Zumutungen dar, mit denen die Paare umgehen müssen. Zudem kann, wie ja auch die Studien zu binationalen Paaren gezeigt haben, nur wenig auf kulturelle Routinen zurückgegriffen werden, vielmehr müssen eigene Wege und Routinen entwickelt werden. Von der neueren Migrationsforschung wird also nach den kreativen und eigensinnigen Bearbeitungen migrationsspezifischer Zumutungen gefragt. Pries hat 1997 auf die Entstehung „,neuer sozialer Wirklichkeiten“ insbesondere hinsichtlich transnationaler, die bisherigen gedachten Zusammenhänge von geographischen und sozialen Räumen auflösenden Räumen und Strukturen hingewiesen. Ein solcher Blick macht die ,blinden Flecke‘ der klassischen Migrationsforschung sichtbar, die Migrationsprozesse als unidirektionale Bewegungen verstehen und die kein mehrdirektionales Verständnis von Migrationspro-

34 Diese Perspektive greifen z.B. Mecheril 1994, 1997, 2003; Apitzsch 1999; Hummrich 2002; Apitzsch 2003, für die interkulturelle Geschlechterforschung insbesondere Schlehe 2000b, 2001a auf. 
zessen besitzen, welche auch die Aufnahmegesellschaft nicht unverändert lassen (Pries 1997: 35). Diese Bearbeitungen sind nicht zwangsläufig als emanzipative zu verstehen. Sie können auch durch den Rückzug auf traditionelle, Sicherheiten versprechende Bezüge und Konstruktionen bearbeitet werden. Individualisierungsprozesse finden insofern nicht nur in der Loslösung aus Bindungen statt, sondern auch in der Hinwendung zu Bindungen.

Festhalten lässt sich, dass Migrationen und Migrationsprozesse binationale Paare vor Zumutungen und Herausforderungen stellen, die ihre Biographien von herkömmlichen Biographien unterscheiden und sie in besonderer Weise zu ,modernen` Biographien machen. Die dabei zu beobachtenden Bearbeitungsformen sind von besonderem Interesse für sozialwissenschaftliche Migrationsforschung, da sich hier (Re-)Konstruktionen von gesellschaftlichen Strukturen und Routinen analysieren lassen, die gesellschaftlichen Wandel be- bzw. vorantreiben.

\section{Das Female Breadwinner Modell: Konkretisierung der Zumutungen}

Als Konkretisierung der Zumutungen für mein Sample lässt sich das $f e$ male breadwinner Modell verstehen. Dieses ist aufgrund der oben skizzierten Schwierigkeiten bei der Einmündung in den deutschen Arbeitsmarkt bei binationalen Paaren regelmäßig zu beobachten, insbesondere wenn es sich um Heiratsmigration handelt, die Paare sich also nicht in Deutschland kennen gelernt haben, und die Beziehung den Migrationsanlass des Mannes darstellt. Die Zeitdauer variiert dabei je nach Paar beträchtlich, da die Dauer nicht nur von ,äußeren ' Faktoren wie beruflicher Vorbildung oder Sprachkenntnissen abhängig ist, sondern selbstverständlich auch von biographischen Plänen und der Bedeutung, die das female breadwinner Modell für das jeweilige Paar hat.

Gesellschaftsstrukturen sind geschlechtlich organisiert, indem sie Zuständigkeiten in einen - weiblich konnotierten - privaten Bereich mit der Betreuung der Reproduktionsaufgaben und in einen - männlich konnotierten - öffentlichen Bereich mit der Betreuung der Produktionsaufgaben einer Gesellschaft aufteilen. Diese Aufteilung ist in zweierlei Hinsicht als strukturierendes Moment von männlichen und weiblichen Lebensläufen maßgeblich. Zum einen gehört die geschlechtlich organisierte Arbeitsteilung als symbolisches Instrumentarium zu einem zentralen Ordnungsprinzip von Lebensläufen. Das Wissen um diese Arbeitsteilung und damit auch um die Ungewöhnlichkeit abweichender Lebensgestaltungen strukturiert sowohl den öffentlichen Diskurs als auch die subjek- 
tiven Lebensplanungen und -gestaltungen. ${ }^{35}$ Zudem ist die Arbeitsteilung auch nach wie vor ganz konkreter Fakt für die überwältigende Zahl der Biographien in Deutschland, spätestens mit Beginn der Familiengründung. ${ }^{36}$ Zwar steigen die Erwerbstätigkeitsraten der Frauen mit Kindern, dies ist aber vor allem auf den Anstieg von Teilzeitarbeit zurückzuführen: „Der Anstieg der Müttererwerbsquote in den alten Bundesländern geht fast ausschließlich auf eine Zunahme der Teilzeiterwerbstätigen und geringfügig Beschäftigen zurück. Der Anteil der in Vollzeit erwerbstätigen Mütter mit Kindern unter 18 Jahren hat sich gegenüber den 70er Jahren sogar etwas verschlechtert" (Bundesministerium für Familie 1999: 109). Zudem zeigt sich regelmäßig, dass die größere Beteiligung von Frauen am produktiven Bereich keine größere Beteiligung von Männern am reproduktiven Bereich nach sich zieht:

„Für die vollzeitberufstätigen Ehemänner ist es nahezu gleichgültig, ob ihre Partnerin Voll- oder Teilzeit arbeitet oder ausschließlich im Haushalt tätig ist, wenn es darum geht, wie viel Zeit sie selbst im Durchschnitt pro Tag für die Hausarbeit aufwenden (ca. 11/2 Stunden)“( Holz 2000: 119).

Dieses konstante Phänomen der Verringerung der Arbeitszeiten der Frauen und damit der beruflichen Karrierechancen mit Beginn der Familiengründung geht einher mit einem positiven Effekt für die Karrieregestaltung der Männer: Männer machen zum selben Zeitpunkt „Berufskarriere im Kindertakt” (Friebel 2000 in Menz/Putschbach 2001: 32). Familiengründung scheint bei Männern das Bedürfnis zu verstärken, beruflich aufzusteigen. Es liegt für sie wesentlich näher, die finanzielle Familienverantwortung zu übernehmen als die Erwerbstätigkeit zu unterbrechen. In gleicher Weise argumentiert auch Elizabeth Gorman, die in ihren Analysen verschiedene Erklärungen für das Phänomen überprüft, dass verheiratete Männer mehr Geld verdienen und schneller Karriere machen als Singles (Gorman 1999). Für sie stellt eines der wichtigsten

35 Aktuelles Beispiel dafür ist die Aufregung um die Einführung des Elterngeldes, welches in voller Höhe nur dann ausgezahlt wird, wenn auch der Vater zwei Monate zu Hause bleibt. Kritik kam nicht nur, wie vielleicht noch zu erwarten gewesen wäre, von männlichen CSU-Abgeordneten. Kritik kam auch von Müttern, die ebenfalls das Selbstbestimmungsrecht der Familie in Gefahr sahen. Auch Ergebnisse der dritten Welle des Familienpanels zeigen, dass 56\% der (west)deutschen, allerdings nur 35\% der ostdeutschen Frauen, der Ansicht sind, dass Kinder unter der Berufstätigkeit der Mutter leiden (vgl. Dornseiff/Sackmann 2003: 327).

36 Tatsächlich scheint eine egalitäre Berufs- und Hausarbeitsteilung für Paare bis zur Familiengründung durchaus realistisch. Spätestens aber der Beginn der eigenen Familiengründung stellt die ,Sollbruchstelle' in der weiblichen Erwerbsbiographie dar (vgl. Friebel/Epskamp/Knobloch et al. 2000). 
Erklärungsmuster ebenfalls der stärkere und längere Arbeitseinsatz und die sorgfältigere Karriereplanung aufgrund der zusätzlichen Verantwortung für Frau und Kind(er) dar.

\section{Exkurs: das male breadwinner Modell}

Die Begriffe und die Konzepte des male breadwinner Modells und des komplementären Modells der female housekeeper entstehen in den 50er Jahren in Folge einer restriktiven Geschlechterordnung und der auf binären Differenzen basierenden Geschlechterverhältnisse. Die Idealisierung und Implementation des Modells in den öffentlichen Diskurs erfolgte über Fernsehserien wie ,Ozzie \& Harriet" in den 50er Jahren (vgl. Deutsch/Saxon 1998: 331) und ist seitdem im US-amerikanischen Raum als Beschreibung des traditionellen Modells der geschlechtlichen Arbeitsteilung bekannt. ${ }^{37}$ Gemeint ist damit also die Bezeichnung einer symbolischen Geschlechterordnung und der damit verbundenen symbolischen und faktischen geschlechtlichen Arbeitsteilung. Daneben dient das male breadwinner Modell aber auch als Grenzziehung zwischen Männern und Frauen und bekräftigt damit die soziale Konstruktion von Geschlechterdifferenzen. Potuchek versteht breadwinning ,as an interpretative gender boundary that continues to divide men from women, even though the behaviour of paid employment has ceased to serve as a means of gender differentiation“" (ebd. 1997 in Winders 2000: 218). Das breadwinner Modell ist also mehr als nur eine Bezeichnung einer bestehenden geschlechtlichen Arbeitsteilung. Sie fungiert auch als identitär besetztes Unterscheidungsmerkmal zwischen den Geschlechtern, das interaktiv hergestellt wird bzw. werden kann. Dieses Verständnis erklärt auch die mögliche Diskrepanz zwischen tatsächlich bestehender Arbeitsteilung in einer Beziehung und dem Selbstverständnis als breadwinner bzw. als Zuverdienerin. Ein solches Selbstverständnis muss eben nicht zwangsläufig mit der tatsächlichen Aufteilung bzw. der Bedeutung des Verdienstes für die finanzielle Situation des Paares oder der Familie einhergehen. Der Begriff stammt zwar aus den USA, auch für Deutschland kann man aber von einer analogen Unterscheidung sprechen: „Man kann [...] davon ausgehen, dass der strukturellen Unvereinbarkeit von Familien- und Erwerbsarbeit in Westdeutschland auch eine ideologische Unvereinbarkeit dieser beiden Sphären entspricht“ (Dornseiff/Sackmann 2003: 317).

Entsprechend der großen Bedeutung, die insbesondere der männlichen Erwerbsarbeit zugesprochen wird, erscheint sie doch als zentrales männliches Identifikationsmuster, wird männliche Arbeitslosigkeit in den

37 Sicherlich ist - eine ähnliche Entwicklung wie in Deutschland - nur eingeschränkt vom traditionellen Geschlechterverhältnis zu sprechen, gilt die Manifestation der uns bekannten Arbeitsteilung und der Geschlechtscharaktere doch erst seit dem II. Weltkrieg. Dennoch gelten sie im allgemeinen Sprachgebrauch als die ,herkömmlichen' und ,traditionellen' Geschlechterordnungen. 
meisten Studien, die sich damit beschäftigen, als pathologisches Phänomen verstanden, welches negative Auswirkungen auf die Paarbeziehung und die Familie hat. So untersuchen Blech und Witte 1992 erhöhte Stressbelastungen durch männliche Arbeitslosigkeit und sehen diese insbesondere dann als gegeben, wenn auch die Frau nicht erwerbstätig ist. Nicht ungewöhnlich sind zudem Untersuchungen über Konfliktbereiche in den Partnerschaften (Uhl 1991) oder sogar über die Zunahme von Gewalt gegenüber Frauen, insbesondere bei bestehender Erwerbstätigkeit der Frau (Macmillan/Gartner 1999). Studien, die sich demgegenüber mit Hilfe von sozialkonstruktivistischen Ansätzen mit (Re-)Konstruktionen von Geschlechterordnungen und -beziehungen beschäftigen, sind rar und in Deutschland fast nicht existent (als Ausnahme in Deutschland Schreyer 1991; für die Türkei Bolak 1997; für die USA Deutsch/Saxon 1998). Diese Studien sehen in der männlichen Erwerbsund Arbeitslosigkeit überwiegend einen Bruch in und mit der bisherigen Lebensplanung und -gestaltung, der eine Neuorientierung hinsichtlich der Arbeitsteilung, der gender-Ordnungen und der Machtverhältnisse in der Beziehung erfordert (zu Machtverhältnissen s. vor allem Streckeisen 1993; Künzler 1995; Wimbauer 2003).

Interessanterweise finden sich aber insbesondere im englischsprachigen Raum deutlich mehr Untersuchungen zum dual earner Modell. Paare, die sich Hausarbeit und Erwerbsarbeit (gerecht) teilen, werden teilweise als „postgender marriages“ kategorisiert (Risman/JohnsonSumerford 1998). Ebenfalls interessant sind Studien, die sich mit gender-Orientierungen berufstätiger Ehefrauen beschäftigen, wie die von Jean Potuchek 1992. Sie untersucht das Selbstverständnis von Ehefrauen und typisiert dabei mit Hilfe von geschlossenen Fragebögen verschiedene Formen der gender-Orientierung Für dieses Vorhaben misst sie das Selbstverständnis hinsichtlich dreier Faktoren: der Bedeutung, die dem finanziellen Zugewinn beigemessen wird (,Wird er für das Grundhaushaltseinkommen verwendet oder dient er für den Erwerb von zusätzlichen, nicht notwendigen Dingen?'), der Bedeutung, die der Beruf für die Familie hat (,Wie viele Stunden arbeitet sie, sieht sie sich als breadwinner?'), und der Perspektive der Frauen auf die Bedeutung von breadwinning als ,gender boundary“ (,Wer sollte ihrer Meinung nach der bzw. die Haupternährer(in) einer Familie sein?‘; Potuchek 1992: 550). Als Ergebnis lässt sich festhalten, dass die tatsächliche Berufstätigkeit der Frau nicht zwangsläufig etwas mit der Einstellung der Frauen zu ihrer Berufstätigkeit zu tun haben muss. Gut ein Fünftel aller Frauen ließ sich nämlich als ,employed homemakers“ kategorisieren: 
„These are women for whom breadwinning remains firmly in place as a gender boundary, despite their labour force participation. These wives do not define their employment as a source for financial support for their family. [...] These interpretations of their employment fit their norms about breadwinning; they see it as a responsibility that ought to be gendered male" (Potuchek 1992: $551)$.

Interessant für meine Untersuchung sind insbesondere auch die ,reluctant providers“ bzw. die „reluctant traditionals“, wie Potuchek zwei der Gruppen von Frauen bezeichnet (ebd.: 552). Bei diesen Frauen klaffen die Einstellungen und die tatsächliche Situation auseinander; sei es, weil ihr Verdienst zwar wesentlich zum Familieneinkommen beiträgt, sie selber aber die Haupternährerrolle eigentlich deutlich dem Mann zuschreiben. Oder sei es, im umgekehrten Fall, dass sie zwar in ihrer Orientierung eine egalitäre Arbeitsteilung anstreben, in der Praxis aber auf die Rolle als Zuverdienerin festgelegt sind. Das male breadwinner Modell als symbolisches Ordnungsmuster hat insofern nach wie vor starken strukturierenden Charakter. Auch die zunehmende Erwerbstätigkeit von Frauen oder die ebenfalls inzwischen alltägliche Arbeitslosigkeit von Männern können daran nur zögerlich etwas ändern, so dass sich nicht zwangsläufig eine Komplementarität zwischen den Einstellungen und der tatsächlichen Situation zeigt. ${ }^{38}$ Vielmehr wird in den Untersuchungsergebnissen vor allem die Interpretationsvariabilität personaler Beziehungen durch die beteiligten Subjekte deutlich. Die Konstellation des female breadwinner Modells kann als Anstoß genommen werden, gender-Orientierungen zu verändern, muss aber weder zwangsläufig zur Umorientierung führen, noch muss sie eine krisenhafte Konstellation zur Folge haben. Ein Ergebnis, das auch durch die deutsche Studie von Schreyer bestätigt wird (Schreyer 1991). Sie hat in Interviews mit Frauen Bearbeitungsstrategien bzw., besser formuliert, Auffangstrategien der Ehefrauen arbeitsloser Arbeiter analysiert. Auch hier zeigt sich, wie ,Normalisierungsarbeit' von Seiten der Ehefrauen geleistet wird, um der männlichen Identität keinen weiteren Schaden zuzufügen und um den Zustand vor der Arbeitslosigkeit aufrecht zu erhalten. Die Ziele und Orientierungen der interviewten Frauen

38 Vgl. zu Erklärungsansätzen für diese langsame Entwicklung z.B. Komter 1989, der den Grund in der Verhinderung von Machtausgleich durch die Ehemänner sieht, oder Sullivan 2004, wonach Diskurse und Praxen in einem komplizierten Verhältnis stehen und sich gegenseitig im Hinblick auf emanzipative Veränderungen unterstützen können, dies aber nur langsam von statten geht. 
„weisen alle mehr oder weniger restaurative Züge auf: keine radikale Neuordung der Ehe wird angestrebt, sondern - soweit unter veränderten Rahmenbedingungen noch möglich - der Erhalt von bzw. die Wiederannäherung an Modi des ehelichen Zusammenlebens, wie sie, den retrospektiven Schilderungen der Frauen zufolge, vor der Arbeitslosigkeit praktiziert wurden“ (ebd.: 14).

Zum Abschluss dieses ersten Teils des theoretischen Zugangs zu meinem Sample sollen daher Überlegungen zur gemeinsamen Wirklichkeitskonstruktion von binationalen Paaren vorgestellt werden, um eine weitere konzeptuelle Grundlage für die Interpretationen zu gewinnen. In der Analyse stellt das Selbstverständnis der Paare die relevante Kategorie dar, nicht eine formale Kategorisierung.

\section{(Binationale) Beziehungen als soziale Systeme und Orte der gemeinsamen Produktion von Wirklichkeit}

Das Paar bzw. die Paarbeziehung können, darauf haben Berger und Kellner 1964 aufmerksam gemacht, als eigenständiges soziales System verstanden werden (deutsch 1965). Sie haben überzeugend nachgezeichnet, wie die Paarbeziehung ,einen im Vergleich zu anderen sozialen Beziehungen privilegierten Status einnimmt. Anders ausgedrückt: Die Ehe ist in unserer Gesellschaft ein entscheidendes nomisches Instrument" (ebd.: 222). Der nomische Bruch, der durch die Heirat bzw. durch eine langfristige Paarbeziehung entsteht, wird durch die dyadische und intime Form der Beziehung ausgelöst, die den Partner bzw. die Partnerin als neuen, zentralen Bezugspunkt der Wirklichkeitsentwürfe in den Mittelpunkt stellt:

„In der Ehe müssen alle Handlungen des einen Partners im Bezug zu denen des anderen entworfen werden. Die Definitionen der Wirklichkeit durch den einen müssen fortwährend in Korrelation zu denen des anderen gesetzt werden. Der andere ist in fast allen Sinnhorizonten des Alltagslebens gegenwärtig“" (ebd.: 226).

Dieser Prozess der Entstehung gemeinsamer Wirklichkeitskonstruktionen wird einerseits durch explizite Gespräche vollzogen, andererseits durch gegenseitige (auch unausgesprochene) Erwartungen und Vorstellungen an und von dem/der Anderen. Die neue, gemeinsame Konstruktion der Wirklichkeit wird notwendig, da sich im Hinblick auf gemeinsame Wirklichkeitskonstruktionen sozusagen zwei ,Fremde' begegnen. Fremd sind sie sich nicht zwangsläufig im Sinne unterschiedlicher Mi- 
lieuzugehörigkeiten oder stark unterschiedlicher Bildungsniveaus. ${ }^{39}$ Die Fremdheit gründet sich vielmehr auf verschiedene biographische Erfahrungen und unterschiedliche Interaktions- und Konstruktionsbereiche. Die Notwendigkeit besteht nun darin, einerseits Übereinstimmungen hinsichtlich der weiteren gemeinsamen Zukunft zu treffen (,Wie wollen wir leben, welche Wünsche können und sollen wann erfüllt werden, wollen wir Kinder, wann wäre der richtige Zeitpunkt?'). Andererseits muss eine solche Übereinstimmung auch über die Vergangenheit erfolgen, der Nomos-Prozess erfolgt also quasi rückwirkend: „Die beiden Realitäten der Ehepartner werden dabei zu einer einzigen zusammengefügt" (Berger/Kellner 1965: 227). ${ }^{40}$ Die Zusammenfügung der Realitäten erfolgt dabei unter der Illusion der Verständigung. Auch in einer Paarbeziehung ist nie ein vollständiges, sondern nur ein scheinbares Verstehen möglich. Die Konstruktionen müssen zwangsläufig unvollständig sein, da man nie alles über den oder die andere wissen kann (vgl. dazu Lenz 2003). Entscheidend ist dabei jedoch, dass Paare von einem umfassenden und gemeinsamen Verständnis ausgehen, dass also erwartet wird, dass aktuelle Wünsche und zukünftige Pläne übereinstimmen. ${ }^{41}$ Die Entwicklung von gemeinsamem Wissen verweist im Übrigen auf die Funktion des Partners als gatekeeper, wie Waldis anhand der Analyse interkultureller Kommunikation zwischen maghrebinisch-schweizeri-

39 Tatsächlich zeigen Untersuchungen zur Partnerwahl eine in der Regel große Homogenität hinsichtlich dieser Kategorien (vgl. z.B. Klein/Lengerer 2001). Eine neuere Studie weist zudem darauf hin, dass die Anzahl der Ehen mit ähnlichem Bildungsniveau ansteigt (vgl. Timm 2004).

40 An dieser Stelle lässt sich an Mannheim und seinen konjunktiven Erfahrungsraum anknüpfen. Die Paarbeziehung wird zu einem solchen, innerhalb dessen sich gemeinsame Verstehensprozesse konstituieren: "Mit dem Terminus des konjunktiven Erfahrungsraums zielt Mannheim auf eine milieuspezifische Ebene des menschlichen Miteinanderseins, die sich im Medium des Selbstverständlichen vollzieht, d.h. einer Ebene des Erlebens, die sich fraglos bzw. präreflexiv und in diesem Sinne a-theoretisch konstituiert" ( Schäffer 2003: 77).

41 Burkart/Koppetsch weisen darauf hin, dass intime Beziehungen andere Regulative beinhalten als öffentliche Geschlechterbeziehungen, wie sie beispielsweise im Beruf vorzufinden sind. Während letztere durch Regulative wie Hierarchie und Herrschaft gekennzeichnet seien, sei die Paarbeziehung durch das Regulativ ,Liebe“ und ,Intimität' gekennzeichnet, was grundlegend andere Strukturierungen zur Folge habe (vgl. Burkart/Koppetsch 2001). Geschlechtsnormen (Erwartungen hinsichtlich der Ausprägung von Männlichkeit und Weiblichkeit) betonen, so ihre These, in der privaten Sphäre die Einheit des Paares und schwächen die Differenz der Geschlechter zugunsten der Komplementarität ab. Hingegen betonen Geschlechtsnormen in der öffentlichen Sphäre Geschlechtsunterschiede, die soziale Unterschiede legitimieren und damit auch eine hierarchisierende Trennung der Geschlechter vorbereiten (vgl. ebd.: 443). 
schen Paaren zeigt: „Gatekeeper ermöglichen und verhindern Dinge für andere Personen“ (Waldis 1998: 185). Die schweizerischen Ehefrauen vermitteln zwischen ihrem Partner und ihrer eigenen Familie, ebenso wie dem maghrebinischen Ehemann ,eine Gatekeeperrolle in Bezug auf seine eigene Gesellschaft“ zukommt: „Es ist auch die Rolle des maghrebinischen Mannes, seiner Frau die Kultur des Maghreb nahe zu bringen“ (ebd.: 186).

Dieses Verständnis des nomischen Prozesses ist hilfreich, um die besondere Konstellation binationaler Paare unabhängig von kulturalisierenden Bezügen fassen zu können. Die von mir betrachteten binationalen Paare stehen vor einem in zweifacher Hinsicht anderen Prozess: Zum einen ist, so ist zu vermuten, die Vergangenheit beider Partner aufgrund unterschiedlicher Sozialisationserfahrungen und unterschiedlicher Sprachen durch eine Vervielfältigung an Selbst- und Wirklichkeitskonstruktionen charakterisiert, die die notwendige Koordination von der mononationaler Paare unterscheidet. Zum anderen sind die zukünftigen Lebenspläne nicht nur ebenfalls durch die Vervielfältigungen von Erfahrungen und Orientierungsmustern gekennzeichnet; auch die Irritationen, wie sie durch den Migrationsprozess und die hier stattfindenden Dequalifizierungsprozesse einerseits oder durch den ,Verstoß' gegen das Homogamiegebot durch die Frau andererseits erfahren werden, haben Auswirkungen für beide und müssen von beiden bearbeitet werden. Die Migration stellt nicht nur für den Migranten selbst eine zu bearbeitende Herausforderung dar, in diesen Prozess werden ebenfalls, wie sich eindrucksvoll am Beispiel des female breadwinner Modells zeigen lässt, seine Partnerin und deren biographische Pläne einbezogen. Das Irritationspotential ist insofern, das zeigen auch übereinstimmend Studien zu binationalen Paaren, höher als bei mononationalen Paaren. Für Untersuchungen wie die vorliegende ist die Paarbeziehung als Ort der Konstruktionen zentral. Die Konstruktionen finden in Auseinandersetzung primär mit dem/der Partner(in) statt, die Erfahrungen in Deutschland und die Bezüge zum Herkunftsland des Mannes werden primär in der Paarbeziehung gemacht. Dies bedeutet in der methodischen Konsequenz auch, beide Partner(innen) zu interviewen, um die gegenseitigen und gemeinsamen Bearbeitungsformen und Wirklichkeitsinterpretationen analysieren zu können (vgl. dazu auch Molnár 2004, die ebenfalls beide Partner(innen) interviewt). 


\section{DiE ForschungsPeRsPEKTIVE: BIOGRAPHIE, GENDER UND NATIO-ETHNO-KULTURELLE}

ZUGEHÖRIGKEIT

\section{Erste Annäherung: Biographie als Ort der Konstruktion}

Maria: „Das ist nicht so, das Leben ist offen sozusagen, also ist viel, nicht so wie bei uns - alles muss geplant sein. [...] Deshalb bin ich auch mal gespannt wie es in Mexiko wird für mich. Und da bin ich auch, merk ich immer noch dass es unterschiedlich ist. [...]- Dieses auch - offen sein nach allen Seiten, Pablo hat mehrere Pläne [...]. Da gibt's viele Möglichkeiten. [...] Also dieses - wo unser_, wir oder ich eben ganz brav, ich bin für das ausgebildet und das mach ich. Und ich arbeite noch als Übersetzerin, aber was anderes kann ich nicht. [...] Eben als Ideelles mach ich das aber - zum Geldverdienen kann man ja auch des machen. Und das find ich schon sehr spannend. - so." (Maria Wesel, 17/32-18/14).

In diesem Teil des Interviews erzählt Maria über die geplante Emigration nach Mexiko, ihrer Unsicherheit hinsichtlich der Veränderung der Lebensumstände und über die Herausforderung, bisherige Planungen und Planungsarten zu verändern und sich neuen, auch ungewohnten Formen der Lebensgestaltung zu öffnen. Sie selber wäre am liebsten erst mit einem Arbeitsvertrag nach Mexiko gegangen. Pablos Pläne und Vorstellungen aber beschränken sich nicht nur auf die eigentlichen Berufsfelder, sondern erweitern die Möglichkeiten nach verschiedenen Seiten hin. ${ }^{1}$ Die Textstelle aus Marias Interview verweist auf die Gleichzeitigkeit von Planungsnotwendigkeiten und Planungsunsicherheiten hinsicht-

1 Auf die kulturalisierenden Deutungen, die in der Textstelle ebenfalls relevant sind, wird an dieser Stelle nicht weiter eingegangen. Siehe dazu die zweite Annäherung in diesem Kapitel. 
lich der weiteren Lebensgestaltung. Dabei pointiert die geplante Migration diese Phänomene der Lebensführung, verweist damit aber gleichwohl auf ein grundsätzliches Phänomen moderner Gesellschaften: Es gibt die wahrgenommene Notwendigkeit der Gestaltung der eigenen Lebenswege und Lebens(ver)läufe (vgl. dazu zuerst Beck 1986, aber auch Hahn 1988; Schimank 2002). In ständisch organisierten Gesellschaften der Vormoderne waren die subjektiven Planungsnotwendigkeiten und möglichkeiten der heutigen Form weder gegeben noch notwendig: Lebens- und Berufswege waren, geschlechts- und milieuspezifisch jeweils unterschiedlich, vorgezeichnet. Aus- und Umwege gab es für die einzelnen Individuen kaum, dafür waren die Lebenswege verlässlich und in gewisser Weise (vor)bestimmt. Dass eine solche Verlässlichkeit heute nicht mehr gegeben ist, dass vielmehr die Aufgabe der Herstellung von Verlässlichkeit und Kontinuität dem Subjekt übertragen wird, das wurde in dem vorhergehenden Kapitel in Bezug auf Zumutungen im Migrationsprozess deutlich. Im folgenden Kapitel werden nun diese Beobachtungen im Hinblick auf ihre Bedeutung für Biographiekonstruktionen konkretisiert, da sich die Arbeit diesem Phänomen der Notwendigkeit von subjektiven Bearbeitungsstrategien und erhöhtem Planungsaufwand biographietheoretisch nähert. ${ }^{2}$ Dieser Zugang ermöglicht es, die Kategorien gender und natio-ethno-kulturelle Zugehörigkeiten als biographisch gebundene Kategorien zu verstehen und somit die Wirkmächtigkeit der Kategorien in und für die Biographien binationaler Paare ernst zu nehmen, ohne aber den Konstruktionscharakter dieser Kategorien aus den Augen zu verlieren. Die folgenden Ausführungen verstehen sich daher als weitere Explikation des sensibilisierenden Konzeptes, womit die Aufmerksamkeitsrichtungen für die Analyse benannt werden. Die zunehmende Pluralisierung und Auflösung traditioneller Sinnbezüge einerseits und die Erosion gesellschaftlicher Institutionen andererseits werden als Phänomen moderner Gesellschaften eingeführt, welches Konsequenzen für die Biographiekonstruktion und Lebensgestaltung von Subjekten haben. Am Wandel bzw. der Konstanz von geschlechtsspezifischer Arbeitsteilug wird die Paradoxie moderner Lebensentwürfe deutlich. Daran anschließend wird das Konzept der Biographie als Schaltstelle zwischen Gesellschaft und Individuum eingeführt. In einer zweiten Annäherung werden die beiden Analysekategorien näher betrachtet und ihre Bedeutung als biographisch gebundene Kategorien erklärt, welche in einem

2 Das bedeutet nicht, dass Leben tatsächlich planbar ist. Vielmehr bezieht sich die Planungsnotwendigkeit auf das subjektive Gefühl und die Anforderung, Leben zu planen und zu organisieren, wie es sich bei Marias Formulierungen zeigt. Aus dieser Planungsnotwendigkeit ist noch nichts über die Planungsmöglichkeiten und -sicherheiten gesagt. 
spezifischen Verhältnis zueinander stehen. Bevor sich mit der Darstellung der methodologischen Besonderheiten der Arbeit der Blick auf die konkreten Biographien der Paare richtet, erfolgt im Abschlusskapitel des theoretischen Teils eine zusammenfassende Einordnung der theoretischen ,Ansichten“ aus den bisherigen Kapiteln.

Ein solch rekonstruktiver Zugang zum empirischen Material schützt dennoch nicht vor Reifizierungen der Kategorien Geschlecht und natioethno-kultureller Zugehörigkeiten. Als Wissenschaftlerin bin ich in Produktionsprozesse eingebunden, die aus der eigenen Standortgebundenheit resultieren und die die eigene Verstrickung in Geschlechterverhältnisse deutlich machen. Dem lässt sich zwar nicht entgehen, jedoch bietet die methodologische Rahmung durch die Grounded Theory (Glaser/ Strauss 1967, vgl. auch Dausien 2002) eine Möglichkeit der Reflexion solcher Konstruktionsprozesse. Interpretationen sind dann keine ,wahren' Erkenntnisse einer außenstehenden Wissenschaftlerin, sondern vielmehr weitere soziale Konstruktionen. Ein solches Verständnis folgt dem Modell der Produktion von „Text als Medium für Text“ (Mecheril 2003: 32), auch wissenschaftliche Interpretationen sind Interpretationen sozialer Prozesse und sind prinzipiell einer weiteren Interpretation zugänglich. ${ }^{3}$

\section{Erhöhte Anforderungen und Kontingenz: vom institutionalisierten Lebenslauf zur "Biographisierung von Erleben und Handeln“4}

Im vorherigen Kapitel wurden Migrant(inn)en als Subjekte eingeführt, die in gewissermaßen pointierter Weise Modernisierungserfahrungen machen. Solche Modernisierungerfahrungen sind eine Folge der Veränderungen von Gesellschaften, wie sie maßgeblich durch Martin Kohli beschrieben wurden. Er hat Mitte der 80er Jahre mit seiner Analyse des Lebenslaufs als eigenständige Institution und als Ablösung der Einbindung in ständische Strukturen ein Kernkonzept sozialwissenschaftlicher Forschung entwickelt (Kohli 1985, 1986). Versteht man Individualisierung (auch) als Freisetzung der Individuen aus ständischen und lokalen

3 Hagemann-White hat als Lösungsvorschlag für das Problem der Reifizierung durch die Geschlechterforschung eine Art doppelten Blick (Hagemann-White 1993, 1995) in die Diskussion gebracht. Um die lebensweltlichen Konstruktionen der Interviewten zu verstehen, müssen wir situativ ,wissen', dass es Männer und Frauen gibt und diese real unterschiedlich sind, auch wenn wir theoretisch wissen, dass es nicht so ist: „Wir müssen gleichsam doppelt hinschauen” (Hagemann-White 1993: 74).

4 Brose/Hildenbrand 1988 
Bindungen, bedeutet dies im Umkehrschluss allerdings keineswegs die oftmals postulierte Freisetzung aus allen Bindungen und sozialen Strukturen. Es entsteht, so Kohli, ein temporal organisierter Lebenslauf, der die Einbindung in ständische Strukturen abgelöst hat (Kohli 1985, 1986). Der Lebenslauf als Institution stellt eine „Sequenzierung des Lebens" (ebd.: 10) dar, die hinsichtlich der Einbindung von Individuen normierend wirkt und Lebensabläufe homogenisiert. Er ist als Reaktion auf die vorrangig ökonomisch strukturierte Arbeitswelt um den Erwerbsverlauf eines Menschen herum organisiert: Die Kindheit und Jugend dient zur Vorbereitung auf das Erwerbsleben, das Erwachsenenalter gilt als aktive Erwerbsphase sowie das Alter als Ruhe- oder Erholungszeit von der Erwerbsarbeit. Die neue Qualität eines solchen Lebenslaufs liegt dabei weniger, das betont Kohli und belegt es mit Zeitstudien früherer Jahrhunderte, in veränderten Lebenserwartungen oder verändertem Familiengründungsalter. Vielmehr liegt die Besonderheit in der Standardisierung des Lebensablaufes. Die Konzentration von Durchschnittswerten auf eng(er) begrenzte Altersjahre führt zu der Implementation von Normen: „Durchschnittswerte entwickelten sich zu tatsächlichen Normen“ (Kohli 1985: 8). Anders zum Beispiel als in früheren Gesellschaften, in denen sich Todesfälle - nach einer hohen Sterblichkeit im ersten Lebensjahr - mit nahezu konstanter Rate über den ganzen Lebenslauf ereignet haben, konzentrieren sich Todesfälle inzwischen auf das höhere Alter der Menschen:

„Unter den Sterblichkeitsverhältnissen von 1871 - 81 starben z.B. von den Frauen, die das 20. Altersjahr erreichten, $17,2 \%$ bis zum 40 . Altersjahr und von den Überlebenden wiederum $29,6 \%$ bis zum 60 . Altersjahr; unter den Sterblichkeitsverhältnissen von 1979 - 81 betragen die Werte 1,5\% und 7,3\%“ (ebd.: 6).

Der Lebenslauf dient als Regulatorium und Korsett des modernen Menschen. Er bietet eine Orientierung und gleichzeitige Begrenzung des Möglichen bzw. Unmöglichen. Zudem stellt er eine Temporalisierung des Lebens dar, die Zukunft als Planbares und Planendes gerät in den Fokus von Entscheidungen: Menschen zu Beginn ihres Arbeitslebens träumen von der Zeit der Pensionierung, Jugendliche gehen in Ausbildungen, deren Strukturen für die weitere berufliche Zukunft entscheidend sind. ${ }^{5}$ Statuspassagen verbinden als Übergänge die verschiedenen

5 So lässt sich auch das Phänomen ,Aussteiger(nnen)` besser erklären: Sie irritieren nicht nur aufgrund ihrer Verweigerung, sich einem solchen Lebenslauf zu unterwerfen, sondern auch aufgrund ihres dahinter liegenden anderen temporalen Verständnisses. Nicht die Zukunft als Versprechen, 
Abschnitte eines Lebenslaufs und dienen als ,Nadelöhr', wie beim Übergang von der Schule in die Berufsausbildung, zur Verteilung von Chancen und Platzierung der Individuen (vgl. dazu z.B. Wahler/Witzel 1995; Witzel/Helling/Mönnich 1996). Das Modell des institutionalisierten Lebenslaufs ist aus verschiedenen Gründen in die Kritik geraten. Durch Analysen insbesondere der Übergange in den Beruf hat die Frauen- und Geschlechterforschung nachweisen können, wie stark solche Statuspassagen vergeschlechtlicht sind und wie die Lebensläufe von Männern und Frauen durch das Zusammenspiel von institutionellen Vorgaben und subjektiven Wünschen als Männer- und Frauenlebensläufe erkenn- und sichtbar sind und bleiben, da der Lebenslauf als eigenständiges Vergesellschaftungsprogramm fungiert: „Geschlechtsspezifische Traditionen [...] verfestigen sich zugleich, sozusagen als ,geronnene Gewalt‘ (wie H. Marcuse es nennt), in den die Gesellschaft strukturell gestaltenden Institutionen“ (Krüger 1991: 141; siehe auch Dausien 1996; Heintz/Nadai/Fischer et al. 1997). Die Art der Verflechtung von männlichen und weiblichen Lebensläufen lässt sich als GeschlechterInstitutionalisierung lesen: Institutionen wie das Berufsbildungssystem gehen mit Männern und Frauen unterschiedlich um und verweisen Männer und Frauen in je unterschiedliche Bereiche. Geschlechtsspezifische Analysen machen auf das Phänomen aufmerksam, dass sich Männer und Frauen

,auf der ,Rolltreppe des Lebenslaufs‘ eben nicht erst ab deren Mitte und auf gleicher Stufe stehend entdecken und als Paare organisieren, sondern sie scheinen sozialstrukturell auf zwei unterschiedlichen Rolltreppen angesiedelt zu sein, deren Verhältnis zueinander erklärungsbedürftig bleibt“ (Born/Krüger 2001: 16).

Nicht nur in dieser Hinsicht ist eine solche Konzeptualisierung geschlechterblind, die als vermeintlich ,typischer' Normallebenslauf (Levy 1977) geschlechtsspezifische Lebens(ver)läufe ignoriert: so ist das Modell von Kohli ein Modell eines auf den Produktionsbereich beschränkten, von persönlichen Bindungen und Verpflichtungen ,gelösten“, scheinbar geschlechtslosen Wesens, tatsächlich jedoch der Verlauf eines männlichen Individuums. Weibliche Lebensläufe aber, so zum Beispiel die Diagnose von Becker-Schmidt 1991, besitzen aufgrund der doppelten Vergesellschaftung von Frauen - in einen Produktions- und Reproduktionsbereich - eine größere Widersprüchlichkeit in der Sozialisation.

als Zeitpunkt der Einlösung von Investitionen stellt den Bezugspunkt dar, sondern die Gegenwart und die zeitlich unmittelbare Einforderung von Lohn und Genuss. 
„Dies gilt sowohl hinsichtlich der historischen als auch hinsichtlich der individuellen Kontinuität" (ebd.: 394). Als zentrales normatives Vergemeinschaftungsmittel und Ordnungsprinzip der weiblichen Normalbiographie stelle sich daher das ,Drei-Phasen-Modell' und ein Prinzip der „doppelten Lebensführung“ (Geissler/Oechsle 1994: 146), des Versuches der Relationierung des Produktions- und Reproduktionsbereiches, dar. Im Entwickeln einer solchen Lebensführung, die quer zu den traditionellen Segregationslinien zwischen Beruf und Familie, Öffentlichkeit und Privatheit verläuft, seien Frauen sogar „,Vorreiterinnen“ des Modernisierungsprozesses“ (ebd.: 300). Auch dieser Gegenentwurf zu Kohlis Modell stellt allerdings die strukturierende und integrationssichernde Funktion des Lebenslaufs in den Mittelpunkt der Argumentation. Das Modell des institutionalisierten Lebenslaufs ist insofern nicht nur aufgrund seiner Geschlechterblindheit kritisch zu diskutieren. Vielmehr stellt es eine strukturierende Vorgabe dar, die im Grunde nur noch von den Individuen verinnerlicht und durchlaufen werden muss. Ein solch abhängiges Verhältnis wird aber durch Deinstitutionalisierungs- und Modernisierungsprozesse aufgelöst:

„Es geht nicht mehr allein um die Abarbeitung gesellschaftlich normierter Lebensereignisse und die Übernahme alters- und geschlechtsentsprechender Rollenerwartungen zur richtigen Zeit, sondern zunehmend um die biographisch stimmige Abfolge und Kombination auch neuartiger Rollenkonfigurationen“ (Heinz 2000: 167).

In dem Maße also, in dem herkömmliche Orientierungsmuster erodieren und Lebensläufe sich vervielfältigen, verliere auch das Modell an symbolischer und realer Bedeutung.

„Als Charakteristikum einer modernen hochkomplexen Gesellschaft gilt nach den erziehungs- und sozialwissenschaftlichen Modernisierungs- und Individualisierungsdebatten etwa der letzten zehn Jahre die Offenheit des Lebensentwurfs, ein großer individueller Handlungsspielraum sowie die Aufweichung sozial institutionalisierter Bildungsverläufe“ (Schlüter 1999: 33).

Diese postulierte Offenheit des Lebensentwurfs muss allerdings hinsichtlich zweier, auf den ersten Blick sich widersprechender Phänomene differenziert werden. Zum einen hat, so zeigt sich in vielen Untersuchungen zu Lebensplanungen (siehe zum Beispiel Hopf/Hartwig 2001), und so zeigt sich auch in Marias Interviewausschnitt zu Beginn dieses Kapitels, die Vorstellung eines linearen, planbaren und verlässlichen Lebenslaufs nicht zwangsläufig an Orientierungsfunktion verloren. Zum 
anderen gewinnt ein solches Modell gerade dann an normativer Bedeutung, wenn es an faktischer verliert:

„Bei der Frage nach der Gültigkeit der ,Normalbiographie“ bzw. der institutionalisierten Muster des Lebenslaufs ist es notwendig, zwischen der normativen und empirischen Gültigkeit zu unterscheiden. Die Hinweise auf die schwindende reale Gültigkeit des Typus der Normalbiographie implizieren ja noch keineswegs notwendigerweise, dass deren normative Bedeutung ebenso in Frage gestellt ist. Das normative Modell kann trotz - oder vielleicht sogar wegen - sozialstruktureller Umbrüche Gültigkeit behalten, ja vielleicht sogar aufgewertet werden. Etwas Ähnliches lässt sich ja bei der Diskussion um das ,Normalarbeitsverhältnis` und auch im Hinblick auf den ,Normalarbeitstag beobachten: Diese Konzepte wurden zu einem Zeitpunkt in die Analyse eingeführt, als die empirischen Verhältnisse, die sie bezeichnen sollten, sich zu verändern begangen“" (Brose/Hildenbrand 1988: 18).

Hier klaffen der praktische Verlust an Planungssicherheit und der normative Bedeutungsgehalt auseinander. Die Ungleichzeitigkeit von Praxis und normativen Erwartungen wird gerade in Bezug auf die geschlechtsspezifische Arbeitsteilung besonders deutlich. Für die von mir interviewten Paare spielt die normative Idee eines kohärenten und verlässlichen Lebenslaufs inklusive geschlechtsspezifischer Arbeitsteilung eine wesentliche Rolle, im Übrigen auch für das soziale Umfeld des Paares. Insbesondere die Eltern sind erheblich von der ,Rollenumkehrung des Paares irritiert. Umgekehrt jedoch finden sich ebenfalls praktische Kontinuitäten in der geschlechtsspezifischen Arbeitsteilung, obwohl oder vielleicht grade weil die normative Bedeutung nachgelassen hat. Dass diese trotz aller Globalisierungs- und Modernisierungsprozesse erstaunlich stabil bleibt, sollte die Aufmerksamkeit für die ,rhetorische Modernisierung“ (Wetterer 2003) schärfen. Wetterer analysiert daher auch die zunehmende Diskrepanz zwischen dem vorherrschenden Gleichheitsdiskurs einerseits und dem, „was nicht zur Sprache kommt, aber u.a. in Gestalt latenter Geschlechternormen und institutionalisierter Strukturvorgaben weiterhin das soziale Handeln bestimmt" (Wetterer 2003: 291). Sie arbeitet mit Goffmans Konzept der ,institutionellen Reflexivität ${ }^{6}$, um den Wandel zu erklären: Passten bislang (idealtypisch)

6 Gemeint ist damit die Beobachtung, dass ,das soziale Geschlecht so institutionalisiert wird, dass es genau die Merkmale des Männlichen und Weiblichen begründet, welche angeblich die differente Institutionalisierung begründen“" (Kothoff 1994: 162). Deutlich macht Goffman dies an seinem berühmten Beispiel der Toiletten: Es existieren unterschiedliche Räume für Männer und Frauen, die als Folge des „natürlichen Unterschieds dargestellt werden, obwohl sie doch eher ein Mittel zur Anerken- 
die verschiedenen Ebenen und Medien der Geschlechterkonstruktion zusammen und schufen einen sinnstiftenden und einheitlichen Bezugsrahmen, fallen heute die Ebene der Praxis und die Ebene des Diskurses auseinander: „ihr Wissen und ihr Tun passen nicht mehr so recht zusammen“ (ebd.: 291). ${ }^{7}$ Umgekehrt müssen eben nicht, wie sich in den Studien zur Arbeitslosigkeit des Mannes und zum dual earner Modell gezeigt hat, ,neue Praxen“ ebensolche ,neuen“ Einstellungen nach sich ziehen. ,Ihr Wissen und ihr Tun passen nicht mehr so recht zusammen", aber hier in Bezug auf eine (normative) Einstellung $\mathrm{zu}$ einer geschlechtsspezifischen Arbeitsteilung, die mir der faktischen Beruftstätigkeit der Frau und deren finanziellen Beitrag zum Familieneinkommen nicht übereinstimmt. So einfach lässt sich also nicht von einem normativen und praktischen Bedeutungsverlust reden. Vielmehr muss je nach Phänomenbereich differenziert werden. Die geschlechtsspezifische Arbeitsteilung stellt dabei einen extrem stabilen Bereich dar, das female breadwinner Modell irritiert nicht nur normative Erwartungen, sondern auch die Lebenspraxis der meisten Familien. Diese Form der Widersprüchlichkeit ist Teil der Lebenswirklichkeit auch der binationalen Paare. Gleichheitsbetonende Diskurse einerseits und differenzkonstruierende Praxen andererseits müssen sich nicht ausschließen. Vielmehr kann ein solcher Egalitätsdiskurs eben auch geschlechtsspezifische Differenzen verschleiern, wie besonders Kaufmann 1995 gezeigt hat. Zudem können Erwartungen und Vorstellungen zum Beispiel über eine gleichberechtigte Partnerschaft durchaus mit den dann erlebten Folgen kollidieren, die eine solche Form der Gleichberechtigung oder sogar Rollenumkehrung in einem anderen Licht erscheinen lassen.

Das Modell des kohärenten Lebenslaufs hat insgesamt an Verlässlichkeit verloren; oder, wie es Dausien formuliert, „das Auseinandertreten von Erwartung und Erfahrung“ (Dausien 2002: 141) bestimmt die soziale Lage der Individuen in (post)modernen Gesellschaften: Ausbildungen bieten keine Garantie (mehr) für einen Arbeitsplatz, von lebenslanger Betriebszugehörigkeit ganz zu schweigen, die Ehe stellt keine

nung oder sogar Hervorbringung dieses Unterschiedes sind“ (Goffman 1994: 134).

7 Vgl. dazu Kaufmann 1995; Koppetsch/Burkart 1999 und zuletzt Bundeszentrale für gesundheitliche Aufklärung 2004: In 90\% der Lebensgemeinschaften mit Kindern tragen die Männer den finanziell höheren Beitrag zum Lebenseinkommen bei, die Frauen sind dafür stärker für den Haushalt zuständig (S. 11), siehe auch Menz/Putschbach 2001. Das Auseinanderfallen von Praxis und Diskurs wird auch für die Interpretation der Paare eine Rolle spielen, lassen sich dort doch ebenfalls gleichheits- und kosmopolitische Diskurse einerseits und differenzkonstruierende Praxen andererseits analysieren. 
Versorgungsgarantie (mehr) für Frauen dar, und Mehrgenerationenfamilien als materielle und lokale Organisations- und Unterstützungseinheit sind ebenfalls aufgrund von zunehmenden Mobilitätsanforderungen und Vervielfältigungen von Familien- und Lebensformen kein verlässliches System (mehr). Berufsabschlüsse und Karriereabläufe verlieren an Erwartbarkeit und gewissermaßen an Vorhersagbarkeit, Investitionen, seien sie beruflicher oder familiärer Art, müssen nicht mehr zwangsläufig $\mathrm{zu}$ den erwarteten Ergebnissen führen. Insgesamt lässt sich sagen, dass Übergänge und institutionalisierte Ordnungssysteme an integrativer Kraft verloren haben, wohl aber nicht, um dies noch mal zu betonen, an normativer.

Gesellschaften geben, so lässt sich resümieren, bestimmte Probleme an die Individuen ab. Moderne Gesellschaften versprechen weniger Sicherheit als vielmehr die Notwendigkeit ständiger Reflexion und biographischer Selbstvergewisserung und die Möglichkeit zur Selbstgestaltung. Die Biographie lässt sich daher als Integrationsleistung der Subjekte verstehen, die so Risiken und Unabwägbarkeiten in den Lebensweg $\mathrm{zu}$ integrieren versuchen (zu möglichen Subjektkonzeptionen bei der Herstellung bzw. dem Herstellungsversuch biographischer Sicherheit s. auch Eßer/Zinn 2002). Die Biographie übernimmt dabei an verschiedenen ,Schnittstellen' des Lebens sichernde Funktionen, an denen zum Beispiel brüchig gewordener Übergänge zwischen verschiedenen Statusgruppen kohärent gestalten werden müssen. Die Planungsnotwendigkeit der Subjekte nimmt daher zu, gleichzeitig nehmen aber paradoxerweise die Planungsmöglichkeiten von Subjekten ab. So müssen Entscheidungen über die Ausbildung oder den Zeitpunkt der Familiengründung selbst getroffen werden, dafür gibt es allerdings keine Garantie, dass die Ausbildung die richtige ist, die zu einer Anstellung oder einer erfolgreichen Selbständigkeit führt, oder dass die Partnerin oder der Partner tatsächlich der Lebenspartner bleibt.

\section{Biographie als ,Ort' von Subjekt und Struktur}

Die Sicherung der Übergänge und die lebenszeitliche Verlässlichkeit, die bislang in Statuspassagen und durch Institutionen angeboten wurden, muss also von den Individuen selbst vorgenommen werden. Damit verschiebt sich auch, wie Brose/Hildenbrand treffend bemerken, das Verhältnis von lebenszeitlichen und alltagszeitlichen Strukturen: „Immer mehr alltägliche Situationen bekommen potentielle biographische Relevanz und werden unter dem Gesichtspunkt selektiver Risiken und Irreversibilität behandelt“ (ebd. 1988: 21). Dieses Phänomen, welches ja in 
besonderer Weise für binationale Paare zutrifft, wird von Brose/Hildenbrand mit dem Begriff der ,Biographisierung' beschrieben. Darunter verstehen sie ,eine selbstreferentielle Behandlung (das kann heißen: Thematisierung) von biographisch relevanten Ereignissen und Situationen“ (ebd.: 21). ${ }^{8}$ Brose/Hildenbrand konstatieren eine erhöhte Rückbindung von Erfahrungen an die eigene Biographie und die zunehmende Selbst-Thematisierung und Reflexion (und damit auch Bedeutungserhöhung) von Erfahrungen. Die potentielle biographische Relevanz muss aber von den Individuen selbst entschieden und getragen werden, nicht nur müssen sie also in gewisser Weise entscheiden, welche Erfahrungen und Wissensbestände für sie in lebenszeitlicher Perspektive Bedeutung bekommen (sollen), sie tragen auch die Verantwortung für die weitere Anschlussfähigkeit ihrer Biographie. Die ,neue Herausforderung' der Biographiekonstruktion in modernen Gesellschaften liegt daher in der flexiblen Neu-Orientierung an neue und evtl. auch unerwartete Anforderungen moderner Wissensbestände. Für diese Neu-Verortungen und Anforderungen wird das Subjekt selbständig verantwortlich: „Für Flexibilität und Kreativität, die neuen Gütesiegel eines guten Lebens, gibt es noch keine Katechismen“ (Brose/Hildenbrand 1988: 17). ${ }^{9}$ Biographien und Lebensläufe werden zunehmend kontingent, da, wie es Eßer und Zinn beschreiben, „Ungewissheit das Kennzeichen gesellschaftlicher Strukturen wird" (Eßer/Zinn 2002: 13). ${ }^{10}$

Die Biographie als soziale Konstruktion und als Vermittlungs- und Integrationsmoment zwischen und von Subjekt und Gesellschaft ist daher weit mehr als ,nur ' die persönliche Lebensgeschichte eines Individuums oder die persönliche und individuelle Ausformung dieses Korsetts. Biographien, wie sie in den Interviews erzählt werden, lassen sich,

8 Vgl. dazu auch schon Kohli, der eine zunehmende Biographisierung hinsichtlich zweier Aspekte postuliert: Zum einen in der Institutionalisierung des Lebenslaufs, die das Individuum als vergesellschaftendes Moment fokussiert, zum anderen in der Veränderung des zeitlichen Horizontes, in welchem nicht mehr das situative Leben im Mittelpunkt steht, sondern eine Orientierung am lebenszeitlichen Horizont (vgl. Kohli 1986: 191).

9 Diese Zuschreibung von Verantwortung führt häufig, wie sich in empirischen Studien zeigt, zum Verlust von Wissen um strukturelle Macht. Einmündungen an der ersten Schwelle werden zum Beispiel, auch von den Mädchen selbst, als ,Berufswahl“ eingeordnet: Damit liegt die Verantwortung für die Einmündung in schlecht bezahlte ,Frauenberufe‘ bei den Mädchen selbst, die institutionalisierte Form der geschlechtspezifischen Berufswahl wird aus den Augen verloren (vgl. dafür die grundlegende Studie von Heinz 1985).

10 ,Kontingenz ist etwas, was weder notwendig ist noch unmöglich ist; was also so, wie es ist (war, sein wird), sein kann, aber auch anders möglich ist" (Luhmann 1984: 152, s. auch Zinn 2001). 
in Unterscheidung zum Lebenslauf, als (Selbst-)Konstruktion eines Lebens verstehen. Sie übernehmen bestimmte Integrationsfunktionen des institutionalisierten Lebenslaufs, wie die Herstellung der Kontinuität in Migrationsprozessen oder in der Entwicklung einer gemeinsamen Lebensplanung als binationales Paar. Die Lebensgeschichte lässt sich daher, so zum Beispiel bei Schimank, als die „Kontinuität und Konsistenz personaler Existenz" konstituierend verstehen (Schimank 2002: 235). Sie ist die lebensgeschichtliche Verarbeitung eines Subjekts und zeigt, wie sich im Umgang mit gesellschaftlichen Institutionen und Strukturen Subjektivität konstituiert und wie durch das biographisch eigensinnige Handeln der Subjekte Strukturen entstehen und sich transformieren. ${ }^{11}$ An Biographien lässt sich verfolgen, wie Menschen in unterschiedlichen sozialen Situationen ihre Biographie herstellen, welche gesellschaftlichen Bezüge in welcher Weise handlungsrelevant und damit verifiziert werden, andererseits aber auch an welchen Stellen Brüche und Widerständigkeiten $\mathrm{zu}$ entdecken sind und gesellschaftliche Lebensmodelle modifiziert und/oder erweitert werden. „Mittels biographischer Studien kann der Frage nachgegangen werden, zu welchen Formen von Sozialität Individualisierungstendenzen im alltäglichen Leben tatsächlich führen und was diese Beschleunigung gesellschaftlicher Veränderungen für den individuellen Lebensvollzug bedeutet" (Scheibelhofer 2003: 90). Der Instabilität der eigenen Lebensplanung und der zunehmenden Unzuverlässigkeit von temporal langfristigen Entscheidungen steht daher der biographische Eigensinn der Subjekte gegenüber. Menschen treffen Entscheidungen, die sie von vorgegebenen Pfaden wegbringen oder sie werden vor Situationen gestellt, die sie dazu zwingen sich neu zu orientieren. Die Arbeitslosigkeit des Mannes kann unerwartet sein, dann müssen die Paare eine Möglichkeit finden damit umzugehen. Solche Entscheidungen, die getroffen werden müssen, enthalten Potential für emergente Prozesse die als Sinnüberschuss ,,weder das lernende Subjekt noch den umgebenden strukturellen Kontext unverändert" lassen (Alheit 1995: 299). ${ }^{12}$ Die Fähigkeit des Subjektes, flexibel mit neuen Herausforderungen umzugehen und sich darin neu zu verorten, die Alheit als

11 Die oft verbreitete Formulierung der ,Spiegelung ' des Lebenslaufs in der Biographie ist auch aus einem anderen Grund falsch: Spiegelung suggeriert Vollständigkeit, eine Biographie ist aber zwangsläufig nur eine Selektion relevant erscheinender Momente, eine Rekonstruktion einer Biographie kann nicht vollständig sein. Insofern handelt es sich bei dem Begriff der ,Spiegelung' bestenfalls um eine unglückliche und schlimmstenfalls um eine theoretisch falsche Wortwahl (vgl. dazu auch Hahn 1988: 93f.).

12 „Der Begriff der Emergenz bezeichnet das Auftreten eines neuen Ordnungsniveaus, das aus den Eigenschaften des materiellen und energetischen Unterbaus nicht erklärt werden kann“" (Kneer/Nassehi 2000: 62). 
neue „Schlüsselkompetenz“ (Alheit 1995: 292) ansieht, bezeichnet er mit dem Begriff der ,Biographizität‘. Er versteht darunter die „Fähigkeit, moderne Wissensbestände an biographische Sinnresourcen anzuschließen und sich mit diesem Wissen neu zu assoziieren“ (Alheit 1990: 327). Sie

„, ist allerdings nicht nur eine spontane Konstruktion, die unser Gedächtnis als Reaktion auf neue Außenimpulse erzeugt, um seine Kontinuität und Konsistenz zu wahren. Sie muss als Konstruktion ,in der Zeit', als biographische Temporalisierung sozialer Strukturen begriffen werden“" (Alheit 2003: 25, Herv. d. Verf.).

In diesem Verständnis von Biographie wird erneut die „Biographizität des Sozialen“ (Alheit/Dausien 2000: 274) deutlich gemacht. Subjekte sind keine autopoietischen Systeme, die unabhängig von anderen Systemen und Eindrücken funktionieren und deren biographische Kommunikation strikt selbstbezogen und von außen nicht nachvollziehbar ist. Sie sind konstitutiv abhängig vom sozialen Raum, innerhalb dessen Erfahrungen gemacht und Handlungen vollzogen werden. Erfahrungshorizonte werden in der jeweiligen gesellschaftlichen Umwelt konstituiert. Das bedeutet, dass biographische Entscheidungen und Handlungen einerseits auf früheren Erfahrungen aufbauen, andererseits aber nicht unveränderliche Handlungsorientierungen darstellen, sondern durch weitere Erfahrungen verändert werden. Die Entscheidung eine binationale Partnerschaft einzugehen, ist, wie jede Entscheidung für oder gegen eine Partnerschaft, auch durch bisherige Erfahrungen strukturiert, durch Erwartungen, die man an die Beziehung und an die gemeinsame Zukunft hat. Auch die weiteren Entscheidungen und Bearbeitungsmodi sind durch frühere Erfahrungen organisiert, verändern sich aber wiederum durch die neuen Erfahrungen, die man in der Beziehung macht. Die Art und Weise, wie das Individuum Informationen aus der Umwelt aufnimmt und verarbeitet, lässt sich mit der ,nach außen hin offenen Selbstreferentialität" (Alheit/Dausien 2000: 264), der biographiespezifischen Logik, beschreiben: Zwar verarbeitet das Gehirn Erfahrungen nach den eigenen Konstruktionsprinzipien, diese sind jedoch nicht unabhängig von seiner sozialen Umgebung zu denken. Vorstellen könne man sich die Verarbeitung von Erlebnissen daher nicht wie ,outputs', die bei allen gleichen und damit erwartbaren Verarbeitungen der ,inputs', sondern vielmehr als ,intakes', als selbstreferentielle Bearbeitungen der Erlebnisse (vgl. dazu Alheit/Dausien 2000). Die nach außen hin offene Selbstreferentialität weist auf die Existenz einer ,gemeinsame[n] Semantik [hin], die ,Soziales` biographisch codierbar und ,Biographisches` sozial 
transportierbar macht" (ebd.: 265), in Abgrenzung zur Unmöglichkeit der Kommunikation zwischen dem Subjekt und der sozialen Umwelt.

Erfahrungen der Subjekte sind in einem solchen Konstruktionsprozess die entscheidenden Brücken ,zwischen biographischer Vergangenheit, biographischer Gegenwart und biographischer Zukunft" (Hoerning 2000: 4). Der Begriff der Erfahrung, wie er hier verstanden wird, ist von Dewey übernommen, wie auch Dausien ihn verwendet. Sie versteht Erfahrung als ,Zusammenhang zwischen Handeln, Erleben und Reflexion, zwischen (inter)aktivem Tun, Erleben mit seinen leiblichen und emotionalen Qualitäten sowie den kognitiven Prozessen von Wahrnehmung und Denken“ (Dausien 2002: 221 nach Dewey 1964). Die Art und Weise der Erfahrungsstrukturierung ist nicht abgekoppelt von gesellschaftlichen Strukturen zu sehen, sondern entsteht vielmehr in der Auseinandersetzung und auch in Abhängigkeit davon. ${ }^{13}$ Alheit konzipiert das Verhältnis zwischen Erfahrungen als Aufschichtung verschiedener Ebenen, deren kontrastierende Dimensionen einerseits „,soziale Rekapitulationsformen mit großer Erlebnisnähe und unmittelbarem Ereignis- und Handlungsbezug“ darstellen, andererseits ,relativ ,universalistische“ normative Orientierungen, die den ideologischen Überbau einer konkreten historischen Gesellschaftsformation bestimmen" (Alheit 1989: 140). Erfahrungen werden dabei zu unseren Erfahrungen, indem wir sie nach einer bestimmten, biographisch jeweils unterschiedlichen Typik ,ordnen'. Neue Erfahrungen sind immer schon durch das bestehende Erfahrungsmuster vorstrukturiert, können aber ebenso die Struktur erweitern oder modifizieren. Das bedeutet, dass in einem neuen Kontext der Erfahrungsverarbeitung immer auch das durchscheint, was vorher schon gegenwärtig gewesen ist. So entstehen kumulierte Handlungs- und Orientierungsmuster, die als subjektspezifische Verarbeitungsmuster verstanden werden können. Die kulturellen und sozialen Zugehörigkeiten, die dabei rekonstruierbar sind, sind Zeugnisse individueller Selbstverortungen und des Verhältnisses zum sozialen Raum. Die aktuelle biographische Situation ist eingebettet in eine lebensgeschichtliche Textur, an die Geschichte (und Zukunftsperspektive) dieser Person.

„Als besondere Geschichte eines Individuums verbürgt sie [die Lebensgeschichte] dessen Einmaligkeit und Unverwechselbarkeit, sie ermöglicht die Konstruktion personaler Identität in Differenz zu anderen. Zugleich sind bio-

13 Abhängig von der sozialen Positionierung werden bestimmte Erfahrungskontexte erst gar nicht zugänglich. Die Wahrscheinlichkeit zum Beispiel, dass man als junger Mann mit abgebrochener Schulausbildung eine universitäre Karriere anfangen wird, ist eher gering (s. dazu auch Alheit/ Hoerning 1989). 
graphische Konstruktionen in einem sozialen Erfahrungsraum verortet, und sie beziehen sich auf geteilte soziale und kulturelle Vorstellungen“ (Dausien 2002: 137).

Biographische Konstruktionen brechen durch ihre Binnensicht die „,objektive[n]' Bedingungen in verschiedener Hinsicht" (Dausien 1996: 105) und lassen Objekt- und Subjektperspektive miteinander verschmelzen. Dabei folgen sie einer spezifischen Zeitstruktur: In einer Biographie können Erwartungen an Ereignisse formuliert und Entscheidungen, die getroffen wurden, expliziert werden. Eine Biographie gibt zum Beispiel Aufschluss darüber, an welchen von der Gesellschaft bereitgestellten Ablaufmustern sich die Individuen wie orientieren (können) und wo sich Veränderungen und Brüche andeuten.

„Die Lebensgeschichte ist, wie der Lebenslauf, primär eine zeitliche Konstruktion, aber die narrativ-biographische Zeitlichkeit folgt nicht den Regeln der linearen, ,objektiven' Zeitmessung, sondern weist eine eigene komplexere Struktur der Zeiterfahrung auf, in der Vergangenheit und Zukunft in der Gegenwart nach den Regeln eines subjektiven Bewusstseins miteinander verschränkt werden“ (Dausien 2002: 138).

Biographie lässt sich so als Konstruktion eines Subjektes im Spannungsfeld von institutionellen Vorgaben, strukturellen Zwängen, bisherigen Erfahrungen und zukünftigen Plänen bezeichnen. Andererseits und zugleich kann sie aber auch ein Ergebnis narrativer Stegreiferzählung sein. Dieses Verhältnis zwischen ,Leben“ und ,Erzählung' ist schwierig zu fassen, biographische Erzählungen sind ein Medium (auch andere autobiographische Medien wie geschriebene Autobiographien kommen dafür in Frage), in welchen Biographie in der narrativen Erzählung und in Reflexionsprozessen erst hergestellt wird: „Die Lebensgeschichte kann [...] zugleich als einmalige biographische Konstruktion eines Subjekts und als Modus der Konstruktion von sozialer und kultureller Zugehörigkeit verstanden werden. Sie interessiert dabei nicht nur als narratives Schema („Erzählung“), sondern auch als narrative Praxis („Erzählen“), mit der soziale Wirklichkeit interaktiv hergestellt wird“ (Dausien 2002: 137). Bevor auf die zweite Dimension von Biographie eingegangen wird, wird in einem Exkurs ein erster Zugang zur erziehungswissenschaftlichen Diskussion um biographische Lernprozesse vorgestellt. Dieser verweist auf das Veränderungs- und Transformationspotential von Subjekten, welches auch in der vorliegenden Arbeit fokussiert wird. 


\section{Exkurs: erziehungswissenschaftliche Biographieforschung und biographische Lernprozesse - ein erster Zugang}

Der skizzierte soziale Wandel und die Folgen für die Individuen hinsichtlich der Planungsnotwendigkeiten bei gleichzeitig zunehmender Planungsunsicherheiten müssen in der Biographie organisiert und bearbeitet werden. Diese Integrationsleistungen können als Lernprozesse in sozialen Kontexten verstanden werden im Sinne von ,sensible[n] Synchronisationsversuche[n] des Außen- und Innenaspektes. Sie finden gleichsam am Schnittpunkt zwischen Subjekt und Struktur statt“" (Alheit 1995: 293). Anders als beispielsweise die soziologische Biographieforschung, deren Interesse stärker auf gesellschaftliche Strukturen und deren Folgen für Individuen gerichtet ist, blickt die erziehungswissenschaftliche Biographieforschung auf das biographische Subjekt und dessen Verarbeitung gesellschaftlicher Prozesse. Theodor Schulze hat in seinen ,Landkarten' der deutschen Biographieforschung die Forschungs- und Interessensschwerpunkte beteiligter Disziplinen systematisiert (Schulze 1995, 1999). Für die erziehungswissenschaftliche Biographieforschung unterscheidet er insgesamt sechs Ansätze, deren Gemeinsamkeit in der Analyse der Verarbeitungen gesellschaftlicher Prozesse durch Subjekte liegt. ${ }^{14}$ Schulze verweist dabei auf die Tradition aus der Pädagogik, die in Form der „Erfahrungsseelenlehre" von Karl Philipp Moritz schon Ende des 18. Jahrhunderts einen Vorschlag gemacht hat, wie (Lebens-)Geschichten zum Anlass und als Möglichkeit für Lernen und Verstehen gesehen werden können (Moritz in Schulze 1995: 11). Hier wird ebenfalls der Gedanke der notwendigen Betrachtung von ,Geschichte von unten“ formuliert. Die Biographie als Bildungsprozess sowohl für die beteiligten Subjekte wie auch für die Gesellschaft ist also als eines der zentralen Themen erziehungswissenschaftlicher Biographieforschung anzusehen.

Im Verständnis von der Biographie als Bildungsprozess steckt eine enge Verbindung von biographischen Entwicklungs- und biographischen Lernprozessen. Lernen betont jedoch eher den aktiven Part des Subjektes, das sich reflexiv mit Erfahrungen auseinandersetzt und dadurch Entwicklungen hervorbringt: „Ich nehme an, [...] dass sich die unterschiedlichen Konzeptualisierungen individuellen Lebens zurückführen lassen auf verschiedenartige Lernprozesse, die die Auseinandersetzungen des einzelnen mit seiner Umwelt, seine Initiativen, auf diese Umwelt einzuwirken und seine Anstrengungen, das Leben zu bewältigen, in unterschiedlicher Weise bestimmen und herausfordern“" (Schulze 1993b: 195). Nach Schulze geht es bei biographischen Lernprozessen um die Frage, ,wie denn das lernende Subjekt im biographischen Bildungsprozess sein Leben entwirft und gestaltet" (ebd. 1995: 21). Lernen als biographischer Prozess und Bildung als emergente, biographisch vermittelte Struktur und „Reflexionsformat" (von Felden 2002: 195) erweitert die klassische Bedeutung dieser Begriffe und ermöglicht es, Biographien von Subjekten als ,Lernbiographien` zu begreifen, ohne einen zielorientierten Kompetenzbegriff von Bildung verfolgen zu müssen. Mehr noch: Die Einführung der Konzepte von Lernen und Bildung in die Biographieforschung irritiert die bisherige kon-

14 1) Einen soziologischen oder sozialisationstheoretischen Ansatz; 2) einen entwicklungspsychologischen Ansatz; 3) einen phänomenologischen Ansatz; 4) einen erziehungstheoretischen Ansatz; 5) einen bildungs- und lerntheoretischen Ansatz 6) einen hermeneutischen und kommunikationstheoretischen Ansatz (vgl. Schulze 1995: 21). 
textuelle und inhaltliche Anbindung der Begriffe an formalisierte Bildungsräume wie die Schule. Zwar finden biographische Lern- und Bildungsprozesse in verschiedenen, auch formalen Kontexten statt und sind an diese gebunden. Lebensgeschichtliches Lernen lässt sich jedoch nicht auf formale Kontexte reduzieren. Es geht darüber hinaus, indem es in formelle und informelle Kontexte als potentiellen Lernanlass begreift. Solche Lernprozesse enthalten dadurch die Chance „der Herstellung neuer kultureller und sozialer Erfahrungsstrukturen" (Alheit/Dausien 2002: 574). Eine solche Perspektive biographischer Lernprozesse, wie sie von der erziehungswissenschaftlichen Biographieforschung eingenommen wird, ermöglicht es Veränderungsprozesse von, wie in dieser Arbeit, Geschlechterkonstruktionen und Konstruktionen natio-ethnokultureller Zugehörigkeit zu konzeptualisieren und als Lernprozesse von Subjekten genauer zu betrachten. Sie bleiben damit nicht mehr ,äußerlich ' oder dem institutionellen Rahmen geschuldet, sondern werden als reflexive Erfahrungsverarbeitungen der Analyse zugänglich. Bislang allerdings wurde diese Perspektive eher als theoretisch-programmatischer Entwurf denn als empirische Konkretisierung verhandelt. Unter Berücksichtigung der vorliegenden Entwürfe zum biographischen Lernen wurde daher für die empirische Arbeit ein heuristisches Modell entwickelt. Durch die (Re-) Konstruktion und Analyse von Formen biographischen Lernens lassen sich Restriktionen, aber auch Modifikationsprozesse durch Subjekte in Beziehung zur Gesellschaft und im Prozess der Produktion und Reproduktion sozialer Strukturen und Strukturierungskategorien nachzeichnen.

\section{Biographie als Produkt von Erzählungen}

Biographien sind also einerseits komplizierte Konstruktionen zwischen vergangenen Erfahrungen, gegenwärtigen Erlebnissen, zukünftigen Lebensplänen und institutionellen Vorgaben. Die Biographie ist Bezugspunkt unserer Handlungen, dieser Bezug bedingt aber im Umkehrschluss nicht eine permanente bewusste Reflexion und die permanente Vergewisserung unserer Selbst. Sie ist uns nicht ständig als ,bewusstes Gestaltungsmoment präsent. ${ }^{15}$ Diese Beobachtung verweist auf eine weitere Dimension der biographischen Konstruktion: Biographien werden, im Reflexionsprozess wie zum Beispiel in narrativen Stegreiferzählungen, erst hergestellt. Erfahrungen werden resümiert und in eine spezifische Abfolge gebracht, es werden Gewichtungen von Erlebnissen vorgenommen und Lebensabschnitte bilanziert. All dies hat wiederum Fol-

15 Zum Element der biographischen Selbststeuerung vgl. den ironisierenden Text von Schimank, in dem er mit Hilfe des Flipperspielens die Begrenztheit und gleichzeitige Notwendigkeit biographischer Entscheidungen und Steuerung(sversuche) deutlich macht (Schimank 2002: 249 - 265): Menschen handeln oftmals, vielleicht sogar in der Mehrzahl der Handlungen, in der vagen Hoffnung, dass dieser oder dann vielleicht der nächste Ball ein Freispiel herausspielen könnte, ohne aber ein solches Freispiel absichtlich herbeiführen zu können. 
gen für die weitere Biographiekonstruktion. Die narrative Stegreiferzählung kann insofern als ein quasi provoziertes Resümee (zu einem bestimmten Zeitpunkt, nicht im Sinne einer abgeschlossenen Lebensgeschichte) verstanden werden. So entstehen zum Beispiel die Erzählungen von Maria nicht zufällig, sie sind Ergebnis eines Interviews, in dem sie der Aufforderung nachkam, ihr Leben zu erzählen. Das Prinzip dieser narrativen Stegreiferzählungen ähnelt dabei dem von Reflexionsphasen des Lebens, in denen Bilanz gezogen wird und Lebenswege betrachtet werden. In solchen Stegreiferzählungen werden durch die Erzählungen die Erfahrungen des Subjekts rekonstruierbar. Dieses Erinnern „ist [...] als eine Leistung zu sehen, die eine Ordnung in biographische Erfahrungen bringt und sie zu biographischen Wissensbeständen und damit zu biographischen Ressourcen werden lässt“" (Schlüter 1999: 47). Solche biographischen Konstruktionen sind daher aktive Konstruktionsleistungen der Subjekte. Sie konstituieren sich durch Ereignisse, denen innerhalb eines sozialen Interaktionsgeschehens Sinn und Bedeutung zugewiesen werden. Biographische Erzählungen sind also ein Medium, durch welches Biographiekonstruktionen rekonstruiert und analysiert werden können. Sie entstehen zum Beispiel in einer Interviewsituation. Dabei bilden biographische Erzählungen nicht einfach eins zu eins das ,tatsächliche“ Leben ab, zwischen Text und Leben besteht ein Abstand, der einerseits wissenschaftliche Texte in dem Sinne legitimiert, dass er Deutungen und Analysen notwendig macht, zum anderen aber nicht zu der Annahme führen darf, dass Texte beliebig sind, also lebensgeschichtliche Erzählungen aus Anlass eines Interviews völlig für sich stehen. In der Biographieforschung wird diese Frage nach dem Verhältnis von Text und Leben intensiv diskutiert. Während Schütze insbesondere in seinen frühen Texten von einer gewissen Homologie zwischen Text und Leben in dem Sinne ausgeht, dass die interviewte Person in der Erzählung in einen Erinnerungsstrom eintaucht, der dem ihrer ,tatsächlichen“ Erfahrungen ähnelt oder sogar entspricht (vgl. v.a. Schütze 1984), argumentiert Nassehi systemtheoretisch und verweigert sich einer solchen Nähe:

„Die biographische Produktion wird nicht durch das gelaufene Leben determiniert. Weder zwingt uns etwas, noch berechtigt uns etwas dazu, von einer vorgängig zu konstatierenden Homologie von Lebenslauf und Biographie [im Sinne einer biographischen Kommunikation, also dem Reden über die Biographie, M.M.] auszugehen“ (Nassehi 1994: 53; vgl. zum Überblick auch Dausien 2002). 
Die Diskussion soll hier nicht in der Tiefe aufgegriffen werden bzw. weder zur einen noch zur anderen Seite hin entschieden werden. Vielmehr folge ich hier Marotzki, der darauf hinweist, dass durch das narrative Interview Material erhoben werden kann,

„aus dem nach bestimmten Auswertungsprozeduren erschlossen werden kann, wie der Informant Ereignisse, in die er - wie auch immer - verwickelt war, verarbeitet hat" (Marotzki 1991: 184).

Damit wird also weder von einer Homologie ausgegangen, von der Vorstellung, dass mir die interviewte Person ihr Leben, so wie es wirklich war, erzählt. Noch gehe ich davon aus, dass die Erzählungen einfach nur Text sind, sozusagen erfundene Biographien, die als Produktion völlig beliebig und in gewisser Weise willkürlich erzählt werden. Vielmehr finden biographische Konstruktionen in einem spezifischen Spannungsfeld statt, stehen in einem nicht zufälligen Verhältnis zum Erlebten. Gleichwohl können in einer Erzählung neue Gewichtungen vorgenommen werden, können Relevanzsetzungen beobachtet werden, die für diese spezifische Situation charakteristisch sind. Die Art und Weise, wie dies geschieht, ist jedoch eben nicht völlig beliebig.

Für den Kontext, in welchem biographische Interviews entstehen, hat Bettina Dausien ein Modell entwickelt, in welchem die verschiedenen Rahmungen, die die Entstehung eines Interviewtextes mitstrukturieren, innerhalb derer also biographische Konstruktionen entstehen, in ein produktives Verhältnis gesetzt werden (Dausien 2002: 176ff.). Ein Verständnis von erzählter Lebensgeschichte als kommunikativ vermittelte und verarbeitete Erfahrungsaufschichtung einer Person hat insbesondere Konsequenzen für das Begreifen der Interviewsituation. Die Interviewsituation stellt als Kontext mehr als nur die Rahmung für die narrative Stegreiferzählung dar. Die Aufforderung an die Interviewerin, sich selbst ganz zurück zu nehmen und der Interviewten die Möglichkeit des Eintauchens in die Erfahrungsrekapitulation zu geben (vgl. Schütze 1984), schützt dabei nicht vor der Wirkung dieser Situation. Die narrative Stegreiferzählung besitzt neben der biographischen Rahmung eben auch eine interaktionelle Rahmung, die die Situation und die Erzählung mit strukturiert. Der biographische Text, in meinem Fall die biographische Erzählung, wird in ,ein Spannungsfeld zu drei Kontexten gestellt, die ihrerseits als Konstruktionsfelder für biographische Texte betrachtet werden können“ (Dausien 2002: 176, Herv. d. Verf.). Die drei Kontexte sind a) die biographische Rahmung, b) der Interaktionsrahmen des Interviews und c) die soziokulturelle Rahmung. Der erste Rahmen bezieht sich auf den biographischen Kontext des Subjekts, und dabei, ana- 
log zur Doppelstruktur der Biographie, sowohl auf den konstativen Aspekt (Was wird erzählt, woran wird sich erinnert?), als auch auf den performativen Aspekt (Wie wird erzählt, was für eine Art von Sprache wird benutzt, welche Metaphern werden verwendet, enthält der Text narrative, argumentative, bilanzierende Passagen, wie werden die kognitiven Figuren des Stegreiferzählens (Schütze 1984) ${ }^{16}$ verwendet?). Der zweite Rahmen bezieht sich auf die konkrete Interaktionssituation: $\mathrm{Zu}$ welcher Zeit im Leben wird erzählt, wie gestaltet sich die Interaktion zwischen der Interviewerin und der/dem Interviewten, welche kommunikativen Zugzwänge entstehen in der Erzählsituation? Der letzte Rahmen bezieht sich auf die soziokulturelle Rahmung: Auf welche sozialen Schemata wird sich wie bezogen, welche strukturellen Rahmungen und institutionalisierten Skripten werden in die Erzählung mit eingebaut, welche nicht? Alle drei Rahmungen spielen eine konstitutive Rolle im Interview. Welche jedoch bei der Interpretation der biographischen Texte explizit einbezogen werden, und welche als implizites Wissen im Hintergrund mitlaufen, hängt von der Fragestellung der Untersuchung ab und entscheidet sich häufig erst im Laufe der Interpretationen. Für die vorliegende Untersuchung sind insbesondere die Zugehörigkeitskonstruktionen der Interviewten wie auch der Bezug auf bzw. die Modifikation von institutionalisierten Skripten und strukturellen Rahmungen relevant. ${ }^{17}$ Dazu gehören vor allem die Kategorien gender und natio-ethnokulturelle Zugehörigkeit. Diese verstehe ich als biographiegebundene Kategorien. Beide Kategorien sind auf allen Ebenen der Konstruktionsrahmungen wirksam, sie werden von Subjekten in ihre biographischen Erzählungen eingebaut. In der Analyse dieser Erzählungen lassen sich Deutungsmuster und Orientierungen rekonstruieren, denen handlungsleitende Funktion zugesprochen wird. Analysieren lässt sich so auch die Einzigartigkeit der jeweiligen Konstruktionen, die Widerständigkeit und ,Eigensinnigkeit‘ von Subjekten, die sich einer eindeutigen Klassifizierung und Rolleneinteilung widersetzen.

16 Kognitive Figuren des Stegreiferzählens ordnen den Erinnerungsstrom und stellen „Figuren der Erfahrungskapitulation“ (Schütze 1984: 80) dar. Schütze unterscheidet vier Formen: 1) den Biographieträger, den Ereignisträger und ihre Beziehung zueinander; 2) die Erfahrungs- und Ereigniskette; 3) den sozialen Rahmen und 4) die Gesamtgestalt der Lebensgeschichte.

17 Die Besonderheiten der methodologischen Anlage, die sich auch durch die Form der entstandenen Interviews ergeben hat, werden im methodologischen Kapitel vorgestellt. 


\section{Zweite Annäherung: gender und natio-ethno-kulturelle Zugehörigkeit}

Nicole: „Am Anfang hatten wir noch das Geld, bis die Sprachschule kam und so weiter, aber - - wir sind nicht Essen gegangen, oder wenn wir Essen gegangen sind hab ich ihm unterm Tisch meinen Geldbeutel gegeben damit er bezahlt hat, u_ und damit es nicht so blöd aussah. Wobei ich immer gesagt hab in Deutschland interessiert es keinen wer zahlt. Aber - in Mexiko würd ich zum Beispiel niemals die Rechnung kriegen. - die Rechnung wird ihm hingelegt. - und - - ja, das Unangenehmste war eigentlich diese Geste, ansonsten - war da nix dabei für mich. Mir war's persönlich egal, weil - was ist wenn ich arbeitslos bin und er nur das Geld verdient? Kommt für mich persönlich aufs selbe raus. Oder wenn ich in Mexiko - wenn ich mit ihm nach Mexiko gegangen wär hätt ich ja erstmal ewig gebraucht bis ich ne Arbeitserlaubnis gehabt hätte und so was, dann hätt ich ja nicht arbeiten können. Also, - für mich gibt's da keine Unterschiede, weiß nicht ob's für ihn das gab aber für mich auf jeden Fall nicht. Besonders die Wohnung und alles war ja schon eingerichtet, von meinem Geld sozusagen. Wenn man dann verheiratet ist, dann ist es ja unser Geld. Ich mein, wir ham keinen Ehevertrag, gar nichts, für mich ist das - meins ist deins, also, deswegen ham wir auch heute ein gemeinsames Konto, da kommen beide Gehälter drauf, und - über Ausgaben wird - diskutiert oder nich.“ (Nicole Ortega Garcia, 30/4 - 18).

Nicole spricht hier über Anfangsschwierigkeiten in Deutschland und über ihre Lösungsstrategien, die sie sowohl für die finanziellen Probleme gefunden hat, wie auch für die Schwierigkeiten hinsichtlich der $\mathrm{Zu}$ ständigkeiten für die Verwaltung und ,Kontrolle ' des Geldes. In ihrer Erinnerung reagiert sie auf ein antizipiertes Unbehagen Carlos` hinsichtlich des Bezahlens der Rechnung durch seine Frau. Zugleich kommt aber auch ein allgemeines Unbehagen zum Vorschein: Es sieht „blöd“ aus, wenn sie (als Frau) die Rechnung für beide bezahlt. Diese Einordnung in eine traditionelle Geschlechterbeziehung wird von ihr mit kulturspezifischen Unterschieden begründet, denen sie durch ihr Handeln Rechnung tragen will. Neben vielen anderen Motiven, die sich analysieren ließen ${ }^{18}$, geht es auch um Verhandlungen von Geschlechterbeziehungen und Modellen natio-ethno-kultureller Zugehörigkeit. Gender wird nicht als abstrakte Kategorie, sozusagen ,kategorial', sondern in einem je ,anderen' konkreten Fall wirksam (vgl. Dausien 2000). Dieser jeweils andere Fall kann in Biographien analysiert werden. Analysiert

18 Man beachte z.B. die Konstruktionen zum Eheverständnis, die eine deutliche Differenz zu einer weniger festen Beziehung in der Gemeinsamkeit und dem Wechsel des Bezugshorizontes (von Ich zum Wir) offenbaren. Auch hier ist eine heterosexuelle Geschlechterbeziehung ihr Bezugspunkt. 
werden kann so auch die Verflechtung von gender mit anderen Differenzkategorien wie natio-ethno-kultureller Zugehörigkeit.

Gender und natio-ethno-kulturelle Zugehörigkeit sind im Grunde nicht zu trennende Kategorien, wird doch Geschlecht in konkreten Biographien immer mit anderer Zugehörigkeitskonstruktionen wie Ethnizität, Klasse oder Generation verflochten. Zudem sind Geschlechterordnungen und Geschlechterbeziehungen Teil kultureller Praxen. Dennoch möchte ich im Folgenden die Überlegungen zu beiden Kategorien analytisch trennen, um auf die Besonderheiten der Modelle einzugehen, die meinen Analysen zugrunde liegen. Der Fokus liegt auf der Spannung zwischen der Bedeutung der Kategorien als Strukturkategorie einerseits und der konkreten und je ,anderen“ (Re-)Konstruktion durch Subjekte. Anschließend wird durch Verweis auf Studien und Konzepte der interkulturellen Geschlechterforschung das Prinzip einer ,intersectional analysis“ vorgestellt.

\section{Gender als biographische Konstruktion}

1995 formulieren Gildemeister/Wetterer die erstmal unverdächtig anmutende Frage, wie es denn zur sozialen Konstruktion der Zweigeschlechtlichkeit komme: „wie die Zweigeschlechtlichkeit im Alltagshandeln als ,moral fact' (Garfinkel) hergestellt, statt von der Natur bereitgestellt wird, und wie ein ,sameness taboo' [Lorber 1975] [...] fortwährend institutionalisiert wird, demzufolge Frauen und Männer zunächst einmal und in allen Belangen verschieden $\mathrm{zu}$ sein haben“" (Gildemeister/Wetterer 1995: 202). Mit diesem Artikel haben die beiden Autorinnen einen Paradigmenwechsel in der deutschsprachigen Frauen- und Geschlechterforschung eingeleitet, durch den der Fokus nicht mehr auf den (biologisch oder sozialisationstheoretisch begründeten) Unterschieden zwischen den Geschlechtern liegt, sondern vielmehr auf der Frage, wie solche Differenzen konstruiert und perpetuiert werden. Geschlecht als soziale Konstruktion zu verstehen, bedeutet die Selbstverständlichkeit und ,Natürlichkeit‘ von Geschlechtszugehörigkeiten zu hinterfragen und stattdessen die ,vielfältigen interaktiven, institutionellen und diskursiven Leistungen [zu analysieren], [...] die die eindeutige Zuordnung der Menschen zu jeweils einem der beiden Geschlechter und die Zweigeschlechtlichkeit als selbstverständlich und als ,natürlich“ herstellen“ (Breitenbach 2000: 19). Solche Analysen nehmen insofern nicht nur die historisch je verschiedenen Erscheinungsformen des ,Weiblichen“ und ,Männlichen', sondern die Grundstruktur der Zweigeschlechtlichkeit selbst in den Blick (vgl. Wetterer 1995: 223. Vgl. zur Etablierung des 
Diskurses auch die Feministischen Studien 2/1993). Diskutiert wird nicht mehr, inwiefern sich Männer und Frauen unterscheiden. Vielmehr treten die Forscher(innen) hinter das selbstverständliche Wissen um Geschlecht und hinter die theoretischen und begrifflichen Voraussetzungen zurück (vgl. Breitenbach 2001: 166). Gefragt wird nach dem Wie der fortwährenden und bestehenden Konstruktion von Zweigeschlechtlichkeit: auf welche Weise greifen Subjekte auf Muster von Geschlechterzugehörigkeiten zurück, wie stellen sie sie dar, in welchen Kontexten werden sie relevant und wie sind sie miteinander und mit anderen Kategorien wie Race oder Class verbunden? Geschlecht ist ein konstitutives gesellschaftliches Ordnungsprinzip, „das wesentliche Lebensbereiche von Männern und Frauen nach geschlechterspezifischen Distinktionsmerkmalen regelt und sich auf die Genese konfligierender Interessen, Machtunterschiede, die Herausbildung von Hierarchien, unterschiedliche Privilegien und Teilhabechancen auswirkt" (Glenn 1999 in BednarzBraun/Heß-Meining 2004, S. 41). Der Verweis auf die soziale Konstruiertheit von Geschlechtszugehörigkeit(en) und der damit einhergehende Verzicht auf die Vorstellung einer biologisch begründeten Unterscheidung in Männer und Frauen ist, darauf weißt z.B. Breitenbach hin, „gleichzeitig ein Verzicht auf die Vorstellung einer Gemeinsamkeit aller Frauen bzw. aller Männer aufgrund ihrer körperlichen Ausstattung“" (Breitenbach 2000: 19).

Für die Perspektive auf Geschlechterkonstruktionen in Biographie kann auch (mindestens) zwei theoretische Zugänge zurückgegriffen werden. Diese schließen sich nicht aus, setzen jedoch jeweils unterschiedliche Akzente und verweisen auf die komplexe Lagerung von gender als sozialer Struktur, als (inter-)aktiv hergestellter und als biographischer Kategorie.

\section{Gender ethnographisch: doing gender}

Der Prozess des ,doing gender‘ (West/Zimmerman 1991), später erweitert zum allgemeineren, doing difference“ (West/Fenstermaker 1995) bedeutet ,creating differences between girls and boys, women and men, differences that are not natural, essential or biological" (West/Zimmerman 1991: 24). Ein solch interaktionistischer Blick wurde in der weiteren Analyse einerseits vor allem in der Tradition der TranssexuellenForschung empirisch weitergeführt (zurückzuführen auf Garfinkel 1967; Hirschauer 1993), zum anderen wurden interaktionistische Studien zu Geschlechterkonstruktionen in der Schule durchgeführt (Thorne 1993; Breidenstein/Kelle 1998, Menz 2001). Doing gender fokussiert die interaktiven Praktiken von Geschlechterkonstruktionen im Sinne der 
Geschlechterarrangements, wie sie Goffman 1994 beschreibt. Die Analyse solcher situativer Geschlechterverortungen und -konstruktionen ermöglicht ein weitergehendes Verständnis des Aspektes der genderPerformance. Der „Imperativ der geschlechtlichen Identifizierbarkeit“, wie Wetterer es bezeichnet hat (1995: 237), beschreibt die Notwendigkeit und auch Verpflichtung, in jeder Interaktion entweder Mann oder Frau zu sein, „Auszeiten“ (Tyrell 1986: 471) oder Geschlechtslosigkeit kann es nicht geben. Ethnographische Studien untersuchen nun eben solche doing gender Prozesse ,,im Hinblick auf je gegenwärtige Praxis wie wird Geschlecht in Interaktionen bedeutsam, welches sind die kulturellen Praktiken, die Geschlecht intersubjektiv relevant machen“ (Dausien/Kelle 2005: 190). Der Blick liegt also eher auf der interaktiven situativen Praxis als auf längerfristigen Prozessen und biographischen Erfahrungsaufschichtungen. Die Permanenz und Dauerhaftigkeit von situativ hergestellten Geschlechtsdarstellungen, die Frage nach dem „Haben des Geschlechts bzw. der Geschlechtsidentität" (Breitenbach 2005: 81, Herv. i.O.) werden nicht bzw. weniger thematisiert (vgl. zur Kritik auch Breitenbach 2000: 30ff). Zudem betonen die empirischen Untersuchungen eher den reproduzierenden als den modifizierenden Part solcher Performances. Zwar entwickeln z.B. sowohl Hirschauer als auch Thorne die Unterscheidung zwischen einer Aktualisierung und einer Neutralisierung von gender (bzw. eines doing und eines undoing genders) in Situationen (z.B. Hirschauer 1993; Thorne 1993), empirisch lässt sich dies allerdings oft nicht nachzeichnen. ${ }^{19}$ Und nicht zuletzt läuft

„die Interpretation gesellschaftlicher Verhältnisse als je ,doing gender' Gefahr [...], die Dimension sozialstruktureller Geschlechterordnung zu personalisieren bzw. die Inkorporation von Machtungleichgewichten zwischen den Geschlechtern in der Sozialstruktur zu übersehen. Es entsteht die Gefahr, die strukturelle Gestaltung von Geschlechterhierarchie, die quasi hinter unserem Rücken wirkt, auf ein aktuelles Interaktionsgeschehen zu reduzieren, sie damit gesellschaftsanalytisch verkürzt zu interpretieren und dem, was Mary Douglas (1987) als sozialstrukturiertes Vergessen bezeichnet, in die Hände zu arbeiten“ (Krüger 2002: 64).

19 Ein Beispiel dafür sind Geschlechterkonstruktionen in der Schule unter aktiver Mitwirkung von Lehrer(inne)n, auch bzw. gerade wenn die explizite Gleichbehandlung von Mädchen und Jungen als Handlungsziel vorgegeben ist: „Solange das internalisierte Wissen von zwei und eben nur zwei Geschlechtern und deren Unterschiedlichkeit die [explizite] Handlungsbasis [der Lehrer(innen)] bildet, wird das zweigeschlechtliche System und das Geschlechterverhältnis in der Gesellschaft bestätigt und fortgeschrieben“ (Menz 2001: 94). Vgl. zur Debatte um Koedukation und die Reifizierung von Geschlechterdifferenzen Breitenbach 2002. 


\section{Gender biographisch: doing biography}

Die Analyse von gender wird von biographisch und sozialisationstheoretisch interessierten Ansätzen mit einer lebensgeschichtlichen Perspektive verknüpft. Dabei wird deutlich, dass sich beide Perspektiven eben nicht ausschließen, sondern aufeinander beziehen. So lassen sich, wie Dausien/Kelle 2005 feststellen, Repräsentationen von Biographischem im ethnographischen Material ebenso finden wir Interaktionen und kulturelle Praktiken im biographischen Material (vgl. ebd.: 190). Doing gender Prozesse lassen sich zum Beispiel, wie bei Eva Breitenbach, mit Konstruktionen von Jugend, also von Lebensphasen, verbinden.

„In einer solchen Auffassung von Jugend und Geschlecht ist die Geschlechtsidentität nichts, das in Auseinandersetzung mit den körperlichen Vorgängen der Pubertät und den gesellschaftlichen Anforderungen ,angeeignet ‘ werden muss und auf geheimnisvolle Weise aus den Tiefenschichten der Person destruiert bzw. stabilisiert wird. Wenn Geschlecht in Bildern und Tätigkeiten dargestellt und wahrgenommen wird, dann könnte die Geschlechtsidentität mithilfe von Erzählungen dieser Tätigkeiten und Beschreibungen der Bilder zusammengehalten werden“" (Breitenbach 2005: 82).

Die Geschlechtszugehörigkeit kann Teil des Erfahrungsraums von Jugendlichen sein, als Käfig oder als Handlungsraum fungieren. Darstellungen von Jugendlichkeit und Darstellungen von Geschlecht sind miteinander verbunden und bedingen sich gegenseitig: „So tanzen Mädchen, weil sie Mädchen sind und auch, um Mädchen zu sein“ (Ebd.). Der Begriff des Erfahrungsraums macht auf die Einbettung im biographischen Prozess aufmerksam. Interaktive Handlungen können lebensgeschichtliche Nachhaltigkeit entfalten, und umgekehrt drücken sich lebensgeschichtliche Prozesse auch in solchen kulturellen Praktiken der Mädchen aus.

Auch Bettina Dausien untersucht den Prozess der lebensgeschichtlichen Konstruktion von Geschlecht. (vgl. v.a. Dausien 1996, 1998, 2000, 2002, in deren Folge Thon 2003, Thon 2006). „Die lebensgeschichtlichen Erfahrungen, Intentionen, Pläne und Selbstinterpretationen der Subjekte spielen [in der Perspektive des doing gender, M.M.] keine (zentrale) Rolle, sondern kommen allenfalls als Randbemerkungen oder Ressourcen für interaktives Handeln in den Blick“ (Dausien 2000: 99). Biographische Konstruktionen aber greifen genau diese ,Tiefendimension` auf. Erfahrungsaufschichtungen strukturieren zukünftiges Handeln und sind individuelle Bearbeitungen gesellschaftlicher und institutionel- 
ler Rahmungen. So wird in biographischen gender-Konstruktionen auf vorherige Erfahrungen verwiesen und wird die Perspektive der Sozialität des Subjekts deutlich, in der sich dessen Positionierung in einem zweigeschlechtlichen System ausdrückt. Nicole verweist einerseits auf eine gesellschaftliche Geschlechterordnung, nach der in Deutschland, anders als in Mexiko, Männer nicht zwangsläufig für das Begleichen der Restaurantrechnung zuständig sind. Gleichzeitig biographisiert und individualisiert sie diese Vorstellung, in dem sie auf ein Leben in Mexiko verweist: Ohne eigene Arbeit und ohne eigenes Geld müsste Carlos schließlich für den Lebensunterhalt aufkommen. Und zu guter Letzt gibt es für sie kein getrenntes Geld, die Eheschließung hat für sie die Trennung zwischen Mein und Dein aufgehoben. Einerseits sind Konstruktionen von Geschlechterdifferenzen, wie sie in der Erzählung von Nicole sichtbar werden, also ein Teil des Modus zur Konstruktion von Biographien:

„Biographische Konstruktionen sind, solange Geschlechterkonstruktionen für die soziale und kulturelle Verfasstheit einer Gesellschaft relevant sind, immer (auch) durch diese eingefärbt, sie sind - auf vielfältige, am empirischen Fall jeweils neu zu rekonstruierende Weise - geschlechtsgebundene Konstruktionen. [...]: Biographien sind eingebunden in gesellschaftliche Strukturbedingungen und Strukturierungsprozesse, in denen soziale Differenzen und Zugehörigkeitskontexte (sozioökonomische Milieus, kulturell und ethnisch definierte Kontexte, religiöse oder säkulare Glaubenssysteme u.a.) erzeugt werden, die für die soziale Positionierung und Identitätskonstruktion eines individuellen Subjekts relevant sind“ (Dausien 2002: 190).

Zugleich aber werden in solchen Bezügen, wie sie Nicole vornimmt, Geschlechterdifferenzen und -beziehungen erst hergestellt. Damit wird deutlich, dass solche Konstruktionen nicht beliebig sind, wie ein oft formuliertes Missverständnis impliziert. ${ }^{20}$ Es lässt sich insofern eine Doppelstruktur der Bedeutung von gender in biographischen Interviews feststellen:

„,Geschlecht' bzw. die gesellschaftliche Geschlechterordnung ist eine Differenz und Zugehörigkeit erzeugende soziale Struktur, die individuell nicht hintergehbar ist. Sie existiert jedoch umgekehrt nur insofern, als sie von konkre-

20 Dieses Missverständnis findet sich ebenfalls in der Vorstellung der „frei flottierenden KonstrukteurInnen“" (wie es Lindemann formuliert, 1993: 27) im Prozess des doing gender. Konstruktionen sind weder beliebig noch frei von Machtverhältnissen. Konstrukteur(nn)e(n) sind eingebunden in strukturelle Zusammenhänge und subjektive Deutungsschemata, die doing gender Prozesse strukturieren und formen. 
ten Individuen ,gelebt' wird, d.h. aufgegriffen, performativ variiert, reproduziert und/oder neu konstruiert wird. Geschlecht kann daher [...] auch als biographische Konstruktion betrachtet werden, und diese Betrachtungsmöglichkeit konstituiert eine eigenständige Analyseperspektive“ (Dausien 2002: 190f).

Dieser zweite Aspekt verweist auf den prozessualen Charakter von Geschlecht in der Lebensgeschichte. Geschlechterkonstruktionen sind, ebenso wie andere handlungsorientierende und wirklichkeitskonstruierende Momente, in den Prozess der Biographiekonstruktion eingebunden. Die biographischen Modi der Erfahrungsaufschichtung und -verarbeitung gelten auch für Konstruktionen von gender. Eben weil biographische Konstruktionen subjektive Bearbeitungen gesellschaftlicher und institutioneller Rahmungen sind, sind biographische Konstruktionen nie einfach nur eine ,Abbildung ' herrschender Normen oder Geschlechterordnungen, sondern eigenwillige und eigensinnige (Re-)Konstruktionen, die Orte für transformative Prozesse und ,Brüche' im scheinbar so holistischen Geschlechtergefüge sein können. ${ }^{21}$ Biographisches Handeln beinhaltet immer beides: die Möglichkeit des anderen Handelns in einer anderen Situation und den Ausschluss von anderen Möglichkeiten durch Handeln. $^{22}$ Nicoles Entscheidung, Carlos unter dem Tisch den Geldbeutel zu zuschieben, beinhaltet auch die Entscheidung gegen andere Handlungsalternativen, so hätte sie auch selber zahlen oder ihm vor dem Besuch im Restaurant genügend Geld für beide geben können. Ebenso beinhaltet diese Entscheidung keine zwangsläufig vorhersagbare Entwicklung für andere Situationen. Sie kann und könnte sich in einer anderen Situation anders verhalten, Carlos und ihr eigenes Unbehagen ignorieren, oder sich reflektierend damit auseinandersetzen.

In einer solchen Perspektive liegt die Chance einer sozialkonstruktivistisch und biographisch argumentierenden Geschlechterforschung. Gender wird immer nur in konkreten Situationen relevant, kein Subjekt ist mit der abstrakten Kategorie ,Geschlecht` konfrontiert, sondern jeweils

21 Nicht sein müssen: Die Hartnäckigkeit, mit der sich Geschlechterordnungen auch in der Lebensplanung halten, zeigen übereinstimmend Studien zur Berufs- und Lebensplanung junger Frauen (vgl. z.B. Hopf/Hartwig 2001). Hier allerdings gilt es zu unterscheiden zwischen diskursivem Anspruch und tatsächlicher Lebensgestaltung. Beides muss in keiner Weise kongruent sein. Vielmehr zeigen sich oftmals große Diskrepanzen zwischen beiden Konzepten, wie im vorherigen Kapitel deutlich wurde.

22 Für den Ausschluss anderer Möglichkeiten wird insbesondere im erziehungswissenschaftlichen Zweig der Biographieforschung der Begriff des ,ungelebten Lebens' benutzt, im Sinne einer Beschränkung und einer „Nicht-Verwirklichung“ des Lebens bzw. der Entwicklungspotentiale (vgl. dazu ausführlich Dausien 1996: 60ff). 
(nur) mit seinen vielfältigen Formen und Spielarten. Damit kann gender auch nur in seiner je konkreten und individuellen biographischen Form der biographischen Konstruktion des Subjektes analysiert werden. ${ }^{23} \mathrm{Zu}-$ dem sind nicht alle biographischen Konstruktionen deutlich mit geschlechterspezifischen Konstruktionen ,aufgeladen'. Es finden sich in Biographien Textpassagen, die, wie die obige, eine nach unserem Alltagsverständnis ,eindeutige‘ Zuordnung zu weiblichen oder männlichen Lebensverläufen ermöglichen. Die Passage oben würde, von einem Mann erinnert, gerade aufgrund der kulturellen Praxis, die in der biographischen Erzählung rekonstruiert wird, schlicht ,unsinnig' erscheinen. Umgekehrt finden sich aber auch Passagen, die, auf den ersten und manchmal auch auf den zweiten Blick, nicht unmittelbar mit geschlechtlichen Konnotationen aufgeladen sind. ${ }^{24}$ Dies hat bedeutende Konsequenzen für ein Verständnis von gender als basaler Kategorie, die jeder Handlung und jeder Situation zugrunde liegt und wesentlich den Ablauf strukturiert. Geschlecht ist eine Ordnungskategorie und eine Form der biographischen Konstruktion, gewinnt aber seine Bedeutung immer erst im Zusammenspiel mit anderen Differenzkategorien wie Milieu, Generation oder eben natio-ethno-kultureller Zugehörigkeit. So verbindet Nicole ihre Deutung der Restaurantsituation mit einem Verständnis von Ehe und Gemeinsamkeit, die ihr als handlungsorientierende Selbstkonstruktion in der konkreten Situation dient. Sie formuliert einen ,RePositionierungsprozess ‘ durch die Eheschließung, um die Sinnhaftigkeit ihrer Handlungen deutlich zu machen. ${ }^{25}$ Ebenso relevant in dieser Situation erscheint aber auch Nicoles Rekurs auf kulturelle Differenzen und länderspezifische Besonderheiten in der Geschlechterordnung. Im folgenden Unterkapitel wird daher dieser Bezug auf natio-ethno-kulturelle Zugehörigkeit als biographische Konstruktion aufgenommen.

23 Solche individuellen Verortungen bleiben aufgrund der Sozialität der Biographie nicht folgenlos für gesellschaftliche Geschlechterordnungen. „Geschlecht und die Identifikation im Geschlechtersystem sind nicht allein als kategoriale (Selbst-)Zuweisungen und Verortungen zu verstehen, sondern können [...] als (Re-)Konstruktionen von Geschichten analysiert werden, eine Perspektive, die sowohl die Kontextualität als auch die historische Veränderbarkeit von Situierungsprozessen im gesellschaftlichen Geschlechterverhältnis von vornherein einschließt“" (Dausien 1998: 265).

24 Für die Interpretation einer Biographie ist nun die Analyse und der systematische Vergleich solcher Passagen erforderlich, um die Eigenlogik des/der Biographieträger(in) erfassen zu können und um eben nicht vorschnell auf Transformationen bzw. auf Perpetuationen von Geschlechterverhältnissen zu schließen.

25 Ein Prozess, der im Grunde idealtypisch den von Berger/Kellner 1965 beschriebenen Nomos-Prozess abbildet. 


\section{Natio-ethno-kulturelle Zugehörigkeit als biographische Konstruktion}

Neben der Analyse von Gender-Konstruktionen werden als zweite Perspektive auf die Biographien der binationalen Paare Konstruktionen von natio-ethno-kulturellen Zugehörigkeiten analysiert. Nicole handelt so, wie sie handelt, aus Gründen, die sie als Respekt gegenüber der , anderen' Kultur ansieht. In diesem Sinne erklärt sie sich und mir ihre Bemühungen, Carlos in der Restaurantsituation nicht bloßgestellt zu sehen und ihm seine Handlungsfähigkeit in einer für ihn richtigen Weise bewahren zu können. Hier finden sich Konstruktionen und Wahrnehmungen einer ,anderen' Kultur und sich daraus ableitenden anderen Handlungsorientierungen. Diese Differenzkonstruktionen sind Teil ihrer Selbstkonstruktionen und werden als Handlungsorientierungen relevant. Ihre Fremdbeschreibung von Carlos als Mexikaner, der durch seine Sozialisation bestimmte Erwartungen an das Verhaltens von Frauen und Männern in Restaurants hat, korrespondiert mit einem Selbstverständnis als ,Kulturtolerante' Frau, die ihrem ,fremden“ Mann zuliebe bestimmte Verhaltensweisen übernimmt. ${ }^{26}$ Für die Interpretation solcher alltagsweltlicher Konstruktionen ist ein sensibilisierendes Verständnis von kulturellen Konstruktionsprozessen vonnöten, welches, ähnlich dem Modell der geschlechtsgebundenen Biographien, die alltagsweltliche Bedeutung aufgreift, ohne sie in der Interpretation zu verdoppeln.

Der begriffliche Umgang mit und die begriffliche Verwendung von ,Kultur' ist in den letzten Jahren wesentlich von Unsicherheit hinsichtlich möglicher Definitionen geprägt, soll doch eine ebensolche „ontologisierende Wesensbestimmung“ (Neidhardt 1986: 11) vermieden, gleichwohl der Begriff auch nicht aufgegeben werden. Matthes weist 1992 auf den Versuch eines Symposiums hin, Kultur von ihrer „raumzeitlichen Fixierung auf Gebilde mit merkmalshaften Andersartigkeiten und deren Festschreibung darauf, je Kultur zu sein“, zu befreien und an dessen Stelle zu fragen, wie sich Kulturen bestimmen lassen, die sich als „ein wechselseitiger Prozess der Fremd- und Selbstbestimmung mit Folgen für alle an ihm beteiligten Seiten“ (Matthes 1992: 5, Herv. d. Verf.) darstellen. Auslöser dafür ist ein von verschiedenen Seiten an die Migrationsforschung herangetragener Vorwurf der Kulturalisierung (vgl. z.B.

26 Diese Selbstzuschreibung geht jedoch, wie sich in der Interpretation des Paares zeigen wird, über eine solche ,Kultur-Toleranz' hinaus. Vielmehr lässt Nicole ihre Interpretation von Carlos auch Möglichkeiten einer korrespondierenden Haltung als ,Frau', die eingeladen wird und die die finanzielle Verwaltungshoheit an ihren Mann abtritt. 
Bohnsack/Nohl 2001). ${ }^{27}$ Kulturalisierung meint dabei die Begrenzung eines Subjektes auf seine kulturelle Zugehörigkeit, ,andere Momente wie soziales Geschlecht, Bildung, sozioökonomischer Status oder Klassenzugehörigkeit, aber auch das Vermögen von Subjekten, ein kritisches und absetzendes Verhalten zu ihrer Herkunft und den sie prägenden Einflüssen zu etablieren, treten demgegenüber weitgehend oder ganz in den Hintergrund“" (Mecheril 2003: 21). ${ }^{28}$ Um solchen Kulturalisierungen nicht durch Interpretationen erneutes Gewicht zu geben und sie wissenschaftlich-naiv zu verlängern und weiterzuschreiben, werden in der folgenden Arbeit solche biographiegebundenen Konstruktionen von natioethno-kultureller Zugehörigkeit als Positionierungen verstanden, die, analog zu biographischen Geschlechterkonstruktionen, durch Erfahrungen und subjektspezifische Modi der Verarbeitung gesellschaftlich strukturierte Bilder und Ordnungen von Kultur und Ethnizität aufnehmen, diese Abstraktionen aber ,eigensinnig‘ verarbeiten. Gefragt wird also nicht nach inhaltlichen Bestimmungen von ,Kultur' und der Unterscheidung der, in Nicoles Fall, deutschen und mexikanischen Kultur. Im Mittelpunkt steht vielmehr die Analyse von Bedeutungszuweisungen von natio-ethno-kultureller Zugehörigkeit und dem Bezug auf spezifische Konzepte bei der Bewältigung und Aushandlung innerhalb der Partnerschaft. Kulturelle und kulturalisierende Fremdzuschreibungen greifen dabei auf gesellschaftlich strukturierte Bilder und Ordnungen über Kultur oder Ethnizität zurück. Der Begriff der ,Kultur' fungiert so als Differenzierungsbegriff und als Begriff der kollektiven Verortung: „Eine Kultur ist [...] die Form der Bearbeitung des Problems, dass es auch andere Kulturen gibt“" (Baecker 2001: 17). ${ }^{29}$ Dennoch spreche ich in dieser

27 Vgl. dazu auch Hamburger (1999), der bezweifelt, dass ,jede Verwendung von Kultur- oder Ethnizitätskategorien in einem praktischen Zusammenhang ideologische Funktion hat" (Hamburger 1999: 171). Gleichzeitig macht er aber auch auf eine analytische Aufgabe der Erziehungswissenschaft aufmerksam: „Es bleibt Aufgabe einer kritischen Erziehungswissenschaft, falsche Verallgemeinerungen, in denen sich Nationalismen verstecken, aufzuspüren, nicht um Besonderes aufzulösen, sondern nur, um ihm einen begründbaren Status zuzuordnen“ (Hamburger 1999: 175f).

$28 \mathrm{Vgl}$. dazu auch Mecheril/Quehl 2006, die auf eine Veränderung im Sprachgebrauch aufmerksam machen, da nach der offiziellen Diskreditierung des Rassismus' im postfaschistischen Europa gegenwärtige rassistische Unterscheidungen ohne explizite Rassekonstruktion operieren, aber mit Bezug auf das Konstrukt ,kulturelle Differenz' (vgl. Mecheril/Quehl 2006: 369).

29 Dies macht die immer wiederkehrende Debatte um die ,Leitkultur Deutschlands deutlich: Eine existierende und für alle in Deutschland lebenden Personen verbindliche ,Leitkultur' folgt aus dem gesellschaftlichen Interesse an Eindeutigkeiten, an dem von Baecker formulierten „Entweder-Oder" (ebd. 2001: 106). Eine solche hegemonial verortete 
Arbeit nicht (nur) von kulturellen Zugehörigkeiten, sondern verwende den von Paul Mecheril entwickelten Begriff der natio-ethno-kulturellen (Mehrfach)Zugehörigkeit (vgl. vor allem Mecheril 2000c, 2003, 2004). Er bezeichnet damit

„Phänomene der sozialen Platzierung, des Selbstverständnisses und der sozialen Einbezogenheit als Prozesse des Verhältnisses zwischen einzelnen Personen und Gruppen mit Bezug auf Verhältnisse der Legitimität und Macht“ (ebd. 2000c: 23f).

Die Begriffsbezeichnung verweist auf die doppelte Schwierigkeit, sowohl unter analytischen wie auch unter alltagspraktischen Gesichtspunkten die Begriffe Ethnizität, Nation und Kultur zu trennen. Auf analytischer Ebene verschwimmen die Begriffe miteinander, wie schon in der Auseinandersetzung mit den Bezeichnungen für binationale Paare deutlich geworden ist. „Staaten ,bringen“ Nationen und Ethnien hervor, dies geschieht eben dadurch, dass der Prozess der Herausbildung von Staaten als Werk von (imaginierten) nationalen Gemeinschaften beschrieben wird“ (Kneer 1997 in Mecheril 2003: 24). Solche ,imaginierte[n] Räume mit territorialer Referenz" (Mecheril 2003: 25) inkludieren die Subjekte in einer umfassenden und (scheinbar) vollständigen Weise. Sie versorgen es mit einer Art Grund(ver)sicherung der Zugehörigkeit, die durch den Nationalstaat vermittelt eine Wir-Gruppe schafft, die

„geographisch gefasst und in dem Sinne einwertig ist, als sie mit relativer normativer Gewissheit, mit sozialer, status- und prestigebezogener Klarheit einhergeht und im Rahmen eines einigermaßen vertrauten Gemeinsamkeitskontextes situiert ist" (Mecheril 2003: 25). ${ }^{30}$

Festschreibung lässt keinen Raum für Mehrdeutigkeiten, Hybriditäten und Polyvalenzen. Sie übersieht zudem, dass ,Kultur' immer mehrdeutig ist, verweist sie doch logisch auf die Möglichkeit des Anderen, des „tertium datur" (ebd.: 106), das sich dem Entweder-Oder verschließt. Kultur ist „systematisch zweideutig und erst in zweiter Linie ein Versuch, die Zweideutigkeiten durch Eindeutigkeiten, Identitäten und Authentizitäten aus dem Weg zu räumen“ (ebd: 9). Durch den Versuch der Bestimmung einer ,Leitkultur' würde Kultur zum Verweis auf eine vergangene, und damit nicht mehr zu ändernde Vergangenheit, die es zu verteidigen gilt, anstelle eines Verweises auf ein durchweg unklares Schicksal (vgl. Baecker 2001: 30f).

30 Ein Beispiel dafür liefert wiederum die Debatte um die Leitkultur: Auf dem CSU-Parteitag im November 2004 wurden Richtlinien für hier lebende „Ausländer“ formuliert. Sie sollten sich doch bitte schön an der liberaldemokratischen Grundhaltung der bundesrepublikanischen Bevölkerung orientieren und ihre sozialisatorischen Prägungen hinsichtlich Ehrenmords, Zwangsheirat und religiösen Fundamentalismus' aufgeben. Ironi- 
Ein solcher gemeinsamer Kontext lässt sich nicht in die einzelnen Elemente wie Nation, Ethnizität oder Kultur aufteilen. Vielmehr gewinnt er seine Bedeutung aus der Melange, die er darstellt. ${ }^{31}$ Der Begriff natioethno-kulturelle Zugehörigkeit bringt die notwendige „Diffusität, Komplexität und Polyvalenz" (Mecheril 2000c: 24) zur Geltung, die bei der Verwendung des sensibilisierenden Konzeptes dieser Untersuchung notwendig ist. Ein solcher Kontext bildet den Rahmen, innerhalb dessen Subjekte aufwachsen und ein „handlungsrelevantes Verständnis ihrer selbst lernen und praktizieren“ (ebd. 2003: 25). Dieses Verständnis kann, wie es in Deutschland üblich war, als eindeutige Zugehörigkeit gekennzeichnet sein. Migrationsprozesse und die Entwicklung Deutschlands hin zu einer „Migrationsgesellschaft“ (ebd. 2005: 312) haben aber zunehmend Mehrfachzugehörigkeiten in dem Sinne zur Folge, dass sich der Bezugsrahmen eben nicht nur aus einem natio-ethno-kulturellen Kontext ergibt, sondern aus zwei oder mehreren. Dies trifft in besonderer Weise auf binationale Paare zu, betrifft die Herausforderung einer uneindeutigen und in gewisser Weise hybriden Zugehörigkeit doch nicht nur den Migrierenden selbst, sondern auch seine Partnerin. Daraus ergibt sich auch die Möglichkeit von Neuverortungen und -positionierungen.

In der alltäglichen Kommunikation wird eine Melange von Konzepten und Begriffen verwendet, um Differenzen und (Nicht-)Zugehörigkeit in natio-ethno-kulturellen Räumen zu markieren und Zugehörigkeitspositionierungen deutlich zu machen. Diese nun in den Interviewpassagen in Aussagen zu Ethnizität, Kultur, und Nationalität zu trennen, erscheint nicht nur aus pragmatischen Gründen wenig Erfolg versprechend. Schließlich müssten bei einem solchen Vorgehen Bedeutungsdimensionen der einzelnen Kategorien analysiert und diese definiert werden, jeder und jede verwendet, gerade aufgrund der Diffusität und Verschwommenheit der Begriffe, diese mit einem anderen Bedeutungshorizont. Verständnis und Nutzung der Kategorien sind abhängig von biographischen Erfahrungen, regionalen Traditionen und inhaltlichem Verständnis hinsichtlich der Verwendung der Begriffe.

Natio-ethno-kulturelle Räume stellen keine feste Größe dar, sie sind wandel- und verhandelbar. Natio-ethno-kulturelle Zugehörigkeitskonstruktionen tragen zwei Elemente in sich, eine gleichzeitige Fremd- und

scherweise wollte die CSU in diesem Zuge zugleich die christliche Ausrichtung Deutschlands wieder stärker sichtbar machen und dafür zum Beispiel das allmorgendliche Schulgebet wieder einführen.

31 Höhne geht 2001 sogar noch einen Schritt weiter, wenn er die These aufstellt, dass ,Kultur ${ }^{6}$ alleine noch nichts über die spezifische Verwendungsweise aussagt, die sich vielmehr erst in der Kopplung mit anderen Merkmalen (wie eben Nation, Ethnie, aber auch Geschlecht) und der dadurch vorgenommenen Spezifik der Zuschreibung einstellt (ebd.: 199). 
Selbstzuschreibung, die die Subjekte zueinander in ein Verhältnis setzen und sowohl in Interaktionen als auch in situative Handlungen überdauernden biographischen Konstruktionen ,Differenzfelder' entstehen lässt, die Deutungsmuster und Handlungsorientierungen organisieren und strukturieren. Das ,Wir', das inkludiert wird und gegen das die ,Anderen' abgegrenzt werden, kann sich verändern und kann neu bestimmt werden. Deutlich zeigt sich dies zum Beispiel an den unterschiedlichen Herangehensweisen zur Erlangung der Staatsbürgerschaft. In Deutschland galt lange das jus sanguinis, mindestens ein Elternteil musste zur nationalen Gemeinschaft gehören, um das Kind ebenfalls einer solch imaginierten Gemeinschaft zugehörig erklären zu können. In den USA hingegen wird man schon durch die Geburt auf dem US-Amerikanischen Territorium zum US-Amerikaner. Eine solche formal-rechtliche Zugehörigkeitserklärung sagt jedoch nichts über die Verschiebung weiterer Grenzen aus. Vielmehr verlaufen Auseinandersetzungen und Kämpfe dann innerhalb dieses Raumes. Natio-ethno-kulturelle Zugehörigkeit meint die idealtypische Konzeption von Zusammenhängen,

„in denen symbolische Distinktions- und Klassifikationserfahrungen, Erfahrungen der Handlungsmächtigkeit und Wirksamkeit als auch biographische Erfahrungen der kontextuellen Verortung nahe gelegt sind" (Mecheril 2003: 25f). ${ }^{32}$

In biographischen Erzählungen können solche Selbst- und Fremdverortungen analysiert werden: „Die Erzählung über die Zugehörigkeit ist ein Bericht, der eine Geschichte darüber erzählt, wie wir uns selbst in Begriffen von sozialen Kategorien platzieren, etwa Geschlecht, Ethnizität und Klasse" (Anthias 2003: 22). In einer lebenszeitlichen Perspektive können Zugehörigkeitskonzeptionen entwickelt, verfestigt oder auch

32 Mecheril analysiert im Folgenden drei Dimensionen dieser Zugehörigkeit: a) die symbolische Mitgliedschaft, zu der sowohl eine formelle Mitgliedschaft wie die Staatsangehörigkeit als auch eine informelle Mitgliedschaft gehört, die in einem selbstverständnlichen Akt des Als-Mitglied-Erkennens festgestellt (oder verweigert) wird; b) die habituelle Wirksamkeit, die auf Handlungsebene Möglichkeitsräume eröffnet, bzw. bei nicht anerkannter Zugehörigkeit verschließt; und c) die biographische Verbundenheit, die das fraglose Verhältnis zwischen Individuum und Kontext, das in dem Begriff der Zugehörigkeit zum Ausdruck kommt, im Hinblick auf z.B. symbolisch-affektive oder kognitiv-pragmatische Verbundenheit kennzeichnet. Diese Verbundenheit flicht die eigene Biographie in den Zugehörigekeitsraum ein (vgl. ebd. 2003: 118ff.) Solche Dimensionen fragloser Zugehörigkeit werden bei Menschen mit (Mehrfach-)Zugehörigkeit prekär, Exklusionseffekte gesellschaftlicher Strukturen und sozialer Interaktionen lassen eine unkomplizierte Zugehörigkeit nicht zu. 
verschoben werden. Ähnlich wie Geschlecht ist natio-ethno-kulturelle Zugehörigkeit ein Konstruktionsmoment einer Biographie. So bezieht sich Nicole auf ihr Wissen über mexikanische Geschlechterordnungen im Restaurant. Ein solches ,Wissen“ kann verschiedene Ursprünge haben: Sie kann bei ihren Aufenthalten in Mexiko beobachtet haben, dass nur Männer die Rechnung bekommen, Carlos kann es ihr erzählt haben oder aber sie stellt es sich so vor. Der Ursprung ist dabei aber nicht entscheidend. Entscheidend ist vielmehr, dass natio-ethno-kulturelle Zugehörigkeit und die damit verbundenen Implikationen oder Imaginationen über Geschlechterverhältnisse Teil des Bedeutungshorizontes sind, vor dem ihre Biographie sich entfaltet. Zugleich verifiziert und konstruiert Nicole in der Erinnerung und in der Erzählung so selbst natio-ethnokulturelle Zugehörigkeiten, Carlos und sie müssen, so ihre Einschätzung, mit dieser kulturellen Differenz eben umgehen lernen. Mit einem solchen Verständnis von natio-ethno-kultureller Zugehörigkeit kann es gelingen, solche (gegenseitigen) Konstruktionen eben nicht als Folge eines holistischen räumlich-begrenzten Kulturkonzeptes zu verstehen, sondern als dynamische und prozessuale Deutungsmuster, welches ihre Wirksamkeit in Relation mit anderen Zugehörigkeitskategorien wie zum Beispiel gender entfalten. ${ }^{33}$

\section{Wechselwirkungen: gender und natio-ethno-kulturelle Zugehörigkeit als verflochtene Kategorien}

In dem einführenden Interviewtext von Nicole zu Beginn dieses Kapitels spielen mehrere Kategorien eine relevante Rolle. So verbindet sie Überlegungen zu einer geschlechtsspezifischen Rollenverteilung mit Hinweisen auf andere, fremde natio-ethno-kulturelle Verhaltensweisen. Die Bedeutungsdimensionen der Textstelle entfalten sich in der Verbindung von geschlechtsspezifischen Verhaltenserwartungen mit der ,anderen Herkunft ihres Mannes. Nicole legitimiert seine und ihre Wünsche hinsichtlich des adäquaten Verhaltens im Restaurant mit seiner natio-ethno-

33 Insofern erscheint das Konzept von Anthias, Erzählungen über Zugehörigkeit als Positionierungen und Differenzkonstruktionen als Imaginationen von Kollektivitäten und Grenzen und Räumen der Zugehörigkeit zu verstehen, zwar auf den ersten Blick einleuchtend (vgl. Anthias 2003). Jedoch wird durch die Fokussierung auf die hegemonialen Bedeutungsvariationen von Race und Ethnizität die biographische Verarbeitung und Transformation durch die Subjekte vernachlässigt. Die Reproduktion essentialisierender und dichotomisierender Konzepte von Kultur, Race, und Ethnizität durch die Subjekte erscheint dadurch zu zwangsläufig. 
kulturellen Sozialisation, umgekehrt bestimmt diese Sozialisation seine Erwartungen hinsichtlich des eigenen Verhaltens und des Verhaltens seiner Frau. Diese Verflechtung von gender mit anderen Differenzkategorien kann empirisch offen legen, wie gegenseitige Naturalisierungen und Differenzbestätigungen funktionieren und in welchem spezifischen Verhältnis solche Konstruktionen stehen (vgl. dazu auch Diehm/Radtke 1999: 95). Ein solches Verständnis der Relationalität der Konstruktionen versteht sich als Weiterentwicklung einer Zeitweise insbesondere in der interkulturellen Frauenforschung präferierten Sichtweise der zweifachen oder sogar dreifachen Unterdrückung der Frau. Die Kategorien race, class und gender wurden als additiv verstanden, je nach Zugehörigkeiten konnten so Abstufungen und Addierungen der Quantität der Benachteiligungen vorgenommen werden (vgl. zum Überblick Bednarz-Braun 2004: 31ff, auch Lutz 2001). Diese Position stellt die Differenzkategorien nebeneinander, ohne sie in ihrem spezifischen Verhältnis genauer zu betrachten:

„An der Mehrfachunterdrückungsthese [...] wurde kritisiert, dass sie Unterdrückung lediglich als die Summe verschiedener Aspekte betrachtete und dabei spezifische Momente der Unterdrückung schwarzer Frauen aus dem Blickfeld gerieten, wie etwa das Zusammenspiel sexistischer und rassistischer Exklusion“ (Lutz 2001: 218).

Deutlich anders hingegen richtet sich die neuere Geschlechterforschung aus:

„Wenn alle gesellschaftlichen Verhältnisse Geschlechterverhältnisse sind, wird auch umgekehrt das Geschlechterverhältnis durch alle gesellschaftlichen Verhältnisse konstituiert. Es wird folglich durch seine Überschneidungen mit weiteren Ungleichheit strukturierenden Differenzierungs- und Hierarchisierungsprozesse geformt" (Gümen 1998: 194). ${ }^{34}$

Die Verflechtung von gender und natio-ethno-kultureller Zugehörigkeit in Biographien zu analysieren bedeutet, ,verschiedene binäre Codierungen in ihrer wechselseitigen Beeinflussung zu untersuchen und andere soziale Kategorien der Differenz in ihrer Interaktion mit der Kategorie Geschlecht zu berücksichtigen“ (Gümen 1998: 193, Herv. d. Verf.). ${ }^{35}$

34 Damit soll nicht die „Totalinklusion“ (Tyrell 1986: 470) in eine Geschlechtszugehörigkeit in Frage gestellt werden. Die Folgen dieser Inklusion allerdings können zu verschiedenen Zeitpunkten und für verschiedene Personen durchaus unterschiedlich sein.

35 Aus der neueren interkulturellen Geschlechterforschung kommen daher Studien, die sich dem Veränderungspotential widmen, das in interkulturel- 
Die Analyse solcher Wechselwirkungen zwischen verschiedenen Kategorien wird in den letzen Jahren mit dem Konzept der Intersektionalität aufgegriffen (vgl. vor allem Knapp 2005). ${ }^{36}$ Die ,intersectional analysis (als Begriff 1987 von Kimberley Crenshaw geprägt) blicken auf race, class und gender als relationale Begriffe:

„Class, race und gender sind relationale Begriffe, wen sie unter welchen Formbestimmheiten und durch welche Mechanismen einschließen und ausschließen, wie die jeweilige Relationalität verfasst ist unter spezifischen soziohistorischen, kulturellen und ökonomischen Bedingungen, kann nicht begriffen werden, wenn man nur eine dieser Kategorien in den Blick nimmt. Sie müssen also sowohl in ihrer jeweiligen Spezifik als auch in ihrem Zusammenhang gesehen werden“" (Knapp 2005: 74).

Damit ist ein forschungsprogrammatischer Zugang entworfen, der die mich interessierenden Wechselwirkungen fokussiert: „Gender, Ethnizität, Klasse, Nationalität und Sexualität gehen stets gleichzeitig und in Wechselwirkung miteinander einher" (Lutz 2004: 482). ${ }^{37}$

Diesen Wechselwirkungen wird in den Interpretationen der Biographien nachgegangen. Dabei werden allerdings nicht die angesprochenen drei Differenzkategorien analysiert, sondern ,nur' gender und natioethno-kulturelle Zugehörigkeit. Wird mit einem solchen Verständnis die Textstelle von Nicole betrachtet, lässt sich zum Beispiel zeigen, wie sich einerseits neue Differenzen herstellen: Nicole übernimmt das ,mexikanische' Restaurantverhalten von Carlos und verbindet und begründet dieses mit einer grundsätzlichen Einstellung und Haltung zu finanziellen Verteilungen in der Ehe: Die Geschlechterbeziehung legitimiert sich durch den Bezug auf natio-ethno-kulturelle Differenzen. Andererseits

len Geschlechterbegegnungen enthalten ist. Sie gehen davon aus, dass interkulturelle Begegnungen Chancen bieten für die Entwicklung neuer, individueller Geschlechterbeziehungen. Dies würde bedeuten, dass binationale Paare gemeinsam neue Bedeutungen entwickeln können (vgl. z.B. Schlehe 2000b), da zwischen den Personen neue Differenzen und Abstände geschaffen werden können und müssen (vgl. auch Lenz 1996).

36 Müller verwendet für die gleiche Idee den Begriff der Interferenzen (vgl. Müller 2003).

37 Intersektionelle Analysen folgen drei Zugangsweisen: 1) anti-kategorial, durch dekonstruktivistische oder nach Leslie McCall poststrukturalistische Zugänge, 2) intra-kategorial, in der Fragen von Differenz und Ungleichheit im Rahmen einer der jeweiligen Kategorien in den Blick genommen werden, und schließlich 3) inter-kategorial, wodurch Verhältnisse und Wechselwirkungen zwischen den Kategorien analysiert werden (aus Knapp 2005: 74f). Krüger-Portratz und Lutz verweisen 2002 auf insgesamt 15 bipolare hierarchische Differenzlinien, die in Analysen Relevanz bekommen können (vgl. ebd.: 88). 
findet Nicoles Konstruktion einer differenten Geschlechterbeziehung durch die ,andere' natio-ethno-kulturelle Zugehörigkeit eine Ausdrucksmöglichkeit. Biographische gender-Konstruktionen und Konstruktionen natio-ethno-kultureller Zugehörigkeit finden sich also in einem subjektlogischen Verhältnis, werden in diesem Verhältnis geformt und bekommen Gültigkeit zugesprochen. Für die binationalen Paare meines Samples ist in diesem Kontext insbesondere die Rahmung durch die Paarbeziehung wichtig, in der Veränderungen oder auch Verfestigungen solcher relationaler Konstruktionen stattfinden. 


\section{BIOgRAPHIEN BINATIONALER PAARE:}

\section{ERSTE ERKENNTNISSE UND WEITERFÜHRENDE}

FORSCHUNGSFRAGEN

In den Anblicken der Arbeit wurden, neben der Vorstellung des Samples, vor allem die theoretischen und konzeptuellen Rahmungen vorgestellt, innerhalb derer sich die Interpretationen der Interviews verorten. In diesen Anblicken auf das Forschungsmaterial wurde zum einen das Forschungsfeld ,binationale Paare' eingeführt, indem auf bereits existierende Studien sowie statistische Grundlagen und rechtliche Rahmenbedingungen zurückgegriffen wurde und die Forschungslandschaft damit skizziert wurde. Das zweite Kapitel beschäftigte sich mit den theoretischen Aufmerksamkeitsrichtungen, die den Analysefokus meiner Interpretationen bestimmen. Bevor nun im zweiten großen Teil der Arbeitden Einblicken - die Biographien der Paare interpretiert werden, dient dieses Kapitel zur zusammenfassenden Referierung der Erkenntnisse der beiden letzten Kapitel. Perspektiven und Fragen können aufgemacht werden, die den ersten Teil der Arbeit charakterisieren und die empirischen Analysen vorbereiten.

Binationale Paare standen und stehen unter besonderer Beobachtung der Gesellschaft. Dies zeigt sich eindrücklich in der Veränderung der wissenschaftlichen Forschung. Wurden sie in den 60er Jahren noch als Katalysatoren für asoziale Tendenzen verstanden, dienen sie inzwischen als Vorbild für moderne, für die Anforderungen komplexer Migrationsgesellschaften bestens gerüstete Familien. Immer noch aber stoßen sie auf Ressentiments, insbesondere bei Frauen wird die Beziehung als ungehörig angesehen, häufig genug auch von den eigenen Eltern. Damit aber nicht genug, gibt es strukturelle Hindernisse und diskriminierende Praxen, die die Biographien kennzeichnen. Binationale Paare stehen also in besonderem Maße vor der Aufgabe, ungewöhnliche und vielleicht 
auch ungewollte Erfahrungen und Konstellationen in ihre Lebensplanung und -gestaltung zu integrieren. Migrationserfahrungen lassen sich (auch) als Zumutungen verstehen, die Subjekten Veränderungen in ihren Positionierungen und Verortungen abverlangen. Die Migration als zentrales lebensgeschichtliches Ereignis (vgl. Fröhlich/Messerschmidt/Walter 2003) erfordert die Integration ungewöhnlicher und vielleicht auch ungewollter Erfahrungen und Konstellationen in ihre Lebensplanung und -gestaltung. Ein typisches Merkmal ist dabei der berufliche Dequalifizierungsprozess, der im Fall der hier interessierenden Gruppe männlicher Migranten sehr deutlich zu Tage tritt. Aufgrund der, institutionellen Zwangslage', die durch die fehlende Anerkennung von Berufsabschlüssen (bzw. nicht vorhandene zertifizierte Ausbildungen), die mangelnden deutschen Sprachkenntnisse und durch Schwierigkeiten bei der Einmündung in den Arbeitsmarkt entsteht, finden sich binationale Paare regelmäßig in der Konstellation des female breadwinner Modells wieder. Dieses Modell widerspricht der nach wie vor dominanten Form der Geschlechterordnung in Deutschland, so wie es auch der normativen Vorstellung widerspricht. Die von mir interviewten Paare müssen sich insofern in irgendeiner Weise $\mathrm{zu}$ der gesellschaftlich ungewöhnlichen Arbeitsteilung verhalten, unabhängig davon, ob diese Konstellation freiwillig gewählt und erwartet wurde oder sich als unerwartete Folge der binationalen Partnerschaft darstellt. Die Situation, in der sich binationale Paare im Zuge des Migrationsprozesses und der Entscheidung, in Deutschland zu leben, (wieder) finden, ist durchaus charakteristisch für das Leben in modernen Gesellschaften: Herauslösung aus bisherigen Bindungen (das gilt insbesondere für den migrierenden Part des Paares, aber auch die soziale Bezugsgruppe der Frauen ist in den Prozess einbezogen und wird unter Umständen aufgegeben oder verändert), notwendig werdende Neu- und Mehrfachintegrationen, die Re-Organisation handlungsleitender Orientierungen und die Re-Organisation Selbst- und Wirklichkeitskonstruktionen. Strukturell also lässt sich die Situation, in der sich binationale Paare befinden, durchaus als Modernisierungslage verstehen. Die binationalen Paare meines Samples irritieren hierbei zudem in mehrfacher Hinsicht die Homogenitätsillusion einer Gesellschaft: hinsichtlich ihrer Partnerschaft, die als natio-ethno-kulturell ,gemischt ${ }^{`}$ markiert und wahrgenommen wird, und hinsichtlich der Arbeitsteilung, die herkömmliche vergeschlechtlichte Familienformen in Frage stellt. ${ }^{1}$ Der Schluss, der jedoch insbesondere von Beck-Gernsheim gezo-

1 Eine Position, die sowohl ihnen wie auch ihrem sozialen und beruflichen Umfeld deutlich bewusst ist, auch wenn sie selber die Konstellation angestrebt hatten: Die Ungewöhnlichkeit ihrer Lebenskonstellation ist zentrales Moment der Selbstwahrnehmung der Paare. Das zeigt sich auch in der Be- 
gen wird, binationale Paare seien ,moderne Romeos und Julias, die nicht den Gesetzen ,von Herkunft und Heimat“ folgen, sondern „dem Gesetz ihres Herzens“ (ebd. 2001: 120), ist so nicht haltbar. Ganz abgesehen davon, dass Romeo und Julia, zumindest hinsichtlich ihrer persönlichen zukünftigen Lebensgestaltung, nicht unbedingt als erfolgreiche Rebellen in die Geschichte eingehen, sind auch binationale Paare, das zeigen die Studien, nicht frei von den Gesetzen von ,Herkunft und Heimat‘. Es ist notwendig, erheblich differenzierter mit der Bedeutung binationaler Paare als Indikatoren einer emanzipierten und egalitären Beziehung, vielleicht sogar als Prototyp moderner Subjekte, umzugehen. Sie stehen tatsächlich in einem Spannungsfeld verschiedener Zumutungen und Anforderungen, die sie auch durch eine Distanzierung von ihrem Herkunftsland und ihren Sozialisationserfahrungen bearbeiten können. Studien zu binationalen Paaren nehmen dieses Spannungsfeld auf. Haben ältere Studien sich in der Regel damit begnügt, verschiedene ,Konfliktfelder ${ }^{6} \mathrm{zu}$ identifizieren, analysieren neuere Untersuchungen vergleichsweise differenziert verschiedene Phänomene binationaler Partnerschaften unter der Perspektive, wie Fremdheit und Gemeinsamkeit konstruiert und verstanden wird. Dabei muss nicht zwangsläufig ein emanzipatives und wegweisendes Partnerschaftsmodell für das 21. Jahrhundert entstehen. Ebenso lässt sich, insbesondere wenn längeranhaltende Konflikte existieren, der Rückbezug auf kulturalisierende Handlungs- und Orientierungsmuster beobachten. Dennoch aber findet sich grundsätzlich Potential zur Grenzverschiebung. Die Schwierigkeit, auf kulturelle und vergeschlechtlichte Routinen zurückzugreifen, und die damit verbundene Notwendigkeit der Entwicklung eigener Handlungs- und Orientierungsmuster beinhaltet die Möglichkeit, geschlechtliche und kulturelle Differenzen zu verschieben bzw. die beiden hier im Mittelpunkt stehenden Differenzkategorien (neu?) ins Verhältnis zu setzen. Gerade das Phänomenfeld ,Liebe‘ als ein Verständigungsmodus der Beziehungspartner(innen), ist dabei erheblich differenzierter als von Beck-Gernsheim angenommen. Liebe dient als Chiffre, die Unvereinbares integriert und die Illusion der vollständigen Verständigung erschafft. Gleichwohl ist auch diese scheinbar freie Kategorie eingebunden in Imaginationen und Variationen des Fremden und des Eigenen, von Zugehörigkeit und Nicht-Zugehörigkeit.

Für meine Studie haben sich insbesondere folgende Erkenntnisse als relevant herausgestellt: Der Beginn der Paarbeziehung scheint, wie die

deutung, die der Konstellation in der Interviewsituation zugesprochen wurde. Alle haben sie von alleine angesprochen und ihr Verhältnis dazu reflektiert. 
Chiffre ,Liebe“ verdeutlich, eine besondere Bedeutung für natio-ethnokulturelle Zugehörigkeitskonstruktionen zu haben. Damit ist eine Nähe zu der Art von Partnerwahltheorien geschaffen, die die Besonderheit von Intermarriage in spezifischen Persönlichkeitsmerkmalen sehen, wie es zum Beispiel Hecht-El Minshawi in der gemeinsamen Außenseiterposition beider Partner(innen) formuliert. Die Beobachtung der Bedeutung der Rahmung der Beziehung zielt jedoch nicht auf kulturalisierende Interpretationen oder auf Persönlichkeitsdispositionen ab. Vielmehr lenkt es den Blick auf die biographische Anschlussfähigkeit und evtl. sogar die Realisierung biographischer Pläne, die durch die Beziehung möglich erscheinen. Dies ist keine Besonderheit binationaler Partnerschaften. Binationale Partnerschaften sind jedoch durch die Vervielfältigungen von Möglichkeiten und Herausforderungen. Ein Konstruktionskontext ist sicherlich die natio-ethno-kulturelle Zugehörigkeit der Partner(innen). Diese ist jedoch nicht unabhängig von anderen Kategorien wie gender zu sehen, sondern gewinnt ihre Relevanz immer in der Verflechtung mit anderen Kategorien. Prozesse der Konstruktion und (Re-) Konstruktion von natio-ethno-kultureller Zugehörigkeit können besonders ergiebig im Zusammenhang mit gender-Konstruktionen analysiert werden, stehen diese doch in einem engen Zusammenhang, ergänzen einander oder werden gleich gesetzt. Neben solchen Aufmerksamkeitsrichtungen, die sich eher auf die Innenperspektive der Paare konzentrieren, spielt, das haben die Studien deutlich gezeigt, auch das soziale Umfeld eine relevante Rolle. Eltern, anderen Verwandten und Freunden einerseits, aber auch Institutionen, Behörden und rechtlichen Regelungen andererseits kommt in den Aushandlungs- und Bearbeitungsprozessen eine entscheidende Rolle zu. Nicht nur, weil die Struktur der Beziehung, Heirat oder nicht, Zusammenleben oder nicht, von binationalen Paaren nicht frei gewählt werden kann, sondern von aufenthaltsrechtlichen Bestimmungen entschieden wird. Soziale Netzwerke, emotionale Unterstützung oder der Eingriff insbesondere der Eltern in die Beziehung spielen eine entscheidende Rolle bei der Lebensgestaltung der Paare. Wesentlich bei der Betrachtung solcher umgebender Bedingungen ist dabei jedoch, dass diese aus der Perspektive der Paare rekonstruiert werden. Deutliches Beispiel dafür ist die ,institutionelle Zwangslage', als die das female breadwinner Modell zu verstehen ist. Wie die Untersuchungen zum dual earner Modell zeigen, ist für die Einstellung der Paare nicht die tatsächliche Verteilung von Berufs- und Familienarbeit entscheidend. Vielmehr scheint das Selbstverständnis vergleichsweise unabhängig von der realen Arbeitsteilung zu sein. Umso notwendiger ist es daher für meine Untersuchung, nicht zwangsläufig einen biographischen ,Bruch“ in der Lebensplanung der Paare anzunehmen, sondern 
vielmehr die Bedeutung für beide Partner(innen) zu rekonstruieren und daraus Bearbeitungsnotwendigkeiten zu analysieren. Dabei sind sowohl mögliche Probleme wie auch die Bewältigungsstrategien wesentlich von der biographischen Situation abhängig: „Eine äußere Veränderung der Lebensumstände erhält für das Subjekt seine dramatische Bedeutung erst durch deren Rekonstruktion in der sozialen Interaktion, sie ist nicht ,natürlich“ gegeben“ (Apitzsch 1999: 9). Eine solche Lesart wird durch die Studien zur geschlechtsspezifischen Arbeitsteilung bestätigt. Die Verarbeitung und daraus resultierende Wahrnehmung der Konstellation ist entscheidend, weibliche Berufstätigkeit muss alles andere als zwangsläufig in einer egalitären Beziehungsstruktur münden. Als letzten, methodisch relevanten Punkt hat die Auseinandersetzung mit der Forschungslandschaft die Entscheidung für die Befragung beider Partner(innen) bestätigt. Die Paare entwickeln ihre Konstruktionen und ihre biographischen Gestaltungen in enger Auseinandersetzung miteinander. Sie sind die signifikanten Anderen füreinander, und die Analyse der Selbst- und Wirklichkeitskonstruktionen beider Partner(innen) ist notwendig. Wenn etwas über Bearbeitungs- und Aushandlungsprozesse binationaler Paare gesagt werden soll, reicht es nicht aus, nur einen Teil dieses Paares zu interviewen. Der Migrationsprozess des Mannes und die Migrationsbiographie schließen die Beziehung ein, auch die Biographien der Frauen sind konstitutiv durch diese Prozesse strukturiert. Wie die Auseinandersetzung mit der Bedeutung der Paarbeziehung für Wirklichkeitskonstruktionen gezeigt hat, sind solche Konstruktionen Konstruktionen zwischen den Bezugspersonen: Zum einen entstehen bestimmte Konstellationen erst aufgrund der Existenz des jeweiligen Partners (so wäre, so ist zumindest zu vermuten, Nicole nicht in der ,Zwangslage‘, ihrem Mann Geld unter dem Tisch zuschieben zu müssen, hätte sie nicht Carlos geheiratet), zum anderen sind die Konstellationen und Konstruktionskontexte entscheidend strukturiert durch den bzw. die Andere(n): Für Nicole z.B. ist die ,Gemeinsamkeit' in der Ehe entscheidend und wirkt handlungsleitend. Die Beziehung fungiert dabei als zentraler Orientierungsort und gleichzeitig als eine Art ,Katalysator für spezifische gemeinsame Interpretationsmuster und exkludiert, durch ihre stark auf die Beziehung gerichtete Struktur, individuelle und gegensätzliche Interpretationsmuster.

Die Forschungsperspektive zeigt, dass Biographien mehr sind als nur Nacherzählungen von Erinnerungen und Erlebnissen. Biographien sind rekonstruierte und dadurch auch konstruierte Lebensgeschichten von Subjekten. Subjekte gestalten ihr Leben unter Zuhilfenahme angebotener Strukturen und institutioneller Vorgaben und verorten sich dabei in unterschiedlichen Kontexten, die durch Differenzkategorien wie gender 
und natio-ethno-kulturelle Zugehörigkeit strukturiert sind. Die Analyse biographischer Interviews und das Verständnis von gender und natioethno-kultureller Zugehörigkeit als biographisch gebundene Kategorien konkretisieren die Perspektive und weisen Veränderungen einen ,Ort ${ }^{\star}$ zu. Die beiden Kategorien lassen sich einerseits als Begrenzungen der Lebensmöglichkeiten von Subjekten, andererseits aber eben auch als Ermöglichungen von Handlungen und der Erzeugung von eigenen Bedeutungszuschreibungen. Wenn Biographien aus einer solchen Analyseperspektive betrachtet werden, lassen sich (Re-)Konstruktionen institutioneller und struktureller Vorgaben entdecken. Es lässt sich analysieren, an welchen gesellschaftlichen Vorgaben sich die Subjekte in welcher Weise orientieren und diese damit verifizieren, andererseits aber auch an welchen Stellen Brüche und Widerständigkeiten $\mathrm{zu}$ entdecken sind und gesellschaftliche Lebensmodelle modifiziert und/oder erweitert werden. Dabei können beide Kategorien in ihrer unmittelbaren und unhinterfragten Bedeutung und Funktion sozusagen ,erschüttert' werden. Diese Erschütterungen wiederum, die sich als Re-Organisierungen von Funktionen und Bedeutungszuschreibungen der Kategorien darstellen (können), tragen Transformationsmöglichkeiten in sich.

Mein heuristischer Zugang für die Analyse der Biographien liegt in dem Konzept der Formen biographischen Lernens. Die erziehungswissenschaftliche Biographieforschung, insbesondere Theo Schulze, fordert schon seit längerem, die „Biographie als Bildungsprozess“ in den Mittelpunkt des Interesses zu rücken. Das Interesse sollte ,zuerst und vor allem den Vorgängen und Bedingungen des Lernens, der Entfaltung einer Biographie als Lerngeschichte und Bildungsprozess" gelten (Schulze 1999: 51). Auch die Bereiche der Biographieforschung, die sich mit der Frage von Migrationsprozessen als Katalysatoren für Lernoder Bildungsprozesse auseinandersetzen, schreiben Migrant(inn)en eine, wie Koller es nennt, ,prototypische Bedeutung “2 (Koller 2002a: 98) im Hinblick auf den kreativen Umgang mit kulturellen Differenzen zu (vgl. dazu auch Apitzsch 1990, Lutz 2001, auch Seitter 1999). ${ }^{3}$ Mi-

2 Diese prototypische Bedeutung ist allerdings nicht ohne gesunden Zweifel zu sehen und erst recht nicht exklusiv, schließlich ließen sich einige Bevölkerungsgruppen vorstellen die in ähnlicher Weise kulturellen und strukturellen Differenzerfahrungen ausgesetzt sind, man denke nur an soziale Aufsteiger(innen) oder Langzeitarbeitslose. Vgl. dazu etwas vorsichtiger Seitter, der von der Herausforderung der „Handhabung von Diskontinuität“" (ebd. 1999: 38) für Migrant(inn)en spricht.

3 Dieser Zweig der Forschung reagiert dabei sicherlich auch auf die Tendenz von Teilen der erziehungswissenschaftlichen Migrationsforschung die, aus der Ausländerpädagogik stammend, dem Interventions- und Unterstützungsbedarf bei Migrant(inn)en starke Beachtung schenkt und 
grationsbiographien lassen sich insofern als ,Lernbiographien“ verstehen. Mit einer solchen Perspektive kann das vorne angesprochene Potential zur Grenzverschiebung konkretisiert und für die Analyse der Interviews fruchtbar gemacht werden. Subjekte machen neue Erfahrungen, positionieren sich neu und verändern dabei auch ihr Verständnis und ihre Funktions- und Bedeutungszuschreibungen von strukturierenden Kategorien wie gender und natio-ethno-kulturelle Zugehörigkeit. Solche Veränderungen, die durch den erhöhten ,Zwang' zur ,biographischen Selbstorganisation“" (Alheit 1994: 33), zum Umgang mit Ambivalenzen und Risiken, entstehen, lassen sich als biographisches Lernen verstehen. So können auch Geschlechterkonstruktionen und Konstruktionen natioethno-kultureller Zugehörigkeit als biographische Ressource verstanden werden, die durch Lernprozesse erweitert, verändert oder aktualisiert werden können. Akteure in Migrationsprozessen werden nicht als Strukturen ausgelieferte Wesen gesehen, sondern als handelnde Subjekte, die behindert oder gefördert werden und unter Rückgriff auf Erfahrungen und kulturelle Deutungsbezüge Pläne entwickeln, verwerfen und sich und ihre Beziehung (neu) positionieren. Solche biographischen Prozesse bergen Rückwirkungspotential auf gesellschaftliche Strukturen in sich, wie Schulze deutlich macht: „Der Einfluss der Individuen auf die Entwicklung der Gesellschaft wird immer noch unterschätzt, die Veränderungen in der Biographie immer noch als abhängig von den Veränderungen in der Gesellschaft angesehen, nicht aber als Faktoren, die ihrerseits auch zur gesellschaftlichen Veränderung beitragen oder sie gar vorantreiben. Nicht nur Konferenzen, Gesetze und Erlasse verändern die gesellschaftliche Wirklichkeit, auch Umwertungen und Neuorientierungen. Die Individuen lernen mehr, als zur Bewältigung ihrer gesellschaftlichen Aufgaben notwendig ist, und aus diesen Lernüberschüssen bilden sich Potentiale, die in bestimmten historischen Situationen wirksam werden können und auch wirksam werden“" (Schulze 1999: 51). Aus dieser programmatisch-konzeptuellen Verortung sind bislang kaum Ansätze einer Systematisierung einer empirischen Analyse von biographischen Lern- und Bildungsprozessen zu erkennen, wie schon in dem Exkurs zur erziehungswissenschaftlichen Biographieforschung angedeutet sind. Für die Analyse der Biographien wurde insofern unter Berücksichtigung der vorliegenden Auseinandersetzungen ein heuristisches Modell von Formen biographischen Lernens entwickelt, um (Re-)Konstruktionen und Modifikationen von gender und natio-ethno-kultureller Zugehörigkeit in den Biographien binationaler Paare analysieren zu können und Elemente

sich so ihr eigenes Betätigungsfeld schafft (vgl. dazu den kritischen Vorwurf von bspw. Diehm/Radtke 1999). 
und Phänomene solcher Formen identifizieren zu können. Sie lassen sich als Ausdrucksform des Verhältnisses zu gesellschaftlichen Prozessen und gesellschaftlichem Wandel und der Konstitution ,neuer sozialer Wirklichkeiten“ (Pries 1997) verstehen. Ein solcher Vorgang entspricht auch dem abduktiven Verständnis der Grounded Theory (Glaser/Strauss 1967), der Entwicklung theoretischer Erkenntnisse in der Auseinandersetzung mit der Empirie.

Als forschungsleitende Fragen für die Einblicke in die Biographien lassen sich aus den bisherigen Überlegungen heraus abschließend folgende formulieren:

- Welche Konstruktionen von Geschlechterbeziehungen und Konstruktionen natio-ethno-kultureller Zugehörigkeit lassen sich zu Beginn der Beziehung analysieren? In welchem Verhältnis stehen diese Konstruktionen zueinander, und in welchem Verhältnis stehen sie zu denen der/des Partners/Partnerin?

- Welche Bedeutung wird dem female breadwinner Modell zugesprochen?

- Was für spezifische Bearbeitungsformen der Konstellation lassen sich beobachten? Verändern sich die Konstruktionen, verstärken oder modifizieren sie sich?

- Was lässt sich aus den analysierten Formen über den Umgang mit gesellschaftlichen Herausforderungen sagen? Welche Bedeutung hat die binationale Partnerschaft? Lassen sich Anhaltspunkte dafür finden, binationale Paare als ,Protoypen“ der Moderne zu verstehen? 
EInBLicke 



\section{DAS METHODOLOGISCHE UND Methodische Design der Studie}

Die ,Einblicke' enthalten das methodologische und methodische Vorgehen, das Interpretationsmodell und die Interpretationen der Biographien. Das Konzept der Grounded Theory stellt die methodologische Grundlage für die Entwicklung des heuristischen Interpretationsmodells der Formen biographischen Lernens dar.

\section{Gegenstandsbezogene Theoriebildung: die Forschungslogik}

Die Studie folgt als qualitativ-empirische Arbeit der Logik rekonstruktiver Sozialforschung. In der Tradition des symbolischen Interaktionismus (vgl. v.a. Blumer 2004 [1973]) steht die Analyse und Nachvollziehbarkeit sozialen Handelns im Mittelpunkt:

„Für mich ist der symbolische Interaktionismus nicht eine philosophische Lehrmeinung, sondern eine bestimmte Betrachtungsweise innerhalb der empirischen Sozialwissenschaft - er ist ein Ansatz, der dazu bestimmt ist, nachprüfbares Wissen über das menschliche Zusammenleben und Verhalten zu erbringen“" (ebd.: 343). ${ }^{1}$

1 Vgl. dazu auch seine „drei einfachen Prämissen“ des symbolischen Interaktionismus: dass 1. Menschen ,Dingen“ gegenüber auf der Grundlage der Bedeutungen handeln, die diese Dinge für sie besitzen; dass 2. die Bedeutung solcher Dinge aus der sozialen Interaktion, die man mit seinen Mitmenschen eingeht, abgeleitet ist oder aus ihr entsteht, und dass 3. diese Bedeutungen in einem interpretativen Prozess, den die Person in ihrer Auseinandersetzung mit den ihr begegnenden Dingen genutzt, gehandhabt und abgeändert werden können (vgl. Blumer 2004 [1973]: 322). 
Die Annahme, dass Wirklichkeit gesellschaftlich konstruiert wird (vgl. Berger/Luckmann 1980 [1966]), hat Konsequenzen für die methodologische Anlage der Arbeit und der Erhebungs- und Auswertungsstrategien. Die (Re-)Konstruktion der Verarbeitung von Erlebnissen und der Art und Weise, wie Subjekte in sozialen Interaktionen Dingen und Phänomenen Bedeutung zuweisen und diese wieder verändern, erfordert ein empirisches Vorgehen, welches vorhandene Theorien nicht verifiziert oder falsifiziert, sondern diese vielmehr in Auseinandersetzung mit dem Material entwickelt und so der (Re-)Konstruktion Vorrang vor der Überprüfung von Hypothesen lässt. Entsprechend ist das Ergebnis solcher Analysen auch als gegenstandsbezogene Theorie, als Grounded Theory, zu verstehen. Ich möchte keine Theorie über binationale Paare allgemein entwickeln, vielmehr geht es mir um spezifische Formen von Bearbeitungsprozessen in Biographien binationaler Paare, meine Analysen besitzen also hinsichtlich ihrer Aussagekraft eine mittlere Reichweite. Das Prinzip einer solchen Grounded Theory wurde insbesondere durch Glaser und Strauss (zuerst 1967) entwickelt. Diese wandten sich gegen das zu der damaligen Zeit vorherrschende hypothetiko-deduktive Paradigma:

„Previos books on methods of social research have focused mainly on how to verify theories. This suggests an overemphasis in current sociology on the verification of theory, and a resultant de-emphasis on the prior step of discovering what concepts and hypothesis are relevant for the area that one wishes to research“ (ebd. 1967: 1f.).

Empirische Forschung sollte sozusagen Handlangerdienste leisten für die Produzenten von Großtheorien, die sich, so Glaser und Strauss ironisch, wie „theoretische Kapitalisten“ verhielten (ebd. 1998: 20). Die Empirie diente der Überprüfung von Hypothesen, die rein deduktiv aus großen Theorien gewonnen und durch Operationalisierung für die empirische Verifizierung ,passend' gemacht wurden. Mit dieser Entwicklung ging die polarisierende (und bis heute bestehende) Diskussion um den Wert und die Beziehung quantitativer und qualitativer Forschung einher. Quantitative Methoden wurden verfeinert und weiterentwickelt, um die unbestätigten Theorien anhand von ,Fakten“ überprüfen zu können. Qualitative Forschung wurde als explorative Forschung verstanden, die nur dazu da sei, Alltagswissen über Sozialstrukturen und soziale Systeme zu registrieren oder um theoretische Ergebnisse zu illustrieren. Das Testen von Reliabilität und Validität bekannter Theorien wurde aber selbstverständlich der quantitativen Forschung zugesprochen. Sofern Studien auf qualitativen Daten basierten, bestanden diese, so die Kritik 
Glasers und Strauss', aus langen detaillierten Beschreibungen, die, wenn überhaupt, relativ geringe Mengen an Theorie erbrachten. Qualitative Forschung sei also entweder nicht theoretisch genug oder die Theorien seien zu „,impressionistisch“ (vgl. ebd. 1998: 24). Folge der „Vorherrschaft des hypothetiko-deduktiven Paradigmas ist dabei eine zunehmende Entfremdung soziologischer Forschung von der sozialen Realität“ (Kelle 1994:285). Es kommt zu einem Bruch zwischen Theorie und Empirie. Die Theorie wirkt abstrakt und leer und verliert den Bezug zur Alltagswirklichkeit, die sie eigentlich zu erklären versucht, weil ihr die empirische Fundierung fehlt. Theoretische Erklärungsansätze und grand theories wurden, so die Kritik Glasers und Strauss', ohne Verbindung zur empirischen Alltagswirklichkeit entwickelt. Das empirische Fundament fand sich nur im Kopf des (in der Regel männlichen) Theoretikers. Umgekehrt fand die empirische Forschung ebenfalls fern von der Alltagswirklichkeit und der Realität statt, die sie erfassen wollte. Die Variablen, mit denen sie operierte, wurden aus den Theorien abgeleitet und verdanken ihre Existenz so ebenfalls ausschließlich deduktiven Prozessen. Glaser und Strauss hingegen wollten neues theoretisches Wissen generieren. Und solches Wissen ,entdeckten“ sie durch intensive Auseinandersetzung mit der empirischen Forschung. Die fruchtbare Beziehung von Theorie und Empirie stand also im Zentrum ihrer Überlegungen. Bei der Entwicklung ihres Verfahrens standen sie dementsprechend vor der Aufgabe, das bislang übliche deduktive Verfahren aufzugeben, ohne aber dem Trugschluss zu unterliegen, neue Schlussfolgerungen über die soziale Wirklichkeit ließen sich durch eine durch theorethische Vorannahmen unbeeinflusste Beobachtung, also induktiv, gewinnen. Der Lösungsvorschlag, den Glaser und Strauss entwickelt haben, um die empirisch fundierte Theoriegenerierung (wieder) in den Mittelpunkt soziologischer Forschung zu rücken und das Verhältnis von Empirie und Theorie anders zu fassen, folgt dementsprechend auch einer abduktiven Logik. Eine solch abduktive Logik liegt auch der vorliegenden Studie zugrunde. Der Vorschlag und das insbesondere von Strauss in Zusammenarbeit mit Juliet Corbin (Strauss/Corbin 1996) entwickelte Verfahren ermöglicht es, abduktive Prinzipien von Beginn des Forschungsprozesses an zu integrieren. ${ }^{2}$

2 Hierbei ist zu beachten, dass Grounded Theory kein in dem üblichen Sinne einheitliches Verfahren darstellt, sondern die Auswertungsverfahren und strategien in den letzten 30 Jahren oft modifiziert und erweitert wurden. Grounded Theory als methodologisches Rahmenkonzept zu nutzen meint also eher, sich den forschungstheoretischen Ideen und dem Prinzip der Datenerhebung und -auswertung zu verpflichten, als den konkreten Auswertungsschritten penibel zu folgen. 


\section{Der abduktive Forschungsprozess}

Das Vorgehen der Grounded Theory lässt sich als ,geplante Flexibilität ${ }^{\circ}$ bezeichnen. Geplant insofern, als dass gewisse hypothetische Vorannahmen auch über ein neues Forschungsfeld notwendig und sinnvoll sind, flexibel und offen, weil sich im Forschungsprozess diese Vorannahmen ändern können und ,in der Konfrontation mit dem empirischen Feld“ (Dausien 1996: 95) bewähren müssen. Vom ersten Kontakt mit dem Feld werden sie mit neuen Informationen angereichert, erweitern sich und postulieren am Ende womöglich das Gegenteil von dem, was sie ursprünglich angenommen hatten. Diese systematische Kreativität wird nun nicht dem Zufall und den Fähigkeiten des und der Forschers/in überlassen, sondern entsteht in einem methodisch kontrollierten Prozess. Strauss und Corbin beschreiben, analog zu dem oben Gesagten, den Forschungsprozess als ,spiralförmige Hin- und Herbewegung zwischen theoretisch angeleiteter Empirie und empirisch gewonnener Theorie" (Dausien 1996: 93). Dabei sollen die ,zentralen Konzepte und theoretischen Annahmen [...] während der Untersuchung aus dem Datenmaterial emergieren" (Kelle 1994: 341). Damit ist jedoch nach Strauss und Corbin keinesfalls das komplette Ignorieren schon vorhandener theoretischer Literatur über den Forschungsgegenstand gemeint. ${ }^{3}$ Die ,theoretische Sensibilität" (Strauss/Corbin 1996: 25) und die Bedeutung von Theorie ist für die Kodierung der Daten wesentlich, um die Aufmerksamkeit des/der Forschenden zu lenken und um eine ,völlige Egalität sämtlicher Beobachtung“ (Dausien 1996: 96) zu verhindern. Kelle definiert diese notwendige theoretische Sensibilität wie folgt:

„Theoretische Sensibilität bedeutet die Verfügbarkeit brauchbarer heuristischer Konzepte, die die Identifizierung theoretisch relevanter Kategorien im Datenmaterial und die Herstellung von Zusammenhängen zwischen diesen Kategorien, d.h. von Hypothesen, ermöglicht” (Kelle 1994: 312).

Um eine solche theoretische Sensibilität und die daraus entstehenden heuristischen Konzepte entwickeln zu können, wurden im ersten Teil der Arbeit das Forschungsfeld und die Forschungsperspektive expliziert und daraus Fragen und Anregungen für die empirische Arbeit gewonnen. Diese Entwicklung sensibilisierender Konzepte bedeutet jedoch nicht

3 Glaser hingegen besteht auf der Forderung, Forschende müssten sich möglichst unwissend, ohne theoretisches Vorwissen, dem Feld nähern, um Deduktivität zu vermeiden (vgl. Kelle 1994: 335). 
„die Formulierung präziser und empirisch gehaltvoller Hypothesen [...], vielmehr sind hiermit Konzepte mit einem hohen Allgemeinheits- und Abstraktionsgrad gemeint, die eine Formulierung empirisch gehaltvoller Hypothesen erst dann zulassen, wenn zusätzliche Informationen hinzukommen, (die sich ein qualitativer Forscher in der Regel erst in seinem Untersuchungsfeld beschaffen muss)" (ebd.: 307).

Die ersten Erkenntnisse führen also nicht zu festen Hypothesen im klassischen Sinne. Vielmehr wurde so Wissen expliziert, welches als Aufmerksamkeitsrichtungen empirieleitend fungiert.

„Welche ,Phänomene‘ ich im Datenmaterial ,erkenne‘ und welche Begriffe und ,Kodes' ich dafür finde, hängt entscheidend von meinem Kontextwissen ab. Dabei gilt es, eine prekäre Balance zu halten zwischen dem ,Ertrinken` im Material einerseits und dem Überstülpen theoretischer Kategorien nach dem Modell des deduktiven Kodieren andererseits“(Dausien 1996:100).

Unterstützt wird das Prinzip des Wechsels von Erkenntnissen aus Theorie und Empirie durch das theoretische Sampling. Bei der Zusammenstellung des Samples interessieren insbesondere solche Fälle, die wesentliche und neue Informationen versprechen, die also über die durch die Theorie gewonnenen Überlegungen hinausgehen und sie in Frage stellen. Um zu solchen Fallvergleichen zu kommen gibt es prinzipiell zwei Möglichkeiten, den maximalen und den minimalen Vergleich. Beim maximalen Vergleich wird das Sample so zusammengestellt, dass ganz gezielt nach Kontrastfällen gesucht wird. Der Kontrast kann sowohl in sozialstatistischen Merkmalen (Alter, Geschlecht, Herkunftsmilieu) als auch beispielsweise in der Art des Erzählens oder in Interaktionsstrategien liegen. Entscheidend ist, zu einem Fall A einen Kontrastfall B und zu beiden unter Umständen weitere Kontrastfälle C oder D zu finden. Beim minimalen Vergleich hingegen werden die durch den maximalen Kontrast gefundenen Kategorien weiter verfeinert, d.h. es wird nach Fällen gesucht, die sich möglichst wenig von den schon analysierten unterscheiden. Für mein Sampling bedeutete dies, dass ich aus den vorliegenden Interviews zuerst für einen maximalen Kontrast zwei Paare aussuchte. Diese, Susanne Frei und Rachid Frei-Hardazadeh sowie Nicole und Carlos Ortega Garcia, unterschieden sich zum einen vor allem hinsichtlich des Alters, der Aufenthaltsdauer in Deutschland und des Vorhandenseins von Kindern. Zum anderen aber, das wurde nach der Erhebung der Interviews deutlich und bestätigte sich in ersten Interpretationen, unterschieden sie sich deutlich im Hinblick auf die Bedeutung des female breadwinner Modells und daraus resultierende Bearbeitungsstrategien. Mit diesen beiden Paaren wurde das Analysemodell entwi- 
ckelt. Als drittes Paar wurden Maria Wesel und Pablo Marmani Rodriguez ausgewählt. Dieses versprach im Sinne einer minimalen Kontrastierung wichtige Ergänzungen und Erweiterungen im Hinblick auf den Paarhorizont und mögliche Bearbeitungsstrategien. Ziel eines solchen Samplings ist die Sättigung der Thesen und der Untersuchungsergebnisse, nicht im Sinne einer ,vollständigen“ Ausschöpfung aller potentiellen Aspekte eines biographischen Interviews, sondern im Sinne einer Sättigung der entwickelten Kategorien und Dimensionen. Diese tritt dann ein, wenn sich im Verlauf der Untersuchung entweder zeigt, dass bereits bekannte Fallkonstellationen auftauchen, also der Fall A mit einem Fall A' verglichen werden kann (insbesondere bei groß angelegten Studien ist dies der Fall), oder wenn, wie in der vorliegenden Arbeit, verschiedene Formen biographischen Lernens analysiert werden konnten, die ausreichend Einblick in Bearbeitungsstrategien geben. ${ }^{4}$

\section{Die Auswertung der Interviews}

\section{Erste Schritte: Transkription, Erstellung des Verlaufprotokolls, biographisches Portrait}

Als ersten Schritt und zur Vorbereitung der Interpretation wurden die von mir durchgeführten Interviews transkribiert. Vollständig transkribiert wurden acht der insgesamt 19 geführten Interviews. Diese Transkriptionen sind einerseits Vorbereitungen für die Interpretation insofern, als dass sie den weiter zu bearbeitenden Text und sozusagen die Grundlage der Interpretation darstellen. Andererseits sind sie schon Teil dieser Interpretation, da die Biographie, die mir in einem Interview erzählt wurde, in gewisser Weise entpersonalisiert und zu einem Fall transformiert wird, der unter einer spezifischen Blickrichtung interpretiert wird. Die Transkriptionsnotationen richten sich nach Ricker 2000:

4 Theoretisches Sampling bedeutet im Übrigen auch, dass die Datenerhebung gerade kein abgeschlossener Vorgang zu Beginn des Forschungsprozesses sein kann, sondern ein sukzessiver Prozess mit deutlichem Schwerpunkt im ersten Stadium der Forschungen, jedoch mit möglichen Ergänzungen und Datenerhebungen selbst während des Auswertungsprozesses. Diese Idealform des theoretischen Samplings ließ sich für die vorliegende Untersuchung nicht durchhalten. Wie in der Dokumentation des Forschungsprozesses beschrieben, war es durchaus schwierig genügend Interviewpartner(innen) zu finden. Ein weiteres Sampling nach spezifischen Merkmalen war so während des Interpretationsprozesses nicht möglich und bleibt der weiteren Forschung vorbehalten. Das theoretische Sampling fand also innerhalb des Gesamtsamples statt, ohne dass nach Beendigung der Erhebungsphase weitere Interviews geführt wurden. 


\title{
Transkriptionsnotationen:
}

\author{
Pausenkennzeichnung \\ - $\quad$ kurze Pause \\ --- mittlere Pause \\ (5 sek) lange Pause mit Angabe der Sekunden
}

\section{Weitere Kennzeichnungen}

. $\quad$ fallende Intonation zur Markierung eines Satzendes

? $\quad$ Frageintonation

Wort Betonung oder besonders deutliche Artikulation eines Wortes oder Syntagmas

Sooo Verdoppelung oder Verdreifachung des Konsonanten und Vokale in Angängigkeit zu der Wortdehnung

(...) unverständliche Textteile

Abbru_ Markierung eines Wortabbruchs

Äh - Gefüllte Pause durch entsprechende Phoneme

\section{Nicht-phonologische akustische Merkmale}

Da bin ich kurz davor gewesen (lachend) In Klammern gesetzte Beschreibung der Erscheinung

Ebenfalls wurden von den Interviews mit den vier Paaren, also von acht Interviews, Verlaufsprotokolle erstellt. Diese bestehen aus einer kombinierten Zusammenfassung von formaler Strukturanalyse (suprasegmentale, segmentale, subsegmentale Gliederung des Textes, Textsorten, vgl. v.a. Schütze 1984) und des thematischem Verlaufs des Interviews. ${ }^{5}$ Es ermöglicht einen systematischen Zugang auf das Interview und im fortschreitenden Interpretationsprozess zugleich forschungsökonomisch den Verzicht auf die gesamte Interpretation der Interviews. Das Verlaufsprotokoll dient einem ersten intensiven Erschließen des Interviews und hat die Funktion, den Aufbau der Erzählung und die jeweilige Struktur (z.B. die ,Vorliebe' für eine bestimmte Textsorte) sichtbar zu machen. Kernstellen können so identifiziert werden. Zugleich dient es als Gliederung für das Interview und ermöglicht so ein rasches Auffinden einzelner Interviewpassagen. Das Verlaufsprotokoll besteht aus folgenden Rubriken: Locus (Kennzeichnung der Interviewstelle durch Zeilennummern), Erzählart, Inhalt und Themen/erste Ideen. Der Interpretationsprozess kon-

5 Lebensgeschichtliche Erzählungen folgen bestimmten „formale[n] und inhaltliche[n] Strukturen der Erfahrungsrekapitulation“" (Schütze 1984: 81, vgl. auch Dausien 1996), welche den Zugang zu inhaltlichen Interpretationen ermöglichen, da sie Aufschluss geben über die Art und Weise der Verarbeitung von Erlebtem und der sozialen Positionierung. 
kretisiert sich hier also insofern weiter, als dass im Verlaufsprotokoll noch stärker Wahrnehmungen der Interpretierenden Relevanz bekommen, der Text durch die Sequenzierungen ,aufgebrochen' und erste Ideen formuliert werden können. Jeder Text, der so produziert wird, enthält verschiedene Textsorten, die für die Interpretation der Interviews relevant sind. Ich habe mit diesen Unterscheidungen gearbeitet (nach Rosenthal 1995: 240f):

- Erzählungen: Beziehen sich auf zurückliegende singuläre Ereignisabfolgen. Abfolge von tatsächlichen oder fiktiven Ereignissen, die in einer Beziehung zeitlicher oder kausaler Aufeinanderfolge zu einander stehen.

- Beschreibungen: Der „entscheidende Unterschied zu Erzählungen liegt darin, dass Beschreibungen statische Strukturen darstellen [...] Der Vorgangscharakter der dargestellten Sachverhalte wird ,eingefroren“" (Kallmeyer/Schütze 1977: 201).

- Argumentationen: theoriehaltige Textelemente, die sowohl innerhalb der Erzählsequenzen auftreten (dann als Evaluationen ausgewiesen) als auch außerhalb anzutreffen sind. Außerhalb: Theorieelemente; Bekundungen allgemeiner Vorstellungen.

- Bericht: ist eine geraffte Erzählung mit sehr niedrigem Indexikalitätsgrad. „Erzählungen, die Ereignisse in Beschränkung auf eine unilineare Ereigniskette ohne Herausarbeitung von Situationen darbieten“ (Kallmeyer/Schütze 1977: 187) eine Art Telegrammstil.

Als letzten vorbereitenden Schritt für die intensive Interpretation wurden auf der Basis der Transkriptionen und der Verlaufsprotokolle biographische Portraits erstellt. Diese dienen zur Erfassung wesentlicher Merkmale der Biographie und zur Präsentation der Personen mit ihren jeweiligen Besonderheiten. Das biographische Portrait, das den Interpretationen vorangestellt ist, soll dem Leser und der Leserin ermöglichen, die Interpretationen nachzuvollziehen, und helfen, die Biographie des Paares zu vergegenwärtigen.

\section{Kernstellenanalyse}

Auf der Grundlage des Verlaufsprotokolls wurden Kernstellen zur intensiven line-by-line Interpretation ausgewählt. Kernstellen sind grundlegende und zentrale Passagen in einem Interview, in denen sich verdichtet die wichtigsten Entwicklungsprozesse zeigen und Veränderungen der Wahrnehmungs- und Deutungsmuster erfasst werden können (vgl. ausführlich Ricker 2000). Für die Entscheidungen, einzelne Passagen als 
Kernstellen auszuwählen, sind neben biographischen Aspekten ebenfalls die Fragestellung der Untersuchung ausschlaggebend. Zu Beginn des Interpretationsprozesses wurden insbesondere die Interviewanfänge als mögliche Kernstellen ausgewählt, da oft schon am Anfang relevante Themen der Biographie angesprochen werden und der ,Aufhänger' der biographischen Erzählung hier zu erkennen ist. Wie gezeigt, unterscheiden sich die Interviewanfänge ganz erheblich voneinander; ob die Erzählung thematisch oder chronologisch in die Erzählung beginnt, entscheidet über die Richtung, die ein Interview nehmen kann, und macht die Relevanzsetzungen der interviewten Person deutlich. Im Verlauf des Analyseprozesses zeigte sich deutlich, dass insbesondere die Erzählungen über den Beginn der Beziehung und die Entscheidung, (zumindest vorläufig) zusammen in Deutschland zu leben, für meine Fragestellung relevant waren. Der Beziehungshorizont kristallisierte sich als integrierende Dimension heraus (vgl. dazu das Interpretationsmodell). Demzufolge wurden letztendlich Kernstellen zu diesem Themenkomplex als Einstieg in die line-by-line Analyse genutzt. Je weiter der Prozess fortschritt, desto deutlicher entwickelte sich das Interpretationsmodell. Dies hatte erhebliche Folgen für den Interpretationsvorgang. Die Interpretationen des ersten Paars, Susanne und Rachid folgten einer ,klassischen“ biographischen Analyse, für das erste Paar wurde auch eine vollständig ausgearbeitete Fallstudie geschrieben. Dimensionen wie Erzählungen über die Kindheit und die Schulzeit wurden ebenso berücksichtigt wie das Verhältnis zu den Eltern. Aus diesen Ergebnissen heraus und unter Zuhilfenahme des zweiten Falls wurde ein immer stärker verdichtetes Interpretationsmodell entwickelt, welches der Besonderheit der Paare Rechnung trug, gleichwohl aber die Vergleichbarkeit auch zwischen den Paaren gewährleistete. Es wurden Kontexte ausgewählt, die sich als thematisch relevant herausgestellt haben: der Paarhorizont und die sich aus der spezifischen Gestaltung dieses Horizontes ergebenden Bearbeitungsfelder. Beim dritten Paar, bei Maria und Pablo, war das Interpretationsmodell schon so weit entwickelt, dass nur noch diese Dimensionen betrachtet wurden. Die Biographien wurden daraufhin analysiert und ,Nebenstränge“ nur insofern berücksichtigt, sofern sie weiteren Aufschluss über die Dimensionen versprachen. Das Interpretationsmodell behält jedoch das Prinzip der Sequenzialität bei, die Erzählung bleibt in ihrer Eigengestalt erhalten und wird in ihrem Verlauf analysiert. Die line-by-line Analyse folgt der „Eigenlogik der autobiographischen Rekonstruktionsleistung“ (Dausien 1996: 106), d.h. die Art und Weise der Verarbeitung von Erlebtem und von Ereignissen steht im Mittelpunkt der Analyse. In der (Re-)Konstruktion solcher Sinnzusammenhänge bleibt der zeitlich-logische Zusammenhang erhalten, Entwicklungen 
können so, besser als bei stark kategorisierenden Verfahren, nachgezeichnet werden. In der (Re-)Konstruktion des Ereignisablaufes stellen die von Schütze entwickelten Prozesstrukturen des Lebenslaufs wichtige Anhaltspunkte für Haltungen dar, mit denen die interviewte Person diese Erlebnisse konstruiert und ermöglichen so eine Einordnung der spezifischen Verarbeitungsmodi (vgl. Schütze 1981). ${ }^{6}$ Relevant wurde für die Interpretation der Interviews zum Beispiel die Haltung der Paare zum female breadwinner Modell und zu dessen Konsequenzen. Entwickelt Susanne Frei ihre Erwerbstätigkeit handlungsschematisch, so entstehen Probleme für sie in dem Moment, in welchem sie als Hausfrau und Mutter einen für sie extrem schwierigen Rollentausch vollzieht. Diese Zeit erlebt sie als Verlaufskurve: „Der Betroffene reagiert auf eine Art, die er an sich selbst bisher nicht gekannt hat und die er sich nicht zu erklären vermag“ (Schütze 1981: 89). Eine solche Zeit des Erleidens, des Eindrucks keine Handlungsmöglichkeiten mehr zu besitzen, endet für Susanne durch eine erneute handlungsschematische Aufnahme der Biographiegestaltung durch die Entwicklung eines individuellen Betreuungsund Vereinbarkeitsmodells.

\section{Biographische Interviews binationaler Paare: Besonderheiten der Interpretation und der Darstellung}

Das methodische Vorgehen, wie es oben beschrieben wurde, hat den Schwerpunkt auf die inhaltliche (Re-)Konstruktion von Handlungs- und Orientierungsmustern gelegt und weniger auf die strukturelle Beschreibung der Kernstellen oder des gesamten Interviews, wie sie Schütze entwickelt hat. Dies war auch eine Folge der besonderen Situation bei der Arbeit mit biographischen Interviews von binationalen Paaren, die diese Studie von anderen unterscheidet. Zum einen wurden Interviews mit Menschen geführt, deren Erstsprache nicht Deutsch ist, zum anderen musste ein Interpretationsmodell entwickelt werden, mit dem die Biographien der Paare aufeinander bezogen werden konnten. Nicht nur die Eigenlogik der jeweiligen Einzelbiographie interessierte für die Untersuchung, sondern auch und vor allem der Bezug aufeinander.

6 Schütze unterscheidet zwischen institutionalisierten Ablaufmustern und -erwartungen, Handlungschemata von biographischer Relevanz, Verlaufskurven und Wandlungsprozessen (Schütze 1981: 67ff). Als biographische Gesamtformung lässt sich in der Regel eine die gesamte biographische Erzählung dominierende Figur bestimmen. 


\section{Biographische Interviews mit Migranten}

Meine eigenen Sprachkenntnisse ermöglichten die Durchführung der Interviews auf Deutsch oder Englisch, zudem bestand noch die Möglichkeit, die Interviews auf Spanisch zu führen. Entsprechend den verschiedenen Herkunftsländern und den sprachlichen Kenntnissen der Migranten wurden die Interviews auf sprachlich sehr unterschiedliche Art und Weise geführt. Im Kernsample wurde ein Interview, mit Rachid Frei-Hardazadeh, auf Deutsch geführt. Rachid ist seit über 20 Jahren in Deutschland und beherrscht die Sprache fließend. Das Interview mit Carlos Ortega Garcia hingegen wurde auf Deutsch und Englisch geführt. Das dritte Interview, mit Pablo Marmani Rodriguez, wurde wiederum auf Spanisch geführt und dann von einer Muttersprachlerin für mich übersetzt. ${ }^{7}$ Biographische Erzählungen sind nun in besonderer Weise durch sprachliche Eigenheiten und Feinheiten strukturiert. Es kann zu Schwierigkeiten kommen, wenn zurückliegende Erfahrungen, die in einem anderen Land und in einer anderen Sprache gemacht wurden, ausgedrückt werden sollen. Der Erfahrungshorizont, so ist zu vermuten, ist nicht in gleicher Weise zugänglich. Zudem gehen bei zum Teil notwendigen ,Übersetzungen“ nicht nur im wortwörtlichen Sinn der Übersetzung von Sprache, sondern auch im Sinne der Übersetzungen von Konzepten, Lebenswirklichkeiten und zum Beispiel politischen Gegebenheiten, Feinheiten und Dimensionen verloren. Darauf weist auch FuchsHeinritz hin, da insbesondere die strukturelle Beschreibung nach Schütze darauf ausgerichtet ist,

„dass Interpret und Befragter die gleiche Sprache sprechen, weil es nur so möglich ist, dass auf der Ebene der Syntax, der Wortwahl usw. liegende Besonderheiten als Indikatoren für die dargestellten Sachverhalte bzw. Haltungen der Befragten zum Sachverhalt interpretiert werden können“ (in Hummrich 2002: 36).

Gerade Textmarker (wie ,äh', ,und“ etc.) können also durch diese sprachlichen Besonderheiten nicht auf gleiche Weise in die Interpretation einbezogen werden wie in einem biographischen Interview, welches in der Erstsprache des/der Interviewten geführt wurde. Sprachanalytisch ist es allerdings fruchtbar zu beobachten, an welchen Stellen zum Bei-

7 An dieser Stelle bin ich Rosa Jimenez Laux und Nurja F. Roja zu großem Dank verpflichtet. Rosa Jimenez Laux hat das Interview mit Pablo Marmani Rodriguez geführt, Nurja F. Roja hat es ins Deutsche übersetzt und mir bei Deutungsmöglichkeiten und Verständnisschwierigkeiten sehr geholfen. Ohne diese beiden gäbe es die Interpretation nicht. 
spiel vom Deutschen ins Englische gewechselt wird oder welche Begriffe in einem spanischen Interview dennoch auf Deutsch verwendet werden. Die sprachlichen Besonderheiten hatten auch Konsequenzen für die Durchführung der biographischen Interviews. Wie dargestellt, lassen sich die Interviews mit den Migranten nur zum Teil als klassische biographisch-narrative Interviews bezeichnen, die der Dreiteilung (Erzählaufforderung, Haupterzählung, Nachfrage) folgen. Zum Teil gewann die Interviewführung ihre Anregungen von der Methodik des problemzentrierten Interviews. Ich entwickelte einen Leitfaden, der sich an der Biographie und an den vorher verfügbaren Informationen des/der jeweiligen Interviewpartners/in orientierte und der dann zum Einsatz kam, wenn die Erzählung ins Stocken geriet oder, nach Zwischendiskussionen, der Erzählfaden wieder zurück zum Interview geführt werden sollte. Der Erzähllogik der Interviewten wurde also immer Vorrang gegeben und Fragen nur dann eingeworfen, wenn sie die Erzähllogik nicht beeinträchtigten (vgl. Diekmann 1998, 451). Insofern ist der Leitfaden eher als ,eine Art Hintergrundfolie [zu verstehen], die zur Kontrolle dient, inwieweit seine einzelnen Element im Laufe des Gespräches behandelt worden sind.“ (Witzel 2000: 9). Die Mischung von unterschiedlichen Interviewsorten machte eine stark sprachanalytische Auswertung schwierig. Schützes Postulat beispielsweise, nur Erzählungen und keine Argumentationen oder Berichte in die Auswertung einzubeziehen, ließ sich schon auf dieser Ebene nicht realisieren. ${ }^{8}$ Eine weitere Dimension hinsichtlich biographischer Interviews mit Migranten ist die damit vorgenommene Unterstellung, das Konzept der ,Biographie“ sei ein weltweites Phänomen:

„Wird nicht mit der Idee, lebensgeschichtliche Erzählungen zu generieren, bereits unterstellt, in der untersuchten Kultur gelte ein ,westlichen' Kulturen vergleichbares, individuumsbezogenes Biographie-Paradigma, über das erlebte Sachverhalte zu unverwechselbaren und kontinuierlichen ,Lebensgeschichten“ organisiert werden?“ (Matthes 1984: 286).

8 Diese Einschränkung ist allerdings noch aus einem anderen Grund schwierig. Schütze vermutet, dass im Fall von dominierenden Argumentationen und Beschreibungen die erlebten Erfahrungen noch nicht verarbeitet sind: „Von einer Zerrissenheit der Identitätskonzeption des Erzählers als Biographieträger kann man in diesem Fall sprechen“ (Schütze 1984 in Marotzki 1990: 251). Marotzki nimmt hingegen an, dass „die verstärkte Krisenanfälligkeit von Subjektivität in der Moderne einen erhöhten argumentativen Bearbeitungsaufwand erzeugt" (ebd.: 251). Eine solche Form von erhöhtem Bearbeitungsaufwand ließ sich auch für mein Sample vermuten. Insofern hat es Sinn, auch bzw. gerade solche Passagen zu analysieren. 
Die in biographischen Interviews enthaltene Vorannahme eines starken Subjekts ist sicherlich nicht unproblematisch. Bei dieser Konzeptualisierung von biographischen Erzählungen steht das Subjekt als Akteur im Mittelpunkt. Eine solche individualistische Sichtweise ist jedoch, wie zum Beispiel Shimada 2001 durch Interviews mit Japaner(innen) zeigt, nicht als universale Voraussetzung zu sehen. Kollektive Bezüge können ebenso die Funktionen übernehmen, die in westeuropäischen Gesellschaften die Ichbezogenheit übernimmt. Es ist eher davon auszugehen, „dass jede Kultur eine eigene Definition und eigene Regeln der Erzählung besitzt" (ebd.: 39). ${ }^{9}$ Die institutionalisierten Skripten, die den Erzähler(inn)en zur Verfügung stehen, unterscheiden sich deutlich, ebenso wie Erzähltraditionen und unterschiedliche Vorstellungen von Zeit und Lebens(ab)läufen. Die Entscheidung für biographische Interviews fiel daher auch nicht aus der Vorannahme heraus, Biographiearbeit sei eine generelle, zeit- und kontextunabhängige Aufgabe für Individuen. Vielmehr entsteht die Notwendigkeit der Biographiearbeit aus verschiedenen Kontexten heraus und lässt sich für das von mir interviewte Sample aus vier Gründen konstatieren. ${ }^{10}$

Migrationsprozesse machen Selbstreflexion notwendig, allerdings in einem paradoxen Zusammenhang: Einerseits steigt die Notwendigkeit der Selbstbestimmung, der Planung und der biographischen Reflexion, andererseits sind Migranten strukturellen Zwängen unterworfen, die die Planung manchmal konterkarieren bzw. eine handlungsschematische Biographiegestaltung unmöglich machen.

Die westliche Moderne, der die Migranten, unabhängig von ihren Herkunftsländern, mit der Migration nach Deutschland begegnen, fordert eine erhöhte Biographisierung und die Übernahme biographischer Verantwortung.

Auch die Paarbeziehung basiert in der westlichen Tradition auf der gemeinsamen Gestaltung der Paarbiographie und, wie Eckert/Hahn/ Wolf 1989 zeigen, auf dem Austausch und der Angleichung biographischer Erinnerungen und der Begegnung von freien Subjekten. ${ }^{11}$

9 Für ein anderes Beispiel vgl. Koch 2006, die bei der Durchführung biographischer Interviews mit Mitgliedern von Roma-Familien ein ebensolches Muster der Konstruktion entdeckte. Biographische Interviews in dem von ihr erwarteten Sinne waren damit nicht möglich.

10 Dies zeigte sich auch im Verlauf der Interviews: Alle interviewten Männer stellten sich selbst als Akteure in den Fokus der Erzählungen und bezogen sich nicht zum Beispiel auf ein Familienkollektiv als Akteur.

11 Das die westliche ,freie' Partner(innen)wahl so frei nicht ist, sondern vielmehr erstaunlich homogen entlang Bildungs- und Altersschichten verläuft, wird dabei gerne vergessen (vgl. zu den sozialstrukturellen Voraussetzungen der Liebe Klein 2001b). 
Und nicht zuletzt stößt die Interviewsituation, in der die Interviewerin explizit nach biographischen Erinnerungen fragt, solche Reflexionsprozesse und eine solche Form von Biographiearbeit an. ${ }^{12}$

\section{Besonderheiten in der Darstellung der Interpretationsergebnisse}

Lernprozesse zwischen den Biographien stehen im Mittelpunkt der Analyse. Dafür müssen die beiden Intervies der Paare aufeinander bezogen werden. Einerseits also stellt die Textmenge eine Besonderheit dar: Schon rein quantitativ hat man in der Analyse mit relativ gesehen mehr Textmaterial zu tun als bei vielen anderen biographischen Studien, die in der Regel nur ein Interview als Fall interpretieren. Andererseits sollte sich aber auch die Analyse entsprechend verändern. Kernstellen können nicht nur nach der Besonderheit des einzelnen Falles ausgewählt werden, sondern müssen auch mit den Kernstellen des/der Partners/Partnerin systematisch verglichen werden können. Die verschiedenen Interviewarten und die daraus entstandenen verschiedenen Textsorten führten dazu, die biographische Analyse noch stärker als vorher geplant an thematischen Besonderheiten auszurichten und sprachstrukturelle Analysen wie die formale Textanalyse zugunsten dieser thematischen Ausrichtung zu vernachlässigen.

Das konkrete Vorgehen wurde insbesondere anhand der Interviews von Susanne und Rachid Frei-Hardazadeh entwickelt und erprobt. Durch Interpretationen in verschiedenen Forschungswerkstätten konnten Interpretationsversuche durchgeführt werden und so das Vorgehen entwickelt werden. Zudem wurden die Interpretationen durch die Arbeit in diesen

12 Bei der Analyse der Interviews muss dennoch sorgfältig mit der Bedeutung sozio-kultureller Kontexte umgegangen werden, da die selbstverständliche Teilung von lebensweltlichen Bedeutungen noch stärker reflektiert werden muss. Zu beachten ist, wie sich die Interviewten selbst $\mathrm{zu}$ als kulturell markierten Kontexten oder Tradierungen verhalten: Wenn Rachid Frei zum Beispiel die Forderung seiner Mutter nach einer arrangierten Ehe kritisch kommentiert, dann tut er das mit Rückgriff auf westliche Bewertungsmuster solcher Ehen. Hier distanziert er sich also von traditionellen iranischen Partnerschaftsverständnissen und sieht sich selber als westlich-modern orientiert. Solche Analysen der Selbstpositionierungen sind auch deshalb möglich, weil die Interviewten in der konkreten Interviewsituation, durch Hintergrundkonstruktionen oder argumentierende Einwürfe, von nicht geteilten Selbstverständlichkeiten ausgingen. So kann ich zwar aus dem Interview heraus nicht ,wissen' und entscheiden, ob, wie in einem der geführten Interviews konstatiert, das kubanische Bildungssystem zu den qualitativ besten der Welt zählt. Ich kann diese Aussage aber als Selbstpositionierung des Interviewten verstehen, der sich als gleichwertig, wenn nicht besser, qualifiziert versteht. 
kollegialen Gruppen methodisch unterstützt und abgesichert. In dieser Arbeit wird der Interpretationsprozess und die Darstellung der Ergebnisse stärker als vielleicht sonst üblich getrennt (vgl. für eine andere Form der Darstellung z.B. Ricker 2000). Die Darstellung folgt nicht dem Prinzip von Kernstelle - Interpretation, Kernstelle - Interpretation, sondern nimmt eine stärkere Verflechtung von einzelnen Interviewstellen zu einem bestimmten thematischen Kontext vor. In diesem thematischen Kontextwurden Kernstellen ausgewählt, in denen die Konstruktion dieses Horizontes deutlich wird; Beim Paarhorizont ist es in der Regel dann der Fall, wenn die betreffende Person vom Beginn der Beziehung erzählt. Häufig haben solche Erzählungen über die Begegnung mit dem/der Partner(in) einen breiten Raum eingenommen. In einigen Interviews jedoch finden sich Erzählungen zum Paarhorizont und zur Bedeutung der Beziehung für den/die Interviewten an verschiedenen Stellen des Interviews. Entsprechend wurden dann auch mehrere Kernstellen interpretiert. Um das Verständnis für den Leser/die Leserin dennoch zu gewährleisten und eine Nachvollziehbarkeit der Interpretationen zu sichern, wurden zum einen ausführliche biographische Portraits erstellt, die einen Überblick über die Biographie der Interviewten gewährleisten sollen und den Interpretationen vorangestellt sind. Zum anderen ermöglichen die in den Interpretationen verwendeten Direktzitate die Nachvollziehbarkeit der Analysen.

\section{Was für ein Text entsteht? Ein methodologisches Verständnis der Interpretationen}

Als Ergebnis und als Grundlage der Interpretationen sind also Interviewtexte entstanden, die als biographische Konstruktionen (auch) als Auseinandersetzungen mit Geschlechterbeziehungen und natio-ethno-kulturellen Zugehörigkeiten gelesen werden können. Die Interpretationen dieser Texte wiederum sind als Texte zu verstehen, die sich einerseits auf die biographischen Interviews als Grundlagen dieser Auseinandersetzungen und Überlegungen beziehen, andererseits durch theoretische Konzepte inspiriert wurden. Interpretationen sind (Re-)Konstruktionen biographischer Konstruktionen. Einem solchen Verständnis liegt die Unterscheidung von Alfred Schütz in Konstruktionen ersten und zweiten Grades zugrunde:

„Die gedanklichen Gegenstände, die von Sozialwissenschaftlern gebildet werden, beziehen und gründen sich auf gedankliche Gegenstände, die durch das 
Common-Sense Denken des im Alltag unter seinen Mitmenschen lebenden Menschen gebildet werden. Die Konstruktionen, die der Sozialwissenschaftler benutzt, sind daher sozusagen Konstruktionen zweiten Grades: Es sind Konstruktionen jener Konstruktionen, die im Sozialfeld von den Handelnden gebildet werden, deren Verhalten der Wissenschaftler beobachtet und in Übereinstimmung mit den Verfahrensregeln seiner Wissenschaft zu erklären versucht" (ebd. 2004 [1971]: 159).

Auch Dausien folgt Schütz: Biographische (Re-)Konstruktionen sind „reflexive, kritisch-analytische (Re-)Konstruktionen des Konstruktionsprozesses ersten Grades sowie der Bedingungen, unter denen diese stattfinden, ausbleiben und sich gegebenenfalls verändern“ (Dausien 2000: 98). Ein solches Verständnis lässt sich zum Beispiel an dem schon vorne verwendeten Interviewausschnitt von Maria Wesel verdeutlichen, in dem sie über die geplante Migration nach Mexiko Auskunft gibt: Marias Erzählung ihrer biographischen Neu-Orientierung und der Vergleich zwischen Mexiko und Deutschland ist ihre Konstruktion der derzeitigen Situation. In diesen Textpassagen kann ich, die an Geschlechterkonstruktionen und Konstruktionen natio-ethno-kultureller Zugehörigkeit interessiert ist, Konstruktionslogiken und Funktionen und Bedeutungen dieser Konstruktionen analysieren. So scheint Marias Deutung unter anderem von einer Gegensätzlichkeit zweier verschiedener Lebensmodelle strukturiert zu sein: Mexiko wird dabei als das flexiblere und offenere Land beschrieben. Gleichzeitig ordnet sie sich Deutschland und ,typisch deutschen' Eigenschaften zu, die ihr Zögern hinsichtlich der geplanten Migration, auch mir als Zuhörerin, verständlich machen (sollen). Solche Analysen sind durch meine theoretischen Vorlieben, Kenntnissen, Entwicklungen in Laufe des Interpretationsprozesses etc. strukturiert, gehen über die Interviewtexte hinaus, erweitern und verändern sie. Paul Mecheril hat für ein solches Verständnis von Texterzeugungsprozessen den Begriff der „Ko-Konstruktion“ (Mecheril 2003: 43) geprägt:

„Die Auslegung der Interviewtranskripte präsentiert eine Geschichte, die erst durch den Bezug auf Text V [den Interviewtext, M.M.] möglich wurde, aber dennoch von ihm unahängig ist. Demnach ist Text $X$ [der Interpretationstext, M.M.] bezogen auf Text V eine Ko-Konstruktion. Der ko-konstruierte Interpretationstext gewinnt sein Profil, indem er im Zuge interessierender Hin-Sichten, im Zuge in den Transkripttext eingreifender, gleichsam dazwischengehender Perspektiven [...] entsteht, welche sich im Verlauf der Modellierung erst herausgeschält haben. Modellierungen präsentieren Lesarten, die sich im Prozess der Modellierung entwickeln. Hierbei fungieren die in den Interviewtexten vorhandenen Auskünfte als auslegungsfähige Konkretisierungen figurativer Muster, die mir im Sinne von hypothetischen als auch sensibilisierenden Kon- 
zepten teilweise bereits vor dem Modellieren bekannt waren, teilweise aber erst durch das Modellieren zugänglich wurden“ (Mecheril 2003: 43, Herv. d. Verf.).

Mit dem Begriff der Modellierungen werden die Interpretationen, stärker noch als bei Schützes Verständnis von Konstruktionen ersten und zweiten Grades, ebenfalls wieder als Konstruktionen erkennbar. Eine solche Sicht betont die relative Unabhängigkeit der entstehenden Interpretationen. Es entsteht ein Text, der wiederum erneuten Analysen zugänglich ist: „Text als Medium für Text“ (Mecheril 2003: 32). Solche Texte sind in gewisser Weise bereinigte Modelle, welche die Vielfalt und die Komplexität sozialen Handelns auf die interessierenden Analysefragen zuspitzen und reduzieren. Wenn auch in dem Interpretationsmodell das Modell des Paarhorizonts die Statik und Gebundenheit des Begriffs des Möglichkeitsraums aufheben soll, bleibt doch das Problem der fehlenden Dynamik dieses Modells. Der Paarhorizont stellt keine starre Größe dar, die, einmal konstituiert, wandlungsresistent ist. Vielmehr folgt er der temporären Dynamik des Beziehungsverlaufes und zirkuliert damit quasi innerhalb der Beziehung. Zwar wird in den Interpretationen auf die Bedeutung für die Veränderung dieses Horizonts aufmerksam gemacht, der Schwerpunkt liegt jedoch auf den Bearbeitungsfeldern, die sich aus dem Paarhorizont ergeben. Die Veränderungen des Beziehungshorizontes werden also in der vorliegenden Interpretation nicht mehr aufgenommen. Ähnlich verhält es sich mit den Verhältnissen zwischen den Interviews der einzelnen Paare. Diese wurden, wie im methodologischen Teil der Arbeit ausgeführt, erst einzeln und dann im Verhältnis zueinander interpretiert. Auch hier finden sich komplizierte temporäre Beziehungen, zum Beispiel im Bezug auf Lernprozesse: Jeder lernt für sich, lernt in der Beziehung, lernt von dem/der Anderen und lernt als Paar. Vollständig lassen sich solche komplexen Prozesse nicht darstellen. In dieser Arbeit wurde sich für eine Forschungsperspektive entschieden, die den Fokus auf das Lernen als Paar gelegt hat, wissend aber um die Idealisierung des Modells zugunsten der Aussagefähigkeit. Das Interpretationsmodell arbeitet daher in gewisser Weise mit Idealtypen, die von bestimmten Problematisierungen befreit worden sind, um zu Aussagen gelangen zu können. Solche Vereinfachungen und Idealisierungen ermöglichen es, Formen biographischen Lernens prägnant zu fassen und Aussagen über Bearbeitungen zu treffen, die sich auf eine bestimmte Konstellation beziehen. 



\section{EINFÜHRUNG IN DAS ANALYSEMODELL: FORMEN BIOGRAPHISCHEN LERNENS IN SOZIALEN KONTEXTEN}

\section{Dimensionen biographischen Lernens}

In dieser Arbeit wird mit einem Konzept des biographischen Lernens gearbeitet, welches biographisches Lernen nicht in ein Definitionskorsett zwängt, sondern es vielmehr als theoretischen Zugang zu Biographien und damit als weitere sensibilisierende Aufmerksamkeitsrichtung nutzt. Es geht also nicht um ein Verständnis im Sinne von ,Lernen ist', sondern darum, dass Prinzip der Offenheit und der Mehrseitigkeit im Hinblick auf die Beobachtung, dass Biographien ohne Lernen nicht möglich sind, ernst zu nehmen. Für die konkrete empirische Analyse können dann verschiedene Modellierungen entwickelt werden. Mit einem solchen eher grundlegenden Verständnis von und Zugang zu ,Lernen“ schließe ich mich weiteren Autor(inn)en an, die sich ebenfalls biographietheoretisch mit Lernprozessen auseinandergesetzt haben. Für die Beobachtung von Bildungsprozessen liegen hingegen Studien vor, in denen ein ausgearbeitetes empirisches Modell entwickelt wurde. In einem ersten Schritt werden daher jetzt die unterschiedlichen Zugänge zu Lernen und Bildung diskutiert, bevor einige grundlegende Aspekte biographischen Lernens ausgeführt werden. Diese Überlegungen liegen dem entwickelten Analysemodell zugrunde, welches im zweiten Teil des Kapitels vorgestellt wird.

\section{Lernen und Bildung: zwei Seiten eines Zusammenhangs}

Nohl verweist auf die von Marotzki getroffene Unterscheidung, in der „Lernen bekanntlich als die Mehrung von Wissen innerhalb gegebener Schemata und Bildung als die Transformation von Lernschemata defi- 
niert wird“ (Nohl 2005: 169). Ecarius schließt sich dieser Unterscheidung an, wie Marotzki orientiert sie sich an der von Bateson entwickelten Unterscheidung von Lernstufen. ${ }^{1}$ Biographisches Lernen wäre auf der zweiten Ebene anzusiedeln: Diese Ebene „bezeichnet Bateson als ,Deutero-Lernen', als eine Veränderung im Lernen des bisher Gelernten. Es bezeichnet sowohl die korrigierende Veränderung der Auswahl von Alternativen als auch eine Änderung in der Art und Weise, wie ausgewählt wird. Lernen auf der zweiten Ebene ist die Fähigkeit, innerhalb schon Bekanntem Neues oder anderes auszusuchen, die bisherigen Schritte zu verbessern oder zu verändern. Auch schließt es ein, dass die Art der Auswahl korrigiert werden kann“ (Ecarius 1998: 139ff). Beide unterscheiden also deutlich zwischen Lern- und Bildungsprozessen. Schulze übernimmt eine solche Unterscheidung, wenn er dem Bildungsbegriff die Unterschlagung der selbstbestimmten Auseinandersetzung eines Individuums mit seiner Umwelt vorwirft:

„Im Begriff der Bildung wird die Vorstellung eines von pädagogischen Einwirkungen unabhängigen Lernens, einer selbsttätigen und selbstbestimmten Auseinandersetzung des menschlichen Individuums mit seiner Umwelt aufgenommen, aber nicht als Voraussetzung der Erziehung, sondern als ihr Ergebnis“( Schulze 1993a: 242).

Erziehung und Bildung sind in einer solchen Konzeption für die Herausbildung einer aktiven Auseinandersetzung notwendig, nicht umgekehrt. Mit seiner Forderung, dass sich die erziehungswissenschaftliche Biographieforschung ,zuerst und vor allem den Vorgängen und Bedingungen des Lernens, der Entfaltung einer Biographie als Lerngeschichte und Bildungsprozess" widmen sollte (ebd. 1999: 51), impliziert er ein zweistufiges Verhältnis, welches Lernen als konstitutiven Bestandteil der Biographie und Bildung als möglichen Teil einer Biographie ansieht. Auch Alheit und Dausien sehen das Verhältnis zwischen Lern- und Bildungsbegriffe als prinzipiell zweistufig. Der von Alheit eingeführte Begriff der Biographizität bezieht sich auf potentielle Bildungsprozesse eines Subjekts. Biographizität meint „die Fähigkeit, moderne Wissensbestände an biographische Sinnresourcen anzuschließen und sich [das

1 Insgesamt unterscheidet Ecarius mit Rückgriff auf Bateson drei Ebenen: Die Ebene $\mathrm{O}$ ist „die elementarste Form des Lernens. [...] [Sie bezeichnet] das Erlernen von Begriffen oder Gegenständen. Hier wird noch keine Korrektur in der Wahrnehmung von Gegenständen wie auch in der Art des Lernens vorgenommen. Auf der Ebene I werden „Korrekturen in der Auswahl innerhalb einer Anzahl von Alternativen vorgenommen“ (Ecarius 1998: 139f). 
Subjekt] mit diesem Wissen neu zu assoziieren“"(Alheit 1990: 327). Biographizität verweist auf die stattfindenden Strukturveränderungen:

„der Übergang in eine neue Qualität des Selbst- und Weltbezuges - ein Prozess, der weder das lernende Subjekt noch den umgebenden strukturellen Kontext unverändert lässt. M.a.W.: Es handelt sich um transitorische Bildungsprozesse“ (Alheit 1995: 299).

Lernprozesse finden häufig quasi nebenher statt, laufen sozusagen mit. Zudem werden durch solche mitlaufenden Lernprozesse ,nicht nur einzelne Erfahrungselemente als Bestandteile der sozialen Welt angeeignet, sondern auch das ,Aneignungssystem“ selbst entwickelt. Es geht also um die Herausbildung übergeordneter, generativer Handlungs- und Wissensstrukturen, die Marotzki z.B. Selbst- und Weltreferenzen nennt. Diese Erfahrungsprozesse bilden den ,biographischen Wissensvorrat", innerhalb dessen wir uns bei unseren Handlungen und Reflexionen bewegen. Dabei haben wir prinzipiell die Möglichkeit, uns große Teile dieses präreflexiven Wissens zu vergegenwärtigen, beispielsweise in Krisensituationen, es explizit zu bearbeiten und u.U. auch Strukturen der ganzen Landschaft zu verändern. Solche reflexiven Prozesse können als Momente von Selbst-Bildung interpretiert werden“. (Alheit/Dausien 2002: 587ff.). ${ }^{2}$ Werden durch Lernprozesse also Selbst- und Weltreferenzen entwickelt, können diese durch Bildungsprozesse transfomiert werden. ${ }^{3}$

2 Von Felden weist allerdings zu Recht darauf hin, dass eine genauere Bestimmung von Lernen bzw. Bildung bei Alheit nicht erfolgt (vgl. von Felden 2003: 78).

3 Dass eine solche Unterscheidung zwischen Lernen und Bildung nicht immer vorgenommen wird, zeigt sich z.B. auch bei Huck, der davon spricht, dass Lernen es ermöglicht, ein ,höheres Niveau an Erkenntnis über die individuellen Beziehungen zur Welt zu erreichen“ (Huck 2005: 21, Herv. M.M.). Hier wird zumindest implizit Lernen als Weg zu einem normativen Erkenntnisziel konzipiert, und nicht als ein anderes Niveau an Erkenntnis. Hormel und Scherr machen hingegen auf die Notwendigkeit aufmerksam, in der Analyse von Migrationsprozessen zwischen Bildung und Lernen zu unterscheiden: „Migrationsprozesse implizieren eine doppelte Lernherausforderung: Einwanderer sind veranlasst, sich auf die Lebensbedingungen in der Aufnahmegesellschaft einzurichten und Einheimische zur Auseinandersetzung mit den Prozessen des sozialen und kulturellen Wandels, die durch Migration mit bedingt sind oder durch die Anwesenheit von Migrant(inn)en in besonderer Weise sichtbar werden. Folglich stellen Migrationsprozesse vielfältige Lernanlässe und Lernangelegenheiten bereit, die keineswegs durchgängig in einem empathischen Sinn als Bildung, sondern nüchterner als pragmatischer Wissens- und Kompetenzerwerb $\mathrm{zu}$ beschreiben sind“" (Hormel/Scherr 2005: 296). 
Die empirische Reformulierung des Bildungsbegriffs ist insbesondere durch Marotzki und Koller erfolgt. Marotzkis unternimmt in seinem ,Entwurf einer strukturalen Bildungstheorie‘ von 1990 (vgl. auch Marotzki 1999) den Versuch, auf Basis von sowohl philosophischer Tradition wie Jean-Paul Sartre als auch biographieanalytischen Implikationen Fritz Schützes den Bildungsbegriff für die Biographieanalyse nutzbar zu machen. Bildungsprozesse stellen für ihn „Lernprozesse besonderer Qualität“" (Marotzki 1990: 224) dar, die notwendigerweise zu einer Modalisierung, im Schütz'schen Sinne einer Wandlung von Selbst- und Weltreferenzen führen (ebd.). Es findet quasi eine vollständige Transformation statt, im Unterschied zum Lernbegriff, in dem für Marotzki ,nur' Veränderungen stattfinden, ohne tatsächliche eine Wandlung nach sich zu ziehen: „Bildungsprozesse beziehen sich als Lernprozesse auf die Interpunktionsprinzipien von Erfahrung; einen Sachverhalt, den ich im weiteren Gang der Arbeit als Verarbeitungsmodus von Erfahrung bezeichnen werden. Damit beziehen sie sich auf die Grundprinzipien der jeweiligen Welt- und Selbstauslegung des Subjektes“ (Marotzki 1990: 52). ${ }^{4}$ Bildung wird als bewusste und reflexive Auseinandersetzung mit den Grundlagen der eigenen Selbst- und Weltkonstruktionen konzipiert. Mit einer solchen Konzeption reformuliert Marotzki den klassischen Bildungsbegriff biographietheoretisch, wie er in der Regel unter Rekurs auf Humboldt und die Weiterentwicklung bei Klafki (z.B. 1993) verstanden wird und wie er auch zum Beispiel von Wagner 1995 nochmals aktualisiert wurde. Wie dieser verweist er auf die Möglichkeit des Subjekts zur Selbstreflexivität und zur Bildsamkeit über die Lebensspanne hinweg, verbunden mit dem Wissen um die prinzipielle Unabgeschlossenheit von Bildungsprozessen, und auf die nicht-umkehrbare Veränderung durch Bildungsprozesse:

„Wir gehen in unserer Selbstbildung zwar von uns selbst und unseren Erfahrungen und Vorstellungen als dem uns schon Bekannten aus, wir kehren aber nicht zu einem unserer Bildung vorgegebenen Ursprung zurück, sondern werden im Bildungsprozess andere, als wir zuvor waren, ohne dass es für unsere frühere Identität und spätere Andersheit irgendeine noch frühere Identität gäbe, bei der wir uns vergewissern, Zuflucht, Sicherheit und Schutz suchen könnten“ (Benner 1990: 20).

Anders als Klafki bezieht Marotzki seine Konzeption nicht zwangsläufig auf formelle, institutionalisierte Bildung. Zudem begreift er den Bil-

4 Marotzki bezieht sich in seiner Konzeption von Bildungsprozessen auf Schütze, der als typische Wandlungsprozesse Konversionserfahrungen anführt (vgl. ebd. 1990:128). 
dungsbegriff ausdrücklich strukturtheoretisch und wehrt sich gegen eine inhaltliche Bestimmung und deren normativen Charakter (vgl. Marotzki 1990: 42).

Hans-Christoph Koller hat 1999 einen zweiten Entwurf für eine biographietheoretische Neuformulierung des Bildungsbegriffs vorgelegt (Koller 1999, vgl. auch 2002a), welchen er als Zuspitzung der These Marotzkis versteht, dass ,neue gesellschaftliche Problemlagen aufgrund ihrer Komplexität solche höherstufigen Bildungsprozesse in zunehmendem Maße erforderlich machen, wenn die damit verbundenen Herausforderungen bewältigt werden sollen“" (ebd. 1993: 81). Er entwickelt mit Rückgriff auf Lyotard ein Modell von Bildung, das der von Lyotard analysierten permanenten Möglichkeit eines Widerstreits von Diskursen (aufgrund der Pluralität von Diskursen in postmodernen Gesellschaften) auf doppelte Weise gerecht werden soll:

„Erstens gilt es in skeptischer Hinsicht, den Widerstreit als solchen anzuerkennen, und d.h. vor allem: anzuerkennen, dass es für Konflikte vom Typ des Widerstreits keine gerechten Lösungen (im Sinne einer universal gültigen Norm) geben kann“. Häufig jedoch wird ein Widerstreit gar nicht erst artikuliert und damit virulent, „weil eine der beiden Konfliktparteien nicht über die Möglichkeiten verfügt, ihr Anliegen in der jeweils vorherrschenden Diskursart zur Sprache zu bringen. [...] Bildung [...] besteht [...] deshalb zweitens in innovativer Hinsicht in dem Versuch, neue Sprachspiele oder Diskursarten hervorzubringen, in denen das artikuliert werden kann, was bislang nicht zur Sprache kommen konnte“ (Koller 2002a: 95f, Herv. d. V.).

Dass auch die Analyse von Bildungsprozessen gerade für die Geschlechterforschung durchaus fruchtbar sein kann, zeigt die Studie von Heide von Felden (von Felden 2002; von Felden 2003). Sie führt biographische Interviews mit Frauen aus dem Studiengang ,Frauenstudien“. „Der Zusammenhang von Bildung und Geschlecht liegt darin, Geschlecht in seiner kulturellen Konstruiertheit zu betrachten und Bildung als Form kultureller Konstruktionen zu sehen“" (von Felden 2003: 121). Auch sie unterscheidet zwischen Lernen einerseits und Bildung im Sinne Marotzkis andererseits und sieht die Möglichkeit, eben weil Bildung die kulturelle Ordnung repräsentiert, durch Bildung geschlechtsspezifische Zuschreibungen zu verändern. Gerade ein Kontext wie der Studiengang „Frauenstudien“, der Geschlecht zum Thema macht, bietet Potential für „Umdeutungs- und Verschiebungsvorgänge“ (ebd.: 244). Solche Konstruktionsprozesse sind wesentlich von „der Erfahrungsaufschichtung, dem Selbst- und Weltbild und der Gegenwartsperspektive der jeweiligen Person abhängig“ (ebd.: 242f). Auch Koller versucht in einem Aufsatz 
(Koch/Koller 1999) sein Bildungskonzept auf Geschlechterverhältnisse zu beziehen. Für ihn kommt es

„erstens darauf an, anzuerkennen, dass es eine Vielzahl widerstreitender Vorstellungen darüber gibt, was ,weiblich` bzw. ,männlich“ ist. Und zweitens geht es darum, Möglichkeiten dafür zu schaffen oder offenzuhalten, dass Heranwachsende neue, vom Bisherigen abweichende Formulierungen oder Inszenierungen ihrer Geschlechterrolle (er)finden können“ (ebd.: 247).

Im anschließenden schriftlichen Streitgespräch mit Martina Koch wird von ihr jedoch vor allem die „Unvermeidbarkeit des Widerstreits“ kritisch hinterfragt:

„Bildung erfüllte sich dann noch nicht im Hervorbringen widerständiger, nicht-subsumierbarer und neuer Sätze und Satzverkettungen, sondern in einem interaktiven Geschehen, in dem das Alternieren von Erfindung, Erneuerung und Wiederherstellung von Sprechweisen auf den Anderen hin kodifiziert bleibt und zugleich die Gerechtigkeitsfrage aufnimmt“ (ebd.: 265).

Bei dieser kurzen Skizzierung der beiden Konzepte, Bildungsprozesse biographietheoretisch zu fassen und sie empirischen Analysen zugänglich zu machen, fallen einige Gemeinsamkeiten auf. Beide sehen die Bildungsprozesse als notwendige Kompetenz von Subjekten, um in modernen Lerngesellschaften (vgl. Marotzki 1990:30), Handlungsfähigkeit zu behalten bzw. zu erwerben. Für beide stellen Bildungsprozesse auch keine Selbstverständlichkeit dar. Zwar wird eine solche Bildungskompetenz immer wichtiger, nicht alle Subjekte aber können sie erwerben. ${ }^{5}$ Die Pädagogik hat sich daher zu fragen, in welcher Weise sie solche Prozesse unterstützend begleiten kann bzw. sie evozieren kann. ${ }^{6}$ Beide

5 Vgl. dazu auch von Felden, die kritisch vor einem Zirkelschluss warnt: „Das Verfahren prüft eher, ob das Denken von Menschen postmodern ist und nicht, wie sich das Denken heutiger Menschen darstellt, was eigentlich als Ziel empirischer Untersuchungen gilt" (von Felden 2003: 73).

6 Koller macht dies explizit mit Bezug auf Bourdieus Gesellschaftstheorie und der Definition von Bildung als Habitustransformation: „In diesem Sinne wäre Bildung angesichts von transnationaler Migration mit und gegen Bourdieu als Transformation des jeweiligen Habitus zu begreifen, soweit sich dieser unter veränderten gesellschaftlichen Bedingungen als nicht mehr angemessen erweist. Die Frage lautet dann, wie der Prozess solcher Habitustransformationen und die Bedingungen, unter denen sie zustande kommen, genauer beschrieben werden können. Auf welche Ressourcen können Individuen zurückgreifen, wenn sie mit Situationen konfrontiert werden, für die ihre Interpretations- und Handlungsschemata nicht mehr ausreichen?“ (Koller 2002b: 186). Die Einführung des Habituskonzepts öffnet die kognitive Ausrichtung des Bildungsbegriffs und 
sehen als Voraussetzung für einen erfolgten Bildungsprozess zudem einen „qualitativen Sprung“ (Marotzki 1990: 131) bzw. die Entwicklung eines dritten Diskurses (Koller). Beide können damit auch als Ergebnis einer biographischen Analyse das Vorhandensein oder eben das NichtVorhandensein eines biographischen Bildungsprozesses konstatieren.

Das Konzept biographischen Lernens und die biographische Bildungskonzeption beziehen sich letztlich auf zwei verschiedene Aspekte des Verhältnisses des Subjekts zu der Welt und dessen Veränderung. Während sich der Lernbegriff auf Veränderungen bezieht, fokussieren biographische Bildungsprozesse Wandlungsprozesse. Biographisches Lernen ist als grundlegendes Konstruktionsprinzip von Biographien zu verstehen, Menschen können nicht ,durchs Leben gehen', ohne Erfahrungen zu machen, diese in spezifischer Weise zu verarbeiten und daraus Haltungen zu entwickeln, mit denen wiederum neue Erfahrungen wahrgenommen und verarbeitet werden. Als biographisches Lernen lässt sich die Art und Weise bezeichnen,

„wie neue Inhalte in eine vorhandene Struktur aufgenommen werden und wie sich dieser Prozess im biographischen Material abzeichnet. Es geht nicht um Veränderungen von Weltanschauungen, sondern um die Verknüpfung von neuen und vorhandenen Inhalten und einer teilweisen Veränderung oder Modifizierung“ (von Felden 2003: 147).

Die Analyse von biographischen Lernprozessen versucht daher gerade solche Fragen zu klären wie: Woran orientieren sich Subjekte? Welche hindernden oder fördernden Bedingungen können analysiert werden? Auf welche Ressourcen können Subjekte zurückgreifen? Lernen ist also einerseits, wie ja auch bei Marotzki und Koller angelegt, in gewisser Weise kleinteiliger als Bildung. Zwar verändern neue Erfahrungen Konstruktionen, dennoch bauen sie auf alten auf, und lassen den Menschen nicht ,als neu' erscheinen. Gleichzeitig erscheint es grundlegender als Bildung. Biographisches Lernen bezieht sich auf ein Verständnis von Lebensgeschichten als Lerngeschichten, so dass Lernen nicht als vorhandene oder fehlende Kompetenz (bei der der Pädagogik die Rolle der Vermittlerin einer solchen Kompetenz zugesprochen bekommt) verstanden wird.

macht auf Bildungsprozesse aufmerksam, die in den Körper eingeschrieben sind und mit dem Körper gemacht werden. 


\section{Lernen in Biographien}

Lernen kann, in Unterscheidung zu einem ,höherstufigen` und nicht zwangsläufigen Bildungsprozess als grundlegendes Konstitutionsprinzip von Biographien gesehen werden. Lernen in Migrationsprozessen wiederum ist keine isolierbare Herausforderung für den Migranten/die Migrantin und lässt sich insofern auch nicht als individuell-personale $\mathrm{Zu}$ mutung an ein isolierbares Subjekt verstehen. Vielmehr sind im Zuge dieses Migrationsprozesses nicht nur Herkunftsfamilien als soziale Bezugsgruppen involviert, auch die ,neue“ soziale Bezugsgruppe, die Ehepartnerin, deren Familie und die in Deutschland lebenden Freunde und Bekannten werden einbezogen. Sie sind Teil des Auseinandersetzungsund Bearbeitungsprozesses und damit Teil des Kontextes, innerhalb dessen sich Formen biographischen Lernens entwickeln. Biographisches Lernen lässt sich hinsichtlich vier verschiedener Dimensionen genauer beschreiben. Diese Dimensionen haben auch für die Entwicklung des Analysemodells eine Rolle gespielt. Insbesondere vier Autor(inn)en haben sich in diesem Zusammenhang intensiver mit biographischem Lernen auseinandergesetzt.

Dazu ist als Erstes Theodor Schulze zu zählen. Er beschäftigt sich seit langem mit Biographien als Lernbiographien. Beim biographischen Lernen geht es ihm

„weniger um das Erlernen von Kenntnissen und Fertigkeiten, mehr um Orientierung in der Umwelt und die Ausbildung von Gefühlen und Interessen, um die Beziehung zu anderen Menschen und um die Einstellung zu bestimmten Sachverhalten oder Ideen, um die Entwicklung von Perspektiven und die Bewältigung von Herausforderungen oder Krisen“ (ebd. 2001: 19).

Schon 1979 vermutete er, dass

„die Beschäftigung mit autobiographischen Materialien einen neuen Zugang $\mathrm{zu}$ einigen wichtigen Bedingungen und Strukturen längerfristigen menschlichen Lernens in unverkürzten Lebenszusammenhängen eröffnet“ (ebd. 1979: 51).

Dies gilt für ihn insbesondere im Verhältnis zur Erziehung, da zwar ,,alle Maßnahmen zur Erziehung [...] Lernen voraus[setzen], aber Lernen auch ohne Erziehung" denkbar ist (ebd. 1993a: 241). Für ihn stellt sich die Frage, wie die von Schleiermacher „Entwicklung der persönlichen Eigentümlichkeit" (in Schulze 1993b: 174) genannte und in der heutigen Diskussion als Entwicklung zur Selbstbestimmung oder der ,Fähigkeit 
zur Selbstverwirklichung' aufgegriffene Aufgabe der Erziehungswissenschaft beschrieben werden kann. ${ }^{7}$ Das bedeutet auch, dass er zuallererst einen theoretischen Blick auf Lebensgeschichten wirft und die Produktion von autobiographischen Äußerungen als lerngeschichtliche Äußerungen begreift. Für ihn steht daher auch die „Biographie als Bildungsprozess“ (ebd.) im Mittelpunkt des Interesses erziehungswissenschaftlicher Biographieforschung.

Auch Peter Alheit und Bettina Dausien sehen den konstitutiven $\mathrm{Zu}-$ sammenhang von Biographie und Lernen. Zum einen ist Lernen für sie der Motor der Entwicklung von Biographien, zum anderen findet Lernen immer in der Biographie statt. Während Schulze den Begriff des lebensgeschichtlichen Lernens in der Auseinandersetzung mit dem curricularen Lernbegriff gewinnt, ist die Perspektive von Alheit und Dausien eine andere. Sie setzten sich im Umkreis der Erwachsenenbildung und dem (politischen) Konzept des lebenslangen Lernens mit dem Lernbegriff auseinander, blicken von der Biographieforschung auf pädagogische Phänomene und fragen, inwiefern biographietheoretische Überlegungen Hinweise auf Lernprozesse geben können. Mit dem Begriff des biographischen Lernens

„meinen [wir] weniger einen empirisch scharf abgrenzbaren Gegenstand - etwa Lernprozesse, die an bestimmte Formen, Orte oder Zeiten gebunden sind als vielmehr eine theoretische Perspektive auf Bildungsprozesse, die im Sinne eines phänomenologischen Lernbegriffs an der lebensgeschichtlichen Perspektive der Lernenden ansetzt“" (Alheit/Dausien 2002: 574). ${ }^{8}$

Insofern fragen sie eher nach den Bedingungen und Möglichkeiten von Lernprozessen als nach dem Vorgang des Lernens selbst, so dass eine

7 Das Verständnis solcher Lernprozesse ist insbesondere dann wichtig, wenn die Erziehungswissenschaft nicht auf die naive Vorstellung, „dass der einzelne Mensch [...] schon allein aus sich und ohne weiteres selbstverständlich zu seiner persönlichen Eigentümlichkeit gelangt“ (Schulze 1993b: 175) vertrauen will. Aufgrund seines theoretischen Ausgangspunkts, den Unterschied zwischen curricularem und lebensgeschichtlichem Lernen herauszuarbeiten und letzteres für die Analyse zugänglich zu machen, entwickelt er den Begriff insbesondere in Abgrenzung zum curricularem Lernen und weist ihm eine eigenständige Bedeutung zu. Curriculares Lernen wird von ihm im "Sinne von vorausgeplantem Lernprozess“ (ebd.: 201) verstanden. Dabei stellt er die beiden Lernformen nicht gegeneinander, vielmehr sind sie konstitutiv aufeinander angewiesen.

8 Für die Weiterentwicklung der Erwachsenenbildung betrachten sie ebenfalls die Ein- und Mitwirkung von Bildungsinstitutionen auf biographische Lernprozesse, eine Diskussion, die nicht notwendigerweise hier nachgezeichnet werden muss (vgl. Alheit/Dausien 2002). 
typologische Unterscheidung von formalen, nicht-formalen und informellen Lernprozessen keinen Sinn mache. Diese Unterscheidung sei eher wichtig im Hinblick auf die sozialen Rahmungen, in denen Lernprozesse stattfinden. Das Verständnis eines biographischen Lernbegriffs beziehen sie aus der Struktur der Biographie als Ort des ,Zusammentreffens“ von Emergenz und Struktur: „Biographische Lernprozesse sind häufig sensible Synchronisationsversuche des Außen- und Innenaspekts. Sie finden gleichsam am Schnittpunkt zwischen Subjekt und Struktur statt" (Alheit 1995: 293). Das bedeutet für sie, besonders die auf das Individuum einwirkenden Strukturen zu betrachten, da diese Grundbedingung für Lernprozesse seien. Dabei greifen biographische Lernprozesse durchaus auf curriculare Lernformen zurück, wenn sie auch anderen Regeln folgen als diese. Bildung als gesellschaftliche Institution formiert und prägt und stellt Modelle für ein mögliches Leben dar (vgl. Alheit/Dausien 2002: 576). Daher sei es notwendig, die in einer Gesellschaft jeweils wirksamen Lebenslaufmodelle zu reflektieren.

„Lebensgeschichtliches Lernen ist also immer an den Kontext einer konkreten Biographie gebunden. Andererseits ist es aber auch die Voraussetzung oder das Medium, in dem biographische Konstruktionen sich überhaupt als reflexive Erfahrungsgestalt herausbilden und verändern können“ (ebd.: 578).

Als letzte hier verwendete Referenz hat sich Jutta Ecarius mit lebensgeschichtlichem Lernen beschäftigt. Sie entwickelt ihr Verständnis in enger Anlehnung an Schulze und an die lerntheoretischen Aussagen von Bateson, die auch für Marotzkis bildungstheoretischen Entwurf einen Bezugspunkt bilden. Auch für sie ist biographisches Lernen für biographische Erzählungen konstitutiv. Die Erfahrungen, die in biographischen Erzählungen offen gelegt und beschrieben werden, ziehen Veränderungen nach sich, die von Ecarius wiederum als Lernen im Sinne von biographischem Lernen verstanden werden. Biographisches Lernen wird von ihr insofern aus zwei Gründen in den Kontext von biographischen (Re-)Konstruktionen gestellt: Zum einen ermöglicht es, in der Analyse die Lernprozesse zu re-konstruieren, die Erfahrungen des Subjekts in seinen verschiedenen sozialen Kontexten nachzuvollziehen und Rückschlüsse auf historische und sozialgeschichtliche Gegebenheiten zu ziehen. Zum anderen werden von den Erzähler(inne)n selbst in der biographischen Stegreiferzählung die für sich selbst relevanten Erfahrungen artikuliert. Für dieses Verständnis der strukturellen Abläufe von Lernprozessen greift sie, wie vorne formuliert, in enger Anlehnung an Marotzki auf die von Bateson entwickelten ,Lernstufen` zurück, von denen insbe- 
sondere die Ebenen I und II in der Lage seien, biographisches Lernen zu verdeutlichen (vgl. Ecarius 1998: 139f.).

\section{Biographisches Lernen als sozial situierte Lernprozesse}

Ein grundlegendes Merkmal biographischen Lernens, welches auch schon die Konzeption der Untersuchungsanlage bestimmte, ist der Umstand, dass es in spezifischen sozialen Rahmungen stattfindet. Ebenso wie Biographien keine rein intersubjektiven Konstruktionen sind, sondern sich in Auseinandersetzung mit Strukturen und gesellschaftlichen Bedingungen entfalten, ist auch biographisches Lernen in diesen Kontexten situiert. Es bezieht sich auf „,bestimmte soziokulturell und historisch bedingte Inhalte und Probleme“" (Schulze 2005: 44). Das bedeutet, dass die Umwelt, historische Ereignisse ebenso wie Umweltwechsel, zum Beispiel durch Migrationsprozesse oder durchlaufene Statuspassagen, Lernprozesse strukturiert. Anstöße für lebensgeschichtliches Lernen kommen immer, so Schulze, „von außen, aus der Umwelt, von anderen Menschen“ (ebd. 1993b: 203). Auch Alheit und Dausien verweisen auf die Sozialität biographischen Lernens. Biographisches Lernen ist eingebunden in Lebenswelten, die unter bestimmten Bedingungen auch als ,Lernumwelten' oder ,Lernmilieus' analysiert werden können. Dies bedeutet, dass die historischen, sozialen und strukturellen Bedingungen, in denen Biographien konstruiert werden und biographisches Lernen stattfindet, als konstitutiv in die Analyse mit einbezogen werden müssen. ${ }^{9}$ Für Susanne Frei und Rachid Frei-Heidarzadeh beispielsweise, dem ersten Paar in meinem Sample, ist diese Kontextualität biographischen Lernens gerade für den Beginn der Beziehung entscheidend: Beide treffen sich an einem ,dritten Ort', also weder in ihrem noch in seinem Herkunftsland, sondern in Sri Lanka. Dieser Ort bietet Susanne einen Anlass und eine Möglichkeit, eigene Positionierungen zu überdenken und sich, zumindest zeitweilig, sowohl sexuell als auch national anders zu verorten, sie stellt sich Rachid zuerst als homosexuelle Schwedin vor. Der dritte Ort hat aber auch, nachdem sich die Beziehung mit Rachid entwickelt hat, insofern Auswirkungen auf die Geschlechterbeziehung, als das Susanne als gatekeeper bezüglich des Wissens über bestehende Geschlechterordnungen in Deutschland fungieren kann. Hier wird eine zweite Dimension der Sozialität biographischen Lernens deutlich, die

9 So bewirken auch, wie Alheit und Dausien 1999 feststellen, institutionelle Lern- und Bildungsangebote mehr als einen reinen Wissenszuwachs. Vielmehr finden dabei auch ,nebenher' sozusagen nicht-intendierte Lernprozesse z.B. hinsichtlich der Milieuverortung oder der Anerkennung biographischer Lebenspläne statt. 
ebenfalls für die Interpretationen relevant ist. Gesellschaftliche Strukturen wirken nicht direkt ein, sondern sind immer sozial vermittelt. ,Das Geschlechterverhältnis der Gesellschaft‘ als abstraktes Modell wirkt ja auch nicht direkt auf Subjekte ein. Vielmehr hat der Partner bzw. die Partnerin eine besondere Bedeutung in biographischen Lernprozessen, grade weil beide Partner(innen) von einer ,Unwissenheit' des Anderen in Bezug auf das Land und Geschlechterordnungen und -modelle ausgehen (können). Diese gatekeeper-Funktion führt insofern, zumindest zu Beginn der Beziehung, zu einer Dominanz der Eigendeutungen gegenüber anderer sozialer Bezugsgruppen. Der Wechsel des Kontexts, die Rückkehr nach Deutschland mit anderen sozialen Bezugsgruppen und anderen strukturellen Voraussetzungen macht wiederum neue Lernprozesse notwendig, da Kontextwechsel die ,Reichweite‘ bzw. die Nachhaltigkeit von Lernprozessen, wie in der Biographie von Susanne, entscheidend begrenzen können. ${ }^{10}$

\section{Biographisches Lernen als Antwort auf Probleme oder Herausforderungen}

Schulzes Hinweis auf den Anstoß von außen, den biographisches Lernen braucht, verdeutlicht einen weiteren Aspekt. Biographisches Lernen braucht, neben der sozialen Kontexualität, einen Anlass. Schulze sieht Lernprozesse als Folge der Konfrontation mit einer neuartigen Aufgabe, einem ungelösten Problem oder einer überraschenden Erfahrung, also im weitesten Sinne als Folge von Differenzerfahrungen. Das kann zum einen durch sich wandelnde Interaktionsbezüge geschehen, diese machen häufig, wie auch Ecarius feststellt, eine Erweiterung oder Korrektur des bisher Gelernten erforderlich (Ecarius 1998: 97). Solche ,Konfrontationen' finden zwar sicherlich im Zuge von größeren Veränderungen wie der Veränderung des sozialen Rahmens, dem Beginn oder Ende einer Statuspassage etc. statt, dennoch ist lebensgeschichtliches Lernen nicht an solche ,großen“ Ereignisse gebunden. Auch ,kleinere' Eindrücke, Wahrnehmungen etc. können lebensgeschichtliches Lernen zur Folge haben (Schulze 2001: 19). Biographische Lernprozesse, wie sie auch in dieser Untersuchung verstanden werden, entstehen in der Akkumulation

10 Diese Sozialität biographischer Lernprozesse bedeutet nach Schulze aber auch, dass die Subjekte durch ihre Lernprozesse, durch ihr Handeln und das Scheitern oder Gelingen ihrer Pläne, durch Umstrukturierungen und Neu-Orientierungen auf ihre Umwelt einwirken und sie dadurch verändern: „Nicht nur Konferenzen, Gesetze und Erlasse verändern die gesellschaftliche Wirklichkeit, auch Umwertungen und Neuorientierungen“" (Schulze 1999: 51). 
vieler einzelner Lernprozesse (vgl. Schulze 2005: 46). In der Analyse der Biographien binationaler Paare hat sich zudem gezeigt, dass sich eine dominante Form biographischen Lernens analysieren lässt, die sich anhand solcher ,kleinerer' Lernprozesse entwickelt. Die spezifische Art und Weise der Verarbeitung von Erfahrungen, die die Form biographischen Lernens bestimmt, findet sich dann, wenn die Entwicklungen von Lebensperspektiven und Plänen auf Widerstand stoßen und mit den realen Bedingungen und Möglichkeiten in Konflikt geraten. Solche Anlässe oder Anstöße müssen nicht zwangsläufig schon zu diesem Zeitpunkt als solche reflektiert werden. Vielmehr können solche Momente auch erst sehr viel später im Leben als für Lernprozesse relevante Erlebnisse verstanden und eingeordnet werden, so dass sie (erst) in der Darstellung der Lebensgeschichte als solche deutlich werden, so zum Beispiel in einer biographischen Erzählung als solche gekennzeichnet oder erst in der Interpretation erkennbar werden. Im Zuge der Entwicklung des Analysemodells wurde dieser Aspekt wesentlicher Teil des Interpretationszugangs. Lässt sich schon der Beginn der Beziehung als ,Lernanlass“ bezeichnen, ließe sich nach der Analyse des Paarhorizonts jeweils ein paarspezifisches Thema benennen, welches in seinen Folgen bearbeitet wurde bzw. werden musste. Dieses Thema und dessen Kontextualisierung bot Anlass für Lernprozesse, sowohl in emanzipativer Form, wie bei Susanne Frei und Rachid Frei-Heidarzadeh, oder auch in reethnisierender Form, wie bei Nicole und Carlos Ortega Garcia, dem zweiten Paar meines Samples.

\section{Biographisches Lernen als eigensinniges Lernen}

Neben der Kontextualisierung und der Notwendigkeit von Lernanlässen ist ein weiterer Aspekt biographischen Lernens implizit angesprochen worden: Einerseits ist biographisches Lernen interaktiv und strukturiert und folgt dabei einer, individuellen Logik', die durch die jeweils besondere biographisch aufgeschichtete Erfahrungsstruktur erzeugt wird. Auch biographische Lernprozesse folgen so dem Prinzip der nach außen hin offenen Selbstreferentialität: „Lebensgeschichtliches Lernen [...] organisiert [sich selbst], indem jede Erfahrung schon auf Vorerfahrung zurückgreift und weitere Erfahrungen nach sich zieht" (Schulze 1993b: 205). ${ }^{11}$ Diese Selbstorganisation bedeutet jedoch nicht die Abschottung

11 Hier wird noch eine weitere Besonderheit biographischen Lernens angesprochen. Die subjektlogische Verarbeitung von Lernanlässen bedeutet auch, dass biographisches Lernens diskontinuierliches Lernen ist: „Lebensgeschichtliches Lernen springt gleichsam von Situation zu Situation, es verschmilzt weit zurückliegende Ereignisse mit späteren und gegenwär- 
von ,außen', sondern betont vielmehr den subjektlogischen Konstruktionscharakter solcher Prozesse. ${ }^{12}$ Auch Ecarius betont den individuellen Charakter biographischer Lernprozesse, wenn sie „Lernen als innere Erfahrung, [als] Ausbildung und Aufrechterhaltung persönlicher Identität“ (Ecarius 1998: 134) konzeptualisiert. Ecarius spricht insofern auch dann von biographischem Lernen, wenn die Erfahrungen für das Selbstkonzept des Subjekts relevant werden (vgl. Ecarius 1999: 97). Biographisches Lernen ist daher vor allem mit Blick auf die Gesamtbiographie zu rekonstruieren. Eine Analyse über den Gesamtverlauf einer Lebensgeschichte kann zeigen, welche Bedeutung Lernprozesse für die Gestaltung und Entscheidung des Subjektes haben und wie diese die innere Logik einer Biographie strukturieren und durch sie strukturiert werden. Die Bedeutung der Beziehung, die für die interviewten Paare in ihrer Funktion als Katalysator für die Verwirklichung von Lebensmodellen liegt, zeigt zudem die Möglichkeit, bisher nicht realisierbare Erwartungen gewissermaßen zu aktivieren und die Beziehung so als Gelegenheitsstruktur zu verstehen. ${ }^{13}$ Die Themen der Beziehungen wiederum folgen aus der Konstruktion des Paarhorizonts. Die Erfahrungen, die zum Beispiel in der Biographie Nicole Ortega Garcias als Anlass für biographische Lernprozesse zu sehen sind, sind nur vor dem Hintergrund ihres Wunsches nach einer starken Beziehung und einem starken Partner zu verstehen. Für andere würde anderes relevant, für Pablo Marmani Rodriguez ist die eigene Kindheitserfahrung des abwesenden Vaters Anlass, sein Verhältnis zu seinen Kindern und sein Engagement zu überdenken und sich, trotz seines Selbstverständnisses als Familienernährer, intensiv um sie zu kümmern und Elternzeit zu übernehmen.

\section{Biographisches Lernen als nicht zu beobachtendes Lernen}

Die Aspekte der Kontextualität, der Lernanlässe und der Eigensinnigkeit konturieren gewissermaßen inhaltlich biographische Lernprozesse. Daneben spielt bei der Interpretation der Biographien noch ein weiterer Aspekt eine Rolle. Biographisches Lernen als solches ist nicht beobachtbar. In der biographischen Erzählung lassen sich zwar nicht die

tigen, und es verbindet scheinbar weit zurückliegende und verschiedenartige Inhalte zu einem bedeutungsvollen Komplex“ (Schulze 1993b: 206).

12 Damit ist aber noch nichts über die Möglichkeit der Selbststeuerung gesagt, vielmehr können stattfindende Lernprozesse den Lernenden überraschen (vgl. Alheit/Dausien 1999).

13 Gelegenheitsstrukturen zeichnen sich dadurch aus, dass sie also solche wahrgenommen und damit erst zu einer gemacht werden, sie werden nicht aktiv ,gesucht', sondern aufgegriffen. 
Lernprozesse ,in vivo', also in den Interaktionen beobachten, analysieren lassen sich aber ,die Wahrnehmung und Verarbeitung biographischen Lernens“ (Ecarius 1998: 137). Denn in der Logik, in der bestimmte Ereignisse und Erinnerungen zusammengefügt werden, lassen sich die für das Subjekt und seine Entwicklung relevanten Lernprozesse analysieren. Auch Schulze sieht insbesondere in der Möglichkeit der (Re-) Konstruktion den Vorteil der Analyse von Lernprozessen: „Lernen kann nicht direkt gesehen werden. Wir wissen, dass wir etwas gelernt haben, aber nicht wie. Das Lernen verschwindet im Gelernten" (Schulze 2001: 18). In den biographischen Erzählungen aber werden Erlebnisse und Erfahrungen thematisiert, die in der Reflexion und Rückschau zu bedeutsamen Ereignissen verwoben und damit der Analyse von lebensgeschichtlichen Lernprozessen zugänglich gemacht werden können. Schulze geht noch weiter, indem er auf Analysemöglichkeiten hinweist, die durch diese Beobachtung entstehen:

„Biographisch relevante Lernprozesse entstehen in der Akkumulation vieler einzelner Lernprozesse. Sie etablieren eine neue Potenz oder Ebene des Lernens. Sie sind in einer Autobiographie in der Regel präsent, nicht in der Beschreibung einzelner Lernvorgänge und auch nicht in kleinen Geschichten, sondern in der wiederkehrenden Thematisierung eines Erfahrungszusammenhangs" (ebd. 2005: 46).

Diesem Verständnis folgt auch das für die Arbeit entwickelte Interpretationsmodell. Das Lernen an sich kann in einer biographischen Rekonstruktion nicht erfasst werden. Dazu wären, wenn überhaupt, psychologische Laborversuche notwendig, um den ,Akt des Lernens' erfassen zu können. Einen solchen Anspruch können und sollen biographische Analysen nicht haben. Vielmehr geht es um die Verarbeitungsprozesse und die biographische Einbettung der Lernprozesse.

\section{Das heuristische Analysemodell: Formen biographischen Lernens in sozialen Kontexten}

Diese vier Dimensionen biographischen Lernens, die soziale Situierung, der Lernanlass, die Eigensinnigkeit und die fehlende Beobachtungsmöglichkeit sind in dem Analysemodell aufgenommen. Sie stellen die Rahmenbedingungen, innerhalb derer sich Modifikationsprozesse von biographisch gebundenen gender-Konstruktionen und Konstruktionen natio-ethno-kultureller Zugehörigkeit rekonstruieren lassen. Als zentrale Fragestellung für die Entwicklung des Modells lässt sich nach dem bis- 
her Gesagten Folgendes formulieren: Welche Bedeutung und welche Funktion bekommen die beiden Kategorien gender und natio-ethnokulturelle Zugehörigkeit im Prozess der biographischen Bearbeitung zugesprochen, in welchem Verhältnis stehen diese beiden Kategorien zueinander, inwiefern verändert sich dieses Verhältnis im Laufe der Biographien, und was sagen solche Bearbeitungsformen sozialer Zumutungen aus über Modifikationen, aber auch über Verfestigungen von Geschlechterverhältnissen? Beide Analysekategorien, gender und natioethno-kulturelle Zugehörigkeit, sind, wie gezeigt wurde, nicht unabhängig voneinander zu sehen, sondern werden in der biographischen Konstruktion in ein spezifisches Verhältnis zueinander gesetzt und sollten daher auch in dieser Wechselwirkung analysiert werden. Für die empirische Analyse müssen diese übergeordneten Fragen jedoch insofern heruntergebrochen und handhabbar gemacht werden, als dass sich das Verhältnis ja jeweils in konkreten Kontexten und in der Bearbeitung konkreter Herausforderungen entwickelt hat und in solchen Kontexten auch analysiert werden kann. Gender und natio-ethno-kulturelle Zugehörigkeit werden ganz konkret wirksam, sie sind in einer Biographie nicht als abstrakte Differenzkategorien bedeutsam. Während also im empirischen Teil der Arbeit verschiedene Formen biographischen Lernens rekonstruiert und analysiert werden, wird dadurch gleichzeitig der Abschluss der Arbeit vorbereitet, indem die von mir empirisch gewonnenen Erkenntnisse über Formen biographischen Lernens im Hinblick auf die übergeordneten Fragen nach der Konstitution neuer sozialer Wirklichkeiten und dem Umgang von Subjekten mit gesellschaftlichen Anforderungen diskutiert werden.

Was ist also mit Formen biographischen Lernens gemeint? Das Konzept der Formen biographischen Lernens bezieht sich auf die biographiespezifischen Konfigurationen der beiden Kategorien gender und natio-ethno-kulturelle Zugehörigkeit, die sich analysieren und beschreiben lassen. Das spezifische Verhältnis zwischen diesen beiden Kategorien, das sich über den Verlauf der Biographie und der Bearbeitungen hinweg entwickelt, wird als Form biographischen Lernens bezeichnet, also die Art und Weise, wie in der biographischen Rekonstruktion auf die beiden Kategorien Bezug genommen wird. Lernen lässt sich, wie Schulze ja nochmals deutlich macht (vgl. v.a. 2001), nicht in der Lernsituation beobachten. Aus Veränderungen lassen sich aber Rückschlüsse auf Lernprozesse ziehen. Die Veränderungen, die daher als biographisches Lernen erfasst werden, drücken sich als Veränderungen der subjektiven Positionierung und der analysierbaren Handlungs- und Orientierungsmuster aus. Damit ist zum einen das jeweilige Verhältnis angesprochen, in 
dem sich biographische Konstruktionen von gender und Konstruktionen von natio-ethno-kultureller Zugehörigkeit zueinander befinden. Welcher Art, hierarchisch oder gleichrangig, ist das Verhältnis, inwiefern wirken sich Veränderungen im Verständnis der einen Kategorie auch auf die andere Kategorie aus, werden beide Kategorien als ver- und aushandelbar verstanden und wie verändern sich die Beziehungen, in die die Kategorien im Laufe der Biographiegestaltung gesetzt werden?

Neben dieser formalen Dimension wird auch die substantielle Dimension angesprochen, die individuelle Form der Bearbeitung biographischer Anforderungen: Wie werden biographische Zumutungen integriert, und wie verändern sich in diesem Prozess die Bedeutungszuschreibungen der natio-ethno-kulturellen Zugehörigkeit des/der Partners/Partnerin? So bleiben viele der im Vorfeld entstandenen Imaginationen auf der Ebene der Erwartungen, sie lassen sich nicht realisieren bzw. stellen einen Kontrast zu tatsächlichen Beziehungswirklichkeiten dar. Zeigen lässt sich ein solcher Verlauf zum Beispiel an der Interpretation der Interviews von Nicole und Carlos Ortega Garcia. Nicole erhofft sich von der Beziehung die Stabilisierung einer traditionellen Geschlechterbeziehung. Interessant ist nun, wie sie mit der Beziehungswirklichkeit, die sie als Haupternährerin fordert, umgeht, die doch anders ist als die Beziehungserwartung, die eine klassische Geschlechterbeziehung favorisierte. Eine dafür prägnante Situation wird von ihr geschildert, wenn sie ihrem Mann während des Restaurantbesuches unter dem Tisch den Geldbeutel zusteckt, damit er bezahlen kann. Der Lernanlass stellt in diesem Fall die für Nicole ungewollte Arbeitsteilung und das daraus resultierende finanzielle Ungleichgewicht dar, ihre Form der Bearbeitung lässt sich, wie sich in der Interpretation zeigen wird, als Normalisierungsmanagement bezeichnen.

Solche sozusagen kleinteiligeren Formen biographischen Lernens, konkrete Situationen, in denen Bearbeitungsformen deutlich werden, lassen sich an vielen Stellen der Biographieerzählungen finden. Mein Blick liegt auf der Gesamtgestalt einer solchen Form biographischen Lernens, wie sie sich über den gesamten Verlauf der Biographieerzählung hinweg darstellt (vgl. zur Gesamtgestalt eines narrativen Interviews auch Schütze 1984). Die Aufmerksamkeitsrichtung liegt also auf der spezifischen Verknüpfung solcher Formen zu einer „,biographischen Gesamtformung“ (ebd.: 104). In den Erzählungen der Paare lässt sich jeweils eine dominante Form biographischen Lernens identifizieren, die gewissermaßen als Kernkategorie die Erfahrungen und Veränderungen integriert und die beobachtbaren Veränderungen erfasst. Diese dominante Form biographischen Lernens bezieht sich jedoch nicht nur auf eine Biographie. Vielmehr werden darin die Biographien beider Part- 
ner(innen) integriert. Das bedeutet, dass die jeweils herausgearbeitete Form biographischen Lernens das Verhältnis der beiden Biographien zueinander und die darin stattgefundenen prozessualen Modifikationen und Verarbeitungen beinhaltet.

\section{Dimension der Bearbeitung}

Die Kontextverbundenheit biographischen Lernens spielt in zweierlei Hinsicht eine Rolle. Zum einen finden solche Bearbeitungen, wie oben bei Nicole beschrieben, in konkreten Situationen statt. Zum anderen lassen sich größere inhaltliche Kontexte unterscheiden, in denen diese Bearbeitungsformen wirksam sind. Als interessant im Hinblick auf die besondere Situation binationaler Paare hat sich in den Interpretationen zum einen die Verflechtung der beiden Kategorien in Bezug auf die Entscheidung füreinander und die Inszenierung der Paarbeziehung herausgestellt. Die Entscheidung für einen bestimmen Menschen und der Beginn einer Beziehung stecken voller Imaginationen und Erwartungen hinsichtlich der Erfüllung von Lebensentwürfen und Wünschen. Platon formulierte, dass man Liebe beschreiben kann als die „Liebe zu etwas, was man noch nicht hat, also ein Begehren“ (in Burkart 1998: 17). Diesem Begehren wird in den Analysen nachgegangen, nicht jedoch im Hinblick auf die Illusion oder die Tatsache der Liebe, sondern vielmehr im Hinblick auf eine biographische Anschlussfähigkeit der Beziehung und der darin stattfindenden Konstruktionen und (Re-)Konstruktionen des ,Anderen“. Zum anderen folgen aus diesem Beziehungshorizont je paarspezifische, thematische Bearbeitungsfelder. Je nach Aufbau des Horizontes, je nach der Bedeutung, die das female breadwinner Modell für das Paar hat und je nach Lebensentwurf ist das Paar mit unterschiedlichen Schwierigkeiten und Bearbeitungsnotwendigkeiten konfrontiert. Daher stehen zwei Kontexte bzw. Dimensionen im Mittelpunkt der Analyse: zum einen die Dimension „Beziehungshorizont der Paare“, zum anderen die Dimension der „Bearbeitungsfelder“. In beiden Dimensionen werden Konstruktionen und Relationen dieser Konstruktionen verhandelt. Während sich jedoch in der ersten Dimension sozusagen als Ergebnis dieser Verhandlungen ein Beziehungshorizont zwischen den Paaren aufspannt, fokussiert die zweite Dimension verschiedene Bearbeitungsfelder, die im Verlauf der Beziehungsbiographie relevant werden. Die erste Dimension stellt insofern eine Voraussetzung in der Form dar, dass sich die Bearbeitungsfelder aus dem jeweiligen Paarhorizont ergeben und die Offenheit oder Geschlossenheit des Paarhorizonts die Art und Weise der Bearbeitungen strukturiert. 


\section{Der Beziehungshorizont der Paare}

Kade und Seitter nehmen 1996 in ihrer Studie zu Bildungswelten Erwachsener eine Idee aus dem dramaturgischen Theater auf, um ihre Vorstellung von Erwachsenenbildung als Möglichkeitsraum zu präzisieren. Dabei verstehen sie die Erwachsenenbildung als Angebot, d.h. als ,Möglichkeitsraum' und die Lernprozesse der Teilnehmenden als Antwort und Rekonstruktion dieses Angebotes und damit als Ausgestaltung des Möglichkeitsraumes Erwachsenenbildung. Einen solchen Möglichkeitsraum stellt auch eine Beziehung dar. Die Beziehung formiert in diesem Fall das Angebot, den Raum, der durch die Beziehungspartner(innen) konturiert wird. In dieser Hinsicht wäre es sinnvoll, von einer Beziehung als einem Möglichkeitsraum zu sprechen. Dennoch erscheint diese Kategorie aus verschiedenen Gründen nicht treffend genug, so dass ich zwar an dieses Konzept anknüpfe, jedoch stattdessen vom Beziehungshorizont der Paare spreche. So erlaubt eine solche Modellierung, anders als bei einem Möglichkeitsraum, die Zirkularität und Dynamik des Geschehens stärker in den Fokus zu nehmen. Einerseits ermöglicht die Beziehung nämlich die Realisierung bestimmter Erwartungen, Hoffnungen, Konzepte etc., andererseits ermöglichen diese Orientierungsmuster wiederum die Beziehung und konstituieren den Horizont der Beziehung. ${ }^{14}$ Während der Raumbegriff also stärker die Abgeschlossenheit und Fixiertheit einer Konstellation betont, erlaubt es die Vorstellung eines Horizontes, die prinzipielle Unabgeschlossenheit solcher Konstellationen mit einzubeziehen. Für eine Arbeit über Formen biographischen Lernens ist insbesondere der Gedanke der wechselseitigen Konstituierung eines Horizontes interessant, an den von beiden Partner(inne)n die Erwartung der Realisierung spezifischer Möglichkeiten der Beziehungs- und Lebensformen geknüpft ist. ${ }^{15}$ Eine solche wechselseitige Konstituierung eines gemeinsam geteilten Horizontes bedeutet die Koordination, die Integration und die Temporalisierung zweier biographischer Entwürfe.

Koordination meint dabei den beständigen Versuch und die beständige Aufgabe, biographische Entwürfe aneinander anschlussfähig zu machen. Damit ist Koordinationsarbeit mit der Konstituierung nicht abgeschlossen, sondern bleibt als Herausforderung für die Dauer der Partnerschaft bestehen. Koordination bedeutet, Entscheidungen $\mathrm{zu}$ treffen, wessen Vorstellungen realisiert werden können und wessen nicht, wo

14 Vgl. dazu auch Burkart, der mit Verweis auf Simmel auf die Wechselseitigkeit als Grundlage von Liebe hinweist (ebd. 1998: 31).

15 Während die Form der Beziehung sich eher nach innen auf die beiden Partner(innen) richtet, bindet sich die Lebensform stärker in die soziale Umwelt ein. 
zukünftig gelebt werden soll und welche Form der Arbeitsteilung vorstellbar ist. Koordinierungsarbeit bedeutet Planungsnotwendigkeit, darf aber nicht verwechselt werden mit Planungsmöglichkeiten. Vielmehr müssen solche Koordinationen immer wieder, insbesondere an ,crucial points' in der Biographie wie der Konfrontation mit ungewollten Ereignissen, aktualisiert und angepasst werden. Integration meint die Entwicklung eines gemeinsamen biographischen Entwurfes. Es gilt dabei, eine Balance zu finden zwischen der Orientierung an einer gemeinsamen Biographie einerseits und der eigenständigen Biographieentwicklung andererseits. Eine solche Balance kann durchaus unterschiedlich erreicht werden, wie sich auch in den Biographieanalysen zeigt. Je nach Lebensphase und je nach den Erwartungen, die an die Beziehung gestellt werden, kann die Konzentration stärker auf dem ,wir' anstelle des ,Ichs ${ }^{6}$ liegen. Gerade zu Beginn einer Beziehung besteht die Notwendigkeit des Rückbezuges: „Im, zumindest zeitweiligen, Rückbezug auf sich selbst bildet sich die gemeinsame Identität als Ehepaar" (Eckert/Hahn/ Wolf 1989: 16). Inwiefern im Verlauf der Beziehung dann jedoch eine andere Orientierung relevant wird, gilt es ebenfalls im Blick zu behalten. Die Temporalisierung schließlich schließt die zeitliche Koordination der biographischen Entwürfe mit ein. Wann wessen Wünsche und Vorstellungen erfüllt werden, gilt es ebenso auszuhandeln wie Entscheidungen über den ,richtigen`Zeitpunkt der Familiengründung oder möglicher beruflicher (Neu)Anfänge.

Solche Notwendigkeiten der Koordination, Integration und Temporalisierung bestehen im gesamten Verlauf der Beziehung. ${ }^{16}$ Insbesondere an den oben genannten ,crucial points' der Beziehung, wenn sich grundlegende Rahmenbedingungen durch Migration oder durch Kinder ändern, wird es oft notwendig, explizite Anpassungsleistungen zu vollbringen und sich über die gemeinsamen Ziele zu verständigen. Aber auch zu weniger dramatischen Zeitpunkten finden, oft implizit, solche Anpassungsleistungen (bzw. deren Überprüfung) statt. $\mathrm{Zu}$ Beginn der Beziehung jedoch sind solche Konstituierungen insofern besonders bedeutsam, als dass die Paare durch gegenseitige Erwartungen und Erzählungen den Horizont verifizieren, der sich als gemeinsam geteilte Wirklichkeitskonstruktion über der Beziehung aufspannt. ${ }^{17}$

16 Dabei ist aber noch nichts über die Art der Verständigung gesagt, zum Beispiel über Machtverhältnisse in Beziehungen, wer also de facto die Entscheidungen trifft.

17 Es lassen sich zwei Formen solcher Erzählungen unterscheiden: zum einen die Erzählungen untereinander zu Beginn der Beziehung, zum anderen die Erzählungen gegenüber der Interviewerin. In letzterer wird ebenfalls eine Version der Beziehungsgeschichte erzählt. Ob von beiden die gleiche rekonstruiert wird, darauf gilt es in der Interpretation zu achten. 
Als ein Ergebnis dieses Interpretationsmodells zeigte sich, dass sich die Beziehung zwischen den Partner(inne)n als Möglichkeit verstehen lässt, spezifische Verknüpfungen von Geschlechterkonstruktionen mit Konstruktionen natio-ethno-kultureller Zugehörigkeit zu realisieren. ${ }^{18}$ Es lassen sich (auch) Imaginationen hinsichtlich des bzw. der Anderen als Vertreter(in) einer anderen natio-ethno-kulturellen Zugehörigkeit analysieren, die die Realisierung spezifischer Partnerschaftsmodelle erlaubt. Die Beziehung dient als Ausdrucksform für Geschlechterbeziehungen, die in einem biographielogischen Verhältnis zu Formen natio-ethnokultureller Zugehörigkeit stehen. Wenn Nicole Ortega Garcia ihren Mann als einen traditionellen (mexikanischen) Partner sieht, den sie so unter deutschen Männern nicht gefunden hat, oder Susanne Frei mit ihrer Beziehung die Möglichkeit einer hierarchischen Machtverteilung in der Beziehung sieht, lassen sich Erwartungen realisieren, die, so zumindest die (wie in Nicoles Fall explizite) Annahme der Paare, in einer mononationalen Ehe nicht möglich zu sein scheinen, die ,andere' Herkunft des Partners und der Partnerin erweitert den Horizont der Möglichkeiten. Der geteilte Sinnhorizont stellt in diesem Fall also eine Art Möglichkeit zur Handlungserweiterung dar. Die Entwicklung eines gemeinsam geteilten Paarhorizontes bedeutet jedoch nicht zwangsläufig, dass die Beziehungspartner(innen) die gleiche Geschichte über den Beginn der Beziehung erzählen. Es bedeutet vielmehr, dass sich ein geteilter Sinnhorizont analysieren lässt, in welchem die Partner(innen) unterschiedliche, aber in spezifischer Weise korrespondierende, Erwartungen und Wahrnehmungen formulieren. ${ }^{19}$ Diese Korrespondenz wurde in den Interpretationen deutlich. Die beiden Partner(innen) müssen nicht die gleichen

18 Für diese Verknüpfungen spielt es keine Rolle, ob Erwartungen und rekonstruierbaren Plausibilitätsmuster schon, vorher' biographisch entwickelt waren oder in der Beziehung interaktiv hergestellt worden sind.

19 Dabei ist dieser Horizont offen für Transformationen und Veränderungen im Laufe der Beziehungszeit. Prioritäten können sich verschieben und andere Erinnerungen an deren Stelle treten. Die Möglichkeit der eigenen Relevanzsetzung und der diskontinuierlichen Verbindung von Erfahrungen lässt sich auch an den Interviews selbst zeigen: In einigen Narrationen, wie der von Susanne Frei oder Pablo Marmani Rodriguez, lässt sich die Herausbildung eines Paarhorizontes deutlich anhand ihrer Kernstellenerzählung zum Beginn der Beziehung analysieren. Bei Rachid FreiHeidarzadeh aber zeigt sich die angesprochene temporale Eigenart des Paarhorizontes sehr deutlich: In seiner narrativen Darstellung werden Erfahrungen aus der Kindheit mit der Begegnung mit Susanne und ihrem späteren Leben zu einem Horizont verwoben, der für ihn in der Rekonstruktion die Bedeutung der Beziehung charakterisiert. Es gibt also kein identifizierbares ,Vorher' und ,Nachher'. Insofern wurden Kernstellen aus dem gesamten Material als Interpretationsgrundlage genutzt. 
Erwartungen an eine Beziehung haben, sie müssen sich allerdings vereinbaren lassen, wichtig ist es daher, den Blick sowohl auf die entwickelte Struktur zu richten als auch auf den Inhalt. Die Erzählungen der Paare, auch wenn sie getrennt voneinander befragt wurden, korrespondieren so in einer nicht-zufälligen Weise. ${ }^{20}$ In einer Paarbeziehung ist eine solche wechselseitige Konstituierung sehr eng an die Vorstellung eines unmittelbaren Verstehens geknüpft. ${ }^{21}$ Zwar ist eine Partnerschaft, wie Koppetsch 1998 zeigt, ,durch und durch auf Reziprozität und Gegenseitigkeit ausgerichtet“ (Koppetsch 1998: 112), es gilt die gemeinsamen Interessen auszuhandeln und in kommunikativer Interaktion die weitere gemeinsame Biographie zu gestalten. Dennoch begründet sich insbesondere der Beziehungsanfang, der Beginn der Konstituierung der Partnerschaft also, im hohen Maße auch auf unausgesprochenen Erwartungen und Imaginationen, die nicht zwangsläufig kongruent sein müssen, von deren Kongruenz jedoch ausgegangen wird. Erwartungen können, müssen den Partner(inne)n aber nicht zwangsläufig bewusst sein. Sehr viel häufiger existieren sie als unformulierte Erwartungshaltungen. Gerade die vorher bereits formulierte Hoffnung, durch Liebe ,immer schon verstanden zu werden“ (Burkart 1998: 34, vgl. dazu Luhmann 1982), kann zur Unaussprechlichkeit von Erwartungen führen. Es kann auch ein gemeinsamer Beziehungshorizont entwickelt werden, ohne dass explizit Grenzen gezogen und Vorstellungen explizit gemacht werden. Andersherum kann vielleicht gerade diese Unaussprechlichkeit eine der Bedingungen für das Zustandekommen der Beziehung sein: Die Annah$m e$ eines geteilten Sinnhorizontes ist entscheidend. Dennoch spielen, gerade zu Beginn der Beziehung, gegenseitige biographische Erzählungen eine große Rolle.

„Der Beziehungsanfang stellt - in den Worten von Alois Hahn (1987) - einen ,institutionellen Kontext‘ für die biographische Erzählung dar, der ihre ,Selektionsmuster' festlegt. Es wird nicht einfach - was auch nicht möglich ist - das Vergangene einer Person reproduziert, sondern es wird zum Thema in einem für den Beziehungsaufbau relevanten Zuschnitt“" (Lenz 2003: 199).

Ausgehend von der Koordinations- und Integrationsnotwendigkeit stellt die so entstehende Narration, als verfestigte Wirklichkeitskonstruktion,

20 Dies ist sicherlich auch eine Folge davon, dass die Paare noch als Paar zusammen sind, und die Koordinations-, Integrations- und Temporalisierungsarbeit auf eine gemeinsame Biographiegestaltung gerichtet ist.

21 Partnerschaft hängt eng mit Motiven wie Liebe, Gleichberechtigung etc. zusammen, die die Illusion der Verständigung verstärken (vgl. dazu auch Oechsle 1998). 
eine gemeinsam entwickelte Übereinkunft der Darstellung und Interpretation dar. ${ }^{22}$

Der Horizont stellt in der aktuellen biographischen Erzählung ein Plausibilitätsmuster dar, das die Beziehungsgeschichte als Ganzes umrahmt und hilft, Erfahrungen vorzubereiten und einzuordnen. Alle Paare weisen in ihren Erzählungen daher der Entstehung ihrer Beziehung einen relativ großen Raum zu. ${ }^{23}$ Der sich so wechselseitig konstituierende Horizont erlaubt die Erweiterung der subjektiven Handlungsmöglichkeiten und auch eine (potentielle) Reorganisation bestehender Geschlechterbeziehungen. Zudem ist diese Form der Modellierung zu Beginn der Beziehung grundlegend relevant für den weiteren Verlauf der Bearbeitungen, der Ausgestaltung der Formen und der Reichweite biographischer Lernprozesse. Zum einen hängt davon die biographische Integrierbarkeit des female breadwinner Modells in der Beziehung ab, zum anderen aber bestimmt sich hierdurch die ,Strukturflexibilität' und Weite des Horizontes (vgl. dazu auch Eckert/Hahn/Wolf 1989). Die Flexibilität, die sich zu diesem Zeitpunkt in den Beziehungsformen und -erwartungen der Partner(innen) analysieren lässt, wirkt, so das Ergebnis der Analysen, temporär nachhaltig und strukturiert die Art und Weise, in der die ,Zumutungen` biographisch bearbeitet werden.

\section{Thema und Bearbeitungsfelder}

Als zweite relevante Analysedimension werden daher die entstehenden Bearbeitungsfelder der Paare begriffen. Wie im obigen Abschnitt entwickelt, sind die Beziehungshorizonte, die sich in der ersten Dimension festhalten lassen, konstitutiv wirksam für die weiteren Bearbeitungen verschiedener ,Zumutungen“ durch die Beziehungspartner(innen). Daher werden in dem Interpretationsmodell nach der Analyse der Merkmale des Beziehungshorizontes die jeweils spezifisch wahrgenommenen $\mathrm{Zu}-$ mutungen und deren Bearbeitungen interpretiert und in Beziehung zu

22 Interessanterweise, aber nicht unerwartet, wandelt sich diese gemeinsame Interpretation bei der Auflösung der Beziehung in durchaus divergierende Einzeldarstellungen der beiden ehemaligen Partner(innen). Plötzlich war alles ganz anders, gemeinsame Wirklichkeitskonstruktionen zum Beispiel über das Ende (und die Gründe für das Ende) der Beziehung sind selten.

23 Diese Erzählungen haben zudem, so lässt sich rekonstruieren, legitimierenden Charakter gegenüber der Interviewerin als ,generalisierter Anderer' im Mead'schen Sinne. Zweierlei wird dadurch aufgenommen: Die Erzählungen dienen als Begründungszusammenhang für die Entscheidung für eine solch ,unpassende' oder ungewöhnliche Beziehung, und die Anschlussfähigkeit der Beziehung zu den bisherigen biographischen Erfahrungen wird thematisiert und sichergestellt. 
dem konstituierten Horizont gesetzt. Diese Zumutungen sind auch Folge der Entscheidung, in Deutschland leben zu wollen, und Folge der Auseinandersetzungsnotwendigkeit mit dem female breadwinner Modell. ${ }^{24}$

Für jede Paarbeziehung lässt sich dabei ein Thema finden, welches als ,Leitmotiv‘ über den Bearbeitungen und Aushandlungen steht. Dieses bestimmt sich aus dem konstituierten Beziehungshorizont. Durch seine gleichzeitige Inklusion und Exklusion bestimmter Handlungs- und Orientierungsmöglichkeiten und durch die ,nicht-intendierten Folgen“ der Handlungen (vgl. Giddens 1995) lässt sich die Konstitution spezifischer Bearbeitungsnotwendigkeiten beobachten. Für ein Paar, für das die Beziehung an sich, wie bei Maria und Pablo, Ausdruck einer kosmopolitischen Haltung darstellt, haben andere Ereignisse biographische Relevanz als für ein Paar wie Nicole und Carlos, welche eine traditionelle Arbeitsteilung erwarten und favorisieren. ${ }^{25}$ Die für ein Paar relevanten Felder müssen jedoch nicht unidirektional aus äußeren Umständen oder Konstellationen wie dem female breadwinner Modell resultieren. Stellt das Modell für das eine Paar tatsächlich eine dramatische Konstellation dar und muss daher an sich bearbeitet werden, sind es für ein anderes Paar eher nicht intendierte Folgen oder Nebenwirkungen beispielsweise in Form der Reaktion der Eltern, die Schwierigkeiten bereiten können. Bearbeitungsfelder beziehen sich jedoch nicht nur auf Probleme und negative Irritationen, die gelöst werden müssen. Es kann sich auch, wie bei Rachid Frei-Hardazadeh, um Herausforderungen positiver Art handeln, die ein Bearbeitungsfeld darstellen. So sieht er in seiner ,gebrochenen Berufsbiographie keine Schwierigkeit, sondern versteht seine Erlebnisse als reiche Erfahrung, die er sonst nicht hätte machen können. Das Bearbeitungsfeld ist insofern in diesem Fall das Verständnis von pluralisierten Berufsbiographien als Bildung.

Im weiteren Verlauf der Entstehung solcher Bearbeitungsfelder bekommen das soziale Umfeld und die Herkunftsfamilien der Partner(innen) erhöhte Relevanz. Nach der Konstituierung der Beziehung, die wie angesprochen häufig mit einem erhöhten Rückbezug auf sich und die Beziehung einhergeht, findet hier die ,Außenwelt' verstärkt Eingang in die biographischen Konstruktionen. So ist diese Dimension wesentlich durch Anforderungen bestimmt, die durch gesellschaftliche Ordnungsmuster und spezifische Erwartungen des sozialen Nahraums an

24 Dazu gehören sicherlich auch die Verortung des Mannes und die Bedeutung, die seine natio-ethno-kulturelle Zugehörigkeit in Deutschland bekommt.

25 Dass die Konstruktion von Lernanlässen wesentlich von der Erfahrungsaufschichtung abhängt, darauf verweist auch von Felden (vgl. ebd. 2003: 242f.). 
die Paare herangetragen werden. Im Vergleich zur ersten Dimension liegt so eine Verschiebung vor: hier erlaubt der Beziehungshorizont eher die Anschlussfähigkeit biographisch entwickelter Wünsche und Erwartungen untereinander, dort sind es eher externe Anforderungen, die, strukturiert durch den Rahmen des Horizontes, bearbeitet werden müssen. So müssen sich die Paare zum Beispiel mit der Haltung der Herkunftsfamilien hinsichtlich geschlechtlicher Arbeitsteilung, binationaler Beziehungen oder zweisprachiger Erziehung der Kinder auseinandersetzen. Insbesondere das female breadwinner Modell wird hierbei gerade von den Eltern der Partnerin regelmäßig als höchst problematisch gesehen. Bearbeitungsfelder können daher in zwei Bezügen entstehen: im Verhältnis zur Herkunftsfamilie und zum sozialen Nahraum und im Verhältnis zur Beziehung selbst. Analysiert werden in dieser zweiten Dimension also notwendige Entwicklungen und Bearbeitungen in der Beziehungsbiographie: Wie werden in den ,Herausforderungen' gender und natio-ethno-kulturelle Zugehörigkeit verarbeitet und verschoben? Wo erweisen sich Modifikationen oder sogar Transformationen als ,nachhaltig'? Wo aber stellen sich zum Beispiel individualisierte Geschlechterbeziehungen als nicht tragfähig oder als langfristig nicht realisierbar heraus? Die Entwicklungen müssen nicht zwangsläufig chronologisch-linear erfolgen. Einerseits kann sich daher ein (vorläufig) abgeschlossener Prozess analysieren lassen, in dem Herausforderungen in einer Art und Weise bearbeitet wurden, die ein (vorläufiges) Fazit ermöglichen. Andererseits kann es sich, grade bei Paaren, die noch nicht so lange zusammen sind, auch eher um einen unabgeschlossenen Prozesse im Sinne eines ,Kreisens um ' Bearbeitungsfelder und relevante Themen handeln. Letzeres ist insbesondere bei Maria Wesel und Pablo Marmani Rodriguez der Fall.

Das Thema des Paares stellt die Abstraktion der spezifischen Ausformung der Bearbeitungsfelder dar. Damit ist jedoch noch nicht die Form biographischen Lernens benannt, wie sie sich in den Interviews analysieren lässt. Die Unterscheidung liegt im Blick auf die biographischen Erzählungen und in der Analyseebene. Die identifizierbaren Themen sind biographisch relevant geworden, daraus ergeben sich Bearbeitungsfelder und darin lassen sich Aushandlungen und Bearbeitungsprozesse analysieren. Die Form biographischen Lernens, die sich analysieren lässt, stellt hingegen die Struktur der Bearbeitung, die Bearbeitungsform dar. Sie beschreibt die Art und Weise des ins-Verhältnis-Setzens der beiden Kategorien und kann im Ergebnis etwas über Modifikationen oder (Re-)Konstruktionen von Geschlechterordnungen und natio-ethno-kulturellen Zugehörigkeiten aussagen. In der Form biographischen Lernens 
werden die beiden Analysedimensionen, der Beziehungshorizont und die Bearbeitungsfelder, daher zusammengeführt.

\section{Schematische Darstellung des Interpretationsmodells}

Abbildung 3: Darstellung des Interpretationsmodells

\section{Zeitdimension}

\section{Analysedimension}

Konstruktion des Paarhorizonts:

Biographische Koordinations-, Integrations- und Temporalisierungsarbeit

\section{Analysedimension}

Verschiedene Bearbeitungsformen

Formen biographischen Lernens: Biographiespezifische Konfigurationen von gender und natio-ethno-kultureller Zugehörigkeit 


\section{DER FREMdE UND DIE ANDERE: SUSANNE FREI UND RACHID FREI-HEIDARZADEH}

Bei den folgenden Interpretationen der Biographien ist die Art der Darstellung bei allen drei Paaren die gleiche: Den Interpretationen sind biographische Portraits der Paare vorangestellt. Die Ergebnisse der Analyse folgen dem Interpretationsmodell, so dass in einem ersten Schritt der Paarhorizont von Susanne und Rachid analysiert wird, bevor das Thema benannt und die Bearbeitungsfelder rekonstruiert werden. Im Anschluss daran lässt sich die Form biographischen Lernens herausarbeiten.

\section{Biographische Portraits Susanne Frei und Rachid Frei-Heidarzadeh}

\section{Susanne Frei}

Susanne Frei wird 1956 als zweites von sechs Kindern in einer Kleinstadt im Süden Deutschlands geboren. Der Vater ist Notar, die Mutter hat nach der Geburt der Kinder aufgehört, als Krankenschwester zu arbeiten. Ihre Herkunftsfamilie ist traditionell eingestellt und zudem sehr religiös. Diese strenge religiöse Einstellung und Erziehung der Eltern erlebt Susanne als sehr einengend und ihrer Entwicklung hinderlich. Sie beendet daher das Gymnasium mit der mittleren Reife, um finanziell möglichst schnell auf eigenen Füßen stehen zu können. Auch wenn sie für ihre Ausbildung als Erzieherin in das Schulinternat zieht, gibt sie den Kontakt zu den Eltern nie ganz auf. Ausdruck ihrer Beschäftigung mit „diesem Ungleichheitsaspekt“ (25/8), der Ungleichheit zwischen Män- 
nern und Frauen, und ihrer Auseinandersetzung mit ihren Eltern ist ihr Engagement in der Frauenbewegung und in linkspolitischen Gruppen. Nach Ende der Ausbildung bewirbt sie sich in ganz Baden-Württemberg, findet aber schließlich nur eine Stelle in ihrem Heimatort. An ihrem Entschluss, in eine Wohngemeinschaft zu ziehen, hält sie trotz der Bedenken der Eltern fest. Nach dreijähriger Berufstätigkeit als Erzieherin möchte sie 1980 für eine Zeitlang ins Ausland gehen. Da sie Entwicklungshilfe aus politischen Gründen ablehnt, andere Möglichkeiten sich aber nicht ergeben, beschließt sie zu reisen. Sie kündigt ihre Stelle, verkauft ihr Auto und reist alleine nach Indien und Sri Lanka. Kurz vor Beginn der Reise hat sie eine Beziehung zu einem Mann begonnen, die sie jedoch schon kurz nach der Abreise wieder beendet, auch weil sie sich nicht sicher ist, ob sie lieber in Frauen- oder in Männerbeziehungen leben möchte:

„Also das war für mich offen. Ich hab in 'ner Frauenwohngemeinschaft gelebt und - ähm meine erste Beziehung war eigentlich zu 'ner Frau und das war so, der erste Kontakt mit 'nem Mann engerer Art und ich dann au so gemerkt ne das war's net" $(5 / 33-6 / 3)$.

In Sri Lanka lernt sie nach kurzer Zeit den Iraner Rachid kennen, der dort auf einen Studienplatz wartet. Beide geben sich als Angehörige einer anderen Nationalität aus (sie als Schwedin, er als Grieche), da ihr das „Deutschtum [...] oft sehr peinlich“ (6/16f) ist. Zudem präsentiert sie sich ihm als homosexuell. Sie reisen zusammen durch Sri Lanka und Indien und fangen nach einiger Zeit eine Beziehung an. Nach etwa drei Monaten beschließen sie, gemeinsam nach Deutschland zu ziehen und zu heiraten. Die Entscheidung zu heiraten wird von Susanne nicht weiter ausgeführt, sie hat den Eindruck, dass es zwischen ihnen gut läuft, kann sich aber nur vorstellen in Deutschland zu leben und zu heiraten, da sie dort die Rechtslage kennt. Gegen den Widerstand ihrer Familie, die aufgrund der schnellen und überraschenden Entscheidung Drogeneinflüsse vermutet, heiraten sie kurz darauf in Deutschland. Die erste Zeit erlebt Susanne als extrem schwierig, da sie die im Ausland geschlossene Beziehung nun definieren und realisieren muss. Hinzu kommt die finanzielle Abhängigkeit Rachids, die Susanne, im Gegensatz zu Rachid, als belastend empfindet, zumal sie Schwierigkeiten hat, das von ihr verdiente Geld als gemeinsames zu definieren. Sie verdient als Erzieherin den Lebensunterhalt, finanziell und praktisch immer wieder von ihren Eltern unterstützt, während er eine Ausbildung als Kfz-Mechaniker macht und dann einige Semester Elektrotechnik studiert. Das Verhältnis zu ihren Eltern bleibt zwar angespannt, dennoch gibt sie den Kontakt nicht auf, 
sie lässt es nicht zu einem endgültigen Bruch kommen. 1986 macht sie eine zweijährige Weiterbildung, Fachlehrerin für geistig Behinderte und arbeitet danach in einer Schule für Körperbehinderte. In dieser Zeit überlegt Rachid, dem sein Studium große Mühe bereitet, ins medizinische Fach zu wechseln. Da Susanne jedoch ablehnt, eine Finanzierungserklärung zu unterschreiben, kann er nicht wechseln, bricht aber dennoch sein Studium der Elektrotechnik ab.

„Und ja, dann hat der das Studium abgebrochen, und dann ist er auch nochmal in 'n Iran gegangen weil das war einfach so klar von seiner Wertigkeit Studium ist das Wichtigste in Leben. Und, das abzubrechen, das ah, das war schon ein längerer Prozess. Und dann hat er's abgebrochen, hat sich ne Stelle gesucht und hatt dann verdient, und dann kam bei ihm sofort so der Wunsch nach Familie“" (12/31 - 13/1).

Im September 1988 wird ihr erster Sohn geboren, acht Wochen später zieht die Familie, zuerst zur Miete, in das Haus von Susannes Eltern. Sie erlebt die darauf folgende Zeit als existenzielle Krise, da ihre finanzielle und berufliche Unabhängigkeit nicht mehr gegeben ist, sie sich vielmehr in der klassischen Frauenrolle gefangen sieht. Ihre ,ganze Frauenrolle [wird] durcheinander gewirbelt" (13/24). Einerseits finden sie eine Lösung, indem beide ihre Arbeitszeiten reduzieren und sich die Betreuung der Kinder teilen. Andererseits leidet ihre Beziehung in der Zeit sehr, und sie nehmen auch externe Beratung in Anspruch, insbesondere da „für mich plötzlich als Mutter da war des so denk ich au mal klar, dass wir, ja, dass meine Rolle ne andere plötzlich ist" (14/12f). Oktober 1991 wird ihr zweiter Sohn geboren, und Susanne kommt erneut an ihre Grenzen: „Nach dem zweiten Kind war noch mal so für mich auch noch mal so 'ne ganz existenzielle Krise. - weil, ähm, weil ich dann ja total angebunden war. Also das war schon heftig“ (14/21 - 24). Susanne überlegt, Mann und Kinder zu verlassen, muss aber feststellen, dass sie sich nicht von gesellschaftlichen Erwartungen und Zwängen frei machen kann, ihrem Eindruck nach würde sie ihre Familie im Stich zu lassen. Nach 15 Monaten fängt sie wieder an zu arbeiten. Rachid kündigt seine Stelle und sucht nach einer neuen beruflichen Möglichkeit. Susanne spricht sich gegen ein erneutes Studium aus, da sie langfristig nicht mehr die finanzielle Sicherung der Familie übernehmen will und sich nicht vorstellen kann, dass er das Studium schnell beenden würde. Schließlich beginnt Rachid eine zweite, vierjährige Ausbildung als Erzieher, in der Zeit sorgt Susanne erneut für die finanzielle Absicherung der Familie. Der Kontakt zu den Eltern von Susanne wird besser, da sie zum einen Rachid sehr gerne mögen und Susanne zum anderen die Lebensweise 
der Eltern besser akzeptieren kann. Auch die Beziehung zwischen Susanne und Rachid verbessert sich im Laufe der Zeit, insbesondere seit Rachid als Gruppenleiter in einer Fachwerkstatt für Behinderte arbeitet und somit finanziell eigenständig ist. Zudem baut er sich einen eigenen Freundeskreis in Deutschland auf, so dass Susanne nicht den alleinigen Bezugspunkt für ihn darstellt. Seitdem sieht sie die Gleichrangigkeit in ihrer Beziehung wieder hergestellt:

„und, vielleicht au die Stabilität also ich denk vom Rachid selber dass er ganz andere Kontakte hat völlig unabhängig von mir. Da au gute Rückmeldungen kriegt, das ist natürlich au a_ hilft einem 'nen Stand hier zu finden, ist ja ganz klar, ja, und das schafft ja au Unabhängigkeit und -- ich hab's Gefühl ich muss mir se au net immer so beweisen ich hab sie (auflachen)“ (19/22 - 26).

\section{Rachid Frei-Heidarzadeh}

Rachid Frei-Heidarzadeh wird 1958 als jüngstes von insgesamt fünf Kindern, alle vier wesentlich älter, in einer iranischen Kleinstadt geboren. Die Familie besitzt viel ökonomisches Kapital, die Mutter ist als Tochter eines Großgrundbesitzers finanziell unabhängig von ihrem Ehemann, einem Einzelhandelskaufmann. Dieser stirbt, als Rachid zwölf Jahre alt ist. Rachid erlebt seine Kindheit insgesamt als ,,sorglos“ (1/26) und frei von materiellen Zwängen: „Ich hab fast auch alles gekriegt was ich wollte, für damalige Verhältnisse und im Iran [...] und, Fahrrad und Moped und Motorrad und überhaupt alles“ (1/27 - 30). Als jüngstes Kind wird er umsorgt und bekommt viel Aufmerksamkeit. Nach dem Abitur möchte er im Ausland studieren, muss aber vorher den Militärdienst ableisten:

„Ich hab immer gewusst, dass ich nach Ausland gehen möchte, dass ich im Ausland studieren möchte. Dann hab ich immer rumgehorcht bei Verwandten, die nach Ausland gegangen sind und studiert haben oder dort studierten, hab ich Briefkontakte gehabt und hab nach Wege gefragt wie das geht und so. Aber im Iran war's so da musst du unbedingt äh zur Bundesweh_äh zum Militär gehen, bevor du Iran, oder dass du Pass kriegst“" $(1 / 31-2 / 3)$.

Nach zwanzigmonatigem Militärdienst bricht die iranische Revolution aus, durch einen Erlass Khoumeinis darf er die Armee vorzeitig verlassen. Auf Anraten und mit Hilfe eines in Sri Lanka lebenden Onkels geht er nach Sri Lanka, um Medizin zu studieren, muss aber ein Jahr auf einen Studienplatz warten. In dieser Zeit trifft er Susanne. Sein Interesse am Ausland ist auch durch sein Verhältnis zu seiner Mutter motiviert: 
„Vielleicht, - ich hab sehr oft nachgedacht warum das so war. Und warum ich auch von dieser Enge - a_ ein beliebtes Kind zu sein, von der Mutter und Geschwister, und dann auch so weit weg und auch so selten jetzt hingeh. - Vielleicht auch ist e_zu viel gewesen die Erwartungen was meine Mutter auf mich überlagert hatte. Da_das ist meine Erklärung. Ähm, vielleicht das war so unbewusst weit weg zu gehen. Möglichst weit, dass diese Frau keine direkten Einfluss auf meine private Leben hat“ (9/7-9/12).

Er beschließt, seine Studiumspläne vorerst aufzugeben und mit Susanne nach Deutschland zu gehen. Seiner Familie teilt er diesen Entschluss brieflich mit - anfangs sind sie enttäuscht, können aber im Nachhinein seinen Entschluss verstehen. Die Mutter ist froh, den Sohn in Sicherheit außerhalb des zerrissenen Irans zu wissen. Die erste Zeit in Deutschland empfindet er als „Traumzeit“ (13/5), die Wohnverhältnisse in einer alten Siedlung mit vielen jungen Leuten und einem großem Gemeinschaftsgefühl erinnern ihn an Iran, er jobbt unter anderem in einem alternativen Gemüse- und Obstladen. Nach einiger Zeit hat er das Gefühl, dass das Leben „ernst“ (13/28) wird und er sich Gedanken über die berufliche Zukunft machen muss. Da das iranische Abitur nur für naturwissenschaftliche Studiengänge anerkannt wird, macht er eine Lehre als KfzMechaniker, um nach dem Erwerb der Fachhochschulreife Maschinenbau zu studieren. Die Lehre beendet er, das Studium bricht er jedoch nach einigen Semestern ab, da es ihm zu schwer fällt. Die zeitweilige Rückkehr in Iran, von der Susanne erzählt, wird von Rachid nicht thematisiert. Kurz vor der Geburt des ersten Sohnes wird er Schichtführer im produzierenden Gewerbe. Durch die Reduzierung seiner Arbeitszeit verbringt er viel Zeit mit seinem Sohn und erlebt die ersten Jahre als sehr intensiv und befriedigend:

„Und, für diese Zeit denk ich war's wichtig gewesen weil Susanne konnt vormittags schaffen - und ich konnt ab zwei Uhr arbeiten wegen Kind damals. Das war - also wenn ich einen anderen Beruf gehabt hätte das wär gar nicht möglich gewesen“ $(15 / 21-23)$.

Trotz beginnender Schwierigkeiten in der Ehe kommt für ihn eine Trennung nie in Frage, er möchte, dass seine Kinder mit Vater und Mutter aufwachsen. Nach knapp sechs Jahren als Schichtführer fühlt er sich unterfordert. Er kündigt seine Stelle und arbeitet in einer Bank als Chauffeur, in der Hoffnung, dort als Quereinsteiger eine Ausbildung beginnen zu können. In dieser Zeit wird der zweite Sohn geboren. Schnell stellt sich heraus, dass ein Aufstieg oder eine Ausbildung in der Bank nicht möglich sein wird, daher kündigt er nach wenigen Monaten wieder. In dieser Umorientierungszeit denkt er über einen zweiten Stu- 
diumsversuch nach, verwirft diesen Gedanken dann aber auf Drängen Susannes wieder. Durch Susanne und ihre Kollegen lernt er den pädagogischen Bereich kennen und beschließt, ebenfalls eine ErzieherAusbildung zu machen. Das Anerkennungsjahr der Ausbildung absolviert er in der gleichen Schule, in der Susanne arbeitet. Seit 1997 ist er Gruppenleiter in einer Fachwerkstatt für Körperbehinderte und sehr zufrieden mit dieser Arbeit. Lange Zeit hat er überlegt, im Ruhestand wieder nach Iran zu gehen. Diese Gedanken hat er allerdings inzwischen aufgegeben, da er dem iranischen Regime sehr kritisch gegenübersteht und die hier kennen gelernten Denk- und Redefreiheiten nicht aufgeben möchte. Aus dem gleichen Grund hat er seinen Kindern kein Persisch beigebracht, er möchte nicht, dass sie etwas mit diesem Regime zu tun haben:

„Die sollen im Iran wenig zu tun haben, - weil, wenn so eine Re_Regierung dort und Religion dort herrscht, - ähm, die sollen lieber fern, ganz weit weg sein von so, so Land. Und alle Möglichkeiten hier haben zu leben, sollen lieber ihre Wurzeln hier schlagen. (4 sek.) Und, deswegen, dort, teilweise denk ich di_das war ein Versäumnis, aber auf der anderen Seite bin ich auch nicht so arg traurig darüber weil ich denk, wenn es jetzt Englisch oder Französisch oder Spanisch oder eine andere lebendige Sprach gewesen wär [...] wäre ich vielleicht mehr nachdenklich gewesen oder traurig warum ich es nicht getan hab, ab_ aber bereu ich es auch nicht. Dass ich die Kinder nicht mit meiner Sprache nicht bekannt gemacht habe“" $(19 / 19-26)$.

Die Beziehung zu Susanne erlebt er inzwischen als sehr bereichernd und stabil, auch wenn beide immer wieder um Gemeinsamkeit und Individualität ringen müssen.

Der Horizont der Paare:

Beziehung als handlungserweiterndes Moment

\section{Konstruktion des Paarhorizontes bei Susanne: Fremdheit als Ressource}

Susannes Erzählungen über die Entstehung ihrer Beziehung zu Rachid und die Entscheidung, in Deutschland zu leben und zu heiraten, beinhalten die Etablierung atypischer Geschlechterbeziehungen auf der Basis struktureller und kultureller Inferiorität ihres Beziehungspartners. Solche Konstruktionen erweitern im Ergebnis Susannes Möglichkeiten hinsichtlich der Aushandlung der geplanten Lebensführung und stärken ihre Position in diesem Aushandlungsprozess. Dieser Prozess der Etablierung 
wird durch zwei dominante Dimensionen charakterisiert: diffizile und komplexe Fremdheitskonstruktionen und der Rückbezug auf ihr Herkunftsland als Ressource.

\section{Fremdheitskonstruktionen und Neu-Erfindung: als Fremde im fremden Land}

Bei der Etablierung der von Susanne entwickelten atypischen Geschlechterordnungen lassen sich zweierlei Formen von Fremdheitskonstruktionen analysieren, die die Beziehung zu Rachid charakterisieren: Ihre Fremdheit in Sri Lanka und Rachids Fremdheit in Deutschland.

\section{Die Fremde in der Fremde}

Fremdheit, so lässt sich mit Simmel (1992 [1908]) sagen, ist keine „Eigenschaft von Dingen oder Personen, sondern die Bezeichnung für die spezifische Qualität eines Beziehungsverhältnisses“ (in Stenger 1998: 19). Dieses Beziehungsverhältnis kann zwischen Menschen bestehen, es kann aber auch zwischen Menschen und Orten existieren. Für Susanne ist insbesondere die örtliche Nicht-Zugehörigkeit zu Sri Lanka entscheidend. Als sich die beiden kennen lernen, gibt er sich als Grieche aus, sie sich als Schwedin. Die Fremdheit in Sri Lanka bedeutet für sie also nicht die Erfahrung der Verringerung von Ressourcen, sondern der Erweiterung von Handlungsmöglichkeiten. Der wechselseitige „Prozess der Fremd- und Selbstbestimmung“ (Matthes 1992: 5) wird zur Maskerade und Reformulierung der natio-ethno-kulturellen Zugehörigkeit genutzt. Sri Lanka bekommt in diesem Prozess die Funktion eines dritten Landes zugesprochen, eines Gegenmilieus, in dem andere Regeln gelten und sich andere Gestaltungsmöglichkeiten ergeben. In Deutschland könnte sie sich wohl nur schwerlich als Schwedin ausgeben, in Indien aber kann sie auf Distanz zur regulären Fremd- und Selbstbestimmung gehen und sich mit Bezug auf eine andere kulturelle Zugehörigkeit frei von imaginären Schemata machen, die ansonsten in Interaktionen mit anderen Leuten soziale Definitionsmacht erlangen könnten. ${ }^{1}$ Susanne nutzt nun dieses dritte Land, um sich von gesellschaftlichen Repräsentationen frei zu machen und um auf Distanz zu gehen zu ihrem Herkunftsland und zum „Deutschtum“ (6/16), dessen Auswirkungen ihr „oft sehr peinlich“ (6/17) sind. Diese natio-ethno-kulturelle Maskerade findet ihren Gegenpart und ihre Ergänzung in der Selbstpräsentation Rachids als Grieche,

1 Eine solche Reformulierung bzw. situative Distanzierung bedeutet für sie jedoch nicht die Auflösung kultureller Zuschreibungen. Im Gegenteil: Für Susanne ist eine solche Rekonstruktion gerade auf Basis der Bestärkung national-kultureller Eigenheiten möglich und sinnvoll. 
nicht als Iraner. Auch er geht auf Distanz zu seinem Herkunftsland, eine Distanz, die auch ihm größeren sozialen Handlungsspielraum ermöglicht: In Susannes Schilderung der Begegnung mit Rachid wird Verständnis für Rachids Verhalten deutlich: Da Iraner zu der Zeit generell „einen ganz schlechten Ruf“ (6/21) hatten, kann Rachid so in Kontakt mit ihr treten ohne zu befürchten, sofort aufgrund seiner Nationalzugehörigkeit abgewiesen zu werden.

Susanne schafft also im ersten Schritt eine Gemeinsamkeit aufgrund ihrer beider Abgrenzung zu ihrer Herkunft. Diese Gemeinsamkeit können sie eigentlich nur in einem dritten Land entwickeln. Solche temporären Re-Positionierungen sind im Übrigen auch deshalb möglich, da bei dem Treffen zwischen Rachid und Susanne auf ,neutralem Boden' die natio-ethno-kulturelle Zugehörigkeit nicht fraglos gegeben ist, Zugehörigkeiten wechselseitig bestimmt und geklärt werden müssen. Beide müssen und können sich in der Interaktion einer Nationalkultur zugehörig erklären, da beide nicht-zugehörig sind. Explizit erwartete Positionierungen ermöglichen so zumindest temporäre Modifikationen bzw. RePositionierungen. Susanne aber nimmt für sich noch eine weitere Abgrenzung vor: „Ja, und außerdem hab ich zu ihm gesagt, also ich habs, mit Männern hab ich nix am Hut quasi“" (6/22f.). Ihre bisherigen erotischen Orientierungen (ihre erste Beziehung führte sie mit einer Frau; kurz vor der Abreise nach Indien führte sie jedoch eine Beziehung mit einem Mann, der sie ursprünglich auch in Asien besuchen wollte) nimmt sie zum Anlass, etwaige romantische Annäherungen von vorneherein auszuschließen. Zusammengefasst lässt sich von einem ganzen Maskenball sprechen, der von Susanne eröffnet wird. Das dritte Land wird von ihr genutzt um sich sowohl von ihrer natio-ethno-kulturellen Zugehörigkeit als auch von einer eindeutigen sexuellen Orientierung abzugrenzen, auf Distanz dazu zu gehen und dadurch mehr Handlungsspielraum zu erlangen bzw. spezifische Interessen realisieren zu können. Erst in dieser zweifachen Abgrenzung kann sie für sich die „Basis“ (6/23) für ein intensiveres Kennenlernen schaffen.

Ihre Beziehung beginnt für sie insofern mit einer doppelten Distanzierung: der Möglichkeit der Neu-Positionierung hinsichtlich zweier zentraler Zugehörigkeitskategorien und der dadurch ermöglichten Schaffung einer ,Basis‘ des Kennenlernens. Eine Dynamik, die sich als Inklusion durch Exklusion bezeichnen lässt. Susanne bezieht Rachid in ihr Leben mit ein, indem sich beide, sie und er, von ,herkömmlichen' und bisherigen Zugehörigkeiten distanzieren. ${ }^{2}$ Solche Handlungen sind dabei

2 Dies birgt sicherlich die Gefahr einer nur prekären Stabilität, sind doch die Positionierungen auf Dauer nicht aufrecht zu halten. Sie stehen im tendenziellen Widerspruch zur Hartnäckigkeit bestehender Zugehörigkeitskon- 
,nur' in einem dritten Land und ,nur' mit einem Nicht-Deutschen möglich. Rachids natio-ethno-kulturelle Zugehörigkeit ist daher durchaus relevant, und zwar in zweifacher Hinsicht: Zum einen ermöglicht sein Nicht-Deutsch-Sein Susanne, ihre Re-Positionierung durchzuführen, zum anderen ermöglicht seine Herkunft als Iraner beiden, in dieser doppelten Distanzierung eine Nähe zu finden. Dies wäre nicht möglich, käme Rachid aus Sri Lanka.

\section{Der Fremde im Vertrauten}

Rachids Fremdheit ist noch in einer anderen Hinsicht entscheidend für die Entwicklung des Paarhorizonts. Susanne etabliert während der Zeit in Sri Lanka und Indien eigene, individualisierte Geschlechterordnungen in der Beziehung zu Rachid. So erzählt sie beispielsweise Rachid, dass es in Deutschland selbstverständlich sei, dass jeder seine eigene Wäsche bügelt (vgl. 28/3 - 28/19). ${ }^{3}$ Sie übernimmt ein „kulturelles Copyright“ (Mecheril 1994) durch Erzählungen über Deutschland und deutsche Gepflogenheiten. Diesem kulturellen Copyright geht eine Fremdheitserfahrung Rachids voraus, wie sie z.B. Stenger beschreibt:

„Die Erfahrung kultureller Fremdheit gründet damit wesentlich im Erleben von Inkommensurabilitäten in der Dimension impliziten Wissens, also jenes nicht mitteilbaren Wissens, das als ,unausgesprochender Bezugsrahmen“ (Polany 1985: 24) Handlungen, Interaktionen und Kommunikationen anleitet" (Stenger 1998: 25).

Ein solch ,unausgesprochener ${ }^{\star}$ Bezugsrahmen wird von Susanne in ihrem Sinne expliziert und Rachid nahe gebracht, wissend aber um dessen Unterscheidung von in Deutschland vorherrschenden Modellen. Rachids Nicht-Zugehörigkeit wird von ihr nutzbar gemacht und kreativ gewendet, die Nicht-Zugehörigkeit dadurch gleichwohl verifiziert. Die Etablie-

zepte und -verantwortungen und im Widerspruch zur lebensweltlichen Realität, die es nicht ohne weiteres erlaubt, die natio-ethno-kulturelle Zugehörigkeit über Bord zu werfen.

3 Dies ist vor dem Hintergrund der damals vorherrschenden Arbeitsteilung durchaus pikant. In einer 1988 durchgeführten repräsentativen Studie wurden Paare nach der Verteilung der Hausarbeiten befragt, mit eindeutigem Ergebnis: ,Sowohl in Single-Earner als auch in Dual-Earner Haushalten werden die Routine-Hausarbeiten bei mehr als der Hälfte der Befragten entweder stets oder meist von der Frau erledigt - mit einer Ausnahme: Jeweils ca. 50\% der Männer in Dual-Earner-Familien meint, sich im gleichen Umfang wie die Partnerin am Geschirrspülen und am Einkaufen zu beteiligen. Die Männer sehen sich stets stärker beteiligt, als es die Frauen ihren Partnern zugestehen. Nur bei der Zuständigkeit der Frauen für die Wäsche ist die Einigkeit eindrucksvoll“" (Künzler 1995: 157). 
rung eigener Arbeitsteilungsmodelle kann so gelingen, fraglich ist allerdings, wie tragfähig sie sind.

Individualisierung ohne Bruch: das Herkunftsland und die Herkunftsfamilie als Ressource

In der Ratifizierung der Differenz zu ihrer Herkunftsfamilie und zu traditionellen Geschlechterverhältnissen, als die sich ihre Hinwendung zur Frauenbewegung und die Reise nach Indien und Sri Lanka lesen lässt, vollzieht Susanne jedoch keinen Bruch mit ihrer Herkunft. Im Gegenteil: Die Erfahrungen im Ausland zeigen ihr, wo ihre Wurzeln liegen:

„In der Fremde gemerkt hab dass mein Wurzeln schon hier liegen. Ja, also ich hab auch sehr große Fremdheit gespürt dort. - Ähm, zwar alles war das sehr aufregend und spannend aber ich hab dann schon das Gefühl gehabt also s_in den Gestalten denen ich begegnet bin, teilweise sie wirklich entwurzelt waren [...] und ähm, also das möchte ich eigentlich nicht" (9/4-9).

Im Begriff der Entwurzelung und der Fremdheit, wie er von Susanne gebraucht wird, wird ihre Notwendigkeit deutlich, auch physisch und geographisch zu Hause zu sein und ein solches zu haben. Der Auslandsaufenthalt nähert sie ihrer Familie und Deutschland wieder an, die unüberbrückbare Unvertrautheit, die sie bei anderen Reisenden erfährt, führt für sie zu einem Reflexionsprozess hinsichtlich ihrer Zugehörigkeit. Daraus folgen für sie zweierlei Konsequenzen: Zum einen will sie sich nicht gegen ihre gesamte Familie stellen, d.h. sie benötigt die Zustimmung der Eltern. Zum anderen kann eine Ehe nur unter ihren Bedingungen und in ihrem Land zustande kommen: „Und dann war mir klar, wenn Heirat dann nur hier. [...] Weil ich dann weiß welche Rechtslage" (8/26 - 28). Eine Ehe kommt für sie nur in Frage, wenn sie sich rechtlich absichern kann und die Fäden in der Hand behält. Dies schließt ein neues, drittes Land als dauerhaften Wohnort aus. In solch einem eher ökonomistischen Prinzip folgenden Verständnis von Ehe findet die Vorstellung einer ,romantischen Liebe‘, die Grenzen überwindet, keinen Platz. Erweitert werden können durch die binationale Beziehung vielmehr konventionelle Lebensläufe. Susannes finanzielle Überlegenheit und ihre Position als kulturell Wissende sichert ihr Handlungs- und Entscheidungsmöglichkeiten insbesondere hinsichtlich von Partnerschaftsmodellen und Lebensentscheidungen zu. Sie kann ihre bisherige Distanzierung von einem geschlechtsspezifischen Lebensentwurf beibehalten, ohne die Integration in ihre Familie oder ihre Herkunft aufs Spiel zu setzen. Vielmehr dient ihre Herkunftsfamilie als Ressource, die ihr den nö- 
tigen Rückhalt gibt. Ihre natio-ethno-kulturelle Zugehörigkeit und die Rachids bekommen in diesem Prozess eine strategische Funktion, basierend auf einem kulturellen Differenzverständnis. Das Verhältnis zwischen gender-Konstruktionen und Konstruktionen natio-ethno-kultureller Zugehörigkeit ist also keinesfalls ein additives, interessanterweise ermöglicht vielmehr gerade das Verständnis von natio-ethnokultureller Zugehörigkeit als Differenzkonstruktion eine Etablierung einer eigenen, individuellen Geschlechterbeziehung. ${ }^{4}$ Diese Modifizierung orientiert sich dabei an einer hierarchischen Ordnung, stellt diese nicht in Frage, kehrt sie aber wohl um.

\section{Konstruktion des Paarhorizonts bei Rachid: die Andere als Katalysator}

In Rachids Erzählungen über die Beziehung zu Susanne und die Entscheidung, mit ihr nach Deutschland zu gehen, finden sich ebenfalls RePositionierungen hinsichtlich traditioneller Geschlechterkonzepte. Anders hingegen als bei Susanne finden diese Modifikationen nicht auf der Basis einer Inferiorität des Beziehungspartners, sondern durch die Distanzierung und die räumliche Trennung von seiner Herkunftsfamilie und deren Erwartungen statt. Relevant ist in diesem Prozess der Horizontkonstruktion insbesondere der stattfindende Individualisierungsprozess, sowohl gegenüber seiner Herkunftsfamilie wie auch gegenüber normativen Beziehungsvorstellungen.

\section{Individualisierung I: die Herkunftsfamilie}

Rachid stellt in seiner Erzählung den ,alten' Iran mit seinen familiären Konzepten einem individualisierten, westlichem Beziehungsmodell entgegen. Diese Gegenüberstellung wird durch ihn und seine Mutter repräsentiert: Seine Mutter vertritt die traditionellen Werte und stellt den alten Iran dar, er selbst ist Repräsentant der jungen, selbständigen und individualistischen Generation, die Traditionen in Frage stellt und ihren eigenen Weg sucht. Die Individualisierungsbestrebungen beziehen sich $\mathrm{zu}$ Beginn der Migration auf den privaten Bereich, auf die Dimension des Individualisierungsbegriffs, der sich auf die Freisetzung bezieht: „Herauslösung aus historisch vorgegebenen Sozialformen und -bindungen im Sinne traditionaler Herrschafts- und Versorgungszusammenhänge“ (Beck 1986: 206). In diesem Zusammenhang stehen für ihn die Ein-

4 Solche Prozesse kann auch Waldis nachzeichnen, die daher von der Instrumentalisierung kultureller Differenz spricht (Waldis 2001: 148). 
schränkungen eines vorgegebenen Lebensweges, den seine Mutter für ihn entwickelt hat:

„Vielleicht auch ist e_zu viel gewesen die Erwartungen was meine Mutter auf mich überlagert hatte [...] Und sie hat auch damals ähm, die Tochter von ihrer Schwester, also meine Cousine mir versprochen gehabt. Also die beiden Schwestern miteinander [...] dass wenn ich fertig bin mit meiner Ausbildung ähm, ähm daß wir miteinander heiraten sollen. Und vielleicht sind dies die Punkte dass ich - ähm, unbewusst so weit weg getrieben hat.“ (9/8ff).

Der Konflikt entsteht für ihn jedoch insbesondere in Bezug auf divergierende Konzepte von Geschlechterordnungen und Eheformen. Die Ehe, die seine Mutter für ihn arrangiert hat, ist für ihn ein weiteres Zeichen des Traditions-Modernitäts-Paradigmas. „Dabei wird die arrangierte Partnerwahl eindeutig dem Bereich des traditionellen, rückwärtsgewandten Fremden zugewiesen, während die so genannte freie Partnerwahl als Eigenschaft des fortschrittlichen emanzipierten Westens fungiert" (Straßburger 2003: 177). Das westliche Ideal kolportiert die Idee der Liebe. Liebe steht dabei außerhalb einer rationalen, kalkulierbaren Basis und macht diese überflüssig (vgl. dazu ausführlich Burkart/Koppetsch 2001). Eine arrangierte Ehe mit einer iranischen Frau zu führen, wäre für ihn undenkbar. Auch im weiteren Verlauf der Beziehung zwischen Susanne und Rachid bestätigt sich die Individualisierung und Distanzierung von seiner Mutter: Als sie das erste Mal (von insgesamt nur drei Besuchen) nach Deutschland kommt, ist sie „entsetzt“ (9/16) über Rachids Mitarbeit in der Küche und darüber, dass Susanne „nicht den Haushalt führt wie im Iran die Frauen tun“ (9/17). Rachids Mutter möchte zu Susannes Vater gehen und sich bei ihm beschweren, so dass Rachid und Susanne viel Mühe haben, ihr das Vorhaben auszureden und die Arbeitsteilung als kulturellen Unterschied zu erklären. Der Konflikt lässt sich für Rachid nur durch räumliche Trennung lösen: „Möglichst weit, dass diese Frau keine direkten Einfluss auf meine private Leben hat" (9/12). Das Auslandsstudium gibt Rachid die Möglichkeit zur räumlichen Distanz und bereitet die endgültige Migration vor. Die Beziehung zu Susanne und die Entscheidung nach Deutschland zu gehen bedeutet für ihn also ebenfalls eine Distanzierung von herkömmlichen Geschlechterkonzepten. Dabei stand für ihn Deutschland als konkretes Migrationsziel vor der Begegnung mit Susanne nicht zur Auswahl: „Als ich jung war, ich wollte nach Ausland aber nie nach Deutschland“" (10/16). Entscheidend war für ihn die Beziehung zu Susanne. 


\section{Individualisierung II: die Beziehung}

Rachids Individualisierungsbestrebungen sind noch in anderer Hinsicht für die Entstehung des Paarhorizonts entscheidend. Für Rachid ist nicht die Binationalität das Besondere, im Grunde seien alle Beziehungen ,interkulturell' oder ,bi“, „weil die Männer sind doch ganz anders als Frauen“ (21/29). Dieser fundamentale Unterschied zwischen Männern und Frauen, der auf unterschiedlichen Denkstrukturen beruht (,also vom Kopf her" (21/29)), nicht auf einem Unterschied in der Intelligenz oder den Fähigkeiten, wird noch gesteigert durch das Aufeinandertreffen zweier Kulturen. Es ist ein additives Verständnis des Verhältnisses von Geschlechterbeziehungen und natio-ethno-kultureller Zugehörigkeit, das hier formuliert wird. Zwischengeschlechtliche Beziehungen sind per se kompliziert und erfordern Arbeit, die interkulturelle Komponente kommt verstärkend aber nicht verändernd hinzu. Im Gegensatz zu den Unterschieden zwischen Männern und Frauen, die sich seiner Meinung nach nicht ändern lassen, sind kulturell bedingte Unterschiede reflexiv zugänglich, individuell verschieden und damit auch bearbeitbar. In diesem Zusammenhang ist für ihn eine Distanzierung von traditionalen Inklusionszusammenhängen Grundlage und Bedingung für die funktionierende Paarbeziehung. Konkret begünstigen insbesondere zwei Faktoren das Eingehen und das Bestehen der Beziehung: die räumliche Distanz zu seiner Herkunftsfamilie, insbesondere zu seiner Mutter, und seine persönliche Distanz zur iranischen und seine sich entwickelnde Nähe zur deutschen Kultur: „Dass ich eher viel mehr von deutscher Kultur übernommen habe. Und das führt zu Erleichterung von unserer Beziehung“" (22/13f.). Im Unterschied zu Susanne, deren Beziehungsvorstellungen den Bruch mit der Familie nicht beinhalten, sieht Rachid in der räumlichen Distanz eine Notwendigkeit, wenn denn die Beziehung in seinem Sinne funktionieren soll. Ihm ist der Einfluss der Umgebung auf die Ausgestaltung von Geschlechterbeziehungen bewusst. Rachid stellt in diesem Zusammenhang eine einengende, klar geschlechtlich definierte Gesellschafts- und Paarstruktur im Iran einer offenen, flexibel aushandelbaren und viele Partnerschaftsmodelle ermöglichende Situation in Deutschland gegenüber. Diese Offenheit ist von ihm erwünscht. Sie bedeutet in der Folge zum einen, die Distanz zu seinem Herkunftsland und seiner -familie aufrecht zu erhalten. Zum anderen muss die Flexibilität hinsichtlich ,kultureller Eigenheiten“ von den Beziehungspartner(inne)n genutzt werden, um die Beziehung aufrecht zu erhalten.

Der Horizont, der von Rachid eröffnet wird, ermöglicht ihm die Realisierung eines individualistischen Beziehungsverständnisses, welches sich von traditionellen Geschlechterordnungen löst. Die Tatsache, dass 
Susanne nicht aus dem Iran stammt, sondern aus einem westlichen Land, ermöglicht die Abkehr von seiner Familie und deren Erwartungen. Susannes Position als kulturell Andere ist also grundlegend notwendig für den Beginn einer Beziehung, die nicht auf einengenden, eindeutigen Geschlechterrollen basiert, in der vielmehr Flexibilität konstitutiv eingebaut ist. Das Verhältnis zwischen gender und natio-ethno-kultureller Zugehörigkeit stellt sich dabei als mehrstufiges Verhältnis dar. Während sich die Unterschiede zwischen Mann und Frau nicht verändern lassen und damit die konstante Basis einer heterosexuellen Beziehung darstellen, sind natio-ethno-kulturelle Routinen reflexiv zugänglich und können bearbeitet und modifiziert werden.

\section{Der Fremde und die Andere: Komplementarität}

Das Verbindende des Paarhorizonts liegt in der Komplementarität der Bedeutung der Beziehung. Spezifische Erwartungen lassen sich durch den bzw. die jeweils andere(n) realisieren. Die Beziehung lässt sich in das jeweilige Lebenskonzept integrieren und erfüllt eigene Erwartungen. Sie fungiert so als handlungserweiterndes Moment. Handlungserweiterung bezieht sich hier zum einen auf Handlungsmöglichkeiten und Realisierungen von Erwartungen und Konzepten, zum anderen auf die Einflussnahme und Reichweite subjektiver Handlungen. Die Handlungserweiterung für Susanne und Rachid ist eine Folge der zusätzlichen Ressource, als die man die Beziehung verstehen kann. Die veränderte biographische Situation erweitert den Handlungsrahmen: Die „biographisch bestimmte Situation erschließt gewisse Möglichkeiten künftiger praktischer oder theoretischer Tätigkeit, die [...] verfügbare Ziele“ genannt werden“ (Schütz 1971: 11). Als ein solches verfügbares Ziel lässt sich bei beiden Beziehungspartner(inne)n u.a. die Loslösung aus traditionalen Geschlechterordnungen analysieren. ${ }^{5}$ Wenn sich in diesem Fall der Handlungsraum durch die Beziehung erweitert, ist damit noch nichts über die Reichweite der Folgen der Handlungen gesagt. Inwiefern sich solche Entwicklungen von Lebensmodellen auch nachhaltig etablieren, lässt sich nur über die biographische Gesamterzählung hinweg verfolgen.

Als Schlüsselbegriffe eines solchen, eher durch Funktionalität strukturierten Paarhorizonts können das Konzept der ,kulturell Anderen“ bei

5 Die Handlungserweiterung bezieht sich auf die beiden zur Diskussion stehenden Kategorien. Dass eine Erweiterung hinsichtlich einiger Möglichkeiten sicherlich auch zur Verengung von Möglichkeiten in anderen Bereichen führen kann, zeigt sich z.B. in den notwendig werdenden Bearbeitungen im Laufe der Beziehung. 
Rachid und des ,kulturell Fremden' bei Susanne gelten. Rachid sucht (und findet in Susanne) eine Beziehungspartnerin, die es ihm ermöglicht, seine Distanz zum Iran und dem dort vorherrschenden Ehe- und Beziehungsmodell aufrecht zuerhalten und seine Vorstellung einer individualistischen Beziehung zu verwirklichen. Dafür ist Susannes Position als kulturell Andere, aus einem anderen kulturellen und gesellschaftlichen Kontext stammend, relevant und wesentlich. Unter anderem kann er so auch die Beziehungsform gegenüber seiner Verwandtschaft rechtfertigen. Susanne dagegen nutzt Rachids Position als kulturell Fremder. Er ist dadurch in verschiedener Hinsicht unterlegen, eine Konstellation, die es ihr erlaubt, ihre Vorstellungen einer Beziehung mit eigenen Geschlechterkonzepten, gekennzeichnet durch ihre Vormacht-Stellung in finanzieller und gesellschaftlicher Hinsicht, zu verwirklichen. ${ }^{6}$

\section{Thema der Beziehung: Nachhaltigkeit}

Das female breadwinner Modell erscheint in diesem Rahmen sicherlich nicht als konfliktuös. Vielmehr lässt es sich bei beiden als biographische Passung verstehen, es folgt aus der Entscheidung für diese Beziehung und für das Leben in Deutschland. In der biographischen Darstellung der beiden erscheint es daher auch nicht als unerwartete Folge, mit der sie konfrontiert werden, sondern vielmehr als spezifischer Ausdruck der Gestaltung des Paarhorizonts der Beziehung: Für Susanne bedeutet das Modell die Fortsetzung ihrer angestrebten Unabhängigkeit und Umkehr der klassischen Geschlechterordnung, für Rachid ist es eine Folge seiner Migrationsentscheidung und der Entscheidung für ein anderes Leben. Als Thema der Beziehung lässt sich für Susanne und Rachid die Problematik einer nachhaltigen Umsetzung eines , selbstbestimmten' Lebens benennen. Die Distanzierung von herkömmlichen Geschlechterordnungen und die Schwierigkeiten bei der Beibehaltung dieser Distanzierung bestimmen die Entwicklungen in der Beziehung und strukturieren die Formen biographischen Lernens.

6 Ein weiteres interessantes Merkmal liegt in dem von ihnen konzipierten Geschlechterverhältnis. Beide gehen, trotz der scheinbar unterschiedlichen Strategien, von einer prinzipiellen Unterschiedlichkeit der Geschlechter aus. Susanne hat eine differenztheoretische Vorstellung einer Geschlechterbeziehung. Auch Rachid geht von dem Aufeinandertreffen zweier Welten in einer heterosexuellen Beziehung aus. 


\section{Bearbeitungsfelder}

\section{Bearbeitungsfelder bei Susanne: Innere und Äußere (Un-)Abhängigkeit}

In der biographischen Erzählung von Susanne zeigt sich deutlich, wie die Entscheidung für ein Leben in Deutschland Zuordnungen notwendig macht bzw. erfordert. Der Horizont ihrer Partnerschaft, der Susanne einerseits bestimmte Möglichkeiten eröffnet hat, bringt im Gegenzug Einschränkungen bzw. Folgen mit sich, mit denen sie sich konfrontiert sieht. Diese liegen sowohl auf der Ebene der Rückkehr in ihren alten Lebenskontext und dessen Erwartungen und Anforderungen, wie auch auf ihrem Changieren zwischen alternativen Lebenskonzepten und dem Integrationswunsch. Nicht das female breadwinner Modell stellt in ihrer Definition den Bruch mit der Lebensplanung dar, vielmehr findet der Bruch bei dem Versuch statt, das Modell langfristig zu realisieren und in ihren alten Kontext zu integrieren.

\section{Ressourcen als Begrenzungen}

Susanne reist nach Asien als bisexuelle links-alternative Feministin, für die Heiraten auf keinen Fall in Frage kommt. Sie kommt zurück und wird Ehefrau eines Iraners. Die Gestaltung dieser Beziehung, die es Susanne überhaupt ermöglicht, eine Beziehung und sogar eine Ehe einzugehen, lässt sich allerdings in der Form in Deutschland nicht mehr aufrechterhalten. Dies folgt aus der Konfrontation mit ihrem sozialen Nahraum, der irritiert auf die ,falsche" Arbeitsteilung in ihrer Partnerschaft reagiert. Die Schwierigkeiten, die ihnen in der Interaktion mit anderen begegnen, lassen sich sehr plastisch und exemplarisch an der Aufteilung der Hausarbeit festmachen. Rachid erlebt in einer Begegnung mit einem Mitbewohner dessen Unverständnis darüber, dass Rachid seine eigenen Hemden bügelt. Als Rachid bei Susanne erstaunt nachfragt, sie habe ihm in Sri Lanka doch von einer egalitären Hausarbeitsteilung erzählt, muss sie ihm erklären, dass „das, was ich hier als Realität verkauf nicht die Realität hier ist“" (28/14f.). In der (Re-)Konstruktion lässt sich zeigen, dass gerade die Aushandlungsnotwendigkeit Chancen zur Selbstgestaltung und Spielraum bei der Definition von geschlechtlich strukturierten Bereichen bietet. Es zeigt sich allerdings auch, dass in Interaktion mit familialen oder außerfamilialen Bezugspersonen die Grenzen eigener Deutungsmacht deutlich werden: Eigene Geschlechterbeziehungen müssen in Auseinandersetzung mit und zum Teil gegen die Wirkungsmacht gesellschaftlicher Geschlechterverhältnisse etabliert und realisiert wer- 
den, strukturelle Geschlechterverhältnisse stellen also deutlich mehr dar als nur den ,kulturellen Kontext", auf dessen Folie subjektive Konstruktionen und (Re-)Konstruktionen stattfinden. Vielmehr müssen die gesellschaftlichen Verhältnisse als ,historische Konfiguration von Austausch- und Interdepenzendverhältnissen und als systematischer $\mathrm{Zu}$ sammenhang“ (Knapp 2002: 41) verstanden werden, die machtvoll in den Konstruktionsprozess eingreifen und diesen strukturieren. Des Weiteren aber erfährt Susanne Folgen ihrer Handlungen, mit denen sie nicht gerechnet hat und deren Folgen sich für sie als durchaus problematisch darstellen. Der Beginn der Ehe ist für sie nicht gleichbedeutend mit dem Beginn einer Partnerschaft. Insofern sind die Folgen, die die Ehe für sie hat, nämlich eine gemeinsame Wohnung bzw. ein gemeinsames Zimmer und die Selbstdefinition als Paar, in ihrer Wirkung bearbeitungsbedürftig. Nach außen hin versucht sie, die Ehe zu verbergen bzw. die damit verbundenen und von ihr vermuteten Reaktionen zu verhindern. Sie behält ihren Namen und verschweigt an ihrer Arbeitsstelle ihren neuen Status (vgl. 10/24 - 26).

Neben diesen Statusirritationen stellt sich insbesondere die finanzielle Situation und das female breadwinner Modell längerfristig als Schwierigkeit heraus. Susannes Verständnis vom Zusammenhang von finanziellem Beitrag und Bestimmung über die Verfügung dieser Beiträge bringt die Beziehung in Schwierigkeiten: „Also, schon auch wer macht was, weil ich hab dann so gemerkt, ich als ich Geld verdient hab (lacht kurz auf) praktisch wollt ich dann auch bestimmen für was man's ausgibt. Also es war für mich nicht unser gemeinsames Geld“" (25/24ff.). Die Vorteile, die sie durch die Beziehung zu Rachid als kulturell und sozial Fremden gesehen hat, verkehren sich im Alltag in Deutschland in Nachteile: Er ist „ewig abhängig letztlich“ (10/31), sowohl finanziell als auch hinsichtlich der sozialen Verortung. Ihr Verständnis von Berufstätigkeit als Garantin biographischer Handlungsfähigkeit (vgl. Thon 2006: 395) zieht Verantwortlichkeiten und Abhängigkeiten nach sich, mit denen sie nicht gerechnet hat, und die sie in ihrer Handlungsfähigkeit wieder beeinträchtigen In dieser Konstruktion zeigt sich die Kehrseite ihrer machtvollen Position: Zwar ist sie im Besitz der finanziellen Ressourcen der Beziehung, diese muss sie jedoch entsprechend dem Primat einer Ehe mit Rachid teilen (vgl. zu Macht und Ressourcen in der Beziehung auch Streckeisen 1993). Diese Verantwortung will sie auf Dauer nicht übernehmen.

Der Paarhorizont, im ,dritten Land' aufgespannt und implementiert, zeigt sich mit seinen Kehrseiten: Susannes Auseinandersetzungen mit (Un-)Abhängigkeiten zeigen ihr die Grenzen ihrer Gestaltungsmöglichkeiten und die mehrdeutigen Folgen, die Migrationsprozesse über die 
individuellen Vorstellungen hinaus mit sich bringen. Die Rückkehr in ihr Herkunftsland, bislang als Machtressource genutzt, bringt nun Schwierigkeiten und Folgen mit sich, die eine Veränderung ihrer Orientierungs- und Handlungsmuster nahe legen.

\section{Integrationsarbeit}

Die Situationen, vor die sich Susanne gestellt sieht, führen zu ihren verstärkten Bemühungen der Integration Rachids in hiesige Gesellschaftskonzepte. Integration bedeutet für sie in diesem Fall auch die Anpassung der Beziehung an bestimmte gesellschaftlich anerkannte Konzepte und Arbeitsteilungen. Die Anerkennung und Annahme solcher Konzepte führen in der biographischen Erfahrung jedoch zu einer Beschneidung der Selbstbestimmung und Unabhängigkeit Susannes, welche sie wiederum als hoch problematisch empfindet. Die Schwierigkeiten, die bei der Annahme dieser Orientierungsmuster entstehen, lassen sich plastisch am Beispiel der Entscheidung für ein Kind und der Aufteilung der Kindererziehung festmachen.

Der Abbruch des Studiums ist für Rachid in Susannes Erinnerung ein einschneidendes Erlebnis, ,weil das war einfach so klar von seiner Wertigkeit Studium ist das Wichtigste im Leben“ (12/31f.). Rachid geht zurück in den Iran, um sich über sich selbst und den weiteren Lebensweg klar zu werden. Bei der Rückkehr nach Deutschland und mit dem Beginn von Rachids finanzieller Unabhängigkeit durch die Aufnahme einer festen, wenngleich statusniedrigen, Erwerbsarbeit, verändern sich auch die Entscheidungsmodi. Das finanzielle Auskommen ist durch Rachid gesichert und sie entscheiden sich für ein Kind. Susanne wird sofort schwanger. Mit der Geburt beginnt ein Prozess, den Susanne eine ,existenzielle Krise“ (14/21) nennt. Sie ziehen in das Haus ihrer Eltern mit ein, und während Susanne die Kindererziehung übernimmt, verdient Rachid das Geld für die Familie: „Also da ist so mein Leben so ziemlich auf den Kopf gestellt gewesen. Da war gar nichts mehr klar. Da gings mir auch net so arg gut muss ich sagen“ (13/28 - 30). Die Rückkehr bzw. die Veränderung ihrer Aufgabenverteilung hin zu einer herkömmlichen Rollenverteilung bringt sie in große Schwierigkeiten. Susannes „ganze Frauenrolle“ wird ,auch noch mal durcheinander gewirbelt“ (13/23f.). Ihr Selbstverständnis einer emanzipierten, unabhängigen Frau wird durch die Entscheidung für die traditionelle Aufteilung in Frage gestellt. Das erste Kind symbolisiert dabei in diesem Prozess den Schlüsselpunkt, der in ihrer Vorstellung und dann auch in ihrer Erfahrung die Unabhängigkeit beschneidet (vgl. 13/15). Für Susanne stehen äußere Ressourcen wie die Erwerbsarbeit und der finanzielle Verdienst einer- 
seits und die Selbständigkeit und Emanzipation andererseits in einem unmittelbaren Verhältnis. Hier zeigt sich ein Deutungsmuster, welches zum Selbstverständnis der Frauenforschung und der Frauenbewegung in den 1980er Jahren gehörte: Emanzipation kann nur durch Erwerbsarbeit und durch finanzielle Unabhängigkeit vom Mann erreicht werden (vgl. kritisch dazu z.B. Budde 1997). Der Verlust des äußeren Status` führt Susanne in eine existentielle Krise, in deren Verlauf sie nur noch reagieren, nicht mehr agieren kann.

\section{Auswege aus der Krise: Modifikation und Annäherung}

Nach dieser Erfahrung der Hilflosigkeit übernimmt sie erneut die Gestaltung ihres Lebens und sucht nach einer Lösung, um baldmöglichst die Erwerbsarbeit wieder aufnehmen zu könnnen. Sie findet sie u.a. in der Aktualisierung Rachids natio-ethno-kultureller Herkunft und damit in der Verwendung von kulturellen Zuschreibungen als strategisches Instrument: „Ich hab zum Rachid gesagt horch einmal, wenn du von deiner Kultur was weitergeben willst, dann werds doch auch gut du täts reduzieren“. Natio-ethno-kulturelle Zugehörigkeit als Differenzkategorie ist nicht in jeder biographischen Situation gleich relevant. Vielmehr ist ihre Bedeutung von der spezifischen Konstellation und Situation abhängig (vgl. dazu auch Hardach-Pinke 1988, die eine zunehmende Individualisierung kultureller Differenzen im Verlauf der Beziehung analysiert, oder auch Breger/Hill 1998). Zu diesem Zeitpunkt wird Rachids ,andere‘ Kultur als eine Art ,Lockvogel' eingesetzt, um ihn zur Mitarbeit bei der Kindererziehung und zur Arbeitsstundenreduzierung zu bewegen. Wie bei der ersten Begegnung in Sri Lanka nutzt sie Rachids ,Fremdheit‘ als Unterstützung ihrer Argumentation. Der Rückgriff auf ,Kultur', auf die kulturelle Differenz und auf die generative Bedeutung von Kultur dient hier sowohl als Argument wie auch als Legitimation. Als Argument, um Rachid von einer veränderten Arbeitsteilung zu überzeugen. Als Legitimation, um sich gegenüber dem sozialen Nahraum abzusichern. Erneut also fungiert die binationale Beziehung als Erweiterung ihres Handlungsraums, nachdem sie in der vorherigen Figur ihre Gestaltungsmöglichkeiten ja eher eingeschränkt hat. Rachid reduziert seine wöchentliche Arbeitszeit, und sie teilen sich die Erwerbsarbeit und die Kindererziehung, das female breadwinner Modell wird durch ein dual earner Modell abgelöst. Auch ein solches Modell wird von ihr als „sehr revolutionär damals“ (13/33 - 14/1) empfunden. Möglichkeiten vervielfältigen sich durch die binationale Beziehung, und durch die gleichberechtigte Teilung von Erwerbs- und Familienarbeit behält für Susanne ihre Distanzierung von traditionellen Geschlechterbeziehungen 
Relevanz. Susannes Wunsch nach Unabhängigkeit und der Realisierung ihrer Distanz zur klassischen Geschlechterbeziehung führt in einer Phase der Abhängigkeit zu einer existenziellen Krise. Dies ist auch eine Folge der (selbstgewählten) Integration und Nähe zu ihren Eltern und ihrem Herkunftsort. Die Rückkehr in ihren alten Kontext macht Anpassungsleistungen erforderlich, die jedoch wiederum Probleme bereiten. Innovative Lebensmodelle müssen gesucht werden, als Susanne mit der Situation nicht mehr klarkommt. Die Möglichkeiten, die sie sich durch die Beziehung eröffnet hat, verschließen sich also nicht wieder, trotz der Schwierigkeiten, denen sich Susanne ausgesetzt sieht. Zudem bleiben ihr durch die binationale Beziehung und die Rückgriffsmöglichkeit auf natio-ethno-kulturelle Kategorien sowohl erweiterte Handlungsmöglichkeiten wie auch eine besondere Stellung in der Wieder-Einordnung in ihren alten Herkunftskontext erhalten, Merkmale wie die besondere Arbeitsteilung und die auch äußerlich sichtbare ,andere' Herkunft Rachids verdeutlichen dies. ${ }^{7}$ So kann sie sich zum Zeitpunkt des Interviews auch vorstellen, ganz aufzuhören zu arbeiten, eine Möglichkeit die für sie lange undenkbar gewesen ist. Sie muss sich ihre Unabhängigkeit „nicht mehr beweisen“ (19/26). Die äußere Unabhängigkeit wird tendenziell durch eine innere Unabhängigkeit abgelöst. Die Bindung zu ihrem Herkunftskontext und die damit für sie notwendig einhergehende Akzeptanz spezifischer Rahmenbedingungen ist konstitutives Element des Aushandlungsprozesses. Sich (zu) deutlich gegen herkömmliche Muster aufzulehnen, war für sie nicht möglich: „Ham wir doch drunter gelitten dass mir so frei wie wir eigentlich dachten wir sind hier dann doch net waren“ (18/1f). Die im ,dritten Land" entwickelten und etablierten Geschlechterbziehungen lassen sich nicht ohne weiteres auf das Leben in Deutschland übertragen. Zu deutlich stehen sie den herrschenden Geschlechterordnungen gegenüber. Zudem muss Susanne bei der nachhaltigen Etablierung des stark hierarchisierten Beziehungsmodells feststellen, dass nicht-intendierte Folgen bearbeitet werden müssen, die ihr die Kehrseite ihrer Erwartungen deutlich machen. An einer solchen Biographie zeigt sich die Zähigkeit herrschender Geschlechterordnungen. Ohne einen Bruch riskieren zu wollen, können eigene Geschlechterbeziehungen nicht unbegrenzt etabliert werden. Vielmehr werden in der Auseinandersetzung mit äußeren und inneren Grenzen Anpassungsleistungen erforderlich.

7 Susanne und Rachid haben die Irritation, die Rachid hervorruft, im gemeinsamen Nachgespräch am Beispiel des Wanderns, ein Hobby der beiden, verdeutlicht und ironisch kommentiert. Das auch Nicht-Deutsche gerne und viel wandern, das scheint den meisten unbegreiflich, so dass die beiden immer wieder erstaunten Blicken ausgesetzt sind. 


\section{Bearbeitungsfelder bei Rachid: (Re-)Positionierungen}

Anders als für Susanne ist für Rachid die Entscheidung nach Deutschland $\mathrm{zu}$ gehen der sichtbare Ausdruck einer deutlicheren Abgrenzung von seiner Herkunftsfamilie und seinem Herkunftsmilieu. In diesem $\mathrm{Zu}-$ sammenhang steht auch die Bedeutung des female breadwinner Modells für ihn. Es handelt sich ebenfalls nicht um einen Bruch, eine unerwartete Wendung bisheriger Planungen. Vielmehr integriert sich das Modell in die Migrationsfolgen und bedeutet für ihn einen Handlungs- und Möglichkeitszuwachs hinsichtlich der gewünschten Realisierung von individuellen Lebensmodellen. Der Möglichkeitszuwachs, den Rachid durch die Migration und die Heirat mit Susanne, der ,Anderen', aufmacht, wirkt auch als handlungsorientierende Kategorie bei der Bearbeitung der beruflichen Dequalifizierungsprozesse. In seiner biographischen Darstellung lässt sich zweierlei zeigen: zum einen die biographisch ,gelungene“ Bearbeitung von ungewöhnlichen sozialen und beruflichen Konstellationen, die herkömmlich als Krisen verstanden werden (vgl. zur Arbeitslosigkeit von Männern z.B. Heinemeier 1991; Schreyer 1991; Uhl 1991; Blech/Witte 1992; Bolak 1997). Zum anderen lassen sich aber auch Verschiebungen von natio-ethno-kulturellen Zugehörigkeiten und eine Entscheidungsnotwendigkeit hinsichtlich seiner natio-ethno-kulturellen Positionierung nachzeichnen. Während für Susanne das Thema ,Nachhaltigkeit‘ vor allem hinsichtlich der Etablierung ihrer ungewöhnlichen Geschlechterbeziehung relevant wird, ist für Rachid die Nachhaltigkeit in der Etablierung seiner Distanzierung von seiner Herkunftsfamilie und der weiteren Entwicklung eines individualisierten Lebens relevant.

\section{Das „reiche Leben“ (26/28): Weiterentwicklungen}

Für Rachid wird die Migration zu einer Möglichkeit der Neuerfahrung und Neu-orientierung. Er versteht sich als privilegiert, insbesondere im Vergleich zu den älteren Generation in Deutschland und im Iran, die „mit zwanzig einen Beruf gelernt hat und bis siebzig durchgeführt“ (26/17f.) hat. Demgegenüber setzt er berufliche Wahlmöglichkeiten und die Möglichkeit, individuellen Bedürfnissen nachgehen zu können: „Und ich denk das gehört auch zu dieser Freiheit hier, dass man, wenn man finanziell Möglichkeiten hat dass man sich nicht festsetzt und sich quält mit einem Beruf" (16/18 - 20). Eine solche Freiheit hätte er so im Iran nicht gehabt: „Das ist auch wieder so positives Punkt von Deutschland das ich's drauf zurückgreif oder ausnutze“ (26/23f.). Die Destandardisierung des Lebenslaufs und der Verlust des inneren Rückgrats der Lebensführung (vgl. Beck 1986: 222), das eine kontinuierliche Berufs- 
biographie bislang darstellte, sind für ihn positiv konnotiert, sie verunsichern ihn nicht. Diese „Berufsfreiheit“ (26/26) gehört zu seinem Selbstverständnis und zu seinen Erwartungen an sich selbst dazu. Voraussetzung für ein „reiches Leben“ (26/28) ist es, Erfahrungen zu sammeln und Dinge auszuprobieren. In diesem Kontext versteht er auch seine unterschiedlichen Arbeitsstellen als Möglichkeit, Einblicke in verschiedene Lebensbereiche $\mathrm{zu}$ bekommen, unabhängig vom Lohnniveau. Sie sind nicht primär Durchgangsstationen auf dem Weg zu einem erfüllenden Beruf, sondern eigenständige Elemente seiner Biographie, die ihm mehr Erfahrungen in unterschiedlichen beruflichen und sozialen Kontexten ermöglichen. Ein individueller Lebensentwurf wird über die (finanziell) gesicherte Existenz gestellt, Umwege und Verlängerungen der finanziellen Abhängigkeit von Susanne nimmt er in Kauf, um einen Beruf zu finden, der nicht „eng“ (4/21) oder „einseitig“ (4/24) ist. Zu einem solch reichen Leben und zu dem bei Rachid analysierbaren Verständnis von Selbstentwicklung gehört ebenfalls die Hinterfragung von einengenden Kategorien und Zugehörigkeiten:

„Und dann seh ich auch bei vielen Leuten [...] damals als ich bei Kreissparkasse geschafft habe wie die dich hochgest_angesehen haben also dass ich, Mensch, schaffst du bei Kreissparkasse obwohl, ich war gleiche Person gewesen. Also bei mir an Persönlichkeit oder bei mir an Person hat sich nichts geändert, nur weil ich jetzt bei einer bestimmten Institution geschafft hab die haben mich woanders gesehen oder eine andere Person gesehen“"(27/4 - 11).

Einem solchen „Schubladendenken von Menschen“ (27/16) versucht er entgegenzuwirken. Die Loslösung der Beurteilung von Personen von ihrem beruflichen Status, die Anerkennung von Vielfalt und Differenz und eine Offenheit gegenüber neuen Erfahrungen fordern zum reflexiven Umgang auf.

Solch einen reflexiven Umgang mit Biographien erwartet er von seiner Herkunftsfamilie nicht. Diese hatte sich von seiner Migration nach Deutschland erheblich mehr an beruflicher Qualifikation erhofft, eine Lehre als Mechaniker sei nicht genug (vgl. 14/31 - 15/5). Rachid distanziert sich von diesen Erwartungen und weist deutlich auf den Mehrwert hin, den er durch seinen Weg erhalten hat:

„,ich hatte Glück oder die Sachen die ich jetzt in meinen Leben gemacht hab, danach die ganze Zeit hab ich, vielleicht wenn ich studiert hätte dann hätte ich keine Zeit gehabt für diese Sachen. [...] mach auch gerade eine dreijährige Ausbildung als Körperarbeit. [...] und das ist erst, sehr emotional und ähm das wär nicht möglich gewesen solche Sachen“ (15/6 - 11). 
Dieses Motiv der intrinsischen Bildungsmotivation und der biographischen Offenheit ist für ihn auch in der Beziehung zu Susanne relevant. Auch hier betont er die Impulse, die er durch Susanne und ihre Freunde erhalten hat und die ihm bei der Weiterentwicklung seiner Persönlichkeit geholfen haben und immer noch helfen.

„Also, allein die Auseinandersetzung im Leben - durch ihr --, ähm, mein Religion oder glaub sie gibt sehr viele Impulse die ich positiv finde und dahinter gehe. Und allein wenn ich mal heimkomme und irgendein Buch irgendwo liegt was sie gelesen hat oder was da sind, das begeistert mich auch und lese ich auch unbedingt miteinander" $(24 / 13-17)$.

Persönlichkeitsentwicklung wird so zum biographischen Entwicklungsprojekt, welches über einen rein intellektuellen Lernprozess hinausgeht und den ,ganzen Menschen“ umfasst (vgl. dazu auch Benner 1990).

\section{Verortung oder: der Preis der Denkfreiheit}

Parallel zu diesem Prozess der Handlungserweiterung und Entwicklung des ,reichen Lebens' lässt sich ein weiterer Entwicklungsprozess in der Biographie Rachids analysieren. Rachids Distanzierung vom Iran und dem dort vorherrschenden Gesellschaftsmodell verstärkt sich im Laufe der Migration. Er vollzieht im Laufe seiner Migrationsgeschichte eine deutliche Zuordnung zu Deutschland, sowohl in der Außenwirkung wie auch in seinem Selbstverständnis. Ausgangspunkt seiner Abgrenzung stellt dabei die sich kurz nach seiner Ausreise etablierende Staatsform des ,Gottesstaates' im Iran (vgl. zur Situation im Iran der 1970er und 1980er Jahre Tilgner 1979; Benard/Khalilzad 1984). Die von ihm formulierte Kritik richtet sich daher insbesondere gegen eine herrschaftsgläubige „engstirnige Auslegung“ (8/20) von Religion und Lebensführung: „dass man die Leute nu_nur so in Schach hält in einem bestimmten Kreis und auch befehlt dass sie nicht anders denken sollen, dass sie nur das was gesagt wird für diese Religion, und außerhalb dieser Mauer gibt's nichts, und darf man nicht denken, darf man nicht fragen“" $(8 / 20$ 25). Das Symbol der Mauer dient hier sowohl zur Verdeutlichung des beschränkten Horizonts wie auch als Bollwerk gegen ,außen', gegen neue und andere Eindrücke. Dagegen stellt er das Bild des aufgeklärten Europas, das sich inzwischen, am Anfang des 21. Jahrhunderts, von seiner Tradition der einengenden Religion verabschiedet hat und nun selbstbestimmtes Leben des Glaubens ermöglicht. Er kann sich nicht mehr vorstellen, in seinem Ruhestand in den Iran zurückzukehren. Es käme einem Rückschritt gleich, die „Denkfreiheit““ (7/30) aufzugeben. 
Hat sich seine Kritik und seine Distanz zu Beginn der Migration also vor allem auf die normativen Erwartungen der iranischen Gesellschaft, vermittelt durch seine Mutter, bezogen, erweitert er sie nun auf den gesamten Staat. Die Kritik an der iranischen Gesellschafts- und Geschlechterordnung aber bleibt. Natio-ethno-kulturelle Zugehörigkeit wird von ihm zu diesem Zeitpunkt unter dem Aspekt des natio-ethno-kulturellen Handelns konzipiert (vgl. Mecheril 2003: 162ff). Er selber sieht sich als „deutscher als deutsch“ (23/9) und verortet sich in seinen Handlungen inzwischen eindeutig in Deutschland. Diese Zuordnung und sein Wille zum Lernen und zur Übernahme der ,deutschen Kultur“ war, so seine Rekonstruktion, entscheidend für den Bestand der Beziehung zu Susanne, ,und denke ich das hilft auch. Das ich eher viel mehr von deutscher Kultur übernommen habe" (22/13f.). ${ }^{8}$ Insbesondere hinsichtlich der geschlechtlichen Arbeitsteilung sieht er hierbei die relevanten Unterschiede. Im Iran würde viel eher erwartet, ,dass die Frau ihre Rolle als Frau übernimmt“ und „der Mann auch so lebt wie der sein soll“ (22/16 - 18), dass also individuelle Entscheidungen hinsichtlich der Lebensführung kaum möglich seien. Die Freiheiten, die er durch die liberalen und individuellen Lebensmöglichkeiten in Deutschland erlebt hat und die die Realisierung ihrer individuellen Lebenskonzepte ermöglicht hat, sind für ihn zu entscheidenden Aspekten seiner Selbstpräsentation und seines Lebensverständnisses geworden, Aspekte, die er nicht mehr bereit ist aufzugeben. So wäre für ihn auch eine zweite Ehe mit einer Iranerin ausgeschlossen (25/13f.), anders als für viele seiner iranischen Freunde. Auch auf anderer Ebene wird ihm seine Zuordnung zu Deutschland bewusst. Seine Nicht-mehr-Zugehörigkeit zum Iran nimmt Rachid deutlich als Fremdheit war. Die Sprache mag zwar noch die gleiche sein, aber sie verbindet nicht mehr, sie rekurriert nicht mehr auf ein gemeinsames Denk- und Handlungsmodell, welches verbindend und inkludierend wirkt. Vielmehr vergrößert sie sogar die Irritation und das Gefühl der Fremdheit. Auch seine Herkunftsstadt schafft keine Heimatgefühle. Keine Straße weckt Erinnerungen an früher, ,die Umwandlung ist extrem“ (7/28), und er fühlt sich zu Hause fremd. Mit diesem Prozess ist für ihn auch die Entscheidung gegen eine eventuelle Rückkehr in den Iran im Ruhestand gefallen, aus einer mehrdeutigen Zugehörigkeit ist für ihn eine eindeutige biographische und soziale Positionierung geworden, die nachhaltig Wirkung entfaltet. Diese Wirkung ist allerdings nicht ungebrochen, in der Außendarstellung wird seine eindeutige Positionierung zum Teil wieder in Frage gestellt. So hat er ja einen Doppelnamen ge-

8 Zur Veränderung von Geschlechterbeziehungen in binationalen Partnerschaften s.a. Refsing 1998. 
wählt. In der Außendarstellung, z.B. am Telefon, verwendet er oftmals nur den Nachnamen von Susanne, so dass er die daraus resultierenden Irritationen $\mathrm{zu}$ spüren bekommt, wenn sein Gesprächspartner ihn das erste Mal persönlich sieht und sein Aussehen nicht mehr mit dem deutschen Nachnamen in Verbindung bringen kann. Der ,Affront', den Rachid begeht, wenn er sich am Telefon mit einem deutschen Nachnamen meldet, beim persönlichen Treffen aber physiognomisch ,augenscheinlich“ Nicht-Deutsch ist, macht deutlich, dass solche Positionierungen immer fragil bleiben und beständig auch gegen das Eindeutigkeitsinteresse anderer Personen behauptet werden müssen.

\section{Form biographischen Lernens: natio-ethno- kulturelle Zugehörigkeit als Handlungsfeld für Konstruktionen von Geschlechterbeziehungen - Individualisierung und Integration}

Literatur zur Arbeitslosigkeit und Nicht-Erwerbstätigkeit von Männern geht in der Regel von einem darauffolgenden ,Bruch“ in der Lebensgestaltung und -planung aus. Sie konzentriert sich auf Untersuchungen zu Verarbeitungsstrategien dieser ,Krise " und den Folgen für die Beziehung und für Kinder (vgl. z.B. Schreyer 1991; Blech/Witte 1992; Macmillan/Gartner 1999). Einige wenige wiederum beschäftigen sich mit dieser Konstellation als eine selbstgewählte dann in der Regel unter dem Fokus der Veränderung von gender-Konzepten in der Beziehung (vgl. z.B. Deutsch/Saxon 1998; Risman/Johnson-Sumerford 1998). Allerdings ist, so lässt sich an der vorliegenden Fallanalyse zeigen, die Trennung zwischen ,selbstgewählt' und ,fremdbestimmt' in dieser Dichotomie nicht haltbar. Analysiert man die Bedeutung der Situation vielmehr im Hinblick auf die biographische Einbettung und Präsentation, so zeigen sich differenziertere und kreativere Handlungsmuster. Weder für Rachid noch für Susanne bedeutet die Konstellation einen Bruch mit ihren bisherigen Lebenskonzepten. Zwar entsteht sie durch die Migration Rachids und ist damit sozusagen von ,außen' initiiert, dennoch kann sie ohne krisenhafte Erfahrungen integriert werden. Beiden erlaubt die Konstellation vielmehr die Realisierung von eigenwilligen Lebensformen. Susanne kann eine eigene Geschlechterbeziehung implementieren, ihre finanziell und sozial überlegene Situation unterstützt diese Realisierung. Die breadwinner Konstellation wird von Susanne nicht als von außen an sie herangetragenes Ereignis verstanden, sondern in der Logik ihrer Beziehung und ihrer eigenen Lebenskonzepte gesehen. Die binationale Partnerschaft und ihre Position als breadwinner stellt für sie überhaupt 
eine Möglichkeit dar, ihren biographischen Entwurf zu leben. Natioethno-kulturelle Zugehörigkeiten werden von ihr vor allem im Hinblick auf die Erweiterung von Handlungsmöglichkeiten gesehen, die Modifikationen von gender-Konstruktionen ermöglichen. Es zeigt sich die Möglichkeit, individuelle Geschlechterbeziehungen zu implementieren und zum Beispiel auf Distanz zur traditionellen geschlechtlichen Arbeitsteilung oder zur herkömmlichen Machtaufteilung in einer heterosexuellen Beziehung gehen zu können. Die natio-ethno-kulturelle Zugehörigkeit dient dabei u.a. als Begründungszusammenhang, der die Individualisierung, auch aufgrund der sich verschiebenden Machtverhältnisse, erst ermöglicht. Rachid hingegen kann durch Susanne seine Vorstellungen eines individualistischen, selbstbestimmten Lebens- und Beziehungskonzeptes realisieren. Die Migration stellt eine Möglichkeit dar, seiner Distanzierung und seinen Schwierigkeiten mit dem Iran und den dort erlebten Geschlechter- und Rollenverständnissen Ausdruck zu verleihen und ein anderes, alternatives Lebenskonzept zu implementieren. Der dafür notwendige Preis, eine verlängerte Ausbildungszeit und die finanzielle und soziale Abhängigkeit von seiner Frau, stellt für ihn kein Hindernis dar. Vielmehr sieht er die verschiedenen Berufsausbildungen und Jobs als Erweiterung seiner Erfahrungen. Sie gehören für ihn zu einem westlich orientierten Lebensstil, der mehr Möglichkeiten eröffnet und Raum für unterschiedliche Erfahrungen lässt. Biographisch integriert werden die verschiedenen Irritationen und Differenzerfahrungen durch die Haltung eines Lernenden, eines für neue Einflüsse und Erfahrungen offenen Menschens, dessen Subjektwerdung durch diese neuen Eindrücke nur gewinnen kann. Hier findet sich eine Form der Emanzipation wieder, die Habermas als „Verschränkung von Zugewinn an Autonomie und Selbstverständigungsprozessen“ (Habermas 2004: 70). bezeichnet. Für diesen in der Präsentation der beiden vergleichsweise unkomplizierten Prozess ist das ,dritte Land' wesentlicher Bestandteil. Beide sind dort ,Fremde ' und müssen daher einerseits ansonsten ,selbstverständliche“ $\mathrm{Zu}$ - und Verortungen vornehmen. Andererseits können sie so auch eigene Konzepte und Gestaltungsmuster einbringen, eine Möglichkeit, die sie im Iran oder in Deutschland in dieser Form nicht hätten.

Schwierigkeiten entstehen eher bei der Rückkehr. Insbesondere Susanne wird ihre besondere Situation deutlich vor Augen geführt. Sie hat sich mit der Heirat mit einem Iraner sowohl von ihrem feministischalternativen Umfeld als auch von ihrer Herkunftsfamilie entfernt. Die Rückkehr in ihr altes Umfeld und ihr Versuch, die Distanz zu ihrer Familie nicht zu einem vollständigen Bruch werden zu lassen, zwingt sie zu Modifikationen in der Gestaltung ihrer Beziehung. Dennoch haben 
auch diese Modifikationen wiederum ,Grenzen“: Wie sich bei der Geburt des ersten Sohnes und der darauf folgenden Arbeitsteilung gezeigt hat, ist für Susanne die äußere, durch die finanzielle Situation gerahmte, Unabhängigkeit ein so wesentlicher Bestandteil ihrer psychischen und sozialen Stabilität, dass sie bei deren Wegfallen in eine existentielle Krise gerät. An diesen Punkten greift erneut die natio-ethno-kulturelle $\mathrm{Zu}$ gehörigkeit als Handlungsfeld, welches sich zu spezifischen Zeitpunkten einsetzen, zu anderen Zeiten aber in den Hintergrund verschieben lässt. Rachid hingegen, dessen Distanz zu seiner Familie und seinem Herkunftsland sich im Laufe der Zeit eher noch verstärkt, wird insbesondere durch Susannes Krisen berührt, weniger durch seine Situation, die ihm im Zuge der Migration als zwangsläufig erscheint und ihn in seinem Selbstverständnis daher weniger in Frage stellt. Die strukturelle und soziale Integration geht nicht mit einer transnationalen Biographiekonstruktion einher. Vielmehr entwickelt er eine zunehmende Distanzierung vom iranischen Staat und von der iranischen Gesellschaft. Er ist in Deutschland angekommen, sieht sich inzwischen „deutscher als deutsch" und vollzieht auch in der generativen Folge eine Zuordnung zu Deutschland. So haben die Kinder zwar iranische Vornamen, sie sollen aber explizit kein Persisch lernen, um mit dem iranischen Staat möglichst wenig zu tun zu haben (19/19ff). ${ }^{9}$ Letztlich lösen Susanne und Rachid das female breadwinner Modell hin zu einem dual earner Modell auf. Dabei kehren sie nicht vollständig zurück in herkömmliche Muster der geschlechtlichen Arbeitsteilung. Vielmehr finden sie eine ,Balance“, die es ihnen erlaubt, ihren Integrationswunsch und die Integrationsnotwendigkeit auszubalancieren mit der individuellen Lebensplanung und gestaltung. Beide resümieren daher auch die Beziehung und den Verlauf durchaus positiv. Trotz oder gerade aufgrund der verschiedenen ,existenziellen Krisen' ist es ihnen gelungen, ein Partnerschaftskonzept zu implementieren, welches eigene, individuelle Aspekte mit der Eingliederung in die Heimatstadt verknüpft. Wenig ist vorgegeben, vieles muss diskutiert und beschlossen werden.

Beide Biographien lassen sich in ihrer Entwicklung als Individualisierungsbiographien lesen. Grenzen werden ihnen durch die Integrationsbedürfnisse gesetzt, Integration bedeutet eben auch Integration in bestehende Geschlechterverhältnisse. Sie selber müssen feststellen, dass sie „,so frei nicht sind“, diese Erkenntnis wird insbesondere während der

9 Das ausschließliche Erlernen der deutschen Sprache ist auch als Zeichen von ,Normalität‘ zu verstehen: nicht nur dass er sich damit vom politischen Regime seines Geburtslandes abgrenzt, sorgt er damit im Hinblick auf seine Kinder auch für mehr Unauffälligkeit und vermeidet eine weitere Kennzeichnung als Mehrfach-Zugehörige. 
Kleinkindzeit ihrer beiden Söhne deutlich. War schon Rachids finanzielle Eigenständigkeit Voraussetzung für seinen Kinderwunsch, so stellt sich in den darauf folgenden Beziehungskonflikten die Nähe zu und ihre Abhängigkeit von ihrer Umgebung heraus. Für Rachid besitzt der Iran als imaginärer oder realer Handlungshorizont keinerlei (positive) Relevanz, vielmehr ist Deutschland für ihn zum (alleinigen) Bezugspunkt geworden. Rachids natio-ethno-kulturelle Zugehörigkeit wird von ihm in den Hintergrund geschoben und soll keine oder nur wenig handlungsrelevante Bedeutung bekommen. In diesem Prozess wird Rachids und Susannes natio-ethno-kulturelle Zugehörigkeit gewissermaßen als Mittel zum Zweck genutzt und damit instrumentalisiert. Susanne ,nutzt ${ }^{`}$ die ,Fremdheit' Rachids, um ein kulturelles Copyright zu übernehmen. Rachid ,nutzt' das ,Anderssein' Susannes, um ein individualistisches Beziehungsideal realisieren zu können. Natio-ethno-kulturelle Zugehörigkeit als differente Eigenschaft zu lesen, erlaubt ihnen im Hinblick auf gender eigene, individuelle Konzepte leben zu können. Auch hinsichtlich Susannes Familie nutzt Rachid seine andere Zugehörigkeit, um Diskussionen aus dem Weg zu gehen und zur Unterstützung der eigenen Vorstellungen: „Das hat auch Vorteile dass ich Ausländer bin, vielleicht die sehen dass auch das manche Sachen wa was die erwarten und ich nicht tue, die schieben auf meine Anders sein, oder meine andere Kultur" (21/19 - 21). Rachids andere Zugehörigkeit erlaubt ihm bestimmte Verhaltensweisen, die ansonsten erklärungs- und rechtfertigungsbedürftig wären. Hier spielt er auf die getrennten Schlafzimmer an, die er und Susanne haben. Susannes Eltern, die das merkwürdig finden, erklären es sich mit Hinweis auf Rachids andere Kultur. Die ihm daraus erwachsenden Vorteile nimmt er an und betont damit erneut die Möglichkeit, natio-ethno-kulturelle Zugehörigkeit als Handlungsfeld zu betrachten. Das bedeutet die Kategorien als eine Art Projektionsfläche zu verstehen, auf der Modifikationen und (Re-)Konstruktionen von gender möglich werden, und lässt sich so als spezifische Relation zwischen gender und natio-ethno-kultureller Zugehörigkeit lesen. Natio-ethno-kulturelle Zugehörigkeit wird dabei nicht primär als identitätsstiftendes Merkmal verstanden, vielmehr wird der produktive Aspekt der Kategorie betont. Dies entspricht der Beobachtung von Lenz, dass Subjekte in interkulturellen Begegnungen ,,konfigurative Handlungsstrategien“ verfolgen, d.h. dass sie einzelne Elemente der Geschlechtsrollen, ihres ethnischen Hintergrundes usw. auswählen, kombinieren oder aber herunterspielen und vermeiden" (Lenz 1996: 219). Ein solches Fazit zu ziehen, bedeutet nicht, die Einschränkungen und Herausforderungen in Form rassistischer und/oder diskriminatorischer Praxis zu ignorieren, die insbesondere Rachid im Verwiesenwerden auf seine ,andere' Zugehörigkeit bearbeiten 
muss. Rachids Position als physiognomisch Anderer bleibt prekär und wird immer wieder thematisiert. Dennoch zeigt sich, dass die binationale Partnerschaft Susanne und Rachid einen Handlungszuwachs ermöglicht.

Die erweiterten Handlungsmöglichkeiten sind wesentlicher Teil der spezifischen Form biographischen Lernens. Sie sind Folge des sich entwickelnden Verhältnisses beider Kategorien, wie es sich im Gesamtverlauf der Biographie analysieren lässt. Die Form biographischen Lernens, die sich als übergeordnete Bearbeitungsform analysieren ließ, lässt sich mit ,natio-ethno-kulturelle Zugehörigkeit als Handlungsfeld für Konstruktionen von Geschlechterbeziehungen - Individualisierung und Integration" bezeichnen. Es ließ sich zeigen, wie die Kategorien gender und natio-ethno-kulturelle Zugehörigkeit in Relation zueinander gebracht werden und im Verlauf der Biographie zu einem spezifischen Verhältnis von Individualisierung und Integration beitragen. Das Handlungsfeld ,binationale Beziehung' wird in dieser Paarbeziehung vorrangig als Erweiterung der Spielräume verstanden. Die Radikalität, mit der bestehende Geschlechterordnungen insbesondere von Susanne aufgebrochen werden, lässt sich auf Dauer allerdings nicht halten. Durch weitergehende Irritationen erfahrene Grenzen wirken daher konturierend und verschiebend. Zeigen ließ sich so auch die Zähigkeit bestehender Geschlechterordnungen, die der dauerhaften Realisierung individueller Geschlechterbeziehungen entgegensteht. In der Rekonstruktion des Paares bleibt die binationale Partnerschaft ein Weg, die Besonderheit des Eigenen zu demonstrieren, sich gegen eine ,vollständige“ Integration zu stellen und den Weg frei zu machen für den programmatischen eigenen Weg: Das ,mehr' an kulturellen Gewohnheiten wird, so Susanne, genutzt, um „das Ureigene rauszufiltern“ (Susanne 42/25). Die „Auseinandersetzungen des Einzelnen mit seiner Umwelt, seine Initiativen, auf diese Umwelt einzuwirken, und seine Anstrengungen, das Leben zu bewältigen“ (Schulze 1993b: 195), münden bei Susanne und Rachid in eine Verschiebung der äußeren Individualisierung nach innen, das Selbstverständnis als progressives Paar ist losgelöst von äußeren Bedingungen und Lebensmodellen. 



\section{SEHNSUCHT UND VeRTRAutheit: \\ Nicole und Carlos Ortega Garcia}

\section{Biographische Portraits Nicole und Carlos Ortega Garcia}

\section{Nicole Ortega Garcia}

Nicole Ortega Garcia wird 1976 als einziges Kind ihrer Eltern in einer mittelgroßen Stadt in Süddeutschland geboren. Ihre Mutter kommt aus einer kleinbürgerlichen Familie, ihr Vater hat nach Ansicht seiner Mutter „unter seinem Stand“ geheiratet. Nicoles Mutter arbeitet zum Zeitpunkt des Interviews als Metzgereifachverkäuferin, der Vater war bis zur Verrentung technischer Angestellter. Nicole erlebt ihre Kindheit als sehr harmonisch und schön, trotz beengter Wohn- und schwieriger finanzieller Verhältnissen: „Ich hab nichts vermisst, obwohl wenn ich jetzt nachdenk was andere Kinder hatten hat ich Sicherheit um einiges weniger, hab's nich vermisst [...] überhaupt nicht.“ (18/18 - 28). Als sie 15 ist, ziehen sie in die Vorstadt, wo die Eltern aufgrund sozialer Kontakte eine deutlich größere, aber günstige Wohnung beziehen können. Nicole beginnt nach der mittleren Reife eine Ausbildung zur Bauzeichnerin und zieht während der Ausbildung in ihre erste eigene Wohnung, zurück in die Stadt. Trotz der sehr guten Beziehung zu ihren Eltern hat sie das Bedürfnis alleine zu wohnen: „Ich hab mir damals vorgestellt ich brauch mehr Freiraum, ich hab so die (auflachend) Rebellionphase gehabt - hab mit meinem Papa meine erste Wohnung gesucht" (2/9 - 11). In dieser Zeit ist ihre Wohnung, die im Stadtzentrum liegt, Anlaufpunkt für ihre Freund(inn)e(n) und Treffpunkt am Wochenende. Schon nach einem halben Jahr wechselt sie den Job innerhalb ihrer Firma und zieht mit ihrem damaligen Freund zusammen nach Berlin. Dort ist sie nicht glücklich; sie findet es schwierig, Kontakt zu Menschen zu bekommen. 
Zudem gestaltet sich die Beziehung zu ihrem Partner problematisch. Sie wohnen zusammen mit einem gemeinsamen Freund in einer WG, in der sie die alleinige Verantwortung für den Haushalt übertragen bekommt. Sie fährt jedes Wochenende zurück zu ihren alten Freunden nach Süddeutschland. Nach einem Jahr trennt sie sich daher von ihrem Freund und zieht wieder ganz zurück, zuerst für drei Monate zu ihren Eltern, dann aber erneut in eine eigene Wohnung. 1998 fährt sie mit einer Bekannten ihrer Eltern nach Mexiko und lernt dort Carlos kennen. Sie beschreibt die Begegnung mit ihm als "Liebe auf den ersten Blick“ (6/12f.), bereits nach zwei bis drei Tagen ist sie sich sicher, den Mann ihres Lebens gefunden zu haben. Insbesondere ist sie begeistert von seinen Manieren und dem, wie sie es nennt, ,altmodischen“ (6/24) Verhalten. So genießt sie es, die Tür aufgehalten zu bekommen und überhaupt Rücksicht und Aufmerksamkeit entgegengebracht zu bekommen. Etwas, was sie von deutschen Männern nicht (mehr) kennt. Zurück in Deutschland, reagieren ihre Freundinnen sehr unterschiedlich. Während eine sie in ihrem Vorhaben unterstützt und ihr Mut zuredet, versucht eine zweite Freundin, ihr andere Männer vorzustellen und sie von der Beziehung abzubringen. Erschwert wird die Situation dadurch, dass Carlos nicht auf Nicoles Briefe reagiert. Erst als sie sich traut, bei seiner Familie anzurufen, stellt sich heraus, dass ihre Briefe nicht angekommen waren und Carlos ihre Adresse verloren hatte. Die Fernbeziehung intensiviert sich, und Nicole bricht den Kontakt zu ihrer zweiten Freundin ab. Im darauf folgenden Jahr kann Nicole ihn für zwei Wochen in Mexiko besuchen. Hier befindet sie sich „wirklich in 'ner anderen Welt" (9/8), ist sehr glücklich und merkt bei der Rückkehr nach Deutschland, dass sie die Fernbeziehung so nicht mehr aufrechterhalten will. Bei ihrem nächsten Besuch im darauf folgenden Jahr vereinbaren sie, dass Carlos mit nach Deutschland kommt, ohne die Aufenthaltsdauer zu besprechen. In Mexiko wird sie von Carlos mit einer Verlobungsfeier überrascht. Sie fliegen zusammen nach Deutschland und beschließen zu heiraten. Die Heiratsvorbereitungen gestalten sich schwieriger als erwartet, da das zuständige Standesamt die Aus- und erneute Einreise von Carlos verlangt. Durch Bekannte ihrer Mutter finden sie jedoch ein anderes Standesamt in der Nähe, wo sie die Hochzeit problemlos durchführen können. Finanziell ist die Situation schwierig. Carlos absolviert einen teuren Sprachkurs, der das Familien-Budget sehr belastet. Zudem hat Nicole ein schlechtes Gewissen, da sie den Eindruck hat, Carlos nicht genügend auf das Leben in Deutschland und auf potentielle Schwierigkeiten vorbereitet zu haben. Da sie ihm seine Arbeitslosigkeit nicht schwerer als nötig machen will, verschweigt sie ihm daher die genaue finanzielle Lage und steckt ihm z.B. bei einem Restaurantbesuch Geld zu, damit er für 
sie beide bezahlen kann. Carlos beginnt ein Praktikum und hat verschiedene kurzfristige Beschäftigungsverhältnisse. In dieser Zeit haben sie immer wieder finanzielle Schwierigkeiten, Nicole versucht aber nach wie vor die psychischen Belastungen für Carlos so gering wie möglich zu halten.

„Ich hab halt - nicht zeigen dürfen dass es mir dabei auch ein bisschen schlecht geht weil ich mir halt dann Sorgen um die Zukunft gemacht hab, schon irgendwo. [...] Finanziell - - klar muss man irgendwo einsparen, und hab dann halt nur noch beim Discounter (auflachend) Läden viel eingekauft, wir sind nicht weggegangen oder sonst wo. - Aber das so zu machen dass es nicht auffällt, das ist das Schwierige an der Sache. [...] Jetzt nicht, weil ich ihn anlügen wollte, sondern ich wollts ihm einfach nicht noch schwieriger machen dass er sich dann grämt ach nur wegen mir können wir können wir da jetzt nicht hin. Zum Beispiel auf ein Konzert oder - so. Sondern, ich ach, ich will da eigentlich gar nich so hin. - Weil ich gewusst hab er würd sich sonst - Vorwürfe machen. - - weil er ja den Job nicht hat, sozusagen." (31/15 - 30).

Die Beziehungen zu ihren alten Freund(inn)en verändern sich. Insbesondere ihre männlichen Bekannten scheinen mit Carlos' höflicher und aufmerksamer Art gegenüber Frauen nicht zurecht zu kommen. Inzwischen hat Carlos jedoch eine feste Arbeitsstelle in der Nähe ihres Wohnorts. Ein Umzug in diese Stadt ist für die nächste Zeit geplant, sobald Nicole dort ebenfalls eine Arbeitsstelle findet. Ein permanenter Umzug nach Mexiko liegt in den nächsten fünf bis zehn Jahren durchaus im Bereich des Möglichen, insbesondere seine Eltern würden sich sehr freuen. Da jedoch ihre Eltern beide sehr krank sind, möchte sie noch nicht zu weit wegziehen. Die Gründung einer Familie macht Nicole von der beruflichen Stabilisierung abhängig. Sie selber möchte in diesem Fall drei Jahre Elternzeit nehmen und danach als Teilzeitkraft wieder einsteigen.

\section{Carlos Ortega Garcia}

Carlos Ortega Garcia wird 1973 als viertes von insgesamt fünf Kindern in einer großen Stadt in Mexiko geboren. Carlos und seine ältere Schwester sind die einzigen leiblichen Kinder des Paares. Seine Mutter bringt eine Tochter und einen Sohn aus erster Ehe mit, die Eltern adoptieren später noch den Sohn einer Bekannten. Carlos' Familie zieht, als er zehn Jahre alt ist, in eine Stadt nordwestlich seiner Geburtsstadt. Der Vater bekommt dort berufliche Aufstiegschancen, er möchte den Kindern eine „bessere Lebensqualität“ (1/17) bieten. Er steigt in den Jahren bis zum Flughafenmanager auf und kann so seinen Kindern eine Universitätsausbildung bezahlen. Carlos sieht seinen Vater nicht als typischen 
Macho (vgl. 8/32), so habe er seiner Mutter erlaubt zu arbeiten und sei auch der bessere Koch. Carlos beginnt nach dem Abitur ein JuraStudium, bricht aber nach einem Jahr ab, da er „zu soft“ (10/27) dafür gewesen sei. Er empfand die Studiumsstruktur als schwierig. Er jobbt als Kellner und in verschiedenen anderen Bereichen, bis ihn seine Schwester ohne sein Wissen in den Studiengang Kommunikationswissenschaften einschreibt. Carlos empfindet die Studienzeit insbesondere hinsichtlich seiner sozialen Stellung als außergewöhnlich, er genießt einen hohen Bekanntheitsgrad und hat einen sehr großen Freundes- und Bekanntenkreis: „Ich war w_wie wie ähm - nicht der König, aber ich war vielleicht de_einer von de_von den meisten bekannten von der Schule, von der Universität“" (11/12f.). Nach und neben dem Studium arbeitet er weiterhin als Kellner und verdient sehr gut. In dieser Zeit, in der er aufgrund der nur saisonalen Arbeitsmöglichkeiten in seiner Stadt überlegt, in eine größere Stadt zu ziehen und sich eine feste Arbeit zu suchen, begegnet er Nicole. Er ist sofort verliebt und hat das Gefühl, der Frau begegnet zu sein, auf die er ,gewartet hat“ (2/26). Ein Umzug ins Ausland, insbesondere nach Kanada, war für ihn in dieser Zeit eine realistische Möglichkeit, seine Lebensqualität zu verbessern und Arbeit zu finden. Mit Unterstützung seiner Eltern macht er Nicole nach zwei Jahren Fernbeziehung einen Heiratsantrag und fliegt mit ihr nach Deutschland. In Deutschland ist er überrascht von den Schwierigkeiten, die ihn dort erwarten. Er lernt sehr schnell Sprachen und ist davon ausgegangen, auch Deutsch so lernen zu können. Er ist sich nicht sicher, ob er im Nachhinein noch einmal eine solche Entscheidung fällen würde:

„Äh, wenn ich denke jetzt was wär wenn ich - mehr Zeit über - über über Gedanken über diese Idee oder weiß nicht, ich glaube ich wär nicht nach äh Deutschland gegangen, oder - weil wenn ich denke es ist nicht so einfach wie ich gedacht habe - und mit der Sprache oder so. Dann, dann hätt ich vielleicht in Mexiko geblieben“ (14/16 - 19).

Die finanzielle Abhängigkeit von Nicole macht ihm zu schaffen. So fällt ihm die Entscheidung zur Hochzeit zunächst schwer, da er sie nicht selber bezahlen kann, er sieht es als seine Aufgabe an, die Hochzeit zu organisieren und finanziell zu bestreiten. Seine Hoffnung, sich hier schnell beruflich etwas aufbauen zu können, realisiert sich nicht. Seine beruflichen Abschlüsse werden nicht bzw. nur zum Teil anerkannt, so dass er verschiedene Praktika machen muss. In dieser Zeit trifft ihn die Krise der Werbebranche sehr direkt. Zwei der Firmen, in denen der arbeitet, müssen Konkurs anmelden, eine andere versucht ihn auszubeuten, so dass er letztendlich kündigt. Zurzeit arbeitet er in einer Firma in der Nä- 
he seines Wohnortes und hofft dort auf eine langfristige Anstellung, zumal diese Firma auch Kontakte nach Südamerika hat, und er so seine Sprach- und Landeskenntnisse nutzen könnte. Er hofft auf eine finanzielle Konsolidierung, so dass er und Nicole bald Kinder bekommen können. Langfristig kann er sich sehr gut vorstellen, zurück nach Mexiko zu gehen. Er möchte die hier erworbenen Qualifikationen nutzen, um dort ein Restaurant aufzumachen:

„Ich will ein Restaurant wie - wie in Deutschland, etwas wie in Deutschland. also wirklich ins Business kommen und, und äh mit guter Qualität, also deutsche Qualität so. Äh - - aber ich weiß nicht, dass, dass ich brauche viele Sachen, viel Geld, viele Investieren und - - weiß nicht. Das dauert noch“ (26/18 21).

\section{Der Horizont der Paare: Beziehung als integratives Moment}

\section{Konstruktion des Paarhorizonts bei Nicole: das Vertraute im Fremden}

In Nicoles Erzählungen über den Beginn der Beziehung zu Carlos lässt sich die Etablierung einer traditionellen, klassischen Geschlechterbeziehung durch Bezug auf natio-ethno-kulturelle Spezifika analysieren. Diese Konstruktionen zeichnen sich durch ihre sinnstiftende Bedeutung hinsichtlich der weiteren Lebensplanung und der Entwicklung von Handlungs- und Orientierungsmustern aus. Der Prozess der Etablierung wird durch zwei dominante Figuren bestimmt: durch Zusammengehörigkeit jenseits von Raum und Zeit und durch Beständigkeit durch Wandel.

Zusammengehörigkeit jenseits von Raum und Zeit:

Liebe auf den ersten Blick

Nicole erlebt mit Carlos die „Liebe auf den ersten Blick“ (6/12f). Sie begegnen sich in einem Restaurant, in dem Carlos arbeitet, und ihre Bekannte muss, da sie sich selbst nicht traut, ihn ansprechen und für sie eine Verabredung treffen. Eine solche Konzeption von Liebe, so lässt sich mit Burkart sagen, folgt keiner kognitiv-rationalen Logik, sondern funktioniert vielmehr

„mittels des ,praktischen Bewusstseins“ (Giddens 1988) oder des ,praktischen Sinns (Bourdieu 1987). Liebe kann so verstanden werden als ein ganz eigener Erkenntnisprozess im Sinne des praktischen Wissens: Hier wird der Andere 
nicht über kognitive Rationalität erkannt, sondern über die Körpersymbolik (Douglas 1970) oder den Habitus. [...] Das könnte das Entstehen der Liebe erklären und gleichzeitig verständlich machen, warum wir diesen Prozess regelmäßig als Mysterium empfinden oder eben als Naturereignis“ (Burkart 1998: 41, Herv. d. Verf.). ${ }^{1}$

Obwohl es bei der einen Begegnung und der einen Nacht bleiben muss, ist sich Nicole hinsichtlich ihrer Gefühle und ihrer Beziehung zu Carlos sicher: „Wir ham wirklich ein paar Stunden miteinander verbracht, und ich war mir sicher. - Ich war mir noch nie so sicher, bei keinem Freund davor“ (8/9 - 11). Das Modell der Liebe auf den ersten Blick macht für sie ein weiteres intensiveres Kennenlernen überflüssig. Das Bild des Naturereignisses, welches andere Erklärungen überflüssig macht, korrespondiert mit einer alltagsweltlichen Vorstellung über binationale Paare, welche sich auch in wissenschaftlichen Veröffentlichungen recht hartnäckig hält, wie sich im Forschungsüberblick gezeigt hat. ${ }^{2}$

Für Nicole besitzt die Rahmung der Liebe jenseits von Zeit und Raum auch im weiteren Verlauf der Fernbeziehung Gültigkeit. Zurück in Deutschland wird sie jedoch mit anderen, gegensätzlichen Interpretationsmustern konfrontiert: Während die eine Freundin sie in ihren Gefühlen bestärkt und unterstützt, akzeptiert eine andere Freundin das Modell der Fernbeziehung nicht, betrachtet sie vielmehr als Single und versucht, sie mit anderen Männern bekannt zu machen. Für Nicole ist die Beziehung zu Carlos jedoch wichtiger als diese soziale Bindung: Sie beendet die Freundschaft und wendet sich denjenigen $\mathrm{zu}$, die sie in ihrer Entscheidung unterstützen, denn ,,in meinem Kopf und in meinem Herzen hatte ich ja 'ne Beziehung“ (23/8f). Der starke Bezug auf die Paarbeziehung führt zur Strukturierung ihres sozialen Umfeldes um die Beziehung herum: Externen Irritationen, die ihr Verständnis der Beziehung in Frage stellen, wird, soweit möglich, ausgewichen. Auch im weiteren Verlauf der Etablierung der Beziehung wirkt das Modell der ,wahren Liebe“ also handlungsorientierend. Dieses ist aber, und hier gilt es BeckGernsheim energisch zu widersprechen, eben nicht akontextuell, frei von Gesetzen von Herkunft und Heimat, zu verstehen (vgl. ebd. 2001: 120). Vielmehr ist es eingebunden in Konstruktionen und Verstrickungen von gender und natio-ethno-kultureller Zugehörigkeit.

1 Das Motiv der romantischen Liebe wird an dieser Stelle vergleichsweise ausführlich besprochen, da es ebenfalls für Carlos Gültigkeit besitzt.

2 Vgl. dazu Root 2001, die die Liebe als zentrales Motiv ansieht, natioethno-kulturelle Grenzen zu überwinden. 


\section{Rückkehr oder: Beständigkeit durch Wandel}

Bei Nicole lassen sich diese Verstrickungen als Sehnsucht nach festen, traditionellen Geschlechterbeziehungen analysieren. Den Beginn der Beziehung versteht sie als Ende ihrer Suche nach solchen Geschlechterbeziehungen. Eine Suche, die in Deutschland, so Nicole, nicht erfolgreich war: „Sind so Sachen, die ich glaub ich an den deutschen Männern - vermiss. Des is jetzt nicht ein Bevormunden oder so, sondern einfach dass er Rücksicht auf mich nimmt, das er auf mich aufpasst“"(6/15 - 18). Die ,Liebe auf den ersten Blick` gründet für Nicole also unter anderem in der natio-ethno-kulturellen Herkunft Carlos“ und der ,altmodischen“ (6/24) Art der Mexikaner in Bezug auf Geschlechterverhältnisse und Umgangsformen mit Frauen. Anders als bei Susanne, für die die Fremdheit Rachids konstitutiv für ihre Beziehung ist, ist bei Nicole vielmehr gerade die Vertrautheit wesentlicher Bestandteil der Bedeutung der Beziehung. Als Verbindungsglied dient dabei die ,altmodische“ (6/24) Art der Mexikaner, die sie positiv überrascht. Altmodisch sind Mexikaner im Verhältnis zu deutschen Männern, Mexikaner besitzen Eigenschaften, die den modernen deutschen Männern inzwischen fehlen. Daher spielt hier weniger Mexiko als Land, als konkreter Bezugsort, eine Rolle als vielmehr die kulturelle Differenz zum modernen Deutschland. Höflichkeit, Rücksichtnahme, Zuvorkommenheit, diese Eigenschaften des mexikanischen Mannes sind für sie konstitutives Element einer Beziehung. Letzere wiederum ließe sich mit deutschen Männern nicht realisieren: „Ich glaub nur nich das man in der heutigen Zeit so jemanden in Deutschland findet, weil das auch schon wichtig ist, diese Kavalierseigenschaften die er hat. - Danach hab ich mich schon gesehnt" (38/22 24). Eine solche Konstruktion des Vertrauten im Fremden findet sich als Analyseergebnis auch in der Studie von Hecht-El Minshawi von 1988. Ihrem Analyseergebnis nach sind ja beide Partner(innen) „outsider“ (ebd.: 237) der bundesrepublikanischen Gesellschaft. Die Frauen hatten, so ihre These, schon vor dem Zusammentreffen mit ihren Männern begonnen, sich aus der deutschen Kultur zu lösen, sie haben ,außenseiterisch gelebt" (ebd.: 21). ${ }^{3}$ Während in ihrem Fall die Frauen Gemeinsamkeiten jedoch in der Distanz zu der bzw. der Exklusion durch die jewei-

3 Gründe dafür sieht sie in der Sozialisation der Frauen, die, z.B. durch häufiges Umziehen oder durch sozialen Aufstieg, zu Fremdheitserfahrungen in der neuen Umgebung geführt hätten. Ebenfalls relevant sind aktuelle Umstände wie Zivilisationsmüdigkeit oder schlechte Beziehungserfahrungen (vgl. Hecht-El Minshawi 1988: 171 - 176). Siehe zum Gefühl von Marginalität und dessen Bedeutung für die Partnerwahl auch KhatibChahidi/Hill/Paton 1998: 57). 
lige(n) Herkunftsgesellschaft schaffen, findet sich bei Nicole keine solche (äußere) Exklusion. Vielmehr stellt die Beziehung zu Carlos die Möglichkeit der Realisierung eines Lebenskonzeptes dar, das ihr die ReIntegration in eine traditionelle Geschlechterordnung in Deutschland ermöglicht. Die binationale Beziehung, die sich daher auf den ersten Blick als Wandel ihres biographischen Entwurfes darstellt, lässt sich bei genauerer Analyse tatsächlich eher als Aufrechterhaltung und Realisierung eines Lebensmodells verstehen, welches Nicole, so ihre Annahme, mit deutschen Männern nicht gelungen wäre.

Bei Nicole ist der Beziehungshorizont vor allem durch ein bewahrendes und rückkehrendes Moment, der Integration in traditionelle Geschlechterverhältnisse, charakterisiert. Modernisierungsprozesse haben für sie zu falschen Entwicklungen in Bezug auf geschlechtsspezifische Höflichkeitsformen geführt, Carlos als „altmodischer“ Mexikaner ermöglicht ihr hier die Rückkehr zu einem klassischen Beziehungsmuster. Die Imaginationen, die sie hinsichtlich des Geschlechterverhältnisses eines vormodernen Deutschland hat, finden ihre Bestätigung in den Handlungen von Carlos. Carlos' natio-ethno-kulturelle Zugehörigkeit stellt für Nicole die Grundlage ihrer Erwartungen an seine geschlechtsspezifischen Handlungen dar. So kann sie im Privaten ihre Geschlechterbeziehung realisieren, unabhängig von deutschen Männern. Der Horizont konstituiert sich dadurch auch durch die starke Betonung der emotionalen Bindung. Die „Liebe auf den ersten Blick“ und das Wissen um die Zusammengehörigkeit nach nur einem Treffen und trotz der Fernbeziehung strukturiert dabei auch schon ihre sozialen Einbindungen in Deutschland. Die Suche nach dem richtigen Partner ist vorbei, und irritierende bzw. in Frage stellende soziale Nahbeziehungen und Freundschaften werden in ein solches Modell nicht mehr integriert, sondern vielmehr beendet. Auch die Entscheidung zur Hochzeit lässt sich in der Logik der Zusammengehörigkeit und ihres Verständnisses von Beziehungen verstehen: „Wenn mich - jemand liebt dann soll er mir beweisen dass er - ein Leben lang mit mir zusammen leben will“" $(10 / 33$ - 11/1). Der Beweis der wahren Liebe soll durch die Ehe angetreten werden, Liebe und Ehe gehören für Nicole unmittelbar zusammen. Diese Erwartung erfüllt sich auch. Carlos schenkt ihr ,so ne richtige Verlobungsfeier mit Verlobungsring“ (11/5) und geht mit ihr nach Deutschland. 


\section{Konstruktion des Paarhorizonts bei Carlos: Gelegenheitsstruktur Beziehung}

In Carlos' Rekonstruktionen über den Beginn der Beziehung finden sich Anschlüsse und Fortführungen seiner bisheriger Lebensentwürfe und seiner Vorstellungen von Geschlechterbeziehungen. In diesem Kontext lässt sich auch die Migration nicht als Umdeutungsnotwendigkeit eines Biographieentwurfs, sondern als Möglichkeitseröffnung verstehen. Die relevante Dimension, die die Konstruktion des Paarhorizonts begleitet, ist die Anschlussfähigkeit.

\section{Die Frau, auf die ich gewartet habe: Anschlüsse}

Wie Nicole rahmt Carlos die Begegnung mit ihr unter dem Motiv der ,Liebe auf den ersten Blick“: „Wir haben nur eine Nacht so unterhalten. Wir waren beide sofort verliebt" (13/21f). Auch für ihn scheint ein weiteres Kennenlernen überflüssig, Nicole ist die Frau, „das $\underline{\mathrm{ich}}$ gewartet habe“ (2/26). Auch für ihn gilt jedoch, dass die ,Liebe auf den ersten Blick` an spezifische Dispositionen anknüpft, die Verknüpfungen von Konstruktionen von gender und natio-ethno-kultureller Zugehörigkeit deutlich machen. Carlos lernt Nicole zu einem Zeitpunkt kennen, zu dem er Überlegungen zu seinem zukünftigen Wohnort und seiner weiteren beruflichen Karriere anstellt:

„Das Problem in B-Stadt, diesem Ort gibt es nur sechs Monate Tourismus. Und sechs Monate gibt es gar nichts. Also kein Tourismus, nix. und das war das Problem. Ia, und - dann hab ich gedacht ah, vielleicht ich geh nach AStadt, oder - nach andere große Städte und - ein Job suchen“ (12/1 - 8).

Anschlussfähig an seinen biographischen Entwurf ist für ihn daher zum einen die Migration, die seine beruflichen Verwirklichungen unterstützt, anschlussfähig ist zum anderen auch die Beziehung zu Nicole, die seiner Vorstellung einer Ehepartnerin entspricht.

\section{Migrationsdisposition}

Carlos arbeitet während seines Studiums und danach als Kellner in einem Restaurant. Zwar kann er dort in der Sommerzeit viel Geld verdienen, der ökonomische Aspekt reicht ihm jedoch nicht aus:

„Aber, ah, naja, ich will nicht - - äh, als Kellner arbeiten immer. Weil ich kenne andere Leute, sie haben 30 Jahre mit demselben Job als Kellner, das ist 
nicht so so gut. Ich habe was studiert dann will ich in meinem Beruf arbeiten, also in meinem was ich gelernt habe“ $(3 / 3-5)$.

Die langfristige Verwertung seiner erworbenen Bildungszertifikate steht für ihn über dem kurzfristigen ökonomischen Zugewinn, auch wenn er als Kellner finanziell gut dasteht (3/2). Für eine solche Verwertung ist für ihn eine Migration eine realistische Perspektive, die sich in seinem Handlungshorizont befindet. Mit Migrationsdisposition ist daher die Vorwegnahme der Möglichkeit einer Migration beschrieben. Bislang wurde sie zwar von ihm nicht aktiv vorangetrieben, dennoch wäre sie für ihn biographisch integrierbar und ist in seinem biographischen Entwurf enthalten. Arbeitsmigration liegt zudem in seinem Erfahrungskontext, seine Eltern sind mit ihnen nach B-Stadt gezogen, um den Kindern sozialen Aufstieg und Bildung zu ermöglichen. Migration bedeutet für ihn einerseits Binnenmigration, der Umzug nach A-Stadt oder in eine andere größere Stadt in Mexiko. Sie könnte aber auch eine Auslandsmigration bedeuten. Kanada zum Beispiel, dessen Sprache er spricht und wo schon Freunde von ihm leben, ist für ihn ein mögliches Ziel. ${ }^{4}$

\section{Genau richtig: Nicole als Gegenentwurf}

Neben der Migrationsdisposition lässt sich Nicole auch noch aus einem anderen Grund als die Frau, auf die er gewartet hat, bezeichnen. Explizit formuliert er seine Vorstellungen über seine zukünftige Frau:

„Ich habe immer gesagt ich heirate nicht mit ner Frau aus diesem Ort. Aus BStadt. [...] Die Frauen sind sehr - - emanzipiert, aber in dem negativ Richtung. Zum Beispiel, ich bin eine Frau, ich will arbeiten, und - und sie wollten immer den Mann - unterdrücken. Und das finde ich nicht so toll. Äh, die Frauen sind immer - vielleicht hast du gehört äh die spanischen - ähm die spanischen Frauen die sind - me_ah Charakter, immer grr, wie kann ich das ausdrücken, äh hm -der Mann muss - - ähm, die Frau muss immer oben sein. Sie muss die Hosen tragen. Und das finde ich nicht so toll. Also ich bin, ich bin nicht, ich habe gar nichts gegen die Emanzipation oder so, ich finde das toll. - - Aber - nicht so extrem. Und - ich hab über diese Frauen immer gesagt, ich wollte nicht mit einer Frau von B-Stadt verheiratet sein. Ich habe auch nicht gedacht mit einer

4 Sowohl Binnenmigration, die Migration vom Land in die Städte, als auch Auslandsmigration betreffen einen Großteil der mexikanischen Bevölkerung. Jährlich wandern etwa 7 Millionen Mexikaner(innen) in die USA aus, so genannte illegale Migration nicht eingerechnet, insgesamt lebt und arbeitet etwa 1/10 der mexikanischen Bevölkerung in den USA (vgl. Mexiko Lexikon 2006). 
Ausländerin. Äh, vielleicht vo_von Südmexiko, die Frauen sind mehr ruhiger, mehr - anders“"(25/24-26/2).

Carlos entwickelt ein Kontinuum von Geschlechterverhältnissen und Emanzipation, in dem die Frauen aus seinem Herkunftsort aufgrund ihrer extremen Emanzipationsausprägung als potentielle Ehefrauen nicht in Frage kommen. Die Art von ,Landkarte', die er zeichnet, unterscheidet zwischen heiratsfähigen und nicht heiratsfähigen Frauen, entscheidendes Kriterium ist dabei eine angemessene emanzipative Haltung. Die Entscheidungshoheit über die Angemessenheit liegt dabei bei ihm. Seine Konstruktionen folgen einem schon klassisch zu nennenden antifeministischen Diskurs: Emanzipation wird als Umkehrung des Unterdrückungsverhältnisses verstanden: „Was bei Männern als Zeichen von Stärke gewertet wird, würde bei Frauen als Aggressivität betrachtet werden, selbst bei identischem Verhalten“ (Knapp 1992: 312). Zu emanzipatives Verhalten ist für ihn nicht nur eine Frage der individuellen Entwicklung, sondern ist an die regionale Zugehörigkeit der Frauen gebunden. Eine solche dispositionale Haltung für eine Heirat außerhalb seines Herkunftsortes eröffnet schon vor der Begegnung mit Nicole die Möglichkeit einer binationalen Ehe.

Für Carlos ist der Beziehungshorizont insofern vor allem durch die Anschlussfähigkeit Nicoles gekennzeichnet. Nicole scheint genau die richtige Frau zu sein. Zwar hat er nicht damit gerechnet, eine „Ausländerin“ (26/1) zu heiraten, aber sowohl hinsichtlich seiner Vorstellungen über Paarbeziehungen wie auch hinsichtlich einer möglichen Migration erfüllt sie seine Vorstellungen. Die Beziehung zu ihr ermöglicht ihm die Weiterführung und die Realisierung seines biographischen Entwurfs. Waldis nennt eine solche Orientierung ,vormigratorische Akkulturation“ (Waldis 2001: 138), die Orientierung an anderen Paarmodellen oder Lebensentwürfen als an denen des bisherigen sozialen Raumes. Carlos entscheidet daher auch, mit ihr nach Deutschland zu gehen, und macht ihr bei ihrem zweiten Besuch in Mexiko einen Heiratsantrag. Auch hier lässt sich also weder die Beziehung noch die sich anschließende Migration nach Deutschland als Bruch mit der bisherigen Lebensgestaltung bezeichnen. Migration, so zeigt sich in den Interviews, ist nur selten trennscharf in Heirats- oder Arbeitsmigration zu unterscheiden (vgl. dazu auch Treibel 2003: 20f. zu verschiedenen Typologisierungsversuchen von Migration und Wanderung). Wenn sich Heiratsmigration als „Migration, die mit einer Heirat gekoppelt ist“" (Ruenkaew 2003: 35), definieren lässt, Arbeitsmigration als Migration, die der Verbesserung der Arbeitsbedingungen dient, so sind beide Motive für Carlos relevant und in ihrer Bedeutung nicht zu trennen. 


\section{Gesehnt und gewartet: Integration}

Hinsichtlich des Verhältnisses des Paarhorizonts der beiden liegt die Gemeinsamkeit in der Bedeutung traditioneller Geschlechterbeziehungen, die natio-ethno-kulturelle Zugehörigkeiten integriert. Für Nicole und für Carlos ist ein Kriterium für die Auswahl des Partners bzw. der Partnerin die Möglichkeit, traditionelle Geschlechterbeziehungen leben zu können. Nicole geht explizit davon aus, solche Höflichkeitsformen und Aufmerksamkeiten bei deutschen Männern nicht mehr anzutreffen, und auch Carlos ist skeptisch, zumindest was Frauen aus Nordmexiko angeht. In dieser Konstellation ist nicht eine natio-ethno-kulturelle ,Fremdheit' und ,Andersheit', wie von Susanne und Rachid entwickelt, entscheidend. Konstitutiv wirken hier vielmehr Imaginationen hinsichtlich der Vertrautheit solcher Ordnungen und der Anschlussfähigkeit an biographisch erwünschte Geschlechterbeziehungen. Natio-ethno-kulturelle Zugehörigkeiten werden so nicht hinsichtlich ihrer Andersartigkeit, sondern hinsichtlich ihrer Ähnlichkeit interessant. Integriert werden können sie durch ihre Ähnlichkeit mit den eigenen Erwartungen und Vorstellungen von Partnerschaftsmodellen. Beide entwickeln so relativ hohe Erwartungen an den bzw. die Andere(n) hinsichtlich der Erfüllung der Imaginationen und Möglichkeiten der Fortführung bzw. Rückkehr zu ,geordneten“ Verhältnissen. Eine neuere Studie zu binationalen Paaren führt als Ergebnis an, „dass die größte Schwäche der interkulturellen Partnerschaften zugleich ihre größte Stärke ist: nämlich, dass die beiden Partner(innen) sich nicht darauf verlassen können, sich wortlos zu verstehen“ (Larcher 2000: 221). Für Nicole und Carlos hingegen trifft diese Form von Unverständnis nicht zu. Vielmehr ist die Übereinstimmung zwischen den beiden durch das Modell der romantischen Liebe gerahmt, welches das wortlose Verstehen, das Erkennen das der/die Andere der Richtige für einen ist, beinhaltet.

\section{Thema der Beziehung: Realisierung}

Im scharfen Kontrast zur Bedeutung des female breadwinner Modells für Susanne und Rachid erscheint es für Nicole und Carlos als konfliktuös und bedrohlich. Beide werden von der Entwicklung in Deutschland überrascht, die Schwierigkeiten kommen unerwartet, rückwirkend wird sogar die gesamte Migration in Frage gestellt (vgl. Carlos 14/16ff). Für Nicole stellt das female breadwinner Modell eine unerwünschte Irritation und zeitliche Verzögerung ihres favorisierten Beziehungsmodells dar, für Carlos eine unerwartete Herausforderung hinsichtlich der Erwar- 
tung eines unproblematischen Migrationsprozesses. Als Thema der Beziehung lässt sich für Nicole und Carlos die Problematik der Realisierung ihres gewünschten Lebensmodells benennen. Der Versuch, das Beziehungsverständnis trotz der irritierenden äußeren Einflüsse aufrechtzuerhalten und dauerhaft zu realisieren, bestimmt die Entwicklung in der Beziehung und strukturiert die Form biographischen Lernens.

\section{Bearbeitungsfelder}

\section{Bearbeitungsfelder bei Nicole: mit den Augen des Anderen}

In der biographischen Erzählung Nicoles zeigt sich die Notwendigkeit der Bearbeitung verschiedener Felder, die sich als Krisenbewältigung darstellt. Die binationale Beziehung eröffnet Nicole zwar die Chancen für eine traditionelle Geschlechterbeziehung, bedeutet jedoch auch, durch die Konstellation des female breadwinner Modells, die Infragestellung der Realisierung eben dieser Beziehung. Die Folgen, mit denen sie konfrontiert wird, finden primär auf der Ebene unerwarteter gesellschaftlicher Einschränkungen und Einwirkungen statt, es gilt, den Bruch in der Lebensplanung zu kitten und negative Folgen der Migration zu heilen.

\section{Distanzierung von Deutschland}

Die Ankunft in Deutschland und die Migration lassen Nicole ihre eigene Kultur und Herkunft ein einem anderen, fremdem Licht erscheinen. Ihr werden rassistische und diskriminatorische Praxen der Deutschen bewusst, bei Carlos vermutet sie gar eine Art „Kultur-Schock“ (27/11). Insbesondere im Vergleich zu der Aufnahme in Mexiko, wo sie sehr freundlich aufgenommen wurde, ist ihr ihr Land peinlich:

„Man ist selbst Deutscher und dann fällt es einem vielleicht nich so auf, aber als wir dann eben zurückgekommen sind aus Mexiko, das hat mir irgendwo die Augen geöffnet, wie wir wirklich sind, wie unfreundlich wir zu Ausländern sind. Und - ich mein jetzt nicht unbedingt die Ausländer - auch die die als Touristen kommen - wir sind so was von unfreundlich zu den Leuten. - im Gegensatz zu anderen Ländern. Also das hat mich schon, das war - - ich hab dann gedacht - oje, musst du dich schon wieder schämen dass du Deutsche bist? - Weil das sind deine Landsleute, - die so schlecht einen behandeln“ $(28 / 13-19)$. 
Interkulturelle Begegnungen sorgen dafür, dass ihr „die Augen geöffnet werden", ,wie wir wirklich sind":

„,Kultur' ist das, was sich an den Lebensweisen der Menschen unterscheidet und in dieser Hinsicht mit den Lebensweisen anderer Menschen verglichen werden kann. Oder kürzer gesagt: ,Kultur' ist das, was unvergleichbare Lebensweisen vergleichbar macht" (Baecker 2001: 47).

Die Beziehung zu Carlos wirft sie zurück auf ihre Herkunft und wird so zu einer selbstreflexiven Erfahrung (vgl. dazu auch Yildiz 2004). Sie blickt mit den Augen des Anderen auf ihr Herkunftsland und kann keine positiven Seiten erkennen, ihr Herkunftsland ist ihr in diesen Handlungen fremd. Ausländer werden erkannt, exponiert, und nicht wie in Mexiko freundlich aufgenommen, sondern zurückgewiesen. Bei der Übernahme der Verantwortung für ,unser' Verhalten ist für Nicole hinsichtlich der Zurechenbarkeit keine, auch reflexive, Distanzierung möglich. Vielmehr setzt sie Staatsbürgerschaft mit nationaler Identität gleich: als Deutsche muss sie sich das Verhalten anderer Deutscher anrechnen lassen, das Individuum ist Repräsentant des Nationalstaates, ist Teil der nationalen Identität. In ihrer Reaktion auf die rassistischen Praktiken lassen sich bei Nicole Verfremdungsprozesse hinsichtlich der eigenen Positionierung analysieren. Sie übernimmt die Verantwortung für die ,eigenen Landsleute' und schämt sich für sie. Zudem hat sie den Eindruck, Carlos nicht genug auf das Leben in Deutschland und die Unfreundlichkeit der Menschen vorbereitet zu haben: „Ich hab mich am Anfang auch wirklich für vieles entschuldigt. - Weil ich - gedacht hab ich hätts ihm erzählen müssen, aber ich hab - damals in Mexiko nicht dran gedacht über diese Dinge“ $(28 / 30$ - 32). Letztendlich entschuldigt sie sich sogar für das Wetter (28/30), aus der Unsicherheit heraus, seine Erwartungen über ein Leben in Deutschland enttäuscht zu haben.

\section{Freundeskreis}

Dieser Verfremdungsprozess wird durch die einsetzende NomosBildung (Berger/Kellner 1965) verstärkt und unterstützt. Für Nicole bedeutet die Eheschließung den Beginn der Gemeinsamkeit. So gibt es für sie beispielsweise auch nur gemeinsames Geld: „Meins ist dein“ (30/16). Für sie war und ist es egal, wer gerade das Geld verdiene: „Wenn man verheiratet ist, dann ist es ja unser Geld“" (30/15). Die Ehe symbolisiert einen neuen Zustand, in welchem die Grenzen zwischen Mein und Dein aufgehoben sind. Die Rahmung durch die romantische Liebe, welche exklusiv und allumfassend ist, verstärkt sich durch die Erfahrungen in 
Deutschland und die stattfindenden Verfremdungsprozesse. ${ }^{5}$ Eine solche eindeutige Positionierung zeigt sich insbesondere in Bezug auf den sozialen Nahraum. Wieder wird mit Personen, die den von ihr konstruierten Horizont in Frage stellen bzw. nicht anerkennen, der Kontakt abgebrochen:

„Die - männlichen Personen aus meinem Freundeskreis - ham Probleme mit $\underline{\mathrm{ihm}}$, weil sie annehmen - das ihre Freundinnen dasselbe wollen dass sie jetzt ihre Freundinnen so behandeln sollen wie er mich behandelt. - Das war damals bei der Hochzeitsfeier ganz lustig - - wir ham uns hingesetzt, und Carlos hat mir den Stuhl vorgeschoben, ich hab mich hingesetzt und er hat ihn mir hingeschoben. Dann kam nur die Bemerkung, des treibn mir auch noch aus. Des, des ist so ne Sache, ich glaub die ham nen bisschen Angst vor ihm. Dadurch hat sich nen neuer Freundeskreis gebildet, also mein alter ist nicht mehr bestehen geblieben“" (40/21-27).

Der Begründungszusammenhang, den Nicole aufmacht, verweist auf kulturelle Differenzen, die jedoch von dem männlichen Teil der Mehrheitsgesellschaft nicht akzeptiert und ausgehalten werden. Vielmehr wird die Differenz als Bedrohung verstanden, der mit Assimilation begegnet werden muss: „Sich assimilieren bedeutet, sich den Erwartungen der Mächtigen unterwerfen, sie sich so gründlich zu Eigen machen, sie so tief zu verinnerlichen, dass man selbst denkt, fühlt, handelt wie die männlichen Träger der Macht" (Larcher 2000: 161). Die Eigenschaften, die sie so an ihm schätzt, die Höflichkeit und Rücksichtnahme, sind jedoch konstitutiv für ihren Paarhorizont. Um ihre Vorstellungen der Partnerschaft und ihren biographischen Entwurf realisieren zu können, werden derartige äußere Irritationen in den Entwurf daher nicht integriert, sondern ausgeschlossen. Auf die Herausforderungen ihrer Freunde reagiert sie mit der Bestärkung ihrer Beziehung, der Freundeskreis verändert sich entsprechend.

\section{Zwangsstruktur female breadwinner Modell}

Für Nicole stellt die Konstellation in Deutschland eine große Herausforderung dar. Für ihre Beziehung hat sie sich die Rückkehr zu einer klassi-

5 Junge Frauen müssen sich, wie Oechsle zeigt, mit widersprüchlichen Leitbildern hinsichtlich der Partnerwahl und Liebe zurechtfinden. Zwischen verständnisorientierter Partnerschaft und (romantischer) Liebe changieren die Bilder, die handlungsleitend wirken. Ein Liebesideal, das die Bedingungslosigkeit einer Liebesbeziehung betont, erschwert das Aufrechnen und Einordnen von Leistung. Man tut, was man tut, der Liebe wegen, und nicht, um etwas zurück zu bekommen (vgl. Oechsle 1998). 
schen Beziehung gewünscht. Die beruflichen Schwierigkeiten von Carlos kamen da unerwartet und vor allem unerwünscht hinzu. Die Zwangsstruktur female breadwinner Modell, welche als drittes Bearbeitungsfeld in der Narration Nicoles sichtbar wird, wird hinsichtlich zweier Dimensionen relevant: zum einen hinsichtlich der aktiven Integrationsarbeit, die von Nicole geleistet wird und die für sie bedeutet, sich um Carlos' berufliche Zufriedenheit zu sorgen, und zum anderen hinsichtlich des Normalisierungsmanagements, welches sie auf der Ebene der privaten Beziehungsgestaltung betreibt.

\section{Berufliche Zufriedenheit}

Die berufliche Integration ist für Nicole und Carlos die derzeit drängendste Aufgabe. Für Nicole steht dabei außer Frage, dass sie Carlos bei der Suche nach beruflicher Beschäftigung behilflich ist. Der Machtvorsprung, den sie aufgrund ihrer Position als natio-ethno-kulturell Wissende hat, bedeutet für sie die Verantwortungsübernahme für das gemeinsame Ziel. ${ }^{6}$ Die traditionell weibliche Aufgabe der Fürsorge für die Familie wird für Nicole zur konkreten Aufgabe, ihre Kenntnisse nutzbringend einzusetzen. So ist es für sie selbstverständlich, die finanziellen Aufwendungen für den Sprachkurs zu tragen. Auch nutzt sie ihre eigenen sozialen Netzwerke, um Carlos zumindest ein Praktikum in einer Werbeagentur zu ermöglichen (vgl. zur Diskussion um care-work insbesondere die Extraausgabe der Feministischen Studien 2000). Dabei ist jedoch nicht die finanzielle Sicherung des Familieneinkommens oberstes Gebot: „Ich mein von meiner Seite aus wär das auch noch gegangen finanziell, ich kann sparen das is es nich, aber ich glaub er war froh arbeiten zu können, doch“ (13/32 - 14/1). Ein solches Verständnis des Primats der beruflichen Zufriedenheit korrespondiert mit seinen Erwartungen an seine berufliche Zukunft, die aufstiegs- und interessensorientiert sind. Als Carlos Unzufriedenheit mit seinem Praktikum wächst, stellt die Konsequenz insofern kein großes Problem für Nicole dar:

„Sin wir zurückgekommen von Mexiko und hab ich mich nachts hingesetzt hab unsere Finanzen alle durchgerechnet, geguckt, wenn es dich nicht glücklich macht - irgendwie schaffen wir des schon. - Und - - dann ham wir nen halbes Jahr für ihn Arbeit gesucht. Ich hatte Gott sei Dank noch [...] Sparbuch, davon ham wir dann teilweise gelebt“" $(14 / 2-7)$.

6 Dies ließ sich in vielen Interviews beobachten, die Integration in den deutschen Arbeitsmarkt erfolgt vorrangig über die eigenen sozialen und beruflichen Kontakte und Ressourcen, so gut wie nie wird auf staatliche Behörden zurückgegriffen. 


\section{Normalisierungsmanagement}

Letztendlich, so lässt sich Nicoles Bearbeitungsstrategie mit ihren eigenen Worten resümieren, will sie es ihm ,so einfach wie möglich machen“ (30/27). Um dies zu realisieren, lassen sich Strategien analysieren, die von Deutsch und Saxon als „Traditional ideologies, nontraditional lives" beschrieben wurden: „When the mother goes to work outside the home, it is not as a breadwinner, regardless of how much money she makes, but to help when her husband can't provide sufficiently. She goes as his proxy“ (ebd. 1998: 358). Die Mythen, wie die Autorinnen die zentralen Überzeugungen der Paare nennen, ermöglichen es, die Handlungsfähigkeit des Paares aufrechtzuerhalten, trotz der ungewollten Konstellation. Solche „traditional gender identities“ (ebd. 1998: 331) beziehen sich auf die Grundkonstante der familiären Arbeitsteilung: Der Mann bleibt, trotz geringerem Verdienst oder Arbeitslosigkeit, der Haupternährer der Familie, und die Frau bleibt, trotz der Erwerbstätigkeit, die Verantwortliche für die Arbeit mit der Familie und den Kindern. Nicole sieht die Tatsache, dass sie das Geld verdient, als „normal“ $(29 / 22)$ an, schließlich gibt es in einer Ehe keine getrennten Kassen, und zurzeit sei sie nun mal diejenige mit dem regelmäßigen Einkommen. Schwierigkeiten entstehen jedoch aufgrund der prekären finanziellen Lage und aufgrund ihres Anspruches, Carlos die schwierige finanzielle Situation zu verschweigen und ihn nichts von ihren Zukunftsängsten und Sorgen wissen zu lassen:

„Und - ja - - ich hab halt - nicht zeigen dürfen dass es mir dabei auch ein bisschen schlecht geht weil ich mir halt dann Sorgen um die Zukunft gemacht hab, schon irgendwo. Wenn kein regelmäßiges Zweit_ 'ne Zeitlang kann man es immer gut mit einem Gehalt überbrücken, - aber - - man möchte halt doch, obwohl er arbeitslos war hab ich halt zugeschaut dass mir halt doch jedes Jahr nach Mexiko konnten. Finanziell - - klar muss man irgendwo einsparen, und hab dann halt nur noch beim Discounter (auflachend) Läden viel eingekauft.Aber das so zu machen das es nicht auffällt, das ist das Schwierige an der Sache" $(31 / 15-22)$.

Sie macht ihn keinesfalls für die Situation verantwortlich, weist vielmehr auf sein schlechtes Gewissen hin, wenn er wüsste, dass aufgrund seiner Arbeitslosigkeit gespart werden muss: „Weil ich gewusst hab er würd sich sonst - Vorwürfe machen. - Weil er ja den Job nicht hat, sozusagen" (31/29f.). Solche Coping-Strategien sind auch aus deutschen Studien zu Arbeitslosigkeit von Männern bekannt (vgl. dazu vor allem Schreyer 1991). Sie stellen aktive Normalisierungsstrategien dar, die komplexe Beziehungsarbeit erfordern, um das prekäre Gleichgewicht 
aufrecht zu erhalten. Da es Carlos unangenehm wäre, wenn sie im Restaurant bezahlt, gibt sie ihm unter dem Tisch den Geldbeutel oder steckt ihm vorher Geld in die Hosentasche, damit er bezahlen kann: „Wenn wir Essen gegangen sind hab ich ihm unterm Tisch meinen Geldbeutel gegeben damit er bezahlt hat, $u_{\text {_ }}$ und damit es nicht so blöd aussah" (30/5f). ,Blöd aussehen“ tut es allerdings nicht nur für Carlos, dem zuliebe sie scheinbar zu solchen Mitteln greift, auch sie möchte solche Momente der Demonstration des Ungleichgewichts und der ,Unordnung' in der Beziehung vermeiden. Was sie verbindet, sind ähnliche Ansichten über Geschlechterbeziehungen und Formen des Ausdrucks, kulturelle Differenz wird hier instrumentalisiert, um solche Geschlechterbeziehungen im Alltag zu implementieren. Beide verstehen sich also, um an den Aspekt der Chance durch Explikation wieder anzuknüpfen, nach wie vor wortlos.

\section{Mexiko als ,Gegenhorizont"}

Verbunden werden diese Formen von gender-Konstruktionen mit der Hervorhebung und der Aktualisierung natio-ethno-kultureller Eigenheiten: „In Mexiko würd ich zum Beispiel niemals die Rechnung kriegen. Die Rechnung wird ihm hingelegt" (30/7f). Damit vollzieht sie nicht nur eine Kulturalisierung der Situation, gleichzeitig öffnet sich ein imaginierter Handlungshorizont, der auf zwei Ebenen als Gegenentwurf zu Deutschland und den existierenden Schwierigkeiten fungiert. Zum einen als langfristige Umzugsoption: Sie liebt „die Mentalität. - - die Kultur“ (26/6) in Mexiko und ist begeistert von den dort existierenden Geschlechterverhältnissen:

„Auch die Art - ähm es stimmt überhaupt nicht wie ses hier oft darstellen dieses Machos - sie behandeln die Frauen einfach besser als in Deutschland. - ich würde nich sagen das die Frauen deswegen nich emanzipiert wären - nur - sie ham sich halt die Vorteile da raus geholt" (26/9ff).

Emanzipation wird von Nicole so nicht in Frage gestellt und wohl auch für sich in Anspruch genommen. In Mexiko aber lässt sich ein emanzipiertes Lebensmodell mit bestimmten ,weiblichen' Vorteilen kombinieren, die deutschen Frauen sind hingegen etwas übers Ziel hinausgeschossen. Zum anderen aber lassen sich so aktuelle Bearbeitungen und Auseinandersetzungen in den unhinterfragbaren Raum der anderen Kultur verlagern. Deutschland schneidet im Vergleich mit Mexiko schlecht $\mathrm{ab}$, die Mexikaner sind offener, das Essen ist besser und der Familienzusammenhalt größer (vgl. 26/15ff). Inwiefern sich diese Vorstellungen im 
alltäglichen Leben dort tatsächlich realisieren würden, bleibt dabei ausgeblendet, solche Imaginationen ermöglichen aber die Fortführung der eigenen Handlungsorientierungen und der ihres Mannes, ohne diese zu reflektieren und eventuell sogar in Frage zu stellen (vgl. zur Imagination des ,ganz Anderen“ auch Schlehe 2000a: 133).

\section{Bearbeitungsfelder bei Carlos: Desillusionierung und Re-Migration}

Ebenso wie für Nicole gestaltet sich für Carlos der Migrationsprozess und die daraus resultierenden Folgen als schwierig. Er selbst versteht sich als ,pluralitätskompetent“" (Mecheril 1997: 306) und hat insofern keine größeren Schwierigkeiten hinsichtlich der Integration erwartet. Vielmehr ist er von der weitestgehenden Anschlussfähigkeit sowohl seiner privaten wie seiner beruflichen Entwürfe ausgegangen, Deutschland stellte in diesem Kontext insofern die Möglichkeit zur Weiterqualifizierung bzw. zur Realisierung seiner beruflichen Qualifizierung dar. Hinsichtlich der Bearbeitungsfelder lassen sich für Carlos daher sowohl die Geschlechterbeziehungen wie auch die berufliche Integration ausmachen. Gerahmt werden diese Felder durch Auseinandersetzungen mit natio-ethno-kulturellen Positionierungen.

\section{Geschlechterbeziehung}

Für Carlos sind die erste Zeit in Deutschland und sein neuer Status als „Hausmann“ (3/14) sehr problematisch. Ohne Geld und ohne Arbeit findet er es schwierig, Nicole zu heiraten: „Zum Anfang hab ich gedacht ich heirate mit der Nicole - aber - so ohne Geld, ohne Job, ohne gar nichts dann das war natürlich schwierig“"(15/23f.). Ehe bedeutet für ihn die Übernahme von Verantwortung und (finanzieller) Fürsorge des Mannes für die Frau. Da dies zu diesem Zeitpunkt aber nicht möglich war, stellt er auch die Hochzeit in Frage. Um seinen Aufenthalt in Deutschland zu sichern, müssen sie jedoch nach drei Monaten eine Entscheidung treffen. Nicole sagt ihm die finanzielle Unterstützung zu, die Heirat findet auch statt, für ihn bedeutet eine Heirat auf Kosten der Frau allerdings eine problematische Situation, die zukünftig revidiert werden muss. Er möchte Nicole an ihrem zehnten Hochzeitstag erneut heiraten: „Und - ja natürlich hab mich nich so, nich so toll äh gefühlt. - Bis jetzt äh - ich will noch mal verheiraten mit der Nicole“ (15/30f). Die finanzielle Abhängigkeit, die von Nicole durch den Verweis auf Liebe und Ehe aufgefangen und in ihrer Wirkung relativiert werden konnte, wird von Carlos als emotionale Belastung empfunden. Eine erneute Hoch- 
zeitsfeier soll die Verhältnisse sowohl nach außen als auch nach innen wieder gerade rücken und seinen Status als auch finanziell erfolgreicher Migrant festigen. Dies gilt es umso mehr zu beweisen, da sich die finanzielle Abhängigkeit durch seine Arbeitslosigkeit fortsetzt. Nicole ist

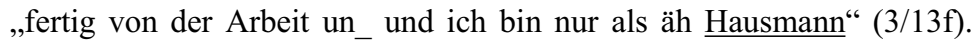
Anders als bei Rachid, der den Bildungsaspekt seiner Migration und seiner verschiedenen beruflichen Erfahrungen betont, sind für Carlos die berufliche Partizipation und der berufliche Aufstieg richtungsweisend. Er kann seiner Situation als Hausmann daher keine positiven Seiten abgewinnen. Die Zeit der Arbeitslosigkeit findet er vielmehr

„schrecklich, schrecklich. Ja, ich äh - ich bin - ich glaube ich bin ein sehr guter ähm - Hausmann, ja aber - es ist nicht so so - für mich. Ähm, ja, hier den ganzen Tag sein, und natürlich hab ich Job, äh Job gesucht - aber, - das ist nicht so einfach. Äh, - ich habe ähm, gekocht für meine Frau, ja aber das Gefühl ist nicht so toll““ $(18 / 26-29)$.

Die ungewollte Situation findet in der Statusumkehrung ihren Kulminationspunkt. Sein Verständnis von Geschlechterbeziehungen in der Ehe beinhaltet die Zuständigkeit des Mannes für den Erwerbsbereich, diese Aufgabenverteilung ist für ihn entscheidend. Einen solchen engen $\mathrm{Zu}-$ sammenhang zwischen Beziehungsmodell einerseits und Entscheidungen über die Arbeitsteilung in der Ehe andererseits sehen auch die Autorinnen einer amerikanischen Studie, die diesen Zusammenhang untersucht haben: „The way couples constructed gender in their marriages permeated their decisions" (Zvonkovic/Greaves/Schmiege et al. 1996: 98). Statt dass er also für den Familienunterhalt aufkommen kann, muss Nicole für Carlos deutliche Mehrkosten in Kauf nehmen: Er besucht eine teure Sprachschule, sie ziehen in eine größere Wohnung, und er braucht neue Kleidung. Carlos ist bis heute nicht klar, wie Nicole die Zeit finanziell überbrückt hat (vgl. 3/17f), das Management der finanziellen Engpässe wird jedoch Nicole überlassen. Anders also als Nicole es der Interviewerin gegenüber andeutet, weiß er von den finanziellen Problemen. Die Coping-Strategien der beiden korrespondieren jedoch miteinander: Die Normalisierungsbestrebungen erfordern die Aufrechterhaltung der Fassade des Nicht-Wissens auf der einen Seite und des Nicht-Sagens auf der anderen Seite. Die Mythen (vgl. erneut Deutsch/ Saxon 1998) helfen auch Carlos, die Handlungsfähigkeit aufrecht zu halten. 


\section{Berufliche Integration}

Hinsichtlich seiner beruflichen Aufstiegsambitionen erfährt Carlos deutliche Dequalifizierungsprozesse. Weder kann er, wie erwartet, seine Englischkenntnisse nutzen, noch ist Deutsch die leichte Sprache für die er sie gehalten hat. Seine ersten Arbeitserfahrungen als Hilfskellner in einem Restaurant verlaufen dementsprechend frustrierend:

„Nachher - vier, fünf Monate dann ich habe als Kellner [...] gearbeitet, nur für äh am Wochenende. - [...] also Hilfe von dem Kellner. - Ähm, aber war nich so toll auch, weil sie -sie haben mir etwas in Deutsch gesagt und ich habe nicht - gar nicht kapiert, und ja, natürlich ich war frustriert. Und dann ich habe gesagt naja warum kann ich nicht verstehen, warum - ich weiß nicht warum die die kommt nicht die Sprache sofort zu mir. Das war etwas äh schwierig. Äh ich kann, also gut Englisch - aber - das hilft mir nicht so ganz in Deutschland. [...] Nein, die_das ist komisch, weil - i_ich habe gedacht viele Deutsche können äh können ähm Englisch, aber das ist nicht so.“ (3/19-28).

Die Deutschen sind nicht die kosmopolitischen Menschen, für die er sie gehalten hat, und auch seine Kompetenzen und sprachlichen Fähigkeiten lassen ihn im Stich. Carlos überraschen diese Fremdheitserfahrungen kultureller und sozialer Art, Unvertrautheit und Nichtzugehörigkeit strukturieren seine ersten beruflichen Erfahrungen in Deutschland (vgl. dazu Stenger 1998) und Deutschland ist für ihn eine ,ganz andere - anderer Welt" (3/6f). Seine bisherigen Qualifikationen sind nicht übertragbar, die Anschlussfähigkeit seiner biographischen Entwürfe damit nicht (mehr) gewährleistet. Trotz dieser ersten Erfahrungen bleibt aber die Motivation der Verwertung seiner beruflichen Qualifikationen bestehen. Er beginnt, vermittelt durch Kontakte von Nicole, in einer Agentur als Praktikant zu arbeiten, wechselt in der weiteren Folge häufiger die Arbeitsplätze und nimmt für eine neue Stelle auch weite Anreisewege und Pendeleien zwischen Wohnung und Arbeitsstätte in Kauf. In seiner derzeitigen Anstellung sieht er das erste Mal die Möglichkeit der längerfristigen Beschäftigung. Er rechnet sich gute Chancen aus, dort langfristig als „Senior Art Director“ (6/17) zu arbeiten. Seine Unvertrautheit mit kulturellen und gesellschaftlichen Entwicklungen in Deutschland, die für die Ausarbeitung von Werbestrategien relevant sind, behindern jedoch den Aufstieg. Unterschiedliche Mentalitäten drücken sich in der unterschiedlichen Verwendung von Farben und Stilmitteln aus (vgl. 6/17ff). Der Spaß und die Leichtigkeit, die mexikanische Werbung auszeichnen, fehlen der deutschen Werbung, ihm fehlen damit entscheidende Kenntnisse. 


\title{
Positionierungen
}

Die Irritationen liegen für Carlos sowohl auf der Ebene von Geschlechtordnungen und -beziehungen als auch auf der beruflichen Ebene. In beiden Fällen ließ sich die Anschlussfähigkeit, die doch ein wesentliches Moment seines biographischen Entwurfs darstellte, nicht realisieren. So verwundert es nicht, dass Carlos rückblickend die Entscheidung für die Migration anzweifelt:

\begin{abstract}
„Äh, wenn ich denke jetzt was wär wenn ich - mehr Zeit über - über über Gedanken über diese Idee oder weiß nicht, ich glaube ich wär nicht nach äh Deutschland gegangen, oder - weil wenn ich denke es ist nicht so einfach wie ich gedacht habe - und mit der Sprache oder so. Dann, dann hätt ich vielleicht in Mexiko geblieben“ (14/16 - 19)
\end{abstract}

Die erwartete einfache Migration und die bessere Lebensqualität ließen sich nicht realisieren, und bei genauerer Planung und genauerer Kenntnis der Schwierigkeiten hätte er sich diesen wohl nicht ausgesetzt. Mexiko und Carlos' natio-ethno-kulturelle Zugehörigkeit als Mexikaner bleiben für ihn im Migrationsprozess und während seiner Erfahrungen in Deutschland die zentralen Bezugspunkte. Sein Herkunftsland gewinnt, wie auch schon bei Nicole zu beobachten war, an Relevanz als Handlungsraum und zwar sowohl hinsichtlich der Wahrnehmung alltäglicher Situationen, wie auch im Hinblick auf Zukunftsentwürfe.

\section{Alltagspraktiken}

Diese Relevanzsetzung wird von Carlos zum einen als Begründungszusammenhang für Alltagspraktiken verwendet:

„Nicole hat alles bezahlt und naja, das ist schwierig im Teilen, ich glaube von uns, als Ausländer. - - weil [...] wir gehen in ein Restaurant un_und der Mann muss, also meiner Meinung nach der Mann muss alles kümmern, oder - alles sagen und - ich konnte das nicht machen, weil - - äh die Sprache und - und auch den den den Währung?“ (16/4-8).

Hier werden deutsche und ausländische Mentalitäten und Geschlechterordnungen einander gegenüber gestellt, und so Carlos' Zugehörigkeit zu der ,anderen' Gruppe verdeutlicht. Heterosexuelle Praxen der Höflichkeit, der Rituale, ,Selbstverständlichkeiten` im Umgang mit Frauen, die Übernahme der Kommunikation im Restaurant sind nicht möglich, seine Fremdheit wird in solchen Momenten sicht- und fühlbar. Der so entstehende Rückbezug auf seine natio-ethno-kulturelle Zugehörigkeit dient als Begründungszusammenhang für sein Unbehagen. Diese essentialisie- 
rende Positionierung beinhaltet jedoch zum anderen und gleichzeitig eine personale Entlastung: Die Verantwortung für sein Unbehagen wird der kulturellen Differenz als drittem, unhintergehbarem Moment zugewiesen. Die vorgenommene kulturalistische Vergemeinschaftung „,von uns, als Ausländer" verschärft und organisiert diese Zuweisung, er ist Vertreter einer ganzen Gruppe (vgl. weiterführend zur Kulturalisierung von Erklärungsmustern und Paarkonflikten z.b. Lauth Bacas 2001: 121ff). ${ }^{7}$ Seine Zugehörigkeit steht daher nicht zur Disposition:

„Natürlich ich will nicht, ich will Mexikaner bleiben u_ und meine mexikanische Meinung und Mentalität bleiben“ (24/5f). ${ }^{8}$ Vielmehr erwartet er die Anpassung an seine Vorstellungen: „Ich sage in Mexiko ist es ganz anders, sie sagt ja aber wir sind nicht in Mexiko. - Wir sind in Deutschland. Aber ich sage ja warum, äh warum sie verändern das nicht" $(16 / 21-23)$.

An solchen Konstruktionen zeigt sich, dass er nach wie vor Anschlussmöglichkeiten fokussiert. Die Fortführung von mexikanischen Konzepten und Modellen, sei es hinsichtlich Geschlechterbeziehungen oder anderer Alltagspraktiken, organisiert nach wie vor seine Erwartungen.

\section{Zukunftsentwürfe}

Neben den Alltagspraktiken findet die Relevanzsetzung ebenfalls im Hinblick auf den weiteren biographischen Entwurf statt. Die Erfahrungen im Zuge des Migrationsprozesses werden also nicht, wie bei Rachid, als handlungs- und orientierungserweiternde Erfahrungen verstanden. Vielmehr lässt sich bei Carlos eine funktionale Orientierung an Deutschland als Möglichkeit der Qualifikationserweiterung analysieren. So möchte er langfristig ein Restaurant in Mexiko aufmachen. Dazu möchte er die Erfahrungen, die er in Deutschland gemacht hat, nutzen, um eine Qualitätssteigerung seines Restaurants zu ermöglichen:

„Und und aber ich will ein Restaurant wie - wie in Deutschland, etwas wie in Deutschland. Also wirklich ins Business kommen und, und äh mit guter Quali-

7 Eine solche Konstruktion lässt sich als interessante Variante des ,strategischen Essentialismus“ (vgl. Spivak 1996 (1985)) lesen. Dies meint die Artikulation von essentialisierenden Haltungen, die jedoch auf dem Wissen des Konstruktionscharakters basieren und denen so eine strategische und gegebenenfalls emanzipatorische Funktion zugesprochen werden kann. Die strategische Funktion liegt für Carlos hier in der Entlastung von aufwendigeren Begründungen wie auch der Entlastung von Reflexionsnotwendigkeiten. Insofern hat diese Konstruktion in gewisser Hinsicht ebenfalls emanzipatorische Funktion.

8 Intensiven Kontakt zu seiner Familie zu halten und jährlich nach Mexiko zu fliegen, ist für ihn daher selbstverständlich. 
tät, also deutsche Qualität so. Äh - - aber ich weiß nicht, das, das ich brauche viele Sachen, viel Geld, viele Investieren und - - weiß nicht. Das dauert noch“" $(26 / 18-21)$.

Während sich also die Qualität ,made in Germany` exportieren lässt, soll die mexikanische Mentalität und Arbeitseinstellung erhalten bleiben:

„Die ist - die Stimmung ist immer toll, e_es ist nicht wie in Deutschland, - Ein Restaurant. Mein Chef war mein Freund, und, er war nie, nie da. Und, und es ist nicht wie in Deutschland das der Besitzer muss dort sein und kochen und. Es ist ganz anders. Mein Chef ist nur abends gekommen und dann eventuell wie wie geht's. Gut. Wie läufts, gut. Ah, ok, komme morgen“ (26/14 - 17).

Carlos schwebt eine Form von längerer Arbeitsmigration vor. Die hier erworbenen Kenntnisse über Marketingstrategien und Qualifikationssteigerungen will er nutzen, um sich in seinem Heimatland eine bessere Ausgangsposition zu verschaffen. Dabei entwickelt er eine eher romantisierende Vorstellung von der Position eines Restaurantleiters. Inwiefern sich diese Vorstellungen tatsächlich realisieren lassen würden, ist in diesem Zusammenhang allerdings nicht die entscheidende Frage, vielmehr dienen solche Entwürfe im Ergebnis der Stabilisierung der aktuell schwierigen Situation und ermöglichen es, handlungsfähig zu bleiben, ohne sich selbst grundlegend in Frage zu stellen.

\section{Form biographischen Lernens: natio-ethno- kulturelle Zugehörigkeit als Handlungsfeld für Konstruktionen von Geschlechterbeziehungen - Individualisierung als Retraditionalisierung und die Entwicklung eines Gegenhorizontes}

Root schreibt über interracial marriage: „Love alone motivated these women and men to cross the color line, despite a lifetime of cultural indoctrination" (Root 2001: 6). Ein solches subjektivierendes Bild versteht Liebe allerdings eher als eine black box, in dessen Inneren unerklärbare Prozesse ablaufen. Dass Liebe vielmehr als Chiffre, als Erklärungsmuster für komplexe Zuschreibungsprozesse fungieren kann, wurde schon im Forschungsüberblick deutlich und lässt sich auch bei diesem Paar analysieren. Liebe dient auch Nicole und Carlos als Erklärungsmuster für ihre Beziehung. Das Motiv der romantischen Liebe beinhaltet insbesondere für Nicole das Entdecken dessen, was sie bei deutschen Männern vermisst hat. Es lassen sich Konstruktionen von gender und natio- 
ethno-kultureller Zugehörigkeit analysieren, die als Antwort auf Modernisierungsanforderungen gelesen werden können. Dennoch stellt für beide die Beziehung die Möglichkeit der Realisierung eigener biographischer Entwürfe dar. Die Partnerschaft mit Carlos bietet Nicole die Möglichkeit, eine Art ,Gegenmodell‘ zum modernen und in ihren Augen falsch-emanzipierten Partnerschaftsmodell Deutschlands zu leben. Realisieren lässt sich so eine Rückkehr zur altmodischen, aber nichtsdestotrotz erstrebenswerten Beziehungsform zwischen Männern und Frauen. Auf bestimmte Rollenverteilungen hätte sie, so Nicole, bei einer Beziehung mit einem deutschen Partner verzichten müssen. In einem kulturalistischen Verständnis sieht sie Carlos und sich als Repräsentant(inn)en des jeweiligen Herkunftslandes und dessen Beziehungs- und Familienmodells. Natio-ethno-kulturelle Zugehörigkeiten werden von ihr daher vor allem in ihrer prägenden Funktion, als handlungsleitende Erzeihung, gesehen. Das von ihr favorisierte Beziehungsmodell beinhaltet für sie auch die unbedingte Positionierung auf der Seite des Partners, dafür nimmt sie auch eine Veränderung des sozialen Nahraums in Kauf. Für Carlos stellt sich die Beziehung zu Nicole als Möglichkeit dar, seine in Mexiko entwickelten Handlungsorientierungen in Deutschland beizubehalten und gleichzeitig seine beruflichen Aufstiegsambitionen und den Wunsch nach einer „,besseren Lebensqualität“ zu realisieren. Dafür ist Nicole sowohl hinsichtlich seiner Imaginationen von Geschlechterverhältnissen wie auch hinsichtlich ihrer natio-ethno-kulturellen Zugehörigkeit die geeignete Frau, um die Anschlussfähigkeit zu gewährleisten. Seine eigene Zugehörigkeit reflexiv in Frage zu stellen, steht für Carlos nicht zur Diskussion. Beiden dient die natio-ethno-kulturelle Zugehörigkeit des bzw. der Anderen als Begründungszusammenhang für die Weiterführung bzw. Realisierung ihres biographischen Entwurfs. Diese Entwicklung des Paarhorizonts war, sagen beide, stark auf Imaginationen aufgebaut. Nicole und Carlos haben sich den Migrationsverlauf und das Leben in Deutschland anders vorgestellt. Sie sind von einer selbstverständlichen Übertragbarkeit ihrer Vorstellungen ausgegangen und haben sich vorher nicht mit den rechtlichen und sozialen Folgen der Migration für sie als Paar auseinandergesetzt. Dementsprechend lassen sich die Folgen der Migrationsentscheidung und des Migrationsprozesses durchaus als Bruch und als deutliche Irritation ihres biographischen Entwurfes fassen. Die female breadwinner Konstellation ist für Nicole und Carlos sowohl unerwartet als auch unerwünscht. Nicht nur die berufliche Situation ist jedoch prekär, auch das soziale Umfeld reagiert nicht in der erwarteten unterstützenden Form.

Bei der Bearbeitung dieser Zumutungen lässt sich bei Nicole allerdings keine Modifikation ihrer Beziehungsgestaltung erkennen wie bei 
Susanne. Vielmehr sind die Bearbeitungen verstärkt auf die dauerhafte Realisierung ihres biographischen Entwurfs gerichtet, die Irritationen fordern sie zu keiner Reflexion dieses Entwurfs und seiner möglichen Grenzen heraus. Innerhalb dieser Bearbeitungen bekommt vielmehr Carlos' ,andere' natio-ethno-kulturelle Zugehörigkeit eine wesentliche Funktion zugesprochen. Für Nicole bedeutet der Beginn der Ehe auch den Beginn des starken Bezuges auf den Partner und der eindeutigen Positionierung auf seiner Seite. So verändert sich der Freundeskreis, Irritationen werden, wie z.B. hinsichtlich der für sie prekären finanziellen Konstellation, ignoriert bzw. möglichst vermieden. Damit geht für Nicole die Übernahme seiner Perspektiven und die dadurch stattfindende Verfremdung ihrer eigenen Kultur und Mentalität einher. ,Kultur' und kulturelle Mentalitäten werden in diesem Prozess zum unhintergeh- und unhinterfragbaren Moment, welches dadurch Auseinandersetzungen und Reflexionen nicht notwendig werden lässt: Kultur wird zum nutzbaren und genutzten Begründungszusammenhang. Wie auch Waldis formuliert, wird der kulturelle Unterschied instrumentalisiert, um eine neue Form von Partnerschaft zu praktizieren (Waldis 2001: 148). Hierbei handelt es sich allerdings nicht, wie von Beck-Gernsheim gehofft, um emanzipativ-moderne Paare, vielmehr dient die Instrumentalisierung als Begründungsstruktur für die Rückkehr zu traditionellen Geschlechterbeziehungen. Auch Carlos nutzt seine natio-ethno-kulturelle Zugehörigkeit, um sein Unbehagen hinsichtlich des female breadwinner Modells $\mathrm{zu}$ erklären und sich den daraus entstehenden Irritationen zu entziehen. Im Zuge der stattfindenden Dequalifizierungsprozesse gewinnt seine „mexikanische Mentalität“ an Bedeutung, und Mexiko rückt als Handlungs- und zukünftiger Wohnort wieder stärker in den Vordergrund. Deutschland spielt so vorrangig hinsichtlich der Qualifikationsmöglichkeiten eine Rolle, um ein Leben in Mexiko zu erleichtern und ihm eine bessere Ausgangsposition zu verschaffen. Diese Form lässt sich als funktionelle Integration beschreiben: Die notwendigen funktionalen Anpassungen werden vorgenommen, die dahinter sichtbaren „Mentalitäten“, wie er es nennt, jedoch nicht in Frage gestellt, eine kulturelle oder emotionale Integration ist nicht gewollt. Zum Zeitpunkt der Interviews stehen beide an einem ,Zwischenziel'. Sie haben eine Stabilität erreicht, die aber dennoch von beiden noch als prekär erfahren wird. So ist auch der nächste Wunsch der beiden, eine „kleine Familie“ (Nicole 39/15), erstmal aufgeschoben, bis sich die Berufstätigkeit von Carlos als dauerhaft erweist und damit die finanzielle und organisatorische Grundlage für die Familiengründung gelegt ist: „Also erst ich bin noch in Probezeit in meiner Arbeit, ich will einen sicheren Job haben. - Und - dann schau- 
en wir weiter, das weiß ich noch nicht. Es ist immer noch unsicher. Aber ja, Kinder sind geplant" (Carlos 18/11-14).

Beide Biographien lassen sich in ihrer Entwicklung in gewisser Hinsicht als Individualisierungsbiographien lesen. Dabei gilt für Nicole und Carlos, wie für Susanne und Rachid, dass sie natio-ethno-kulturelle $\mathrm{Zu}$ gehörigkeit als Handlungsfeld für Geschlechterbeziehungen nutzen. Die Ergebnisse der Studien von Waldis 2001 oder Larcher 2000, dass binationale Ehen „dynamisierend auf die bestehenden Geschlechterbeziehungen in einer Gesellschaft“ wirken, ,weil sie durch ihre Existenz und Praxis deren Grenzen aufzeigen und sie so in Frage stellen" (Waldis 2001: 148), lassen sich insofern durchaus auf Nicole und Carlos übertragen. In der Regel werden damit jedoch zumindest implizit ,moderne“ Geschlechterbeziehungen gemeint. Hier jedoch ist die Beziehung auf die Rückkehr zu traditionellen Geschlechterbeziehungen gerichtet. Die Notwendigkeit zur Auseinandersetzung mit natio-ethno-kultureller $\mathrm{Zu}$ gehörigkeit ermöglicht also neue biographische Entwürfe und biographische Ausdrucksformen, die ansonsten für die Partner nicht ohne weiteres, oder wie Nicole sagt sogar aller Wahrscheinlichkeit nach, in ihrem jeweiligen Herkunftsland nicht realisierbar gewesen wären. Zwar ermöglicht die Konstellation Handlungserweiterungen, diese aber eben nicht nur in einem gesellschaftlich-emanzipatorischen Sinne, sondern auch hinsichtlich einer Retraditionalisierung von Geschlechterverhältnissen. Diese Individualisierungsprozesse verlaufen jedoch durchaus nicht unproblematisch. Anders als bei Susanne und Rachid, deren erfahrene Irritationen zu Modifikationen ihrer Beziehungspraxis führten, findet insbesondere bei Nicole eine starke Abgrenzung von der sozialen Umwelt und dem sozialen Nahraum statt. Der Freundeskreis, der der Beziehung kritisch bzw. eher skeptisch gegenübersteht und Nicoles starke Orientierung an der Beziehung in Frage stellt, wird sukzessive aufgegeben.

Die Form biographischen Lernens, die als übergeordnete Bearbeitungsform analysiert wurde, lässt sich daher als ,natio-ethno-kulturelle Zugehörigkeit als Handlungsfeld für Geschlechterbeziehungen: Individualisierung als Retraditionalisierung und die Entwicklung eines Gegenhorizonts ' bezeichnen. Es ließ sich zeigen, wie natio-ethno-kulturelle Zugehörigkeiten und Imaginationen über ,fremde‘ Mentalitäten zur Handlungserweiterung mit dem Ziel der Sicherung der biographischen Anschlussfähigkeit genutzt werden können. Die binationale Beziehung führt in der Rekonstruktion des Paares zu Grenzverschiebungen hinsichtlich Geschlechterbeziehungen, in diesem Fall jedoch zu deren Retraditionalisierung. Als im Migrationsverlauf die Anschlussfähigkeit durch äußere Irritationen und unerwartete Entwicklungen in Frage ge- 
stellt wird, findet im Verlauf der Beziehung der Bezug auf Mexiko und auf die mexikanische Mentalität als die bessere statt. ${ }^{9}$ Einerseits externalisieren und legitimieren sie damit bestimmte potentielle Konfliktfelder wie z.B. die finanziellen Aushandlungen oder die kritischen Äußerungen der Freunde. Nicole und Carlos können so äußere Irritationen ignorieren und Freundschaften beenden, ohne den Paarhorizont und die biographischen Entwürfe in Frage stellen zu müssen. Gleichwohl ist ein solches Bearbeitungsprinzip, welches die Paarbeziehung als entscheidenden Bezugspunkt setzt, durchaus kritisch zu sehen: Die Aufgabe, Risiken subjektiv abzusichern und institutionelle Zwänge individuell zu bearbeiten, führt und verführt zum Rückzug ins private, natio-ethno-kulturell aufgeladene Beziehungsmodell. Wenn Risiken und Herausforderungen nicht biographisch anschlussfähig erscheinen und integriert werden können, werden sie so weit wie möglich ignoriert. Insofern ist dieses Paar tatsächlich individualisiert, allerdings um den Preis der Abschottung von ,störenden' und irritierenden sozialen Einflüssen bei Verlagerung des Bezugshorizonts auf einen imaginierten ,Gegenhorizont", der die Retraditionalisierung ${ }^{10}$ begünstigt: Mexiko wird als Bezugskontext relevanter und spielt auch in den Alltagspraktiken eine große Rolle. Während Carlos seine mexikanische Mentalität explizit beibehalten will, passiert bei Nicole so etwas wie eine Zugehörigkeitsverschiebung und die Entwicklung einer Mehrfachverbundenheit: Sie übernimmt Carlos' Sichtweisen, blickt mit seinen Augen auf ihr Herkunftsland und orientiert sich auch in der Zukunftsplanung an ihm. Damit verbunden ist eine Form von transnationaler Biographieausrichtung. Mehrfachverbundenheit, weil es symbolische und alltagspraktische Zugehörigkeitsdeklamationen sind, die sich auf mehr als einen sozialen Raum mit geographischer Referenz beziehen (Mecheril 2000b.: 231). Transnational, weil so Räume geschaffen werden, die sich über nationale Grenzen hinwegspannen. Inwiefern sich Carlos` Pläne für ein Leben in Mexiko realisieren lassen würden, spielt nicht die entscheidende Rolle. Entscheidend ist dieser Gegenhorizont vielmehr in seiner Entlastungsfunktion, die er in der Bearbeitung der aktuellen Schwierigkeiten bekommt Nicole und Carlos sind, so ließe sich resümieren, vielleicht nicht ambiguitätskompetent, aber ambiguitätsresistent.

9 Damit bekommt der Integrationsbegriff auch eine neue Bedeutung im Sinne faktischer Partizipationsfähigkeit in bestimmten sozialen Räumen, die weder von einer Totalinklusion in alle Teilbereiche noch von der Übernahme von Normen und Werten abhängig ist (vgl. ausführlich De Carlo/Menz/Walter 2006).

10 Retraditionalisierung sowohl im Sinne einer traditionellen Geschlechterbeziehung wie auch im Sinne eines kulturalistischen Verständnisses von Personen. 
PROGRAMMATIK UND LEBENSWIRKLICHKEIT: Maria Wesel und Pablo Marmani Rodriguez

\section{Biographische Portraits Maria Wesel und Pablo Marmani Rodriguez}

\section{Maria Wesel}

Maria Wesel wird 1969 als drittes Kind in einer großen Stadt in Süddeutschland geboren. Ihre Eltern sind beide Professor(inn)en an einer Fachhochschule, sie hat zwei deutlich ältere Brüder. Die Kindheit erlebt sie als sehr frei und ungezwungen, sie ist sehr sportlich und will „also ganz, ganz stark ein Junge sein. Also ich glaub einfach durch die beiden Brüder, - irgendwie glaub ich durch das Fußballspielen [...]" (1/23ff). Mit 13 Jahren erkrankt sie an Rheuma. Die Krankheit stellt einen entscheidenden Einschnitt in ihrem Leben dar, sie muss ihre sportlichen Aktivitäten einschränken, gleichzeitig führt die Erkrankung zu einer Distanz von ihrem Körper. In dieser Zeit intensiviert sich ihr Verhältnis insbesondere zu ihrer Mutter. Durch den Aufenthalt in einer RheumaKlinik für Jugendliche lernt sie Rheuma als Normalität kennen und lernt selber über Einschränkungen zu entscheiden, auch gegen den Rat der Ärzte. Seit der Schulzeit hat sie ein Interesse an Lateinamerika und am Ausland generell: In der elften Klasse verbringt sie ein Austauschjahr in den USA, nach dem Abitur geht sie für einige Zeit nach Spanien. Sie beginnt ein Journalistikstudium, um Auslandskorrespondentin zu werden, und zieht dafür in eine große mitteldeutsche Stadt. Während des Studiums arbeitet sie in der Flüchtlingshilfe und engagiert sich auch bei 
Amnesty International. Im Rahmen des Studiums geht Maria für ein Auslandspraktikum nach Mexiko. Auch wenn sie ursprünglich nicht nach Mexiko, sondern lieber nach Argentinien wollte, da sie die dortige politische Situation spannender fand, ist sie sofort von Mexiko fasziniert:

„Also ich hab mich da viel mehr zu Hause gefühlt als in den USA. - und ich hatte das Gefühl es ist mehr, europäischer, - die legen auch Wert auf - alte Geschichten [...] Ähm vielleicht auch die katholische Kirche ich weiß es nicht, vielleicht. Ja, und oft auch dieses, also nicht nur dieses - - kapitalistisch, also Geld orientierte, also doch sehr familiäre Werte, so andere Werte auch noch ne Rolle spielen. - Dass in den USA zum Teil auch gibt, aber man nicht so stark sieht. [...] Und da habe ich in Mexiko also - diese alte Kultur und dieses aufeinander Treffen der Kulturen - dieses Vermischen der Kulturen, [...] den Rassismus gibt's schon auch, aber - lang nicht so wie wie woanders. Also du kommst wenn du heller bist schneller in ne Disko rein, als wenn du dunkel bist. Das gibt's da auch. - aber das ist nicht, diese ständige Auseinandersetzung mit den Kulturen“ (11/9-24).

In Mexiko lernt sie Pablo kennen und ist von Anfang an von seiner Offenheit und seinem Interesse beeindruckt. Er organisiert für sie Wochenendausflüge und versucht ihr möglichst viele ihrer Wünsche zu erfüllen. Nach zwei Jahren Fernbeziehung erfährt sie, dass sie mit Zwillingen schwanger ist. Da es sich aufgrund der Einnahme zellschädigender Medikamente um eine Risikoschwangerschaft handelt, ist es unsicher, ob die Kinder überleben werden. Pablo beeilt sich sein Studium abzuschließen und so schnell wie möglich nach Deutschland zu kommen. Wieder ist Maria beeindruckt von den ,bedingungslosen“ (15/21) Prioritäten, die er setzt: „Also wo ich auch das Gefühl hatte das hätte auch hier jemand also - so leicht nicht gemacht. Der hätte dann gesagt ja wir müssen mal schauen, und ich muss das erstmal mit meiner Arbeit“ (15/21 - 23). Die Kinder bestimmen die Beziehung von vorneherein mit. Während er die Kinder erzieht, arbeitet sie wieder in ihrem alten Beruf als Journalistin. Sie findet es schade, das Pablo Deutschland nur über die Kinder kennen lernt, und kaum einen eigenen Zugang, durch den Beruf oder durch mehr Freunde, bekommt. Für sie selber war die Konstellation nicht besonders irritierend, Pablo hatte eher das alte Bild im Kopf „,dass wenn er 'ne Familie er ein Vater ist der für die Familie sorgt" (28/6). Die Beziehung bringt Maria manchmal emotional und intellektuell an ihre Grenzen, insbesondere in politischen Diskussionen hat sie das Gefühl, keinen Zugang mehr zu ihm zu bekommen. Dennoch kann sie jetzt Sachen akzeptieren, die sie vorher nicht akzeptiert hätte, sie ist deutlich toleranter geworden. Ihre Eltern, insbesondere ihre Mutter, stehen der 
Beziehung sehr skeptisch gegenüber. Ihre Mutter wollte sie von der Hochzeit abbringen, und auch später erwartet sie von Pablo mehr berufliches Engagement, auch wenn er keine seiner Qualifikation angemessenen Arbeitstelle bekommt. Maria und Pablo wollen auf jeden Fall eine Zeit lang in Mexiko leben, sie möchten, dass beide das Herkunftsland und das soziale und familiäre Umfeld des jeweils anderen kennen. Maria freut sich auf den Umzug und ist fasziniert von der offenen Zukunftsperspektive, die sie durch Pablo kennenlernt.

„Pablo hat mehrere Pläne, dann nehmen wir eben irgendwie Babysachen mit und verkaufen eben Babysachen. Das gibt's viele Möglichkeiten. Babysachen geht immer. [...] Also dieses - wo unser_, wir oder ich eben ganz brav, ich bin für das ausgebildet und das mach ich. Und ich arbeite noch als Übersetzerin, aber was anderes kann ich nicht." $(18 / 7-12)$.

Für den Aufenthalt in Mexiko hat sie unbezahlten Urlaub genommen, so dass sie jederzeit zurück zu ihrer alten Stelle kann. Langfristig wäre ihr Traum, zwischen beiden Kontinenten zu pendeln. Sie könnte als Auslandskorrespondentin arbeiten, Pablo kann sich vorstellen eine deutschmexikanische Firma aufzumachen. Wahrscheinlich aber wird sich dieser Plan nicht realisieren lassen, da die Kinder in der Schulzeit nur in Deutschland leben sollen.

\section{Pablo Marmani Rodriguez}

Pablo Marmani Rodriguez wird 1973 in einer großen Stadt in Mexiko als jüngstes von insgesamt sechs Kindern geboren. Während seine Mutter aus einer hohen Bildungsschicht stammt, besitzt sein Vater nur „wenig ökonomischen Ressourcen [...] es gab immer sehr viele Unterschiede in meiner Familie auch wegen dieser ökonomischen Unterschiede, immer viele Streiten und, ja es war sehr unterschiedlich“ (1/16 - 20). Pablo erinnert sich insgesamt an eine glückliche Kindheit, hat aber ein schwieriges Verhältnis zu seinem Vater, den er als abweisend erfährt. Während der Schulzeit, die ihm Spaß macht, fühlt er sich fehl am Platz und erlebt eine „sehr chaotische Adoleszenz“ (2/22). Familiäre Schwierigkeiten setzen ihm zu, und er hat den Eindruck nicht zu wissen wo sein Platz ist. In dieser Zeit fährt er für einige Wochen nach Korsika, und sieht in dieser Fahrt einen Wendepunkt für seine weitere Biographie. Er lernt andere Jugendliche und andere Perspektiven kennen und kann sich selbst und seine Probleme besser einordnen: „eine Perspektive, eine Möglichkeit zu eröffnen, meine Augen zu öffnen, für das, was die andere Seite der Welt war“ (3/7f). Nach dem Abitur fängt er an, Architektur 
zu studieren. Das Studium fällt ihm sehr schwer, die Leistungserwartungen sind hoch und es gibt strenge Bewertungskriterien. In dieser Zeit trifft er auf einem Kulturfestival Maria. Seit seinem Korsikaaufenthalt war er an kulturellem Austausch interessiert, und so bemüht er sich, sie besser kennen zu lernen und ihr Mexiko zu zeigen. Sie halten Kontakt, und er besucht sie in Deutschland, um auch ihren Hintergrund kennen zu lernen. Nach zwei Jahren Fernbeziehung möchte er sie heiraten:

„,ich als Mexikaner sagte, gut, das Normale ist dass _, wenn, wenn _ wir schon planen zusammenzuleben, dass in Mexiko freie Beziehungen nicht akzeptiert werden, d.h. wohnen, mmhh, ohne zu heiraten, zusammenwohnen ohne zu heiraten und ich sagte zu ihr, gut, wenn du möchtest, dass wir zusammenleben, meine Familie, weil sie sehr konservativ ist, ich weiß nicht, wie sie das finden werden und ich möchte nicht, weil ich der Jüngste bin, so was, wie ein schlechtes Beispiel (lacht) setzen und dann sagte ich zu ihr, weißt du? Lass uns lieber heiraten“ $(5 / 19-6 / 2)$.

Die Auseinandersetzungen gestalten sich allerdings als schwierig, da Maria mit einer Heirat zuerst nicht einverstanden ist. In dieser Zeit wird sie schwanger, und damit ändert sich für Pablo die Situation. Da Maria aufgrund ihrer rheumatischen Erkrankung nicht nach Mexiko reisen kann, beeilt er sich sein Studium abzuschließen, um dann nach Deutschland kommen $\mathrm{zu}$ können. Von vornherein ist jedoch ein Leben in Deutschland und Mexiko geplant. Nach ,vielen, vielen, vielen gemeinsamen Gesprächen, fast philosophisch und magisch" (8/16f), ist Maria mit der Hochzeit einverstanden. Er war mit der Arbeitsteilung einverstanden, ist aber davon ausgegangen, dass er selber bald arbeiten könnte. Die Erziehung der Kinder ist ihm auch deshalb wichtig, weil er ein schwieriges Verhältnis zu seinem Vater hatte und für seine Kinder da sein möchte: „Der Vater muss für die Kinder da sein und ich fühle mich jetzt sehr glücklich, wenn ich mit meinen Kindern bin“" (23/17f). Die berufliche Integration in Deutschland hat sich als sehr viel komplizierter herausgestellt, als er erwartet hatte. Eine Stelle im Norden Deutschlands lehnte er ab, da er die Familie nicht alleine lassen wollte. Weder durch Kontakte von Maria noch durch eigene Bemühungen findet er eine andere Stelle. Die Formalität des Bildungssystems ist für ihn überraschend, seine in Mexiko entwickelten Strategien des Nutzens sozialen Kapitals greifen nicht. Auch eine Weiterqualifikation durch ein Aufbaustudium ist nicht möglich, da sein Abschluss nicht als Studienabschluss anerkannt wird. Zurzeit arbeitet er im Kindergarten seiner Kinder, ohne Bezahlung. Zugleich hat er den Kontakt nach Mexiko nicht abbrechen lassen, und hat zum Beispiel ein Haus für eine seiner Schwestern ent- 
worfen. Trotz der fehlenden bezahlten Arbeit versucht er jeden Tag sechs bis acht Stunden zu arbeiten, um einen normalen Arbeitsrhythmus $\mathrm{zu}$ behalten und sich selbst als beschäftigt zu verstehen. Zum Zeitpunkt des Interviews sind Maria und Pablo dabei, den Umzug nach Mexiko zu planen, der im kommenden Frühjahr stattfinden soll. Für beide war immer klar, dass sie in beiden Ländern leben wollen. Für Pablo ist der Umzug auch deshalb wichtig, weil die Familie von Maria sehr kritisch ihm gegenüber ist und von ihm verlangt, wenigstens als Taxifahrer zu arbeiten. Er wiederum erwartet von ihnen, dass sie seine „Kultur respektieren“ (26/21) und nicht von ihm verlangen, sich vollständig anzupassen. Langfristig hofft Pablo ein deutsch-mexikanisches Unternehmen aufbauen zu können. Die Kinder sollen jedoch in Deutschland zur Schule gehen, auch aufgrund Marias Erkrankung ist ein dauerhafter Umzug nach Mexiko nur schwer möglich. Er findet es wichtig, den Kindern die Möglichkeit zu geben, beide Kulturen kennen zu lernen, auch wenn das für die Eltern Einschnitte bedeutet: „Für sie ist es ein Gewinn und am Ende, klar, ein Elternteil muss das Risiko eingehen_, in einem anderen Land zu leben. Die Eltern sind diejenigen, die etwas riskieren, aber die Kinder müssen etwas gewinnen“ (19/19-21). Für sich selber sieht er in Mexiko nach wie vor die emotionale, in Deutschland die ökonomische Heimat:

„Dann möchte ich sehr viel in Deutschland arbeiten, mit diesem Projekt von der Firma, aber sehr viel arbeiten, und danach in Rente gehen, nach Mexiko, mit Maria. Das ist mein Traum, ich meine, der richtige Traum, sehr viel hier arbeiten und sich danach in Mexiko ausruhen“ (42/10 - 13).

\section{Der Horizont der Paare: Beziehung als bestätigendes Moment}

\section{Konstruktion des Paarhorizonts bei Maria: interkulturelle Begegnungen}

In Marias Erzählungen über den Beginn der Beziehung mit Pablo lässt sich die Konstruktion eines Paarhorizonts analysieren, der sich durch eine biographische Kontinuität hinsichtlich ihres Interesses an interkulturellen Begegnungen auszeichnet und so die Kontinuität und Sicherung ihrer bisherigen sozialen und politischen Positionierung zum Ausdruck bringt. Desweiteren lassen sich bei ihr Neupositionierungen in Geschlechterbeziehungen analysieren. Zusätzlich zu dieser Anschlussfähigkeit nach außen zeigt sich im Prozess der Horizontkonstruktion auch 
ein Blickwechsel auf ihr Herkunftsland, der sie im Ergebnis in ihrer transnationalen Biographieausrichtung bestärkt. Dem Blickwechsel liegt ein Prinzip eines Kulturvergleichs zugrunde, welcher Reflexionsprozesse anstößt.

\section{Programmatische Kulturoffenheit}

Die erste Dimension, die sich in den Interpretationen Marias analysieren lässt, ist die der Fortführung einer von mir als programmatisch bezeichneten Kulturoffenheit. Diese Offenheit findet einen Ausdruck in der Beziehung zu Pablo. Programmatisch ist sie deshalb, da sie vor allem diskursiv rekonstruiert wird und ihre Grenzen in den konkreten praktischen Aushandlungssituationen findet. „Interkulturalität setzt [...] beides voraus, schreiben Rieger et al. 1993: eine Grenze zwischen den Kulturen und ihre Überschreitung“ (Schlehe 2001b: 13). Einerseits stellt die Beziehung als interkulturelles Setting, von Maria auch als solches explizit gekennzeichnet, für sie eine Ressource der Reflexivität und des AnstoBes zur Neu-Orientierung dar. Andererseits findet diese reflexive Grundhaltung ihre Begrenzung in konkreten Aushandlungssituationen, wie der Entscheidung über den gemeinsamen Wohnort.

\section{Interkulturelle Begegnungen I: Anschlussfähigkeit}

Für Maria steht die Beziehung mit Pablo in einer biographischen Kontinuität, die durch ihr Interesse an politischen Entwicklungen mit einer Offenheit und einer Suche nach interkulturellen Begegnungen gekennzeichnet ist. Diese Offenheit zeichnet sich durch ein Verständnis von Kulturen aus, die von- und miteinander lernen können (vgl. zum habituellen Handeln auch Bohnsack/Nohl 2001: 22). Die Faszination der anderen Kulturen besteht für Maria vor allem in der Irritation durch ungewohnte und andere Denkweisen in interkulturellen Begegnungen und in den Reflexionsanstößen durch fremde Überzeugungen: „Und - wo man auch - so nicht die eigenen Überzeugungen vertritt. - Aber - ähm, andererseits denkt. Ne, es ist spannend und ist auch interessiert, und ist offen, kriege immer neue Sachen (9/25ff).“ Diese Suche nach neuen Erfahrungen schlägt sich sowohl in ihrer bisherigen Berufsbiographie wie auch in den verschiedenen Reisen nieder, die sie unternommen hat. Das Fremde ist ihr unvertraut, und ,seine Unvertrautheit kann uns zugleich ängstigen wie anziehen“ (Boesch 1998 in Straub/Garz/Krüger 2001: 9). Neben dieser Form der Erweiterung des eigenen Horizonts durch interkulturelle Begegnungen findet sich jedoch der Versuch der Zugänglichkeit des Fremden wieder, indem in diesem das Vertraute, Bekannte gesucht wird. Deutlich wird eine solche Dimension des Paarhorizonts, wie sie auch 
aus den Interviews zum Beispiel von Nicole bekannt ist, an Marias Wahrnehmung von Mexiko. Vor ihrem ersten Besuch dort stand Mexiko für die Wahl ihre Auslandsstation nicht an erster Stelle, als Nachbarland der USA und aufgrund der Tatsache, dass VW dort gebaut wird (10/23ff) und es insofern eine Verbindung zu Deutschland aufwies, schien es ihr zu vertraut zu sein. Argentinien, ,mit der Diktatur und dem Umbruch und so" (10/24f), schien ihr das spannendere und fremdere Land zu sein. Ihre Erfahrungen in Mexiko ändern jedoch das Bild. Ihre eher negative Einstellung gegenüber Mexiko wandelt sich grundlegend bei ihrer Ankunft. Sie findet Mexiko „umwerfend“ (11/4) und betont die Anschlussfähigkeit an Deutschland. Anders als bei Nicole steht also nicht das ,ganz Andere“ (Schlehe 2000a: 135), der Kontrast im Vordergrund. Vielmehr betont Maria einerseits die Anschlussfähigkeit an ihre bisherigen Erfahrungen, erlebt jedoch andererseits hinreichend Differenzen, um Mexiko als ,fremd' zu erleben und damit interkulturelle Erfahrungen machen zu können. Diese Form von ,vertrauter Fremdheit", macht sie an verschiedenen Elementen fest. Sie findet die ihr unsympathische Geschichtslosigkeit der USA nicht wieder, vielmehr kommt ihr das Land „europäischer“ (11/10) vor, sie hat das Gefühl sich mit Mexikanern in der Sicht auf die Welt treffen zu können. Wie auch Nicole ist sie fasziniert von der hohen Bedeutung, die der Familie zugesprochen wird, insbesondere im Unterschied zu den USA. Im Vergleich zu Deutschland sieht sie zwar einerseits die Nähe durch ein gemeinsames europäisches Gefühl, andererseits zeigt ihr Mexiko andere Möglichkeiten des Umgangs mit Migranten und mit Patriotismus:

„Diese ständige Auseinandersetzung mit den Kulturen und dieses ständige sich Reiben an den USA - das fand ich - extrem spannend, also. Und auch an den Spaniern, [...] also diesen Patriotismus den es da gibt - da_das in 'nem Land wo se alle sagen boah ist das korrupt hier und diese Polizei, trau nie der Polizei, aber Mexiko ist das Größte. [...] Und das ist, für mich was ganz Neues. Grade für uns als Deutsche wo man sich also diese Patriotismus sich so ganz schwer tut aber - des ist gleichzeitig verbunden is mit 'ner Kritik an der eigenen Regierung, die - ganz massiv ist“ (11/24-32).

Mexiko fungiert in ihrer Rekonstruktion also nicht, wie bei Nicole, als Gegenpol zu Deutschland. Wohl aber lässt es sich als positives Vorbild lesen, welches durch die Widersprüchlichkeiten und die gleichzeitige Differenzierung zwischen der Regierung einerseits und dem Patriotismus andererseits neue Möglichkeiten der Positionierung aufzeigt. Mexiko ist nicht $\mathrm{zu}$ fremd, es stellt sich als anschlussfähig und verständlich heraus, nicht alles ist anders, aber vieles besser gelöst. 


\section{Interkulturelle Begegnungen II: Begrenzung}

Diese programmatische Offenheit und die Suche nach Reflexionsanstößen ist aber nur die eine Seite der Konstruktion des Paarhorizonts. Auf der anderen Seite der großen Bedeutung interkultureller Begegnungen stehen Grenzziehungen, die von Maria vollzogen werden. Mit der Beziehung werden auch die zukünftigen Wohnorte verhandelt. Wie für Susanne ist für Maria ihr emanzipatives Selbstverständnis an äußere Rahmenbedingungen gebunden, insbesondere an ihre Erwerbstätigkeit. Für sie ist eine solche Einstellung Teil ihrer nationalen Mentalität:

„Des war auch vorher haben wir immer diskutiert und haben gesagt naja, ähm, er hat gesagt komm du doch nach Mexiko und ich war dann eben die Deutsche, ja, ich hab da nichts zu tun oder ich brauch 'nen Job und i_ich will da erst hinkommen wenn ich wieder Arbeit hab“ (16/8 - 10).

Der Begründungszusammenhang der kulturellen Differenz steht zwar nicht außerhalb eines reflexiven Zugangs - sie sieht sich selber als typisch deutsch - er wird jedoch von ihr nicht modifiziert bzw. in Frage gestellt, sondern dient hier als argumentative Absicherung. Ein solcher Rückbezug auf nationale Gepflogenheiten als unhintergehbaren Hintergrund verifiziert die Grenze interkultureller Begegnungsmöglichkeiten und schreibt Differenzen fest. Die Beobachtung, dass Maria etwas als kulturell geregelt erkennt, bedeutet eben nicht zwangsläufig, wie Larcher schreibt, dass es damit ,,auch als veränderbar erkannt wird“ (ebd. 2000: 12). ${ }^{1}$ Der Rückbezug auf nationale Eigenheiten ist aber nicht nur situativ relevant, auch für die Zukunft der Beziehung sind für Maria das Wissen um das Herkunftsland und die bisherigen biographischen Bezugspunkte höchst relevant:

„Wollte aber auch dass er mal in Deutschland gelebt hat, ich wollte dass er Deutschland kennt und - die Deutschen kennt, sozusagen. Also dass er wei das er meine Kultur kennt und auch meine Freunde, also. Das war mir immer, für mich, sozusagen dieser Kontrast. Für ihn ist es sehr stark die Familie und für mich die Freunde also Bezugskreis. Und dass er meine Freunde kennenlernt das war für mich enorm wichtig“ $(16 / 11-15){ }^{2}$

1 Dies entspricht eben nicht der Vorstellung einer kulturellen Kompetenz, wie sie Baecker formuliert: „Es nimmt jede Kultur nicht als Verweis auf eine vergangene und daher nicht mehr zu ändernde, also entweder abzulehnende oder zu verteidigende Vergangenheit, sondern als Verweis auf ein durchweg unklares Schicksal nicht nur dieser Kultur, sondern der Weltgesellschaft insgesamt“" (ebd. 2001: 30f).

2 Die transnationale Ausrichtung ihres biographischen Entwurfes wird jedoch nicht in Frage gestellt: „Und - ähm aber wir ham dann auch als er 
Die interkulturelle Beziehung zu Pablo basiert für Maria auf dem Wissen über beider kultureller Herkunft, der Verweis auf die Herkunft dient als Rahmung, die nicht in Frage gestellt wird, fungiert jedoch nicht im Sinne einer Verhinderungs-, sondern vielmehr einer Ermöglichungsstruktur der Beziehung. Die Kenntnis über kulturelle Eigenheiten soll, davon geht Maria aus, Verständnis für den anderen ermöglichen, sie stellt für sie eine Grundlage der Beziehung dar. Damit steht sie im deutlichen Kontrast insbesondere zu Susanne, für die der Iran als konkretes Herkunftsland von Rachid kaum eine Rolle spielt.

\section{Soziale Erweiterungen: neue Beziehungserfahrungen}

Als zweite Dimension lässt sich bei Maria eine Erweiterung im Hinblick auf ihre Erfahrungen mit sozialen Beziehungen beobachten. Nicht nur Mexiko als Land fasziniert Maria. Auch die Beziehung zwischen den Geschlechtern, von ihr am Beispiel von Höflichkeits- und Aufmerksamkeitsritualen festgemacht, stellt für sie eine positive Unterscheidung zu deutschen Männern dar. In der Begegnung mit Pablo erlebt sie neue Umgangsformen, die ihre eigenen Vorstellungen von Geschlechterbeziehungen verändern und für sie positiv erweitern, sowohl innerhalb der Beziehungsdyade, als auch hinsichtlich der Bedeutung von Familie. Aufgrund ihrer rheumatischen Erkrankung kommt sie nicht gut alleine durch die Menschenmenge beim interkulturellen Festival hindurch, und so hilft er ihr dabei:

„Irgendwie nich so wie die Deutschen die sozusagen auf Höflichkeit bedacht sind, war mehr Praxis orientiert, das hat sich ergeben - ich geh vor dir und halt dich an mir fest, ich geh vor dir durch und dann kannst hinter mir, schlag ich dir 'ne Schneise oder so (12/26 - 29)“.

Eine solche Praxisorientierung setzt sich auch in Pablos Umgang mit ihrer Krankheit fort: „Dass er mich sofort drauf angesprochen hat auf das Rheuma. Also als $w_{-}$am Tag als er mich kennen gelernt hat. Ach du hinkst ja, und zeig mal deine Hände, [...] und auch mit Interesse, aber irgendwie nicht mit - mit abstoßend“ (25/10 - 13). Maria möchte sich von ihrer rheumatischen Erkrankung nicht einschränken lassen, sie versucht, in Auseinandersetzung mit Ärzten und Selbsthilfegruppen, ihren eigenen Weg und ihre eigenen Ziele zu realisieren, ohne die körperlichen Einschränkungen. Insofern wird Höflichkeit von ihr durchaus differenziert gesehen. Nach Goffman, der darauf aufmerksam macht, das

gekommen ist haben wir immer gesagt dass wir auch nach Mexiko gehen“ $(16 / 26 f)$. 
„das System der Höflichkeiten ein Zeugnis von der hohen Wertschätzung der Frauen“ (Goffman 1994: 123) abgibt, verbindet sich damit der Eindruck, dass Frauen ,als wertvoll, dekorativ und zerbrechlich“ (ebd.) gelten, und vor den Widrigkeiten des Lebens zu schützen seien. Während dieserart ,leere" Höflichkeitsrituale von Maria abgelehnt werden, hebt sich für sie die Form der praktischen Höflichkeit von Pablo positiv von der deutscher Männer ab. Er versucht nicht ihre Krankheit und die sich daraus ergebenden Behinderungen zu ignorieren, sondern baut sie unsentimental und selbstverständlich in den Umgang mit ein. Nicht nur auf dieser Ebene ist sie jedoch von der anderen Form der Geschlechterbeziehung positiv überrascht. Auch die Art und Weise, in der Pablo ihren Aufenthalt gestaltet und versucht ihre Wünsche zu erfüllen, imponiert ihr:

„Irgendwie so beeindruckt dass er immer so. Ich hab gesagt naja ich will am Wochenende irgendwo hinfahren, ich will das Umland sehen. - Und er hat gesagt ja ok, ich schau ob ich das möglich machen kann. - und dann hat er das hingekriegt. Und dann, möchte er, ja am Schluss möchte ich noch mal ne halbe Woche Urlaub machen oder ne Woche, ja ich schau ob ich das möglich mache. Und dann sind wir am Schluss noch, ah genau an die Karibik“" (13/4 9).

Pablo realisiert Marias Wünsche, diese Form der Aufmerksamkeitszuwendung beeindruckt sie. In der Konzentration auf sie und ihre Wünsche stellt sich für Maria die natio-ethno-kulturelle Zugehörigkeit Pablos vor allem als Erweiterung ihrer bisherigen Erfahrungen mit Geschlechterbeziehungen dar. Sowohl hinsichtlich der körperlichen Beeinträchtigungen wie auch hinsichtlich der Aufmerksamkeit, die sie erfährt, zieht sie eine positive Bilanz, er unterscheidet sich in positiver Hinsicht von deutschen Männern. Die neue Form der Beziehung kennzeichnet sich für sie insbesondere durch die Aufmerksamkeit ihr gegenüber und den Versuch, ihr das Leben schöner zu gestalten. Neben einer Konstruktion der Dyade, die durch die Hervorhebung von Marias Wünschen gekennzeichnet ist, sieht Maria auch in Pablos Umgang mit Familie deutliche Unterschiede zu deutschen Männern. Seine Orientierung am Notwendigen und die gleichzeitige Familienorientierung bestätigen sich erneut, als die ungeplante Schwangerschaft eine Entscheidung über den gemeinsamen Wohnort virulent macht. Die „bedingungslose“ (15/21) Entscheidung für Maria und für die Kinder beeindruckt sie sehr. Deutsche Männer, so ihre Vermutung, hätten ihre berufliche Sicherheit der Familie vorgezogen bzw. wären nicht so schnell bereit gewesen, ihre Karriere für die Familie aufzugeben (15/21ff.). Auch hier stellt die Konzentration 
auf seine Familie einen positiven Bezugspunkt für Maria dar, der ihn zudem von deutschen Männern unterscheidet.

Der Horizont, der von Maria eröffnet wird, lässt sich einerseits in der Fortführung und Bestätigung ihrer bisherigen biographischen Handlungsorientierungen sehen, andererseits aber auch in der Erweiterung bisheriger Erfahrungen, insbesondere hinsichtlich der Realisierung von Geschlechterbeziehungen. In der Beziehung zu Pablo manifestiert sich ihre Faszination gegenüber anderen Kulturen und anderen Ländern, dafür ist seine natio-ethno-kulturelle Zugehörigkeit zu einem anderen Land entscheidend. Daneben führen ihre interkulturellen und interkulturell vergleichenden Handlungsorientierungen zu einem Verständnis von anderen Kulturen als Herausforderung und Reflexionsmöglichkeit. Hierbei integriert Maria ihr bislang fremde Erfahrungen und Einstellungen, und betont insbesondere die Anschlussmöglichkeiten solcher Fremdheiten, Unvertrautes wird dadurch vertraut. Gleichwohl gerät eine solch programmatische Offenheit insbesondere durch die sozialisatorische Betonung nationaler Zugehörigkeiten an ihre Grenzen, das gemeinsame Wissen um das andere Herkunftsland betont eben auch die identitäre Bedeutung, die in der Konstruktion von Zugehörigkeit für Maria im Vordergrund steht . Pablos natio-ethno-kulturelle Zugehörigkeit stellt daher für Maria eine Möglichkeit der Auseinandersetzung und Reflexion dar, auch hinsichtlich der Geschlechterbeziehungen, ohne das damit jedoch zwangsläufig, wie bei Susanne oder Nicole, deutliche Modifikationen in der spezifischen Beziehungsform zwischen den beiden verbunden werden. Wohl aber genießt sie die andere Rolle, die sie in der Beziehung zu Pablo einnehmen kann. Die Aufmerksamkeit, die sie bekommt, und die ihre rheumatische Erkrankung so unproblematisch einbezieht, und die große Bedeutung, die er seiner (werdenden) Familie zumisst, sind für sie Teil und Ausdruck seiner mexikanischen Sozialisation und stellen eine positive Erfahrung im Unterschied zu deutschen Männern dar.

\section{Konstruktion des Paarhorizonts bei Pablo: Fortsetzungen}

Auch für Pablo stellt die Beziehung die Weiterentwicklung und Fortführung relevanter biographischer Dispositionen dar. Ähnlich wie bei Maria spielt die programmatische Öffnung gegenüber anderen Kulturen und anderen Ländern eine große Rolle in seinem Selbstverständnis. Maria ist dabei vor allem als Vertreterin einer anderen Kultur bedeutsam, im Ergebnis zeigt sich für ihn die Möglichkeit der Realisierung seiner interkulturellen Interessen. Anders als bei Rachid oder Carlos lässt sich bei ihm keine Migrationsdisposition analysieren, vor der Begegnung mit Maria blieb der Kontakt zu anderen Ländern auf Reisebeziehungen be- 
schränkt. Die zentrale Dimension in der Konstruktion des Paarhorizonts lässt sich daher als Fortsetzung und ebenfalls als Erweiterung bezeichnen.

\section{Programmatische Kulturoffenheit}

Reisen können den Blick auf das Herkunftsland verändern. Reisen ermöglichen die Distanzierung vom Alltäglichen, das Heraustreten aus der unmittelbaren Involviertheit und die Neusichtung und Neubewertung der eigenen Situation (vgl. dazu z.B. Weigel 1992). Für Pablo trifft dies zu. In dieser Dimension ähneln sich die Konstruktionen Marias und Pablos sehr: Beide sehen sich in ihrem Selbstverständnis als interkulturell interessiert und damit offen und kompetent im Umgang mit anderen Kulturen und Personen anderer natio-ethno-kultureller Zugehörigkeit. Und beide verstehen fremde Länder als Reflexionsmöglichkeit über die eigenen Positionierungen und das eigene Land. Für Pablo stellte, wie er selber sagt, insbesondere die Reise nach Korsika mit 17 Jahren einen Wendepunkt dar. Durch die Reise gewinnt er einen neuen Blick auf Mexiko:

„Eines der Dinge, die ich als junger Mann machte war meine erste Reise nach Europa. [...] Diese Reise er_mir, ermöglichte mir [...] meine Augen zu öffnen, für das, was die andere Seite der Welt war, andere Gedanken mit anderen Jugendlichen auszutauschen, und, zu denken, dass, gut, auch wenn mein Land von der Fläche her sehr groß ist, gut, es gibt andere Gedanken, und [...] zu sehen, was es auf der anderen Seite gab und wieso die Dinge unterschiedlich sein konnten, in einem anderen Ort" $(3 / 2-12)$.

Diese Reise stellt für ihn einen Wendepunkt in seiner Orientierung dar, ab diesem Zeitpunkt „war ich sehr überzeugt, dass ich andere Kulturen kennen lernen wollte“ (5/3f). Andere Kulturen dienen ihm hier als Chiffre für neue Gedanken und positive Irritationen. Für ihn war es daher nur logisch, Maria, als blonde Frau sichtbar als Nicht-Mexikanerin zu erkennen, auf dem Kulturfestival anzusprechen (5/7ff). Die Begegnung verleiht seinem Interesse Ausdruck, gleichzeitig findet so vor der ersten Begegnung eine Fokussierung auf die kulturelle Andersartigkeit Marias statt. Eine gewisse Form der Entpersonalisierung lässt sich hier festhalten: Pablo fokussiert auf die natio-ethno-kulturelle Zugehörigkeit der Person und versteht diese als Vertreterin der anderen, interessanten Kultur. Marias andere Kultur stellt so den Anlass zur Begegnung mit ihr dar. 


\section{,Gleicher unter gleichen': Aushandlungen zwischen den Kulturen}

Eine solch interkulturelle Handlungsorientierung lässt sich, wie auch bei Maria, in weiteren Aushandlungen und Entscheidungen rekonstruieren und weiter differenzieren. Dabei stehen für Pablo beide Kulturen gleichberechtigt nebeneinander, Differenzen, die auf kulturelle Unterschiede zurückgeführt werden, müssen kommunikativ bearbeitet und gelöst werden. Relevant ist die kulturelle Herkunft auch noch aus einem anderen Grund: Die Entscheidung für den zukünftigen Wohnort steht an, und anders als bei den anderen beiden Paaren ist dies in der biographischen Rekonstruktion von Maria und Pablo ein länger dauernder und durchaus konfliktreicher Prozess, der durch das Spannungsfeld zwischen interkultureller Offenheit bei gleichzeitiger starker kultureller Verortung organisiert ist.

\section{Das Herkunftsland als biographischer Bezugspunkt}

Für Pablo ist es wichtig, ebenso wie für Maria, Deutschland als Marias Herkunftsland kennen zu lernen: „Und sie auch _ ,sie war einen Monat in A-Stadt, und sie hatte eine sehr schöne Zeit, ich gab mir auch Mühe, dass sie eine schöne Zeit hatte und dann war die andere Probe dran, zu erfahren, wie es hier wäre, mit ihr, in Berlin“ (5/11 - 13). Nicht nur will er Marias Herkunftsland kennen lernen, er will im Gegenzug auch, dass sie Mexiko auf positive Art und Weise erlebt und sich dort wohlfühlt. Maria ist für Pablo nicht losgelöst von ihrer Herkunft und ihrem Land zu denken. Anders also als z.B. für Carlos, der sich ohne weitere Informationen für ein Leben in Deutschland entschieden hat, ist für Pablo die Kenntnis über den möglichen Wohnort und den Herkunftsort Marias entscheidend. Ein solches Motiv einer ,informierten Migration“ ist Teil der Konstruktion eines Horizonts, der auf interkulturellen Begegnungen auf Basis kultureller Differenz aufbaut. Marias Herkunftsland kennen zu lernen ist Teil des Interesses an anderen Kulturen generell und dem konkreten kulturellen Bezugssystem Marias. Ohne Kenntnisse darüber kann Pablo nur schwer mit einer Person zusammenleben, die sozialisatorischen Erfahrungen und Marias soziale und räumliche Umgebung sind für ihn für die gemeinsame Zukunft entscheidend. Ein solches Interesse vor der Eheschließung scheint eher untypisch für binationale Paare zu sein, die sich in einer Urlaubssituation kennen lernen, wie z.B. KhatibChahidi/Hill/Paton 1998 feststellen: „Very few of them (der Frauen, M.M.) bothered to find out about their future spouse's culture, his family or country before marriage, as this did not seem important to them" (in Breger/Hill 1998: 12). 


\section{Heirat als Kulturkonflikt}

„Kultur ist im Normalfall eine latente Hintergrundgröße. Erst wenn sie in ihrem eigenen Geltungsbereich strittig wird, nimmt ihre Sichtbarkeit zu. [...] Kultursoziologisch instruktiv sind also Konstellationen, in denen durch Konflikte auf mehreren Seiten Begründungszwänge sowohl hinsichtlich der Rechtfertigung eigener wie der Verwerfung abweichender Positionen entstehen“ (Neidhardt 1986: 17). Für Pablo stellt insbesondere die Diskussion um eine mögliche Heirat einen solchen Eingriff in den Geltungsbereich seiner Kultur und seiner Traditionen dar:

„Ich als Mexikaner sagte, gut, das Normale ist dass _, wenn, wenn _ wir sogar schon planen zusammenzuleben, dass in Mexiko freie Beziehungen nicht akzeptiert werden, [...] und dann sagte ich zu ihr: Weißt du? Lass uns lieber heiraten. Und sie sagte genau das Gegenteil, das war ihre Kultur: Nein, nein, nein, wir heiraten nicht, wir, mhhh, probieren zuerst, wie es ist' und dann, seitdem kommt das Schwierigste, immer der Konflikt zwischen Kulturen“" $(5 / 19-6 / 5)$.

Hier prallen zwei Kulturen, nicht zwei Personen aufeinander. Interkulturelle Begegnungen tragen für Pablo also insofern ihr Konfliktpotential in sich, als dass in den alltäglichen Auseinandersetzungen die Unterschiede und die Grenzen zwischen den Kulturen am virulentesten werden. Die Schwierigkeit sieht er nun darin, diesen kulturalistischen Diskurs auf eine reflexiv bearbeitbare Ebene zu bringen. Pablo organisiert natio-ethnokulturelle Zugehörigkeit dabei vorrangig als sozialisatorische Prägung, die durch den Aspekt der generativen Verpflichtung noch ein weiteres Moment erhält, die Kultur als abstraktes Normengefüge findet eine Konkretisierung in der Herkunftsfamilie: Gerade Hochzeiten und andere Feste sind stark mit kulturellen Traditionen verbunden und stellen daher in vielen binationalen Partnerschaften den Kontext großer Auseinandersetzungen zwischen den Partner(innen) und deren Familienangehörigen dar (vgl. dazu z.B. Breger/Hill 1998; Scheibler 1992). Auch für Pablo ist die Haltung seiner Familie ein weiteres Argument für die Hochzeit:

„meine Familie, sie ist sehr konservativ, ich weiß nicht, wie sie das finden werden und ich möchte nicht, weil ich der Jüngste bin, so was, wie ein schlechtes Beispiel setzen“ $(5 / 22-6 / 2)$. ,[Ich] respektiere [...] das Heiraten als Basis der Familie und für mich sehr, ich denke, es ist nicht schlimm und es gibt keinen Grund es nicht zu machen, na ja, es war etwas was ich meiner Familie geben wollte“ $(8 / 9-13)$.

Gerade aufgrund seiner Beziehung zu einer Deutschen will er die Bindung an seine Herkunftsfamilie und seine Herkunftskultur nicht aufge- 
ben, Heiraten würde für ihn diese Bindung sichtbar werden lassen. „Das Schwierigste, immer der Konflikt zwischen den Kulturen“, muss in der jeweiligen konkreten Situation gelöst werden, ohne dass aber die grundsätzliche Differenz aufgehoben werden kann.

Der Paarhorizont, der sich in Pablos biographischer Erzählung rekonstruieren lässt, ähnelt stark dem von Maria. Er lässt sich am besten als Weiterführung und Realisierung von Pablos interkulturellen Interessen verstehen. Die Möglichkeit der Perspektivenvervielfältigung und der Blickwechselveränderung, die Pablo auf der die Reise nach Korsika erlebt hat, findet er auch bei Maria, wie er in irritierender und in bestärkender Weise feststellt. Die natio-ethno-kulturelle Herkunft Marias stellt für Pablo die Ressource und den Schlüssel für ihr Verständnis dar, dieses kulturalistisches Verständnis gilt es in konkreten Konfliktsituationen kommunikativ zu bearbeiten. Pablos enge Anbindung von Personen an ihre jeweilige Kultur begrenzt jedoch auch die Verhandlungsmöglichkeiten. Eine Trennung oder eine Distanzierung von der durch seine Eltern symbolisierten Tradition der Heirat kommt so für ihn nicht in Frage. Die Spannung zwischen seiner selbst-postulierten Freiheit von kulturellen Bindungen (,,sage ich, dass ich in bestimmten Sachen, irgendwie, ja, sehr radikal bin, ja, ich bin sehr frei, was das Erfahren angeht" [8/9 11]) einerseits und die gleichzeitige Verifizierung solcher Bindungen andererseits löst sich nicht auf, sondern bleibt bestehen. Geschlechterbeziehungen stehen für ihn dabei nicht so im Mittelpunkt seiner biographischen Rekonstruktion wie zum Beispiel für Carlos. Vielmehr sind sie Teil der kulturellen Verortung, Ausdruck und Konkretisierung. So versteht er auch die Ablehnung des Heiratens durch Maria nicht als persönliche Neigung, sondern als Teil ihrer kulturellen Verortung. Dies entlastet die Situation von persönlichen Schuldzuschreibungen, schreibt aber die dominante Bedeutung von Kultur als Hintergrundsnorm weiter.

\section{Interkulturelle Begegnungen: Öffnungen und Grenzen}

Anders als bei den vorhergehenden Paaren korrespondieren die von Maria und Pablo aufgespannten Paarhorizonte nicht nur strukturell, sondern auch inhaltlich. Beide, Maria und Pablo, verstehen sich als interkulturell kompetente Personen, die kulturelle Begegnungen suchen und offen sind für neue Erfahrungen und Blickwechsel. Die jeweils ,andere' natioethno-kulturelle Zugehörigkeit wird in diesem Zusammenhang als faszinierendes Moment eingeführt, welches Interesse weckt. Das Konzept von Interkulturalität ist daher für die Konstruktion des Paarhorizonts entscheidend. Interkulturalität hat, das hat sich in der Konstruktion deutlich gezeigt, dass Grundproblem das ,der Versuch, der Verschiedenheit 
,Rechnung zu tragen“ (Prengel), eine spezifische Verschiedenheit immer schon voraussetzt" (Mecheril 2004: 16) und damit Differenz fördert. Einerseits ergeben sich durch interkulturelle Orientierungen neue Reflexionsanstösse, andererseits begrenzen sie und schreiben die und den jeweils Anderen in dieser Andersheit fest. Beides findet sich auch in dem Paarhorizont von Maria und Pablo. Die Beziehung ist Ausdruck der interkulturellen Haltung der beiden und dient als eine Ressource für die weitergehende Beschäftigung mit anderen Kulturen. Beide Partner(-innen) werden damit einerseits stark an ihre natio-ethno-kulturelle Zugehörigkeit gebunden, sie dient als Erklärungsmuster für und bei Konflikte(n) und entlastet damit die personale Ebene, Kultur als ,ultimo ratio“ setzt aber konkreten Auseinandersetzungen enge Grenzen. Andererseits basiert der Paarhorizont auch auf dem Interesse an und der Suche nach einer Möglichkeit der Überwindung solcher kultureller Grenzziehungen. Eine solche Überwindung wird unter anderem in der kognitiven Reflexion der durch Sozialisation entstandenen Erwartungen und Vorstellungen gesehen. Fast „magische Gespräche“ (Pablo 8/17) werden geführt, um die verschiedenen Vorstellungen über das Zusammenleben vereinen zu können und den Konflikt über die Hochzeit zu lösen. Im Verhältnis der Konstruktionen natio-ethno-kultureller Zugehörigkeiten zu genderKonstruktionen stehen hier erstere als dominante Konstruktionen im Vordergrund. Diese latente und omnipräsente Hintergrundsnorm findet einen Ausdruck in geschlechtsspezifischem Verhalten, letzteres dient als Explikation und weitere Differenzierung der (als positiv oder negativ) gesehenen kulturellen Unterschiede, und ist insofern Teil der kulturellen Praxis und Traditionen.

\section{Thema der Beziehung: Transmigration}

Die Auseinandersetzungen über den zukünftigen Wohnort werden durch die (ungeplante) Schwangerschaft Marias entschieden. Aufgrund ihrer rheumatischen Erkrankung kann es zu Komplikationen kommen, und sie muss sich, zumindest für die Zeit der Schwangerschaft, dauerhaft in Deutschland aufhalten. Für Pablo ist es daher keine Frage, dass er so schnell wie möglich sein Studium abschließt und nach Deutschland kommt. Die große Bedeutung, die die Kinder insbesondere für Pablo haben, löst und entschärft so vorläufig den Konflikt. Letztendlich führen die „,magischen Gespräche“ über die Ehe für Pablo zum Erfolg, sie heiraten kurz vor der Geburt der Zwillinge. Damit ist aber nur die erste Etappe ihres gemeinsamen Lebens entschieden, beide planen einen Umzug nach Mexiko und sind, zum Zeitpunkt des Interviews, kurz davor. 
Langfristig wollen sie in beiden Ländern leben, wobei die Schulzeit der Kinder in Deutschland stattfinden soll. Das female breadwinner Modell ist insofern Teil eines größeren Plans, als dass mit dem Wechsel des Wohnortes auch Zeiten der Arbeitslosigkeit zu erwarten sind, sich dabei Pablo um die Kinder kümmern soll, während Maria weiterhin arbeitet. Unerwartet für Pablo ist jedoch die Länge seiner Arbeitslosigkeit, er ist von einer baldigen beruflichen Integration ausgegangen. Als Thema der Beziehung lässt sich daher die Perspektive der Transmigration, die Realisierung und die Realität einer gemeinsamen transnationalen Biographie benennen. Sowohl die Erwartungen zum Beispiel der Eltern von Maria wie auch eigene Vorurteile und interkulturelle Grenzziehungen konterkarieren zum Teil die interkulturelle Programmatik des Paares. Die Spannungen zwischen kulturalisierten Zwängen und individuellen Bearbeitungen bestimmen die Entwicklung der Beziehung und strukturieren die analysierbare Form biographischen Lernens. Mit Kulturalisierung meine ich hier die Reduzierung von Erklärungen von Differenzen zwischen Personen auf ihre kulturelle Herkunft: „Problematisch wird es, wenn ein Partner [...] Differenzen zwischen den Partnern immer kulturalistisch - d.h. mit Hinweis auf die Herkunftskultur des jeweils anderen - erklärt werden.“ (Larcher 2000: 92). Betrachtet man die in den Biographien von Maria und Pablo analysierbaren Bearbeitungsformen und felder fällt auf, dass die Problematik einer kulturalisierenden Konstruktion nur eine Seite des Begriffs darstellt. Auf der anderen Seite ermöglicht gerade die starke Verbindung der Personen mit ihrer jeweiligen Herkunftskultur die Respektierung bestimmter Entscheidungen und Verhaltensweisen.

\section{Bearbeitungsfelder}

\section{Bearbeitungsfelder bei Maria: Konkretisierungen}

Für Maria lässt sich die Zeit in Deutschland, ähnlich wie bei Nicole und Carlos, als von Schwierigkeit mit der Realisierung ihres Lebensmodells geprägt beschreiben. Das für sie biographisch relevante Thema der interkulturellen Begegnung konkretisiert sich durch die Beziehung im Hinblick auf eine mögliche und gewünschte transnationale Biographie. Durch diese notwendig werdende Konkretisierung lassen sich Bearbeitungsfelder rekonstruieren, in denen die interkulturelle Haltung an ihre Grenzen hinsichtlich der Anschlussfähigkeit und der Verständigung stößt und so neue Wege der Realisierung gesucht werden müssen. Innerhalb des sich schon im Paarhorizont abzeichenden Spannungsfeldes 
spielen sich auch die weiteren Entwicklungen ab. Für Maria zeigen sich die Grenzen der Anschlussfähigkeit insbesondere in unterschiedlichen Wissensbeständen und Haltungen, wie sie bei der Erziehung der Kinder oder in politischen Diskussionen virulent werden. Gleichwohl aber differenziert sich hier auch die ,andere' Seite der interkulturellen Haltung, die Ermöglichungsstruktur, weiter aus. Einerseits ermöglicht ihr die Beziehung die Fortsetzung ihrer Berufstätigkeit, und andererseits, durch die Migration nach Mexiko neue Formen der Erwerbsarbeit auszuprobieren.

\section{Interkulturelle Grenzen}

Schwierigkeiten bei der Realisierung ihrer interkulturellen Beziehung entstehen für Maria vor allem auf der Ebene der Kindererziehung, politischer Diskussionen und der Aushandlungen von Geschlechterbeziehungen.

\section{Kindererziehung als grundlegender Konflikt}

Trotz des interkulturellen Habitus erlebt Maria in der Beziehung zu Pablo deutliche Grenzen ihrer Toleranz und ihres Reflextionsvermögens hinsichtlich kultureller Differenzen. Als grundlegenden kulturellen Konflikt bezeichnet Maria die Erziehung der Zwillinge. Hierbei geht es nicht mehr ,nur' um die eigenen Charakterzüge und das eigene Weltverständnis, sondern auch um die Frage, was man den eigenen Kindern mit auf den Weg geben will. Insofern sieht sie die Streitereien um die Erziehung als ,mit die heftigsten“ (35/33) an. ${ }^{3}$ Die Erziehung der Kinder als Weitergabe der eigenen Lebenseinstellungen und -erfahrungen stellt die Integrierbarkeit der kulturellen Unterschiede immer wieder in Frage. Pablo betont die Bedeutung körperlicher Kraft und möchte, dass die Jungen „,ihren Mann“ (35/33) stehen und sich durchsetzen können, da dies in Mexiko sehr viel wichtiger als in Deutschland sei. Sie merkt an solchen Auseinandersetzungen, ,wie man sozusagen gepolt ist“ (35/32f.) und wie sich in konkreten Feldern wie der Kindererziehung die unterschiedliche Sozialisation ausdrückt. Die Notwendigkeit der Aushandlung besteht insbesondere deswegen, weil die Erziehung auch auf ein Leben in Mexiko vorbereiten soll, die transnationale Ausrichtung spiegelt sich insofern in der konkreten Ausgestaltung der Erziehung wieder.

3 Kinder als augenfälligstes Zeichen einer Grenzüberschreitung durch binationale Paare stehen häufig im Mittelpunkt empirischer Studien. Während die einen ihnen besondere multikulturelle Kompetenz zusprechen (vgl. Khounani 2000), beschäftigen sich andere mit den Konsequenzen für das Kind hinsichtlich seines rechtlichen und sozialen Status (vgl. zusammenfassend Thode-Ahora 1999: 390ff). 


\section{Politische Diskussionen}

Neben der Kindererziehung stellen politische Diskussionen einen weiteren Grenzpfeiler in der interkulturellen Kommunikation dar. Maria bemerkt bei sich in Diskussionen mit anderen ein Befremden gegenüber manchen Haltungen und Äußerungen Pablos. Sie bekommt das Gefühl, plötzlich „keinen Zugang mehr“ (37/31) zu ihm zu haben, und wird so wütend, „wie ich es sonst von mir nicht kenne“ (37/32). Grundlegende Widersprüche werden von ihr als kulturelle Grenzen bewertet und können von ihr nicht überschritten werden. Solche Beobachtungen lassen etwas euphorisierende Proklamationen wie die der Europäischen Konferenz der binationalen/bikulturellen Beziehungen 1996 in Paris in einem differenzierteren Licht erscheinen. In der Abschlussresolution der Konferenz wird eine Anerkennung internationaler Familien gefordert, da sie „konkrete Stätte[n] des Dialoges zwischen den Zivilisationen“ seien (vgl. ebd. 1996: 40). An dieser Stelle zeigt sich, dass der Dialog der Zivilisationen an seine Grenzen geraten kann. Die Fremdheit scheint zumindest situativ unüberbrückbar, zumal Pablo ihr, stellvertretend für alle Deutschen, Vorwürfe macht: „dass ich manchmal das Gefühl hab er weist sehr schnell mir und mit mir allen Deutschen die Schuld zu“ $(36 / 32 f)$.

\section{Geschlechterbeziehungen}

So sehr Maria also in politischen Diskussionen die Nachteile solcher stereotypen Zuschreibungen erfährt, so sehr ist sie selber in solche Konstruktionsprozesse verstrickt. „Group characteristics are presented as if they are universal to the group, often as specific group or national ,characters' that are inherent, ,natural', and therefore unchangeable" (Breger/Hill 1998: 11). Hinsichtlich der Erwartung an Geschlechterverhältnisse hantiert sie, wie sie merkt, mit Vorurteilen. „Also ich so das Vorurteil hab die Mexikaner - in bestimmten Sachen ist er bestimmt ein Macho, - und aus diesen Dingen vorsorglich sofort reagiere, und dann reagiert er natürlich ganz scharf, weil er gar nicht unbedingt so reagiert hätte“ (37/14-16). Kulturalisierungen und vorweggenommene Zuschreibungen führen in solchen Situationen zu Überreaktionen, wie Maria selber kritisch anmerkt. Entpersonalisierte Kulturalisierungen fördern insofern nicht nur die Auseinandersetzung, sondern führen auf beiden Seiten zum Gefühl des Nichtverstandenwerdens und zum Einfordern einer entkulturalisierten Sicht. 


\section{Interkulturelle Erweiterungen}

In verschiedenen Bearbeitungsfeldern erfährt Maria so etwas wie unüberbrückbare Differenzen, das Gefühl, den anderen nicht mehr zu kennen und nicht mehr die Person, sondern nur noch die imaginierte Kultur zu betrachten bzw. ihr zu begegnen. Gleichwohl erweitert die Beziehung gerade im Hinblick auf Marias Berufsbiographie ihre eigenen Handlungsmöglichkeiten. In einer deutsch-deutschen Beziehung, so vermutet sie, hätte sie mehr Schwierigkeiten mit der Aushandlung gehabt:

„Ja, bestimmte Sachen die ich jetzt, also dass ich jetzt wieder angefangen hätte zu arbeiten das wär mit 'nem Mann aus Deutschland ziemlich unwahrscheinlich, also es wär vielleicht so gegangen dass wir es irgendwie regeln das wir beide, aber ich hätte bestimmt den kleineren Teil gemacht. [...] Und dass - Pablo so was akzeptiert also das find ich schon sozusagen, wo er aus nem Kulturkreis kommt wo das eher noch viel weniger so is, - find ich das ist ein noch viel größerer Schritt““ (38/15 - 22).

Das „alte Bild“ (28/9) eines berufstätigen Ehemannes hat er zwar noch im Kopf, umso mehr wiegt aber sein Wille zu Hause zu bleiben. Die Festschreibung der ,anderen' Zugehörigkeit Pablos bekommt hier eine interessante Doppelfunktion: Zum einen kann sie ihn für bestimmte Entscheidungen noch stärker respektieren, da diese ihm, aus einem anderen Kulturkreis stammend, noch schwerer fallen müssen. Andererseits wäre ihre derzeitige Arbeitsteilung, so Marias Einschätzung, mit einem deutschen Mann gar nicht möglich gewesen. Marias Schilderung der Erfahrungen verweist auf eine Gelegenheitsstruktur, die auch bei Susanne zu beobachten war. Die natio-ethno-kulturelle Zugehörigkeit Pablos erleichtert ihr, wie Susanne, die Aufrechterhaltung eines individualisierten Lebenswegs. Seine Schwierigkeiten, hier in Deutschland eine Stelle zu finden, stellen Gelegenheitsstrukturen dar, die sie nutzen kann und nutzt, um ihre Vorstellungen ihrer Berufstätigkeit zu realisieren. Mit ihrem Status als Alleinverdienerin in der Familie hat sie keine Schwierigkeiten, Schwierigkeiten entstehen für sie vor allem aus der Tatsache, dass Pablo Deutschland so nur als Vater kennen lernt. Eigene, insbesondere berufliche, Zugangsmöglichkeiten sind ihm größtenteils versperrt. Die berufliche Integration würde ihm, so ihr Eindruck ,noch ganz viel öffnen, und er würde mehr kennen lernen“" (29/9). Der Wunsch des Zugangs und der Partizipation in verschiedenen Feldern entspricht der Vorstellung beider, das andere Land kennen zu lernen und so ein besseres Verständnis vom jeweils Anderen zu erhalten. Da die Perspektive der Biographie transnational ausgerichtet ist, gibt es keinen aktuellen Handlungsdruck. Der 
Umzug nach Mexiko steht unmittelbar bevor und Pablo orientiert sich beruflich dorthin, so dass die grundsätzliche Arbeitsteilung oder die Möglichkeiten einer Einmündung in den ersten Arbeitsmarkt derzeit nicht ausgehandelt werden muss.

\section{Kinder als Integrationsmoment}

Neben der Transmigrationsperspektive sind auch noch die Kinder starkes Integrationsmoment. Die Diskussionen über die Erziehung der Kinder werden mit dem Hinweis auf das Recht beider Seiten auf Weitergabe kultureller Tradierungen gelöst. Die Betonung der Interkulturalität der Beziehung dient hier also wieder zur Integration auch scheinbar unintegrierbarer Einstellungen.

„Ach dass ich, also für mich ist das ganz wichtig für die Kinder, das sie beide Kulturen mitkriegen. Und das find ich schon, dass ist für sie ne ganz große Chance, - und - ähm - ja also so wo ich auch wirklich hinwill. Also auch für die Kinder dass wir nach Mexiko gehen. Dass die das auch da mitkriegen und - ähm - das (unverständlich) und das die auch was von beiden Eltern was dabei haben“ $(40 / 18-21)$.

Für Maria stehen die Kinder im Mittelpunkt der Beziehung, sie sind Gegenstand der heftigsten Auseinandersetzungen, sie haben aber auch erst dafür gesorgt, dass die Eltern zusammenleben können (36/18f). Die Kinder bieten für Maria auch die Möglichkeit, die Migration nach Mexiko von einer anderen Perspektive aus zu sehen. Bislang stand für sie die Sicherung des Lebensunterhalts durch Erwerbstätigkeit, und zwar in der zertifizierten Ausbildung, an erster Stelle: „Also dieses - wo unser_, wir oder ich eben ganz brav, ich bin für das ausgebildet und das mach ich. Und ich arbeite noch als Übersetzerin, aber was anderes kann ich nicht" (18/10 - 12). Durch die Kinder sieht sie nun eine neue Möglichkeit:

„Also ich wollte, aber ich wollte sozusagen, ich bin ja auch immer mehr auf Sicherheiten bedacht, war, also es war mit den Kindern wirklich schon einfacher weil ich gesagt hab na gut, da hab ich was zu tun sozusagen, ich sitz da nicht nur rum. - Und - inzwischen hab ich auch das Gefühl ich kann mir da schon was zu tun - beschaffen, aber selbst wenn ich mich erstmal nur mit den Kindern beschäftige“"(16/26 - 30).

Einen neuen Blick auf berufliche Möglichkeiten gewinnt sie auch durch Pablos Haltung. Er legt sich nicht auf ein Berufsfeld fest, sondern ist offen auch für ungewöhnliche Ideen, wie z.B. deutsche Babysachen in 
Mexiko zu verkaufen (vgl. 18/8f). Pablos mexikanische Mentalität zeigt ihr einen anderen Umgang mit Berufstätigkeit auf. Die deutsche Engführung von Ausbildung und Berufsausübung wird der mexikanischen Offenheit und Spontaneität gegenübergestellt: „Das ist nicht so, das Leben ist offen sozusagen, also ist viel, nicht so wie bei uns - alles muss geplant sein" (17/32f). In der geplanten Migration und dem gemeinsamen transnationalen Biographieentwurf kulminiert für Maria die Notwendigkeit der biographischen Integration ihrer interkulturellen Programmatik. Sie gibt einen Anlass zur Reflexion über ihre eigene berufliche Positionierung. Die Beziehung zu Pablo gibt ihr die Möglichkeit und fordert gleichzeitig von ihr, sich ,eben auch mit mir mit meinem eigenen Bild auseinanderzusetzen“ (38/29f). Dass dieser Auseinandersetzungsprozess, der von Ermöglichungen und Begrenzungen durch Kulturalisierungen begleitet wird, dennoch nicht im Unverständnis und in einer Verfremdung mündet, wird bei Maria auch durch ihre interkulturelle Grundhaltung erklärbar. Die transnationale Perspektive macht es notwendig, scheinbar Unvereinbares zu integrieren, macht es aber umgekehrt auch möglich, Fragen wie die der Kindererziehung zu lösen, indem die Berechtigung beider Eltern an der Weitergabe der eigenen Kultur betont und nicht in Frage gestellt wird.

\section{Bearbeitungsfelder bei Pablo: Tourist oder Migrant}

Auch für Pablo ist die Realisierung einer transnationalen Biographie das relevante Thema. Für ihn gilt wie für Maria, dass er in Deutschland bzw. eigentlich schon in der Vorbereitung auf die Migration nach Deutschland mit Konsequenzen seines programmatischen Ideals konfrontiert wird, die er nicht erwartet hatte und die ihn vor Bearbeitungsnotwendigkeiten stellen. Die Realisierung des dauerhaften Aufenthalts ändert seinen Blick auf Deutschland. „In Berlin _ gut, ich fand es sehr schön, weil es hier immer Urlaub war (lacht) und die Stadt als Tourist zu sehen, im Urlaub, ist immer was anderes als die von innen zu sehen“"(5/14f). Insbesondere die berufliche Situation ist für ihn ein zentraler Bezugspunkt der Auseinandersetzungen. Zudem stellen Fremdheitserfahrungen und Zuschreibungen, wie sie unter anderem von Marias Eltern vorgenommen werden, seinen Aufenthalt in Deutschland in Frage.

\section{Berufliche Partizipation}

Pablo ging in seinen Erwartungen an Deutschland von einer adäquaten Fortführung seiner bisherigen beruflichen Karriere aus. Die stattfindenden beruflichen Exklusions- und Dequalifizierungsprozesse treffen ihn 
unvorbereitet: „Und so änderten sich meine Erwartungen zum schlechten, hier, als ich nach Deutschland kam“ (34/8f). Seine berufliche Integration will er mit Hilfe des ihm zur Verfügung stehenden sozialen Kapitals bewältigen. ${ }^{4}$ Er versucht, erfolglos, über Freunde und Bekannte von Maria Hinweise auf freie Stellen oder andere Arbeitsmöglichkeiten zu bekommen. Viele kennen zwar Architekten, sowohl auf dem freien Markt als auch in der Universität, konkrete Beschäftigungsmöglichkeiten ergeben sich jedoch daraus nicht. Auch seine eigenen Recherchen, die er im Vorfeld in Mexiko durchgeführt hat, führen zu keinem Ergebnis. Pablo hatte von einer Gruppe mexikanischer Architekten gehört und ging davon aus, durch sie ebenfalls berufliche Kontakte knüpfen zu können. Auch dies aber stellte sich als Sackgasse heraus. Zwar sind sie in Vorträge und Kolloquien involviert, haben aber keine oder nur „sehr schlechte Arbeit“ (11/4). Seine Erfahrungen in Deutschland stehen für ihn in einem deutlichen Gegensatz zu Mexiko, wo soziale Beziehungen den Grundstein für berufliche Erfolge legen:

„In Mexiko war es genau das Gegenteil, weil wenn du in Mexiko fragst, ich will dieses oder jenes, sagen sie sofort: Ja, klar, geh dahin oder dort_und sie halfen dir Beziehungen aufzubauen, klar, es war auch sehr schwer, aber es gab mehr Beziehungen und es war _ _offener. [...] Viele offene Wege [...] und in Deutschland genau das Gegenteil“" $(11 / 14-19){ }^{5}$

Für Pablo stellen sich so die Unterschiede der beruflichen Einmündungsmöglichkeiten vor allem als kulturelle Unterschiede dar. Die unterschiedliche Bedeutung der Kapitalien in Deutschland und in Mexiko verweist für ihn auf kulturelle Differenzen, die ihm die berufliche Partizipation erschweren. Während in Mexiko das soziale Kapital und informelle Wege wesentlich für die Einmündung sind, erfährt er hier die Exklusionsmechanismen eines formellen Sektors mit zertifizierten $\mathrm{Zu}$ gangsmöglichkeiten und wenig informellen Wegen. Das kulturelle Kapital, und insbesondere dessen institutionalisierte Form, wie es seinen Ausdruck z.B. in Bildungstiteln findet (vgl. Bourdieu 1983), wird im Verlauf des Migrationsprozesses Dequalifizierungsprozessen unterwor-

4 Mit sozialem Kapital rekurriere ich auf Bourdieus Unterscheidung verschiedener Kapitalsorten. Soziales Kapital meint den Nutzen, den man aus einem funktionierenden Netz aus „Beziehungen gegenseitigen Kennens und Anerkennens“ (Bourdieu 1983: 190) ziehen kann. Zu weiteren Definitionen des Begriffs s. den Überblick bei Haug 1997.

5 Die geschätzte Arbeitslosenquote lag 2002 in den mexikanischen Städten bei ungefähr 3\%, es ist aber von einer großen Zahl an ,underemployed“ Personen auszugehen, $40 \%$ der Bevölkerung leben unterhalb der Armutsgrenze (Quelle: Konsulate und Botschaften 2006). 
fen und lässt sich nicht mehr, oder wie in Pablos Fall, nur in geringerer Form verwenden. Bewerbungen auf offene Stellen führen zu keinem Ergebnis, der Beginn eines Aufbaustudiums an der Universität ist aufgrund der fehlenden Anerkennung seines Abschlusses nicht möglich. Erst nach längerem Verhandeln kann er erreichen, dass die Universität seinen Universitätsabschluss immerhin als Fachhochschulabschluss anerkennt. Dies eröffnet ihm die Möglichkeit, sich wenigstens Visitenkarten mit seiner Berufsbezeichnung (mexikanischer Architekt) drucken zu lassen. Die paradoxe Situation, in der er sich befindet, verweist auf institutionalisierte Ausgrenzungsmechanismen, die Migrant(inn)en die Eingliederung in den Arbeitsmarkt erschweren oder sogar unmöglich machen. Das deutsche System mit stark formalisierten Zugangsbeschränkungen lässt nur wenig Spielraum für die Wirkung sozialen Kapitals. Gleichzeitig ist ihm auch der Einstieg in den formalisierten Ausbildungsmarkt in Form eines Aufbaustudiums verwehrt.

Sein beruflicher Ehrgeiz und die Suche nach einer ausbildungsadäquaten Beschäftigung bleiben aber bestehen. Eine dauerhafte Erwerbslosigkeit bzw. eine dauerhafte Übernahme der Erziehungsaufgabe kommt für ihn nicht in Frage. Die Perspektive der Re-Migration unterstützt dabei sein berufliches Interesse, indem er zukünftig deutsche und mexikanische Solartechnologien verbinden und eine eigene Firma gründen möchte. Bis dahin versucht er, durch verschiedene Möglichkeiten eine kontinuierliche Berufsbiographie zu erhalten. So arbeitet er, unentgeltlich, in der Kita der Kinder mit und hat von Deutschland aus für eine seiner Schwestern ein Haus mit entworfen. Die Kontinuität der Berufsbiographie ist für ihn auch durch den Arbeitstag gewährleistet. Ob mit oder ohne Bezahlung spielt dabei keine Rolle:

„Ich muss meine Arbeitszeiten einhalten, weil sonst bin ich nicht zufrieden, ich, ich denke, ich habe gar nichts getan, ich muss die Stunden einhalten für die Arbeit, auch wenn ich nicht eingestellt bin, oder ich nicht bezahlt werde oder ich keinen Lohn bekomme“ (35/17 - 19).

\section{Assimilation, Fremdenfeindlichkeit und Normalitätserwartungen}

Fremdheit lässt sich als spezifische Qualität eines Beziehungsverhältnisses verstehen. In Pablos Narrationen zeigen sich deutlich die Grenzen von Zugehörigkeitsverschiebungen, rekonstruieren lässt sich, wie natioethno-kulturelle Grenzen verfestigt und aufrechterhalten werden. Das Beziehungsverhältnis zwischen Pablo und Deutschland bleibt für ihn exklusiv: „Ich bin immer Ausländer“ (42/17). Für ihn bestätigt sich das Vorurteil, dass Deutsche generell unfreundlich gegenüber Fremden sind: 
„Aber von Deutschland wird auch gesagt - ja, die Deutschen sind nicht freundlich zu den Ausländern, der Ausländer muss sich immer verteidigen“ (16/18f). Diese Notwendigkeit der ständigen Legitimation und der Verteidigung erlebt er sowohl auf der rechtlichen Ebene gegenüber einer ausländerfeindlichen Gesellschaft wie auch auf der personalen Ebene. In mehreren Begegnungen schildert er, wie er selbst sagt, ,,immer dieselbe Geschichte“: „Manchmal sage ich zu mir, ich kenne die Geschichte schon, es ist immer gleich“ (15/19f). Sei es beim Bezahlen mit einer ECoder Kreditkarte, sei es bei der Einreise nach Deutschland, seine rechtmäßige Anwesenheit muss von ihm jedes Mal aufs Neue nachgewiesen werden. Solche rassistischen Ausgrenzungen lassen sich, wie Neidhardt schreibt, als „Gegenbewegungen“ bezeichnen, mit denen eine Gesellschaft ihre Normalität gegen deren Alternativen verteidigt (vgl. ebd. 1986: 17; zu einem Verständnis von rassistischen Inhalten als Ausdruck symbolischer Kämpfe vgl. Weiß 2001: 48). ${ }^{6}$ In solchen Gegenbewegungen werden dichotome Grenzen erstellt, an denen ,die Ausländer ${ }^{6}$,den Deutschen“ gegenüber stehen. In einer solchen Konstruktion gibt es nur ein Entweder - Oder, für Pablo gibt es keine Möglichkeit in diesem Sinne als ,Normal' angesehen zu werden, da seine dunkle Hautfarbe ihn sichtbar macht und somit aus der unsichtbaren Normalität exkludiert. Auch das Verhältnis zu seinen Schwiegereltern, insbesondere zu seiner Schwiegermutter, ist durch kulturalisierende Konstruktionen und Assimilationsanforderungen geprägt. Die Schwiegereltern, insbesondere die Mutter, erwarten von ihm die Aufgabe seiner mexikanischen Verhaltensweisen: „Sie fordern von mir sogar, ich müsse mich benehmen als wäre ich deutsch, weil ich in Deutschland bin, [...] ich soll mich sogar ändern“ (26/16 - 18). ${ }^{7}$ Die Assimilationserwartungen der Schwiegereltern, als welche sie von Pablo wahrgenommen werden, beziehen sich dabei nicht nur auf die Erziehung der Kinder (so rufen sie täglich bei

6 Rassismus ist immer verbunden mit der Vorherrschaft, wie Auernheimer mit Rückgriff auf Memmi ausführt. „Der Rassismus besteht in einer Hervorhebung von Unterschieden, in einer Wertung dieser Unterschiede und im schließlichen Gebrauch dieser Wertung im Interesse und zugunsten des Anklägers“" (Memmi 1987 in Auernheimer 2004: 91).

7 Assimilation, verstanden als vollständige Übernahme der Haltungen und Einstellungen der Mehrheitsgesellschaft, wird nach wie vor von einigen Autor(inn)en, trotz der ideologischen und theoretisch-simplifizierenden Implikationen, als für Migrationsprozesse relevant betrachtet. So definieren Alba/Nee 1997 Assimilation ,as the decline, and at its endpoint the disappearance, of an ethnic/racial distinction and the cultural and social differences that express it" (ebd.: 845) und wollen den Begriff so explizit für zweiseitige Veränderungsprozesse, also auch seitens der Mehrheitsgesellschaft, öffnen. Ein solcher Versuch stärkt jedoch im Ergebnis die Homogenitäts-Illusion einer Gesellschaft. 
ihm an und fragen nach den Kindern, für ihn ein Zeichen der Kontrolle), sondern auch auf die Arbeitsteilung des Paares. Sie erwarten von ihm mindestens einen Taxischein, damit er überhaupt erwerbstätig sein kann: „Du musst arbeiten, auch wenn du nur als Chauffeur arbeitest. Besorge dir eine Lizenz. Das ist das Mindeste, was wir wollen“ (29/2f). Einem solch öffentlichen Dequalifizierungsprozess verwehrt sich Pablo allerdings deutlich: „Ich bin kein Taxifahrer [...] ich bin Architekt, auch wenn es hier [mit den beruflichen Chancen] nicht gut aussieht" (29/6f). Für Pablo stellt die eigene kulturelle Verwurzelung den Rückhalt für interkulturelle Öffnungen dar: „Vielleicht ist es ein bisschen so, dass ich meinen Wurzeln sehr treu bin, wie meine Frau sagt aber _ im allgemein, ja, ich glaube, dass ich ein Mensch bin, der offen ist, für andere Sachen“ (2/17f). Die eigene Herkunft will er nicht aufgeben, diese ist für ihn die Basis seiner Verortung und seines Selbstverständnisses. Einer solch assimilatorischen und rassistischen Erwartung, wie er sie bei Marias Eltern wahrnimmt, stellt er die Perspektive seiner eigenen Familie gegenüber, die Marias Kultur und damit sie als Person respektiere und sich nicht in ihre Familienangelegenheiten einmische. Diese Form von interkultureller Toleranz, die kulturalisierend Unterschiede anerkennt und akzeptiert, erwartet er allerdings nicht mehr von seinen Schwiegereltern, für ihn ein Grund mehr bald nach Mexiko zu gehen (vgl. 29/2).

\section{Kinder als Integrationsmoment}

Auch für Pablo stellen die Kinder den entscheidenden Handlungsbezug dar. Er sieht in ihnen nicht nur eine Brücke zwischen Mexiko und Deutschland, sondern auch zwischen Maria und ihm. Im Leben in Deutschland und in Mexiko sieht er einen „Gewinn“ (19/18) für die Kinder. Er möchte ihnen die biographischen Möglichkeiten zweier Staaten eröffnen und nicht derjenige sein, der Maria und den Kindern ihren ,deutschen' Anteil wegnimmt. Deutlich wird aber auch, dass die Entscheidung für ein Leben in beiden Ländern eine Entscheidung für die Kinder ist, die er selber für sich so nicht treffen würde, er sieht es als „Risiko“ an, „,in einem anderen Land zu leben. Die Eltern sind diejenigen die etwas riskieren aber die Kinder müssen etwas gewinnen“ (19/19f). Für dieses Ziel opfert er in gewisser Weise auch seine freie Entscheidung: „Ich mache es alles für die Familie, für meine Kinder“ (19/20f). Die Kinder und seine eigenen Erfahrungen mit einem abwesenden Vater waren für ihn der Grund, nach Deutschland zu ziehen und das female breadwinner Modell zu realisieren. Für sie bleibt auch die transnationale Biographieausrichtung bestehen. Anders als bei Carlos, für den Mexiko zum Handlungshorizont wird und der langfristig gerne 
ganz nach Mexiko remigrieren würde, ist für Pablo Deutschland als auch zukünftiger Wohnort relevant. Einerseits stellen die Kinder also für ihn den Grund dar, in Deutschland zu bleiben, andererseits bieten sie ihm auch Zugangsmöglichkeiten: Über die Kita der Kinder hat er Freunde gefunden, dort kann er, wenn auch unentgeltlich, arbeiten. Das pragmatische Verhältnis, welches er zu Deutschland und zu seinem Leben in Deutschland gefunden hat, spiegelt sich auch in seiner Zukunftsplanung wider: Deutschland und das Leben in Deutschland werden für ihn vor allem hinsichtlich der schulischen und medizinischen Qualität relevant, ähnlich wie bei Carlos lässt sich hier von einer Teilinklusion sprechen, die Partizipation an bestimmten Systemen muss eben nicht mit einer Vereindeutlichung der identitären Zugehörigkeit einhergehen (vgl. dazu auch Pries 1996: 469). Die Kinder sollen eine gute Ausbildung erhalten, und Maria soll weiterhin die medizinische Unterstützung bekommen, die sie braucht. Für sich selber strebt er eine Weiterqualifizierung in Mexiko an, mit der er seinem Traum, eine „mexikanisch-deutsche Firma“ (32/11) aufzubauen, näher kommen kann. Er hofft auf eine veränderte Wirtschaftslage in der Zukunft, die eine solche Firma auch in Deutschland möglich macht, um danach wieder nach Mexiko zurückkehren zu können: „Das ist mein Traum, ich meine, der richtige Traum, sehr viel hier arbeiten und sich danach in Mexiko ausruhen“ (42/12f).

\section{Form biographischen Lernens: gender als Ausdruck natio-ethno-kultureller Zugehörigkeit - Transmigration als Integrationsmodus}

Gogolin und Pries konzeptionalisieren 2004 Transmigration als ,ein[en] Modus, in dem die Betroffenen selbst den Wanderungsprozess unabgeschlossen halten Gogolin/Pries 2004: 6). In so entstehenden hybriden, multiplen und offenen Bewegungen lässt sich eine Neubestimmung des „Verhältnisses von geographischem Raum und sozialem Raum vornehmen“ (vgl. Pries 1997: 16), die es den Akteuren des Migrationsgeschehens erlaubt, in vielfältiger Weise individuelle Verortungen vorzunehmen. Eine solche Transmigrationsbewegung lässt sich auch in der Konstruktion des Paarhorizonts von Maria und Pablo analysieren. Maria und Pablo sehen sich schon vor ihrer Begegnung und vor Beginn der Beziehung als interkulturell interessiert und kompetent. Beide suchen die Begegnung mit ,fremden Kulturen“ und nutzen diese als Reflexionsanstoß über die eigene Herkunftskultur. Maria versteht dabei natio-ethnokulturelle Zugehörigkeit als dominantes Merkmal und Ergebnis der Sozialisation. Entsprechend ist diese nur bedingt reflexiv zugänglich, sie 
wird vor allem als handlungsleitende und -erklärende Funktion verstanden. In einem engen Verhältnis werden so auch gender und natio-ethnokulturelle Zugehörigkeitskonstruktionen gesehen. Formen von Geschlechterbeziehungen werden als Ausdruck natio-ethno-kultureller Zugehörigkeit konzipiert, Pablos Erwartungen an die Beziehung sind für Maria eindeutig kulturell erklärbar und zuordenbar. Anders als beispielsweise bei Nicole, für die die ,altmodische“ Art der Mexikaner eine große Anziehungskraft darstellt, der Schwerpunkt also auf anderen Geschlechterkonstruktionen liegt, haben jedoch solche ,anderen“ Geschlechterordnungen in den Erzählungen von Maria keine so große Bedeutung für den Paarhorizont. Vielmehr stellt die Aufmerksamkeit, die Pablo ihr schenkt, eher so etwas wie einen zusätzlichen Pluspunkt dar, der sie in ihrer interkulturellen Haltung bestärkt. Die Beziehung stellt keine Umbruchsituation in Marias Biographie dar, sie bietet ihr auf mehreren Ebenen Anschlussmöglichkeiten und Realisierungen ihrer bisherigen politischen und kulturellen Einstellung, die sie in ihrer interkulturellen Öffnung und Orientierung bestärken. Ganz ähnlich lässt sich die Konstruktion des Paarhorizonts bei Pablo lesen. Er ist, nach dem einschneidenden Erlebnis seiner Reise nach Korsika, auf der Suche nach interkulturellen Begegnungen, um Reflexions- und Lernanstöße zu erhalten. Auch für ihn ist Maria in diesem Sinne die richtige Frau, die ihm die Anschlussmöglichkeit und die Weiterentwicklung seiner interkulturellen Orientierung ermöglicht. Und auch für ihn können seine und ihre natio-ethno-kulturelle Zugehörigkeit nicht grundsätzlich in Frage gestellt werden. Vielmehr dient sie als Hintergrundfolie, vor der Diskussionen und Entscheidungen über den zukünftigen Wohnort und eine mögliche oder tatsächliche Heirat stattfinden. In diesem Zusammenhang wird die Relation zwischen gender und natio-ethno-kultureller Zugehörigkeit hergestellt: gender ist auch für ihn ein Teil des kulturellen Sozialisationspakets, welches handlungserklärend fungiert und einen Großteil der interkulturellen Problematik ausmacht, primär werden für ihn aber die Aushandlungen auf kultureller Ebene geführt.

Anders als bei den beiden Paaren zuvor wird die Entscheidung für den (zeitweiligen) Umzug nach Deutschland im Rahmen einer ausführlichen und durchaus konfliktreichen Auseinandersetzung getroffen. In dieser Auseinandersetzung hilft die Entpersonalisierung durch die starke kulturelle Rückbindung der Argumente, den Konflikt zu entschärfen. Dennoch findet sich erst mit der ungeplanten Schwangerschaft ein Weg, mit der die Entscheidung über den ersten Wohnort quasi aus der Hand genommen ist. In den (Re-)Konstruktionen wird deutlich, dass die Folgen der Migrationsentscheidung zwar nicht als Bruch zu sehen sind, dass sie aber dennoch nicht-intendierte Folgen nach sich ziehen, die bei- 
de, trotz der Diskussionen und trotz der ,informierten Migration', in der Form nicht erwartet hatten. Die Notwendigkeit des ,Umgangs mit der Kultur', wie es Pablo formuliert, wird in den alltäglichen Auseinandersetzungen nicht mehr nur programmatisch, sondern auch lebenswirklich notwendig. Der Wechsel vom Touristen zum Migranten, der Wechsel vom Besuch zum dauerhaften Leben in Deutschland stellt insbesondere für Pablo ein Problem dar, während Maria sich auf Ähnliches in Mexiko gefasst macht. Bei der Bearbeitung der Zumutungen, insbesondere hinsichtlich der Begrenzungen in der Kommunikation, die Maria in der Beziehung zu Pablo erlebt, wird natio-ethno-kulturelle Zugehörigkeit vor allem als Mittel zum Verständnis konzipiert. Schwierigkeiten entstehen vor allem aufgrund der nun notwendig gewordenen Realisierung der vorher eher programmatisch geäußerten interkulturellen Kompetenz. Auch hier bleibt die Konstruktion von der spezifischen Form der Geschlechterbeziehungen als Ausdruck der natio-ethno-kulturellen Zugehörigkeit bestehen. Die Vorstellungen darüber sind eben Folgen der kulturellen Sozialisation, ,wie man gepolt ist“" (25/4), wie Maria sagt, entscheidet eben auch darüber, was für Erwartungen an die Beziehungsgestaltung geknüpft sind. Das gemeinsame Feld, welches die Integration situativer Inkompatibilitäten ermöglicht und die Beziehung strukturiert, sind die gemeinsamen Kinder und die große Bedeutung, die sie ihnen beimessen. Die Transmigration als Thema der Beziehung wird aufgrund der Kinder verhandelt und realisiert. Insbesondere im Hinblick auf die Kindererziehung und die Arbeitsteilung hat Maria großen Respekt vor Pablos Einsatz und Bereitschaft, das female breadwinner Modell zu akzeptieren. Mit einem Deutschen, so Maria, wäre diese Entscheidung wohl nur sehr schwer möglich gewesen. Pablo sei es noch höher anzurechnen, schließlich sei seine Kultur noch traditioneller. Die Beziehung stellt auch hier eine Möglichkeit für sie dar, emanzipiert im Sinne von selbstbestimmt leben zu können. Mit einem deutschen Mann hätte sie solche eigenen Wege wohl nicht realisieren können. Gender hat jedoch auch hier nicht die dominante Rolle als handlungsermöglichende Kategorie, die es in den anderen Biographien bekommt. Vielmehr stellt es ein Bearbeitungsfeld dar, welches aber der natio-ethno-kulturellen $\mathrm{Zu}$ gehörigkeit untergeordnet ist. Natio-ethno-kulturelle Zugehörigkeit wird hier als Ressource im Sinne einer Zumutbarkeitserwartung genutzt. Verständnis füreinander funktioniert in diesem Fall vor allem über kulturelle Differenz, eine Differenz die es in ihrer Unterschiedlichkeit zu respektieren, aber auch kommunikativ zu bearbeiten gilt. Für Maria bedeutet die Notwendigkeit der biographischen Integration der Beziehung eben auch, sich mit ihren eigenen Bildern auseinandersetzen zu müssen. Sei es hinsichtlich ihrer Einstellung zur Erwerbsarbeit und Lebensplanung, 
oder sei es hinsichtlich der Erziehung der beiden Söhne. Die Auseinandersetzungen, die rekonstruiert werden konnten, sind weniger, wie beispielsweise bei Susanen und Rachid, chronologische Entwicklungen, die zum Zeitpunkt des Interviews zu einem (vorläufigen) Ende gekommen sind. Vielmehr präsentieren beide, Maria und Pablo, Themenfelder, die nach wie vor Aktualität besitzen. Das ,Kreisen um‘ bestimmte Problemfelder bleibt in der Beziehung bestehen. Anders als bei den beiden anderen Paaren findet sich hier keine Instrumentalisierung eines kulturellen Unterschieds, um individualisierte Partnerschaftsbeziehungen zu realisieren. Die natio-ethno-kulturelle Zugehörigkeit Pablos wird zwar auch hier instrumentalisiert, in dem Sinne, dass sie eine Funktion bekommt. Sie ermöglicht aber in diesem Fall die biographische Integration dessen, was ansonsten wahrscheinlich, so Maria, bei einem deutschen Mann nicht annehmbar gewesen wäre: ,tatsächlich also Sachen zu akzeptieren die ich vorher nicht akzeptiert habe. Oder das die ich ähm - auch nicht wüsste ob ich sie bei einem Deutschen akzeptieren würde (Maria 38/3f). Auch hier entlastet die kulturelle Differenz, Fremdartigkeit ermöglicht die Akzeptanz ansonsten kritischer geschlechtsspezifischer Handlungen. Die Notwendigkeit des Perspektivenwechsels durch kulturelle Differenzen führt insgesamt, so resümiert Maria, zu mehr „Höhen und Tiefen“ (Maria 38/18) in der Beziehung, führt aber auch zur Erweiterung biographischer Möglichkeiten und zur Realisierung einer transnationalen Biographie: „Wo du manchmal auch der Nationenstreit. [...] Ja ähm es ist also das auch - sozusagen dass man zu bestimmten Sachen mehr Reibungsflächen hat - aber auch mehr - mehr Sachen kennen lernen kann“ (40/18 - 24). Für Pablo liegen die Schwierigkeiten ebenfalls bei der biographischen Integration der bisher vorrangig programmatisch geäußerten interkulturellen Offenheit. Die von ihm vorgefundene Situation, ein ausländerfeindliches Umfeld und ein geschlossener Arbeitsmarkt, lassen die Realisierung seines interkulturellen Biographieentwurfs nicht ohne weiteres zu. Die Fremdheitserfahrungen bleiben, trotz beginnender Freundschaften und trotz seiner Mitarbeit in der Kita der Kinder hat er nicht den Eindruck der Möglichkeit zur Grenzüberschreitung, er wird immer Ausländer bleiben. Anders als Carlos lässt sich bei Pablo jedoch keine Verfestigung seiner natio-ethno-kulturellen Zugehörigkeit analysieren. An sich stellt er sie nicht in Frage, und weist Assimilationsgesuche seiner Schwiegermutter empört zurück. Die Zugehörigkeitserklärung geht jedoch nicht mit einer Verschiebung seines Handlungshorizonts nach Mexiko einher. Vielmehr findet sich hier eine pragmatische Form der Mehrfachzugehörigkeit, die Optionen in beiden Ländern offen hält und zwischen emotionaler und beruflicher Zugehörigkeit unterscheidet. Die Möglichkeit der biographischen Anschlussfähigkeit findet auch er in 
seinen Kindern. Diese entscheiden über den ersten Wohnsitz, und diese entscheiden auch über den weiteren Verlauf der (transnationalen) Migrationsbewegungen. Natio-ethno-kulturelle Zugehörigkeit wird hier als Sozialisationsmoment verstanden, welches sowohl handlungsleitend in den Diskussionen mit Maria wirkt als auch den Kindern als doppelte Ressource zugestanden werden soll. Der beständige Kontakt zu Mexiko ist für ihn höchst relevant: „Und auf der anderen Seite habe ich mein Land nicht verlassen, über Internet gibt es immer Kommunikation“ (17/14f.), und bestätigt die transnationale Biographieausrichtung. Mexiko bleibt als emotionaler Halt relevant, insofern als dass er sich wünscht im Alter mit Maria dort leben zu können. Dennoch will er sich interkulturellen Irritationen nach wie vor in der Beziehung aussetzen. In einer Ehe mit einer Mexikanerin, so seine Einschätzung, wäre ihm wahrscheinlich langweilig geworden: „Ich denke, dass ich ein bisschen gelangweilter gewesen wäre, ich hätte mich eher ___abgefunden mit den Zuständen“"(41/7f).

Die Biographien von Maria und Pablo lassen sich als Individualisierungsbiographien bezeichnen. Anders als für die beiden vorhergehenden Paare liegt der Individualisierung allerdings nicht eine Instrumentalisierung der natio-ethno-kulturellen Zugehörigkeit in den bisherigen Formen zugrunde. Natio-ethno-kulturelle Zugehörigkeit dient hier vielmehr als Ressource sowohl zur Entwicklung einer eigenen reflexiven Grundhaltung wie auch für die Anerkennung von kommunikativen oder emotionalen Grenzen zwischen den Partner(inn)en. Für Maria und Pablo stellt die binationale Beziehung nun eine Möglichkeit dar, eine transnationale Paarbiographie zu realisieren. Relevant werden für sie insbesondere die Sequentialität der Migrationsbewegungen und die gleichzeitige Verortung in Deutschland und in Mexiko (Gogolin/Pries 2004: 10). Für beide stellen die Kinder den Referenzpunkt dieser transnationalen Biographie dar. Die Kinder haben dafür gesorgt, dass die Eltern an einem Ort wohnen, die Kinder ermöglichen es Maria, auch ohne Arbeitsstelle nach Mexiko zu gehen, und die Kinder sind für Pablo ein Grund sich mit Deutschland und dem female breadwinner Modell zu arrangieren.

Die Form biographischen Lernens, die sich als übergeordnete Bearbeitungsform analysieren ließ, lässt sich daher mit ,gender als Ausdruck natio-ethno-kultureller Zugehörigkeit: Transmigration als Integrationsmodus' bezeichnen. Es ließ sich zeigen, wie natio-ethno-kulturelle Zugehörigkeit sowohl als Ressource als auch als Grenze in interkultureller Kommunikation genutzt werden konnte und durch die Betonung der Differenz biographische Anschlussfähigkeit gewährleistet werden kann. Die Perspektive der Transmigration integriert auch die situativen und interakiven Fremdheitskonstruktionen, die sowohl Pablo als auch Maria 
erleben. Pablo sieht sich selber als „Landstreicher“, der „,mit beiden Füße an beiden Orten“ (40/12f.) lebt und leben muss. Die permanente Mehrdeutigkeit von Situationen unterscheidet dieses Paar von Nicole und Carlos, die die Mehrdeutigkeit eher zu Gunsten von Eindeutigkeit auflösen. Maria und Pablo versuchen mit der Mehrdeutigkeit zu leben und nutzen dafür grade ein Verständnis von kultureller Differenz. Gerade die Konstruktion von natio-ethno-kultureller Zugehörigkeit erlaubt es, Mehrdeutigkeiten zuzulassen und Toleranz, wie es Maria formuliert, $\mathrm{zu}$ entwickeln. Auch wenn es Situationen gibt, in denen Maria meint, Pablo nicht mehr verstehen zu können, erlaubt ihr Verständnis von Pablo als ,Fremdem‘ die nachträgliche und nachhaltige Integration solcher Irritationen. Eine solche Integration ist jedoch nicht unkritisch zu sehen. Das Spannungsfeld zwischen kultureller Zugehörigkeit und individueller Autonomie bleibt bestehen, beide erheben den Anspruch, nicht mit Stereotypen betrachtet zu werden, beide nutzen aber eben solche Stereotype. Insofern setzen Verfremdungsprozesse der Wahrnehmung des und der Anderen als autonomes Subjekt Grenzen. Einerseits werden so Konflikte gefördert, wenn zum Beispiel Maria Pablo ,vorschnell' unterstellt, als Macho zu handeln, und insofern im Vorfeld schon auf diese Imaginationen reagiert. Andererseits entschärft es auch Konflikte, indem Maria Handlungsweisen ,toleriert', die sie bei einem deutschen Mann in Frage gestellt hätte. Die transnationale Ausrichtung der Biographie ermöglicht es, den Zustand zu stabilisieren und auch Dequalifizierungsprozesse, wie sie Pablo erlebt, auszuhalten. Noch ist nicht klar, wie es konkret weitergehen wird, ob sich das Verhältnis zwischen geographischem und sozialem Raum tatsächlich neu verortet, oder ob sich langfristig nicht doch eines der Länder als fester Wohnort herausstellen wird. Die transnationale Perspektive, die sich durch die Kinder realisiert hat, ist jedoch die relevante Kategorie, die die Beziehung charakterisiert und die es erlaubt, Irritationen und Fremdheitserfahrungen zu integrieren. 
Ausblicke 



\section{NEUE SOZIALE WIRKLICHKEITEN? \\ BIOGRAPHIEN BINATIONALER PAARE}

„Indem Menschen ihre Biographie in bestimmter Weise darstellen und sie sie damit je eigensinnig konstruieren (doing biography), dabei allerdings nicht rein individuell verfahren, sondern als vergesellschaftete Wesen ihre Auseinandersetzungen mit kulturellen Normen und gesellschaftlichen Zuschreibungen dabei ausdrücken, haben sie Teil an der Umdeutung bzw. Verschiebung von Bedeutungen der kulturellen Konstruktionen. Sie konstruieren sich selbst in Lern- und Bildungsprozessen, indem sie neue Inhalte in ihr bisheriges Wahrnehmungssystem integrieren und es dadurch verändern und indem sie ihr Welt- und Selbstbild verändern. Dabei performieren sie auch gesellschaftliche, geschlechtstypische Zuschreibungen. Sie legen sie in ihren Auseinandersetzungen mit Welt im Rahmen von Lern- und Bildungsprozessen neu aus oder sie stabilisieren sie teilweise" (von Felden 2003: 249).

Von Felden verweist hier auf die Bedeutung biographischer Prozesse für gesellschaftliche Veränderungen. Biographische Lernprozesse können kategoriale Zuschreibungen verändern. Die in der Arbeit analysierten Formen biographischen Lernens geben Auskunft über biographische Bearbeitungen der Herausforderungen, die sich durch die Migration und das female breadwinner Modell stellen. Diese Formen beziehen sich auf die biographiespezifischen Verflechtungen der beiden Kategorien gender und natio-ethno-kulturelle Zugehörigkeit, die sich beschreiben und analysieren lassen. In den Erzählungen der Paare lässt sich jeweils eine dominante Form biographischen Lernens identifizieren, die gewissermaßen als Kernkategorie Erfahrungen und Veränderungen integriert und die beobachtbaren Veränderungen erfasst. Die Herausforderung der „Handhabung von Diskontinuität“ (Seitter 1999: 38) für Migrant(inn)en, in der Koller ja sogar eine ,prototypische Bedeutung“, im Hinblick auf kreativen Umgang mit kulturellen Differenzen sieht (vgl. ebd. 2002a: 
98), konnte also durch das Analysemodell konkretisiert und empirisch (nach-)gezeichnet werden. Ein zentrales Ergebnis der Arbeit ist, dass die Art und Weise der Erfahrungsverarbeitung, die Erfahrungsaufschichtung, wesentlich die Rezeption weiterer Erfahrungen und damit die Reichweite und die Richtung von Lernprozessen strukturiert.

Für den Ausblick der Arbeit werden die Leitfragen, die die Entstehung und den Verlauf der empirischen Analyse begleitet haben, erneut aufgegriffen. Dafür werden zuerst die Formen biographischen Lernens in der Biographiegestaltung der Paare zusammenfassend verglichen und diskutiert. In diesen Entwicklungen werden spezifische Verhältnisse von ,Kultur' und ,Geschlecht‘ deutlich, die als Ressourcen der Biographiegestaltung fungieren und daher darauf folgend genauer betrachtet werden. Abschließend wird die Frage nach der Bedeutung binationaler Paare für soziale Veränderungen in der Weise aufgegriffen, dass die Vorstellung der Prototypen moderner Gesellschaften kritisch hinterfragt wird. Sind binationale Paare tatsächlich als Vorreiter differenzkompetenter und demokratischer Subjekte zu sehen, die eigenständig und eigenwillig ihre Biographie gestalten? Welche Möglichkeiten eröffnen dabei natio-ethno-kulturelle Zugehörigkeiten und was für (neue?) Geschlechterbeziehungen sind zu beobachten? Auch wenn ein Verständnis von binationalen Paaren als im besonderen Maße modernen Paaren sicherlich kritisch zu betrachten ist, bleibt eben auch festzuhalten, dass alle drei Paare die Beziehung als Möglichkeit verstehen, ,andere‘ Biographien zu entwerfen bzw. zu realisieren und damit nationale, kulturelle, ethnische oder auch Geschlechtergrenzen zu erweitern bzw. für sich neu zu definieren. Wie am Anfang der Arbeit beschrieben, stehen die Paare vor der Notwendigkeit, eigene Bezüge zu entwickeln, der Rückgriff auf bekannte Strukturen fällt schwer, lebensweltliche Gewissheiten lassen sich, für den Migranten ebenso wie für seine Partnerin, nicht unhinterfragt aufrechterhalten. Bei der Analyse fällt nun auf, dass genau das aber auch (zumindest zu Beginn der Beziehung) nicht das Interesse der Paare ist. Vielmehr, das ließ sich an der Konstruktion des Paarhorizonts der Paare zeigen, sehen sie in einer binationalen Beziehung im- aber meistens explizit die Möglichkeit zur Veränderung im Sinne von Erweiterung ihrer Lebensentwürfe. Alle von mir interviewten Paare betonten zudem die Möglichkeit, das Eigene zu entwickeln. An den konkreten Entwicklungen von Bezugssystemen lässt sich so zeigen, wie mit Herausforderungen der Moderne umgegangen werden kann und wie sich solche Formen der ,neuen sozialen Wirklichkeiten' beobachten und beschreiben lassen. 


\section{Formen biographischen Lernens in Biographien binationaler Paare}

Die Auseinandersetzungen und die Bearbeitungen, die unter anderem aufgrund des female breadwinner Modells notwendig werden, wurden unter der Perspektive der Konstruktion von natio-ethno-kultureller $\mathrm{Zu}$ gehörigkeit und Geschlechterkonstruktionen betrachtet. Hinsichtlich der Bedeutung beider Kategorien fällt auf, dass insbesondere natio-ethnokulturelle Zugehörigkeit in ihren Dimensionen flexibilisiert und pluralisiert wird. Waldis und Ossipow beobachten in ihrer Studie über in der Schweiz lebende binationale Paare ein Herunterspielen der, wie sie es nennen, kulturellen Differenz: „Einerseits legen die Befragten den Akzent auf geschätzte Gemeinsamkeiten, andererseits bemühen sie sich, Unterschiede zu relativieren“" (ebd. 2003: 405). Dies erscheint insbesondere vor dem Hintergrund schlüssig, dass die Paare ja noch in einer Beziehung zusammen leben, und es insofern problematisch wäre, wenn Differenzen als unüberbrückbare Unterschiede wahrgenommen werden würden. Die Bestärkung der Gemeinsamkeiten ist vorrangig vor der Betonung grundsätzlicher Unterschiede. Kulturelle Differenz wird daher auch, so ein weiteres Ergebnis von Waldis und Ossipow, in den Erzählungen der Paare vor allem dann hervorgehoben, wenn die Beziehung konfliktuös ist (vgl. ebd.). Die Entwicklung eines gemeinsamen Verständnisses von natio-ethno-kulturellen Zuschreibungen und eines gemeinsamen Verständnisses des/der jeweils ,Anderen' findet sich auch in meinen Analysen. Die von Waldis und Ossipow beobachtete Relativierung kultureller Unterschiede findet insofern statt, als dass alle drei Paare Zuschreibungen als gemeinsamkeitsstiftendes Moment begreifen und ihnen in diesem Sinne Bedeutung verleihen. Es muss sich dabei jedoch nicht um eine Relativierung im Sinne eines ,undoing' handeln, die Bedeutung kultureller Differenz muss also nicht zwangsläufig in den Hintergrund geschoben werden, vielmehr kann die Relativierung auch in der Betonung der inkludierenden Funktion von Differenz liegen. Dabei wird die grenzherstellende Funktion relativiert, nicht aber ihre Bedeutung als subjektbedeutsame Kategorie. Diese Haltung ist insofern eine gemeinsame, als dass sie, wie in den Interpretationen deutlich wurde, als korrespondierend zu verstehen ist. Korrespondieren kann sie, wie bei Nicole und Carlos, auf einer inhaltlichen Ebene. Beide stimmen in ihren Erwartungen an die Beziehung und an das gemeinsame Leben in Deutschland überein, hier findet sich eine Komplementarität hinsichtlich geschlechtsspezifischer Erwartungen, welche die Gemeinsamkeit ausmacht. Korrespondieren kann die Haltung aber auch, das wurde bei Susanne und Rachid deutlich, auf einer eher strukturellen Ebene. Auch hier ergänzen 
sich die Erwartungen und die Konstruktionen des Paarhorizonts. Dabei unterscheiden sie sich jedoch inhaltlich deutlich voneinander, sie sind zudem weniger auf den Zusammenhalt als Paar als vielmehr auf die Weiterentwicklung als Individuum gerichtet. Innerhalb dieses Prozesses lässt sich ein kreativer Umgang mit Zuschreibungen und dem Spielen mit solchen Zuschreibungen beobachten. Kreativ in dem Sinne, dass eigenwillige Bedeutungen produziert werden: Wenn Susanne Rachid erzählt, dass in Deutschland jeder Mann seine Hemden selber bügelt, verlieren kollektive kulturelle Zuschreibungen den Anschein der Vorgängigkeit und der Naturwüchsigkeit und werden vielmehr in ihrer funktionalen Bedeutung sichtbar. Für die Paare werden Zuschreibungen und Imaginationen hinsichtlich der , anderen“ Kultur vorrangig als Ermöglichungskategorie relevant. Die binationale Beziehung wird so zu einer Ressource kreativer Produktion (vgl. dazu auch den Band von Fröhlich/Messerschmidt/Walter 2003). Dies gilt für den Beginn der Beziehung ebenso wie für die in Deutschland stattfindenden Bearbeitungsund Aushandlungsprozesse. ${ }^{1}$ Ein Bedeutungsverlust geht damit jedoch nicht einher. Ebenso wenig wie kollektive Zugehörigkeiten völlig beliebig wählbar und interpretierbar sind, sind personale Identitäten eben nicht, wie auch Bronfen und Marius anmerken, „Effekt eines Spiels frei flottierender Zeichen“ (ebd. 1997: 3). Dies gilt umso mehr, als dass die Bedeutung von natio-ethno-kultureller Zugehörigkeit in der Paarbeziehung, wie bei Susanne und Rachid, relativiert werden kann, dennoch aber in der Außenwirkung die kollektive Zugehörigkeit (zum Ausländer, wie es Pablo formuliert), virulent bleibt.

Für zwei der Paare fungiert die binationale Beziehung als Grundlage für Individualisierungsbestrebungen. Die Biographiearbeit ist insofern auf die Entwicklung einer gemeinsamen Biographiegestalt gerichtet, die Grenzen einer mononationalen Beziehungsform überschreitet und neue Beziehungsmodelle entwickelt. Eine solche Form von Pluralisierung ist Spezifikum der Moderne und gleichzeitig besondere Herausforderung für binationale Paare, Merkmale wie die Herauslösung aus traditionalen Gemeinschaftsformen und sozialen Bezügen gelten für Migrant(inn)en in besonderer Weise (vgl. zu weiteren Merkmalen von Individualisierungs- und Pluralisierungsprozessen auch Neuendorff 1998). Allerdings verbirgt sich darin die Gefahr eines konzeptuellen Kurzschlusses, wenn darunter zum Beispiel wie von Gemende die Notwendigkeit verstanden

1 Insofern stimmt die Annahme von Müller-Dincu 1981, dass binationale Paare den gemeinsamen Lebensentwurf erst erschaffen müssen. Allerdings gilt dies für mononationale Paare auch, wenn auch unter anderen Vorzeichen. 
wird, eine ,mehr oder weniger befriedigende Balance [zu] finden zwischen der Freiheit des Ich und der Sicherheit in kollektiven WirBezügen“" (Gemende 2001: 50). ${ }^{2}$ Das ist insofern problematisch, als dass hier suggeriert wird, dass Individualisierung (nur) als Emanzipation von kollektiven Bezügen und Gruppen zu verstehen ist und persönliche Freiheit nur in der Trennung von Kollektiven zu finden ist. In was für einem Verhältnis stehen aber Individualisierungsprozesse einerseits, also die Freisetzung der Individuen aus vorgängigen Bindungen wie Familie, lokale Kontexte oder Traditionen, und die Rückbindung an natio-ethnokulturelle Zugehörigkeiten andererseits (vgl. dazu auch Scherr 2000)? Trotz der ,Befreiung' der Individuen von kollektiven Identitäten und Bezugssystemen treten Subjekte eben nicht als isolierte und einzelne auf, sondern bauen sich in ihren „kleinen sozialen Lebenswelten“ (ebd.: 400) Netzwerke auf und legen Zugehörigkeiten fest. Der Begriff der ,kleinen sozialen Lebenswelten“ geht auf Hitzler und Horner 1988 zurück, „um auf die vielfältigen ,Sinnprovinzen“ mit ,je eigenen Relevanzen, Regeln und Routinen' hinzuweisen, in und zwischen denen sich Individuen bewegen“ (ebd.: 400). Individualisierung meint also nicht die vollständige Freisetzung des Subjekts und damit einhergehend die zunehmende Irrelevanz kollektiver Identitäten wie race, class und gender. Aus den Analysen der Interviews lässt sich vielmehr ein differenzierteres Verständnis von Individualisierung entwickeln, welches Individualisierung als Chance und Herausforderung in dem Sinne begreift, dass es um die Entwicklung eigener, spezifischer Selbst- und Wirklichkeitskonstruktionen geht, um Positionierungsarbeit also. Solcherart biographische Arbeit umfasst dabei unter anderem Zugehörigkeitsarbeit, wie sie von Mecheril (2003) formuliert wird: „Zugehörigkeitsarbeit umfasst Strategien der aktiven Auseinandersetzung mit dem Zugehörigkeitsstatus und Strategien der Absetzung von diesem Status“ (ebd.: 385). Sie umfasst aber auch biographische Gestaltungsansprüche, Erwartungen an die eigene Beziehung genauso wie die Entwicklung von beruflichen Karrieremustern:

„Menschen sind im Übergang zur Informationsgesellschaft in hohem Maße auf biographische Arbeit verwiesen. Das liegt nicht nur daran, dass eine so genannte Normalbiographie ihre normative Kraft weitestgehend eingebüßt hat,

2 Dies ist auch insofern problematisch, als dass sich hier eine Verdoppelung des Modernitäts-Traditionsparadigmas wiederfindet. Die Migration nach Deutschland ermöglicht, so Gemendes Interpretation der Interviews mit Frauen aus Vietnam, Emanzipationsprozesse im Sinne der Stärkung der individuellen Freiheit durch die Distanzierung von kollektiven Bezügen. Emanzipationsprozesse in kollektiven Bezügen können mit einem solchen Blick nicht erfasst werden. 
sondern es hat auch viel damit zu tun, dass beispielsweise Lernen lebenslang in die Biographie integriert werden muss, und es nicht bei einer einmaligen beruflichen Ausbildung bleiben kann. In dieser Hinsicht erweist sich also der Begriff der ,biographischen Arbeit‘ als geeignet, die Bildungsanforderungen der Menschen in der Moderne biographietheoretisch $\mathrm{zu}$ thematisieren“ (Kraul/Marotzki 2002: 8).

In solchen Positionierungen werden Verhältnisse zwischen dem Ich und dem Wir verhandelt, ohne dass aber damit das Ergebnis dieser Prozesse bestimmt wäre. Das Ergebnis muss eben nicht in der Entfernung von kollektiven Bezügen liegen. Umgekehrt kann gerade in einem deutlichen Bezug auf kollektive Gruppen und Gruppenzugehörigkeiten eine Individualisierung in dem von mir skizzierten Sinne stattfinden, indem sich dadurch von bisherigen kollektiven und auch singulären Bezügen distanziert wird. Individualisierung meint insofern durchaus eine Neuordnung, jedoch im Sinne einer Pluralisierung, die Freilassung der Subjekte aus traditionellen Sinnbezügen bedeutet eben auch die Freiheit, sich neuen kollektiven Identitäten anzunähern.

Die in den Interviews der beiden ersten Paare, Susanne und Rachid und Nicole und Carlos, analysierten Individualisierungsbestrebungen sind maximal kontrastiv, gemeinsam ist ihnen aber, dass sich die Individualisierungsbestrebungen auf die Geschlechterbeziehungen beziehen. Natio-ethno-kulturelle Zugehörigkeit fungiert hier also als Handlungsfeld für Geschlechterkonstruktionen. Zu Beginn der Beziehung ließen sich zwei sehr unterschiedliche Individualisierungsbestrebungen analysieren: Susanne und Rachids Biographiearbeit ist auf die Erweiterung von Geschlechterbeziehungen im Sinne einer Auflösung klassischer Geschlechterordnungen gerichtet. Im Fall von Susanne ist damit jedoch noch nicht gesagt, dass ihr als Emanzipation deklariertes Interesse zwangsläufig eine gleichberechtigte Beziehung zur Folge hätte. Dennoch ist beiden, Susanne und Rachid, an einer eigenen Gestaltung der Geschlechterbeziehung gelegen, die sich von der Deutschlands und von der des Iran abgrenzt. Spezifische Kulturalisierungen im Sinne der Reduzierung eines Partners auf seine Zugehörigkeit zu einer fremden Kultur finden in der Form nicht statt, vielmehr geht es um Unwissenheit (in Bezug auf Rachid) und der Zugehörigkeit zum Westen (in Bezug auf Susanne). Individualisierung ist hier daher als Distanzierung von bisherigen, ,eigenen' Geschlechterverhältnissen zu verstehen, ohne sich dabei jedoch anderen kollektiven Bezügen hinzuwenden. Anders hingegen verhält es sich bei Nicole und Carlos. Diese sind an der Realisierung einer individualisierten Geschlechterbeziehung interessiert, die hinter die Errungenschaften der Frauenbewegung zurückfällt. Nicole möchte 
aber nicht nur eine traditionelle Geschlechterbeziehung leben, sie erwartet in dieser Beziehung auch Eigenschaften ihres Mannes, die sie in Deutschland nicht mehr findet: Höflichkeitsformen, „diese Kavalierseigenschaften“ (Nicole 38/23) insgesamt, sind Teil des ,Gesamtpaketes“, welches sie mit Carlos zu realisieren versucht. Dies ist eine Form der Kulturalisierung die zwar einerseits dem anderen Handlungsautonomie abspricht, andererseits aber durchaus nützlich für die Beziehung sein kann. Handlungsautonomie spricht sie dem anderen insofern ab, als dass Carlos" Verhaltensweisen unter der ,Brille der Kultur' betrachtet werden und er sich auch analog zu solchen Erwartungen verhalten sollte. Das ,typisch Mexikanische“ wird so zum Maßstab. Nützlich wird es dann, wenn damit, wie bei Nicole, Imaginationen über das andere Land und dessen Bewohner verknüpft sind, die als positives Gegenbild gegenüber Deutschland verstanden werden. Die Gemeinsamkeit liegt dann in der Abgrenzung zu Deutschland und der Hinwendung zum anderen Land, Individualisierung findet eben durch die Betonung kollektiver Zugehörigkeiten statt. Ein solcher Prozess macht auf eine weitere Dimension von Individualisierung aufmerksam: Sie überschreitet nationale Grenzen. Die (emotionale und symbolische) Hinwendung zu Mexiko ermöglicht es, sich mit der ungewollten Situation in Deutschland zu arrangieren, grenzüberschreitende Beziehungen ermöglichen auch eine solche Form des grenzüberschreitenden Bezuges.

Das dritte Paar, Maria und Pablo, unterscheidet sich hinsichtlich der Instrumentalisierung natio-ethno-kultureller Zugehörigkeiten (vgl. Waldis 2001: 148) und hinsichtlich des biographischen Lernprozesses. Anders als bei den beiden vorhergehenden Paaren werden hier nicht kulturelle Zuschreibungen genutzt, um Geschlechtergrenzen zu erweitern. Vielmehr sind für beide geschlechtsspezifische Charaktereigenschaften des/der jeweils anderen Ausdruck der spezifischen Sozialisation des Herkunftslandes und als solche auch Teil des ,kulturellen Rucksacks“, den beide mit in die Beziehung bringen. So sind sie selbstverständlicher Teil der Beziehung. Der interkulturellen Kompetenz Ausdruck zu verleihen und sich dadurch von mononationalen Gesellschaftskonstruktionen zu distanzieren, ist Teil ihres Paarhorizonts. Dies erfordert, wie die Aushandlungen über eine mögliche Heirat gezeigt haben, eine klare Festlegung kultureller Grenzen. Nicht die Individualisierung durch den Verweis auf mögliche andere Geschlechterbeziehungen steht insofern im Vordergrund, sondern die ,Multikulturalisierung ' der eigenen Biographie und der Entwurf einer transnationalen Biographie, die Irritationen als kulturelle Irritationen wahrnimmt und versucht, mit einem (gleichberechtigten) Nebeneinander von kulturellen Identitäten Diffe- 
renzen zu integrieren. ${ }^{3}$ Wie in der Interpretation gezeigt, entstehen aus einer solchen multikulturellen Perspektive bestimmte Schwierigkeiten:

„Allgemein kann der Begriff Multikulturalität dahingehend problematisiert werden, dass mit ihm eine Festschreibung und ein Starkmachen von Unterschieden - etwa auf der Ebene von Nation oder Ethnie - betrieben wird“ (Mecheril 2000a: 1f.).

Um eine multikulturelle Beziehung leben zu können, müssen die Unterschiede betont werden, Grenzen werden so festgeschrieben. Gleichwohl eröffnet diese Festschreibung für Maria und Pablo auch Möglichkeiten, sie engt Handlungsmöglichkeiten nicht nur ein, sondern erweitert sie auch. Wenn die gleichberechtigte Koexistenz gelingt, eröffnet sich die Perspektive eines reflexiven Miteinanders, in dem sich mit verschiedenen Bezugshorizonten auseinandergesetzt werden kann. Dass dies in der Biographiegestaltung von Maria und Pablo gelingt, liegt in der transnationalen Ausrichtung, die die Gleichbehandlung beider kultureller Identitäten und Herkünfte zumindest diskursiv sichert. Beide können so an ihre interkulturellen Kompetenzen anknüpfen. Durch die Planung des Lebens in beiden Herkunftsländern ist für sie nicht nur die symbolische, sondern auch die reale Notwendigkeit gegeben, beiden Kulturen genügend Raum zu geben und keine zugunsten der einen, im Alltag präsenteren, zu vernachlässigen. Die Perspektive einer multikulturellen Biographieausrichtung ermöglicht also die Fortführung des Selbstverständnisses der interkulturellen Kompetenz. Anders aber als in der Perspektive einer reflexiven Multikulturalität werden von Maria und Pablo in den Interviews vor allem Irritationen benannt, die aber nicht als bedrohlich wahrgenommen werden, sondern als kulturell gekennzeichnet und damit von der Notwendigkeit der Auseinandersetzung befreit werden. ${ }^{4}$

3 Interkulturell und Multikulturell werden hier mit zwei unterschiedlichen Bedeutungen genutzt: Interkulturell bezieht sich auf das Interesse von Maria und Pablo an anderen Kulturen und ihrem Verständnis von sich als Personen, die mit anderen Kulturen gut umgehen können, der Fokus liegt also auf der Begegnung der Kulturen. Multikulturell hingegen bezieht sich auf den Biographieentwurf der beiden, beider kultureller Herkunft zu respektieren und ihnen Platz zu verschaffen im Sinne eines Miteinanders dieser Kulturen. $\mathrm{Zu}$ den Unterschieden und der Entwicklung eines Konzepts von Transkulturalität vgl. auch Welsch 1997.

4 „In multikulturellen Gesellschaften bedeutet die gleichberechtigte Koexistenz der Lebensformen für jeden Bürger eine gesicherte Chance, ungekränkt in einer kulturellen Herkunftswelt aufzuwachsen und seine Kinder darin aufwachsen zu lassen, die Chance, sich mit dieser Kultur - wie mit jeder anderen - auseinanderzusetzen, sie konventionell fortzusetzen oder sie zu transformieren, auch die Chance, sich von ihren Imperativen gleich- 
Der Beginn der Beziehung ist also bei allen Paaren durch kreative Neuproduktionen gekennzeichnet. Das zentrale lebensgeschichtliche Ereignis der Migration und der binationalen Partnerschaft bietet Anlass zur Reflexion über bisherige Erfahrungen und die Möglichkeit, neue Wege einzuschlagen. Die Beziehung wird so zur biographischen Ressource, die paarspezifisch eingesetzt und genutzt werden kann. Insgesamt ist diese erste Analysedimension durch eine stärkere Selbstbestimmtheit gekennzeichnet, die Entwicklung des Paarhorizonts kann auch entgegen gesellschaftlicher Anforderungen stattfinden. In der zweiten Analysedimension, die der thematisch organisierten Bearbeitungsfelder, ist eher die Bearbeitung ,fremdbestimmter' Anforderungen, der Umgang mit gesellschaftlichen und sozialen Irritationen wie den Reaktionen des Freundeskreises und der Verwandtschaft oder mit der Arbeitslosigkeit des Mannes, notwendig. Diese Bearbeitungen lassen sich bei allen drei Paaren als Konkretisierung einerseits und Modifikationen andererseits lesen. Bei der Art der Bearbeitung finden sich die Unterschiede zwischen Lern- und Bildungsprozessen wieder. Es lassen sich eher Kontinuitätslinien analysieren. Qualitative Sprünge und grundlegende Wandlungen des Selbst- und Weltverhältnisses, wie sie als Voraussetzungen von Bildungsprozessen verstanden werden, ließen sich in den Interpretationen nicht finden. Die Formen biographischen Lernens, die sich analysieren ließen, sind dementsprechend Entwicklungsprozesse, die bestimmte Konstruktionen verstärken und sie nicht grundlegend in Frage stellen. Für alle drei Paare gilt, dass sie mit gesellschaftlichen und sozialen (vermittelt durch ihr soziales Umfeld, die Verwandtschaft etc.) Anforderungen und Korrekturversuchen konfrontiert werden, die ihnen eine Neubewertung der Konstellation nahe legen. Für alle drei gilt aber auch, dass sie das jeweilige Thema der Beziehung, welches es zu bearbeiten oder $\mathrm{zu}$ verteidigen gilt, weiter verfolgen und versuchen es zu realisieren. Biographisches Lernen meint also bei diesen Paaren Kontinuitätswahrung unter den Vorzeichen einer hindernden Umwelt. Spannend zu beobachten ist vor allem, wie und ob der Versuch gelingt, trotz aller Widrigkeiten eine eigenständige Beziehungs- und Biographiegestaltung zu realisieren.

Für Susanne und Rachid endet die Realisierung einer eigenen Geschlechterbeziehung erst einmal in der Zeit, in der sie nach Deutschland und in Susannes Herkunftsort (zurück-)ziehen. Die Auseinandersetzungen sowohl mit Susannes eigenen Begrenzungen als auch mit sozialen Anforderungen zeigen deutlich die Grenzen der Gestaltungsfreiheit auf,

gültig abzuwenden oder selbstkritisch loszusagen, um fortan mit dem Stachel eines bewusst vollzogenen Traditionsbruchs oder gar mit gespaltener Identität zu leben“ (Habermas 1993: 175). 
soziale Strukturen in Form der persönlichen Involviertheit in ihre bisherigen Lebenswelten, in die sie zurückkehren will, schränken ein. Im Konflikt zwischen Anpassung und Auflehnung wird in einer Schlüsselszene in Susannes biographischer Erzählung sichtbar, dass sie sich nicht gegen eine der Grundregeln der Gesellschaft stellen kann. Sie kann ihre Familie, also ihren Mann und ihr Kind, nicht verlassen. Susanne und Rachid sind, wie sie konstatiert, nicht „so frei“ (Susanne 18/2), wie sie selber dachten. Die Form biographischen Lernens bewegt sich daher auch in dem Spannungsfeld zwischen Anpassung an und Veränderung bestehende(r) Verhältnisse. Trotz der stattfindenden Integrationsbemühungen, die auf ein dual earner Modell hinauslaufen, bleibt die Beziehung an sich Ausdruck der Distanzierung von ihren bisherigen sozialen und kulturellen Bezugssystemen. Es ändert sich aber die Richtung der Gesellschaftskritik: War sie bislang nach außen gerichtet, gegen Deutschland (durch Susanne) und gegen Iran (durch Rachid) und wurde durch die Beziehungsführung der Emanzipation und einer geschlechtersensiblen Haltung Ausdruck verliehen, ist in der Beziehungspraxis zum Zeitpunkt des Interviews eher eine Angleichung an herkömmliche Arbeitsteilungen festzustellen. So wird die binationale Beziehung selbst, weniger die Praxis dieser Beziehung, zum Teil und zum Ausdruck der gesellschaftskritischen Distanz. Gerade für Susanne, die sich zu Beginn der Beziehung deutlich gegen eine traditionelle Arbeitsteilung gestellt hat und in der Beziehung zu Rachid eine Möglichkeit sah, hierarchische Rollen umzukehren, wird nicht mehr die faktische Arbeitsteilung relevant, sondern die Möglichkeit „das Ureigene“ (Susanne 42/25) herauszufinden und zu realisieren. Diese Verlagerung und tendenzielle DeDramatisierung kultureller Zuschreibungen bedeutet nicht unbedingt, dass die Kategorie ihre funktionale Bedeutung vollständig verliert. Sie wird jedoch vor allem gegenüber Dritten betont, um zum Beispiel den Schwiegereltern die für sie ungewohnte Form des Getrenntschlafens des Paares plausibel zu machen. Einen deutlichen Bedeutungsverlust können Zugehörigkeitszuschreibungen auch deswegen nicht erfahren, da Rachid in Deutschland sichtbar fremd ist. Wenn er sich am Telefon nur mit dem deutschen Teil seines Nachnamens meldet, erlebt er bei einer folgenden persönlichen Begegnung regelmäßig Erstaunen oder sogar Befremden: Sein Äußeres stimmt nicht mit den Erwartungen des Gegenübers überein, die Erfahrungen des körperlichen ,Anderssein“ bleiben Teil seines Erfahrungshorizontes und machen ihm so eine eindeutige Zuordnung zu Deutschland als Bezugshorizont zumindest in dieser Hinsicht unmöglich.

Für Nicole und Carlos endet die Erwartung einer Beziehungsgestaltung nach ihren Vorstellungen ebenfalls mit der Migration nach 
Deutschland. Carlos findet keine Arbeitsstelle, und beide können so eine traditionelle Geschlechterbeziehung erst einmal nicht realisieren. Auch bei ihnen irritieren sowohl gesellschaftliche Strukturen wie die Exklusionsstrategien des deutschen Arbeitsmarkts als auch der soziale Nahraum insbesondere in Gestalt der Freund(inn)e(n) die Beziehungsgestaltung. Die Bearbeitungsstrategie ist jedoch eine andere als bei Susanne und Rachid: Was bei diesen als aktive Auseinandersetzung mit gesellschaftlichen und eigenen Grenzen und als Versuch der Aushandlung zu bezeichnen ist, findet sich bei Nicole und Carlos als Rückzug ins Private. Freundinnen, die Nicole in der Zeit der Fernbeziehung nicht ernst nehmen und sie nicht unterstützen, oder Freunde, die sich über die Höflichkeitsformen von Carlos lustig machen, werden aufgegeben. Die finanziellen Schwierigkeiten werden nicht als Anlass genommen, um über die aktuelle Situation und/oder Wünsche zu reflektieren, vielmehr wird, mit stillschweigendem Wissen beider Seiten, die Situation verschwiegen damit es, wie es Nicole formuliert, „nicht auffällt" und da Carlos sich sonst „Vorwürfe machen“ müsste (Nicole 31/15ff). Ziel der beiden bleibt die Realisierung einer klassischen Geschlechterbeziehung und die vollständige und geglückte Einmündung Carlos' am ersten Arbeitsmarkt. Dies kann, muss aber nicht, der deutsche Arbeitsmarkt sein. Entscheidend für die Bedeutung natio-ethno-kultureller Zugehörigkeiten ist, dass eine Zugehörigkeitsverschiebung möglich ist, Mexiko wird für beide zum (imaginären) Handlungs- und Gegenhorizont. Während natioethno-kulturelle Zugehörigkeit bei Susanne und Rachid entdramatisiert wird, fungiert sie bei Nicole und Carlos als Lösung aus dem Dilemma und gewinnt so an Bedeutung für die Beziehungspraxis. Biographisches Lernen ist hier auf die Bestätigung und Verfestigung bestehender Geschlechterbeziehungen gerichtet. Zugleich verfestigen sich in diesem Prozess die Grenzen zwischen der Beziehung und dem sozialen Umfeld, ,innen' und ,außen' werden deutlich getrennt und die Beziehung wird gestärkt. Dies geht einher mit der Aufgabe von sozialen Bezügen die diese Konstruktionen irritieren oder in Frage stellen. ${ }^{5}$

Bei Pablo und Maria unterscheidet sich die Konstruktion des Paarhorizonts deutlich von der Konstruktion der anderen beiden Paare. Für sie steht die Transmigration, das Leben in zwei Orten, als wesentliche Ori-

5 Dieser Prozess wird durch zweierlei verstärkt, so eine Vermutung: zum einen durch die Wirkung der gatekeeper-Funktion Nicoles. Nicole ist für das Nahebringen Deutschlands und der ,deutschen Kultur' zuständig. Dies korrespondiert zum anderen nun mit Nicoles Verständnis von care-work (vgl. Feministische Studien 2000). Sie ist für den emotionalen Zusammenhalt in der Beziehung zuständig, für Carlos soll die Situation nicht noch schwerer werden, als sie ohnehin schon ist. 
entierung für die Beziehungsgestaltung von vornherein fest, Auseinandersetzungen über den aktuellen und zukünftigen Wohnort werden daher auch schon zu dem Zeitpunkt virulent, an dem die Entscheidung über eine gemeinsame Zukunft getroffen wird. Das female breadwinner Modell ist bei ihnen in eine längerfristige Planung eingebunden und trifft sie nicht, wie Nicole und Carlos, überraschend. Dennoch ist die Realisierung nicht so einfach, wie vielleicht erhofft. Anforderungen werden insbesondere durch Marias Mutter gestellt, die auf eine traditionelle Arbeitsteilung beharrt, zudem erlebt Pablo, dass seine Zustimmung zur Arbeitsteilung doch nicht so einfach ist wie erwartet. Die langfristige Planung einer transnationalen Biographie fungiert in diesem Fall jedoch als Möglichkeit, im Sinne eines ,größeren Plans‘ die aktuellen Schwierigkeiten zu relativieren und die Situation auch für Pablo aushaltbar zu machen. Da die (Re-) Migration nach Mexiko geplant ist und zum Zeitpunkt des Interviews kurz bevor steht, kann die Arbeitslosigkeit bzw. die Berufstätigkeit ohne Bezahlung weniger als grundlegendes Problem bzw. als grundlegende Herausforderung als vielmehr vorübergehende Schwierigkeit verstanden werden. Auch die rassistischen Eingriffe, die Kennzeichnung als Ausländer, als Nicht-Zugehöriger, die Pablo regelmäßig erfährt, bestätigen ihn in der Re-Migration. In diesem Sinne ist auch seine Äußerung „Ich bin immer Ausländer“ (Pablo 42/17) zu verstehen. Ankommen kann er hier nicht, insofern ist es für ihn nur logisch dass Mexiko praktisch und symbolisch Teil des Handlungshorizonts bleibt, Weiterqualifikationen wie der Hausbau für die Schwester sind daher auch sinnvolle Investitionen. Innerhalb dieses Plans gewinnen die Kinder als Nutznießer einer binationalen Beziehung an Bedeutung und treten in den Vordergrund. Die jeweils andere Kultur als Bereicherung und als (positive) Ressource soll insbesondere den Kindern zur Verfügung stehen. Für die Kinder kann Pablo es akzeptieren, keiner bezahlten Arbeit nachzugehen, für die Kinder kann Maria situative Inkompatibilitäten aufgrund kulturell verschiedener Sozialisationen biographisch integrieren. Kulturelle Unterschiede, die auf unterschiedliche Sozialisationserfahrungen zurückzuführen und Teil der Identität sind und somit nur schwer veränderbar erscheinen, werden so zum grundlegenden Bestandteil der Beziehung, die Formel des ,Lebens in und zwischen zwei Kulturen' scheint auf dieses Paar in seiner Konsequenz zuzutreffen und bezeichnet auch die Form biographischen Lernens im Sinne der Transmigrationsperspektive als Integrationsmöglichkeit. Das permanente ,Mitlaufen“ des Irritationspotentials erhöht die Reflexionsnotwendigkeit des Paares, Reflexion führt aber in diesem Fall nicht zu einer Infragestellung von Grenzen, sondern zu einer Selbstvergewisserung der interkulturellen Kompetenz des Paares. 
Als Ergebnis dieser ersten Diskussion lässt sich festhalten, dass sich in Biographien binationaler Paare soziale Wirklichkeiten wieder finden lassen, die sich insbesondere in den Augen der Paare abseits klassischer Biographiemodelle bewegen. Das ,Eigene‘ zu entwickeln und dieses auch im Umgang mit gesellschaftlichen Herausforderungen beizubehalten scheint insofern in einer binationalen Beziehung (leichter?) möglich als in einer mononationalen. Die Modifikationen und (Re-)Konstruktionen von gender und natio-ethno-kultureller Zugehörigkeit, die dabei zu beobachten sind, zeigen Grenzverschiebungen und Wege zur Realisierung von eigenen Lebensmodellen auf. In diesem Prozess können natio-ethno-kulturelle Zugehörigkeiten durchaus flexibel eingesetzt und variabel instrumentalisiert werden. Differenzen sind nicht naturgegeben und vorgängig, sondern entstehen als kulturelle Zuschreibungen in den Aushandlungen und biographischen Bearbeitungen der Paare. Sie werden situationsbezogen herangezogen und fungieren zum Beispiel als Legitimation, sowohl innerhalb der Beziehung als auch gegenüber Dritten oder auch als Handlungsorientierung im Sinne einer impliziten Erwartungshaltung. Dies kann, wie bei Maria und Pablo, zu Problemen führen, wenn Maria ihm machistisches Verhalten unterstellt. Das kann aber auch, wie bei Nicole und Carlos, zu einem stillschweigenden Agreement und so zu einer Entlastung des alltäglichen Handelns führen. Das Argument der kulturellen Differenz wird insofern in der Regel dazu verwendet, den Alltag interpretierbar und bewältigbar zu machen (vgl. Waldis 2003: 98). Trotz oder vielleicht gerade aufgrund des Wissens um die Verschiebbarkeit kultureller Grenzen werden Zugehörigkeiten und Differenzen als Erklärungsmuster herangezogen. Entscheidend für die Bedeutungsvariationen, die natio-ethno-kulturelle Zugehörigkeit in den Biographien zugesprochen bekommt, ist dabei die Konstruktion des Paarhorizonts. Gemeinsam ist den Paaren, dass sie in der Beziehung die Möglichkeit sehen, an eigene biographische Entwürfe anzuknüpfen und bestimmte Modelle oder auch, wie bei Maria und Pablo, Selbstbilder zu realisieren und zu verstärken, die sie in einer mononationalen Beziehung nicht hätten realisieren können. Aus den sich entwickelnden Paarhorizonten ergeben sich, wie die Interpretationen gezeigt haben, die weiteren Bearbeitungsfelder. In der Art und Weise der Bearbeitung und der Form, die die biographischen Lernprozesse annehmen, lässt sich auch zeigen, dass der Grad der Offenheit bzw. der Geschlossenheit des Paarhorizonts eine wichtige Rolle spielt. Bei Nicole und Carlos lässt sich von einem im vergleichsweise hohen Maße geschlossenen Paarhorizont sprechen. Er betont die Beziehung als Paar, beide beziehen sich in ihren Konstruktionen stark auf den bzw. die jeweils andere(n). Hier scheint es, so die Ergebnisse der Interpretation, naheliegend $\mathrm{zu}$ sein, diese Abgrenzung 
weiter zu verstärken, gesellschaftliche Irritationen oder soziale Reflexionsanstöße werden nicht als solche aufgenommen, sondern abgewehrt. In einer anderen Konstruktion des Paarhorizonts, wie bei Susanne und Rachid, ist die Beziehung von vornherein stärker auf die Interessensrealisierung der beiden Individuen ausgerichtet. Beider Konstruktionen legen den Schwerpunkt eher auf die Weiterführung und -entwicklung der individuellen Biographien in der Beziehung als auf die Beziehung als solche. In einem solchen Modell scheint es mehr Möglichkeiten zu geben, soziale Anforderungen in den Paarhorizont aufzunehmen und Selbst- und Wirklichkeitskonstruktionen in Frage zu stellen und gegebenenfalls zu verändern, als in Paarbiographien, die stark aufeinander gerichtet sind. Den Paaren gelingt also die Durchsetzung eigener Lebensmodelle. Die Formen biographischen Lernens spiegeln zwar die Widerstände wieder, denen sie begegnen, sie zeigen aber vor allem die Hartnäckigkeit, mit der die eigenen Ziele verfolgt werden. Die Erweiterung der Möglichkeiten und Handlungsspielräume, die die binationale Beziehung mit sich bringt, wird also aufrechterhalten und funktioniert auch über den Verlauf der Beziehung hinweg.

\section{Zum Verhältnis von ,Kultur' und ,Geschlecht" als Ressourcen der Biographiegestaltung}

Gender und natio-ethno-kulturelle Zugehörigkeit können als Ressource der Biographiekonstruktion und -gestaltung gesehen werden, beide wirken sowohl hinsichtlich der Ermöglichung als auch hinsichtlich der Begrenzung. Dabei handelt es sich jedoch nicht um ein additives Verhältnis, kulturelle Zuschreibungen verstärken nicht zwangsläufig geschlechtsspezifische Ungleichheiten. Vielmehr entwickeln beide Differenzkategorien ihr spezifisches Verhältnis in einer spezifischen Biographie. Die Entwicklung eines solchen Verhältnisses wurde in der vorliegenden Arbeit als Form biographischen Lernens verstanden. In den hier analysierten Biographien ließen sich zwei Grundstrukturen des Verhältnisses finden: Zum einen dient natio-ethno-kulturelle Zugehörigkeit als Handlungsfeld für gender-Konstruktionen, ,kulturelle Differenz' wird hier instrumentalisiert, wie Waldis sagt, um Möglichkeiten der eigenwilligen Beziehungsgestaltung zu erweitern. Dies gilt für Susanne und Rachid ebenso wie für Nicole und Carlos, wenn auch mit ganz anderen Intentionen und ganz anderen Folgen. Zum anderen, wie bei Maria und Pablo, erscheint gender als Ausdruck natio-ethno-kultureller Zugehörigkeit. Nicht die Überschreitung oder Neudefinition von Geschlechtergrenzen steht bei einer solchen Konzeption im Vordergrund, weniger al- 
so ein instrumenteller Zugang zu ,kultureller Differenz', vielmehr wird natio-ethno-kulturelle Zugehörigkeit als Teil der Identität verstanden, die eben auch spezifische Verständnisse von Geschlechterbeziehungen mit sich bringt.

Das female breadwinner Modell stellt für alle Paare eine Herausforderung dar. Auch wenn es nur für Nicole und Carlos wirklich unerwartet kommt, ist die Umkehrung der klassischen geschlechtsspezifischen Arbeitsteilung nicht unproblematisch. Migrationsprozesse von Familien können leicht, wie z.B. Sakamoto anhand von Migrationen japanischer Akademikerfamilien in die USA zeigt, zur Verfestigung der klassischen Zuständigkeiten in der privaten und der öffentlichen Sphäre führen (vgl. Sakamoto 2006). Einen solchen Verlauf können die Biographien meines Samples auch deshalb erst mal nicht nehmen, da die Sphärenzuständigkeit ,verstauscht‘ ist. Vielmehr gibt es die Möglichkeit und die Notwendigkeit, Zuständigkeiten neu $\mathrm{zu}$ verteilen. Solche geschlechtsspezifischen Zuständigkeiten und Zuschreibungen werden, das ist ein weiteres Ergebnis meiner Arbeit, bei den ersten beiden Paaren mit Hilfe von erklärungs- und handlungsorientierenden Mustern entwickelt und ausgehandelt, die auf ,kulturelle Differenz' zurückgreifen. Bei den ersten beiden Paaren fungiert natio-ethno-kulturelle Zugehörigkeit also als Ressource im produktiven Sinn. Sie kann als Möglichkeit des Spielens mit Zuschreibungen und Zugehörigkeiten gesehen werden, die Wege der eigenständigen Biographiegestaltung eröffnet und Distanzierungen von Teilsystemen und Strukturen des Herkunftslandes ermöglicht. Kulturelle Differenz erscheint so einerseits als flexibles und pragmatisch nutzbares Interpretationsmuster. Sie kann in ihrer Bedeutung entdramatisiert werden, sie kann aber auch als Entscheidungshilfe genutzt und damit in den Vordergrund geschoben werden. Andererseits ist damit ein weiteres Merkmal kultureller Differenz angesprochen, welches sich in den Analysen beobachten ließ. So fällt auf, dass Verfestigungen von kulturellen Zuschreibungen und Re-Ethnisierungsprozesse, wie sie insbesondere bei Nicole und Carlos zu beobachten sind, zu Handlungserweiterungen hinsichtlich geschlechtsspezifischer Lebensmodelle führt. Gerade Kulturalisierungen, wie sie bei Nicole und Carlos zu finden sind, ermöglichen also auf einem anderen Gebiet Grenzerweiterungen. Solche individuellen Geschlechterbeziehungen sind allerdings auf traditionelle Geschlechterbeziehungen gerichtet, es geht also nicht um eine emanzipative oder auf die Weiterentwicklung zu einer demokratischen oder gleichberechtigten Beziehung gerichtete Konstruktion, sondern um die Rückkehr zu Sicherheit versprechenden Mustern der geschlechtlichen Arbeitsteilung und der Beziehung zwischen den Geschlechtern. Solche Formen von 
Geschlechterbeziehungen sind für Nicole wesentlich durch Aufmerksamkeitsrituale gekennzeichnet, die Frauen in gewisser Weise Schutz bieten. Solche Höflichkeitsrituale sind, wie auch bei Maria und Pablo beschrieben, zugleich eine Verständigung darüber, Frauen als „wertvoll, dekorativ und zerbrechlich" (Goffman 1994: 123) anzusehen. Damit drückt sich ein spezifisches Geschlechterverhältnis auch in der Form des körperlichen Umgangs miteinander aus, eine Form die stark mit kulturellen Zuschreibungen verbunden und für Nicole (nur noch) bei mexikanischen Männern zu finden ist.

Eine solche Verstärkung der Retraditionalisierung und Re-Ethnisierung lässt sich bei Susanne und Rachid nicht beobachten, wohl aber schwächt sich auch hier der emanzipative Anspruch des Paares durch die Konfrontation mit gesellschaftlichen Strukturen und eigenen Widersprüchen ab. Anders aber als bei Nicole und Carlos findet sich bei Susanne und Rachid eher so etwas wie eine entkernte oder strukturelle Kulturalisierung: Kulturalisierung, weil der/die jeweils Andere als kulturell Fremder bzw. Anderer relevant wird. Entkernt, weil nicht, wie bei Nicole, konkrete Imaginationen hinsichtlich mexikanischer Männer relevant sind, sondern eher die Positionen eines Nicht-Wissenden (Rachid) und einer Nicht-Iranerin (Susanne). Diese entkernte Kulturalisierung steht ebenfalls ,in Diensten“ der Realisierung einer individuellen Geschlechterbeziehung, auch hier ermöglicht die Verfestigung der einen Kategorie die Pluralisierung der anderen. Solche Interpretationsergebnisse verfeinern den Blick auf Kulturalisierungsprozesse als reine Verhinderungsprozesse und bestätigen so einerseits die These der Flexibilität kultureller Zuschreibungen. Andererseits zeigt es eben auch die ContainerFunktion von Kultur und kulturellen Zuschreibungen: Alles kann als kulturell relevant verstanden werden. Dem Grundparadoxon von Kulturalisierungsprozessen entgeht also auch eine solche Handlungsorientierung nicht. In diesem Spannungsfeld zwischen kreativer Neuordnung und Omnipotenz können sich Susanne und Rachid die meiste Zeit sehr selbstbestimmt bewegen, gleichwohl wird Fremdheit auch durch ihr Handeln festgeschrieben: Wenn sie ihre getrennten Schlafzimmer gegenüber Susannes Eltern mit Rachids ,Anderssein“ erklären, so muss Rachid damit rechnen, damit einerseits Rechtfertigungen aus dem Weg gehen zu können, andererseits wird so auch eine eindeutige und einwertige Zugehörigkeit verhindert.

Bei Maria und Pablo ist das Verhältnis zwischen ,Kultur' und ,Geschlecht' ein anderes. Bei ihnen handelt es sich nicht um Kulturalisierung (entkernt oder nicht entkernt) als Möglichkeit der Erweiterung von Geschlechterordnungen. Vielmehr stellt die binationale Beziehung eine Ausdrucksform für ihre multikulturelle Orientierung dar. Geschlechter- 
ordnungen und deren Entwicklung und Veränderung spielen hier nicht die dominante Rolle wie bei den beiden anderen Paaren. Zwar ist auch bei den beiden anderen Paaren gender ein Teil und damit auch eine Form des Ausdrucks kultureller Differenzen, allerdings ist der Zusammenhang ein anderer. Bei Maria und Pablo werden Geschlechterbeziehungen nicht produktiv, unter Rückgriff auf ,kulturelle Differenz' verhandelt und/oder entwickelt. Vielmehr werden sie mit Rückgriff auf eben diese ,kulturelle Differenz' erklärt und zu einer nicht verhandelbaren Charaktereigenschaft gemacht. ,Kulturelle Differenz' dient als Erklärungsmodell, welches ermöglicht, Differenzen zu überwinden oder, wenn das nicht möglich ist, sie auszuhalten, geschlechtsspezifische $\mathrm{Zu}-$ schreibungen sind ein Teil dieser kulturellen Differenz. Die transnationale Biographieausrichtung führt dabei jedoch nicht zu einer reflexiven Grundhaltung. Vielmehr begrenzt interessanterweise gerade die Transmigration das Infragestellen von Selbstverständlichkeiten. Die Möglichkeit, sich an verschiedenen Bezugssystemen gleichzeitig zu orientieren, bedeutet zum Beispiel, dass Pablo an den von ihm geschätzten deutschen Gesundheitssystem partizipieren, die Erziehung seiner Kinder aber an seiner eigenen Erziehung orientieren kann. Pries spricht hierbei von „segmentierten Identitäten“ (ebd. 1996: 469), bezogen auf biographische Lern- und Bildungsprozesse ist die transnationale Biographieausrichtung eine Möglichkeit, in der kein Bildungsprozess angestoßen wird, sondern nur kleinere Modifikationen und Erweiterungen in den Selbst- und Wirklichkeitskonstruktionen vorgenommen werden. Wenn transnationale soziale Räume aufgespannt werden, ergibt sich also hinsichtlich der Entstehung von heterogenen und hybriden Zugehörigkeitsformen ein widersprüchliches Bild. Einerseits werden so nationale Grenzen aufgeweicht oder ad absurdum geführt, Verortungen (und, wie sich gezeigt hat, Individualisierungsprozesse), machen nicht an Staatsgrenzen halt. ${ }^{6}$ Andererseits können sich so individuelle Positionierungen verstärken. Die mexikanische Identität muss nicht in Frage gestellt werden, da sie vergleichsweise unabhängig von der Partizipation an bestimmten Systemen ist.

6 Ein anderes Beispiel zeigt Goeke 2006, der Bildungsbiographien von kroatisch-deutschen Jugendlichen analysiert. Staatsgrenzen und dadurch definierte Unterschiede von Bildungssystemen können sogar Voraussetzung für transnationale Biographien sein, in dem der Abschluss im einem Land weiterführende Bildung im anderen Land ermöglicht. Transnationale Biographien nutzen nationale Grenzen für ihre diese überspannenden Bewegungen. 



\section{Binationale PaARE ALS ,PROTOTYPEN DER MOdERne'?}

Binationale Paare setzen sich mit unterschiedlichen, sich zum Teil widersprechenden oder auch ergänzenden Bezugssystemen auseinander. In ihrer Biographiegestaltung müssen sie mit den umgehen, was moderne Gesellschaften ausmacht: wandelnde Lebensräume, divergierende Bezugssysteme, hohe Ambiguitäten, unsichere Biographieverläufe bei gleichzeitiger Aufgabe der Individuen, die Sicherung und Kontinuität der Biographiegestaltung selbst zu übernehmen. Aus den vorgestellten Gesellschaftsanalysen lässt sich eine Art Anforderungsprofil an Subjekte erstellen, welches sich mit ,Differenzkompetenz' bezeichnen ließe. Über eine solche Differenzkompetenz verfügen binationale Paare. Macht es sie damit zu ,Prototypen der (Post-)Moderne"? Sind sie also in besonderer Weise für die Anforderungen gerüstet, und können sie in insofern als Vorbilder gelten, wie die Herausforderungen einer Migrationsgesellschaft zu bewältigen sind? Sind die Erwartungen, die von verschiedenen Seiten an sie gestellt werden, also berechtigt? Sind sie nicht nur diejenigen, die sich aufgrund ihrer Lebenssituation Differenzkompetenz aneignen müssen, sondern sind sie auch diejenigen, die eine solche Differenzkompetenz nutzen, um, wie es Straub formuliert, nicht nur „die Selbstund Weltauffassungen, Lebensformen und Praktiken anderer Menschen in ihrer Eigenart erkennen und anerkennen zu können", sondern auch den „Blick für das Andere oder Fremde im Eigenen“ zu schärfen (vgl. Straub 1999: 194). Sind sie also diejenigen, für die die Notwendigkeit und die Möglichkeit biographischer Bildungsprozesse gegeben ist? Einerseits und vorschnell müssten diese Fragen nach der Interpretation der Interviews mit ,Nein' beantwortet werden. Pluralisierung wird, wie bei Nicole und Carlos,mit Eindeutigkeiten beantwortet, steigende Differenzerfahrung mit Verfestigungen zentraler Kategorien, Re-Ethnisierungsprozesse lassen sich ebenso beobachten wie Festschreibungen kulturel- 
ler Differenzen. Gleichwohl ergibt sich bei genauerer Betrachtung ein durchaus differenzierteres Bild.

Binationale Beziehungen, das hat sich deutlich in den Interpretationen gezeigt, lassen sich als Möglichkeitsraum für Grenzerweiterungen, für die Realisierung individueller und von der Herkunftsgesellschaft sich distanzierender Lebensentwürfe verstehen (vgl. dazu auch Menz 2007). Die Paare sehen in der Beziehung Möglichkeiten, die sie, so ihre Einschätzung, in einer mononationalen Beziehung nicht hätten. Insofern findet sich hier eine Form der Pluralisierung von Lebens- und Beziehungsformen, die durch Migrationsprozesse erst ermöglicht wird, binationale Paare sind so wesentlicher Teil einer solchen Pluralisierung. Die neuen sozialen Wirklichkeiten, die ja Pries in Bezug auf transnationale soziale Räume sich entwickeln sieht, lassen sich auch in den Biographien der binationalen Paare wiederfinden. Scheibelhofer spricht in diesem Zusammenhang mit Bezug auf Müller-Schneider (1998) von innen gerichteter Modernisierung, die auf die ,positiven Selbstzustände der Subjekte" gerichtet ist (ebd. 2003: 185). Die Realisierung eines individuellen Lebensprojekts ist dabei zentral (vgl. ebd.: 186). Tatsächlich lässt sich in den von mir geführten Interviews so etwas wie eine nach außen gerichtete Modernisierung im Sinne von einer expliziten Auseinandersetzung mit gesellschaftlichen Strukturen oder Zwängen nur bei Susanne und Rachid finden, Susannes Modell der binationalen Beziehung ließe sich auch als politisches Statement lesen. Dennoch überwiegt bei allen Paaren die Realisierung des eigenen Lebensmodells und weniger die Veränderung gesellschaftlicher Zustände. Natio-ethno-kulturelle Zugehörigkeit erscheint in diesem Zusammenhang als zusätzliche Ressource, die einen Begründungs- und Handlungszusammenhang konstituiert und dabei dem Anschein der Vorgängigkeit entledigt wird. Ein solch kreativer, eigene Spielräume und Interpretationsmöglichkeiten entdeckender Umgang ließ sich als eine Form der Instrumentalisierung bezeichnen, die den Paaren nutzt bzw. von den Paaren genutzt wird. In der Beziehungskonstruktion können bislang nicht aufgegriffene Perspektiven der Biographiegestaltung realisiert, andere Sichtweisen entwickelt und neue Schwerpunkte gesetzt werden. Solchen Konstruktionsprozessen liegt das Motiv der Anschlussmöglichkeiten zugrunde, die Beziehung wird als folgerichtige Entscheidung in die Biographierekonstruktion eingefügt: „Egal, ob die Partnerwahl nun auf die Konditionierung durch das Herkunftsmilieu, auf eine zufällige Begegnung oder eine politische Position zurückgeführt wird, sie wird so in die Lebensgeschichte integriert, dass diese neu erfunden, neu gestaltet oder neu interpretiert wird“ (Waldis/Ossipow 2003: 393). Die Beziehung fungiert in diesem 
Sinne als Gelegenheitsstruktur, die genutzt wird: Dominante Geschlechterverhältnisse können in Frage gestellt werden, dem Selbstverständnis von kulturkompetenten Individuen kann ebenso Ausdruck verliehen werden wie der Sehnsucht nach dem Vertrauten im Fremden.

Was sich an Bezügen analysieren lässt, die in der Konstruktion Bedeutungszusammenhänge und Positionierungen erweitern, lässt sich mit Mecheril als natio-ethno-kulturelle (Mehrfach-)Zugehörigkeit bezeichnen: die Gleichzeitigkeit des Bezugs auf (mindestens) zwei Kontexte (Mecheril 2003: 26). Eine solche mehrfache Positionierung, die nationale Grenzen überschreitet und neue Sinnzusammenhänge entwickelt, schafft Lebenswirklichkeiten, die bisherige Migrationsmodelle nicht fassen konnten. Migration nach Deutschland bedeutet kein Entweder-oder, keine Alternativlosigkeit zwischen vollständiger Assimilation oder vollständiger Integrationsverweigerung. Vielmehr sind, wie bei Pablo, gleichzeitige Verortungen in mehreren Kontexten möglich: Das deutsche Gesundheits- und Bildungssystem ist für ihn ebenso handlungsrelevant wie das mexikanische Berufssystem. Einerseits also konnte in den Interviews die Schaffung von erheblichen Handlungsfreiräumen interpretiert werden. Auf der anderen Seite aber lässt sich mit Susanne formulieren, dass die Paare ,so frei nicht sind', wie sie glauben. Notwendig werdende Konzessionen entstehen zum einen aufgrund des schon erwähnten gesellschaftlichen und sozialen Umfeldes, sie entstehen aber auch aufgrund der „unbeabsichtigten Folgen“ (Giddens 1995: 63), die Menschen mit ihren intentionalen Handlungen produzieren und mit denen sie dann im weiteren biographischen Verlauf konfrontiert sind, die Handlungsmöglichkeiten einschränken und strukturieren. Dies muss nicht zwangsläufig in eine Krise führen. Maria und Pablo sind mit den Schwierigkeiten ihrer interkulturellen Beziehung konfrontiert, geben diese jedoch nicht auf, sondern können die situativ entstehenden Differenzen in ihr langfristiges Biographiemodell integrieren, welches die Gleichberechtigung beider kultureller Herkünfte betont.

Differenzerfahrungen wie die Bearbeitung des female breadwinner Modells und die Erwartungen des sozialen Nahraums an die Beziehungsführung werden sehr unterschiedlich gelöst. Die Art und Weise der Bearbeitung solcher strukturellen Zwänge erscheint insbesondere bei Nicole und Carlos problematisch. Der starke Bezug auf die Beziehung und auf Mexiko als Gegenhorizont, der zum Ausschluss von die Beziehung in Frage stellenden Freunden ist eine Antwortmöglichkeit auf gesellschaftliche Anforderungen und steigende Unsicherheiten. Hier findet sich also kein nach außen gerichteter, produktiver Umgang mit Differenzen. Vielmehr wird, in Zeiten der Krisen, die Kernfamilie als Rück- 
zugsort entdeckt. Die starke Abgrenzung zwischen Innen und Außen bleibt bestehen und verstärkt sich sogar noch, Bearbeitungen finden so auf der Ebene des Normalisierungsmanagements statt, indem Nicole Carlos den Geldbeutel unter dem Tisch zuschiebt, und nicht auf der Ebene der kritischen Auseinandersetzung mit eigenen Erwartungen und Plänen. Argumentativ ermöglicht wird solch eine Form der Privatisierung, die den Schwerpunkt der Biographiegestaltung auf die private Sphäre legt, durch Carlos mexikanische Herkunft. Sie bietet Anschlussund Begründungsmöglichkeiten für diese Schwerpunktsetzung, in einer mononationalen Beziehung wäre eine solche Bearbeitung nicht möglich. ${ }^{1}$ Ein solches Ergebnis modernisierter Biographien stimmt nachdenklich. Mehrdeutigkeiten werden durch Eindeutigkeiten aufgelöst, Irritationen durch Rückzug. Wenn, so scheint es, die Gestaltungsmöglichkeiten in der öffentlichen Sphäre als gering oder gar nicht vorhanden eingeschätzt werden, wenn mit den Irritationen also nicht lösungsorientiert umgegangen werden kann oder sie als nicht zu bewältigen angesehen werden, dann eröffnet die binationale Partnerschaft die Möglichkeit, andere Bezugssysteme zu entwickeln. Dies ist, wie deutlich geworden ist, auch eine Folge der geringeren Flexibilität des Paarhorizonts, der bei Nicole und Carlos von vornherein stark auf die Beziehung ausgerichtet ist. Das Mehr an Möglichkeiten eröffnet eben auch die Möglichkeit sich gegen bestimmte Entwicklungen zu stellen und sich seine „kleine Familie“ (Nicole 39/15) mit der Rückkehr zu traditionellen Geschlechterverhältnissen zu erhalten bzw. zu erkämpfen. Susanne und Rachid hingegen setzen sich von Beginn an mit dem ,Außen', gesellschaftlichen und sozialen Anforderungen, auseinander ebenso wie mit den eigenen Erwartungen und hinterfragen sie, wie Susanne, auch selbstkritisch: Die Erfahrungen der ,Heimatlosen“ in Indien bringt sie dazu, sich ihrer Herkunftsfamilie und ihrem Herkunftsort wieder anzunähern, ihre Erfahrung von sich in der Position als Alleinverdienerin bringt sie dazu, von ihrem ursprünglichen Modell Abstand zu nehmen und Möglichkeiten zur Erwerbsarbeit für Rachid zu suchen. Die eigene Position und die eigene Erwartung an sich und an die Beziehung kann in diesem Fall sehr viel flexibler reflektiert und gegebenenfalls auch in Frage gestellt werden.

Was lässt sich für ein Fazit ziehen? Lässt sich an Biographien binationaler Paare die Entstehung erweiterter Möglichkeiten beobachten, die durch die als zusätzliche Gestaltungsressource gesehene natio-ethnokulturelle Zugehörigkeit des und der jeweils Anderen eröffnet werden,

1 Wahrscheinlich lassen sich dort andere Formen des Rückzugs finden, für binationale Paare bieten aber natio-ethno-kulturelle Zugehörigkeiten eine geeignete Möglichkeit. 
entstehen so auch neue soziale Wirklichkeiten. Diese können in mehrfacher Hinsicht transnational sein: wie bei Maria und Pablo als Biographieentwurf einer Pendelmigration, oder wie bei Nicole und Carlos als Orientierung an Mexiko beim Leben in Deutschland. Ob solche Möglichkeiten zur Lebensgestaltung die Paare nun zu Prototypen der Moderne macht, kann einerseits - im Hinblick auf die zunehmende Wahlfreiheit und die Möglichkeiten von selbst gewählten Mehrfachzugehörigkeiten - mit ja beantwortet werden. Hier sind Lebensmodelle entstanden, die über mononationale Bezüge hinausgehen und Vervielfältigungen von Geschlechterbeziehungen ermöglichen. Andererseits ist aber die damit verbundene Hoffnung, binationale Paare seien sozusagen Vorreiter einer demokratischen und pluralisierungskompetenten Gesellschaft, wohl eher skeptisch zu beurteilen. Dies ist sicherlich zum Teil, das hat sich deutlich gezeigt, auch gesellschaftlichen Anforderungen geschuldet. Sich dauerhaft, wie Susanne und Rachid, gegen die normativen Erwartungen zu stellen, ist kaum möglich bzw. mit einem Preis verbunden, der insbesondere Susanne zu hoch erscheint. Traditionelle Geschlechterverhältnisse sind stabil und setzen sich als solche - v. a. auf der Praxisebene - durch. Zum anderen aber müssten, um den Erwartungen zu entsprechen, binationale Paare die Beziehung aus solchen, demokratischen und/oder emanzipativen, Gründen eingehen. Dass die Paare durch ihre Bearbeitungen der Herausforderung und durch die Lernprozesse, die sich darin analysieren lassen, Geschlechterordnungen verändern, neu entwickeln und sich darin neu positionieren, bedeutet jedoch noch nicht dass sie sich selber als besonders gleichberechtigt verstehen. Der Zivilisationsdialog im Kleinen, der diesen Beziehungen oft unterstellt wird bzw. der von ihnen erwartet wird, ist insofern eher als nicht intendierte Folge zu verstehen. Auch Maria und Pablo, die ja beide durch ihre interkulturelle Programmatik noch am ehesten einem solchen Bild entsprechen, sind weniger an der Realisierung einer pluralisierten Gesellschaft als an einer gleichberechtigten Weitergabe ihrer Kultur an ihre Kinder interessiert. Die Motive für die Beziehungen sind insofern deutlich komplexer und entspringen eben nicht (immer) dem Wunsch nach einer gesellschaftskritischen Äußerung oder gar einer Veränderung bestehender Verhältnisse. Beziehungen, auch binationale Beziehungen, sind nicht zwangsläufig Ausdruck politischer Orientierungen. Sie können vielmehr, zumindest bei den von mir interviewten Paaren, als Ausdruck vielfältiger Erwartungen und Hoffnungen gelesen werden, die sich jedoch vorrangig auf die Erfüllung individueller Lebenspläne beziehen. 



\section{ABBILDUNGS - UND TABELLENVERZEICHNIS}

Abbildung 1: Eheschließungen nach der Staatsangehörigkeit der Ehepartner von 1955 - 2000 (Quelle: IAF 2006)

Abbildung 2: Erteilte Visa zum Zweck des Ehegatten- und Familiennachzugs nach Deutschland von 1996 bis 2003 (Quelle: Beauftragte der Bundesregierung für Migration 2004)

Abbildung 3: Darstellung des Interpretationsmodells (eig. Darstellung)

Tabelle 1: Kernsample (eig. Darstellung) 



\section{LITERATUR}

AiD - Integration in Deutschland (2004): Arbeitslosigkeit und Sozialhilfebezug. Unter: http://www.isoplan.de/aid/2004-3/statistik.htm vom 16.4.06

Alba, R./Nee, V. (1997): Rethinking Assimilation Theory for a New Era of Immigration. In: International Migration Review 4/1997, S. 826 849

Aldrige, D. P. (1978): Interracial Marriages: Empirical and Theoretical Considerations. In: Journal of Black Studies 3/1978, S. $255-268$

Aleemi, J. (1991): Zur sozialen und psychischen Situation von Bilingualen. Frankfurt a. M.

Alheit, P. (1989): Erzählform und „soziales Gedächtnis“: Beispiel beginnender Traditionsbildung im autobiographischen Erinnerungsprozeß. In: Alheit, P./Hoerning, E. M. (Hg.): Biographisches Wissen. Frankfurt a. M./New York, S. $123-147$

Alheit, P. (1990): Der ,biographische Ansatz' in der Erwachsenenbildung. In: Mader, W. (Hg.): Weiterbildung und Gesellschaft. Grundlagen wissenschaftlicher und beruflicher Praxis in der Bundesrepublik Deutschland. Bremen, S. 289 - 337

Alheit, P. (1994): Arbeit und Bildung im Modernisierungsprozeß: Entkopplung oder neue Synthese? In: Alheit, P./u.a. (Hg.): Von der Arbeitsgesellschaft zur Bildungsgesellschaft? Bremen, S. 23 - 47

Alheit, P. (1995): Biographizität als Lernpotential: Konzeptionelle Überlegungen zum biographischen Ansatz in der Erwachsenenbildung. In: Krüger, H.-H./Marotzki, W. (Hg.): Erziehungswissenschaftliche Biographieforschung. Opladen, S. $276-307$ 
Alheit, P. (2003): Biographizität. In: Bohnsack, R./Marotzki, W./Meuser, M. (Hg.): Hauptbegriffe Qualitativer Sozialforschung. Opladen, S. 25

Alheit, P./Dausien, B. (1999): Biographieforschung in der Erwachsenenbildung. In: Krüger, H.-H./Marotzki, W. (Hg.): Handbuch erziehungswissenschaftliche Biographieforschung. 2. überarbeitete Auflage. Opladen, S. $407-432$

Alheit, P./Dausien, B. (2000): Die biographische Konstruktion der Wirklichkeit. Überlegungen zur Biographizität des Sozialen. In: Hoerning, E. M. (Hg.): Biographische Sozialisation. Stuttgart, S. 257 282

Alheit, P./Dausien, B. (2002): Bildungsprozesse über die Lebensspanne und lebenslanges Lernen. In: Tippelt, R. (Hg.): Handbuch Bildungsforschung. Opladen, S. $565-585$

Alheit, P./Hoerning, E. M. (1989): Biographie und Erfahrung: Eine Einleitung. In: Alheit, P./Hoerning, E. M. (Hg.): Biographisches Wissen. Frankfurt a. M./New York, S. 8 - 23

Anthias, F. (2003): Erzählungen über Zugehörigkeit. In: Apitzsch, U./Jansen, M. M. (Hg.): Migration, Biographie und Geschlechterverhältnisse. Münster, S. $20-37$

Apitzsch, U. (1990): Lernbiographien zwischen den Kulturen. In: Gieseke, W. (Hg.): Ethische Prinzipien der Erwachsenenbildung. Kassel, S. $156-169$

Apitzsch, U. (1999): Traditionsbildung im Zusammenhang gesellschaftlicher Prozesse. In: Apitzsch, U. (Hg.): Migration und Traditionsbildung. Opladen/Wiesbaden, S. 7 - 20

Apitzsch, U. (2003): Einleitung. In: Apitzsch, U. (Hg.): Migration, Biographie und Geschlechterverhältnisse. Münster, S. 7 - 19

Auernheimer, G. (2004): Einführung in die interkulturelle Pädagogik. Darmstadt

Ausländerbeauftragte (2003) = Ausländerbeauftragte der Bundesregierung (2003): Migrationsbericht der Ausländerbeauftragten im Auftrag der Bundesregierung. Berlin

Baecker, D. (2001): Wozu Kultur? Berlin

Beauftragte der Bundesregierung für Migration = Beauftragte der Bundesregierung für Migration, Flüchtlinge und Integration (2004): Daten - Fakten - Trends. Migrationsgeschehen. Berlin

Beck, U. (1986): Risikogesellschaft. Auf dem Weg in eine andere Moderne. Frankfurt a. M.

Becker, W. (1974): Ehen mit Ausländern. Informationen für junge Frauen, die einen Ausländer heiraten wollen. Hamm 
Becker-Schmidt, R. (1991): Individuum, Klasse und Geschlecht aus der Perspektive der Kritischen Theorie. In: Zapf, W. (Hg.): Die Modernisierung moderner Gesellschaften. Verhandlungen des 25. Deutschen Soziologentages in Frankfurt am Main 1990. Frankfurt a. M./New York, S. $383-394$

Beck-Gernsheim, E. (2001): Liebe kennt keine Grenzen. Vom Leben in binationalen und bikulturellen Beziehungen. In: Kursbuch 144/2001, S. $111-121$

Bednarz-Braun, I. (2004): Entwicklungen von Theorieansätzen im Schnittpunkt von Ethnie, Migration und Geschlecht. In: BednarzBraun, I./Heß-Meining, U. (Hg.): Migration, Ethnie und Geschlecht. Theorieansätze - Forschungsstand - Forschungsperspektiven. Wiesbaden, S. $21-94$

Benard, C./Khalilzad, Z. (1984): „The government of god“ - Iran's Islamic Republik. New York

Benner, D. (1990): Wilhelm von Humboldts Bildungstheorie. Eine problemgeschichtliche Studie zum Begründungszusammenhang neuzeitlicher Bildungsreform. Weinheim/München

Berger, P. L./Kellner, H. (1965): Die Ehe und die Konstruktion der Wirklichkeit. In: Soziale Welt 1965, S. 220 - 235

Berger, P. L./Luckmann, T. (1980 [1966]): Die gesellschaftliche Konstruktion der Wirklichkeit. Eine Theorie der Wissenssoziologie. Frankfurt a. M.

Blau, P. M./Becker, C. ,et al. (1984): Intersecting Social Affiliations and Intermarriage. In: Social Forces 3/1984, S. 585 - 604

Blech, C./Witte, E. H. (1992): Zu Veränderungen in der Paarbeziehung bei Erwerbslosigkeit des Mannes. In: Kölner Zeitschrift für Soziologie und Sozialpsychologie 4/1992, S. 731 - 746

Blumer, H. (2004 [1973]): Der methodologische Standort des symbolischen Interaktionismus. In: Strübing, J./Schnettler, b. (Hg.): Methodologie interpretativer Sozialforschung. Klassische Grundlagentexte. Konstanz, S. $321-385$

Bohnsack, F./Nohl, A.-M. (2001): Ethnisierung und Differenzerfahrung: Fremdheit als alltägliches und methodologisches Problem. In: Zeitschrift für qualitative Bildungs-, Beratungs- und Sozialforschung $1 / 2001$, S. $15-36$

Bolak, H. C. (1997): When Wifes are Major Providers: Culture, Gender and Family Work. In: Gender and Society 4/1997, S. $409-433$

Born, C./Krüger, H. (2001): Das Lebenslaufregime der Verflechtung: Orte, Ebenen und Thematisierungen. In: Born, C./Krüger, H. (Hg.): Individualisierung und Verflechtung. Geschlecht und Generation im deutschen Lebenslaufregime. Weinheim/München, S. 11 - 26 
Bourdieu, P. (1983): Ökonomisches Kapital, kulturelles Kapital, soziales Kapital. In: Kreckel, R. (Hg.): Soziale Ungleichheiten. Sonderband 2 der Sozialen Welt. Göttingen, S. 183 - 198

Breger, R. (1998): Love and the State: Women, Mixed Marriages and the Law in Germany. In: Breger, R./Hill, R. (Hg.): Cross-Cultural Marriage. Identity and Choice. Oxford/New York, S. 129 - 152

Breger, R./Hill, R. (1998): Introducing Mixed Marriages. In: Breger, R./Hill, R. (Hg.): Cross-Cultural Marriage. Identity and Choice. Oxford New York, S. 1 - 32

Breidenstein, G./Kelle, H. (1998): Geschlechteralltag in der Schulklasse. Ethnographische Beobachtungen zur Gleichaltrigenkultur. Weinheim

Breitenbach, E. (2000): Mädchenfreundschaften in der Adoleszenz. Eine fallrekonstruktive Untersuchung von Gleichaltrigengruppen Opladen

Breitenbach, E. (2002): Geschlecht im schulischen Kontext. Theoretische und empirische Fragen an die Koedukationsdebatte. In: Breitenbach, E./Bürmann, I. et al. (2002) (Hg.): Geschlechterforschung als Kritik. Bielefeld, S. $149-164$

Breitenbach, E. (2005): Vom Subjekt zur Katagorie. Veränderte Denkfiguren. In: Casale, R./Rendtorff, B. et al. (2005) (Hg.): Geschlechterforschung in der Kritik. Opladen, S. 73 - 86

Bronfen, E./Marius, B. (1997): Hybride Kulturen. Einleitung zur angloamerikanischen Multikulturalismusdebatte. In: Bronfen, E./Marius, B./Steffen, T. (Hg.): Hybride Kulturen. Beiträge zur anglo-amerikanischen Multikulturalismusdebatte. Tübingen, S. 1 - 29

Brose, H.-G./Hildenbrand, B. (1988): Biographisierung von Erleben und Handeln. In: Brose, H.-G./Hildenbrand, B. (Hg.): Vom Ende des Individuums zur Individualität ohne Ende. Opladen, S. 11 - 30

Buba, H.-P./Ueltzen, W. et al. (1984): Gemischt-nationale Ehen in der Bundesrepublik Deutschland. In: Zeitschrift für Bevölkerungswissenschaft 4/1984, S. 421 - 448

Budde, G.-F. (1997). Frauen arbeiten. Weibliche Erwerbstätigkeit in Ost- und Westdeutschland nach 1945. Göttingen.

Bukow, W.-D./Heimel, I. (2003): Der Weg zur qualitativen Migrationsforschung. In: Badawia, T./Hamburger, F./Hummrich, M. (Hg.): Wider die Ethnisierung einer Generation. Beiträge zur qualitativen Migrationsforschung. Frankfurt a. M./London, S. 13 - 39

Bundesministerium für Arbeit $=$ Bundesministerium für Arbeit und Sozialordnung (1995): Repräsentativuntersuchung '95. Situation der ausländischen Arbeitnehmer und ihrer Familienangehörigen in der Bundesrepublik Deutschland. Bonn 
Bundesministerium für Familie $=$ Bundesministerium für Familie, Senioren, Frauen und Jugend (Hg.) (1999): Die Familie im Spiegel der amtlichen Statistik. Bonn

Bundeszentrale für gesundheitliche Aufklärung (Hg.) (2004): männer leben. Eine Studie zu Lebensläufen und Familienplanung. Köln

Burian, E. (1999): Wer macht welche Frauen glücklich? Eine Untersuchung des Einflusses von Werten und Zielen der Frauen auf deren Partnerwahl sowie Zusammenhänge mit der Geschlechtsrollenausprägung der Partner unter dem Gesichtspunkt der Ehezufriedenheit der Frauen anhand einer Gegenüberstellung monokulturell-österreichischer und österreichisch-afrikanischer Ehepaare. Unter: www. binational-in.de/archive/Wer_macht_welche_Frauen_gluecklich.pdf vom 16.04.2006

Burkart, G. (1998): Auf dem Weg zu einer Soziologie der Liebe. In: Hahn, K./Burkart, G. (Hg.): Liebe am Ende des 20. Jahrhunderts. Opladen, S. 15 - 49

Burkart, G./Koppetsch, C. (2001): Geschlecht und Liebe. Überlegungen zu einer Soziologie des Paares. In: Kölner Zeitschrift für Soziologie und Sozialpsychologie. Sonderheft 2001, S. 431 - 453

Close, E. (2003): The Black Gender Gap. In: Newsweek Magazine March 3/2003, S. 46 - 55

Dausien, B. (1996): Biographie und Geschlecht. Zur biographischen Konstruktion sozialer Wirklichkeit in Frauenlebensgeschichten. Bremen

Dausien, B. (1998): Die biographische Konstruktion von Geschlecht. In: Schneider, N./Lohmar, D. (Hg.): Einheit und Vielfalt: das Verstehen der Kulturen. Amsterdam, S. 257 - 277

Dausien, B. (1999): Geschlechterkonstruktionen und Körpergeschichten. Überlegungen zur Rekonstruktion leiblicher Aspekte des ,doing gender' in biographischen Erzählungen. In: Alheit, P./Dausien, B./Fischer-Rosenthal, W./Hanses, A./Keil, A. (Hg.): Biographie und Leib. Gießen, S. $177-200$

Dausien, B. (2000): „Biographie“ als rekonstruktiver Zugang zu „Geschlecht". Perspektiven der Biographieforschung für die empirische und theoretische Analyse von Geschlechterkonstruktionen. In: Lemmermöhle, D./Klika, D./Fischer, D./Schlüter, A. (Hg.): Lesarten des Geschlechts: zu De-Konstruktionsdebatte in der erziehungswissenschaftlichen Geschlechterforschung. Opladen, S. 96-115

Dausien, B. (2002): Sozialisation - Geschlecht - Biographie. Theoretische und methodologische Untersuchung eines Zusammenhangs. Unveröff. Habilschrift. Bielefeld 
Dausien, B./Kelle, H. (2005): Biographie und kulturelle Praxis. Methodologische Überlegungen zur Verknüpfung von Ethnographie und Biographieforschung. In: Völter, B./Dausien, B. et al. (2005) (Hg.): Biographieforschung im Diskurs. Wiesbaden, S. $189-212$

De Carlo, S./Menz, M. et al. (2006): Begrenzung und Ermöglichung: Migrationsprozesse in modernen Gesellschaften. In: Walter, A./ Menz, M./De Carlo, S. (Hg.): Grenzen der Gesellschaft? Migration und sozialstruktureller Wandel in der Zuwanderungsregion Europa. Göttingen, S. 9 - 36

De Hart, B. (2003): Foolhardy Women. Mixed relationships in Nationality Law and Immigration Law. Unveröff. Manuskript. Nijmegen, S. $279-290$

Deutsch, F. M./Saxon, S. E. (1998): Traditional Ideologies, Nontraditional Lives. In: Sex Roles 5/6/1998, S. 331 - 362

Diehm, I./Radtke, F.-O. (1999): Erziehung und Migration. Eine Einführung. Stuttgart

Diekmann, A. (1998): Empirische Sozialforschung. Grundlagen, Methoden, Anwendungen. Hamburg

Dornseiff, J.-M./Sackmann, R. (2003): Familien-, Erwerbs- und Fertilitätsdynamiken in Ost- und Westdeutschland. In: Familien Survey 11 (Hg.): Partnerschaft und Familiengründung: Ergebnisse der dritten Welle des Familienpanels. Opladen, S. 309 - 348

Ecarius, J. (1998): Biographie, Lernen und Gesellschaft. Erziehungswissenschaftliche Überlegungen zu biographischem Lernen in sozialen Kontexten. In: Bohnsack, R./Marotzki, W. (Hg.): Biographieforschung und Kulturanalyse. Transdisziplinäre Zugänge qualitativer Forschung. Weinheim, S. $129-151$

Ecarius, J. (1999): Biographieforschung und Lernen. In: Krüger, H.-H./ Marotzki, W. (Hg.): Handbuch erziehungswissenschaftliche Biographieforschung. Opladen, S. $89-105$

Eckert, R./Hahn, A. ,et al. (1989): Die ersten Jahre junger Ehen. Verständigung durch Illusionen? Frankfurt a. M./New York

Eßer, F./Zinn, J. (2002): Subjektkonzeptionen bei der Herstellung biographischer Sicherheit. Arbeitspapier 7 des SFB 536 „Reflexive Modernisierung“. München

Europäische Konferenz der Binationalen /Bikulturellen Beziehungen (1996): Die internationale Familie in Europa. Paris

Felden, H. v. (2002): Bildungsdiskurse der (Post)Moderne. Zum Zusammenhang von Bildungs, Biographie- und Genderforschung. In: Zeitschrift für qualitative Bildungs-, Beratungs- und Sozialforschung 2/2002, S. $191-214$ 
Felden, H. v. (2003): Bildung und Geschlecht zwischen Moderne und Postmoderne. Opladen

Feministische Studien (2/1993): Kritik der Kategorie Geschlecht. Opladen

Feministische Studien (2000): Feministische Studien extra: Fürsorge Anerkennung - Arbeit. Opladen

Fischer-Rosenthal, W./Rosenthal, G. (1997): Warum Biographieanalyse und wie man sie macht. In: Zeitschrift für Sozialisationsforschung und Erziehungssoziologie 4/1997, S. 405 - 427

Friebel, H./Epskamp, H. ,et al. (2000): Bildungsbeteiligung: Chancen und Risiken. Eine Längsschnittstudie über Bildungs- und Weiterbildungskarrieren in der ,Moderne'. Opladen

Fröhlich, M./Messerschmidt, A. ,et al. (Hg.) (2003): Migration als biographische und expressive Ressource. Beiträge zur kulturellen Produktion in der Einwanderungsgesellschaft. Frankfurt a. M.

Garfinkel, H. (1967): Studies in ethnomethodology. Englewood Cliffs, NJ

Geissler, B./Oechsle, M. (1994): Lebensplanung als Konstruktion: Biographische Dilemmata und Lebenslauf-Entwürfe junger Frauen. In: Beck, U./Beck-Gernsheim, E. (Hg.): Riskante Freiheiten. Frankfurt a. M., S. $139-167$

Gemende, M. (2001): Frauen und Migration. Und: Überlegungen zur These von der Individualisierung in (post)modernen Gesellschaften. In: Zeitschrift für Frauenforschung und Geschlechterstudien 3/2001, S. $40-57$

Giddens, A. (1995): Die Konstitution der Gesellschaft. Frankfurt a. M.

Gildemeister, R./Wetterer, A. (1995): Wie Geschlechter gemacht werden. Die soziale Konstruktion der Zweigeschlechtlichkeit und ihre Reifizierung in der Frauenforschung. In: Knapp, G.-A./Wetterer, A. (Hg.): TraditionenBrüche. Entwicklungen feministischer Theorie. Freiburg, S. $201-254$

Glaser, B./Strauss, A. L. (1967): The Discovery of Grounded Theory. Strategies for Qualitative Resarch. Mill Valley

Glaser, B./Strauss, A. L. (1998): Grounded Theory. Strategien qualitativer Forschung. Bern/Göttingen

Goeke, P. (2006): Transnational assimiliert? Transmigranten der zweiten Generation zwischen Kroatien und Deutschland. In: Walter, A./ Menz, M./De Carlo, S. (Hg.): Grenzen der Gesellschaft? Migration und sozialstruktureller Wandel in der Zuwanderungsregion Europa. Göttingen, S. 337 - 360

Goffman, E. (1994): Das Arrangement der Geschlechter. In: Goffman, E. (Hg.): Interaktion und Geschlecht. Frankfurt a. M., S. $105-158$ 
Gogolin, I./Pries, L. (2004): Stichwort: Transmigration und Bildung. In: Zeitschrift für Erziehungswissenschaften 1/2004, S. 5 - 19

Gorman, E. H. (1999): Bringing Home the Bacon: Marital Allocation of Income-Earning Responsibility, Job Shifts, and Men's Wages. In: Journal of Marriage and the Family 1/1999, S. 110 - 122

Gümen, S. (1998): Das Soziale des Geschlechts. Frauenforschung und die Kategorie ,Ethnizität‘. In: Das Argument 1998, S. 187 - 203

Habermas, J. (1993): Anerkennungskämpfe im demokratischen Rechtsstaat. In: Taylor, C. (Hg.): Multikulturalismus und die Politik der Anerkennung. Frankfurt a. M., S. 147 - 196

Habermas, J. (2004): Philosophie in Zeiten des Terrors. In: Habermas, J./Derrida, J. (Hg.): Philosophie in Zeiten des Terrors. Zwei Gespräche, eingeleitet und kommentiert von Giovanna Borradori. Berlin/ Hamburg

Hagemann-White, C. (1993): Die Konstrukteure des Geschlechts auf frischer Tat ertappen? Methodische Konsequenzen einer theoretischen Einsicht. In: Feministische Studien 2/1993, S. 68 - 78

Hahn, A. (1988): Biographie und Lebenslauf. In: Brose, H.-G./Hildenbrand, B. (Hg.): Vom Ende des Individuums zum Individuum ohne Ende. Opladen, S. 91 - 105

Hamburger, F. (1999): Zur Tragfähigkeit der Kategorien ,Ethnizität‘ und ,Kultur' im erziehungswissenschaftlichen Diskurs. In: Zeitschrift für Erziehungswissenschaften 2/1999, S. 167 - 178

Hardach-Pinke, I. (1988): Interkulturelle Lebenswelten. Deutsch-Japanische Ehen in Japan. Frankfurt a. M.

Haug, S. (1997): Soziales Kapital. Ein kritischer Überblick über den aktuellen Forschungsstand. Arbeitspapier des Mannheimer Zentrums für Europäische Sozialforschung. Mannheim

Haug, S. (2000): Klassische und neuere Theorien der Migration. Arbeitspapier des Mannheimer Zentrums für Europäische Sozialforschung. Mannheim

Hecht-El Minshawi, B. (1988): Wir suchen, wovon wir träumen: Zur Motivation deutscher Frauen einen Partner aus dem islamischen Kulturkreis zu wählen. Frankfurt a. M.

Heinemeier, S. (1991): Zeitstrukturkrisen. Biographische Interviews mit Arbeitslosen. Biographie und Gesellschaft 12. Opladen

Heintz, B./Nadai, E. ,et al. (1997): Ungleich unter Gleichen. Studien zur geschlechtsspezifischen Segregation des Arbeitsmarktes. Frankfurt a. M./New York

Heinz, W. R. (1985): Hauptsache eine Lehrstelle. Jugendliche vor den Hürden des Arbeitsmarktes. Weinheim u.a. 
Heinz, W. R. (2000): Selbstsozialisation im Lebenslauf. Umrisse einer Theorie biographischen Handelns. In: Heinz, W. R. (Hg.): Übergänge : Individualisierung, Flexibilisierung und Institutionalisierung des Lebensverlaufs. Weinheim, S. $165-186$

Herwartz-Emden, L. (1997): Die Bedeutung der sozialen Kategorien Geschlecht und Ethnizität für die Erforschung des Themenbereichs Jugend und Einwanderung. In: Zeitschrift für Pädagogik 6/1997, S. $895-913$

Hirschauer, S. (1993): Die soziale Konstruktion der Transsexualität: über die Medizin und den Geschlechtswechsel. Frankfurt a. M.

Hoerning, E. M. (2000): Biographische Sozialisation. Theoretische und forschungspraktische Verankerung. Eine Einleitung zu den Beiträgen. In: Hoerning, E. M. (Hg.): Biographische Sozialisation. Stuttgart, S. $2-20$

Höhne, T. (2001): Kultur als Differenzierungskategorie. In: Lutz, H./ Wenning, N. (Hg.): Unterschiedlich verschieden. Differenz in der Erziehungswissenschaft. Opladen, S. $197-213$

Holz, E. (2000): Zeitverwendung in Deutschland - Beruf, Familie, Freizeit. Statistisches, B. Schriftenreihe Spektrum Bundesstatistik 13. Wiesbaden

Hopf, C./Hartwig, M. (2001): Liebe und Abhängigkeit: Partnerschaftsbeziehungen junger Frauen. Weinheim

Hormel, U./Scherr, A. (2005): Migration als gesellschaftliche Lernprovokation - Programmatische Konturen einer offensiven Bildung für die Einwanderungsgesellschaft. In: Hamburger, F./Badawia, T./ Hummrich, M. (Hg.): Migration und Bildung. Über das Verhältnis von Anerkennung und Zumutung in der Einwanderungsgesellschaft. Wiesbaden, S. $295-310$

Huck, L. (2005): Möglichkeiten und Behinderungen kooperativen Lernens. Anmerkungen zu Frigga Haugs ,Lernverhältnissen`. In: Forum Kritische Psychologie 48/2005, S. 14 - 25

Hummrich, M. (2002): Bildungserfolg und Migration. Opladen

IAF (2006) Unter: http://verband-binationaler.de/zahlenundfakten/index .htm vom 07.04.06.

Jacob, G. (1997): Das narrative Interview in der Biographieforschung. In: Friebertshäuser, B./Prengel, A. (Hg.): Handbuch qualitative Forschungsmethoden in der Erziehungswissenschaft. Weinheim/München, S. $445-458$

Kade, J./Seitter, W. (1996): Lebenslanges Lernen. Mögliche Bildungswelten. Opladen 
Kallmeyer, W./Schütze, F. (1977): Zur Konstitution von Kommunikationsschemata der Sachverhaltsdarstellung. In: Wegner, D. (Hg.): Gesprächsanalysen. Hamburg, S. 159 - 274

Kaufmann, J.-C. (1995): Schmutzige Wäsche: zur ehelichen Konstruktion von Alltag. Konstanz

Kelle, U. (1994): Empirisch begründete Theoriebildung. Zur Logik und Methodologiebildung interpretativer Sozialforschung. Weinheim

Kennedy, R. J. R. (1944): Single or Triple Melting Pot? Intermarriage Trends in New Haven, 1870 - 1944. In: The American Journal of Sociology 1944, S. 56 - 59

Khatib-Chahidi, J./Hill, R. ,et al. (1998): Chance, Choice and Circumstance: A Study of Women in Cross-Cultural Marriages. In: Breger, R./Hill, R. (Hg.): Cross-Cultural Marriage. Identity and Choice. Oxford/New York, S. $49-66$

Khounani, P. M. (2000): Binationale Familien in Deutschland und die Erziehung der Kinder. Eine Vergleichsuntersuchung zur familiären Erziehungssituation in mono- und bikulturellen Familien im Hinblick auf multikulturelle Handlungsfähigkeit. Frankfurt a. M.

Kienecker, S. (1993): Interethnische Ehen. Deutsche Frauen mit ausländischem Partner. Hamburg

Klafki, W. (1993): Neue Studien zur Bildungstheorie und Didaktik. Weinheim und Basel

Klein, T. (2000): Partnerwahl zwischen sozialstrukturellen Vorgaben und individueller Entscheidungsautonomie. In: Zeitschrift für Soziologie der Erziehung (ZSE) 3/2000, S. 229 - 243

Klein, T. (2001a): Intermarriages between Germans and Foreigners in Germany. In: Journal of Comparative Familiy Studies 3/2001a, S. $325-346$

Klein, T. (Hg.) (2001b): Partnerwahl und Heiratsmuster. Sozialstrukturelle Voraussetzungen der Liebe. Opladen

Klein, T./Lengerer, A. (2001): Gelegenheit macht Liebe - die Wege des Kennenlernens und ihr Einfluss auf die Muster der Partnerwahl. In: Klein, T. (Hg.): Partnerwahl und Heiratsmuster. Sozialstrukturelle Voraussetzungen der Liebe. Opladen, S. 265 - 285

Knapp, G.-A. (1992): Macht und Geschlecht. Neuere Entwicklungen in der feministischen Macht- und Herrschaftsdiskussion. In: Knapp, G.A./Wetterer, A. (Hg.): TraditionenBrüche. Entwicklungen feministischer Theorie. Opladen, S. $287-325$

Knapp, G.-A. (2001): Dezentriert und viel riskiert... Anmerkungen zur These vom Bedeutungsverlust der Kategorie Geschlecht. In: Wetterer, A. (Hg.): Soziale Verortung der Geschlechter: Gesellschaftstheorie und feministische Kritik. Münster, S. 15 - 62 
Knapp, G.-A. (2005): ,Intersectionality“ - ein neues Paradigma feministischer Theorie? Zur transatlantischen Reise von ,Race, Class, Gender'. In: Feministische Studien 1/2005, S. $68-81$

Kneer, G./Nassehi, A. (2000): Niklas Luhmanns Theorie der sozialen Systeme. München

Koch, M./Koller, H.-C. (1999): Geschlechter im Widerstreit? Ein Streitgespräch über Bildung und Geschlechterverhältnisse. In: Behm, B./ Heinrichs, G./Tiedemann, H. (Hg.): Das Geschlecht der Bildung Die Bildung der Geschlechter. Opladen, S. 239 - 268

Koch, U. (2006): Errichtung und Management von Grenzen: Roma in einer westdeutschen Großstadt. In: Walter, A./Menz, M./De Carlo, S. (Hg.): Grenzen der Gesellschaft? Migration und sozialstruktureller Wandel in der Zuwanderungsregion Europa. Göttingen, S. 201 218

Kohli, M. (1985): Die Institutionalisierung des Lebenslaufs. Historische Befunde und theoretische Argumente. In: Kölner Zeitschrift für Soziologie und Sozialpsychologie 1/1985, S. 1 - 29

Kohli, M. (1986): Gesellschaftszeit und Lebenszeit. Der Lebenslauf im Strukturwandel der Moderne. In: Soziale Welt. Sonderband 4 1986, S. $183-208$

Koller, H.-C. (1993): Bildung im Widerstreit. Bildungstheoretische Überlegungen im Anschluß an Lyotards Konzeption pluraler Diskurse. In: Marotzki, W./Sünker, H. (Hg.): Kritische Erziehungswissenschaft: Moderne - Postmoderne Bd.2. Weinheim, S. 80 - 104

Koller, H.-C. (1999): Bildung und Widerstreit. Zur Struktur biographischer Prozesse in der (Post-)Moderne. München

Koller, H.-C. (2002a): Bildung und kulturelle Differenz. Zur Erforschung biographischer Bildungsprozesse von MigrantInnen. In: Kraul, M./Marotzki, W. (Hg.): Biographische Arbeit. Perspektiven erziehungswissenschaftlicher Biographieforschung. Opladen, S. 92 116

Koller, H.-C. (2002b): Bildung und Migration. Bildungstheoretische Überlegungen im Anschluss an Bourdieu und Cultural Studies. In: Friedrichs, W./Sanders, O. (Hg.): Bildung/Transformation. Kulturelle und gesellschaftliche Umbrüche aus bildungstheoretischer Perspektive. Bielefeld, S. $181-200$

Komter, A. (1989): Hidden Power in Marriage. In: Gender and Society 2/1989, S. $187-216$

Konietzka, D./Kreyenfeld, M. (2001): Die Verwertbarkeit ausländischer Ausbildungsabschlüsse. Das Beispiel der Aussiedler auf dem deutschen Arbeitsmarkt. In: Zeitschrift für Soziologie 4/2001, S. 267 282 
Konsulate und Botschaften 2006. Unter: http://www.konsulate.de/zahlen _und_fakten/zahlen_und_faktenmexiko/php vom 14.08.2006

Koppetsch, C. (1998): Liebe und Partnerschaft: Gerechtigkeit in modernen Paarbeziehungen. In: Hahn, K./Burkart, G. (Hg.): Liebe am Ende des 20. Jahrhunderts. Opladen, S. $111-129$

Koppetsch, C./Burkart, G. (1999): Die Illusion der Emanzipation: zur Wirksamkeit latenter Geschlechtsnormen im Milieuvergleich. Kon$\operatorname{stanz}$

Kothoff, H. (1994): Geschlecht als Interaktionsritual? In: Goffman, E. (Hg.): Interaktion und Geschlecht. Frankfurt a. M./New York, S. 159 $-194$

Kraul, M./Marotzki, W. (2002): Bildung und biographische Arbeit Eine Einleitung. In: Kraul, M./Marotzki, W. (Hg.): Biographische Arbeit. Perspektiven erziehungswissenschaftlicher Biographieforschung. Opladen, S. 7 - 21

Kriechhammer-Yagmur, S./Pfeiffer-Pandey, D. ,et al. (2001): Binationaler Alltag in Deutschland. Ratgeber für Ausländerrecht, Familienrecht und interkulturelles Zusammenleben. Frankfurt a. M.

Krüger, H. (1991): Doing Gender - Geschlecht als Statuszuweisung im Berufsbildungssystem. In: Brock, D./Hantsche, B. (Hg.): Übergänge in den Beruf. Zwischenbilanz zum Forschungsstand. Weinheim/München, S. 139 - 169

Krüger, H. (20029: Gesellschaftsanalyse: der Institutionenansatz in der Geschlechterforschung. In: Knapp, G. A./Wetterer, A. (2002) (Hg.): Soziale Verortung der Geschlechter: Gesellschaftsanalyse und feministische Kritik. Münster, S. $63-90$

Krüger, S. (Hg.) (1997): Zusammen sind wir bunt: interkulturelle Projekte in der Kinderbibliothek. Berlin

Krüger-Portratz, M./Lutz, H. (2002): Sitting at a crossroads - rekonstruktive und systematische Überlegungen zum wissenschaftlichen Umgang mit Differenzen. In: Tertium Comparationis. Journal für International und Interkulturell vergleichende Erziehungswissenschaft. 2/2002, S. $81-92$

Kugler, R. (2000): Ausländerrecht. Ein Handbuch. Göttingen

Künzler, J. (1995): Familiale Arbeitsteilung in der Bundesrepublik Deutschland 1988. In: Gerhardt, U./Hradil, S./Lucke, D./Nauck, B. (Hg.): Familie der Zukunft. Opladen, S. 149 - 169

Larcher, D. (2000): Die Liebe in den Zeiten der Globalisierung. Konstruktion und Dekonstruktion von Fremdheit in interkulturellen Paarbeziehungen. Klagenfurt/Celovec 
Lauth Bacas, J. (2001): Binationale Ehen im neuen Haus Europa. Das Beispiel deutsch-griechischer Paare in Athen. In: Schlehe, J. (Hg.): Interkulturelle Geschlechterforschung. Identitäten - Imaginationen Repräsentationen. Frankfurt a. M., S. $111-131$

Lenz, I. (1996): Grenzziehungen und Öffnungen: Zum Verhältnis von Geschlecht und Ethnizität zu Zeiten der Globalisierung. In: Lenz, I./Gerner, A. (Hg.): Wechselnde Blicke. Frauenforschung in internationaler Perspektive. Opladen, S. $200-228$

Lenz, K. (2003): Soziologie der Zweierbeziehung. Eine Einführung. 2., aktualisierte und überarbeitete Auflage. Wiesbaden

Levy, R. (1977): Der Lebenslauf als Statusbiographie. Stuttgart

Lindemann, G. (1993): Das paradoxe Geschlecht. Transsexualität im Spannungsfeld von Körper, Leib und Gefühl. Frankfurt a. M.

Lüdi, G. (1996): Mehrsprachigkeit. In: Goebl, H. (Hg.): Kontaktlinguistik. Berlin/New York, S. 233 - 245

Luhmann, N. (1982): Liebe als Passion. Zur Codierung von Intimität. Frankfurt a. M.

Luhmann, N. (1984): Soziale Systeme. Grundriss einer allgemeinen Theorie. Frankfurt a. M.

Lutz, H. (2001): Differenz als Rechenaufgabe: über die Relevanz der Kategorien Race, Class und Gender. In: Lutz, H./Wenning, N. (Hg.): Unterschiedlich verschieden. Differenz in der Erziehungswissenschaft. Opladen, S. $215-230$

Lutz, H. (2004): Migrations- und Geschlechterforschung. In: Becker, R./Kortendiek, B. (Hg.): Handbuch Frauen- und Geschlechterforschung. Theorie, Methoden, Empirie. Wiesbaden, S. 476 - 484

Macmillan, R./Gartner, R. (1999): When She Brings Home the Bacon: Labor-Force Participation and the Risk of Spousal Violence against Women. In: Journal of Marriage and the Family 4/1999, S. 947 958

Marotzki, W. (1990): Entwurf einer strukturalen Bildungstheorie. Biographietheoretische Auslegung von Bildungsprozessen in hochkomplexen Gesellschaften. Weinheim

Marotzki, W. (1991): Bildungsprozesse in lebensgeschichtlichen Horizonten. In: Hoerning, E. M./Schulze, T./Marotzki, W./Tietgens, H. (Hg.): Biographieforschung und Erwachsenenbildung. Bad Heilbrunn, S. $135-181$

Marotzki, W. (1995): Forschungsmethoden der erziehungswissenschaftlichen Biographieforschung. In: Krüger, H.-H./Marotzki, W. (Hg.): Erziehungswissenschaftliche Biographieforschung. Opladen, S. 55 89 
Marotzki, W. (1999): Bildungstheorie und Allgemeine Biographieforschung. In: Krüger, H.-H./Marotzki, W. (Hg.): Handbuch erziehungswissenschaftliche Biographieforschung. Opladen, S. $57-68$

Marotzki, W. (2003): Biografieforschung. In: Bohnsack, R./Marotzki, W./Meuser, M. (Hg.): Hauptbegriffe Qualitativer Sozialforschung. Opladen, S. $22-24$

Matthes, J. (1984): Über die Arbeit mit lebensgeschichtlichen Erzählungen in einer nicht-westlichen Kultur. In: Kohli, M./Robert, G. (Hg.): Biographie und soziale Wirklichkeit. Neue Beiträge und Forschungsperspektiven. Stuttgart, S. $284-295$

Matthes, J. (1992): ,Zwischen` den Kulturen? In: Soziale Welt. Sonderband 1992, S. 3 - 9

McFadden, J./Moore, J. L. (2001): Intercultural Marriage and Intimacy: Beyond the Continental Divide. In: International Journal for the Advancement of Conselling 23/2001, S. $261-268$

Mecheril, P. (1994): Die Lebenssituation Anderer Deutscher. Eine Annäherung in dreizehn thematischen Schritten. In: Mecheril, P./Theo, T. (Hg.): Anderer Deutsche. Berlin, S. 57 - 93

Mecheril, P. (1997): Zugehörigkeitserfahrungen von Anderen Deutschen. Eine empirische Modellierung. In: Pries, L. (Hg.): Transnationale Migration. Soziale Welt Sonderband 12. Baden-Baden, S. $293-313$

Mecheril, P. (2000a): Anerkennung des Anderen als Leitperspektive interkultureller Pädagogik. Unter: http://www.forum-interkultur. net/fileadmin/user_upload/pdf/21.pdf\#search=\%22mecheril $\% 20$ aner kennung\%22 vom 21.08.2006

Mecheril, P. (2000b): Doppelte Heraussetzung und eine Utopie der Anerkennung. Mehrfachverbundenheit in natio-ethno-kultureller Pluralität. In: Jakobs, K./Wießmeier, B./Frieben-Blum, E. (Hg.): Wer ist fremd? Ethnische Herkunft, Familie und Gesellschaft. Opladen, S. $231-250$

Mecheril, P. (2000c): Prekäre Verhältnisse. Über natio-ethno-kulturelle (Mehrfach) Zugehörigkeit. Unveröff. Habilschrift. Bielefeld

Mecheril, P. (2003): Prekäre Verhältnisse. Über natio-ethno-kulturelle (Mehrfach) Zugehörigkeit. Münster

Mecheril, P. (2004): Einführung in die Migrationspädagogik. Weinheim/Basel

Mecheril, P. (2005): Pädagogik der Anerkennung. Eine programmatische Kritik. In: Hamburger, F./Badawia, T./Hummrich, M. (Hg.): Migration und Bildung. Über das Verhältnis von Anerkennung und Zumutung in der Einwanderungsgesellschaft. Wiesbaden, S. 3113 28 
Mecheril, P./Quehl, T. (2006): Sprache und Macht. Theoretische Facetten eines (migrations)pädagogischen Zusammenhangs. In: Mecheril, P./Quehl, T. (Hg.): Sprache und Macht. Englische Perspektiven auf die mehrsprachige Schule. Münster, S. 355 - 381

Menz, M. (2001): Schulalltag und Geschlecht. Eine empirische Studie zu Geschlechterkonstruktionen in der 5. Klasse. Göttinger Beiträge zur erziehungswissenschaftlichen Forschung. Göttingen

Menz, M./Putschbach, R. (2001): Unbegrenzter Lebensentwurf - begrenzte Lebensgestaltung. Mädchen zwischen Schule und Arbeitswelt. In: Niedersächsisches Modellprojekt, Mädchen in der Jugendarbeit (Hg.): Up to Date. Mädchenarbeit präsentiert sich. Hannover, S. $30-34$

Menz, M. (2007): Grenzverschiebungen? Formen biographischen Lernens in Biographien binationaler Paare. In: bildungsforschung, Jahrgang 4, Ausgabe 1 http://www.bildungsforschung.org/Archiv/200701/grenzverschiebungen

Merton, R. K. (1972 [1942]): Intermarriage and the social structure: fact and theory. In: Barron, M. L. (Hg.): The Blending American. Patterns of Intermarriage. Chicago, S. $12-35$

Mexiko Lexion 2006. Unter: http://mexiko-lexikon.de vom 10.5.2005

Molnár, C. (2004): Binationale Paare: Eine qualitative Studie zur Relevanz der ,Kulturdifferenz'. Radolfzell

Müller, M. (2003): Geschlecht und Ethnie. Historischer Bedeutungswandel, Interaktive Konstruktion und Interferenzen. Wiesbaden

Müller-Dincu, B. (1981): Gemischt-nationale Ehen zwischen deutschen Frauen und Ausländern in der Bundesrepublik: eine familiensoziologische Analyse ihrer Situation und Problematik. Wiesbaden

Nassehi, A. (1994): Die Form der Biographie. Theoretische Überlegungen zur Biographieforschung in methodologischer Absicht. In: bios $1 / 1994$, S. $46-63$

Nauck, B. (2001): Solidarpotenziale von Migrantenfamilien. Expertise für die Friedrich-Ebert-Stiftung. Gesprächskreis Migration und Integration. Chemnitz

Neidhardt, F. (1986): Kultur und Gesellschaft. Einige Anmerkungen zum Sonderheft. In: Kölner Zeitschrift für Soziologie und Sozialpsychologie, Sonderheft 1986, S. $10-18$

Neuendorff, H. (1998): Pluralisierung und Partikularisierung in der Moderne. In: Rapp, F. (Hg.): Globalisierung und kulturelle Identität. Bochum, S. $25-37$

Nohl, A.-M. (2005): Lernen und Biographie: Sammelrezension zu einem vernachlässigten Thema. In: Zeitschrift für qualitative Bildungs-, Beratungs- und Sozialforschung 1/2005, S. 163 - 171 
Oechsle, M. (1998): Ungelöste Widersprüche: Leitbilder für die Lebensführung junger Frauen. In: Oechsle, M./Geissler, B. (Hg.): Die ungleiche Gleichheit: Junge Frauen und der Wandel im Geschlechterverhältnis. S. $185-200$

Potuchek, J. L. (1992): Employed Wives‘ Orientation to Breadwinning: A Gender Theory Analysis. In: Journal of Marriage and the Family 3/1992, S. $548-558$

Pries, L. (1996): Transnationale Soziale Räume. Theoretisch-empirische Skizze am Beispiel der Arbeitswanderungen Mexiko-USA. In: Zeitschrift für Soziologie 6/1996, S. 456 - 472

Pries, L. (1997): Neue Migration im transnationalen Raum. In: Pries, L. (Hg.): Transnationale Migration. Soziale Welt Sonderband 12. Baden-Baden, S. $15-44$

Pusitz, H./Reif, E. (Hg.) (1996): Interkulturelle Partnerschaften. Begegnungen der Lebensformen und Geschlechter. Frankfurt a. Main

Refsing, K. (1998): Gender Identity and Gender Role Patterns in CrossCultural Marriages: The Japanese-Danish Case. In: Breger, R./Hill, R. (Hg.): Cross-Cultural Marriage. Identity and Choice. Oxford/New York, S. $193-208$

Richard, M. A. (1991): Ethnic Groups and Marital Choices. Ethnic History and Marital Assimilation in Canada, 1871 and 1971. Vancouver

Ricker, K. (2000): Migration, Sprache und Identität. Bremen

Risman, B. J./Johnson-Sumerford, D. (1998): Doing it fairly: a study of postgender marriages. In: Journal of Marriage and the Family 1/1998, S. $23-40$

Root, M. P. P. (2001): Love‘s Revolution. Interrracial Marriage. Philadelphia

Rosenthal, G. (1995): Erlebte und erzählte Lebensgeschichte. Gestalt und Struktur biographischer Selbstbeschreibungen. Frankfurt a. M.

Ruenkaew, P. (2003): Heirat nach Deutschland. Motive und Hintergründe thailändisch-deutscher Eheschließungen. Frankfurt a. Main

Sakamoto, I. (2006): Akkulturation or Negotiation? What Japanese academic migrants teach us about family processes and gendered experiences of cultural adaptation. In: Mahalingam, R. (Hg.): Cultural psychology of immigrants. New Jersey, S. $337-364$

Schäffer, B. (2003): Generationen - Medien - Bildung: Medienpraxiskulturen im Generationenvergleich. Opladen

Scheibelhofer, E. (2003): Migration und Integration. Frankfurt a. M.

Scheibler, P. (1992): Binationale Ehen. Zur Lebenssituation europäischer Paare in Deutschland. Weinheim

Scherr, A. (2000): Ethnisierung als Resource und Praxis. In: Prokla Zeitschrift für kritische Sozialwissenschaft 120/2000, S. 399 - 414 
Schily, Otto (1999): Vom christlichen Abendland zum multikulturellen Einwanderungsland? Unter: http://www.h-quandt-stiftung.de/root /index.php?lang=de\&page_id=229 vom 07.04.06

Schimank, U. (2002): Das zwiespältige Individuum. Zum Person - Gesellschaft - Arrangement der Moderne. Opladen

Schlehe, J. (2000a): Reiseromanzen. Beziehungsstrukturen zwischen westlichen Frauen und indonesischen Männern. In: Schlehe, J. (Hg.): Zwischen den Kulturen - zwischen den Geschlechtern. Kulturkontakte und Genderkonstrukte. Münster, S. 125 - 141

Schlehe, J. (Hg.) (2000b): Zwischen den Kulturen - zwischen den Geschlechtern. Kulturkontakte und Geschlechterkonstrukte. Münster

Schlehe, J. (2001a): Interkulturelle Geschlechterforschung. Identitäten Imaginationen - Repräsentationen. Frankfurt a. M.

Schlehe, J. (2001b): Lebenswege und Sichtweisen im Übergang. Zur Einführung in die interkulturelle Geschlechterforschung. In: Schlehe, J. (Hg.): Interkulturelle Geschlechterforschung. Identitäten - Imaginationen - Repräsentationen. Frankfurt a. M., S. 9 - 26

Schlüter, A. (1999): Bildungserfolge: eine Analyse der Wahrnehmungsund Deutungsmuster und der Mechanismen für Mobilität in Bildungsbiographien. Opladen

Schramm, K./Steuer (1965): Ehen zwischen deutschen und ausländischen Arbeitnehmern. Sozialkritische Erhebung aus dem Bereich eines Gesundheitsamtes. In: Der öffentliche Gesundheitsdienst. Monatsschrift für Gesundheit und Verwaltung und Sozialhygiene 12/1965, S. $487-493$

Schreyer, F. (1991): Weibliche familiale Arbeit und männliche Dauererwerbslosigkeit im Arbeitermilieu. ,...des wird hart für a Frau, wenn der Mo sei Stell verliert“. Beiträge zur Arbeitsmarkt- und Berufsforschung 149. Nürnberg

Schulze, H. (2002): When Interviewer and Interviewee speak different Languages. The method of biographical analysis for understanding something which cannot be understood at first sight. Unveröff. Manuskript. Kassel

Schulze, T. (1979): Autobiographie und Lebensgeschichte. In: Baacke, D./Schulze, T. (Hg.): Aus Geschichten Lernen. München, S. 51 - 98

Schulze, T. (1993a): Zum ersten Mal und immer wieder neu. Skizzen zu einem phänomenologischen Lernbegriff. In: Bauersfeld, H./Bromme, R. (Hg.): Bildung und Aufklärung. Studien zur Rationalität des Lehrens und Lernens. Festschrift für Helmut Skowronek. Münster/New York, S. $271-286$ 
Schulze, T. (1993b): Lebenslauf und Lebensgeschichte. In: Baake, D./ Schulze, T. (Hg.): Aus Geschichte lernen: zur Einübung pädagogischen Verstehens. Weinheim/München, S. $174-226$

Schulze, T. (1995): Erziehungswissenschaftliche Biographieforschung Anfänge - Fortschritte - Ausblicke. In: Krüger, H.-H./Marotzki, W. (Hg.): Erziehungswissenschaftliche Biographieforschung. Opladen, S. $10-31$

Schulze, T. (1999): Erziehungswissenschaftliche Biographieforschung. Anfänge - Fortschritte - Ausblicke. In: Krüger, H.-H./Marotzki, W. (Hg.): Handbuch erziehungswissenschaftliche Biographieforschung. II. Opladen, S. $33-55$

Schulze, T. (2001): Die außerordentliche Tatsache des Lernens. Jörg Schlee zum 60. Geburtstag. Hans-Joachim Oldenburger Universitätsreden Vorträge - Ansprachen - Aufsätze Nr. 132. Oldenburg

Schulze, T. (2005): Strukturen und Modalitäten biographischen Lernens. Eine Untersuchung am Beispiel der Autobiographie von Marc Chagall. In: Zeitschrift für qualitative Bildungs-, Beratungs- und Sozialforschung $1 / 2005$, S. $43-64$

Schütz, A. (1971): Gesammelte Aufsätze I. Das Problem der sozialen Wirklichkeit. Den Haag

Schütz, A. (2004 [1971]): Common-Sense und wissenschaftliche Interpretation menschlichen Handelns. In: Strübing, J./Schnettler, b. (Hg.): Methodologie interpretativer Sozialforschung. Klassische Grundlagentexte. Konstanz, S. 157 - 197

Schütze, F. (1977): Die Technik des narrativen Interviews in Interaktionsfeldstudien: dargestellt an einem Projekt zur Erforschung von kommunalen Machstrukturen (Manuskript). Bielefeld

Schütze, F. (1981): Prozessstrukturen des Lebensablaufs. In: Matthes, J./Pfeifenberger, A./Stoßberg, M. (Hg.): Biographie in handlungswissenschaftlicher Perspektive. Nürnberg, S. 67 - 129

Schütze, F. (1984): Kognitive Figuren des autobiographischen Stegreiferzählens [1]. In: Kohli, M./Robert, G. (Hg.): Biographie und sozial Wirklichkeit. Neue Beiträge und Forschungsperspektiven. Stuttgart, S. $78-114$

Schwingel, M. (1998): Pierre Bourdieu zur Einführung. 2. Auflage. Hamburg

Seitter, W. (1999): Riskante Übergänge in der Moderne. Vereinskulturen, Bildungsbiographien, Migranten. Opladen

Shimada, S. (2001): Wissenssoziologie der kulturellen Wechselwirkung. Eine Skizze zur Methodologie einer interkulturell angelegten Sozialforschung. In: Zeitschrift für qualitative Bildungs-, Beratungs- und Sozialforschung $1 / 2001$, S. $36-48$ 
Simmel, G. (1992 [1908]): Exkurs über den Fremden. In: Rammstedt, O. (Hg.): Georg Simmel. Soziologie. Untersuchungen über die Formen der Vergesellschaftung. Georg Simmel Gesamtausgabe Bd. 11. Frankfurt a. M., S. 764 - 771

Spivak, G. C. (1996 (1985)): Subaltern Studies. Deconstructing Historiography. In: Landry, D./MacLean, G. (Hg.): The Spivak Reader. Selected Works of Gayatri Chakravorty Spivak. New York, S. $203-$ 235

Stenger, H. (1998): Soziale und kulturelle Fremdheit. Zur Differenzierung von Fremdheitserfahrungen am Beispiel ostdeutscher Wissenschaftler. In: Zeitschrift für Soziologie 1/1998, S. 18 - 38

Straßburger, G. (2003): Heiratsverhalten und Partnerwahl im Einwanderungskontext. Eheschließungen der zweiten Migrantengeneration türkischer Herkunft. Busch, F. W. W., Hans-Joachim/Nauck, B./ Nave-Herz, R. Familie und Gesellschaft 10. Würzburg

Straub, J. (1999): Handlung, Interpretation, Kritik: Grundzüge einer textwissenschaftlichen Handlungs- und Kulturpsychologie. Berlin

Straub, J./Garz, D. ,et al. (2001): Begegnung mit dem Fremden. Einführung in den Themenschwerpunkt. In: Zeitschrift für qualitative Bildungs-, Beratungs- und Sozialforschung 1/2001, S. 3 - 13

Strauss, A. L./Corbin, J. (1996): Grounded Theory. Grundlagen qualitativer Sozialforschung. Weinheim

Streckeisen, U. (1993): Weiblicher Lebenslauf und eheliche Machtverhältnisse. Überlegungen aus ressourcentheoretischer Sicht. In: Born, C./Krüger, H. (Hg.): Erwerbsverläufe von Ehepartnern und die Modernisierung weiblicher Lebensläufe. Weinheim, S. 53 - 69

Sullivan, O. (2004): Changing Gender Practices Within the Household. A Theoretical Perspective. In: Gender and Society 2/2004, S. $207-$ 222

Thode-Ahora, H. (1999): Interethnische Ehen. Theoretische und methodische Grundlagen ihrer Erforschung. Berlin/Hamburg

Thon, C. (2003): Frauenbewegung - Bewegungsgenerationen - Generationenbruch? Generationenkonzepte in Diskursen der Frauenbewegung. In: Feministische Studien 1/2003, S. $111-122$

Thon, C. (2006): Frauenbewegung und intergenerationaler Wandel. Eine qualitativ-empirische Studie über Frauenbiographien aus drei Generationen. Unveröff. Doktorarbeit. Bielefeld.

Thorne, B. (1993): Gender Play. Girls and boys at school. Buckingham Tilgner, U. (Hg.) (1979): Umbruch im Iran. Augenzeugenberichte Analysen - Dokumente. Hamburg

Timm, A. (2004): Partnerwahl und Heiratsmuster in modernen Gesellschaften. Der Einfluss des Bildungssystems. Wiesbaden 
Treibel, A. (2003): Migration in modernen Gesellschaften. Soziale Folgen von Einwanderung, Gastarbeit und Flucht. Weinheim/München

Tseng, W.-S./McDermott, J. F. ,et al. (Hg.) (1984): Adjustment in intercultural marriage. Hawaii

Tyrell, H. (1986): Geschlechtliche Differenzierung und Geschlechterklassifikation. In: Kölner Zeitschrift für Soziologie und Sozialpsychologie 1986, S. 450 - 489

Uhl, E. (1991): Über den Zusammenhang zwischen männlicher Arbeitslosigkeit und Konfliktbereichen in der Partnerschaft. Eine empirische Untersuchung an langzeitarbeitslosen Männern und ihren Partnerinnen. Trier

Wagner, H.-J. (1995): Die Aktualität der strukturalen Bildungstheorie Humboldts. Weinheim

Wahler, P./Witzel, A. (1995): Berufswahl - ein Vermittlungsprozess zwischen Biographie und Chancenstruktur. In: Schober, K./Gaworek, M. (Hg.): Berufswahl: Sozialsations- und Selektionsprozesse an der ersten Schwelle. Nürnberg, S. 9 - 35

Waldis, B. (1998): Trotz der Differenz. Interkulturelle Kommunikation bei maghrebinisch-europäischen Paarbeziehungen in der Schweiz und in Tunesien. Münster

Waldis, B. (2001): Zur Analyse von geschlechtstypischen Migrationsstrategien bei binationalen Paaren. In: Schlehe, J. (Hg.): Interkulturelle Geschlechterforschung. Identitäten - Imaginationen - Repräsentationen. Frankfurt a. M., S. $132-152$

Waldis, B. (2003): Alles relativ? Kultur als Argumentationsressource in der Gestaltung binationaler Partnerschaften. In: Schweizerische Akademie der Geistes- und Sozialwissenschaften (Hg.): Muslime in der Schweiz. Bern, S. $97-104$

Waldis, B./Ossipow, L. (2003): Binationale Paare und multikulturelle Gesellschaften. In: Wicker, H.-R./Fibbi, R./Haug, W. (Hg.): Migration und die Schweiz. Ergebnisse des Nationalen Forschungsprogramms ,Migration und interkulturelle Beziehungen`. Zürich, S. 390 $-420$

Weigel, S. (1992): Literatur der Fremde - Literatur in der Fremde. In: Literatur, H. S. d. (Hg.): Gegenwartsliteratur seit 1968. 12. München, S. $182-229$

Weiß, A. (2001): Rassismus wider Willen: Ein anderer Blick auf eine Struktur sozialer Ungleichheit. Wiesbaden

Welsch, W. (1997): Transkulturalität. In: Universitas. Zeitschrift für interdisziplinäre Wissenschaft 1/1997, S. 16 - 24

West, C./Fenstermaker, S. (1995): Doing difference. In: Gender and Society $1 / 1995$, S. $8-37$ 
West, C./Zimmerman, D. (1991): Doing gender. In: Lorber, J./Farrell, S. (Hg.): The Social Construction of Gender. Newbury Park

Wetterer, A. (1995): Dekonstruktion und Alltagshandeln: Die (möglichen) Grenzen der Vergeschlechtlichung von Berufsarbeit. In: Dies. (Hrsg.) (1995): Die soziale Konstruktion von Geschlecht in Professionalisierungsprozessen. Frankfurt a.M., New York, S. 221-246

Wetterer, A. (2003): Rhetorische Modernisierung: Das Verschwinden der Ungleichheit aus dem zeitgenössischen Differenzwissen. In: Knapp, G.-A./Wetterer, A. (Hg.): Achsen der Differenz. Gesellschaftstheorie und feministische Kritik II. Münster, S. 286 - 319

Wimbauer, C. (2003): Geld und Liebe. Zur symbolischen Bedeutung von Geld in Paarbeziehungen. Frankfurt a. M./New York

Winders, R. M. (2000): Potuchek, Jean (1997): Who supports the family? Gender and Breadwinning in Dual-Earner-Marriages. Book review. In: NWSA 1/2000, S. 216 - 219

Witzel, A. (2000): Das problemzentrierte Interview (26 Absätze). In: Forum Qualitative Sozialforschung/Forum: Qualitative Social Research [Online Journal), 1(1). Unter: http://qualitative-research.net/fqs vom 10.5.2006

Witzel, A./Helling, V. ,et al. (1996): Die Statuspassage in den Beruf als Prozess der Reproduktion sozialer Ungleichheit. In: Bolder, A./ Heinz, W. R./Rodax, K. (Hg.): Jahrbuch Bildung und Arbeit: Die Wiederentdeckung der Ungleichheit. Aktuelle Tendenzen in Bildung für Arbeit. Opladen, S. 171 - 187

Wolf-Almanasreh, R. (1979): Die soziale Lage der mit Ausländern verheirateten deutschen Frauen in der Bundesrepublik. In: Evangelische Akademie Hofgeismar (Hg.): Ehen zwischen Ausländern und Deutschen. Hofgeismar, S. 3 - 35

Yildiz, E. (2004): Konstruktion des Anderen als ethnisch Fremder. In: Karakadoglu, Y./Lüddecke, J. (Hg.): Migrationsforschung und Interkulturelle Pädagogik. Aktuelle Entwicklungen in Theorie, Empirie und Praxis. Münster, S. 145 - 157

Zinn, J. (2001): Zwischen Gestaltungsanspruch und Strukturvorgaben. Junge Fachkräfte in den ersten Berufsjahren - Erwerbsverläufe, Handlungskontexte und biographische Gestaltungsmodi. Baden-Baden

Zvonkovic, A. M./Greaves, K. M. ,et al. (1996): The Marital Construction of Gender through Work and Family Decisions: A Qualitative Analysis. In: Journal of Marriage and the Family 1/1996, S. 91 - 100 


\section{Gender Studies}

Ursula Mihciyazgan

Der Irrtum

im Geschlecht

Eine Studie zu Subjektpositionen im westlichen und im muslimischen

Diskurs

Januar 2008, 288 Seiten,

kart., 29,80 €,

ISBN: 978-3-89942-815-5

\section{Katrin Oltmann}

\section{Remake | Premake}

Hollywoods romantische

Komödien und ihre

Gender-Diskurse, 1930-1960

Januar 2008, ca. 336 Seiten,

kart., ca. $29,80 €$,

ISBN: 978-3-89942-700-4

\section{Sylvia Pritsch}

\section{Rhetorik des Subjekts}

Zur textuellen Konstruktion des Subjekts in feministischen und anderen postmodernen

Diskursen

Januar 2008, 516 Seiten,

kart., 39,80 €,

ISBN: 978-3-89942-756-1

\section{Margarete Menz}

Biographische

Wechselwirkungen

Genderkonstruktionen und

»kulturelle Differenz"

in den Lebensentwürfen

binationaler Paare

Januar 2008, 312 Seiten,

kart., $29,80 €$,

ISBN: 978-3-89942-767-7

\author{
Rita Casale, \\ Barbara Rendtorff (Hg.) \\ Was kommt nach der \\ Genderforschung?
}

Zur Zukunft der feministischen

Theoriebildung

Dezember 2007, ca. 280 Seiten,

kart., ca. $27,80 €$,

ISBN: 978-3-89942-748-6

Ulrike Brunotte,

Rainer Herrn (Hg.)

Männlichkeiten und Moderne

Geschlecht in den

Wissenskulturen um 1900

Dezember 2007, 294 Seiten,

kart., $28,80 €$,

ISBN: 978-3-89942-707-3

Ute Frietsch,

Konstanze Hanitzsch,

Jennifer John,

Beatrice Michaelis (Hg.)

Geschlecht als Tabu

Orte, Dynamiken

und Funktionen

der De/Thematisierung

von Geschlecht

Dezember 2007, 270 Seiten,

kart., zahlr. farb. Abb., 25,80 €,

ISBN: 978-3-89942-713-4

Daniel Devoucoux

Mode im Film

Zur Kulturanthropologie

zweier Medien

November 2007, 350 Seiten,

kart., zahlr. Abb., $34,80 €$,

ISBN: 978-3-89942-813-1

Anette Dietrich

Weiße Weiblichkeiten

Konstruktionen von "Rasse« und Geschlecht im deutschen Kolonialismus

Oktober 2007, 430 Seiten,

kart., $29,80 €$,

ISBN: 978-3-89942-807-o

Leseproben und weitere Informationen finden Sie unter: www.transcript-verlag.de 


\section{Gender Studies}

Corinna Tomberger Das Gegendenkmal

Avantgardekunst, Geschichtspolitik und Geschlecht in der bundesdeutschen

Erinnerungskultur

September 2007, 362 Seiten, kart., zahlr. Abb., 34,80 €, ISBN: 978-3-89942-774-5

\section{Sven Glawion,}

Elahe Haschemi Yekani, Jana Husmann-Kastein (Hg.)

Erlöser

Figurationen männlicher Hegemonie

September 2007, 218 Seiten, kart., 24,80 €,

ISBN: $978-3-89942-733-2$

\section{Nadja Sennewald}

Alien Gender

Die Inszenierung von Geschlecht in ScienceFiction-Serien

August 2007, 314 Seiten, kart., zahlr. Abb., 29,80 €, ISBN: 978-3-89942-805-6

\section{Tanja Maier}

\section{Gender und Fernsehen}

Perspektiven einer kritischen Medienwissenschaft

August 2007, 280 Seiten, kart., $27,80 €$,

ISBN: 978-3-89942-689-2

\section{Marit Cremer}

\section{Fremdbestimmtes Leben}

Eine biographische Studie über Frauen in Tschetschenien August 2007, 204 Seiten, kart., $21,80 €$,

ISBN: 978-3-89942-630-4
Claudia C. Ebner

Kleidung verändert

Mode im Kreislauf der Kultur

Juli 2007, 170 Seiten,

kart., $20,80 €$,

ISBN: 978-3-89942-618-2

Marion Mangelsdorf

Wolfsprojektionen:

Wer säugt wen?

Von der Ankunft der Wölfe in der Technoscience

Juli 2007, 312 Seiten, kart., zahlr. Abb., 29,80 €, ISBN: 978-3-89942-735-6

Carmen Leicht-Scholten (Hg.) "Gender and Science"

Perspektiven in den Naturund Ingenieurwissenschaften Juli 2007, 188 Seiten,

kart., $21,80 €$,

ISBN: 978-3-89942-674-8

Hedwig Wagner

Die Prostituierte im Film Zum Verhältnis von Gender und Medium

Juli 2007, 324 Seiten,

kart., $29,80 €$,

ISBN: 978-3-89942-563-5

Ingrid Hotz-Davies, Schamma Schahadat (Hg.)

Ins Wort gesetzt, ins Bild gesetzt

Gender in Wissenschaft, Kunst und Literatur

Mai 2007, 310 Seiten,

kart., $30,80 €$,

ISBN: 978-3-89942-595-6

Leseproben und weitere Informationen finden Sie unter: www.transcript-verlag.de 


\section{Gender Studies}

Lutz Hieber, Paula-Irene Villa Images von Gewicht

Soziale Bewegungen,

Queer Theory und Kunst

in den USA

Mai 2007, 262 Seiten,

kart., 26,80 €,

ISBN: 978-3-89942-504-8

Marion Hövelmeyer

Pandoras Büchse

Konfigurationen von Körper

und Kreativität.

Dekonstruktionsanalysen

zur Art-Brut-Künstlerin

Ursula Schultze-Bluhm

April 2007, 284 Seiten,

kart., zahlr. Abb., $30,80 €$,

ISBN: 978-3-89942-633-5

Bettina Bock von Wülfingen

Genetisierung der Zeugung

Eine Diskurs- und

Metaphernanalyse

reproduktionsgenetischer

Zukünfte

Februar 2007, 374 Seiten,

kart., 30,80 €,

ISBN: 978-3-89942-579-6

Jürgen Martschukat,

Olaf Stieglitz (Hg.)

Väter, Soldaten, Liebhaber

Männer und Männlichkeiten

in der Geschichte Nordamerikas.

Ein Reader

Februar 2007, 432 Seiten,

kart., $32,80 €$,

ISBN: 978-3-89942-664-9

Roswitha Muttenthaler,

Regina Wonisch

Gesten des Zeigens

Zur Repräsentation von Gender

und Race in Ausstellungen

Januar 2007, 268 Seiten,

kart., zahlr. Abb., 26,80€,

ISBN: 978-3-89942-580-2

Leseproben und weitere Informationen finden Sie unter: www.transcript-verlag.de 